\title{
Report to the American Physical Society by the study group on nuclear fuel cycles and waste management ${ }^{\star}$
}

Utilization of nuclear fuels and management of nuclear wastes have become major topics of public discussion. Under the auspices of the American Physical Society this study was undertaken as an independent evaluation of technical issues in the use of fissionable materials in nuclear fuel cycles, together with their principal economic, environmental, health and safety implications. Reprocessing and recycling in light water reactors were examined, along with technical measures proposed as possible safeguards; advanced reactor fuel cycles were also studied for their resource and safeguards implications. Much of the work of the group centered on the principal alternatives for disposal of radioactive wastes and control of effluents. The group examined the research and development programs sponsored by government agencies along with associated relationships among agencies and between government and private industry. Available information was also considered on nuclear fuel resources, and on important economic and environmental aspects of the various fuel cycles in order to strive for a balanced comparative study. The report presents many conclusions on various aspects of the nuclear fuel cycles and also provides recommendations concerning present utilization and future improvement of fuel cycle technology.

APS Study Group Participants

L. Charles Hebel, Chairman

Xerox Corporation, Palo Alto, California 94304

Eldon L. Christensen

Los Alamos Scientific Laboratory, University of California, Los Alamos, New Mexico 87545

Fred A. Donath

University of Illinois, Urbana, Illinois 61801

Warren E. Falconer

Bell Telephone Laboratories, Murray Hill, New Jersey 07974

Leon J. Lidofsky

Columbia University, New York, New York 10027

Ernest J. Moniz

Massachusetts Institute of Technology, Cambridge, Massachussetts 02139

Thomas H. Moss

Staff, U.S.House of Representatives

and (formerly, IBM Research and Columbia University)

Robert L. Pigford

University of Delaware, Newark, Deleware 19711

Thomas H. Pigford

University of California, Berkeley, Califormia 94720

Gene I. Rochlin

University of California, Berkeley, Calfornia 94720

Robert H. Silsbee

Cornell University, Ithaca, New York 14850

McDonald E. Wrenn

New York University Medical Center, Tuxedo, New York 10987

APS Council Review Committee

Hans Frauenfelder, Chairman

University of Illinois, Urbana, Illinois 61801

Theodore L.Cairns

E. I. duPont de Nemours, Wilmington, Delware 19898

W. K. H. Panofsky

Stanford Linear Accelerator Center, Stanford California 94305

M. Gene Simmons

Massachusetts Institute of Technology, Cambridge, Massachusetts 02139

* Supported by the National Science Foundation. 
Rev. Mod. Phys., Vol. 50, No. 1, Part II, January 1978 
CONTENTS

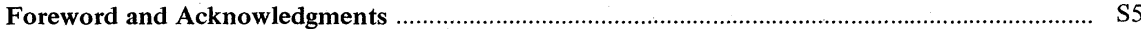

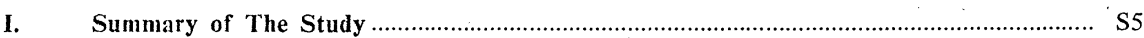

A. Scope and Approach $\quad$ S5

B. Principal Conclusions $\quad$ S5

$\begin{array}{ll}\text { C. Principal Recommendations } & \text { S7 }\end{array}$

$\begin{array}{ll}\text { D. Specific Questions and Answers } & \text { S7 }\end{array}$

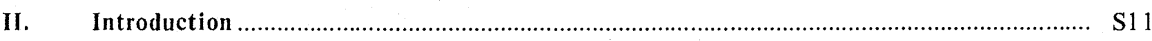

A. Background of the Study $\quad$ S11

B. Organization of the Report $\quad$ S12

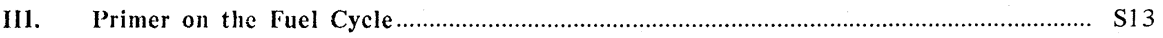

A. Nuclear Reactor Fundamentals $\quad$ S13

B. The LWR Fuel Cycle--A Description of Options $\quad$ S21

C. Radiation Exposures and Biological Effects on Humans $\quad$ S24

$\begin{array}{ll}\text { D. Fuel Cycle Safeguards and Security } & \text { S27 }\end{array}$

E. LWR Fuel Cycle--Organizations and Interactions $\quad$ S30

IV. LWR Fuel Cycle--Technology and Economics of Reprocessing and Recycle................. S35

A. Issues, Conclusions and Recommendations $\quad$ S35

$\begin{array}{ll}\text { B. Perspective on the Technology } & \text { S37 }\end{array}$

C. The Chemical Reprocessing of Spent Fuel $\quad$ S37

D. Commercial Reprocessing Plants $\quad$ S42

E. The Cost of Commercial Fuel Reprocessing $\quad$ S47

F. Mixed Oxide Fuel Fabrication $\quad$ S53

G. Fuel Cycle Economics and Resource Utilization $\quad$ S56

V. LWR Fuel Cycle--Assessment of Radiation Exposures............................................... S71

A. Issues, Conclusions and Recommendations $\quad$ S71

B. Assessment of Risk Associated with Radiation Exposure $\quad$ S72

C. Environmental, Biological, and Dosimetric Aspects of Specific Radionuclides $\quad$ S76

D. Exposure and Source Terms for Specific Fuel Cycle Facilities $\quad$ S77

E. Summary of Occupational and Public Expsoure from the Fuel Cycle $\quad$ S85

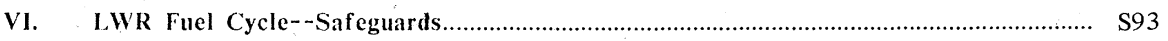

A. Issues, Conclusions and Recommendations $\quad$ S93

B. Perspective on Nuclear Safeguards $\quad$ S94

C. Physical Security Measures as Safeguards Barriers $\quad$ S96

D. Technical Measures for Safeguards Barriers $\quad$ S97

E. International Safeguards -- "Denatured" LWR Fuel Cycles S101

VII. High-Level and TRU Waste Management ................................................................... S107

A. Issues, Conclusions and Recommendations $\quad$ S107

B. Alternate Fuel Cycles and Waste Management $\quad$ S109

C. Currently Available HLW Management Technologies $\quad$ S112

D. Alternative Options of Waste Isolation $\quad$ S114

E. Potential Modes of Breaching Geologic Containment $\quad$ S119

$\begin{array}{ll}\text { F. Transport to the Biosphere } & \text { S120 }\end{array}$

$\begin{array}{ll}\text { G. Improved Wasteforms } & \text { S128 }\end{array}$

H. ERDA High-Level Waste Management Program $\quad$ S132

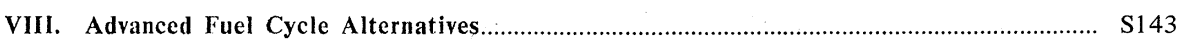

A. Issues, Conclusions and Recommendations $\quad$ S143

B. LWR--Thorium and Advanced LWR Cycles $\quad$ S144

C. CANDU and Advanced Heavy Water Reactor Cycles $\quad$ S147

D. High Temperature Gas Reactor (HTGR) $\quad$ S149

E. Fast Breeder Fuel Cycles $\quad$ S151

F. Technical Safeguards Features of Advanced Fuel Cycles . $\quad$ S154

IX. Institutional and Organizational Issues of the Nuclear Fuel Cycle and Waste

$\begin{array}{ll}\text { A. Issues and and Conclusions } & \text { S159 }\end{array}$

B. Historical Evolution of Civilian Nuclear Development and Regulation $\quad$ S160

C. Current Status of Jurisdiction at Interfaces in Nuclear Devleopment and S161

D. Advantages \& Problems of the Current Framework of Dispersed Responsibility S163

E. Implications for Fuel Cycle Decisions $\quad$ S163

$\begin{array}{ll}\text { F. Organization of the Fuel Cycle Management } & \text { S104 }\end{array}$ 
Appendices .

$\mathrm{S} 165$

A1. The Fuel Cycle as a Source of Waste

$\mathrm{S} 165$

AlI. Reprocessing of Thorium-containing Spent Fuel from HTGRs

S169

Alll. Actinides and Thorium Cycles

$\mathrm{S} 171$

AIV. Dose Commitment and Information on Individual Nuclides $\quad$ S173

AV. Start-up of Fast Breeders

S179

AVI. Protactinium as a Safeguards Issue

List of Abbreviations..... 


\section{FOREWORD AND ACKNOWLEDGMENTS}

Use of nuclear fuels and the management of nuclear wastes have become major topics of public discussion as part of broadened public consideration of all aspects of commercial nuclear power. Until recently, plans for completion of the nuclear fuel cycle emphasized reprocessing of spent reactor fuel, recycling the recovered plutonium and unused fissionable uranium, and disposing of the high-level and transuranic nuclear waste in terminal geologic storage to isolate the waste from the biosphere. The public discussion now has widened to question not only the technical feasibility and potential benefit of such plans, but also the viability of the safeguards that might be necessary to achieve secure domestic operation as well as negotiations and institutions needed to control international proliferation of nuclear weapons.

This study was undertaken by a study group for the American Physical Society as an independent evaluation of technical issues in the use of fissionable materials in nuclear fuel cycles. The effort is another step by the Society to extend its contribution beyond its traditional activities to address an important and timely subject that involves substantial scientific and technological content. The study group participants were selected under the auspices of the Society to provide a wide range of scientific and engineering training and viewpoints. Whereas a few of the group had some background in aspects of the fuel cycle, the majority had not previously considered these issues. Several had participated in other technical assessments of broad national issues; for several others, this study was their first such experience. In view of this diversity, it is noteworthy that a high degree of consensus was achieved within the group concerning both conclusions and recommendations.

The work of the study group was monitored and the technical content of their report to the Society was reviewed by a Review Committee established by and reporting to the APS Council. The report was also examined with respect to its public release by the APS Panel on Public Affairs. Both groups have recommended release and publication of the study group report by the Society.

The group met at Los Alamos throughout the month of July 1976, and also held approximately a dozen three-day meetings and conducted much correspondence during the past year to complete their work. The hospitality of the Los Alamos Scientific Laboratory and the administrative assistance of the American Physical Society are gratefully acknowledged. The study was supported by the National Science Foundation.

The cooperation of the nuclear fuel cycle community, and of experts in related fields such as biological effects of radiation, was outstanding; we acknowledge the help of many experts, including both proponents and opponents of nuclear power, who supplied us with information and presented briefings. The individuals involved are too numerous to mention by name. We also appreciated the cooperation which we received from many major U.S. laboratories and from the staffs of the Energy Research and Development Administration and the Nuclear Regulatory Commission. It is fair to say that we were not denied access to any information which we felt was important for our task.

\section{CHAPTER I. Summary of the Study}

\section{A. Scope and Approach}

This study has been carried out under the auspices of the American Physical Society and provides an independent evaluation of the technical issues in nuclear fuel cycles and waste management, together with their principal economic, environmental, health, and safety 'implications. The topics and emphasis were chosen by the study group as it proceeded. Technology for fuel reprocessing and for plutonium and uranium recycling was examined, along with costs and fuel cycle economics. Technical measures proposed as safeguards were also evaluated for their potential in preventing misuse of the materials. Emphasis was given to light water reactors; but attention was also devoted to fuel cycles for some advanced reactors having potential resource or safeguards significance, especially, fast breeder advanced converter, and so-called "denatured" fuel cycles. Much of the work centered on the principal alternatives for storage and disposal of radioactive wastes--in particular, high-level and transuranic wastes, and tailings from uranium mills. Occupational exposures in fuel cycle facilities and public health effects from fuel cycle effluents were studied. The group also-examined the research and development programs sponsored by government agencies, along with associated relationships among agencies and between government and private industry.

Many important issues were addressed in the course of the study, including the following:

--How safely can nuclear waste be managed and effluents be controlled? Is the technology ready for these tasks, or is more development needed? What are the potential environmental and health effects? Is the choice of fuel cycle an important consideration for effective waste management; in particular, is reprocessing a necessity? Are government programs focused on the critical issues and making timely progress?

- - Is the technology ready for reprocessing options and for recycle? Can they be practiced reliably at full industrial scale? Is recycle economic? How long can we expect uranium resources to support LWR fuel cycles with and without recycle? Can technical measures contribute to a safeguarded LWR fuel cycle if plutonium is recycled?

--Are advanced converters and improved L.WR's significant options for future resource utilization? How important is the fast breeder relative to advanced converter options for the longterm? Do advanced fuel cycles present any special features that can influence domestic or international safeguards? Is reprocessing technology for advanced cycles keeping pace with advanced reactor development?

The effort centered on normal fuel cycle operation and did not reexamine reactor accidents; thus it is complementary to the previous study of light water reactor safety carried out for the American Physical Society (APS, 1970) Overall, the study group looked for features and differences among various fuel cycle assumptions that could influence choice of waste management alternatives, improve use of nuclear fuel resources, or influence effective implementation of safeguards. The study group did not address the complex political and institutional considerations necessary for complete evaluation of fuel cycles on a national or international scale. Neither did they examine nuclear versus non-nuclear energy alternatives. The group felt that, as members of the technical community, they could make their most valuable contribution by independent evaluation of the present technical foundation of nuclear fuel cycles as well as prospects for future improvement.

\section{B. Principal Conclusions}

Detailed conclusions are presented in the first section of each chapter. We summarize here our overall conclusions and the main conclusions of each principal topic area.

\section{B1. Overall Conclusions}

For all LWR fuel cycle options, safe and reliable management of nuclear waste and control of radioactive effluents can be accomplished with technologies that either exist or involve straightforward extension 
of existing capabilities. However, technical choices, incl:rding those for geologic waste disposal, require further delineation of regulatory policies. For normal operation of all fuel cycle options studied, potential radiation exposures from either wastes or effluents do not appear to limit deployment of nuclear power.

The decision to reprocess nuclear fuel does not depend significantly on waste management considerations but rather on resource and economic incentives and on international and domestic safeguards constraints. The technology for LWR reprocessing-recycle options is well advanced. However, the present recycle economics are uncertain and depend on technical, regulatory, and policy choices. As uranium- ore availability becomes an important future constraint, advanced reactors, including breeders, could provide more effective options than do present LWR's; such options require reprocessing.

Safeguarding the fuel cycle raises important unresolved institutional and political issues. Pending their resolution, recoverable storage of spent fuel rods (stowaway) offers a fuel cycle alternative requiring minimal safeguards and preserving energy- resources; safe interim stowaway measures exist, and geologic stowaway could be safely continued indefinitely. If permitted by resolution of safeguards issues, reliable industrial-scale operation could be attained for reprocessing and refabrication for recycle in LWR's or for use in advanced reactors, with safe isolation of high-level and transuranic waste in geologic repositories.

B2. Management of Nuclear Wastes and Radiation Exposure, and Control of Effluents

Federal regulations and standards are not yet complete concerning required solidification, processing, transport, and subsequent storage or isolation of high-level and transuranic wastes. Encapsulated spent fuel can be an acceptable wasteform. The technology exists for recoverable storage of the spent fuel with minimal deterioration to preserve the associated resources, but full-scale implementation is required. Where reprocessing is employed, solidification of liquid high-level waste is currently a required step. Technology is well developed to immobilize such waste in borosilicate glass cast in stainless steel canisters for handling and transport. Present understanding is not sufficient to rely upon this wasteform, in itself, as a principal long-term barrier to release.

Effective long-term isolation for spent fuel, high-level or transuranic waste can be achieved by geologic emplacement. A waste repository can be developed in accord with appropriate site selection criteria that would ensure low probability that erosion, volcanism, meteorite impact and other natural events could breach the repository. The possibility of inadvertent human intrusion also can be made remote and limited in consequences. Hydrogeologic transport is the most important mechanism for potential transfer of radionuclides from a geologic repository to the biosphere. We conclude that many waste repository sites with satisfactory hydrogeology can be identified in continental U.S., in a variety of geological formations. Bedded salt, proposed for the first repository in current ERDA plans, can be a satisfactory medium for a repository, but certain other rock types, notably granite and possibly shale, could offer even greater long-term advantages. Irrespective of the time scale adopted for reprocessing, two geologic test facilities in different media should be completed. As a possibly superior disposal option for the future, rock melting concepts are attractive, and techniques associated with superdeep drilling could provide an effective means of waste emplacement.

The dominant fuel cycle gaseous effluents that affect long-term public exposure are ${ }^{14} \mathrm{C},{ }^{85} \mathrm{Kr}$ and, to a lesser extent, tritium and ${ }^{129} \mathrm{I}$. Even as the number of facilities becomes large, the increment to background dose rate is small and can be reduced even further by suitably engineered controls. More attention should be given to the control of collective occupational dose, which exceeds collective public dose. We find no evidence to justify major reductions in plutonium inhalation concentration limits, proposed with the "hot particle" hypothesis. For long-term waste management, the hazard associated with radium is more significant than that for plutonium. In addition, for regional population exposure, radionuclides in uranium mill tailings are potentially at least as important as the actinide chain elements in high-level waste; the relative accessibility of mill tailings contrasts with the isolation proposed for other actinide-containing wastes.

\section{B3. Reprocessing, Refabrication and Recycle}

An essentially complete technology is at hand for industrial-scale chemical reprocessing of present LWR fuel. The chemistry and engineering are well understood and have been tested in several plants. Mixed-oxide fuel fabrication also has been demonstrated at the level of a pilot-scale batch process. Subsequent reprocessing of mixedoxide fuel requires and is receiving further work. Reliable operation of an industrial-scale reprocessing-refabrication system has not yet been attained and represents an important step early in industrial deployment. Operation of the industrial-scale reprocessingrefabrication system would yield a much broader understanding of the operating features and a firmer design base for future facilties.

Recycle would provide significant reduction in ore requirements, but resource considerations alone for LWR fueling provide little urgency to begin industrial-scale reprocessing within the next decade. At this time various uncertainties preclude an unequivocal assessment of the possible economic benefits of recycle options. Sensitivity analysis of the fuel cycle cost-benefits indicates that the dominant uncertainty is the cost of reprocessing and the second most important uncertainty is the future price of uranium ore. The reference case defined in Chapter IV indicates a net benefit, of order $9 \%$ of fuel cycle cost or $1 \%$ of the net cost of nuclear electric energy; other reasonable cases lead to an increased benefit or even a loss. None of these cases include the cost of safeguards.

Another important reprocessing consideration is provided by the fast breeder program, which is predicated on start-up with plutonium from LWR reprocessing. Breeder commercialization in 1993 would require industrial-scale reprocessing of LWR fuel by the late 1980's. If breeder commercialization were delayed or start-up based on other resources, present estimates of uranium ore resources and projections of LWR growth indicate that industrial-scale reprocessing would not be necessary for resource extension until near the end of this century. LWR reprocessing experience provides useful background for the reprocessing required for advanced fuel cycles, but breeder fuel and thorium fuel reprocessing and refabrication need further development before industrial-scale operations can be undertaken.

\section{B4. Fuel Cycle Safeguards}

Technical measures for safeguards, by themselves, do not provide adequate protection against theft or diversion of fissile material, but such measures can play an important role in complementing and reinforcing necessary conventional physical security measures. For this purpose real-time accountability systems are attractive and merit further development. Coprecipitation of plutonium and uranium at the reprocessing plant is technically feasible, albeit more costly, and would have the important safeguards advantage that plutonium never would appear separately in the entire fuel cycle. Effectiveness of either domestic or international safeguards depends as much on political agreements and choice of institutions as it does on physical security and technical safeguards measures. We regard long-term and short-term considerations as equally important; design for long-term effectiveness of safeguards appears to be the more difficult task.

We have considered isotopically denatured fuel cycles involving uranium and thorium cycles with denatured reactors located at "national sites" and with plutonium-burning reactors, reprocessing and enrichment restricted to "international sites". Such cycles might contribute to safeguards by diminishing the threat of sub-national theft and by impeding the use of fuel cycle facilities and materials for weapons production. Even so, nuclear weapons capability can be attained independent of the spread of fuel cycle facilities. Denatured uranium cycles, with low-enriched uranium in national reactors, provide near term alternatives. Plutonium inevitably is present in the spent fuel; denatured thorium-uranium cycles reduce such plutonium production by about a factor of seven and may have long-term safeguards benefits. However, thorium cycle costs are uncertain; in addition, potential safeguards problems are introduced by the use of highly enriched uranium make-up fuel and in the use of ${ }^{233} U-T h$ fuel which can be enriched to weapons grade with comparatively little effort. The nature of the necessary institutional and political safeguards arrangements are not substantially different from those required for denatured uranium fuel cycles. 


\section{B5. Advanced Fuel Cycles}

For the long term, significant resource extension could be obtained from several advanced reactors utilizing mixed-oxide fuels with uranium and thorium cycles. Fast breeders are the most resource efficient of all options. Breeders can be started with $\mathrm{Pu},{ }^{233} \mathrm{U}$ and ${ }^{235} \mathrm{U}$; $\mathrm{Pu}$ start-up is much more economic than ${ }^{235} \mathrm{U}$ start-up; ${ }^{233} \mathrm{U}$ is intermediate but supplies are not available. Advanced heavy water reactors seem to be the next most resource efficient. Such options require development and would become competitive as uranium ore costs rise.

Shorter term advantages are possible through improved LWR's, especially with thorium. Significantly improved uranium resource utilization can be achieved thereby in LWR's with higher conversion ratios in the 0.7 to 0.8 range. However, versions with even higher conversion ratios, such as the light water breeder reactor, have no short-term advantage primarily because of the long time required to achieve a net resource saving.

\section{Principal Recommendations}

Our detailed recommendations are presented at the beginning of each chapter. We summarize a few of the major ones here, according to each main topic area.

\section{C1. Management of Wastes and Effluents}

a) High priority should be given to timely completion of Federal regulations and standards, including the required data base, concerning appropriate wasteforms, transport, and subsequent storage or isolation of high-level and transuranic wastes and spent fuel elements.

b) Waste management programs should complete and implement the technology for recoverable interim storage, either surface or geologic, as well as provide for long-term geologic isolation for spent reactor fuel.

c) Whatever time scale is adopted regarding a decision on reprocessing, two test facilities for geologic isolation should be developed in different media. The program should include hydrogeologic measurement and modeling, as well as geological exploration. Upon completion of two satisfactory test facilities, procedures should be initiated for licensing of the more favorable of the two. When the needs for storage dictate, that facility can be expanded into a full repository.

d) Criteria for selection of a waste repository site should include specifications of appropriate hydrogeologic parameters that must be satisfied by present-day hydrogeology and by the projected bounds of the future hydrogeology of any specific site.

e) The waste solidification program should complete the implementation of present technology for the treatment of high-level wastes and continue to develop advanced wasteforms. Transuranic waste deserves comparable attention, especially with regard to compaction and wasteform suitable for geologic disposal. The goals of such programs, especially regarding immobilization, should be defined more clearly and quantitatively.

f) We urgc active research on rock melting concepts as a possible future alternative for waste disposal. The technology should be evaluated for emplacement of waste canisters using superdeep drilling.

g). We support re-examination of the criteria and practices for management of uranium mill tailings, to make their treatment consistent with that for other actinide wastes.

h) The major sources of collective occupational dose in the entire fuel cycle should be identified, and design effort should be directed to dose minimization. Regulatory guides should be developed for collective occupational dose in future facilities.

i) Technology should be completed for control and sequestering of ${ }^{14} \mathrm{C},{ }^{85} \mathrm{Kr}$, and ${ }^{129} \mathrm{I}$, and eventually ${ }^{3} \mathrm{H}$; steps should be taken toward development of international agreements for the degree and timing of control.

\section{C2. Reprocessing, Refabrication and Recycle}

a) If reprocessing is to be a major component of the U.S. nuclear industry in the near future, we recommend that appropriate existing reprocessing facilities be completed and operated to gain experience with integrated technology on industrial scale; further, the corresponding refabrication facility should be built and operated with a similar goal. We emphasize that resolution of the issues involved in the GESMO decision and in international fuel cycle safeguards strongly influence the timing of such operation.

b) The technology and engineering of the dissolution and separations steps needed to reprocess fuels for advanced fuel cycle should be carried forward to a state of readiness sufficient for future engineering scale-up.

\section{C3. Fuel Cycle Safeguards}

a) We urge evaluation of safeguards fuel cycles using lowenrichment uranium fuel, especially the economic, institutional and technical arrangemeints associated with co-located reprocessing, refabrication, and plutonium-fueled reactors.

b) Real-time nondestructive assay-accountability systems should be developed further for possible use for control of special nuclear materials in industrial-scale reprocessing and refabrication plants. Design criteria should be set, and costs involved in meeting these criteria should be evaluated.

c) The economic impact on the fuel cycle of coprecipitating to a fixed $\mathrm{Pu} / \mathrm{U}$ ratio should be evaluated.

\section{C4. Advanced Fuel Cycles}

a) We urge evaluation of advanced heavy water thermal reactors and also of improved light water reactors with higher conversion ratio, as candidates for development in addition to the already existing program to develop fast breeder reactor technology.

b) The development programs for future reactors should include their associated reprocessing-refabrication technology and should emphasize providing options, so that future commercialization can be chosen from several nuclear fission power alternatives to fit circumstances now too uncertain to assess accurately.

\section{Specific Questions and Answers}

1. Should there be a moratorium placed on the construction and licensing of new reactors in view of uncertainties about how to dispose of nuclear waste?

We see no need for such a moratorium. We are confident that long-term isolation could be effective for either spent fuel or the high-level and transuranic waste, and that there are no important technical barriers to the development of a repository on a pilot plant scale by 1985 . The corresponding regulatory and licensing basis is not yet available, but we see no reason why it cannot be completed in an orderly way.

2. What about high-level waste from the Nuclear Fuel Services plant in New York State?

The bulk of the high-level waste from the NFS plant operations were neutralized and stored on the site in large tanks. Their chemical form is quite similar to military program wastes stored at the Hanford and Savannah River military plants and is very different from that of the future commercial practices examined by the APS group. We did not study the neutralized wastes in detail, nor did we examine the range of options that might be available for their disposal. Our examination was sufficient to satisfy us that management of future commercial high-level waste would not be limited by the major waste disposal problems encountered at the NFS site.

3. What is the value of a full-scale demonstration of high-level waste disposal?

The technology for waste solidification and for constructing and operating a geologic nuclear waste repository has been developed to a 
point where a test facility can be carried out. A test facility would provide checks on short-term stability of wasteforms and encapsulation, operational experience with waste handling equipment, and initial verification of the predicted effects of the emplaced waste on the immediate geologic environment. Such a facility also could be used for retrievable storage of spent fuel.

The demonstration by itself cannot establish the long-term viability of the site for a waste repository. Confidence would be based on a combination of measurements and calculations of which considerations of the groundwater hydrology and pathway's to man are paramount. Some additional factors, e.g. anthropogenic concerns, will be evaluated in the course of the licensing procedures. We believe that the need for storage should dictate whether a test facility should be developed into a full repository.

\section{What does the APS Study Group think of the ERDA plan for dealing with high-level waste?}

We are confident of the ability to develop and deploy the technology to treat and dispose of high-level and transuranic waste from reprocessing plants. We are concerned that insufficient attention may be given to procedures to store spent fuel in a fully retrievable fashion in the event of an extended deferral of reprocessing. Technologies exist for such a "stowaway" cycle but need full-scale implementation.

We recommend emplacement of high-level and transuranic wastes in a geologic repository. If reprocessing were to be deferred for the long term, we recommend that provision be made for the storage of spent fuel in a geologic repository. We expect that many waste repository sites with satisfactory hydrogeology can be found in continental U.S. in a variety of geological formations, including bedded salt. We consider it important that two satisfactory test facilities, as contrasted with full repositories, be developed at the earliest possible time consistent with an adequate data base. We believe that bedded salt may be a satisfactory medium for a repository but that granite, and possibly shale, could provide even greater longterm advantages. Thus, we consider it important to evaluate fully other geologic formations for repository sites in addition to salt. Current projections of waste accumulation would allow for the orderly and timely development of a second test facility in a medium other than salt. We favor an orderly program involving hydrogeologic measurements and modeling followed by development of test facilities. Two sites, developed in different geologic media, represent a minimum number for adequate demonstration of acceptability of different geologies.

5. Is reprocessing required to avoid accumulation of spent reactor fuel and have orderly disposal of wastes?

Reprocessing is not an essential step in the management of nuclear wastes but rather a means of extending fuel resources. Safe interim measures exist for spent fuel storage. If longer-term measures are needed, the design and operation of facilities for the recoverable geologic storage of spent fuel are of comparable difficulty to that for geologic isolation of solidified waste from reprocessing plants. Reprocessed high-level waste has lower actinide content than spent fuel, but miscellaneous transuranic waste is created as a result of reprocessing and refabrication; on balance the two waste disposal situations are comparable.

\section{Does the APS Study Group favor an industrial-scale demonstration of reprocessing?}

Operation of a reprocessing plant makes sense to us primarily in the context of industrial deployment to utilize the reprocessed material. Operation of such a plant would yield valuable information on costs, plant reliability, occupational and public radiation exposures, and achievable security levels. However, operation without intent to use the reprocessed material is not warranted. We emphasize that resolution of the issues involved in the $\mathrm{GESMO}^{+}$decision and in international fuel cycle safeguards strongly influence the timing of such operation.
7. How long will U.S. uranium supplies permit nuclear power to continue if reprocessing and use of plutonium are not permitted.

Projections of uranium ore supply for the long-term are one of the most uncertain aspects of nuclear power. Present ERDA estimates of present reserves and potential uranium resources up to $\$ 30 / \mathrm{lb}$, approximately 3.6 million short tons, would supply the lifetime ore commitment of at least 500 LWR's without recycle. ERDA's 1977 projections of LWR growth indicate that such a deployment would be reached between 2000 and 2010 A.D., and those reactors would remain in service for their 30 year life. We are unaware of dependable information concerning how much uranium ore may be available in the U.S. at prices up to the several hundred dollars per pound which would still permit further LWR use or growth.

We strongly support efforts to improve our knowledge of available future uranium supply. We also recommend serious evaluations of improved LWR's and other options to stretch our resources as far as possible.

8. Does the APS Study Group think that fuel cycle safeguards are likely to be effective enough to permit use of plutonium?

In the absence of a GESMO ${ }^{+}$Supplement on Safeguards, we did not attempt a comprehensive evaluation, but centered on possible contributions of technical measures. If safeguards for plutonium use were strictly a U.S. domestic matter, we think it possible that effective security could be imposed throughout the fuel cycle by a combination of technical and conventional physical security measures. Nevertheless, an overall assessment of plutonium utilization, including its international ramifications for weapons proliferation, involves far broader evaluation than we have made.

9. Even given a GESMO ${ }^{+}$resolution permitting commercial use of plutonium, does the APS Study Group think that we should defer its recycle in LWR's in favor of more efficient future use in advanced converters or fast breeders?

Under such circumstances one need not be concerned about using some of the LWR plutonium for recycle. There would be sufficient plutonium available from LWR's at a later time for fast breeder startup.

10. What does the APS Study Group think of the U.S. program to develop the liquid metal fast breeder?

We have not examined the reactor safety issues of the liquid metal fast breeder, which comprises a complex study in itself. We do analyze the role of several fast breeder fuel cycle alternatives and find fast breeders to be the most resource efficient of all the long-term nuclear fission alternatives. To realize the benefits of fast breeders, resolution of the reactor safety, the fuel cycle safeguards and the plutonium utilization issues must be accomplished.

\section{Should the U.S. develop an advanced converter reactor?}

Advanced converters would not be necessary for resource conservation in this country if the fast breeder were to be commercialized in this century. Therefore, development of advanced converters should proceed with the near-term goal of identifying welldefined options which can be selected for more intensive development, should there be a significant deferral in commercialization of the fast breeder and/or a significant shortage in uranium resources. Candidates for development are improved LWR-thorium reactors, the HTGR, and the plutonium-thorium fueled heavy water reactors. Cooperation with the longer-range Canadian heavy water reactor development program would be mutually beneficial and should include the examination of U.S. licensing criteria for such reactors. Development of advanced converter alternatives should include the development, at the pilot-plant scale, of associated reprocessing and refabrication operations.

${ }^{+}$NUREG-0002, Final GESMO (Generic Environmental Statement on Mixed Oxide Fuels). 
12. What does the APS Group use as a standard of comparison in evaluating potential public health effects from the fuel cycle?

We have examined potential doses in terms of (1) comparison with natural background, (2) the calculation of health effects by linear dose response techniques, and (3) by examination of various hazard indices. The typical hazard indices have limited usefulness. The second approach is a well known technique but runs into difficulty because of the uncertainty in the frequency of occurrence of health effects at very low radiation levels and because of the necessity to make asșumptions about future generations.

We find that the most meaningful comparisons are with natural background, primarily because one can compare the increment to generalized background which would be associated with any assumed magnitude and time scale of the nuclear power industry. This is particularly useful with respect to natural fluctuations in background which occur in a typical person's lifetime or which occur over the same time scale as increments from the fuel cycle.

13. Do the present regulations need to be tightened to reduce gaseous radioactive effluents and occupational exposures in existing plants?

There are recent EPA regulations (40CFR190) limiting gaseous effluents. We see no need for a further tightening of regulations prior to formulation of international agreements governing the extent and timing of controls. Reduction of effluent releases may become desirable in the future if the number of facilities grows very large. For example, even without additional controls on gaseous effluents, it would take 500 years of normal operation of 600 reactors and associated reprocessing plants to produce increments in average global whole body dose rates that reach even $0.1 \%$ of that from natural background. With feasible controls in the future, such doses could be made much smaller if desired.

The collective occupational dose from fuel cycle facilities exceeds the collective public dose, and occupational dose occurs at dose rates typically much higher than background. Of all aspects of the fuel cycle, improvements here may be most effective in reducing potential biological effects. Regulatory guides should be developed for future collective occupational exposure limits, to guide design and operation of future reactors and reprocessing plants.

14. How do different parts of the fuel cycle contribute to potential public radiation exposures? What parts are most significant?

The parts of the fuel cycle that contribute the most to public radiation exposure are fuel reprocessing, uranium mining and milling. For normal operations, we expect reprocessing plants to contribute more than reactors to average global whole body dose commitment; even as the number of such facilities becomes large, the increment to background dose rate is small and can be reduced even further by suitably engineered controls. The long-term potential for national public radiation exposure from uranium milling and mining is estimated to be less than that from reactor reprocessing facilities. Accessibility of uranium mill tailings, as handled in the past, appears undesirable from the point of view of local and regional exposure. Accordingly, procedures and criteria for handling mill tailings need to be reexamined. For high-level and transuranic wastes, we are confident that geologic waste repositories can be effective in isolating such wastes from the biosphere with little risk of public radiation exposure. 


\section{CHAPTER II. Introduction}

\section{A. Background of the Study}

Until recently the near term focus of U.S. nuclear power has been the demonstration of the safety, technical adequacy and economic viability of the use of slightly enriched uranium fuels in commercial light-water reactors (LWR). In the longer term both government and nuclear industry have been considering a fuel cycle that would utilize plutonium which is created as a by-product of the normal LWR operation. Industry has planned to recycle such plutonium to augment uranium resources in LWRs. Many people also have recognized the potential of plutonium to fuel the liquid metal fast breeder reactor (LMFBR) which is being developed to extend the nation's energy resources.

The options for a future breeder reactor give an important perspective on the fuel cycle. Early work on nuclear reactors had indicated that one might transform abundant uranium and thorium isotopes into useful nuclear reactor fuels by either of two breeding cycles: ${ }^{238} \mathrm{U}$ producing ${ }^{239} \mathrm{Pu}$, or ${ }^{232} \mathrm{Th}$ producing fissile ${ }^{233} \mathrm{U}$. In principle, a long term source of nuclear energy could be based on either cycle. However, the first cycle, producing plutonium, was found to have a significantly higher ratio of nuclear fuel produced to fuel consumed. Thus, R and D efforts in the U.S. and in several foreign countries turned toward development of special fast neutron reactors like the LMFBR to breed the plutonium and open the way to a plutonium-powered economy with fuel enough to extend indefinitely in to the future.

The long range plans anticipated commercial utilization of mixed oxide fuels in LWRs by the 1980's. The AEC began to lay the technical groundwork at Hanford, Washington in the late 1950's. During the 1960's additional experiments were conducted in several existing reactors in the U.S. and in many countries overseas to demonstrate the viability of the use of mixed oxides in commercial reactors. Of course to obtain the plutonium one must reprocess the spent nuclear fuel. The Purex chemical extraction process for uranium and plutonium had been worked out by the AEC to supply material for its weapons programs. In the early 1960's the first commercial plants were built and operated in the U.S. by Nuclear Fuel Services at West Valley New York, and in Belgium by Eurochemic, in order to separate uranium and plutonium for recycle. Larger reprocessing plants with improved fuel handling technology were built in the early 1970's at Barnwell, South Carolina, and at La Hague, France. Effort was also under way to obtain a more accurate assessment of the overall fuel cycle which ultimately must include treatment and permanent storage or disposal of nuclear waste.

However, events of the past few years have changed the situation considerably. Both reactor technology development and plans for its utilization received growing public attention in the 1960's and early 1970 's. Many people were convinced of the critical need for a strong nuclear component in the U.S. power picture and publically advocated its rapid adoption. Other members of the public were skeptical of the overall value of nuclear power. While public participation in the 1960 's was of ten limited by availability of public information, passage of the Freedom of Information Act and the National Environmental Policy Act of 1970 (NEPA) did a great deal to open up the debate. A number of individuals and groups raised questions about the safety of power reactors themselves. By the mid 1970's public attention broadened to include the whole back end of the nuclear fuel cycle-spent fuel storage, reprocessing, recycling and waste disposal.

The plutonium recycle controversy came to a head with public release of the AEC's August 1974 draft of the Generic Environmental Statement on Mixed Oxide Fuels (GESMO), which was required under the procedures of NEPA. Plutonium has been the subject of a great deal of study since its discovery in World War II. Plutonium health hazards to lung tissue and to bone marrow are extensively documented, and international standards (e.g., for occupational exposures) have been developed to regulate the nuclear industry, albeit with some debate remaining on specific points.

The main public concern over plutonium centers on its possible contribution to the international proliferation of nuclear weapons as well as on its potential attraction to terrorists or criminal organizations. One potential reason for theft would be the commercial value of the element. But the biggest fear arises from its potential use as a weapon that could have enormous impact. There is the well publicized risk of construction of a nuclear bomb by a determined group, were they to gain access to sufficient quantities of plutonium. Even conventional explosives could disseminate plutonium into the atmosphere, where it could pose both acute and long-term radiological hazards to those who inhaled the air-borne particles. Thus, utilization of plutonium in commercial reactors requires. both domestic and international safeguards and poses dilemmas for society which were recognized long ago by the nuclear community itself. Similar considerations arise with highly enriched uranium considered for some advanced reactors.

The original GESMO draft was criticized as emphasizing the economic benefits of plutonium recycle while incompletely evaluating environmental, health and safety risks and, especially, the safeguards issues. The controversy was settled by the Federal Council on Environmental Quality which reviewed the draft and related commentary as a part of NEPA proceedings. The Council returned the GESMO draft to the Nuclear Regulatory Commission with instructions to evaluate the environmental impact in more detail, especially the feasibility of plutonium safeguards as well as their economic, social, legal and institutional impact.

In addition to the plutonium recycle issues, public attention has focused on the radioactive waste, either the spent fuel rods themselves or high-level waste, which arises from nuclear fuel reprocessing. The U.S. weapons programs have already generated a significant amount of high-level nuclear waste stored in tanks at government facilities near Hanford, Washington, Idaho Falls, Idaho and Savannah River, Georgia. Similar waste from commercial reactors is comparatively much smaller in amount; the commercial waste is projected to grow to equal volume by the mid to late 1990's with an order of magnitude greater specific activity. Nuclear waste contains both fission products and long-lived transuranic elements created by the nuclear reaction, and both must be isolated until the various radioactive isotopes have decayed to insignificant levels. For fission products this requires a period of perhaps six hundred years; some of the actinides have sufficiently long half-lives that hundreds of thousands of years may be required for adequate isolation from the biosphere. While there are theoretical possibilities for transforming these elements into less long-lived forms, such a process poses great technical difficulties as well as occupational hazards.

Thus, all solutions proposed in the near-term focus on geological isolation of nuclear wastes. Since the time scales for waste disposal transcend man's experience and the viability of his social and governmental institutions, it is clear that institutional as well as technical aspects of waste management require very careful attention. Well-institutionalized long term procedures must be put in place not only for transuranic and high-level waste but also for low-level waste, now placed in burial grounds, and tailings from the uranium milling operations, along with decommissioning of fuel cycle facilities at end of life.

These problems and their prospective solutions are at the center of attention in the federal government. Following their creation in 1974 the Nuclear Regulatory Commission (NRC) and the Energy Research and Development Administration (ERDA) broadened and accelerated their technical and administrative reviews of the wide range of fuel cycle issues. The NRC undertook extensive studies of nuclear energy centers, of nuclear security forces and of safeguards and overall safety consideration for plutonium recycle in order to guide policy decision. The NRC has released recently a revision of the GESMO dealing with the health, safety and environment issues. As of this writing a special supplement dealing with the safeguards issues has yet to be presented to the public. The NRC had expected to conduct reviews and reach a decision on the use of mixed oxide fuels in 1977. However, policy statements in late October 1976, by the Winite House raised the possibility of a ban on reprocessing until a better international basis can be worked out to avoid the risks of weapon proliferation. This policy has developed further under the new administration. 
ERDA has also conducted a review of the whole fuel cycle and broader aspects of nuclear waste management, published as a Task Force Report (ERDA-33) in March 1975. The ERDA Task Force focused attention on the many unresolved issues in the fuel cycle and urged a vigorous program to demonstrate the technical feasibility and acceptability of recycle technology and waste management alternatives. The October White House policy statement put additional emphasis on a demonstration of high-level waste management and established a time table for licensed operation of a federal high-level waste repository by 1985 .

It is our intent to carry out a careful assessment of the principal technical features of fuel cycle alternatives and of the factors entering into evaluation of the risks. We recognize that the hazards and risks posed by widespread commercial use of nuclear materials raises questions of a social, political and ethical nature that transcend physical science and technology. ${ }^{+}$Nevertheless, we hope that an independent assessment of the technical issues and their implications, by a broadly constituted technical group, could be a useful contribution to public debate on the nuclear fuel cycle as well as to the members of government who are responsible for the conduct of research and development programs. It is with this spirit that we present the information contained in this report.

\section{B. Organization of the Report}

The bulk of our conclusions and recommendations are found in the main text of the report and are collected at the beginning of each Chapter. A summary of the more important appear in Chapter I. The background and organization of the study are contained in Chapter II. Chapter III of the report provides a primer on the fuel cycle and relevant issues--technical and institutional. In the first sections, III-A and $\mathrm{B}$, we provide background on the various parts of the fuel cycle. In III-C we review the basic aspects of the biological effects of radiation. Fuel cycle safeguards are introduced in Section III-D, and the private and governmental bodies involved in development, operation and regulation of the fuel cycle are discussed in Section III-

The main review and critique of the LWR uranium-plutonium fuel cycle is found in Chapters IV through VII. Chapter IV examines the technology for reprocessing and refabrication for LWR cycles in comparison with that for advanced alternatives; LWR fuel cycle economics and recycle are also analyzed there. Chapter $V$ provides an evaluation of potential public and occupational radiation exposures for various L.WR fuel cycles including both plant effluents and mill tailings. Chapter VI treats L.WR fuel cycle safeguards centering on technical measures but reviewing important aspects of physical safeguards measures for context. Chapter VII provides the main discussion of alternatives for management of high-level and transuranic waste, including FRDA and NRC plans. Our analysis and critique of advanced fuel cycle alternatives is contained in Chapter VIII and centers on improved LWR's, advanced converters and the fast breeder as well as "denatured" cycles proposed for safeguard purposes. Finally, Chapter IX provides an evaluation of fuel cycle institutional responsibilities and relationships, including a critique of their coordination and its effect on fuel cycle management. Following the report are Appendices and a list of commonly used abbreviations.

${ }^{+}$For a broader discussion of the nature of safety and the dilemmas that must be faced in evaluating benefits and risks, we refer the reader to: Lowrance, W. W., Of Acceptable Risk, (William Kaufmann, 1976).

Rev. Mod. Phys., Vol. 50, No. 1, Part II, January 1978 


\section{Chapter III. Primer on the Nuclear Fuel Cycle}

\section{A1. Nuclear Reactor Fundamentals--Physical Principles}

In nuclear fission power reactors, the source of energy is the fission of uranium or plutonium into two lighter elements, several neutrons, and a spectrum of beta particles, neutrinos, and gamma radiation. The rest mass of the products is less than that of the initial reactants, and the most of the mass loss appears as released energy. A typical fission reaction is illustrated by

$$
\mathrm{n}+{ }^{235} \mathrm{U}-\cdots>^{236} \mathrm{U}-\cdots{ }^{147} \mathrm{La}+{ }^{87} \mathrm{Br}+2^{1} \mathrm{n}
$$

There are a large number of possible fission reactions through which the intermediate excited state of ${ }^{236} \mathrm{U}$ proceeds. Figure $3 \mathrm{~A}-1$ provides a diagram of the fission yield, that is, the percentage of fissions that result in fission products of a given mass number. Most of the energy released in the reaction resides in the kinetic energy of the fission products. As shown in Table $3 \mathrm{~A}-1$, the fissioning of one atom of ${ }^{235} \mathrm{U}$ leads to the release of about $200 \mathrm{MeV}$ of heat; in more practical units, complete fissioning of $1 \mathrm{~g}$ of ${ }^{235} \mathrm{U}$ releases about 1 megawatt-day of thermal energy.

In order to sustain a chain reaction, one of the neutrons emitted in the fission must cause another fission before it is captured by some nonfission process or leaks out of the reactor core. The number of neutrons emitted in a fission is given in Table $3 \mathrm{~A}-2$. The average energy of the emitted neutrons is about $2 \mathrm{MeV}$, spread out over the spectrum shown in Figure 3A-2. Figure $3 \mathrm{~A}-3$ shows the fission cross section for ${ }^{235} \mathrm{U}$ and ${ }^{238} \mathrm{U}$ as a function of neutron energy. For ${ }^{235} \mathrm{U}$ the fission cross section for neutrons in thermal equilibrium with the medium $(\leq 0.1 \mathrm{eV})$ is more than an order of magnitude higher than for fast neutrons. With such thermal neutrons, the fission cross section for ${ }^{238} \mathrm{U}$ is zero, whereas the fast neutron fission cross section is comparable to that for ${ }^{235} \mathrm{U}$ above the ${ }^{238} \mathrm{U}$ fission threshold. ${ }^{233} \mathrm{U}$ also has suitable thermal neutron cross sections for fission; although it is very scarce in nature, it can be produced in reactors.

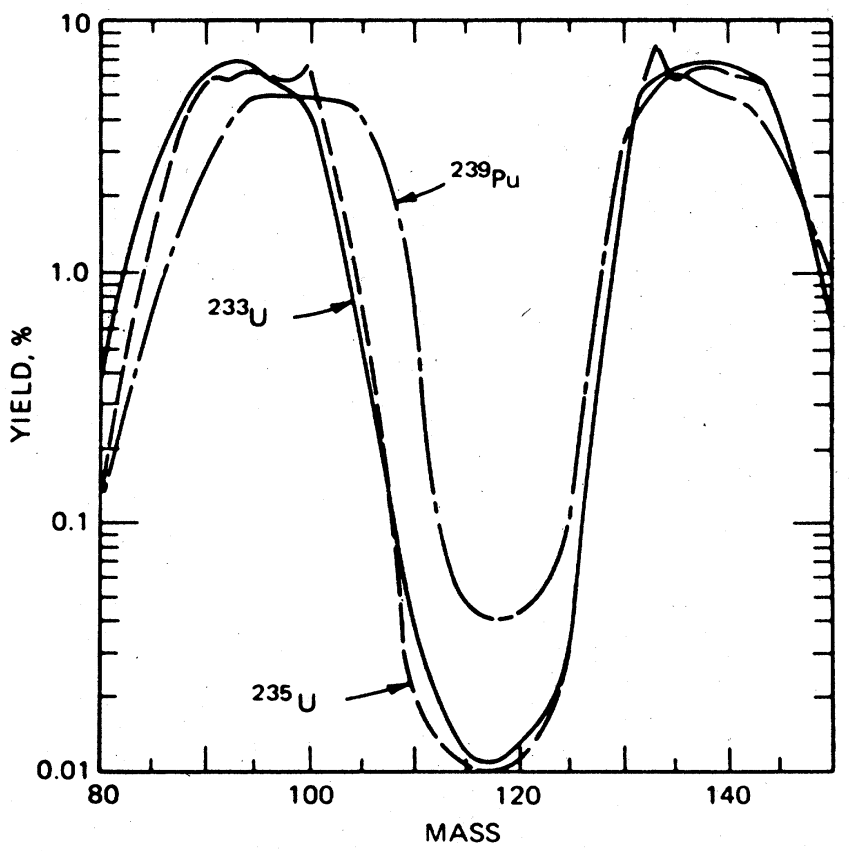

FIG. 3A-1. Mass-yield curves for thermal neutron fission of ${ }^{233} \mathrm{U},{ }^{235} \mathrm{U}$, and ${ }^{239} \mathrm{Pu}$.
To take advantage of the increased fission cross section at thermal energies, present-day commercial reactors contain a moderating material, chosen to slow down neutrons to thermal energy through elastic collisions, while at the same time having a small cross section for neutron absorption. The principal moderators are water, heavy water, beryllium, beryllium oxide, and graphite. Any neutron moderator will be characterized by an average logarithmic energy loss for the neutron per collision with a moderating nucleus. The lighter the moderator nucleus, the greater the energy loss, and the fewer coltisions needed to slow the neutron to thermal energies. When this logarithmic energy decrement is multiplied by the scattering cross section and divided by the cross section for neutron absorption, the result is a dimensionless figure of merit called the moderating ratio. The moderating ratio for several moderators is shown in Table $3 \mathrm{~A}-3$. The first reactor built at Chicago in the early 1940's used graphite. Canadian power reactors typically use heavy water; power reactors in the U.S. use ordinary light water (hence the terminology light-water reactor, LWR).

Control of the reaction is made possible by the existence of "delayed" neutrons that come from fission products or their daughters. Since these have relatively long half-lives for neutron decay, ranging from a few tenths of seconds to several seconds, the control sensing mechanism can anticipate the neutron flux in time to react. The ratio of delayed neutron yield to prompt thermal fission neutrons is about 0.0065 for ${ }^{235} \mathrm{U}, 0.0026$ for ${ }^{233} \mathrm{U}$, and 0.0021 for ${ }^{239} \mathrm{Pu}$. As a result, control systems for reactors using the latter two isotopes must be designed somewhat differently than those for present reactors.

Natural uranium consists of about $0.715 \%$ of thermally fissile ${ }^{235} \mathrm{U}$ and $99.285 \%$ of ${ }^{238} \mathrm{U} .{ }^{233} \mathrm{U}$ is a rare isotope in nature. The more abundant ${ }^{238} \mathrm{U}$ has several resonances in its neutron absorption cross section that rapidly reduce the number of neutrons passing through those energy regions while being thermalized. Unless a moderator with unusually low absorption cross section is used (such as heavy water in the Canadian power reactors), it is necessary to enrich the ${ }^{235} \mathrm{U}$ content of the fuel. In present day LWR's uranium fuel is enriched to about $3 \%{ }^{235} \mathrm{U}$ content by means of the gaseous diffusion process. The iuel is spatially separated from the water moderator, allowing the fission neutrons to escape into the moderator and slow down, with a smaller chance of absorption, before diffusing back to the fuel. As the reactor is operated, fissile ${ }^{239} \mathrm{Pu}$ is produced by neutron absorption in ${ }^{238} \mathrm{U}$; that is,

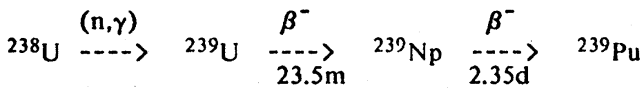

TABLE 3A-1. End products and energies from fission of ${ }^{235} \mathrm{U}$ (from Bennett, 1973).

\begin{tabular}{lc}
\hline End-product & $\begin{array}{c}\text { Emitted Energy } \\
(\mathrm{MeV})\end{array}$ \\
\hline Fission products & 168 \\
Fission neutrons & 5 \\
Prompt $\gamma$ radiation & 7 \\
Fission product decay & \\
$\beta$ radiation & 8 \\
nradiation & 7 \\
Capture $\gamma$ radiation & 12 \\
Total & $\underline{5}$ \\
\hline \hline
\end{tabular}


TABLE 3A-2. The number of neutrons emitted per fission (from Bennett, 1973).

\begin{tabular}{lll}
\hline Isotope & $\begin{array}{c}\text { Incident } \\
\text { neutron energy }\end{array}$ & $\begin{array}{c}\text { Number of neutrons } \\
\text { emitted per fission }\end{array}$ \\
\hline${ }^{235} \mathrm{U}$ & $0.025 \mathrm{ev}$ & 2.44 \\
& $1 \mathrm{MeV}$ & 2.50 \\
${ }^{239} \mathrm{Pu}$ & $0.025 \mathrm{eV}$ & 2.87 \\
& $1 \mathrm{MeV}$ & 3.02 \\
${ }^{233} \mathrm{U}$ & $0.025 \mathrm{eV}$ & 2.48 \\
& $1 \mathrm{MeV}$ & 2.55 \\
${ }^{232} \mathrm{Th}$ & $1.5 \mathrm{MeV}$ & 2.12 \\
${ }^{238 \mathrm{U}}$ & $1.1 \mathrm{MeV}$ & 2.46 \\
\hline \hline
\end{tabular}

The ${ }^{239} \mathrm{Pu}$ so produced is also fissionable with thermal neutrons and therefore represents an important energy source in the thermal reactor as its concentration builds up during the life of the fuel. ${ }^{239} \mathrm{Pu}$ has the highest number of neutrons emitted per fission of all the isotopes listed in Table $3 \mathrm{~A}-2$, whether in a fast or thermal neutron reactor However, in the very tight neutron economy of a reactor core, it is important to consider the correction due to neutron captures in the fuel. Taking $\nu$ to be the number of neutrons emitted per fission, and $\alpha$ to be the ratio of neutron captures to fissions, the average fission neutron yield per neutron absorbed, $\eta$, is given by

$$
\eta=\nu /(1+\alpha)
$$

which is a better index of fuel performance. Figure $3 A-4$ plots $\eta$ as a function of neutron energy for ${ }^{233} \mathrm{U},{ }^{235} \mathrm{U}$, and ${ }^{239} \mathrm{Pu}$. At thermai energies, ${ }^{2.33} \mathrm{U}$ is the most favored, with $\eta=2.28$.

Note also that only ${ }^{233} \mathrm{U}$ has an $\eta$ greater than 2.0 over most of the energy range. From the point of view of neutronics, ${ }^{233} \mathrm{U}$ would be the first choice for a thermal reactor. However, given the natural abundances of uranium isotopes, the ${ }^{235} \mathrm{U}$ cycle had to be developed first, and it was simple to then turn to ${ }^{239} \mathrm{Pu}$ bred from the fertile ${ }^{238} \mathrm{U}$. (Equation 2). Nevertheless, it is also possible to breed ${ }^{233} \mathrm{U}$ by neutron absorption in naturally abundant and fertile ${ }^{232} \mathrm{Th}$; that is,

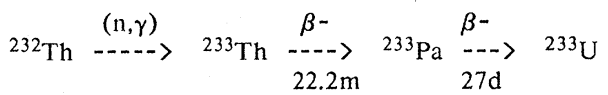

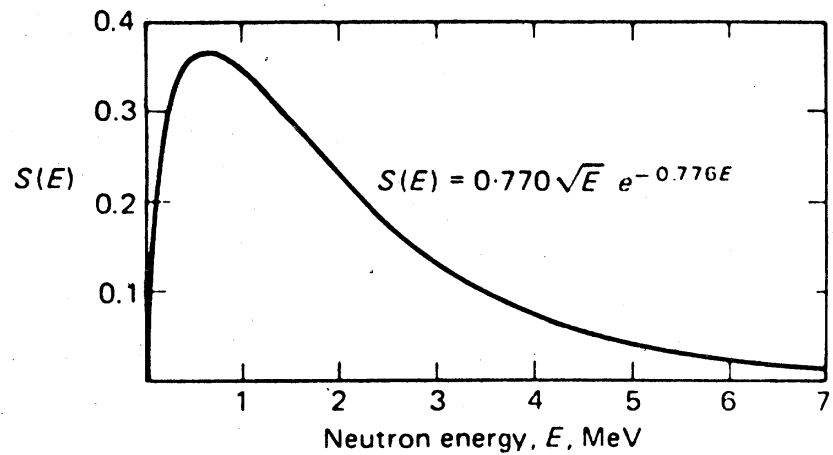

FIG. 3A-2. The fission neutron energy spectrum for ${ }^{235} \mathrm{U}$ (Bennett, 1973).

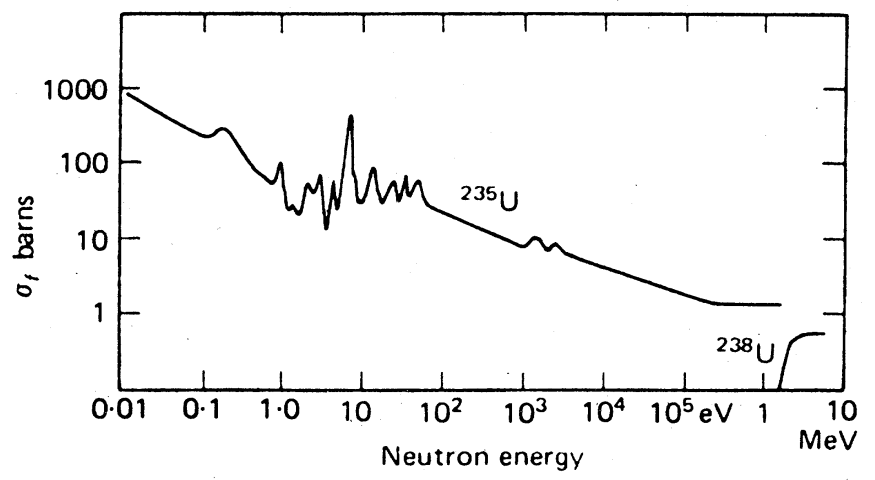

FIG. 3A-3. Fission cross-section of ${ }^{235} \mathrm{U}$ and ${ }^{238} \mathrm{U}$ (Bennett, 1973).

Because of the properties of ${ }^{233} \mathrm{U}$, the virtues of thorium as the fertile material in thermal reactors has been discussed by many investigators over the years. This cycle was chosen for use in the High Temperature Gas Reactor (HTGR), developed by General Atomic, as a possible advanced thermal reactor having a greater uranium ore resource efficiency than existing LWRs. The use of thorium can also improve the resource utilization of either light water or heavy water reactors. (See Section III-A2 for a description and Chapter VIII for an evaluation.) The thorium cycle has even been considered for a thermal breeder reactor, one that could produce a net amount of fissile material $\left({ }^{233} \mathrm{U}\right)$ with a thermal neutron spectrum. However, this type of breeder does not have the potential for as high a breeding ratio as do those operating on a fast neutron spectrum.

The production of new fissile material, by neutron absorption and the resultant conversion of fertile to fissile material in the core, is an important source of energy during the residence of the fuel in the reactor. The additional fissile isotope production per unit fissile isotope fission is expressed by the conversion ratio (CR), where

$\mathrm{CR}=\eta \varepsilon-1-$ losses.

The losses include not only neutrons leaking out of the core without capture or fission, but also the absorption of neutrons by the moderator, by the reactor components and cladding, and by the fission products and actinides produced in the fuel during operation; the neutron required to sustain the reaction is also subtracted. The factor $\varepsilon$ is the ratio of total fissions to fissile fissions; it is inserted to take into account the fission of fertile isotopes at higher neutron energies. Thus $\mathrm{CR}$ is an important index of the net efficiency of use of fissile material in the reactor.

At thermal neutron energies, $\boldsymbol{\varepsilon}$ is very close to 1 . For present commerical LWR's, CR is usually about 0.6 , while for other present types such as the CANDU (deuterium moderated) and the HTGR (graphite moderated) it is somewhat higher. Reactors with CR less

TABLE 3A-3. Moderating ratio of several moderators (from Glasstone and Sesonske, 1963).

\begin{tabular}{ll}
\hline Moderator & Moderating Ratio \\
\hline Water & 58 \\
Heavy Water & 21000 \\
Heliuma & 45 \\
Beryllium & 130 \\
Graphite & 200 \\
\hline \hline
\end{tabular}

a At atmospheric pressure and temperature. 


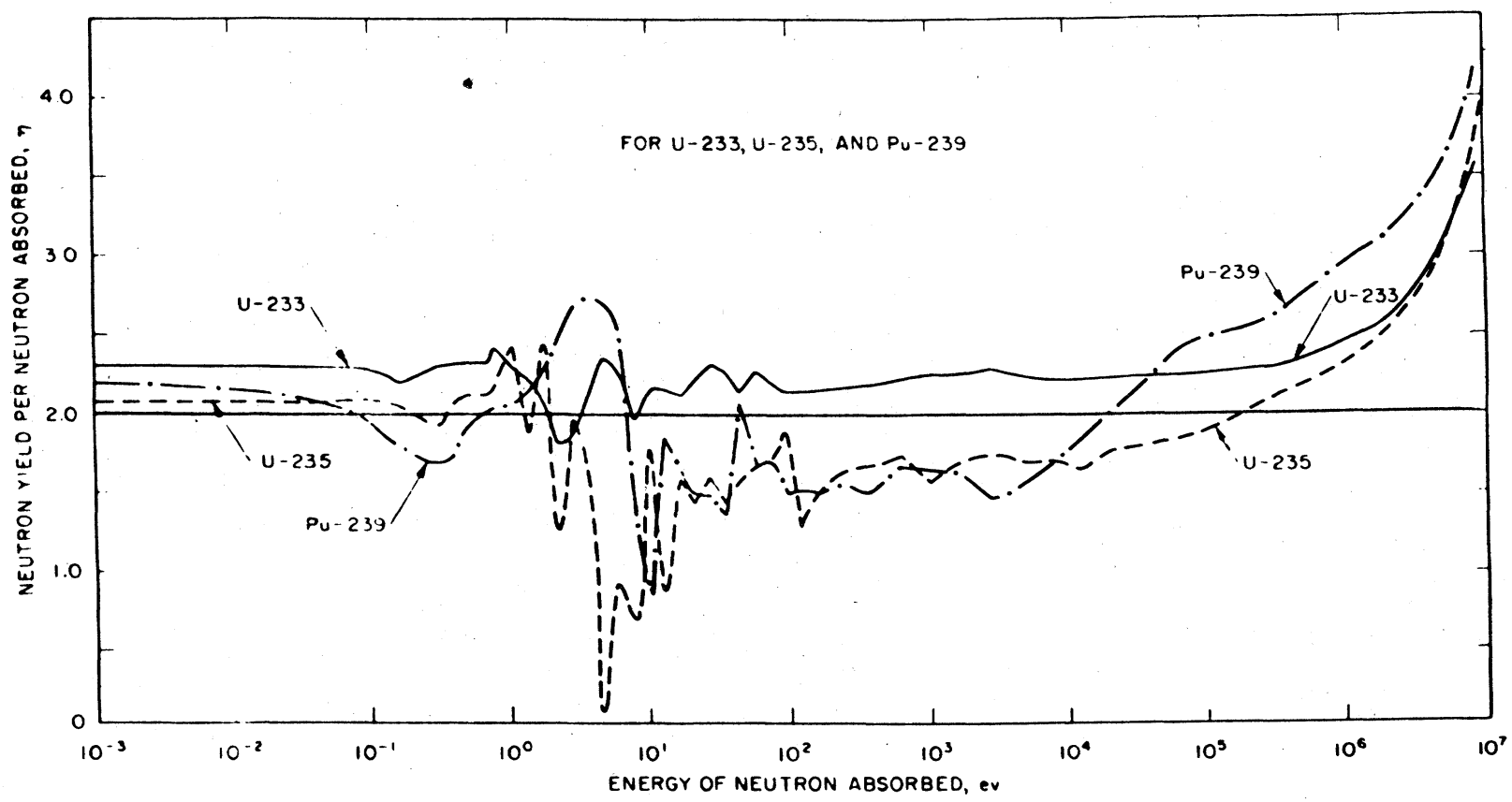

FIG. 3A-4. Neutron yield $(\eta)$ per neutron absorbed for ${ }^{233} \mathrm{U},{ }^{235} \mathrm{U}$, and ${ }^{239} \mathrm{Pu}$ (from ERDA-1541).

than 1 are usually referred to as converter or "burner" reactors, since there is a net consumption of fissile material during operation.

Reactors with a CR greater than 1.0 are called "breeders", since they produce more fissile fuel than they consume. Since ${ }^{2.3 .} \mathrm{U}$ has an $\eta$ greater than 2.0 over much of the neutron spectrum, a thermal breeder using the ${ }^{232} \mathrm{Th}-{ }^{233} \mathrm{U}$ cycle is possible. However, the rapidly increasing value of $\eta$ for all three isotopes in the vicinity of the fission neutron spectral peak, favors the use of a fast neutron spectrum for breeding. Such reactors, known generically as fast breeders, can have $\mathrm{CR}$ values high enough not only to self-sustain, but to produce additional fuel to start up new reactors.

For a reactor designed to operate with a fast neutron spectrum, the low capture ratio advantage of ${ }^{233} \mathrm{U}$ does not exist, and the larger value of $\eta$ for ${ }^{239} \mathrm{Pu}$, as shown in Fig. $3 \mathrm{~A}-4$, gives it an edge. There is an additional advantage to the $\mathrm{U}-\mathrm{Pu}$ cycle at high energies in that, as

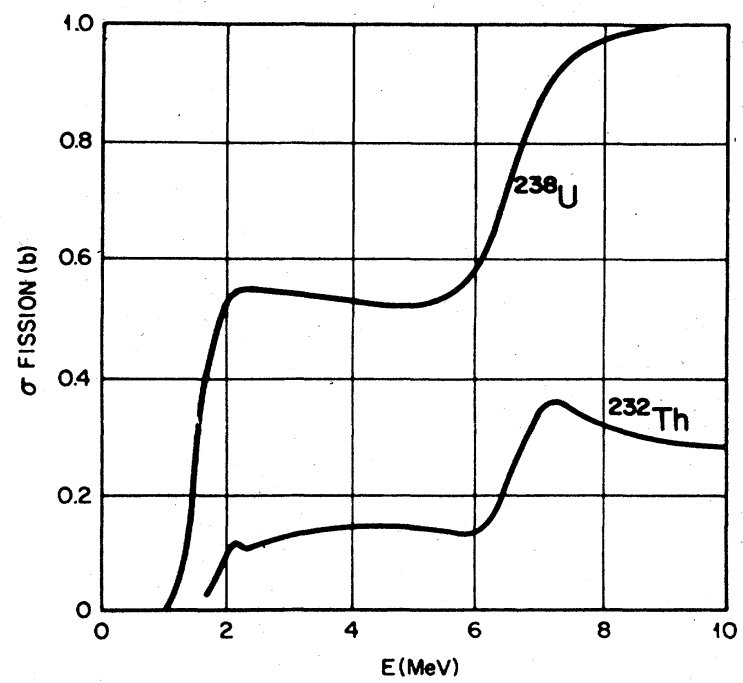

FIG. 3A-5. Fission cross-section of ${ }^{232}$ Th and ${ }^{238} \mathrm{U}$ (ORNL/ TM-5565). shown in Figure $3 \mathrm{~A}-5,{ }^{238} \mathrm{U}$ has a somewhat higher cross section for fission, which increases $\varepsilon$ and therefore the CR.

The higher $\mathrm{CR}$ possible with the ${ }^{238} \mathrm{U}-{ }^{239} \mathrm{Pu}$ cycle makes it the most resource efficient choice for a fast breeder cycle. In addition, ${ }^{239} \mathrm{Pu}$ is available from spent LWR fuel to start up the cycle. Nevertheless, ${ }^{233} \mathrm{U}$ has sufficiently good characteristics to provide fast breeding with $\mathrm{CR}$ considerably greater than 1.0. However, since it is not abundant in nature, the use of ${ }^{233} \mathrm{U}$ would entail a new program to generate start-up cores. Such reactors could, in principle, be started with ${ }^{235} \mathrm{U}$ cores with significant penalty, as discussed in Chapter VIII. The attractiveness of the ${ }^{239} \mathrm{Pu}$ fast breeder has led to major development efforts both in the U.S. and abroad. However, recent concern over the safeguards implications of plutonium use has led to increasing consideration of isotopically denatured fuel cycles based on ${ }^{233} \mathrm{U}$ or ${ }^{235} \mathrm{U}$, diluted to less than $20 \%$ concentration in ${ }^{238} \mathrm{U}$. Fast breeder, as well as advanced converter, cycles based on ${ }^{233} \mathrm{U}$ are also discussed in Chapter VIII.

For a given initial fuel composition, depletion of fissile material and, to a lesser extent, the build-up of neutron-absorbing fission products, mandates eventual replacement of the fuel. This "spent" fuel still contains an inventory of fissile material that is an appreciable fraction of the initial fissile content. This is the source of the potential economic benefit of reprocessing the spent fuel to recover fissile material. As the primary purpose of a breeder reactor is to generate additional fissile material, reprocessing is required for all breeder cycles. In addition, many of the advanced converter cycles discussed in Chapter VIII will also require reprocessing, particularly those based on ${ }^{233} \mathrm{U}$ bred from ${ }^{232} \mathrm{Th}$.

In a typical reprocessing operation, more than $99 \%$ of the uranium and plutonium (and thorium when applicable) are recovered from the spent fuel by chemical dissolution and separation, as discussed in Chapter IV. The recovered products may then be treated further for re-use of their fissile values. The remainder of the spent fuel, consisting of the residual uranium and plutonium, all of the neptunium, americium, curium, and the non-volatile fission products, makes up what is usually referred to as the high-level wastes (HLW).

\section{A2. Commercial Power Reactors}

The preceding discussion focussed almost entirely on the physical neutronic processes underlying reactor design. The translation of these principles into a commercial design, however, involves a wide 
range of physical and engineering considerations beyond the scope of this primer. Among the more significant are operating temperature and heat transfer considerations, net core reactivity and its control, operational reliability and safety, maintenance, and capital cost. In many ways, such factors have had much to do with the history of reactor design and development. The two primary conditions for a commercial reactor in a competitive energy market are economic and resource efficiency. The first of these, measured as lifetime average cost per $\mathrm{kw}-\mathrm{hr}$ of delivered electricity, dominates commercial investment and planning. An economic analysis for present reactor types and fuel cycle plans is performed in Chapter IV, Section G. For the alternate fuel cycles discussed in Chapter VIII, precise economic determinations are difficult. Resource efficiency, measured in units of metric tons of ore mined to produce a certain amount of electrical energy, most conveniently expressed in terms of $\mathrm{Mw}(\mathrm{e})$-yrs, provides a useful and socially meaningful method for comparison.

As is described in Chapter VIII, the resource efficiency and, therefore, the fuel cycle economics of different reactors depend upon thermal efficiency, CR, and the specific core power density (expressed in $\mathrm{Mw}(\mathrm{th}) / \mathrm{Mg}$ ). The latter enters not only for breeder reactors, where the doubling time will depend on both $C R$ and specific power, but for converters as well. Reactors with low specific power have larger initial core inventories, larger reloads, and longer equilibrium times than those with high specific power. It is important to note, however, that fuel costs are typically less than $15 \%$ of the cost of nuclear generated electricity, so that utility preferences are more likely to be determined by the capital costs and reliability of different reactor types than by details of fuel cycle economics.

\section{A2a. Light Water Moderated Reactor (LWR)}

At the present time, the commercial power reactor market in the U.S. and abroad is dominated by the LWR. There are two distinct varieties, the boiling-water reactor (BWR) in which steam is generated directly within the core, and the pressurized-water reactor (PWR), in which a pressurized water coolant circulates to an external heat exchanger, generating stream in a second, independent water circuit. Generically, however, the two types are quite similar.

In an I.WR, ordinary (light) water is used as both moderator and coolant. The use of water as a coolant has obvious advantages; light water is the most effective neutron moderator. However, hydrogen nuclei also have a fairly large cross section for neutron absorption. This combination of factors not only allows, but requires, a fairly compact core design. The fuel is in the form of narrow rods. The fission neutrons escape the rods, are slowed in the moderator, and then diffuse back into the rods where they induce new fissions. The combination of physical design considerations and neutron losses in the moderator mandates the use of fuel with a higher concentration of fissile isotopes per unit volume than natural uranium. Present day fuel for LWR's consists of uranium that has had its content of fissile ${ }^{235} \mathrm{U}$ enriched to $2.5-3 \%$. It is also possible to operate LWR's on fuel in which the fissile content has been increased by the addition of fissile ${ }^{239} \mathrm{Pu}$ or ${ }^{233} \mathrm{U}$.

Present design LWR's have a CR of about 0.6. As discussed in Chapter VIII, however, modifications to existing LWR designs are possible that would increase the $\mathrm{CR}$ to roughly $0.7-0.85$.

\section{A2a.1. The Pressurized-Water Reactor (PWR)}

In a typical present-day PWR, heat from the core is conveyed via the primary pressurized water circuit to a secondary circuit used to generate steam to drive the turbines. Table $3 \mathrm{~A}-4$ lists the characteristics of a model $1000 \mathrm{Mw}(\mathrm{e}) \mathrm{PWR}$. The fuel consists of small pellets of sintered $\mathrm{UO}_{2}$ placed in zirconium alloy (zircalloy) tubes about $1 \mathrm{~cm}$ in diameter and 4 meters long. A large PWR contains about 40,000 fuel rods, bundled into fuel rod assemblies; a typical assembly consists of a $15 \times 15$ array.

The reactor is shut down annually for refueling. At that time, the one-third of the fuel that has had the highest exposure in the core (burnup) is removed as spent fuel. Fresh fuel is then inserted to make up for the one-third removed. As shown in Table 3A-4, for a nominal $80 \%$ load factor with annual refueling, the spent fuel will have been expcsed for an average burnup of $33 \mathrm{Mw}(\mathrm{th})-$ days $/ \mathrm{kg}$. and
TABLE 3A-4. Assumed characteristics of model PWR and BWR enriched uranium reactors (ORNL, 1976).

\begin{tabular}{lll}
\hline \hline & PWR & BWR \\
\hline Electric Power, Mw(e) (net) & 1000 & 1000 \\
Thermal power, Mw(th) & 3077 & 3067 \\
Avg. spec, power, Mw(th)/Mga & 37.5 & 23.8 \\
Avg. burnup, Mw(th)-days/kg & 33. & 27.5 \\
Refueling interval, days ${ }^{b}$ & 365.25 & 365.25 \\
& & \\
Steady State Charge, $\mathrm{kg}^{\mathrm{C}}$ & & \\
U-234 & 9.57 & 10.1 \\
U-235 & 903 & 838.5 \\
U-236 & 76.6 & 90.3 \\
U-238. & 26450 & 31315 \\
Total & 27350 & 32250 \\
& & \\
Steady State Discharge, $\mathrm{kg}^{\mathrm{C}}$ & & \\
U-235 & 219 & 233 \\
Total U & 26,150 & 31100 \\
Fissile Pud & 170 & 198 \\
Total Pue & 248 \\
\hline \hline
\end{tabular}

a Based upon full power and total fuel charged.

b At $80 \%$ load factor

c Annual charge and discharge of one third of PWR and one quarter of BWR.

d Plutonium isotopes ${ }^{239} \mathrm{Pu}$ and ${ }^{241} \mathrm{Pu}$.

e $238 \mathrm{Pu}+{ }^{239} \mathrm{Pu}+240 \mathrm{Pu}+241 \mathrm{Pu}+{ }^{242} \mathrm{Pu}$.

will contain about $390 \mathrm{~kg}$ of fissile material as compared to an annual fresh fuel charge of about $900 \mathrm{~kg}$ fissile.

\section{A2a.2. Boiling-Water Reactor (BWR)}

In a BWR, there is only a single water circuit, and steam is generated directly by circulation of water within the core. Table $3 \mathrm{~A}-4$ also lists the characteristics of a model $1000 \mathrm{Mw}(\mathrm{e}) \mathrm{BWR}$. The fuel rods differ only in detail from those of a PWR. As a rule, they are somewhat larger in diameter -- about $1.5 \mathrm{~cm} \mathrm{--,} \mathrm{and} \mathrm{are} \mathrm{assembled}$ into $7 \times 7$ or $8 \times 8$ arrays. A large BWR contains about 40,000 such rods. The core is somewhat larger than for a PWR and the core average specific power is lower, in the range of $24 \mathrm{Mw}(\mathrm{th}) / \mathrm{Mg}$.

Fuel management schedules and shutdowns differ only in detail from those of a PWR. One-fourth of the core is replaced at each fueling, compared to one-third for a PWR, and fuel burnup at discharge is somewhat lower. As shown in Table $3 A-4$, a model BWR discharges about $430 \mathrm{~kg}$ of fissile material per year, compared to an annual charge of roughly $840 \mathrm{~kg}$ fissile.

\section{A2b. Heavy-Water Moderated Reactor (HWR)}

The use of deuterated (heavy) water as a moderator has some advantages for thermal reactor operation, owing to the lower capture cross section as compared to light water. The improvement in neutron economy allows for the use of natural uranium as a fuel, eliminating the need for enrichment, as well as simplifying adaptations to the use of other fissile materials as fuel. However, two problems are introduced: the neutron energy loss per collision is less for heavy than for light water, requiring a larger spacing between the fuel rods to allow for moderation; heavy water also has been fairly expensive. A number of conceptual designs exist for HWR's. Single pot systems similar to current LWR designs would entail very large and correspondingly expensive pressure vessels.

The only commercial heavy water reactor at present is the CANDU, designed and developed by Atomic Energy of Canada, Ltd. (AECL), in which fuel and coolant are confined to a system of 
pressure tubes, with a heavy water moderator at low pressure in the intervening spaces. In the presently commercialized CANDU, heavy water is used in both coolant and moderator circuits. But the separation of moderator and coolant circuits allows for a variety of possible coolants. AECL has designed several variants in which different coolants, such as organic liquids, are used. The British have also designed a similar reactor, the steam-generating heavy water reactor, that is modified to allow the use of light-water as a coolant despite its relatively large neutron absorption cross section.

A typical CANDU fuel bundle consists of an array of rods considerably larger than those for an LWR, and containing natural rather than enriched $\mathrm{UO}_{2}$. The low fuel burnups in natural uranium CANDUs necessitates frequent fuel rod replacement, and one of the most important features of the Canadian design is the use of individual fuel rod tubes to allow for continuous refueling without shutting down the reactor. A pair of refueling machines replace individual fuel rods according to a programmed fuel burn schedule. Present CANDUs have a CR of about 0.75 . Thermal efficiency is about $30 \%$, somewhat lower than for LWRs. As discussed in Chapter VIII, considerably higher CR's are possible particularly through the use of ${ }^{233} \mathrm{U}-\mathrm{Th}$ fuels.

A $1000 \mathrm{Mw}(\mathrm{e})$ CANDU would contain about $130 \mathrm{Mg}$ of natural uranium, as $\mathrm{UO}_{2}$ in zircalloy rods about $1.2 \mathrm{~cm}$ in diameter. These are assembled into circular arrays containing typically, 37 rods each. Operation with natural uranium, with only $0.7 \%{ }^{235} \mathrm{U}$, limits the fuel residence time to about a year. Fuel burnups are therefore far lower than for an LWR, on the order of only $7500 \mathrm{Mw}$-days(th)/Mg. The annual natural uranium charge has a fissile content of about $940 \mathrm{~kg}$, and the annual discharge contains about $220 \mathrm{~kg}$ of remaining ${ }^{235} \mathrm{U}$ and $350 \mathrm{~kg}$ of ${ }^{239} \mathrm{Pu}$. (See Chapter VIII).

At the present time, CANDU reactors are not licensed for use in the U.S. Pending an application for such a license, it has not been determined what modifications to present designs would be required to adapt CANDU-type reactors to meet U.S. criteria.

\section{A2c. The High-Temperature Gas-Cooled Reactor (HTGR)}

Water-cooled reactors have a thermal efficiency limited by the temperature to which the coolant can be raised. The HTGR was designed to use helium gas as a coolant, allowing higher heat exchange temperatures and improved thermal efficiency. Current design HTGRs have a core consisting of a massive assembly of hexagonal graphite moderator blocks, each containing fuel made up of small coated particles. The total core mass is about an order of magnitude higher than for an LWR, since the relatively large mass of the carbon nuclei requires many more collisions to thermalize neutrons in graphite than is the case for water.

Alternate means of fueling HTGRs are described in Chapter VIII. Current designs use a combination of highly-enriched (93\%) uranium for fissile pellets, and thorium as fertile. As the intention has been to recover the ${ }^{233} \mathrm{U}$ bred in the fertile pellets for recycle as future fuel, the HTGR cycle as presently conceived requires reprocessing. A representative HTGR has a thermal efficiency of almost $40 \%$, compared to roughly $32 \%$ for an LWR and $28 \%$ for a CANDU. Fuel burnup is very high, approaching $100 \mathrm{Mw}(\mathrm{th})$-day/kg; one-fourth of the core is to be replaced with fresh fuel each year. Present HTGR designs have a CR of about 0.66 and, as described in Chapter VIII, much higher CRs are possible. The use of thorium fuel and especially, the use of a graphite fuel matrix, imposes an additional set of requirements upon reprocessing for this reactor, as described in Appendix II. At the present time, the only commercial manufacturer of HTGRs has suspended production.

\section{A2d. Thermal Breeder Reactors}

As previously discussed, it is possible to design a reactor with a $\mathrm{CR}$ greater than 1.0 (breeders) using ${ }^{233} \mathrm{U}$, since $\eta$ is greater than 2.0 for this isotope over almost the entire range of neutron energies. This is the basis for many of the breeder and near-breeder alternate fuel cycles discussed in Chapter VIII. There is, in addition, a conceptual design for the U-Th breeder cycle using light water moderation and a thermal neutron spectrum -- The Light-Water Breeder Reactor (LWBR). Because light water is used as a moderator and coolant, breeding is already limited by neutron absorption in the water, so that other neutron absorbers such as control rods, must be avoided. The LWBR achieves control by moving fuel assemblies with relatively high enrichment in and out of the core, rather than the usual procedure of inserting and removing neutron absorbers. The core is intended for insertion in the pressure vessel of current PWR designs, as a substitute or replacement. In order to obtain the necessary quantities of ${ }^{23.3} \mathrm{U}$ to start up the cycle, it would be necessary to run a series of "prebreeder" reactors on the U-Th cycle for a number of years; the tight neutron economy of the LWBR would not allow its initial fueling on other isotopes for startup.

One other thermal breeder design, the Molten-Salt Breeder Reactor (MSBR) has been studied, but has not been commercialized. It was to have used a fluid fuel-coolant combination consisting of ${ }^{232} \mathrm{Th}$ and ${ }^{233} \mathrm{U}$ dissolved in a $\mathrm{LiBeF}_{2}$ molten salt; the combination was to circulate through a graphite moderator matrix. A small experimental MSBR was operated for several years, but no subsequent work has been done -- largely because of the maintenance problems entailed by corrosion due to the molten salt and the circulation of highly radioactive fuel throughout the coolant and heat exchange systems.

\section{A2e. Fast Breeder Reactors}

Generically, fast breeder reactor designs involve the use of a core and blanket. The blanket consists of assemblies of fertile materials surrounding the core, so that the fast neutrons that leak from the core can efficiently breed fissile material. It is necessary to operate the core with unmoderated (fast) neutrons in order to achieve CRs appreciably greater than 1.0

Because of the smaller fission cross section for fast neutrons than for thermal, a fast reactor core requires more hichly enriched fuel than for a thermal reactor, typically in the range of 15-20\% enrichment. The core must be compact and operated at high power density, typically three to five times that of an LWR. This requires a coolant with excellent heat transfer properties, but one which is a poor moderator. Currently, two choices are under consideration. Liquid metal is an excellent coolant, and most developmental work is currently being done on Liquid-Metal Cooled Fast Breeder Reactors (LMFBRs). Considerations of low neutron moderation and good heat transfer properties has led to the choice of sodium as a coolant in such designs. This requires very careful attention to design, as sodium will burn rapidly in air at the temperatures maintained in the primary coolant circuit. Sodium is also used in the secondary circuit, and must be carefully isolated from water, with which it is very reactive.

An alternate choice is the Gas-Cooled Fast Breeder Reactor (GCFR) which, like the HTGR, uses helium as a coolant. However, the core of a GCFR is more compact than that of an HTGR, and uses no graphite. Unlike sodium, helium does not absorb or moderate neutrons, and is not reactive with air or water. The lack of a liquid coolant to act as a thermal sink requires that very careful attention be paid to heat transfer and to the prevention of sudden loss of coolant in the high pressure helium gas circuit.

The neutron economy of fast breeder reactors is based on neutrons in the vicinity of the fission neutron spectral peak (c.f. Fig. 3A-2). The large value of $\eta$ for these neutrons (c.f. Fig. 3A-4) provides for enough excess neutrons to overcome the increased absorptive losses in coolant, fission products, and structural materials. In addition, there is an appreciable contribution from the fast fission of fertile isotopes, (c.f. Fig. $3 \mathrm{~A}-5$ ), leading to values of $\varepsilon$ in the neighborhood of 1.1 to 1.2.

Since the intention of the fast-breeder is not merely to be selfsustaining, but to provide new fuel for the cores of additional reactors, CRs in the vicinity of 1.4 or greater are sought. From the point of view of technical efficiency and resource use, both factors favor the ${ }^{238} \mathrm{U}-{ }^{239} \mathrm{Pu}$ cycle. ${ }^{239} \mathrm{Pu}$ has the highest value of $\eta$ of the three fissile isotopes considered, and the fast fission cross section for ${ }^{238} \mathrm{U}$ is significantly greater than that for ${ }^{232} \mathrm{Th}$. The combined value of $\eta \varepsilon$ for the U-Pu cycle is in the vicinity of 3.0, somewhat higher than for the Th-U cycle. As discussed in Chapter VIII, however, it is also possible to operate $\mathrm{Th}-\mathrm{U}$ breeder cycles at sufficiently high $\mathrm{CR}$ to allow for breeding gain. Such fuel cycles are currently being given consideration in response to concerns about the nuclear proliferation implications of the widespread use of plutonium in the nuclear fuel cycle; we discuss them in Chapter VIII. 
There are some substantial differences between fuel cycle operations for the breeder and those used for thermal reactors, even with recycle. The projected very high burnup of breeder core fuel will require modifications to existing reprocessing plant designs, as discussed in Chapter IV. The high fuel burnup and high plutonium content, impose more stringent conditions on handling and transportation systems and on reprocessing technology than do present LWR fuel cycles.

\section{A2e.1. The Liquid-Metal Cooled Fast Breeder Reactor ( $L M F B R)$}

Current LMFBR designs use molten sodium as the coolant. Because the sodium in contact with the core will become radioactive, an intermediate sodium loop is used to convey heat from the primary coolant to the steam-generating water system. There are two basic design philosophies. In the "pot" type LMFBR, both core and primary coolant loops are immersed in a sodium bath at roughly atmospheric pressure. This reduces the amount of external piping, and provides fluid coverage for the core at all times. The "loop" arrangement currently favored in the U.S. is similar to current PWR systems in that only the core and associated equipment are contained in the reactor vessel, simplifying maintenance. Elevation of loops and the provision of guard tanks is to provide core submergence. Two types of fuel rods are to be used in LMFBR's. Blanket assemblies will have rods consisting only of fertile material. Core assembly rods will have fissile loads in the center, but fertile (axial blanket) loads at the ends. Thus the core is totally surrounded with fertile material.

\section{A2e.2. The Gas-Cooled Fast Breeder Reactor (GCFR)}

Helium-cooled GCFRs have received some attention. Higher CRs than for the LMFBR are possible, owing to the absence of neutron moderation or absorption in the helium coolant. Helium has other advantages in that it is not chemically active, and because a gaseous coolant minimized reactivity changes due to voids in the coolants. However, a massive prestressed concrete vessel is needed for helium retention, and coolant provides little heat capacity for stablizing thermal excursions. The behavior of fuel cladding at the higher fuel

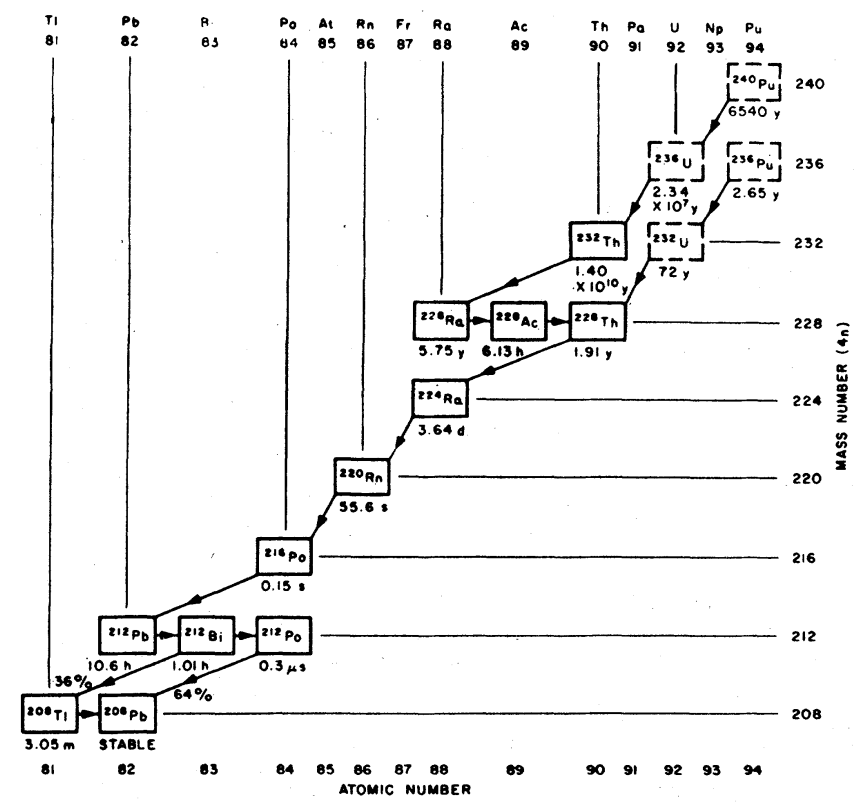

FIG. 3A-6. Urnaium-232* ( $4 n$ series) and Thorium-232 [from ERDA-1541; Adapted from R. D. Evans, The Atomic Nucleus (McGraw-Hill, 1965)]. The solid boxes denote naturally occurring nuclides. Diagonal arrows indicate $\alpha$, and horizontal arrows $\beta$ decay. temperatures and high irradiation characteristic of the GCFR is less well understood than for the LMFBR.

\section{A3. Fission Products and Transuranic Elements}

\section{A3a. Natural Radioactivity}

Natural radioactivity is an important source of background ionizing radiation. As discussed in Section $\mathrm{C}$ of this Chapter, natural radiation currently accounts for about two-thirds of the average per capita dose in the United States.

Naturally occurring radionuclides can be divided into those that occur singly, such as long-lived ${ }^{40} \mathrm{~K}$ of terrestrial origin or short-lived ${ }^{3} \mathrm{H}$ produced by cosmic rays, and those that are components of three distinct chains of decay of naturally occurring actinides. These chains are: the thorium $(4 n)$ series, originating in nature with ${ }^{232} \mathrm{U}$; the uranium $(4 n+2)$ series, originating with $238 U$; and the actinium $(4 n+$ 3) series, originating with ${ }^{235} \mathrm{U}$. A fourth series, the neptunium $(4 n+$ 1 ) series, completes the set but is not found in nature. The decay schemes of all four of these series are diagrammed in Figs. 3A-6 through $3 A-9$, with naturally occurring radionuclides denoted by solid boxes. The abundance of these elements in nature is determined by the half-lives of the parents compared to the age of the earth and the equilibrium between parents and daughters in the decay chain. Uranium is fairly common in nature, and is present at the $1-5 \mathrm{ppm}$ level in most surface rock, as well as in the richer ore deposits. Thorium is about four times as abundant, on the average. In general, the greatest potential biological hazard is presented by one of the intermediate daughters in the chain, such as ${ }^{226} \mathrm{Ra}$ in the uranium $(4 n+2)$ series.

${ }^{226} \mathrm{Ra}$ is an alpha-emitter of special concern because of its high solubility and ready biological takeup, and because its daughter ${ }^{222} \mathrm{Rn}$ is an alpha-emitting noble gas that diffuses readily into the atmosphere. ${ }^{226} \mathrm{Ra}$ is present in many foods and in almost all public water supplies in the U.S., usually at levels below $0.2 \mathrm{pCi} /$ liter. This figure is exceeded only at a very few locations in the U.S., although it does range as high as several $\mathrm{pCi} / \mathrm{liter}$. ${ }^{222} \mathrm{Rn}$ from decay of ${ }^{226} \mathrm{Ra}$ is readily identified in the atmosphere. Both directly and through the deposition of $210 \mathrm{~Pb}$, it contributes a measurable amount to natural

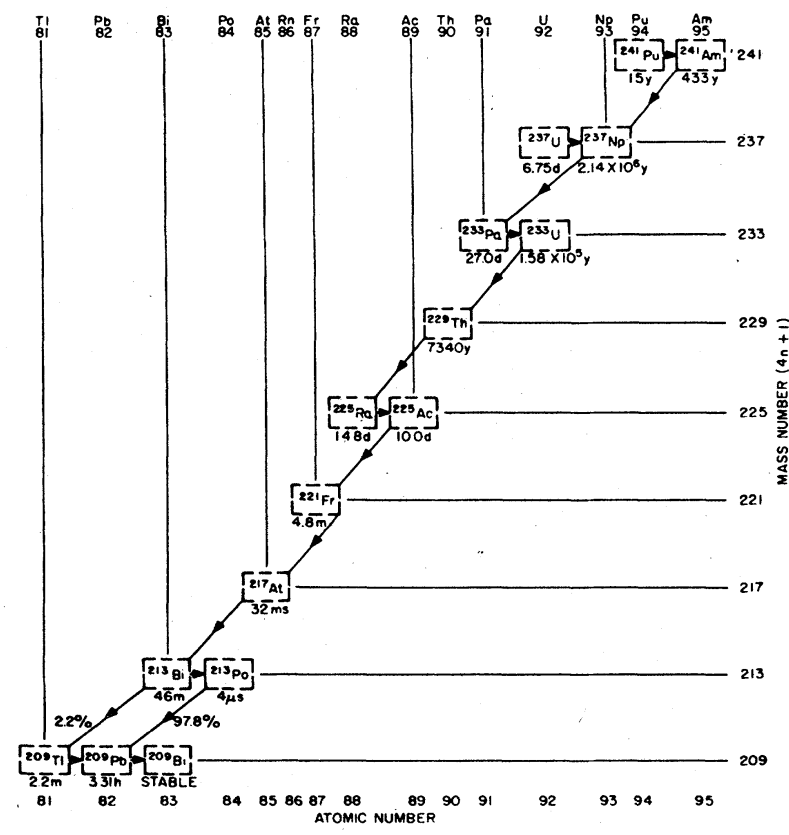

FIG. 3A-7. Uranium-233* $(4 n+1)$ series, [from ERDA-1541, adapted from R. D. Evans, The Atomic Nucleus (McGraw-Hill, 1965)]. The solid boxes denote naturally occurring nuclides. Diagonal arrows indicate $\alpha$, and horizontal arrows $\beta$ decay. 


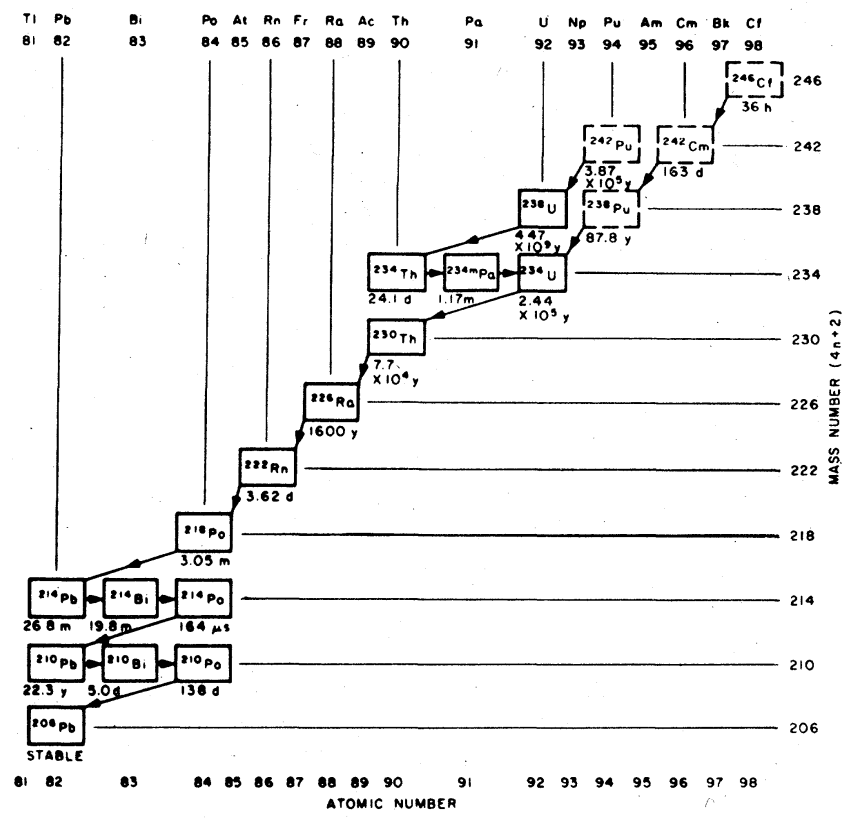

FIG. 3A-8. Uranium-238* $(4 n+2)$ series, [from ERDA-1541; adapted from R. D. Evans, The Atomic Nucleus (McGraw-Hill, 1.965)l. The solid boxes denote naturally occurring nuclides. Diagonal arrows indicate $\alpha$, and horizontal arrows $\beta$ decay.

dose. The contribution of ${ }^{228} \mathrm{Ra}$ from the thorium ( $4 \mathrm{n}$ ) decay series is about an order of magnitude less important, on the average, primarily because the thorium chain decays quickly all along the series without the holdup (at ${ }^{226} \mathrm{Ra}$ and ${ }^{210} \mathrm{~Pb}$ ) characteristic of the uranium series.

\section{A3b. Uranium Cycle}

As was shown in Fig. 3A-1, fission results in a broad distribution of radioactive fission products divided between two mass peaks. All of the longer-lived products must be considered wastes whose halflives, chemistry, and biological effects vary widely. They are predominantly emitters of beta and gamma radiation. As described in Chapter VII, the time scale for the decay of the fission products is such that, after roughly 600 years, the alpha-emitting actinides and their daughters plus ${ }^{99} \mathrm{Tc}$ and ${ }^{129} \mathrm{I}$ become the dominant radiological concern.

A wide range of actinides is also produced by neutron absorption processses in the fuel. As is shown in Fig. 3A-10, the absorption of neutrons not entering into fission reactions can eventually build up significant quantities of transuranic elements. As these are increasingly higher-order processes, the greater the fuel burnup, the greater the production of higher isotopes. One of the most important reactions for the fuel cycle is the production of ${ }^{239} \mathrm{Pu}$ from ${ }^{238} \mathrm{U}$ by the reaction of Eq. (2). Non-fission capture of neutrons by ${ }^{239} \mathrm{Pu}$ produces ${ }^{240} \mathrm{Pu}$ which is not fissile by thermal neutrons. In turn, capture by ${ }^{240} \mathrm{Pu}$ produces thermally fissile ${ }^{241} \mathrm{Pu}$. Some of the ${ }^{241} \mathrm{Pu}$ will decay to form ${ }^{241} \mathrm{Am}$, leading to the ultimate production of higher actinides, and some will be converted to ${ }^{242} \mathrm{Pu}$ by neutron absorption, leading to yet another higher actinide branch, as is also shown Fig. 3A-10. The net result is that the thermally fissile plutonium isotopes, ${ }^{239} \mathrm{Pu}$ and ${ }^{241} \mathrm{Pu}$, are inevitably mixed with other isotopes. It is important to note that ${ }^{240} \mathrm{Pu}$ is not fissile by thermal neutrons, but is fissile for fast neutrons, such as those characterizing a nuclear weapon. The desirability of low fuel burns for "weaponsgrade" plutonium is a consequence of the spontaneous neutron emission of ${ }^{240} \mathrm{Pu}$, and not because it constitutes a non-fissile diluent. (See Section D of this Chapter.)

At higher fuel burns, increasing quantities of the transuranics are formed, including ${ }^{238} \mathrm{Pu}$ which is a principal source of ${ }^{226} \mathrm{Ra}$ in the

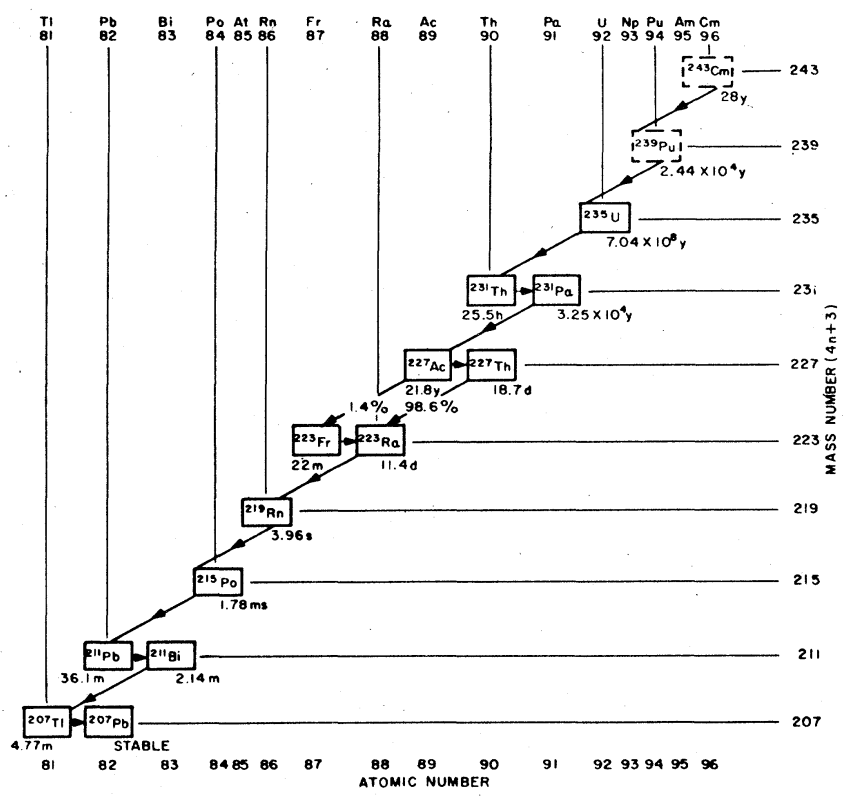

FIG. 3A-9. Uranium-235* $(4 n+3)$ series, [from ERDA-1541; adapted from R. D. Evans, The Atomic Nucleus (McGraw-Hill, 1965)]. The solid boxes denote naturally occurring nuclides. Diagonal arrows indicate $\alpha$, and horizontal arrows $\beta$ decay. Chain branches of $<1 \%$ omitted.

wastes. It should be noted that fueling with mixed-oxides increases the inventory of transplutonic isotopes due to irradiation of plutonium throughout the cycle. The inventories of americium and curium are important not only because of their intrinsic alpha-decay, but because, as is shown in Figs. 3A-6 through 3A-10, they are also the parents of plutonium isotopes during their decay.

\section{A3c. Uranium-Thorium Fuel Cycles}

Figure 3A-11 illustrates the principal actinide chains for the use of thorium-uranium fuel cycles, and should be compared to the uranium-plutonium characteristics shown in Fig. 3A-10 Because the primary fueling isotopes are lighter than those used from the uranium-plutonium cycle, relatively little plutonium, americium, and curium are formed during irradiation of thorium-uranium fuel. As a result, the actinide alpha-activity of high-level and other actinide wastes will be considerably lower for this case. The net activity of the wastes will be smaller initially for periods in excess of 1000 years, when the fission products have decayed away.

However, after roughly $10^{5}$ years, the theoretical ingestion hazard for both cycles is governed by ${ }^{226} \mathrm{Ra}$ from the decay of ${ }^{238} \mathrm{Pu}$ or ${ }^{234} \mathrm{U}$ in the wastes (see Fig. 3A-8). The larger production of ${ }^{234} \mathrm{U}$ and ${ }^{238} \mathrm{Pu}$ in the thorium-uranium cycle results in the buildup of ${ }^{226} \mathrm{Ra}$ activity between $10^{5}$ and $10^{6}$ years to a level greater than that for uraniumplutonium high-level wastes, and comparable to that for unreprocessed discharge uranium fuel. The decay chain originating with ${ }^{237} \mathrm{~Np}$ or residual ${ }^{233} \mathrm{U}$ (Fig. 3A-7) results in the additional buildup of ${ }^{225} \mathrm{Ra}$, which governs the potential ingestion hazard at times between $10^{6}$ and $10^{7}$ years. This is discussed further in Chapter VII of this report.

The primary reaction of importance in using thorium-uranium cycles is that which produces ${ }^{233} \mathrm{U}$ from ${ }^{232} \mathrm{Th}$, equation (4). The relatively long 27 -day half-life of ${ }^{233} \mathrm{~Pa}$ affects the time the fuel must be stored before reprocessing. Accordingly, most flowsheets for this cycle specify reprocessing after one year of storage to minimize contamination with highly radioactive ${ }^{233} \mathrm{~Pa}$. This results in a greater inventory of fissile material in the thorium-uranium cycle, delays the time to reach equilibrium recycle, and increases total uranium ore requirements, when compared to the uranium plutonium cycle. 


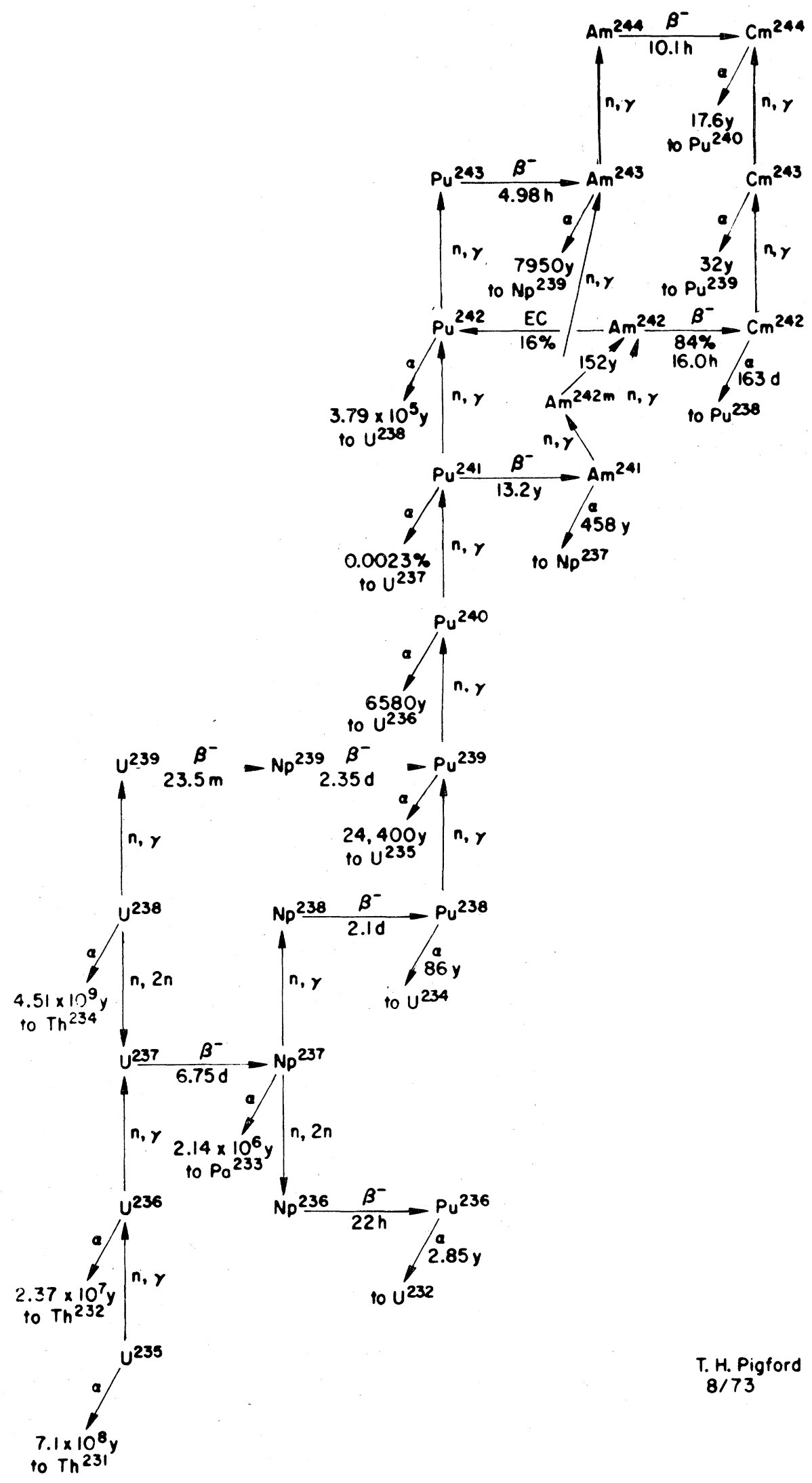

FIG. 3A-10. Actinide chains in the uranium-plutonium nuclear fuel cycle. 
A second problem of the thorium-uranium cycle results from the activity of ${ }^{232} \mathrm{U}$ and its daughters (shown in Fig. 3A-6). ${ }^{232} \mathrm{U}$ is produced in thorium-uranium fuels by a number of reactions:

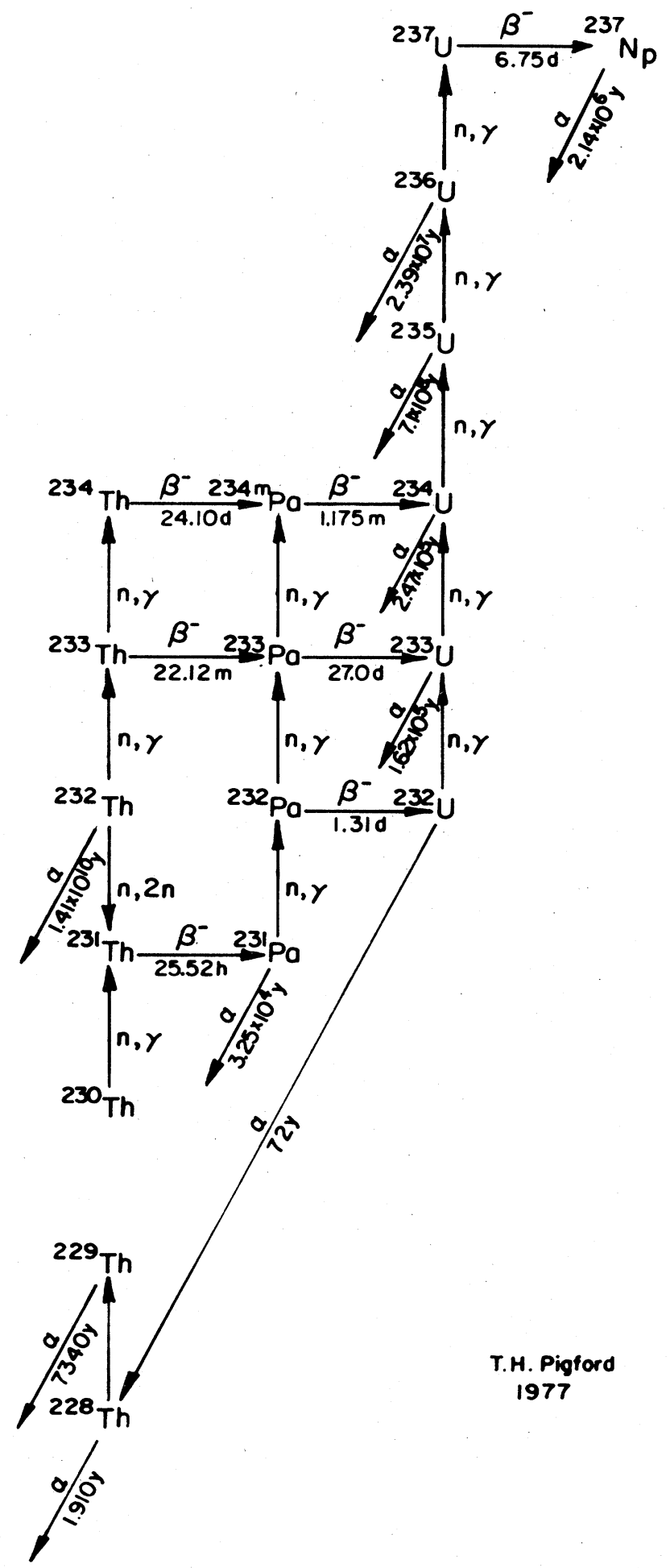

FIG. 3A-11. Actinide chains in the uranium-thorium nuclear fuel cycle.

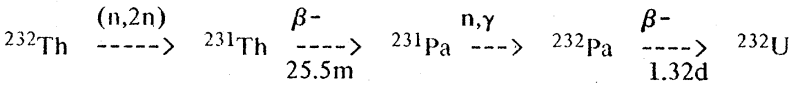

$$
\begin{aligned}
& { }^{233} U \quad \stackrel{(n, 2 n)}{-\cdots-->>}{ }^{232} U
\end{aligned}
$$

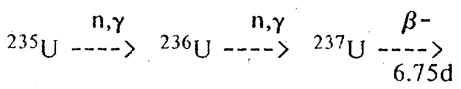

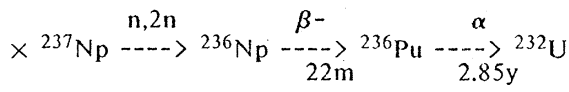

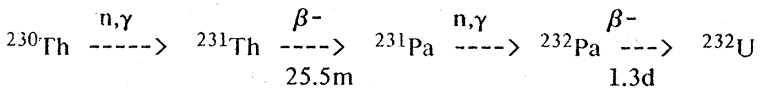

The last of these is important when thorium is a byproduct of uranium mining, since $230 \mathrm{Th}$ is a daughter of $238 \mathrm{U}$. The alpha activity of the 72-year half-life ${ }^{232} \mathrm{U}$ is less of a problem than the activity of its daughters. As is shown in Fig. 3A-6, the decay daughters are all short lived compared to ${ }^{228} \mathrm{Th}$, and come to secular equilibrium with it. The decay of ${ }^{212} \mathrm{Bi}$ and ${ }^{208} \mathrm{Tl}$ are accompanied by very energetic and penetrating gammas, so that extensive shielding is required for the handling, processing, and fabrication of recycle fuel containing ${ }^{232} \mathrm{U}$.

At the time of reprocessing, all the ${ }^{228} \mathrm{Th}$ will be separated from the uranium. The subsequent buildup and decay of the gamma activity in the separated uranium product, owing to ${ }^{232} \mathrm{U}$ decay, is described in Appendix III. There is considerable incentive to refabricate and recycle the uranium quickly, to avoid the gamma buildup. On the other hand, the ${ }^{228} \mathrm{Th}$ does go off with the thorium product, so that it is not practical to recover and recycle the thorium for about 10 years. Owing to the comparatively large inventories of ${ }^{238} \mathrm{Pu}$ and the relatively low production of $239 \mathrm{Pu}$ and $241 \mathrm{P}_{11}$, it is expected that there will be no incentive to recover plutonium from the thorium-uranium cycle. However, if ${ }^{238} \mathrm{U}$ in appreciable quantities is used to denature the ${ }^{2.3 .} \mathrm{U}$ isotopically, as has been suggested for safeguards fuel cycles, appreciable quantities of ${ }^{239} \mathrm{Pu}$ will be produced, as discussed in Chapter VIII.

\section{B. The LWR Fuel Cycle--A Description of Options}

As an LWR operates and fissile uranium is progressively consumed, fissile plutonium is produced by the transmutation of ${ }^{238} \mathrm{U}$, as described in Section IIIA. The plutonium in turn contributes to the nuclear fission reaction and the energy produced by the reactor. The average conversion ratio, CR, for an LWR is about 0.6 , which means that 6 atoms of plutonium are produced in the fuel for every 10 atoms of ${ }^{235} \mathrm{U}$ that are fissioned. At the time the spent fuel is discharged (with a typical burnup of about $30 \mathrm{Mw}(\mathrm{th})$-days/kg) more than half the fissions occurring in that fuel are from self-produced plutonium rather than ${ }^{235} \mathrm{U}$.

Fuel is considered spent when it can no longer sustain the neutron chain reaction at economic power levels because of the depletion of fissile materials and the accumulation of neutron-absorbing byproducts in the fuel and fuel hardware. At that point, however, the fuel still contains appreciable quantities of fissile isotopes, typically about 6 grams of fissile plutonium and 8 grams of unused ${ }^{235} \mathrm{U}$ per kilogram of spent fuel. The fissile isotopes can be recovered from the spent LWR fuel after it is removed from the core by treatment in a reprocessing plant. At this point several options are possible:

1. The spent fuel rods can be disposed of without reprocessing. This is the no-recycle option, with two variations depending on whether the disposition is regarded as permanent or recoverable. We will refer to these as "throwaway" in the first case or "stowaway" in the second.

2. Uranium can be recovered by reprocessing the spent fuel. Such fuel can be enriched or used as is to replace some of the virgin natural uranium in the preparation of new fuel assemblies. This option is called uranium-only recycle. Again, there are two variations with 
respect to the plutonium contained in the spent fuel: permanent disposal (throwaway) or recoverable storage (stowaway).

3. Plutonium can be recovered from spent fuel and combined with uranium having a lower $235 \mathrm{U}$ content than that of normal fresh uranium fuel; that is, a substitution of recovered plutonium can be made for some of the $235 \mathrm{U}$ fissile content of the fuel. Further, it is possible to make useful fuels using plutonium in combination with any predominately $238 \mathrm{U}$ uranium, including a low-enriched uranium product from an enrichment plant, recovered uranium from spent fuel, virgin natural uranium, or depleted uranium enrichment plant tails. Any such fuel is called plutonium-uranium mixed-oxide or simply mixed-oxide fuel, and the use of plutonium for this purpose is known as plutonium recycle.

It is important to examine the fuel cycle operations that occur in each of the three options to describe their similarities and differences. It is useful to assume a particular size and rate of growth for the nuclear industry to help the reader understand the scale of the various operations. For illustrative purposes we use the same case treated in the GESMO, which projects 507 nuclear reactors of $1000 \mathrm{Mw}(\mathrm{e})$ each by the year 2000. The illustrative numbers provide a snapshot reflecting the projected production quantities and varying lead times for such a growing industry, not steady state numbers. For more detail the reader is referred to the GESMO. The wastes associated with various cycles are described in detail in Appendix I.

\section{B1. No Recycle (Throwaway or Stowaway)}

LWR's require many supporting operations, most of which relate to the supply of fresh fuel and treatment of spent fuel. Where no uranium or plutonium is recycled, all LWR fuel comes from virgin natural uranium enriched to about $3 \%$ in ${ }^{235} \mathrm{U}$ content. Fuel cycle operations are diagramed in Fig. $3 \mathrm{~B}-1$ and include the following:

--Mining of uranium ores, both underground and in open pit. --Milling, to concentrate uranium values from the ores and to

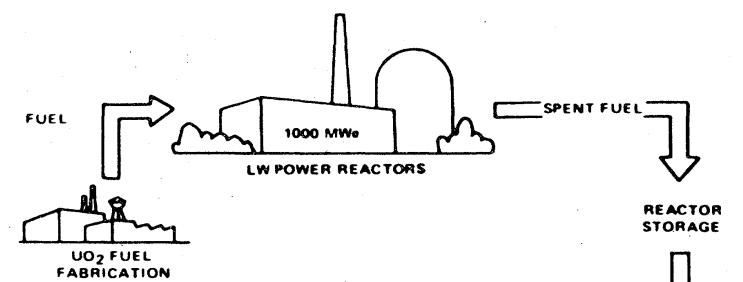

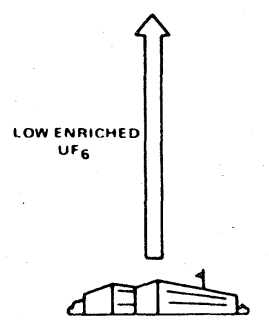

ENAICHMENT
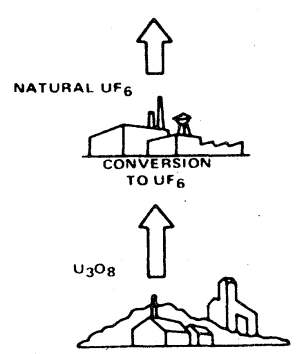

URANIUM MINES \& MILLS

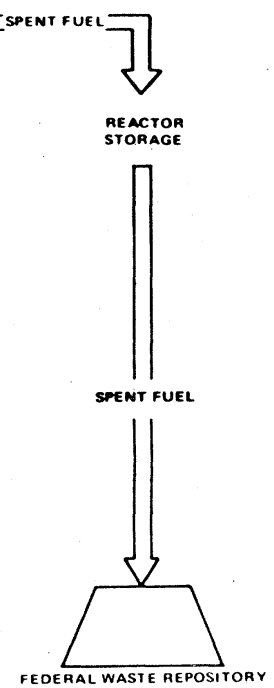

FIG. 3B-1. Light water reactor fuel cycle-no uranium or plutonium recycle (GESMO). produce a semi-refined uranium oxide product called yellowcake (assayed as equivalent $\mathrm{U}_{3} \mathrm{O}_{8}$ ).

--Refining and converting yellowcake to volatile uranium hexafluoride $U_{6}$, which is the feed for isotopic enrichment facilities.

--Enrichment of $\mathrm{UF}_{6}$, (currently by the gaseous diffusion process) to yield a product enriched in the fissile isotope ${ }^{235} \mathrm{U}$ and a depleted stream (enrichment plant tails).

--Conversion of enriched $\mathrm{UF}_{6}$ to oxide.

--Fabrication of the oxide into pellets, encapsulation into fuel rods, and assembly into fuel elements.

--Spent fuel storage, to permit fuel to cool thermally and radioactively to levels suitable for transportation.

--Long-term storage or disposal of spent fuel, which is permanent in the case of a "throwaway" option or recoverable for possible future use in the case of a "stowaway" option. It should be noted that the throwaway option would result in spent fuel being designated as high-level waste.

B2. Uranium-only Recycle (Throwaway or Stowaway of Plutonium)

If spent fuels are reprocessed for recovery and recycle of uranium, additional operations (designated by ${ }^{*}$ ) are added to the fuel cycle. The overall cycle is diagrammed in Figure $3 \mathrm{~B}-2$ and includes:

--Mining.

--Milling.

--Refining and conversion.

--Enrichment.

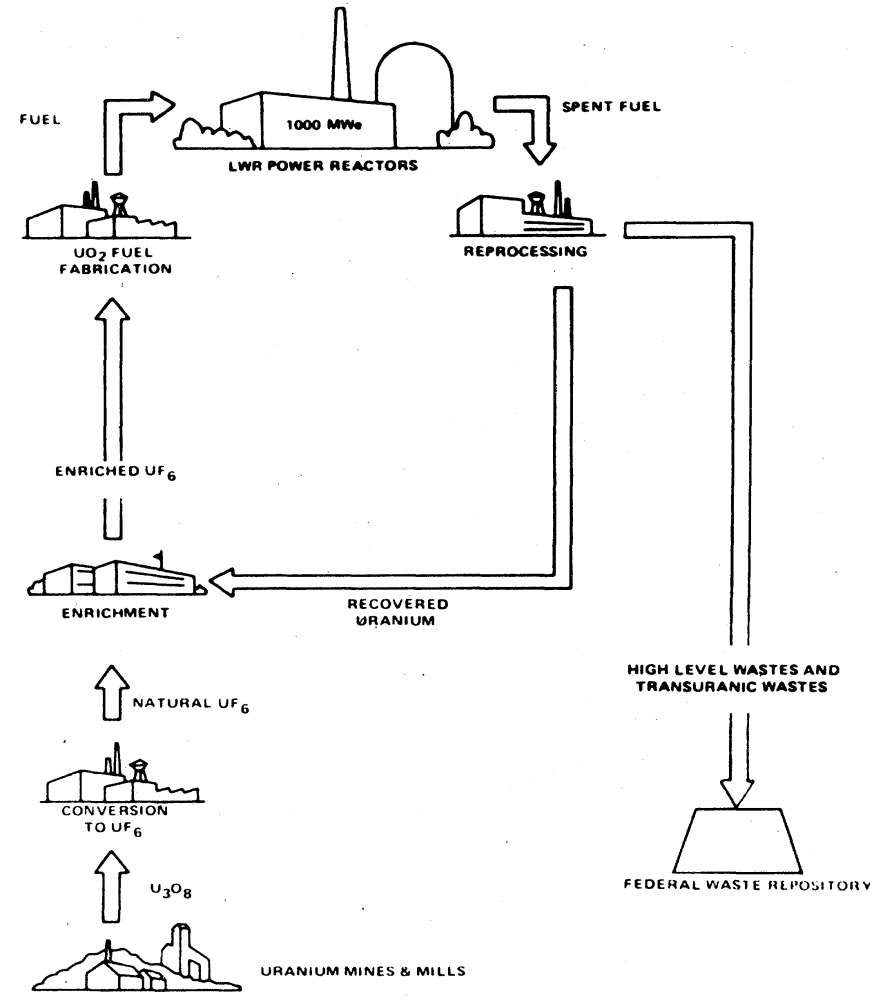

FIG. 3B-2. Light water reactor fuel cycle-uranium recycle only (GESMO). 
--*Chemical reprocessing of spent fuels to separate and recover residual uranium from plutonium and radioactive wastes.

$-{ }^{*}$ Conversion of the recovered uranium to $U_{6}$.

--*Re-enrichment of that recovered uranium in the enrichment plant simultaneously with enrichment of virgin natural $U_{6}$ to make a combined enriched product.

--Conversion of combined $\mathrm{UF}_{6}$ product to oxide.

--Fabrication.

--Spent fuel storage, to cool sufficiently for subsequent reprocessing.

--*High-level and transuranic waste disposal in Federal Repository. The plutonium is discarded in this waste in the case of the throwaway option. For stowaway of plutonium for future recovery, one would expect to separate the plutonium but store it indefinitely, rather than remine the plutonium from the high-level waste at a later date.

The chemical reprocessing step opens up many new features relative to the No-Recycle option. In the fuel reprocessing plant, the spent fuel is chopped into pieces, the fuel is dissolved in nitric acid, and the Purex solvent extraction process is used to separate the constituents into separate dissolved material streams. The whole process has been practiced on a large scale by the AEC and by private industry for 20 years, and many refinements in technique and technology have been introduced, as described in Chapter IV. The uranium is converted to the hexafluoride for shipment. The plutonium and nuclear wastes streams are converted into suitable chemical and physical forms depending on whether the plutonium is to be recycled, stored, or merged with the waste.

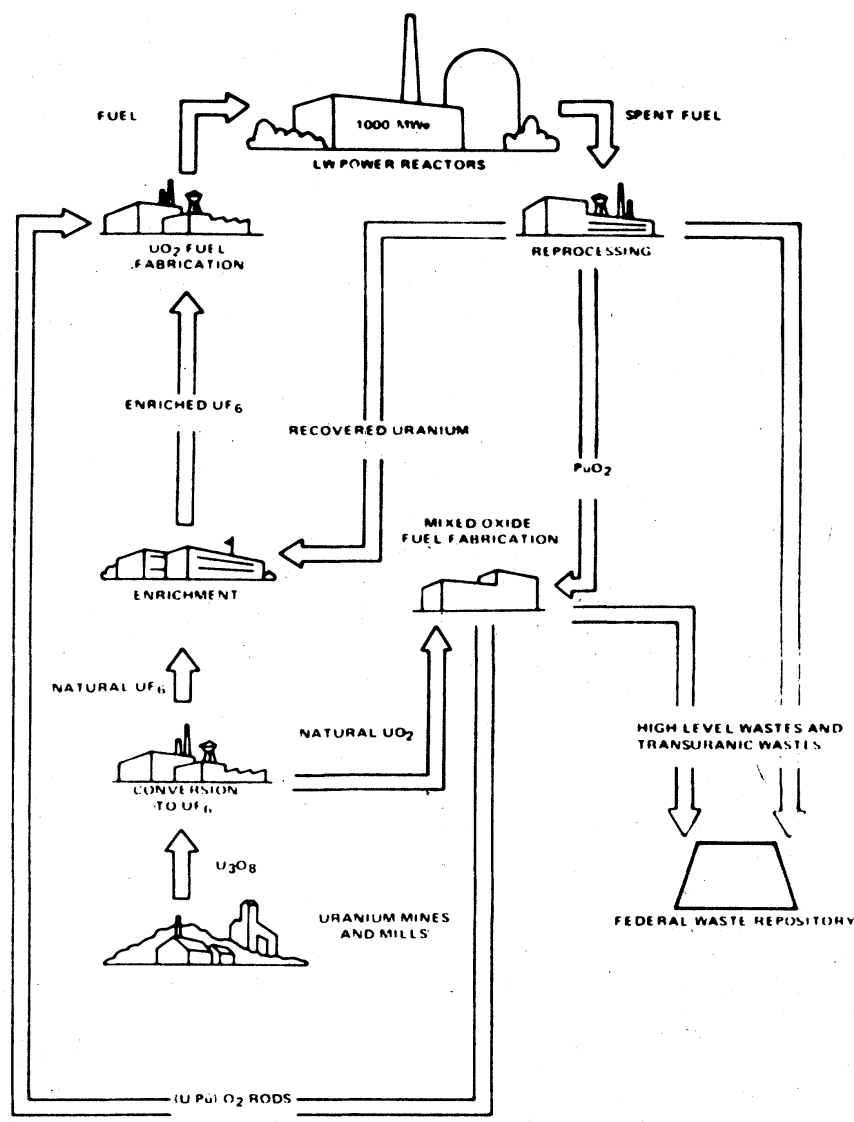

FIG. 3B-3. Light water reactor fuel cycle-uranium and plutonium recycle (GESMO).

\section{B3. Plutonium-Uranium Recycle}

With plutonium recycle, two new operations - plutonium conversion and mixed oxide fuel manufacture - are added to the fuel cycle, and the reprocessing step modified somewhat from that of the uranium-only-recycle. The overall fuel cycle is diagrammed in Figure $3 \mathbf{B}-3$. Individual steps are as follows:

--Mining.

--Milling.

--Refining and conversion.

--Enrichment.

--*Reprocessing of spent fuels to separate and recover uranium and plutonium from radioactive wastes and from one another.

--*Conversion of recovered uranium.

-*Reenrichment of recovered uranium.

--Conversion (of enriched $\mathrm{UF}_{6}$ ).

--Fabrication of enriched uranium pellets.

--*Conversion of recovered plutonium into a solid form (e.g., $\mathrm{PuO}_{2}$ ).

- ${ }^{*}$ Combination of recovered plutonium with uranium to make mixed oxide fuel, fabrication of pellets, encapsulation in fuel rods and assembly into fuel elements.

--Spent fuel storage.

$-{ }^{*}$ High-level and transuranic waste storage and disposal. For this option permanent disposal is required.

If recovered uranium and plutonium are recycied to LWR's, the separative work and yellowcake requirements for sustaining the LWR economy are substantially reduced. However, there will not be enough plutonium to make mixed oxide fuel for all LWR requirements, and there will be a continuing need for the present type of slightly enriched $\mathrm{UO}_{2}$ fuel. For the GESMO model estimate of uranium and plutonium recycle over the period $1975-2000$, it is projected that $87 \%$ of the LWR fuel over this period would be the standard $\mathrm{UO}_{2}$ fuel, whereas $13 \%$ would be mixed oxide. At the end of the period this model shows the quantity of plutonium being recycled to be such that $20 \%$ of the LWR fuel in the year 2000 would be mixed-oxide.

\section{B4. Comparison of the Three Fuel Cycle Options}

Study of the projections made in the GESMO shows the following major effects for the year 2000. These will be discussed at length in our report.

1. When both plutonium and uranium are recycled, enriched uranium supply operations for the year 2000 are reduced by about $20 \%$ compared to the uranium-only-recycle option; $\mathrm{U}_{3} \mathrm{O}_{8}$ and natural $\mathrm{UF}_{6}$ requirements are reduced by about $30 \%$ compared with the norecycle option; and uranium enrichment supply operations are reduced by about $20 \%$ :

2. The recycle of plutonium and uranium introduces a commercial traffic in purified plutonium, with all the attendant safeguards requirements;

3. If all spent fuel is reprocessed and the plutonium recycled promptly, but no mixed-oxide fuel is reprocessed, the quantity of spent fuel plutonium discarded in various nuclear wastes is about 1 $2 \%$ of what it would be without recycle. Since americium and curium decay to various plutonium isotopes, and since the amount of plutonium as well as of americium and curium in mixed-oxide fuel is greater than for $\mathrm{UO}_{2}$ fuel, the reduction in plutonium in high-level wastes depends upon the fuel mix and the time that has elapsed since the fuel was reprocessed. The overall reduction will be about an order of magnitude for most cases. However, recycling, the plutonium requires reprocessing, mixed oxide fabrication, and management of various additional types of waste, all of which raise serious questions of economics, health and safety, and environmental matters. 


\section{B5. Wastes from the Nuclear Fuel Cycle}

Each step of the nuclear fuel cycle generates wastes, much of which is non-radioactive and is handled in the normal manner. Appendix I provides more comprehensive details on the various fuel cycle steps and the origin of the several wastes from each. Miscellaneous waste is contaminated at low level with fission products, and is customarily buried below the surface in special low-level burial sites. Mill tailings are a potentially significant source of long-term public radiation exposure and are discussed in Chapter V. Particular attention is given in Chapter VII to those wastes that are likely to be required to be shipped to a federal repository for disposal. This includes either spent fuel or high-level wastes from reprocessing operations, the fuel cladding hulls and other miscellaneous hardware from which the fuel has been dissolved if reprocessed; another very important portion includes miscellaneous wastes contaminated by transuranics from both reprocessing and mixed-oxide fuel fabrication operations. As the discussions in Chapter $\mathrm{V}$ and Chapter VII point out, the most significant issues arise less from the quantity of the waste than from the viability and long-term predictability of the technical alternatives and institutional procedures to manage them.

\section{Radiation Exposures and Biological Effects on Humans}

Mans' laboratory experience with highly ionizing radiation dates from the discovery of x-rays by Roentgen in 1895. The research of Becquerel and the Curies concerning radiations from uranium pitchblende ore attracted world-wide scientific attention, and their isolation of radium is a landmark in twentieth century science. The level of understanding expanded greatly in the twenties and thirties with extensive research on the electron, positron, and neutron as well as on a wide spectrum of ionized nuclei. In addition, studies of radium watch dial painters in the 1920 's, and later of uranium miners and of people subjected to intense $\mathrm{x}$-radiation, laid the basis for the extensive study of biological radiation effects that was to follow the discovery of fission and the creation and use of nuclear weapons.

At this time, no complete, integrated theory of the biological effects of ionizing radiation exists. Even the simplest organism displays radiation effects which are exceedingly complicated. Of necessity, the classical scientific pattern of experiment is not available in studying radiation effects on humans. In fact, information on the reactions of humans to radiation is limited to the rare cases of exposure in wartime, under accident conditions, or in specific medical treatment regimes. Accordingly, much of the experimental work that does exist is based on effects on relatively short-lived animals.

Two approaches may be used in dealing with the effects. The first, is to consider physical and chemical views of the interactions of ionizing radiation with matter, emphasizing those features considered to be fundamental to subsequent biological effects. The second, considers gross pathological effects of radiation exposure and attempts to relate them to the conditions of exposure.

\section{C1. Radiation and Its Interaction with Matter and Dose}

The term ionizing radiation includes both directly-ionizing particles (charged particles with sufficient kinetic energy to ionize by coulomb collisions) and indirectly-ionizing particles (neutral particles which can interact to free directly-ionizing particles or which can initiate nuclear transformations). The directly-ionizing particles of interest include beta and alpha radiations such as are emitted from fission products and from naturally occurring radionuclides. These particles lose energy continuously and so have a finite energydependent range in matter. Typically, alpha particles can penetrate $<<$ $1 \mathrm{~mm}$ of tissue, while beta rays have ranges up to a few $\mathrm{cm}$ of tissue. Thus, effects are likely to be more pronounced for radiation sources internal to the body and close to relevant organs. The indirectlyionizing particles with which we are concerned include gamma rays and neutrons. The fission process itself is a prolific source of both of these; gamma rays are also emitted in the decay of many radioactive substances. Gamma radiation is very penetrating, and gamma rays characteristic of fission product decays are generally attenuated only slightly by tissue of the thickness of the human body. Neutrons also penetrate the body relatively freely, being scattered and slowed (moderated) by the light atoms in tissue. As a result, the effects of gainma radiation and neutrons usually can be expressed in terms of whole body radiation, in which all tissue is assumed to be exposed to a roughly uniform flux.

Biological effects are caused by the loss of energy along the path of the radiation; whether by collisions or ionization, there will be molecular damage. The energy loss is of ten expressed in terms of the linear energy transfer (LET), which is a measure of the density of ionizing events along the radiation path. A high LET implies large local biological damage. Beta and gamma radiation typically have a low rate of energy transfer to tissue, and are characterized as low-LET radiation. Alpha particles are high-LET because of their short range. Neutrons are also classified as high-LET; although their average energy loss rate over the path is small, when they are scattered by nuclei a large local energy transfer occurs. The recoiling nuclei have a very high LET. Thus, a neutron track is a series of relatively widely spaced high-LET events.

The effects of radiation exposure result from deposition of energy, conventionally specified as dose. Ideally, the unit of dose should be a measure of the effect produced. Current international recommendations attempt such a defintion in several stages. Historically, the problem of radiation protection and the need for radiation protection standards arose in connection with $\mathrm{x}$-ray (equivalent to gamma ray) exposure. The unit introduced at that time was defined in terms of resulting ionization density in air. In terms of energy deposition, the unit of gamma radiation exposure is the roentgen $=.0877 \mathrm{~J} \mathrm{~kg}^{-1}$ dry air at STP. However, the energy dependence of energy loss to tissue differs from that to dry air. A unit with more general significance is that of dose absorbed in the material of interest. The unit for Absorbed Dose is the rad, defined as the amount of radiation depositing $.01 \mathrm{~J}$ per $\mathrm{kg}$ of tissue.

Equal absorbed dose alone may not imply equal risks of any given biological effect since biological effectiveness may be affected by differences in types of radiation or in irradiation conditions. An indication of the effect on a given organ may be inferred by weighting the absorbed dose in that organ by certain modifying factors. The quantity thus obtained is called the Dose Equivalent. The modifying factors include a Quality Factor $(Q)$, which takes into account the differences in LET along the track of the particle in the tissue. Further modifications may be necessary to account for spatial nonuniformities in tissue absorption, radiosensitivity and the effect of dose rates (among others). Dose equivalent is defined as $H=D \cdot Q$. $\mathrm{N}$, where $\mathrm{D}$ is the absorbed dose, $\mathrm{Q}$ the Quality Factor and $\mathrm{N}$ comprises all other modifying factors.

The Quality Factor is determined by LET independent of all other exposure factors. LET for electrons (either beta radiation or resulting from gamma radiation) is nearly independent of energy and is the smallest of those for radiation. Accordingly, $Q$ for low LET radiations is defined $=1$. Alpha particles and recoils from neutron interactions are classified as high LET radiations and have values for $Q$ as a function of energy as high as 10 .

The modified radiation unit that takes into account different dose equivalents per unit energy loss is the rem (radiation dose equivalent, man). Although lacking the precise definition of the rad, use of the rem allows at least a rough comparison between differing radiations. One rad of alpha particles at energies characterizing fission processes is usually assigned a dose equivalent of $20 \mathrm{rem}$. Neutrons are assigned a value in the neighborhood of $10 \mathrm{rem}$ per rad.

Most of the radiation to which the public is exposed as a result of normal operation of the nuclear fuel cycle is low LET, as shown in Table $3 \mathrm{C}-1$. The sources are primarily released fission products and activation products, all of which emit beta and gamma radiation. The radiation exposure to which radiation workers are exposed may include high LET as well as low LET radiation. Neutron exposure is possible at the outside surfaces of reactor shields but is at completely negligible levels outside the reactor building. Alpha exposure is possible in the mines and mills as a result of emission from naturally radioactive uranium and its decay products and in reprocessing and fuel fabrication plants, where there is additional alpha emission from transuranic elements (particularly $\mathrm{Pu}$ ) produced as a result of neutron activation of the fuel. 
TABLE 3C-1. Identification of the major contributors to dose from the nuclear fuel cycle by portion of cycle, radiation type, and exposed group.

\begin{tabular}{|c|c|c|c|c|c|c|}
\hline & \multicolumn{2}{|c|}{ Radiation Type } & \multicolumn{2}{|c|}{ Exposure Group } & \multicolumn{2}{|c|}{ Exposure Mode } \\
\hline & Low Let & High LET & Occupational & Public & Internal & External \\
\hline Mining & & $\mathrm{x}$ & $x$ & & $\mathrm{x}$ & \\
\hline Milling & & $\mathrm{x}$ & $\mathrm{X}$ & (b) & $x$ & \\
\hline Refining & & & $X^{(a)}$ & & $\mathrm{x}$ & \\
\hline Enriching & & & $\mathrm{X}$ & & $\mathrm{X}$ & \\
\hline Fabrication & & & $\mathrm{x}$ & (c) & $x$ & \\
\hline Reactor Operation & $x$ & & $\mathrm{x}$ & $x$ & & $x$ \\
\hline Fuel Processing & $x$ & & $x$ & $x$ & & $x$ \\
\hline Waste Management & $\mathrm{x}$ & $\mathrm{X}$ & $x$ & & $x$ & $x$ \\
\hline
\end{tabular}

(a) Most of this work is confined to the processing of natural, depleted, and slightly enriched uranium which is generally agreed to be more of a chemical than radiological hazard. (b) Public exposure can occur if mill tailings are not properly controlled.

(c) If $\mathrm{Pu}$ is used in recycle fuel, then the potential for occupational and environmental exposure will exist.

\section{C2. Radiobiological Effects and Mechanisms}

Among the additional factors capable of influencing the likelihood of inducing biological effects are: rate of delivery of dose, the fractionation (i.e., periods of intermittent non-exposure), the spatial inhomogeneity of radiation and the radiation sensitivity of various organs. It is necessary to invoke modeling or inferences from the observation of actual biological effects in order to evaluate the roles of these latter factors.

Radiation effects manifesting themselves in the exposed individual are called somatic. The effects are called acute if they occur within several weeks of the exposure, or latent, in which case they may occur tens of years later. Acute effects follow the delivery of very large doses (many hundred rem) to all or most of the body within a time interval of hours. There is roughly a $50 \%$ chance that death will result. within several weeks from failure of blood forming tissue for doses of about 400 rem, while at much higher doses (thousands of rem) death will almost certainly occur in much shorter time as the result of failure of the gastro-intestinal system or even the central nervous system. For doses much below $100 \mathrm{rem}$, there are usually no acute somatic symptoms. Latent somatic effects in specific cases may include the appearance of leukemia and/or other malignant diseases, opacities of the lens of the eye, impairment of fertility, defective development of the fetus and non-specific life-shortening. Studies of somatic effects in animals have been carried out with dose rates ranging from about $0.3 \mathrm{rem} /$ day to about $100 \mathrm{rem} / \mathrm{min}$. Effects below $0.3 \mathrm{rem} / \mathrm{h}$ are inf requent and difficult to detect, as is discussed in Chapter V.

It is generally conceded that the most important somatic effect in man is cancer. For that reason, the analysis of health effects per unit of radiation dose has tended to focus on estimation of carcinogenic potential. Most quantitative estimates for this come from epidemiological studies of latent effects in which populations exposed either occupationally, purposefully in medical treatment, or in war. In such studies care has been taken to estimate the doses received. However, the degree of uncertainty in the dosimetric estimates depends on the study, conditions of exposure and on the observed effects in humans. Further uncertainties are introduced in extrapolating these observations to other conditions.

The radium dial painters have been a particularly important group in that the occurrence of bone sarcoma (cancer) in this group has been the basis for setting of standards for the body burden of bone-seeking alpha-emitting radionuclides. Uranium miners have been studied for the effects of radon daughters and other heavy-metal alpha emitters in the lungs. The survivors at Hiroshima and Nagasaki, the group accidentally exposed in the Marshall Islands, the groups who have undergone extensive external irradiation treatements for the treatment of various diseases--all have been studied to determine the induction of leukemia and other cancers as a function of exposure. These studies have been supplemented by animal studies, in particular, to infer the relative guidlelines for bone-seekers other than radium and the relative toxicities of various radionuclides.

Equally important are the mutagenic effects of radiation, which have been recognized since 1927. Early protection guidelines were based solely on somatic effects of radiation. Subsequently, both classes of effects have been considered. There is very little information which would allow one to estimate the mutagenic effects of ionizing radiation on man. The only mammalian work with a sufficient number of animals for valid statistics is based on studies on the mouse, where it has been observed that dose-rate effects are of importance. The data from which radiation standards have been derived are based on exposures in the range of $1 \mathrm{rem} / \mathrm{day}$ for female mice and slightly lower for male. It was noted in that work that repair of the oocyte damage appears to take place under conditions of chronic irradiation. Thus, most of the mutations originate in males. Mutagenic effects include both point and chromosome changes. Where the change occurs in germ cells, hereditary consequences are expected among the descendants of the irradiated individual. For those mutations which persist through many generations, it is immaterial whether the changed genes are introduced by many individuals who have received a small dose or a few individuals who have received a larger dose.

\section{C3. Internal Radiation Sources}

We have noted that the biological effect of a particular quantity of beta and alpha-emitting radionuclides is likely to be maximal if the miaterial has somehow entered the body. National governments generally have codified standards for whole-body radiation exposure, usually based on the recommendation of international or national committees on radiation protection (ICRP, NCRP, FRC) as shown in Table 3C-2. In order to construct recommendations for internal sources, it is necessary to consider the behavior or metabolism of the materials once inhaled or ingested. Depending on their entrance route and chemical form, individual elements may tend to concentrate nonuniformly within the body. Thus, their effect depends on the radiosensitivity of their effective host. Further, the time over which they may deliver their effect depends on their biological half-life. For instance, tritiated water remains in the body with an effective half-life of about eight days as compared to its inherent half-life of about 12 years. Thus, secondary standards, referred to as Radiation Concentration Guides, have been promulgated that specify limits to the concentration of various nuclides in air or in water for various chemical forms consistent with the radiation protection guide limits. These may differ from guides that refer to whole-body radiation, since internal activities may be distributed inhomogeneously and 
TABLE 3C-2. Dose-limiting recommendations (C. F. NCRP, 1971).

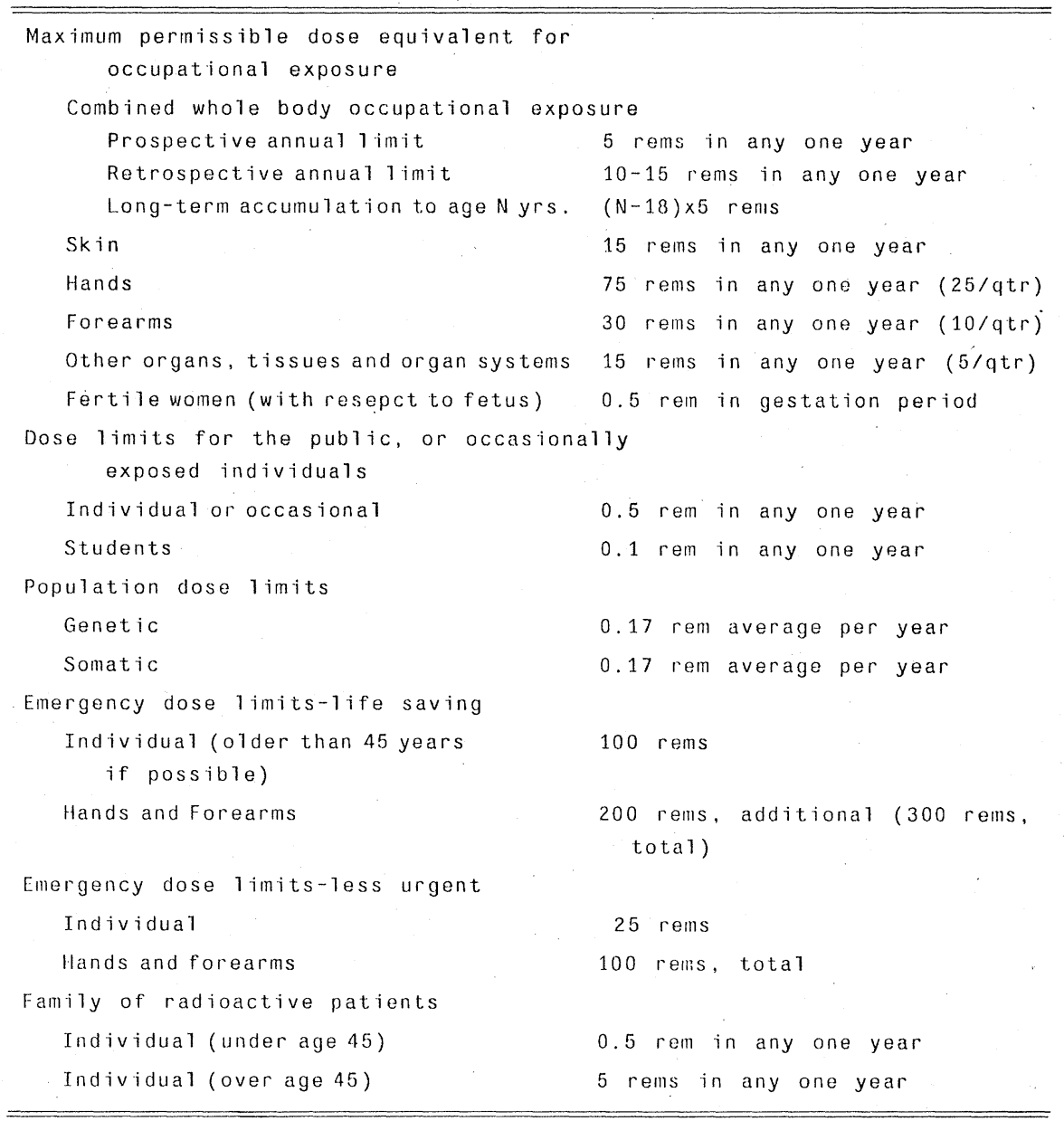

organs have differing sensitivities. For specific cases (such as iodine and the thyroid) for which there is known to be very inhomogeneous distribution and for which there is some knowledge of radiation sensitivity, these considerations are taken into account. But human data tend to be meagre, and there is continuing reassessment of methodology and standards. Additional uncertainty is introduced by the need to determine pathways for introduction of radionuclides into the biosphere, in order to utilize the concentration guides to estimate the extent of potential effects in a given situation.

\section{C4. Plutonium}

In Chapter V, we review plutonium as an example of an internal radiation source. Plutonium has been chosen because of a widely held perception regarding its toxicity. The biological effects of plutonium are due to its alpha radiations, which are classified as high LET. For reactor grade plutonium, the alpha activity over the first few centuries is dominated by ${ }^{238} \mathrm{I}$ 'u; at later times first ${ }^{240} \mathrm{Pu}$ then ${ }^{239} \mathrm{Pu}$ become relatively more important (c.f. Chapter VII). Like all heavy metals, plutonium would remain in the body were it to be taken up by tissue. The primary pathways into the body are by ingestion or by inhalation. Inhalation of fine plutonium particles is an especially important consideration in assessing potential plutonium health hazards. Small insoluble particles tend to remain in the lung if inhaled, although there are mechanisms that move the particles toward the lung periphery. Insoluble plutonium, when ingested, tends to be eliminated readily by the body. However, the potential radiotoxicity of soluble plutonium compounds was recognized when they were first produced over 30 years ago. It was found that the main sites for the deposition of soluble plutonium entering the body were the bones. Experiments on dogs have shown plutonium produces five to ten times as many osteosarcomas as radium delivering the same average dose. The increased effectiveness is because plutonium collects on the bone surface, irradiating the cells lining the bone, while radium disperses more uniformly through the bone, where its radiations are less effective. Thus, the maximum body burden for plutonium is $4 \times 10^{-}$ ${ }^{8} \mathrm{Ci}$, while that for radium is $2 \times 10^{-7} \mathrm{Ci}$. In Chapter $\mathrm{V}$, we discuss recent suggestions that these standards should be altered.

\section{C5. Radiation Exposure and Assessment of Risk}

Radiation dose is not a new, artificial experience. As shown in Table $3 \mathrm{C}-3$, humanity has existed in a radiation background from external cosmic rays and radioactivity of primordial and cosmogenic origin. The dose equivalents from these sources include internal emitted radiation primarily from beta active (low LET) ${ }^{40} \mathrm{~K}$ and alpha and beta active (high LET) decay products of the natural uranium and thorium chains ( 25 to $30 \mathrm{mrem} / \mathrm{y}$ ) and external radiation from the same sources. Smaller contributions are provided by cosmogenically produced ${ }^{14} \mathrm{C},{ }^{3} \mathrm{H},{ }^{7} \mathrm{Be}$ and ${ }^{22} \mathrm{Na}$. The dose equivalents from cosmic rays vary with altitude and latitude over a range of about 25 to 90 mrem/y in populated areas of the continental United States. Those from terrestrial radioactivity will depend on local soil composition and vary from 15 to $55 \mathrm{mrem} / \mathrm{y}$. The summed dose, averaged over population, is about 80 to $85 \mathrm{mrem} / \mathrm{y}$ with minima of about 65 mrem/y along the Atlantic Coast plain to about $125 \mathrm{mrem} / \mathrm{y}$ in Denver and even higher in other Colorado areas. Thus, there is broad spatial variability in the radiation environment which we inhabit. In addition to changes which occur as one moves from one place to another, variations in effective background dose may be attributed to 
TABLE 3C-3. Average body tissue does rates in "normal" regions of the United States (rem per year) (BEIR, 1972).

$\begin{array}{lc}\text { Natural Radiation } & \\ \text { External irradiation: } & \\ \text { Cosmic rays } & 44 \\ \text { Terrestrial radiation } & \underline{40} \\ & \\ \text { Internal irradiation } & 15 \\ 40 \mathrm{~K} & 1.6 \\ { }^{14} \mathrm{C} & <0.3 \\ { }^{87} \mathrm{Rb} & \sim 1\end{array}$

Man-Made

Medica1 and Dental (1970)

Diagnostic

Radiopharmeceutica 1

$72^{b}$

Other

Fallout (1970)

Occupationa 1 (1970)

Miscellaneous

0.8

7

Total:

commercial nuclear fuel cycle excluded.(1970)

Nuclear fuel cycle (1970)

Nuclear fuel cycle (2000) (estimated)

0.003

$<1$ a primarily ${ }^{226} \mathrm{Ra}$, ${ }^{222} \mathrm{Rn}$, and ${ }^{210} \mathrm{~Pb}$ and its daughters.

based on the abdominal dose.

material and design choices in housing, to fat/muscle ratio, and to sex. Time variability of dose rate occurs on seasonal time scale with moisture content of soil (up to $30 \%$ of ${ }^{40} \mathrm{~K}$ contribution to external terrestrial radiation source) and on a sunspot cycle scale (about 11 years) in which the cosmic radiation component of dose varies from a few percent to factors of several depending on latitude and altitude. Finally, the possible association of galactic cosmic radiation production with the occurrence of supernovae indicates that there may be small step changes in the level of cosmic radiation with subsequent changes in cosmogenically produced activities. Current evidence is that the average level has remained constant to within $10-20 \%$ over the past 30,000 years, with temporal variations of order a few percent of the average over the 70 year typical lifetime of an individual.

In Chapter V we compare the average public dose rate to present and any future generation due to the nuclear fuel cycle with that already due to natural background and with the fluctuations in natural background. It is worth noting here that, at present, man-made radiation sources (particularly diagnostic radiation sources) provide an average dose rate in the U.S. of roughly half average natural background rates.

The objectives of radiation protection guides are both to, prevent acute radiation effects and to limit the risks of latent effects to an acceptable level. However, the various ways in which dose can be turned into effect, as well as varying definitions of "acceptable", make consensus difficult to achieve. For instance, the effects can be expressed on a per capita or a total population basis, and can be evaluated annually or integrated over all future times; they can also be expressed either as a total effect or as an incremental effect superimposed on that arising from natural background. Further, the production of nuclear power from LWR's has benefits that accrue primarily to present populations whereas at least part of the risk, e.g., from waste, is delayed to generations in the distant future. Thus apart from the usual uncertainties in source terms and pathways to the biosphere, which make the radiation dose itself somewhat uncertain, the evaluation of effects and the basis for using conventional riskbenefit analysis are open to debate.

If the quantitative relationship between dose and the risk of an effect were known, an absolute calculation of risks would be possible, and societies or individuals could judge the degree of risk that would be acceptable in order 10 gain some benefit relative to other alternatives. At this time the relationship between dose and risk is not known precisely, nor are the risks associated with alternative procedures which might result in the same benefit. Most bodies responsible for setting limits have assumed that a linear relationship exists between dose and effect, on the basis that such a procedure is unlikely to lead to an underestimate of risks. However, it is important to distinguish between an estimate of risk as opposed to an estimate of upper limit of risk. The hypothesis used amounts to interpolation from observed effects to predicted effects. Observable somatic effects occur at doses greater than tens of rem delivered over periods on the order of hours, whereas for nuclear fuel cycle analyses, it is desired to make assessments of the effect of incremental doses and dose rates many orders of magnitude smaller than such reference values. We discuss these points further in Chapter $\mathrm{V}$ and consider the relative merit of the various procedures that may be used.

The final resolution of the cost-benefit decision clearly revolves about the public perception of short and long-term risks and benefits from nuclear power in comparison to those afforded by other viable energy alternatives. In Chapter $\mathrm{V}$, we indicate one basis for assessing the level of biological risk that seems to us to be most useful for such a purpose.

\section{Fuel Cycle Safeguards and Security}

Even while they were designing and constructing the first atomic bomb, the developers of nuclear energy viewed nuclear fission as a potentially limitless resource, one that could supply arbitrarily large quantities of energy for society. They also recognized that commercial nuclear development carried with it the seed of weapons proliferation. After the end of World War II, the U.S. sought to embargo the spread of nuclear technology and information unless strict international controls were established, as contained in the Baruch Plan of 1946. However, other nations refused to negotiate on that basis; the Soviet Union, in particular, created its own nuclear programs for both weapons and civilian power. By 1950, the U.S. shifted from its strategy of containing foreign nuclear development unilaterally. In a major new initiative the U.S. launched the Atoms for Peace program, modifying the Atomic Energy Act of 1946 to allow certain information to be released to its allies.

Under the 1954 revision of the Atomic Energy Act, the U.S. embarked on a policy of active dissemination of information on reactor and fuel cycle technology, and promoted the spread of commercial nuclear power throughout the world. Much U.S. information was declassified and released, and many foreign scientists and technologists were trained in the U.S. At the same time as it was promoting the dissemination of the technology, the U.S. sought commitments against the development of military uses of nuclear power and, both through bilateral treaties and through the creation of the International Atomic Energy Agency (IAEA), sought to provide for safeguards on the nuclear fuel cycle. It was recognized that both domestic and international safeguards were needed to control access to enriched uranium and plutonium and to prevent diversion of fuel cycle materials. Codification of adequate, comprehensive and reliable safeguards remains a critical task facing the world today.

It is not our purpose in this report to dwell on the complex political and institutional considerations required for protection of fissionable materials on a national or international scale. Instead, we essay in Chapter VI to perform an independent evaluation of some technical aspects of nuclear safeguards and of possible contributions of selected technological approaches toward protection of fissionable materials. As the context in which such technology would be used has a great deal to do with its practicality, we review below some salient features of the history of weapon and critical material control as a prelude to discussion of the possible role of technology.

\section{D1. Historical Perspective}

In the 1940's and early 1950's control of proliferation of weapon technologies and weapon knowledge was considered of highest priority, and all the work on nuclear energy remained under tight classification. In addition, the U.S. nuclear laboratories searched for 
TABLE 3D-1. Critical mass of uranium vs enrichment: Sphere, $19 \mathrm{~g} / \mathrm{cm}^{3}, 15 \mathrm{~cm}$ natural uranium reflector (Taylor, 1975).

\begin{tabular}{ccc}
\hline \hline Enrichment $\left(\%{ }^{235} \mathrm{U}\right)$ & Critical Mass of Core $(\mathrm{kg})$ & ${ }^{235}$ Content $(\mathrm{kg})$ \\
\hline 100 & 15 & 15 \\
80 & 21 & 17 \\
60 & 37 & 22 \\
40 & 75 & 30 \\
20 & $\sim 250$ & $\sim 50$ \\
10 & $\sim 1300$ & $\sim 130$ \\
\hline
\end{tabular}

various technical means to "control" a nuclear explosion. For uranium, dilution of ${ }^{235} \mathrm{U}$, "isotopic denaturing", with ${ }^{238} \mathrm{U}$ can serve as a means of protection because isotopic separation capability is required to obtain weapons-grade material. The critical mass of ${ }^{235} \mathrm{U}$ as a function of concentration is shown in Table 3D-1 for natural uranium used as a "tamper" to reflect neutrons back into the core. One should note that below $20 \%$ enrichment, the critical mass rises prohibitively. This is the basis for one technical strategy for protection of fissile ${ }^{235} \mathrm{U}$, or, with somewhat different dilution factors, for ${ }^{233} \mathrm{U}$ as well. The means for isotope separation presently used are based on slight differences in the mechanical motions of different mass isotopes, e.g., slightly different diffusion rates of gaseous isotopes through porous barriers. To achieve significant mass flow needed for separation from natural uranium, an advanced technology is involved with large capital expenditures and power requirements. So long as efficient small-scale isotope separation methods remain unavailable, isotopic denaturing of fissile uranium isotopes can be an important tactic for protection.

It was hoped initially that the ${ }^{239} \mathrm{Pu}$ used for weapons could also be "denatured" through the addition of ${ }^{240} \mathrm{Pu}$ in appropriate quantities. Although ${ }^{240} \mathrm{Pu}$ is not fissile by thermal neutrons, it has a fairly large fission cross-section at high energies; unlike ${ }^{238} \mathrm{U},{ }^{240} \mathrm{Pu}$ has a finite bare critical mass of about $50 \mathrm{~kg}$. Therefore, this is not dilution in the same sense that ${ }^{238} \mathrm{U}$, with its very high fission threshold and large absorption cross-section, dilutes ${ }^{235} \mathrm{U}$. The general principle of spherical implosion bombs is the compression of a less than critical mass to a denser, and therefore more than critical one. There is, at some point along the compression, an optimal time to initiate the chain reaction in order to maximize the yield. ${ }^{240} \mathrm{Pu}$ is a spontaneous neutron emitter at a rate of about 1000 neutrons per second per gram. The higher the concentration of ${ }^{240} \mathrm{Pu}$, the greater the probability that less than optimal initiation (preinitiation) will occur, limiting the weapon yield and making it rather unpredictable. It is for this reason that the distinction is of ten made between "weapons-grade" and "reactor-grade" plutonium. Tables 3D-2 and $3 \mathrm{D}-3$ list the critical mass of plutonium metal spheres as a function of the concentration of various plutonium isotopes. This should be compared with the isotopic concentrations predicted for reactor plutonium in the GESMO, as listed in Table 3D-4. It can be seen that the critical mass of plutonium does not depend strongly on isotopic composition over the range of mixes likely to occur in the fuel cycle.
Further work on the problem of preinitiation induced by ${ }^{240} \mathrm{Pu}$ showed that more sophisticated designs could reduce the difficulties. Other possibilities were investigated to seek either a plutonium isotope that could prevent the chain reaction from occurring, or one that would guarantee predetonation at very low "fizzle" yields. After many years of work, such efforts are generally conceded to have been unsuccessful. (IRT, 1975) No new diluent for plutonium has been discovered that cannot be separated by conventional, albeit sometimes risky and/or expensive, chemical procedures. Indeed, even plutonium oxide can be used directly for a nuclear explosive, with a critical mass of about $50 \mathrm{~kg}$ with tamper, (Willrich and Taylor, 1974), compared to less than $10 \mathrm{~kg}$ for pure plutonium metal.

Indeed, what the U.S. experts accomplished at so much cost and effort during World War II has now been repeated by expert design teams in other countries. Some have even gone to the effort of obtaining or producing the necessary materials and testing weapons to prove out their designs -- the USSR in 1949, the UK in 1953, France in 1960, China in 1964, and, af ter a long hiatus, India in 1974. The case of India is a particularly troubling one, in that the necessary plutonium was obtained by chemical separation of fuel irradiated in a research reactor originally obtained to explore the peaceful uses of atomic energy. However, it has also been argued that France did not set out solely to develop a weapons program (Scheinmann, 1965); their commercial nuclear research and development program provided them with an important technological and personnel base. This base already exists in many other countries.

It is important to distinguish carefully between the weaponmaking capabilility of subnational groups and states. A government wishing to embark on a nuclear weapons program can devote the resources necessary to produce the required material, either by constructing the large and expensive isotopic separation facilities or by the less expensive route of constructing a production reactor and the associated reprocessing facilities to produce weapons-grade plutonium. Neither route is likely to be within the means of a sub-national group, especially since they would have to operate clandestinely.

Widespread commercial use of plutonium, on the other hand, would create a new situation because of the large amounts of plutonium that would be involved. The concerns most frequently expressed center on (1) diversion of plutonium in shipment or from a

TABLE 3D-2. Critical Mass Data (Paxton, 1975). Plutonium (1975). Plutonium Metal (o Phase) Spheres.

\begin{tabular}{|c|c|c|c|c|c|c|c|}
\hline $\begin{array}{l}\text { Critical } \\
\text { Mass } \\
(\mathrm{Kg})\end{array}$ & $\begin{array}{l}\text { Neutron } \\
\text { Reflector } \\
\text { (Tamper) }\end{array}$ & $\begin{array}{l}P u-239 \\
(\%)\end{array}$ & $\begin{array}{l}\text { Pu }-240 \\
(\%)\end{array}$ & $\begin{array}{l}P u-241 \\
(\%)\end{array}$ & $\begin{array}{l}\text { Pu-242 } \\
\text { (\%) }\end{array}$ & "Other" & $\begin{array}{c}\text { Calculated } \\
\text { or } \\
\text { Measured }\end{array}$ \\
\hline 16.85 & bare & $\sim 94$ & 4.5 & 0.3 & -- & $1 \% \mathrm{Ga}$ & M \\
\hline 18.8 & bare & 75 & 21 & 3 & -- & $1 \% \mathrm{Ga}$ & M \\
\hline 19.68 & bare & 65.4 & 23.3 & 8.0 & 2.3 & $1 \% \mathrm{Ga}$ & C \\
\hline 23.02 & bare & 46.3 & 21.6 & 15.1 & 16.0 & $1 \% \mathrm{Ga}$ & c \\
\hline 25.83 & bare & 39.7 & 19.2 & 13.9 & 26.2 & $1 \% \mathrm{Ga}$ & C \\
\hline
\end{tabular}


TABLE 3D-3. Critical mass of plutonium vs isotopic composition (Taylor, 1975).

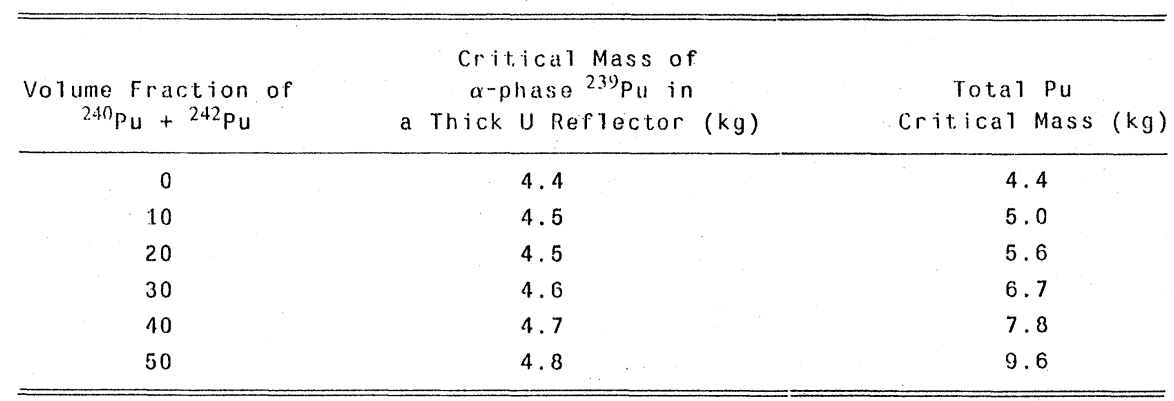

commercial separations facility by either national or subnational groups, or (2) covert or overt utilization of a commercial separations facility located within the territory of a nation which wants to move quickly to nuclear weapons status. A range of safeguards methods have been proposed to deal with such situations. We have already discussed the limitations of isotopic denaturing of plutonium. Reactor-grade plutonium, particularly if denatured with traces of high neutron emission rate isotopes, might be difficult for terrorist or other subnational groups to handle or separate. These problems are not much of a deterrent to a state, which has much greater resources at its disposal. It is of ten argued that a subnational group does not need a high-yield weapon. But this is to some extent true for a state, provided that the yield is not below the threshold of external detection; the demonstrated capability to design and construct a nuclear weapon is sufficient to elevate a state (e.g., India) to nuclearweapons status.

In spite of classification of weapons technology and design concepts, information gradually has become available not only to groups of experts in various countries but also to individual members of the public. As we review in more detail in Chapter VI of this report, the design principles for fission explosives are distributed quite widely in the open literature. A good example is provided by the Encyclopedia Americana article by John S. Foster, who is a well known expert on nuclear weapon technology and formerly the Director of one of the AEC's weapons development laboratories. His article presents a broad view of the nature of the nuclear explosion and requirements for its initiation. In addition, skilled people can use other information now in the public domain that was originally classified, concerning the measured and critical masses of various fissionable materials with several types of neutron reflectors, as well as the properties of explosives and other materials used in fission weapons.

A wide range of information is published in technical literature concerning the chemistry and metallurgy of plutonium and uranium. Of the two metals, uranium is the easier to handle by far, with respect to both radiological hazards and practical concerns like combustibility; given isotopic enrichment capability, uranium would offer the simplest and most straightforward route to a weapon. However, the procedures for handling plutonium metal also are well described. The required chemical and metallurgical apparatus for small scale operation also is available on the open market for those willing to practice in its use and subject themselves to some radiological hazards. In addition to the chemistry and metallurgy, details of the Purex process and even flow sheets of separation plants are public information readily obtainable. Of course such a design group would lack the advanced mathematical and experimental tools and data needed for sophisticated weapon designs. Many months of study and practice no doubt would be required. Nevertheless, based on the wide range of information and equipment available, a determined and intelligent group probably could create a credible fission weapon if they could gain access to 10 kilograms or so of plutonium or highly enriched uranium. The role of safeguards is to prevent or inhibit such access by either national groups or groups of individuals.

\section{D2. Physical. Security Measures and Technical Measures for Safeguards}

Proposed safeguards systems consist of both conventional physical security measures and technical measures whose aim is to prevent misuse of the fissile materials present in the nuclear fuel cycle. Physical security barriers are intended to contain fissile material in authorized channels and generally consist of standard measures, such as guard forces, which might be used in the protection of any valuable and/or potentially dangerous commodity. The level of implementation and therefore the cost required for adequate protection remains to be set by the Nuclear Regulatory Commission. Presumably, the GESMO Safeguards Supplement will help resolve such questions.

Technical safeguards refer to measures designed to account accurately for fissile material or to modify the fissile material so as to hinder its misuse. In Chapter VI, we provide an evaluation of some

TABLE 3D-4. Calculated fuel cycle plutonium composition (NUREG-0002, 1976).

\begin{tabular}{|c|c|c|c|c|}
\hline & 1 & 2 & 3 & 4 \\
\hline & Pu Recovered & Pu After & Pu After & Pu Recycle \\
\hline & From Spent U & One 4-year & Two 4-year & Mode1 BWR \\
\hline & Fuel & Recycle & Recycles & \\
\hline${ }^{238} \mathrm{Pu}$ & 1.9 & 3.46 & 4.87 & 3.4 \\
\hline${ }^{239} \mathrm{Pu}$ & 57.9 & 38.2 & 29.4 & 41.7 \\
\hline${ }^{240} \mathrm{Pu}$ & 24.7 & 29.4 & 33.5 & 29.2 \\
\hline${ }^{241} \mathrm{Pu}$ & 11.0 & 17.2 & 17.4 & 15.2 \\
\hline${ }^{242} \mathrm{Pu}$ & 4.4 & 11.7 & 14.9 & 10.4 \\
\hline$P u_{f} *$ & 68.9 & 55.4 & 46.8 & 57.0 \\
\hline
\end{tabular}

$* \mathrm{Pu}_{\mathrm{f}}={ }^{239} \mathrm{pu}+{ }^{241} \mathrm{Pu}$

Rev. Mod. Phys., Vol. 50, No. 1, Part II, January 1978 
proposed technical safeguards measures. Accountability obviously directly complements the conventional physical security safeguards; we will examine real-time accountability systems in considerable detail. Some perspective can be gained on requirements for accountability systems by comparing the amount of plutonium produced from reprocessing versus a critical mass needed for a plutonium weapon. A typical $1500 \mathrm{Mg} / \mathrm{yr}$ reprocessing plant produces about $30 \mathrm{~kg}$ of fissile plutonium per day. Comparison with Tables $3 \mathrm{D}-2$ and $3 \mathrm{D}-3$ shows this to be equivalent to two to five critical weapon masses depending on whether or not an efficient tamper is used. We consider in Chapter VI the potential contribution of real-time nondestructive assay methods for improving accountability.

Modification of the fuel form centers upon "spiking" or denaturing the fuel. Spiking refers to placing in or near the fuel materials which would make unauthorized access to the fuel dangerous; for example, intense gamma ray sources, such as ${ }^{60} \mathrm{Co}$, have been proposed. Denaturing would degrade the value of nuclear fuels for use in a nuclear explosive. As already noted in section D1, incorporation of neutron emitters might limit crudely designed implosion weapons to low-yields. The fact that different elements can be separated chemically has led to emphasis on isotopic denaturing, meaning denaturing with isotopes of the fissile material under consideration. We discussed that the most abundant isotope of uranium, ${ }^{238} \mathrm{U}$, serves to denature ${ }^{235} \mathrm{U}$; in fact $10 \mathrm{CFR} 73$ based on data such as given in the critical mass table (Table 3D-1), defines uranium with $20 \%{ }^{235} \mathrm{U}$ or greater as 'special nuclear material'. As discussed in the preceeding subsection, the situation for plutonium unfortunately is different. While only ${ }^{239} \mathrm{Pu}$ and ${ }^{241} \mathrm{Pu}$ are thermally fissile, all plutonium isotopes are fissionable in the fast neutron spectrum of a nuclear explosive device. Consideration of plutonium isotopic denaturing has focused upon the neutron background, which is fairly large for plutonium produced in high burn-up commercial nuclear fuel and which could be further increased with the addition of other isotopes such as ${ }^{252} \mathrm{Cf}$. Such approaches are examined in Chapter VI.

International safeguards considerations, especially reduction of the dangers associated with nuclear weapons proliferation, are having significant impact on domestic fuel cycle policy. One approach for reducing the possible impact of commercial nuclear activities upon proliferation is that offered by isotopically denatured fuel cycles. In particular, considerable attention has focused recently upon avoiding or at least limiting the "plutonium economy" by employing the ${ }^{233} \mathrm{U} / \mathrm{Th}$ cycle with denatured uranium. The main idea is to supply only isotopically denatured fuel to "national" reactors, with all plutonium-related activities restricted to "internationally controlled" fuel cycle centers. We provide a partial evaluation of a wide range of potential denatured fuel cycles, including the present ${ }^{235} \mathrm{U} /{ }^{238} \mathrm{U}$ cycle, in Chapters VI and VIII, together with some of the technical information needed for evaluating some of the benefits and debits of such proposals.

\section{E. Nuclear Fuel Cycle - Organizational and Institutional Structure}

On October 11, 1974 President Ford signed into law the Energy Reorganization Act of 1974, which abolished the Atomic Energy Commission and created two new agencies: the Energy Research and Development Administration (ERDA) and the Nuclear Regulatory Commission (NRC). The main purpose of the Act was to bring together in one agency, ERDA, federal activities relating to research and development of the various sources of energy, and to increase the efficiency and reliability of the use of energy. It was also an important purpose of the Act to separate the nuclear licensing and regulatory functions of the former AEC from the other nuclear power and development functions which were assigned to ERDA. ERDA's mandate in energy was broadened to include strong emphasis on all energy forms, as opposed to specializing on nuclear areas.

In addition to these two principal agencies, several other organizations of the federal government have responsibilities with respect to the fuel cycle; along with them the various states and the International Atomic Energy Agency (IAEA) also play important roles. Finally, the focus of economic concern is on utilities and private industry. It is important that the reader understand the roles of each organization and how they interact, since the institutional aspects of the fuel cycle have a major bearing on the efficacy of its options and alternatives. We will briefly outline here the defined responsibility of each of the individual organizations, and then in Chapter IX of the report we will focus on their interactions and problems related to these interfaces.

\section{E1. Energy Research and Development Agency (ERDA)}

ERDA is headed by an Administrator appointed by the President with the advice and consent of the Senate. Under the responsibilities of the Administrator the general functions of ERDA are spelled out as follows:

--Existing central responsibility for policy planning, coordination, support and management of research and development programs for all energy sources, including assessment of and policy planning for long-range energy research and development needs.

--Conducting and encouraging research and development of extraction, conversion, storage, transmission and utilization phases of all energy sources.

--Supporting and conducting environmental biomedical, physical, and safety research related to the development of energy sources and their utilization.

--Coordinating with other public and private R\&D activities.

--Developing, collecting, distributing and disseminating scientific and technical information on energy manufacture, development, extraction, conversion, transmission and utilization.

--Encouraging and conducting $R \& D$ in clean and renewable energy sources and on energy conservation.

--Encouraging and participating in international cooperation in energy and environmental R\&D.

--Increasing the supply of manpower for energy R\&D through assistance to education and training programs.

The 1974 Act established six areas of responsibility, each headed by an Assistant Administrator. One of these is the office of the Assistant Administrator for Nuclear Energy. Within the office is the Division of Waste Management, Production and Reprocessing with which we will be especially concerned in this study; it is this organization that is responsible for ERDA's programs on the uranium supply and enrichment as well as on improved reprocessing and waste management technologies for the back half of the fuel cycle. The responsibility of ERDA is to bring fuel cycle technology to a level that can be transferred to private industry, should that decision be made with regard to a particular portion of the fuel cycle. We consider ERDA's waste management technology program in detail in Chapter VII of this report.

\section{E2. Nuclear Regulatory Commission}

In Title II of the Act the duties and organization of the Nuclear Regulatory Commission are described. Five commissioners give direction to the organization and are selected by the President with the advice and conisent of the Senate. The Chairman is designated by the President and acts as the Chief Executive and Administrative Officer. A Director of Regulation is appointed to serve as a coordinator of the functions of the three main offices of the Commission which are described as follows.

1. The Office of Nuclear Reactor Regulation has the responsibility of licensing and regulating all facilities and materials within nuclear power plants. It also reviews safety and safeguards of such facilities including monitoring and testing. It may recommend changes in systems for safety purposes as well as research necessary for the discharge of the NRC's duties.

2. The Office of Nuclear Materials Safety and Safeguards has the responsibility for licensing and regulation involving all facilities and materials handling outside nuclear reactors. This involves primarily processing, transport handling of nuclear materials and includes provisions for safeguards against threats, thefts, and sabotage of such licensed facilities and materials. Licensing and regulation of waste management also fall under this office's responsibility. It also has 
safety and safeguards review responsibilities like those described for the Office of Nuclear Reactor Regulation.

3. The Office of Nuclear Regulatory Research is to engage in, or contract for, research which the Commission deems necessary for the performance of its licensing and regulatory functions. It is also directed to develop recommendations for research deemed necessary for the Commission.

Thus the Nuclear Regulatory Commission has responsibilities under the Act for regulating all parts of the nuclear fuel cycle. Under the Energy Reorganization Act, the NRC was given licensing responsibility for long-term storage and disposal of future high-level radioactive wastes arising from commercial operations. In addition, the National Environmental Policy Act (NEPA) provides the NRC the opportunity to review the environmental impacts associated with fuel cycle developments or facilities for which an environmental impact statement has been filed.

When the NRC was created, the regulatory basis for power reactors was already in existence. The NRC assumed the responsibility for developing similar regulations for commercial utilization of plutonium and for high-level waste management. In the preparation and evaluation of the Generic Environmental Statement on Mixed Oxide Fuels (GESMO), the NRC is charged with considering a broad range of health, safety, and environmental problems involved with reprocessing and plutonium use in LWRs, including the matter of safeguards that would be appropriate to the protection of the plutonium for wide-spread use. This review was expected to result in a ruling by the Commission sometime in the latter part of 1977. If the decision is for recycle, performance goais and regulations, standards and guides for the transport and safeguarding of plutonium will be required. If the decision is against it, then plutonium issues will become moot for the time being, and attention will focus on the management of spent reactor fuel--either on an interim basis or as a nuclear waste.

Recognizing that priorities need to be clearly assigned to meet the growing need for definition of waste management goals, the Commission has moved to establish waste management as a highpriority effort, and has made the commitment to develop waste management performance goals, specific regulations, standards and guides for a licensed high-level waste management facility. The management of low-level and transuranic waste is also undergoing extensive review by the NRC.

\section{E3. Environmental Protection Agency (EPA)}

A jurisdiction in the field of radiation protection standards was established by the Federal Reorganization Plan of 1970. It transferred to EPA the authority to advise the President on all radiation matters affecting public health. In addition, EPA was given a broad general mandate to insure the protection of public health from hazardous materials in the environment. This, as well as the opportunity to review environmental impact statements as defined under the National Environmental Policy Act, gives EPA considerable authority to review in detail the radiation protection and other environmental aspects of projects proposed by ERDA or industry, or NRC licensing proposals In practice, the jurisdictional relationship between EPA and the NRC has been defined such that the EPA has broad authority for setting standards for acceptable total radiation levels and ambient standards for the environment; the Nuclear Regulatory Commission has the responsibility for radiation emission standards for each particular facility of the nuclear fuel cycle. As indicated above, problems at this interface will be addressed in Chapter IX.

EPA's program thus has the following basic parts: (1) establishing the underlying principles for the evaluation and setting of general radiation standards to protect the public; (2) assessing health effects from such radiation; (3) evaluating the economics of risk reduction in order to advise the President and other agencies with regard to radiation standards.

EPA's role recently has been apparent from its advocacy of tighter general environmental standards for several specific effluents from the nuclear cycle. It has proposed new general environmental standards, in particular for ${ }^{85} \mathrm{Kr}$, and ${ }^{129} \mathrm{I}$. These guidelines are now being discussed in other parts of the executive branch. It is anticipated that the final ruling on these guidelines will be provided by EPA sometime in 1978 as part of final general ambient standards for the entire nuclear fuel cycle, including guidelines for high-level waste disposal.

\section{E4. Council on Environmental Quality (CEQ)}

The Council on Environmental Quality acts as a clearing house within the Executive Office of the President on all matters related to the environment. Its most important function concerns the review and recommendation to the President on the adequacy and implications of all environmental impact statements prepared by the federal agencies. As an example, when ERDA prepares an Environmental Impact. Statement concerning a federal waste repository, it will be reviewed by the NRC and by EPA as well as other agencies. The comments of these agencies as well as comments by members of the public will be considered by FRDA in issuing the final draft of the Statement. This draft and all of the commentary is then considered by the Council on Economic Quality in making its recommendation to the President on approval or disapproval of the impact statement in question. The Council already has had a major impact on preparation of documentation in this field through its review of the original GESMO draft. By returning that document to the NRC for further analysis, the CEQ in effect called for a much broader treatment of environmental matters and set the tone for all subsequent treatment of such matters by the agencies.

\section{E5. The States}

State governments historically have exercised the first controls over radiation and radioactive materials. States were originally concerned largely with the registration of X-ray machines and radium sources, and in some cases the licensing of $X$-ray technicians. In the 1950 's some State governments entered into agreements with the Atomic Energy Commission to take over the regulation of possession of small amounts of radio isotopes and of the disposal of low-level nuclear waste materials other than those classified as transuranic wastes. These are the so-called "Agreement States," which numbered 24 by the end of 1972 and had regulatory responsibility for about 8,500 licenses to regulate various kinds of nuclear materials. In addition, there are five waste burial facilities on State land which are licensed by State regulatory agencies.

In the last few years states have become more active in nuclear policy areas, especially with regard to waste management. New York and Kentucky have taken the initiative with regard to locations for the deposition of transuranic waste in their states. It is well known that the State government of Kansas played an important role in the rejection of the proposed Lyons, Kansas, waste facility which had been considered by ERDA for the deposit of high-level nuclear waste. In other states, Michigan as one example, the Governor has reserved for himself a role in decisions to locate any nuclear waste facility in State territory. The states certainly look to the federal government for development, consistent implementation, and regulation of the nuclear waste practices, but nevertheless wish to play an important role in the site selection for any future waste facilities.

\section{E6. International Organizations}

Decisions taken by international organizations and foreign atomic energy agencies have a direct influence on domestic policies and actions. Within the Organization of Economic Cooperation and Development, OECD, there are two agencies with nuclear interests and responsibility, the Nuclear Energy Agency. NEA, and the International Energy Agency, IEA. The other major European nuclear organization is the International Atomic Energy Agency, IAFA. In the case of NEA, IEA, and IAEA, the United States is a participating member, and therefore held to certain decisions made by these agencies.

\section{a. Nuclear Energy Agency (NEA)}

The NEA is primarily a coordinating agency, and is responsible neither for policy formation nor direct research. Its job is to facilitate agreements and cooperation between countries, to collect data and perform surveys, and to arrange joint programs. Its charge is specifically nuclear. NEA has studies on the international demand for reprocessing of plutonium, on fast breeder reactor development, on 
waste management, and on safety and regulations, as well as other areas. The agency has attempted to stay out of areas which are too heavily politicized, and to operate primarily on a technical level. With the U.S. recently joining the NEA, even more cooperation on waste management is expected. It is hoped that cooperative international programs on waste management will be developed further. Because countries have been reluctant to consider handling foreign-generated wastes, NEA envisions that each country will have its own national program in waste management, at all levels, low, medium and high. The desire of the NEA, however, is to foster cooperative agreements on finding one or two best sites, either within or outside of Europe.

\section{b. International Energy Agency (IEA)}

Unlike the NEA, the IEA is primarily a policy coordination agency, originally formed originally to coordinate OECD policy on fossil fuels and conservation in response to the 1973 OPEC oil embargo. It has since expanded its role to include some aspects of nuclear energy policy, particularly with regard to surveying $R$ \& D efforts in member states. The IEA also has a cooperative program concerning nuclear safety with which the U.S. is involved as a member.

\section{c. International Atomic Energy Agency (IAEA)}

For a number of years the IAEA has been the principal international coordinating agency for the nuclear power field. It has a wide span of activities ranging from information services through coordination of different national programs to facility inspection and safeguards verification. Of particular importance to this report is the IAEA role in the reprocessing and waste management aspects of the fuel cycle.

The IAEA was established in Vienna in 1957, largely as a consequence of U.S. initiatives under the Atoms for Peace plan in the early 1950's. The agency is part of the United Nations system, and presently has a membership of over 100 nations. Under its statutes, the agency is charged both with promotion of the peaceful uses of nuclear technology and with the responsibility to ensure that the technical assistance provided is not used in any way to further mililtary purposes. Such issues, as technology transfer, nuclear waste disposal, and safeguards fall under its aegis as a coordinating, inspecting, and verifying agency. The first set of agency safeguards, in 1964, was largely limited to control of items provided by the IAEA in its nuclear assistance role, and to voluntary arrangements. The safeguards role of the agency was expanded greatly with the advent of the Treaty on the Non-Proliferation of Nuclear Weapons (NPT) in 1970. Under this treaty, the IAEA has the special responsibility of providing safeguards to ensure that non-nuclear weapons states party to the treaty do not enter into nuclear explosive programs. In general, IAEA safeguards concentrate on verification of national programs, through the vehicle of an international inspectorate, and on the regulation and registry of transfer of nuclear materials. In exchange for acceding to the treaty, non-nuclear weapons states insisted on an even larger role for the IAEA in promoting the transfer of nuclear technology and equipment for peaceful uses of atomic energy.

A number of countries now have plans to proceed with nuclear power generation programs at an accelerated pace. These countries will also have to decide at an early stage of their program what should be done with the spent fuel from the power reactors; that is, whether to reprocess the fuel and, if so, how to manage that process. Thus, the same issues that concern the U.S. are being raised around the world with regard to the back-end of the fuel cycle. If a decision is made in a particular situation to store the spent fuel, long-term storage must be carried out under specific arrangements between the country contracting to supply reactor facilities and the country in which the reactors and the fuel will be located. However, if their desire is to recycle the fuel, then arrangements must be considered that will allow all the countries involved to realize their national goals in a way consistent with adequate safeguards for the materials.

The IAEA recently examined whether the best way to solve this complex of interrelated problems might be through international cooperation on a regional basis, in order to coordinate effectively the development and regulation of all steps of the fuel cycle. The advantages were stated to be not only economic benefits from large regional centers, but also improved manpower utilization, more efficient technological operations, more reliable waste management and disposal, better security of materials, and more effective international safeguards to be applied to plutonium separation and storage facilties. However, others (Chayes and Lewis, 1977) in the field have been more skeptical of the effectiveness of intermational control procedures and international safeguards. Views vary widely, ranging from those who are optimistic on the value and effectiveness of regional centers to those who feel that such centers may actually promote the widespread use of plutonium, thereby promoting the very proliferation of nuclear weapons they are intended to control.

Special fuel cycles intended to limit weapons proliferation will be examined in Chapter VIII of this report. The role of international organizations and institutions has been newly emphasized, however, by the Ford administration's October pronouncement concerning its new nuclear fuel cycle policy. After many months of study, the administration concluded that fuel reprocessing and recycling of plutonium should not proceed unless there was sound reason to conclude that the world community can effectively overcome the associated risks of proliferation. The present administration is following a similar course. This policy, and the international negotiations it supports, attempt to harmonize specific U.S. nuclear policies with much broader foreign policy considerations with major, albeit uncertain, implications for long-range U.S. domestic energy policy.

\section{E7. Utilities and Private Industry}

One of the cardinal tenets of U.S. domestic nuclear policy since the early 1950's is promoting involvement and leadership of U.S. private industry in the nuclear power field. In the case of LWRs, technology transfer to private industry was essentially complete. The nuclear portion of an LWR power station is manufactured by one of four private U.S. vendors, each of which has been in business since the early days of nuclear power and carries out its own extensive R\&D program. The power plants themselves are owned and operated by utilities which are responsible, as licensees, for safe design, construction and operation. The NRC's responsibility is that of regulator to ensure that the licensee and his contractors take all the necessary steps to protect the health and safety of the public.

In the front (fuel supply) end of the fuel cycle, mining and milling operations as well as fabrication of oxide fuel pellets and fuel rod assemblies are all carried out by private industry under NRC regulation. In the U.S., enrichment has always been performed at government facilities. The future of recent proposals to encourage the entry of private industry into enrichment in the U.S. is uncertain.

For the back end of the fuel cycle, both industry and government have assumed until recently that the same approaches to private entry would apply as had been the case for power reactors. Information on fuel reprocessing was declassified in the 1950 's, and a private company, Nuclear Fuel Services, built and operated the first U.S. commercial reprocessing plant in New York State in the early 1960's. General Electric constructed a plant at Morris, Illinois that was never operated owing to design problems with a new process. A larger reprocessing plant with advanced Purex technology was constructed in the early 1970's at Barnwell, S.C., by a consortium of Allied Chemical and Gulf General Atomic. Other private plants are on the drawing boards. Key portions of the Barnwell plant are completed, and the decision as to whether to complete and operate it is the most immediate practical matter involved in the overall reprocessing debate.

The importance of preparing adequate environmental impact statements under NEPA, as well as the role of Federal environmental agencies in their review, are well known to private industry. Nevertheless, there was still considerable shock at the course of events in which the original GESMO draft was rejected by CEQ and the Barnwell plant operating license approval held up by the NRC. The nuclear industry had not anticipated the extent of the broad public fuel cycle debate and the larger issues of safeguards, weapon proliferation and waste management which have been raised.

Spokesmen for private nuclear industry have generally stated that, in their view, further development of the nuclear fuel cycle is impeded by the lack of consistent industry-governmental relationships and the 
lack of a firm and reliable regulatory basis. As we have already noted, the Nuclear Regulatory Commission has been concerned with the widespread utilization of plutonium for some time, but it will be well into 1977 before a ruling can be given. Even if that ruling permits plutonium recycle, more time will be required to work out the specific regulations that will have to be followed with regard to packaging of the separated plutonium, safeguards, procedures, requirements for transportation, and requirements and approved technologies for waste disposal.

Because of the difficulties manifest in the private sector's role in the heavily regulated fuel cycle, it has sometimes been suggested that all parts of it in which plutonium or enriched uranium are handled should be under direct governmental control and operation Presumably this would include enrichment, reprocessing and mixed oxide fabrication. Others still advocate a strong role for private industry in fuel cycle supply and reprocessing-fabrication functions once the regulatory requirements and constraints are clarified. The choice of institutional relationships can have a significant effect on the way technology is developed and on the manner in which improved technology is introduced. Following our discussion of the status of fuel cycle technology, we will seek in Chapter IX to point out where the present relationships have a positive or negative influence in working toward safe and effective practice. 
Rev. Mod. Phys., Vol. 50, No. 1, Part II, January 1978 


\section{Chapter IV. LWR Fuel Cycle - Technology And Economics Of Reprocessing And Recycle}

\section{A. Issues, Conclusions and Recommendations}

\section{A1. Issues}

This chapter discusses and evaluates the technology and economics of fuel cycles, that would utilize reprocessing. It begins with an examination of the chemical treatment of spent fuel, especially the Purex process and its immediate derivatives, to recover either uranium alone or uranium and plutonium for further use as nuclear fuel. Conversion of uranium and plutonium into oxides and the refabrication of these oxides into mixed-oxide fuel elements are also considered. All safeguards issues are relegated to Chapter VI.

The individual steps of reprocessing and refabrication of uranium and plutonium have been practiced for many years. We ask are they now well understood? All have been implemented at the pilot-plant level, and many have been incorporated in full-scale plants. Does a sound basis now exist for the design of commercial reprocessing plants? No commercial reprocessing plant has yet operated in the U.S. at design capacity; in fact, no commercial reprocessing plants are operating in the U.S. today. Three have been built, of which one has operated for a time at reduced capacity and with some problems. A second has never operated because design deficiencies were uncovered during cold testing. Another plant stands ready to begin operation as soon as equipment can be added for the conversion of plutonium nitrate solution to plutonium oxide and for the soldification of the fission product waste stream. Thus, what can we say about the very important subject of operational reliability at industrial scale?

The reprocessing of spent fuels from reactors using thorium and fuels from breeder reactors is known to present some special problems. We ask what is the present status? Although the chemistry of the individual primary compounds is well known and the separation steps are understood, there is little if any operating experience with separation plants for such spent fuels. How adequate is experience available on the refabrication of recycle ${ }^{233} \mathrm{U}$ fuels, which leads to the buildup of ${ }^{232} \mathrm{U}$ and the need for additional shielding? Are further engineering and pilot-plant testing needed before industrial-scale plants to reprocess these materials should be built?

Such issues and the technical problems associated with the processes used for dissolving, purification, and refabrication both of today's LWR spent fuel and of possible future mixed-oxide and breeder fuels are summarized in this chapter. Although the methods of manufacture are tried and reliable for conventional LWR fuel, several questions arise concerning modifications that may be required to handle fuels produced when plutonium is recycled or when breeder reactors are employed. These are given special attention in the body of the Chapter.

The overall economics of various fuel cycle options are evaluated for both existing and proposed facilities. The evaluation includes assessment of the sensitivity of economic estimates to uncertainties in the cost of spent fuel reprocessing, in the future price of uranium ore, and in other factors. The effects of delaying the implementation of commercial reprocessing are also considered.

Because of the similarities in the chemistry and enginering of reprocessing spent fuel from reactors using thorium and from breeder reactors to those for LWR's, we have departed from our format of considering only the U-Pu LWR fuel cycle in Chapters IV, V, VI, and VII to present a summary of the reprocessing technology for these advanced fuel cycles here. For spent fuels containing thorium, questions similar to those for the Purex process are addressed, as well as others arising from additional complications. We consider to what extent we stand ready to practice the reprocessing and refabrication technology for advanced cycles in comparison to the LWR cycle.

The enhanced difficulties in dissolving the spent fuel containing thorium oxide, and of the greater corrosivity of the dissolvent, are discussed. The difficulties in separating radioactive zirconium from thorium are considered, as is the complication of large quantities of ${ }^{14} \mathrm{C}$ from HTGR fuels. Especially, we assess whether fuel from thorium cycles can be processed reliably using existing technology and engineering.

In the same sense, we consider the technologies available and proposed for reprocessing spent fuels from breeder reactors. The important question of fuel solubility is again addressed. The effects of the high fissile content in spent breeder fuels are evaluated, including an assessment of greater radiation damage to the solvents in the extraction steps potentially resulting from the higher burn-up and fission product content of these fuels.

The viability and reliability for refabrication of MOX fuel rods is likewise evaluated. We consider if mixtures of $\mathrm{UO}_{2}$ and $\mathrm{PuO}_{2}$ can be made in a way to ensure their easy solubility in nitric acid af ter use in a power reactor. We ask if a refabrication plant can be operated safely, recognizing the toxicity of air-borne plutonium oxide, the gamma and neutron activity of refabricated mixed-oxides, and the need to safeguard separated plutonium with great care.

From our considerations of the technological aspects of reprocessing and mixed oxide fuel refabrication, and of the associated economics, we arrive at a number of conclusions and recommendations.

\section{A2. Conclusions}

1. Present information on U.S., uranium ore leads to a very uncertain supply picture for the long-term. Nevertheless, available information on proven reserves plus potential resources indicates that lifetime ore commitments for LWR's through the year 2000 can be satisfied without recycle for all but highest currently projected LWR growth rates.

We have considered the effect of fuel reprocessing on the uranium resource requirements for light-water reactors if constructed according to a high-growth case of $600 \mathrm{GW}(\mathrm{e})$ by the year 2000 and a lowgrowth case of $300 \mathrm{GW}(\mathrm{e})$ over the same period. Without fuel reprocessing, proved uranium resources at $\$ 30 / 1 b$., i.e., "reserves", are adequate for the cumulative consumption through 2000 for the lowgrowth case but inadequate for the high-growth case. The ERDA estimates of potential U.S. resources of uranium at a cost up to $\$ 30 / \mathrm{lb}$ $\mathrm{U}_{3} \mathrm{O}_{8}$ indicate sufficient resources for the lifetime commitment for the high-growth case only if there is recycle of spent fuel, but for the low-growth case, potential resources will be adequate to fuel the reactors without recycle. However, even with absence of recycle, the larger potential resources of uranium available at much higher costs could be used for the high growth case without destroying the economic viability of nuclear power. In this later case, while fuel reprocessing and recycle are not required for lifetime commitments, the higher uranium costs introduce greater economic incentive for fuel reprocessing.

2. There are four major options for the disposition of spent fuel elements from the light water reactor cycle. Our analysis indicates that only $b$ ) and d) below offer suitable alternatives when both resources and fuel cycle economics are considered.

a) "throwaway", in which spent fuel is treated as waste for permanent disposal.

b) "stowaway", in which spent fuel is stored in a manner which does not preclude the later recovery and utilization of its energy content.

c) reprocessing to recover uranium only.

d) reprocessing to recover both uranium and plutonium.

Discharged fuel from light water reactors is an energy resource whose long term value presently cannot be determined. From both economic and resource points of view, stowaway of spent fuel is a reasonable alternative to reprocessing at this time. Irreversible disposal or throwaway of a major portion of the spent fuel would be inappropriate, at least for the next few decades pending the 
development of alternative sources of energy. Similarly, we can see no purpose at present to reprocessing and letting the plutonium follow the high level waste.

3. At this time, various uncertainties preclude an unequivocal assessment of the possible economic benefits of various recycle options.

3a. Sensitivity analysis of fuel cycle costs indicates that the dominant uncertainty in evaluating the economic benefits is that associated with the cost of spent fuel reprocessing; also very important is the uncertainty in future uranium ore costs. Especially important are uncertainties in the costs of constructing and operating future plants, uncertainties in the means of financing these plants, and uncertainties in the costs of required safeguards measures. With a $\mathrm{U}_{3} \mathrm{O}_{8}$ price of $\$ 28 / \mathrm{lb}$, we find an economic benefit if reprocessing costs are less than $\$ 292 / \mathrm{kg}-\mathrm{HM}$; a $\mathrm{U}_{3} \mathrm{O}_{8}$ price of $\$ 56 / \mathrm{lb}$ raises this breakeven cost to $\$ 474 / \mathrm{kg}-\mathrm{HM}$.

3b. A reprocessing cost of $\$ 165 / \mathrm{kg}-\mathrm{HM}$ gives a $9 \%$ reduction in fuel cycle costs and a $1 \%$ reduction in the net cost of nuclear generated electrical energy (a cumulative thirty-year saving of several billion dollars). This is our reference case, in which we assume semi-direct maintenance and median industrial financing for the reprocessing plant, no costs for safeguards, and a $\mathrm{U}_{3} \mathrm{O}_{8}$ price of $\$ 28 / \mathrm{lb}$. Other assumptions being the same, government financing with semi-direct maintenance leads to a reprocessing cost of $\$ 90 / \mathrm{kg}-\mathrm{HM}$ (14\% fuel cycle cost reduction), while high-risk industrial financing of a remotely maintained plant could lead to a reprocessing cost as high as $\$ 450 / \mathrm{kg}-\mathrm{Hm}$ (resulting in a net loss of $11 \%$ ). Safeguards costs will increase reprocessing costs but cannot be estimated prior to establishment of safeguards performance criteria (see Chapter VI). Future uranium ore costs are uncertain but are likely to increase over the long term.

3c. Only for government financing of a reprocessing facility or for a future era of very high uranium costs do we predict a financial incentive for uranium recycle only. Deferred reprocessing does not appreciably increase fuel cycle costs over that for prompt reprocessing and is more cost effective than reprocessing for uranium recycle-only with storage of recovered plutonium.

4. Reprocessing of spent fuel from water reactors has potential technical and resource benefits that must be weighed against social and political costs that are more difficult to quantify. The benefits include: the acquisition of experience in industrial-scale reprocessing which, with further development and modification, is important for advanced fuel cycle alternatives; the production of the plutonium that may be needed to fuel various advanced reactors; and significant reductions in uranium ore requirements for water reactors if uranium and plutonium are recycled. Separative work requirements would also be reduced.

Social and political costs arise from considerations such as: efforts to prevent the proliferation of nuclear weapons states and requirements for domestic surveillance for physical safeguards. Public and occupational health and safety in normal operation do not appear to be controlling variables. (See Chapter V).

5a. An essentially complete technical base exists for industrialscale chemical reprocessing of spent uranium fuel elements from light water reactors. The chemistry and engineering involved are well understood, have been tested in a variety of plants, and are unlikely to produce problems. The engineering of large plants is well advanced, having gone through several evolutionary stages. Large scale experience is needed in the chopping operation, the dissolving of $\mathrm{PuO}_{2}$, and in the conversion of $\mathrm{Pu}\left(\mathrm{NO}_{3}\right)_{4}$ to $\mathrm{PuO}_{2}$.

There presently exists one industrial-scale separations facility at Barnwell, S.C. This plant has been designed carefully and competently to minimize operational hazards and radioactive emisions. Proof of the reliability of some segments on a large scale has not been reached, but will very likely be obtained if this plant is operated. Such operation would test predictions of reliability and performance of an industrial-scale facility which integrates the best currently available technology and which depends on a carefully chosen combination of remote and hands-on maintenance and operation.
$5 \mathrm{~b}$. The technology of chemical reprocessing of mixed-oxide fuel rods from light water reactors is not complete; however, the logical extension and development of present designs shou!d lead to reliable and safe technology. Although the reprocessing chemistry is essentially unchanged from spent $\mathrm{UO}_{2}$ fuel, the presence of $\mathrm{PuO}_{2}$ in the starting fuel may make dissolution in nitric acid more difficult, requiring control of fuel rod refabrication or the addition of fluoride ion to the dissolvent. In addition, increases in the $\alpha$-activity in the process streams may complicate the separations chemistry. Experience is needed to understand these aspects of MOX spent fuel reprocessing more fully. The need to reprocess recycle fuel will not arise for many years because of the increasing accumulation of first cycle spent fuel. Therefore the need for this technology is less urgent.

5c. Reprocessing of spent fuel from reactors which use thorium requires extensive engineering development for the separation of uranium, plutonium, and thorium, from fission products and from each other. Enhanced difficulty in the dissolution of the spent fuel elements, requiring more corrosive dissolvents than for $\mathrm{UO}_{2}$ fuel elements, must be overcome. A more complicated solvent extraction technology will be required for thorium-containing fuels. Implementation of engineering and technology at the pilot-plant scale is required for reprocessing fuel elements containing thorium before advancing to full industrial-scale deployment.

5d. Reprocessing of spent fuel from breeder reactors also requires extensive engineering development, followed by experience at the pilot-plant scale. A modified Purex process seems promising for this purpose, but substantial modification of the dissolution and separation steps will be needed. The high plutonium content of the breeder fuels necessitates additional redesign for criticality contol. These modifications should be demonstrated before proceeding to full industrial-scale breeder fuel reprocessing.

6. Mixed-oxide fuel fabrication for LWR's has been developed and demonstrated at the pilot-scale level.

Commercial plants have not yet been needed on a full industrial scale, but designs are already much advanced for this purpose. We expect that reliable industrial-scale operation could be attained in conjunction with fuel reprocessing when and if commercialization is approved.

7. The technology of removing the gaseous radioactive effluents ${ }^{14} \mathrm{C},{ }^{129} \mathrm{I}$, and ${ }^{85} \mathrm{Kr}$ is available, although it has not been demonstrated at the plant level for ${ }^{14} \mathrm{C}$ and ${ }^{85} \mathrm{Kr}$. We believe these isotopes could be removed from the critical gas streams. A technology for removal of ${ }^{3} \mathrm{H}$ has been suggested, but has not yet been proved. The technology for safely sequestering these four effluents, once removed, has not been completely developed.

\section{A3. Recommendations}

1. If reprocessing-refabrication is to be a major component of the U.S. nuclear industry in the near future, we recommend that appropriate existing reprocessing facilities be completed and operated to gain experience with integrated technology on an industrial scale and, further, that the corresponding refabrication facilities be built and operated with a similar goal. We emphasize that resolution of the issues involved in the GESMO decision and in international fuel cycle safeguards strongly affects the timing of such operations.

2. Because of significant differences between the processing technology needed for LWR spent fuel and that for advanced reactor cycles, such as breeder and thorium fuel cycles, we recommend that technology and engineering of the dissolution and separations steps involved in the reprocessing of these later cycle fuels be advanced to a state of readiness sufficient for future engineering scale-up (See Chapter VIII).

3. Consistent with the recommendations in Chapter V, we recommend that adequate technology be developed for concentration, disposal, and isolation of the gaseous effluents ${ }^{129} \mathrm{I},{ }^{14} \mathrm{C},{ }^{85} \mathrm{Kr}$, and eventually for ${ }^{3} \mathrm{H}$.

4. We endorse the philosophy followed in the design of the Barnwell reprocessing plant that only solids and gases should leave the plant in radioactive form. The absence of a contaminated liquid effluent stream greatly enhances the control of radioactive wastes. 
5. We urge the prompt delineation of policies which are prerequisite to sound assessment of the benefits of reprocessing spent reactor fuels, especially with regard to safeguards constraints, direct versus indirect maintenance, and policies for government or private financing.

\section{B. Perspective on the Technology}

Spent fuel rods from today's light-water reactors contain plutonium, some higher actinides, and highly radioactive fission product elements, in addition to a residue of fissile uranium. At the present time about $2500 \mathrm{Mg}$ of irradiated spent fuel are being stored existing reactors are discharging spent fuel at the rate of $900 \mathrm{Mg}$ per year. The uranium and plutonium in this spent fuel are potentially valuable national energy resources. However, to be utilized they must be separated from the fission products and from each other, the uranium enriched, and new reactor fuel elements formed. Also, the fission products and residual transuranic elements from processing must be separated as waste and sent to permanent storage or disposal If this were accomplished, the need for freshly mined uranium would be reduced.

There are many technical difficulties associated with such operations on a scale sufficient to support the nuclear power industry, either at today's level or especially at the levels projected for future years. The separations must be performed in a chemical plant of special and secure design; new-type fuel-rod refabrication plants mus be designed and operated; the plutonium product must be protected very carefully from theft and sabotage; the fission products must be put in a form suitable for handling and isolation; and secure, safe long-term waste isolation must be provided.

For our evaluation it is important to distinguish several stages of evolution of nuclear technology toward a mature industrial-scale process. Another important consideration is the relationship between process maturity and the maturity of the corresponding regulatory requirements and constraints. Early development efforts typically take place on a small laboratory scale where a few people can examine the prospects and problems at minimum cost with rapid response to new ideas. Successful demonstration at laboratory scale of ten leads to an initial engineering scale-up where practical concerns can be examined but where the scale is still small enough and the approach flexible enough to accomodate innovation. Continued success may then lead to further scale-up to a pilot-plant demonstration, where investment and size result in an emphasis on evaluation of practical operation and verification of yield and cost projections. As a broader base of experience and data are gained during scale-up, it may be necessary to develop an enlarged or even a new regulatory methodology to deal with the new process. While an initial regulatory basis usually exists, the principal regulatory requirements and constraints generally evolve and mature along with the process itself.

Finally, a full industrial-scale demonstration may be undertaken to confirm the process and its costs prior to extensive industrial deployment. It is important to work toward reliable industrial-scale operation early in the deployment, but extensive experience with successfully demonstrated technology, i.e., a maturity or learning curve, is usually necessary to attain the ultimate in process yield, cost reduction and day-in and day-out reliability. We will try to clarify the degree of technological maturity of the processes for the various fuel cycles in our discussion, including aspects where regulatory issues have significant impact.

\section{The Chemical Reprocessing of Spent Fuel:}

\section{C1. The "Purex" Process for Uranium/Plutonium:}

The Purex solvent-extraction process, still thought to be the best and most reliable available, originated in the early fifties. A closely related process was used at the Hanford Engineering Works, the Savannah River Plant, and elsewhere for the separation of plutonium for military use; this process was modified and extended to recover purified uranium and isolate fission products. Although there are small variations in the types of equipment used in some process steps in modern plants, the chemistry of the process has not changed much since its origin. It is now a well developed production method.
The principal steps of the process, illustrated in Fig. 1, are:

1) Cutting the zirconium-clad fuel rods into short sections so that the contents can be dissolved in nitric acid. The chopped cladding material ("hulls") remains undissolved and, af ter washing, must be disposed of as a portion of the trans-uranic contaminated (TRU) waste.

2) Dissolution of the fuel into an aqueous nitric acid solution.

3) Separation of uranium and plutonium nitrates from the fission products by extracting the aqueous phase with a solution of tributyl phosphate ("TBP" or $\left.\left(\mathrm{n}-\mathrm{C}_{4} \mathrm{H}_{9}\right)_{3} \mathrm{PO}_{4}\right)$ in a hydrocarbon solvent. The reactions

$$
\begin{aligned}
& \mathrm{UO}_{2}^{++}+2 \mathrm{NO}_{3}^{-}+2 \mathrm{TBP} \rightleftarrows \mathrm{UO}_{2}\left(\mathrm{NO}_{3}\right)_{2}(\mathrm{TBP})_{2} \\
& \mathrm{Pu}^{+4}+4 \mathrm{NO}_{3}^{-}+2 \mathrm{TBP} \rightleftarrows \mathrm{Pu}\left(\mathrm{NO}_{3}\right)_{4}(\mathrm{TBP})_{2}
\end{aligned}
$$

reach an equilibrium which favors the transfer of the uranium and plutonium ions into the light organic phase when the aqueous phase has a high concentration of nitrate ion, as in concentrated nitric acid solutions. Tetravalent plutonium is more easily extracted than the trivalent state of the element. The ions of the fission products are strongly retained in the aqueous nitric acid phase. (For details, see Cleveland, 1970.)

4) Reduction of plutonium to the trivalent state, either with a chemical reducing agent or electrolytically, followed by reverse extraction with nitric acid. Now, because of the change in valence, the plutonium returns preferentially to the aqueous phase, which is easily separated from the organic phase still containing most of the uranium.

5) Purification of both uranium and plutonium nitrates by additional solvent extraction. to $\mathrm{UF}_{6}$

6) Chemical conversion of $\mathrm{Pu}\left(\mathrm{NO}_{3}\right)_{4}$ to $\mathrm{PuO}_{2}$ and $\mathrm{UO}_{2}\left(\mathrm{NO}_{3}\right)_{2}$

Figures 2 and 3 give additional details about the principal steps listed above. Other processing steps include recovery and concentration of nitric acid from several solvent extraction and dissolving steps, purification of the organic solvent, concentration of the highly radioactive liquid waste before temporary storage at the reprocessing plant, and decontamination of evolved gas streams before they are discharged through a tall stack.

The feed material to a commercial Purex plant would be typically a mixture of oxides containing about $96 \% \mathrm{U}, 1 \% \mathrm{Pu}$, and $3 \%$ fission products as obtained from LWR's in which the "burn-up" of fuel has reached about 30 to $40 \mathrm{MW}(\mathrm{t})$ day $/ \mathrm{kg}$. Such plants are ordinarily designed to handle feed material having somewhat higher ${ }^{235} \mathrm{U}$ content or higher Pu content from more prolonged irradiation, although at somewhat smaller plant capacity. They can also accommodate mixedoxide fuels, also at a reduced throughput.

Since it is essential in the Purex process to obtain nearly complete separations in the many solvent extraction steps, efficient countercurrent extractions are required. They require extensive interfacial areas between the phases. Moreover, to minimize radiation damage to the solvent and to avoid problems with near-critical masses in the units, small-volume equipment capable of high mass transfer rates is very desirable. Several kinds of extractors have been tried, starting with the conventional packed column (a vertical tube filled with irregular solid packing over which one of the liquid phases is dispersed to obtain a large interfacial area). This kind of equipment is used frequently for conventional chemical processing where the requirements are less stringent. For the Purex extractions, however, packed columns have to be very tall, complicating the design of a plant that must be shielded and cannot use external mechanical pumps.

An alternative extractor is the "mixer-settler", a succession of pairs of stirrers for mixing the phases with each other and settling vessels in which fluid motion is gentle so that the phases can separate from each other. Here the chief disadvantage is the large volumes of liquid which must be kept in the settler units, leading to chemical or radiolytic changes and possibly to critical masses. An advantage; however, is the ease of design for remote maintenance. 


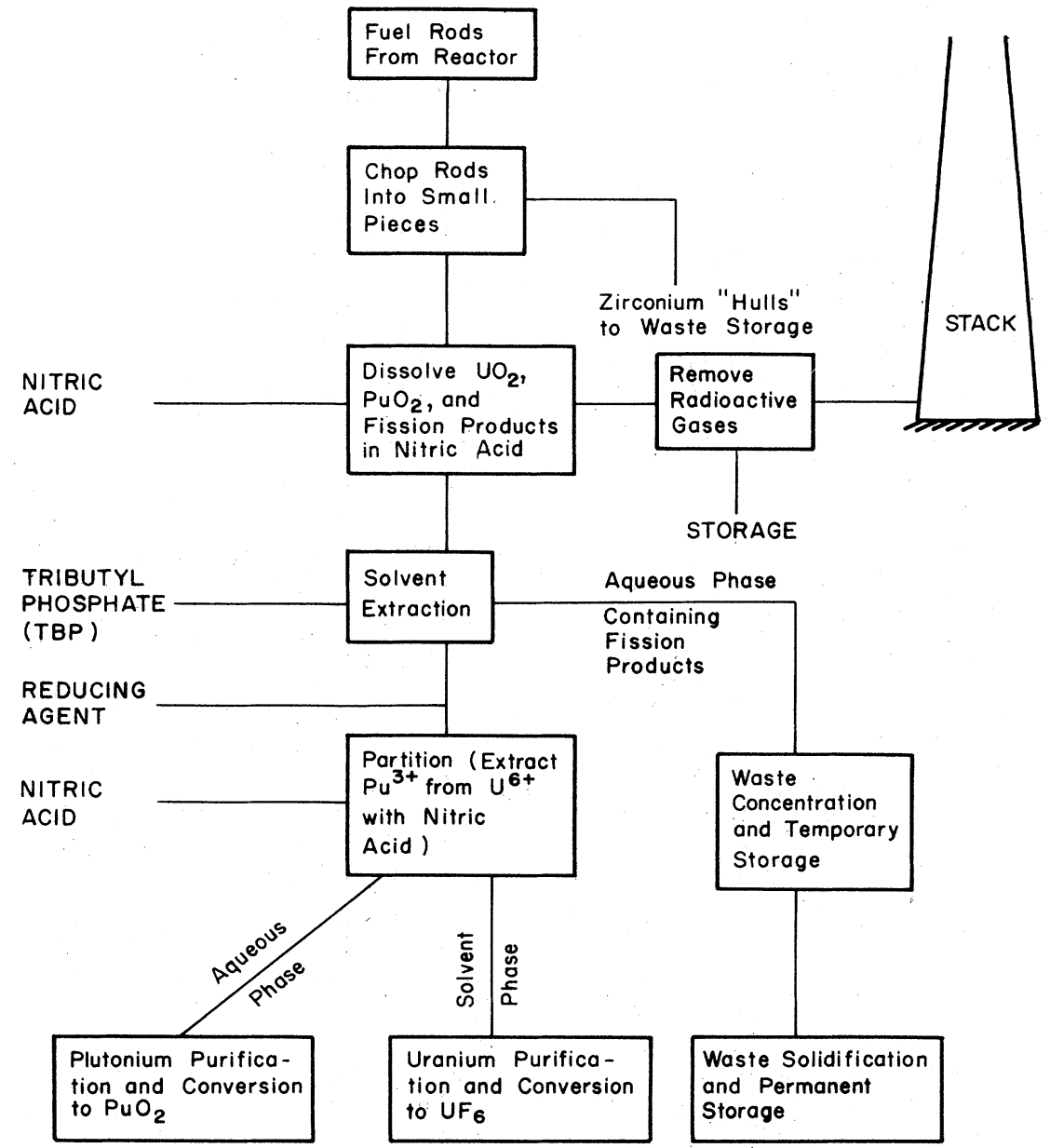

FIG. 1. Elementary steps in nuclear fuel reprocessing by the purex process.

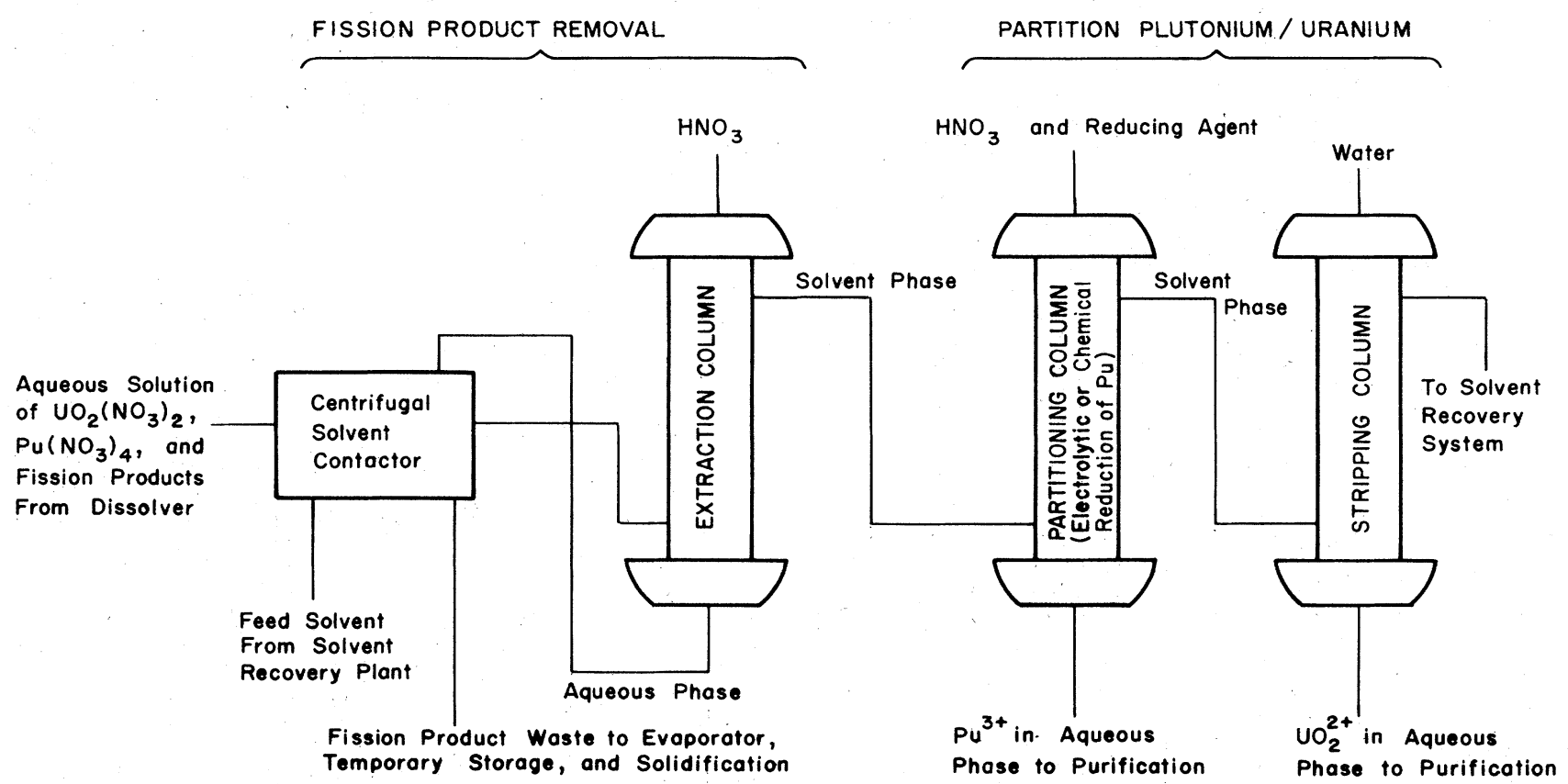

FIG. 2. Flowsheet for separation of uranium, plutonium, and fission products. 


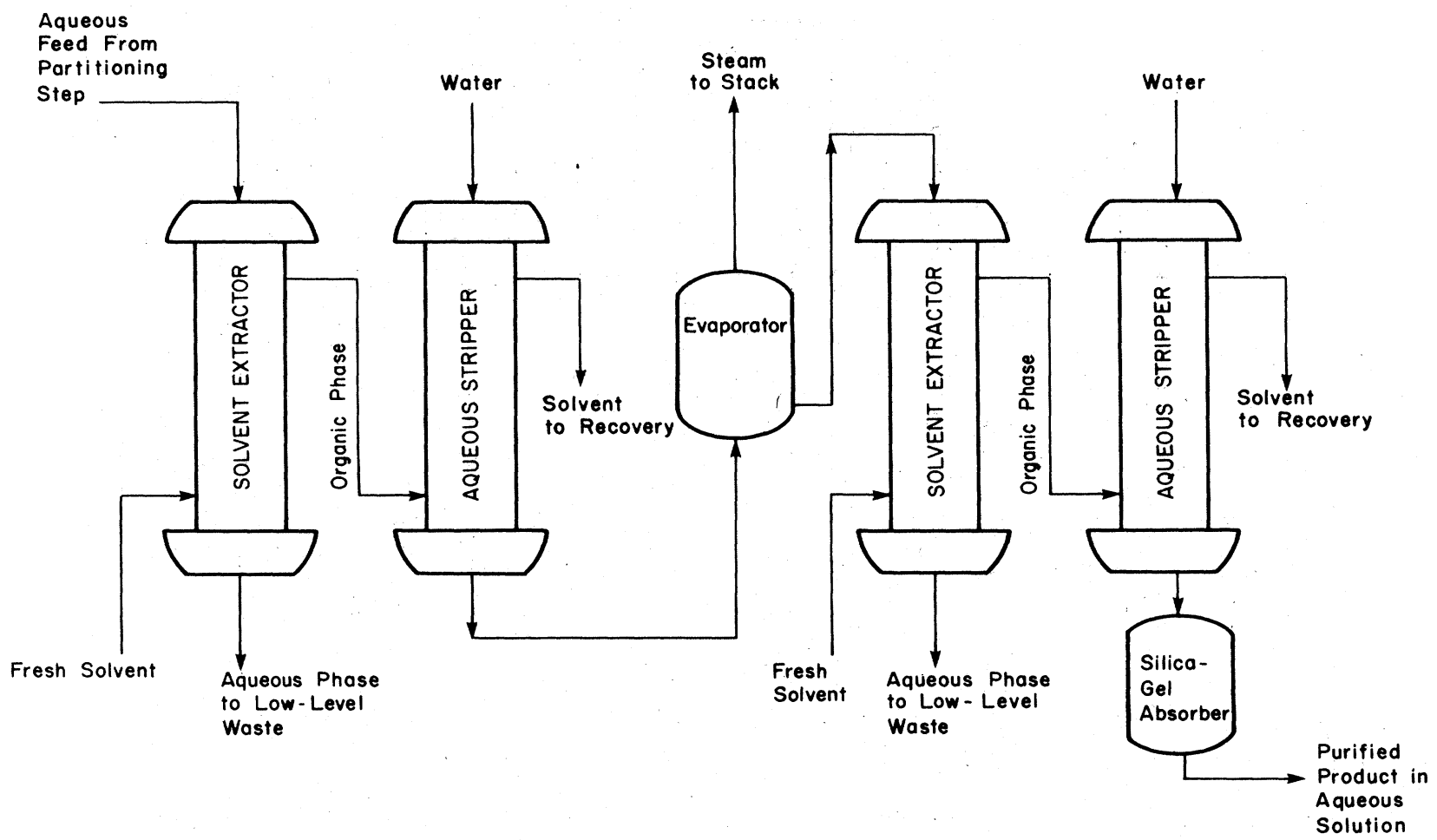

FIG. 3. Flowsheet for purification of uranium or plutonium.

An extractor generally preferred today is the "pulse column", which consists of a vertical tube containing horizontal perforated plates. The light and heavy phases pass steadily through the perforations but, in addition, the fluid motion is given an oscillating component. This causes the fluid to pass up and down through the perforations and increases the interfacial' surface area considerably without requiring a large liquid volume in the apparatus. Still another unit of recent design is the centrifugal contactor, in which phase separation is assisted by centrifugal force.

Such progressive development has produced machinery in which mass exchange between the phases and their separation from each other is rapid, even in equipment having small volume (Bebbington, 1976). Therefore, the time of exposure of the organic fluids to radiation leading to degradation is substantially reduced. Moreover, remote operation is possible.

\section{C1a. Solubility of Recycled Fuels}

A frequent question raised about the reprocessing of mixed oxide fuels centers on the solubility of the fuel rods when mixed oxides, not $\mathrm{UO}_{2}$ alone, are used for the feed. Results and experience of 5 years ago or longer have identified difficulties with complete dissolution of all of the plutonium oxide contained in MOX fuel rods.

If plutonium oxide is in solid solution with uranium oxide, it is soluble in nitric acid. In fact, evidence shows that irradiated fuel elements are more readily soluble than before irradiation (ORNLTM-3534, 1971). Tests run at Kerr-McGee showed 15\% Pu-85\% U oxide fuel dissolved readily in nitric acid, but $30 \% \mathrm{Pu}-70 \% \mathrm{U}$ did not. Homogeneity and solubility of mixed-oxide fuels manufactured from blended powders can be enhanced by sintering in an argon-air atmosphere at $1600^{\circ} \mathrm{C}$, followed by hydrogen reduction to control oxygen stoichiometry. Electron microprobe analysis of the sinter shows no traces of the original $\mathrm{PuO}_{2}$ particles and indicates a uniform distribution throughout the mixture (IAEA, 1967). The initial homogeneity of the fuel pellet is also an important factor in its later solubility.
If plutonium is not fully dissolved in the $\mathrm{HNO}_{3}$ process, the dissolver must be flushed and the solids filtered out. Alternately, $\mathbf{H F}$ can be added with $\mathrm{HNO}_{3}$ to increase solubility. The mixed $\mathrm{HF} / \mathrm{HNO}_{3}$ solvent is extremely corrosive. Furthermore, the presence of fluoride is undesirable.

It is probable that solubility difficulties can be avoided by careful control and preparation of mixed oxide fuels. (Schiefelbein and Lerch, 1971; Lerch, 1972) However, the technology has not been demonstrated on an industrial scale. Proper monitoring and detection capabilities for undissolved materials must be incorporated into the dissolver and related downstream piping and reservoirs. Timely corrective measures can then be taken.

\section{C1b. Radiation Damage to Solvents in the Extraction Train}

In the Purex separation process, dissolved uranium and plutonium are purified in sequential cycles to obtain the required decontamination factors of $\approx 10^{7}$. The concentrated plutonium is extracted with a solution of TBP in kerosene, $n$-dodecane, or some other hydrocarbon solvent and stripped with dilute nitric acid.

Several potential problems occur in this process. One involves the radiolysis of TBP, yielding dibutyl phosphoric acid. This compound forms very stable complexes with tetravalent ions, thereby inhibiting strongly the stripping of $\mathrm{Pu}^{+4}$. The quality of the separation between uranium and plutonium in the partitioning column is impaired, and plutonium travels with the solvent to process steps where the solvent is purified. Another problem arises from the reoxidation of $\mathrm{Pu}^{+3}$ by nitric acid in the aqueous phase after plutonium has been transferred from the organic phase. This reaction is catalyzed by one of its products, nitrous acid, causing plutonium to reenter the organic phase. Moreover, nitrous acid is soluble in the organic phase and tends to be carried toward the uranium-rich end of the partitioner and to promote production of organic-soluble $\mathrm{Pu}^{+4}$ throughout the column (Burkhart, et al., 1977), thus complicating control of the partitioning step. The strongly complexed $\mathrm{Pu}^{+4}$, and the ambivalence of the oxidation state, also complicates the separation of plutonium from zirconium, which occurs preferentially in the +4 valence state. 
With extended raliolysis, a third liquid phase called "red oil" is formed. Although this material is poorly characterized, it contains TBP, nitric acid, and uranium, or their radiolysis products. "Red oil," if formed, presents a special hazard in the separation stream as it is highly explosive and detonates readily. Problems of radiolysis of the hydrocarbon diluent are less serious, leading simply to chemical degradation of the solvent, which can be repurified by acid and alkaline scrubbing and if necessary by suitable fractionation.

Spent fuel assemblies are stored before reprocessing to permit decay of fission product radionuclides. Nevertheless, contact time of the radionuclides with the TBP must be minimized to reduce side effects due to radiolysis. Techniques for this are known, e.g. centrifuge extractors are available, and flow photolysis schemes are being investigated. The usual mixer-settlers and even pulse extraction columns may not be suitable, however, for high burn-up fuels because of relatively long contact times with TBP phase.

\section{C2. Reprocessing Spent Fuel from Reactors Using Thorium}

\section{C2a. General Features of Reprocessing Thorium Fuels}

Among the options for alternate fuel cycles available to the nuclear power industry, the incorporation of thorium into the fuel assembly to generate additional fissile material offers some important advantages. These thorium fuel options are described and evaluated in Chapter VIII. To take advantage of a fuel cycle with thorium, reprocessing to recover the bred fissile material is essential. The thorium-containing fuel cannot simply be reprocessed in a Purex uranium-plutonium separation plant because the chemistry of thorium-containing materials is different. Fuel with thorium oxide is more difficult to dissolve in nitric acid, and added fluoride is usually employed. Thorium separation is more difficult to control, and satisfactory partitioning of uranium from thorium presents extra difficulties. The presence of protactinium ftirther complicates the decontamination of uranium and thorium. Thorium reprocessing technology has been developed in the Thorex process, and limited experience with this process technology has been obtained.

An attractive implementation of thorium-containing fuels is in the high temperature gas reactor, the HTGR. Spent HTGR fuels will require reprocessing operations beyond those of the simple Thorex process. These differences arise because the high irradiation of HTGR thorium and the special design of HTGR fuel require special head-end reprocessing treatment. A more detailed description of HTGR thorium reprocessing is given in Appendix II.

\section{C2b. Thorium Reprocessing Technology}

The general features of the Thorex process are given here. The engineering philosophy of the Thorex process for treating spent reactor fuels containing thorium parallels that used in the Purex separation scheme, with additional complications described below. The principal steps are:

1. Chopping the fuel rods into short sections and dissolving the spent fuel. To dissolve thorium, a very concentrated $13 \mathrm{M}$ nitric acid solution with $0.05 \mathrm{M}$ hydrofluoric acid is used. In the early version of Thorex, $0.10 \mathrm{M}$ aluminum nitrate was also included. The presence of the fluoride ion to enhance dissolution results in a much more corrosive solution than that used for the dissolution of $\mathrm{UO}_{2}-\mathrm{PuO}_{2}$ fuel. The highly acid solution must be denitrated by evaporation to about $10 \mathrm{M} \mathrm{HNO}_{3}$ prior to solvent extraction. The highly oxidizing conditions of denitration require formic acid addition to suppress the oxidation and subsequent dispersion of fission-product ruthenium. Furthermore, $\mathrm{Al}\left(\mathrm{NO}_{3}\right)_{3}$, if used, adds to the ultimate solids in the high-level radioactive wastes.

2. Separation of thorium, uranium, and plutonium from the fission products by extracting the denitrated aqueous phase with a solution of TBP in a hydrocarbon solvent. Because the nitrate-TBPthorium complex is relatively weak, a concentrated TBP solution is used as the extractant, and concentrated nitric acid is added below the feed point of the extraction column to increase nitrate ion concentration. Careful control of conditions is required to separate fission product zirconium, including 65 -day ${ }^{95} \mathrm{Zr}$ from thorium in Thorex processing, because thorium, present in the tetravalent state, is chemically similar to zirconium.
Another problem in Thorex processing arises because of the relatively long half life (27.4 days) of ${ }^{233} \mathrm{~Pa}$, the precursor of the bred ${ }^{233} \mathrm{U}$, as contrasted with the 2.33 day half life of ${ }^{239} \mathrm{~Np}$, the precursor of ${ }^{239} \mathrm{Pu}$ in the uranium-plutonium fuel cycle. Consequently, a greater fraction of the potential uranium fuel will be in the form of its undecayed precursor at the time of reactor discharge in the thorium fuel cycle. 'If the potential fissile material is to be recovered, either the discharged fuel must be stored longer prior to reprocessing, or an additional separation must be added in Thorex processing to recover protactinium from the fission product raffinate from solvent extraction. Also, unless relatively long preprocessing cooling periods are specified for thorium fuel, the presence of chemically similar protactinium contributes to the difficulty of the achieving low levels of radioactive contamination in the separated uranium and thorium.

3. Reverse extraction to separate the uranium from the thorium. Highly irradiated uranium-thorium fuel will also contain appreciable quantities of plutonium, both from neutron absorption in the ${ }^{238} \mathrm{U}$ present in the enriched ${ }^{235} \mathrm{U}$ make-up as well as ${ }^{238} \mathrm{Pu}$ from the neutron-absorption chain initiated by non-fission capture in ${ }^{235} \mathrm{U}$. Plutonium will extract more easily than thorium, so it will follow the uranium stream and must ultimately be partitioned in a Purex extraction step to achieve a sufficiently decontaminated product.

4. Purification of the uranium (and possibly plutonium) nitrates, and subsequent processing to provide suitable fuel.

The technological basis for reprocessing thorium fuels 'is well understood, but much less experience has been obtained in its implementation than with the Purex separation scheme. However, a Thorex pilot plant was operated sucessfully on solutions of irradiated thorium at Oak Ridge over twenty years ago, and ${ }^{233} \mathrm{U}$ production campaigns with Thorex separations have been carried out in the AEC, now ERDA, facilities at Hanford, Savannah River, and Oak Ridge. Also, some of the first fuel reprocessed in the NFS plant, the first commercial reprocessing plant in the U.S., was thorium-uranium fuel from the first core of the Indian Point-1 nuclear power plant. Thus, small-scale ope:ation of the process has been achieved, but full-scale engineering design and subsequent demonstration would be needed to provide confidence in the Thorex process as a reliable element of the technology to provide nuclear energy in the United States.

Reprocessing of HTGR fuel will involve many additional operations not found in the earlier Thorex systems. (See Appendix II.) The problem of plutonium decontamination is only one of these. Another difference from earlier Thorex technology is the greater irradiation exposure of the HTGR thorium. When first developed, the Thorex process was used with fuels with relatively low exposure, in the range of 10 to $30 \mathrm{MW}(\mathrm{t})$ day $/ \mathrm{kg}$. The HTGR thorium will have been exposed to about $95 \mathrm{MW}(\mathrm{t}) \mathrm{day} / \mathrm{kg}$. Other differences arise from the design of the HTGR fuel and the special head-end treatments.

\section{C3. Reprocessing of Breeder Fuels}

Various breeder reactor cycles have been proposed to extend the resources of fissile materials. Some of these breeder options are described and evaluated in Chapter VIII. All breeder fuel cycles would require reprocessing to recover the fissile material generated in the reactor.

The first generation liquid metal fast breeder reactors (LMFBR) would use mixed plutonium and uranium oxides (MOX) as the fuel. Candidate fuels that are now being developed and tested as advanced fuels for future breeder reactors are the mixed plutonium and uranium carbides $(\mathrm{U}, \mathrm{Pu}) \mathrm{C}$ and the mixed nitrides $(\mathrm{U}, \mathrm{Pu}) \mathrm{N}$.

Because of high plutonium content, and because of higher irradiation, the complete dissolution of spent MOX breeder fuel can be difficult to attain. Furthermore, direct dissolution of carbides (U,Pu)C and nitrides (U,Pu)N in nitric acid is unlikely to be suitable (Tennery, 1976). For example, dissolution of carbide fuels in nitric acid results in the formation of soluble organic compounds that couple complex plutonium and may interfere with the solvent extraction steps of the separation processes. Therefore most proposed flowsheets for carbide and nitride fuels include an oxidation step at the head end to convert the plutonium and uranium to oxides before dissolution. 
The basic Purex separation process has many advantages over alternative separation schemes. Therefore, it would be desirable to adapt this process to high burn-up, high plutonium-content fuels. Baumgartner (Baumgartner, 1976) has done this successfully for kilogram quantities of fuel, but larger scale experience would be essential before proceeding to a full industrial-scale breeder reprocessing plant. The major problems to be addressed are the insolubility of $\mathrm{PuO}_{2}$, the radiolysis of TBP to form dibutyl phosphate with its concommitant adverse effects on the separations steps, and criticality control with the higher concentration of plutonium.

If the MOX were not completely soluble in nitric acid, then headend treatment in the reprocessing plant will be more difficult. Eithe fluoride would need to be introduced to catalyze the dissolution of $\mathrm{PuO}_{2}$, or the undissolved $\mathrm{PuO}_{2}$ would have to be removed by filtration or centrifugation. The $\mathrm{PuO}_{2}$ could then be dissolved in a smaller dissolver designed to minimize equipment corrosion.

The dissolver solution would go to a Purex type solvent extraction system to recover the uranium and plutonium. The larger radiation flux from the high burn fuel and the increased quantity of fission products will cause greater radiation damage of the organic solvent The radiolysis products will tend to complex with the plutonium and complicate efficient separation.

Thus, while modifications of the known Purex technology will probably be satisfactory for breeder fuel reprocessing, and indeed have been used for kilogram quantity processing, redesign of the head-end treatment and of the separation steps is required. Different of $f$-gas treatment may be necessary, and revised engineering for criticality control is required. Considerable scaling up is therefore needed to proceed from bench top technology to a pilot plant and eventually an industrial-scale reprocessing plant.

\section{C4. Plutonium Conversion Facilities}

In 1974 a Federal regulation was issued to prohibit the shipment of liquid plutonium nitrate as of 1978. Consequently all reprocessing plants are required to convert the liquid plutonium product from their plants to a shippable form. Technology is available for conversion to solid $\mathrm{PuO}_{2}$ by either direct denitration or oxalate precipitation and calcination.

One of the proposed reprocessing plants is considering the use of direct denitration, but such a process has never been used on more than laboratory or small pilot plant scale experiments.

-The Barnwell (AGNS) plant selected continuous oxalate precipitation followed by calcination to the oxide $\left(\mathrm{PuO}_{2}\right)$ for conversion. Their stated reasons for selecting this process were that it has been used for more than 20 years in many nuclear installations, the reactants and products are stable, and the process can be safely controlled. In addition, it is a process that can be operated in a semiremote mode. In this process, all of the solutions are moved by vacuum or by pumps, controlled by remote switches. The precipitate is removed on a drum filter which is scraped so that the solids fall into the feed end of a rotary calciner. The proposed Barnwell design is semi-remote in that normal operation is remote but maintenance of equipment will be done from a maintenance aisle through a glovebox type arrangement. Such an arrangement is designed to reduce occupational radiation exposure.

The conversion plant will accept plutonium nitrate solution from the reprocessing plants. The solution will be placed in feed preparation tanks where the acidity and plutonium valence will be adjusted. The acidity will be 3 molar, the plutonium, predominantly in the tetravalent state, will be about $150 \mathrm{~g} /$ liter, and the solution will be heated to the desired temperature. The adjusted feed and a $1 M$ oxalic acid stream will be fed continuously to a precipitation vessel. The slurry from the precipitation vessel will flow to a digester, where crystal growth is achieved, and from there to a rotary drum filter. The precipitate is fed to a rotary calciner where it is heated to $750^{\circ} \mathrm{C}$ to convert the $\mathrm{Pu}$ oxalate to $\mathrm{PuO}_{2}$. The calcined oxide is screened and blended to achieve a uniform product. The oxide is then sampled for analysis, packaged, and stored until the analytical data are available to determine if the product can be shipped to a fuel fabricator or if the $\mathrm{PuO}_{2}$ must be recycled to meet fabricator specifications. The filtrate will be concentrated by distillation and the evaporator bottoms returned to the reprocessing plant for recovery of the plutonium by solvent extraction. This arrangement will eliminate liquid discharges from the conversion plant.

This continuous precipitation process has been used successfully at Hanford for many years but on a smaller scale. Nevertheless, the Hanford experience plus the experience obtained in batch precipitation processes used at several other sites assures us that the technology is well known and workable. What remains to be worked out is the design, construction and operation of a large scale, semiremote operation as is proposed for the Barnwell plant.

C5. Release of Radioactive Substances in the Gaseous Effluent from Fuel Reprocessing Plants (Bonka, et al., 1974; Draft Environmental Impact Statement for LMFBR Appendix, Chapt. IV; ERDA-43, 1976; Trotter, et al., 1958; USEPA, 1973; UNSCEAR, 1972).

Fine particulates carried in the gas stream, including $\mathrm{PuO}_{2}$, are removed effectively with high efficiency filters and become part of the TRU waste. The release of radioactivity as gas from the fuel reprocessing step depends both on the type and history of the fuel and on the details for the reprocessing plant. A discussion of these wastes from proposed HTGR fuel reprocessing is given in Appendix II.

For light water reactors, essentially all of the radioactive fission gases are trapped physically or chemically in the zirconium cladding, in the fuel matrix itself, and in the fission gas plenum. These gases are released in the early stages of reprocessing, usually during chopping or dissolution. ${ }^{85} \mathrm{Kr}$ is released in the chopping and dissolving stages and goes into the off-gas system. Under current practice, some of the tritium is released from fuel or cladding during dissolution and goes into solution as HTO with only a small fraction (1 to $10 \%$ ) gcing into the off-gas. The remainder of the ${ }^{3} \mathrm{H}$ is retained by Zircaloy hulls. ${ }^{129}$ I exists in a wide variety of chemical forms within the fuel and accordingly is released in many forms, but usually as elemental iodine or organic iodides. It has been found largely in the off-gas, but signficiant amounts can enter a liquid effluent stream if one exists. ${ }^{14} \mathrm{C}$ is produced as an activation product from the action of neutrons on ${ }^{17} \mathrm{O}$, naturally occurring as a constituent of $\mathrm{UO}_{2}$, and on ${ }^{13} \mathrm{C}$ and ${ }^{14} \mathrm{~N}$ impurities. Its chemical form in the fuel or clad is unknown, although it is believed that release to off-gas would be as $\mathrm{CO}$ or $\mathrm{CO}_{2}$.

Representative values for the principal radioactive gaseous effluents released in the reprocessing of LWR fuel are given in the following table. The releases would also be expected to be appropriate for conventional natural $U$ fueled CANDU reactors.

\begin{tabular}{ll}
\hline Isotope & $\begin{array}{c}\text { Range of Activities } \\
\text { Ci } / \mathrm{GW}_{\mathrm{e}} \mathrm{yr}\end{array}$ \\
\hline${ }^{3} \mathrm{H}$ & $1.5-2.7 \times 10^{4}$ \\
${ }^{14} \mathrm{C}$ & $15-40$ a \\
${ }^{85} \mathrm{Kr}$ & $2.4-3.8 \times 10^{5}$ \\
& $2.3 \times 10^{-3}$ to $3 . \times 10^{-2} \mathrm{~b}$ \\
\hline
\end{tabular}

\footnotetext{
${ }^{a}$ The wide variation for ${ }^{14} \mathrm{C}$ is in part due to varying assumptions concerning the level of ${ }^{14} \mathrm{~N}$ impurity in fuel and cladding, including no release from cladding.

Value depends on the decontamination factor achievable at the reprocessing plant; the lower value corresponding to a D.F. of 500 represents current state of the art.
}

Techniques for removing each of these elements from the off-gas stream are known and many are discussed in the TAD (ERDA-43, 1976). Others which will merit consideration are being developed in Europe. lodine scrubbers have been incorporated into reprocessing plants in uxistence in the United States. The technology for high efficiency removal of ${ }^{129} \mathrm{I}$ is at hand. The difficulty of removal of the other elements increases in the order ${ }^{14} \mathrm{C},{ }^{85} \mathrm{Kr}$, and ${ }^{3} \mathrm{H}$.

Tritium is most easily handled as an effluent if it is in gaseous form. Once in solution, recovery becomes much more difficult, if not 
impossible. A method for retaining ${ }^{3} \mathrm{H}$ in gaseous form, called voloxidation, has been proposed. In this process, chopped, undissolved fuel is heated in air to $450-550^{\circ} \mathrm{C}$. $\mathrm{UO}_{2}$ undergoes chemical oxidation and a crystal phase change takes place. The fuel disintegrates and trapped gases are released. Approximately $99 \%$ of the ${ }^{3} \mathrm{H}$ and $50-75 \%$ of $I$ are released under these conditons. Air concentrations of several vppm are expected but seeding with hydrogen carrier to concentrations of $100-1000 \mathrm{vppm}$ makes it possible to trap HTO quantitatively on metal catalytic converters while maintaining the total volume of trapped gas at an acceptable level. Subsequently, HTO can be driven off and retained on molecular sieves. Voloxidation has been demonstrated only at the laboratory scale. It can not be considered suitable for industrial-scale use until pilot-scale and demonstrationscale studies have been made. If the humidity is low, the iodine released could be removed using molecular sieves. Several problems associated with the possible use of voloxidation in a reprocessing plant are identified in the TAD (ERDA-43, 1976). In particular, its use tends to increase the flow of air out of the plant and require much larger gas handling systems to maintain the plant at a pressure negative with respect to the outside ambient pressure.

The form in which ${ }^{14} \mathrm{C}$ is present in spent LWR fuel is not well understood and is a subject of current concern. ${ }^{14} \mathrm{C}$ is expected as a product from known nuclear activation processes. It has been found in spent LWR fuel in small scale laboratory experiments, but has not been observed directly in commercial reprocessing wastes. The sites and magnitudes of ${ }^{14} \mathrm{C}$ releases in reprocessing, and the chemical form in which it is released, will depend in detail on the design of the specific reprocessing plant and the head-end treatment. If voloxidation were used, ${ }^{14} \mathrm{C}$ present in the fuel and some of that from the cladding would be converted into $\mathrm{CO}_{2}$ and released at that stage. Methods for trapping and isolating $\mathrm{CO}_{2}$ are known, and could be incorporated into the off-gas stream.

Krypton is a noble gas and does not ordinarily enter into chemical reactions. Accordingly its removal may be effected only by the use of physical techniques such as adsorption on solids, absorption in liquids, or cryogenic trapping and distillation. Although these techniques are known in the cryogenic industries and have been used at Idaho and tested in Europe, they have not yet been used on industrial-scale reprocessing plants. Since Federal regulations now require the control of ${ }^{85} \mathrm{Kr}$ effluent release at future reprocessing plants, they will probably be part of any reprocessing plant operating in the U.S.

The process gas may need a pretreatment clean-up to prevent overloading or plugging of the piping associated with the cryogenic surfaces, and to limit the volume of trapped gas. Subsequent cyclic distillation and trapping allow decontamination factors of several thousand to be reached. The krypton can then be transferred to cylinders for isolation. Storage of gas for about a century will assure that the decontamination factor attained would not be negated by subsequent release. (Bohnenstingle, et al., 1976; Broothaerts, et al.; Glass, et al., 1974; ERDA-43, 1976; USNRC Report, 1976).

\section{Commercial Reprocessing Plants}

Three plants for the reprocessing of spent commercial fuel rods have been built in the United States to date and at least one proposal has been made to construct an additional plant by industry. One large engineering study of the cost of construction of a new plant is under way. The existing plants are (a) the Allied-General Nuclear Services plant (AGNS) at Barnwell, S.C.; (b) the General Electric Midwest Fuel Recovery Plant or Morris Operation (MFRP) at Morris, I11.; and (c) the Nuclear Fuel Services, Inc. facility (NFS) at West Valley, N.Y. The proposal for a new plant construction license is by EXXON Nuclear Co., to be built at Roane County, Tenn. The engineering study is in progress at the Engineering Department of E.I. duPont deNemours and Co. in Wilmington, Del., and is part of a larger LWR fuels reprocessing study which ERDA has contracted out to a number of National Laboratories, universities, and industrial firms.

None of these plants is operational today. Only the NFS plant has processed commercial spent fuel rods, but at present it is being used as a storage facility only. Tables 1 and 2 list some of the characteristics of the existing facilities.

\section{D1. Nuclear Fuel Services (NFS), West Valley, N.Y.}

\section{Dla. History}

An implicit policy for the total nuclear power industry in the United States over the past two decades has been that certain functions, including fuel rod fabrication, power generation, and spent fuel reprocessing, would be carried out by private industry. The AEC first attempted to have private industry undertake fuel reprocessing in 1956. In seeking bids, the AEC offered its already working defense reprocessing technology, assured a supply of spent fuel and a market for the product, set specifications for the products, set standards for in-plant operation and effluent releases to the environment, and agreed to let the reprocessor negotiate charges with its customers.

In 1962, Nuclear Fuel Services, Inc. (at that time Davison Chemical Co., a subsidy of W.R. Grace and Co.) negotiated to build and operate a privately owned demonstration (sic) commercial fuel reprocessing plant in West Valley, New York. The plant was designed to meet the established criteria for safety and security of the early 1960 's as laid down by the AEC, but the proposed construction was at a Spartan level, adequate to stay within the regulations, but not aspiring. to state-of the art technology.

The plant was to be used for a variety of spent fuels, partly because of their availability and partly to demonstrate the range of feasible reprocessing. It is located on a 3345 -acre site in a relatively remote area 28 miles from Buffalo, N.Y. On the site is the NFS reprocessing plant, a commercial solid waste burial ground operated by NFS, and a discontinued plutonium storage facility operated by the New York Atomic and Space Development Authority, ASDA,

TABLE 1. Irradiated fuel reprocessing plant site data and demography (USNRC, 1976).

\begin{tabular}{|c|c|c|c|c|c|}
\hline Plant and Location & $\begin{array}{l}\text { Plant } \\
\text { Capacity } \\
\text { Mg/day }\end{array}$ & $\begin{array}{l}\text { Site Size } \\
\text { Acres }\end{array}$ & $\begin{array}{l}\text { Major Nearby } \\
\text { Cities }\end{array}$ & Population & $\begin{array}{l}\text { Distance } \\
\text { Miles }\end{array}$ \\
\hline $\begin{array}{l}\text { Nuclear Fuel Services } \\
\text { West Valley, N.Y. }\end{array}$ & 1 & 3,500 & $\begin{array}{l}\text { Buffalo, N.Y. } \\
\text { Olean, N.Y. } \\
\text { Springville, N.Y. }\end{array}$ & $\begin{array}{r}463,000 \\
19,000 \\
4,350\end{array}$ & $\begin{array}{l}28 \\
25 \\
4.5\end{array}$ \\
\hline $\begin{array}{l}\text { Morris Operation }{ }^{\mathrm{b}} \\
\text { Morris, III. } \\
\text { (General Electric) }\end{array}$ & 1 & $890^{c}$ & $\begin{array}{l}\text { Morris, Ill. } \\
\text { Joliet, III. } \\
\text { Aurora, III. }\end{array}$ & $\begin{array}{r}8,000 \\
79,000 \\
74,000\end{array}$ & $\begin{array}{r}8 \\
15 \\
27\end{array}$ \\
\hline $\begin{array}{c}\text { Barnwell Nuclear Fuel Plant } \\
\text { Barnwell, S.C. } \\
\text { (Allied-General } \\
\quad \text { Nuclear Services) }\end{array}$ & 5 & $1,700^{d}$ & $\begin{array}{l}\text { Barnwell, S.C. } \\
\text { Aiken, S.C. } \\
\text { Augusta, Ga. }\end{array}$ & $\begin{array}{r}4,500 \\
16,000 \\
60,000\end{array}$ & $\begin{array}{l}7.5 \\
26 \\
33\end{array}$ \\
\hline
\end{tabular}

a NFS applied for a license to operate at $2.5 \mathrm{Mg} /$ day. This application was subsequently withdrawn.

b Formerly called the Midwest Fuel Recovery Plant, now used to store spent fuel.

c Adjacent to the Dresden nuclear reactor site of 2,230 acres.

d Adjacent to ERDA's Savannah River Plant exclusion area. 
TABLE 2. Fuel reprocessing plants-Major waste systems (USNRC, 1976).

\begin{tabular}{|c|c|c|c|c|}
\hline \multirow[b]{2}{*}{ Plant } & \multirow[b]{2}{*}{ Gaseous Effluents } & \multicolumn{2}{|c|}{ Liquid Wastes } & \multirow[b]{2}{*}{ Solid Wastes } \\
\hline & & High Level & Other than High Level & \\
\hline NFS & $\begin{array}{l}\text { To atmosphere via } \\
\text { iodine scrubber, } \\
\text { HEPA filters, } \\
61 \text { meter stack. }\end{array}$ & $\begin{array}{l}\text { Stored onsite as } \\
\text { neutralized } \\
\text { liquid in tanks } \\
\text { in vault. }\end{array}$ & $\begin{array}{l}\text { Controlled release to } \\
\text { creek. }\end{array}$ & $\begin{array}{l}\text { Stored onsite } \\
\text { in drums in } \\
\text { impervious } \\
\text { clay deposits. }\end{array}$ \\
\hline MFRP & $\begin{array}{l}\text { To atmosphere via } \\
\text { iodine scrubber, } \\
\text { sand filter, } \\
91 \text { meter stack. }\end{array}$ & $\begin{array}{l}\text { Converted to solid. } \\
\text { Stored onsite in } \\
\text { containers under } \\
\text { water in storage } \\
\text { basin. b }\end{array}$ & $\begin{array}{l}\text { Stored onsite in tanks } \\
\text { in vault as immobile } \\
\text { salt cake. }\end{array}$ & $\begin{array}{l}\text { Stored onsite in } \\
\text { in underground } \\
\text { lined vault. }{ }^{b}\end{array}$ \\
\hline AGNS & $\begin{array}{l}\text { To atmosphere via } \\
\text { iodine scrubber, } \\
\text { silver zeolite } \\
\text { adsorber, HEPA } \\
\text { filters, } 100 \text { meter } \\
\text { stack. }\end{array}$ & $\begin{array}{l}\text { Stored onsite as } \\
\text { acidic liquid in } \\
\text { tanks in vault. }^{c}\end{array}$ & $\begin{array}{l}\text { Stored onsite in tanks } \\
\text { in vault. }\end{array}$ & $\begin{array}{l}\text { Stored onsite } \\
\text { in concrete or } \\
\text { steel drums. }\end{array}$ \\
\hline
\end{tabular}

Note: Information in this table has been compiled from the safety analysis reports for these facilities. Only the NFS plant has actually generated waste to date.

a Plans were to add tanks for storage of high level radioactive liquids in acidic conditions for ultimate conversion to solids and transfer to a Federal storage facility.

b Prior to shipment to Federal storage facility.

c Plans for solidification of high level radioactive liquid wastes for transfer to Federal storage facility.

subsequently reorganized as the New York State Energy Research and Development Authority, NYSERDA. The land was leased from ASDA.

$$
\text { , }
$$

In April 1966 the NFS plant was issued an operating license. As agreed, the AEC then withdrew from commitments to reprocess fuel from privately owned reactors. Through 1971, NFS produced both purified $\mathrm{UO}_{2}\left(\mathrm{NO}_{3}\right)_{2}$ and $\mathrm{Pu}\left(\mathrm{NO}_{3}\right)_{4}$

\section{D1b. Technology}

The NFS plant recovered uranium and plutonium from spent fuel rods using the Purex separation method. Fuel elements removed from reactors were held for a minimum of 150 days to permit the decay of short-lived radionuclides. Much of the spent fuel processed by NFS had been aged considerably longer.

Processing proceeded by cutting off the nonfueled ends of the fuel rod assemblies and mechanically chopping the remainder into twoinch pieces, which were collected in a perforated stainless steel basket with a mild steel sacrificial liner to retain the chopping fines. The basket was loaded into the dissolver, $\mathrm{HNO}_{3}$ added and heated, and the solution analyzed for accountability purposes. The hulls and basket were intermittently monitored for residual $\mathrm{U}$ and $\mathrm{Pu}$ and were sealed with the assembly ends in scrap containers for burial.

The nitric acid solution was then processed using the conventional Purex method with pulsed extraction columns, and the separated solutions were concentrated, $\mathrm{Pu}$ to $250 \mathrm{~g}^{-1}$ and $\mathrm{U}$ to $300 \mathrm{~g}^{-1}$. Waste was concentrated to about 60 gal per MTU and committed to high level waste storage tanks as neutralized waste.

The facilities were a mechanical process cell, a chemical process cell, four Purex extraction cells plus equipment to clean up and recover $\mathrm{HNO}_{3}$ and solvents, six evaporators, two for waste, one for rework, two for $\mathrm{U}$, and one for $\mathrm{Pu}$, analytical laboratories with five hot cells, one storage cell, and six backup radiochemical laboratories, two 750,000-gallon mild steel storage tanks for neutralized high level waste, two 15,000-gal stainless steel tanks for acid waste, two lagoons for low level liquid waste, and the necessary water, ventilaiton, and utilities support. Criticality was controlled by geometry, solution concentration or mass control, and by the use of neutron absorbers. Maintenance was remote for the mechanical and chemical process cells, and contact for other operations.

The uranyl nitrate product from NFS was of adequate quality for conversion to $\mathrm{UF}_{6}$ and subsequent enrichment by gaseous diffusion; however most was taken by the AEC and reduced to uranium metal to meet government needs. The $\mathrm{Pu}\left(\mathrm{NO}_{3}\right)_{4}$ product was stored as an aqueous solution in plastic bottles in a facility provided by the New York State Atomic and Space Development Authority.

The wastes from the NFS plant include the fuel rod hulls, the high level fission products, transuranics and unrecovered $\mathrm{Pu}$, and processing wastes, in solid, liquid, or gaseous forms. The gaseous effluents have been released to the atmosphere through a 61-meter stack after passing through a series of HEPA filters to remove radioactive particulate matter with high efficiency. The fuel was aged sufficiently to allow ${ }^{131}$ I to decay. ${ }^{129} \mathrm{I}$ was not recognized as an important harmful effluent and was vented. High level wastes have been and are being stored on-site in tanks as neutralized liquids. This waste form is similar to that used by military plants at Hanford, Savannah River, and Idaho Falls. Low level liquid wastes have been released from the plant after proceeding through a system of lagoons. Solid wastes have been stored on site, in an inventoried burial ground, and low level solids have also been buried on site.

\section{D1c. Experience}

The NFS facility was used for a variety of fuels, including many not typical of commercial power reactors. Numerous technical difficulties arose, which were on the whole accommodated. The NFS plant capacity was $1 \mathrm{Mg}$ per day, with an expected working schedule of 300 days per year. During its active period it processed about 600 $\mathrm{Mg}$ of fuel, $100 \mathrm{Mg}$ per year, one-third of its design capacity. Most of the spent fuel, about two thirds, came from the Hanford N-reactor. This fuel had a high burn for a $\mathrm{Pu}$ production reactor but a low burn for a power reactor. It was metallic fuel, a $\mathrm{U}$ alloy in $\mathrm{Zr}$, not the usual oxide, and the mechanical configuration was also atypical. This spent fuel was contracted to NFS as part of the AEC agreement to guarantee feed for processing. Most of the remaining assemblies processed were conventional LWR spent fuels except one batch of uranium-thorium fuel from the Indian Point 1 reactor. This fuel was processed by the Thorex process using mixed $\mathrm{HNO}_{3} / \mathrm{HF}$, and the waste from this core was stored in the 15,000-gal stainless steel tank in acid form.

The NFS plant operated for over 5 years without exceeding the AEC limits of 10 CFR 20 required by its license. The liquid waste level in Catarraugas Creek was typically in the range of $10-25 \%$ of allowable limits. Nevertheless, some incidents did occur. One fire burned a hole in the dissolver tank. This was believed to result from $\mathrm{N}$-reactor fuel cladding becoming defective in transport and hydride being formed in the fuel assembly, which ignited on contact with acid in the dissolver. In another incident, an Allied Chemical $\mathrm{HNO}_{3}$ tank truck crashed into an NFS building. An operational problem was encountered when a line became plugged with solids. Direct 
maintenance was required, in which repairs had to be effected using short-term entry of personnel. These occupational exposures are reviewed in Chapter $V$. There were 13 occasions on which a total of 39 people were affected by inhalation of radionuclides. At one time, a HEPA filter bank failed owing to inadequate mechanical support.

\section{D1d. Changes in Performance Requirements}

NFS recieved a great deal of bad publicity, both on the grounds that its technology did not keep radioactive effluent levels "low enough", and on the questions of operator exposure and the use of temporary personnel. Increasing awareness and a changing social climate led to the implementation of "as low as reasonably achievable" as an operating criterion, as well as to major changes in AEC licensing requirements.

In response to these changes, NFS announced its intention to modify the plant and operations to:

1. reduce radioactive effluents

2. reduce personnel exposure

3. expand annual capacity from $300 \mathrm{Mg}$ to $750 \mathrm{Mg}$

4. increase security towards natural phenomena such as storms and earthquakes.

5. provide HLW storage compatible with waste solidification.

In 1972, recognizing its poor competitive position in the future reprocessing market, NFS shut its plant down for enlargement and modifications. They continued to operate a storage facility, and to prepare a revised and extended license application.

In October 1973, NFS (now a subsidiary of Getty Oil Corp.) applied for a license to modify the West Valley facility to comply with current and proposed regulatory practice and to increase capacity. They sought permission to process spent fuel for an additional 40 years. They proposed to follow the Federal regulation concerning effluents, and to redesign and reconstruct the plant accordingly. They also proposed to add a $\mathrm{UF}_{6}$ conversion facility, a $\mathrm{PuO}_{2}$ conversion facility, and a HLW soldification and storage unit. This would include six stainless steel tanks, each of 150,000 gal capacity, to be used for high level acid waste prior to solidification. Capacity would be increased to $750 \mathrm{MTU}$ per year.

\section{Dle. Present Status}

Much opposition was raised to this relicensing, partly on the grounds of the bad publicity from earler NFS operations. NFS stated that it could not meet the próposed seismic requirements without unacceptable cost penalties and construction difficulties.

The initial lease to NFS by the State of New York was from May 15, 1963 to December 31, 1980. Thereafter, at the option of NFS, one to ten renewals of ten years each could be made extending the effective lease to at least 2080. Significantly, an option for NFS to turn over custody of the site and of the wastes to the State was written into the lease. The detailed questions of decommissioning the NFS plant are less clear.

Four principal options were open to NFS:

1. withdraw completely from reprocessing.

2. limit the plant to its present level at the existing site.

3. expand operations at the West Valley site.

4. relocate, at the same or at some different level of operation.

In its 1973 application, NFS sought to pursue option (3). It has since, however, of ficially withdrawn its application, and has informed the N.Y. State - ERDA of its intention to transfer custody of 600,000 gallons of neutralized high level waste and low- and high-level solid waste burial grounds to the NYS-ERDA in accordance to the provisions of the lease.

\section{D2. General Electric Midwest Fuel Recovery Plant (MFRP) - Morris, Ill.}

\section{D2a. History}

The Midwest Fuel Recovery Plant or Morris operation of the General Electric Company was the second commercial reprocessing facility to be built in the United States. It was designed as a development project to determine whether a small reprocessing plant could be an economically viable entity, and if so, whether it could prove feasible and desirable to colocate a smaller reprocessing plant with a group of nuclear power reactors. The Morris Operation is located on an 890 acre site adjacent to the 2230 acre Dresden nuclear reactor site. It is about 15 miles from Joliet, Ill., and about 27 miles from Aurora. At this time the General Electric Company has elected not to operate its Morris facility, which is currently being used only to store spent fuel assemblies.

\section{D2b. Technology}

The Morris Operation is the only reprocessing plant not designed about the Purex separation. scheme; it used the "aqua-fluor" scheme. In this design, fuel elements removed from reactors would be held for a minimum of 150 days to permit decay of short-lived radionuclides. It was proposed to disassemble the spent fuel assemblies and pull the active fuel rods prior to chopping. The chopped rods would be transferred to the dissolver and the fuel dissolved in $\mathrm{HNO}_{3}$.

A single solvent extraction step would concentrate the uranium, plutonium, and neptunium fractions, and reject $98-99 \%$ of the fission products and unwanted trans-neptunium elements. The actinides would then be separated into three streams - $\mathrm{U}, \mathrm{Pu}$, and $\mathrm{Np}-\mathrm{by}$ ion exchange. The U-stream would be calcined to $\mathrm{UO}_{3}$ in a hightemperature fluidized bed, but the product would still be quite hot with $\approx 1 \%$ of the fission products. Therefore remote handling would be required for this and the next stage, a high temperature fluorination with $F_{2}$ gas to give $U_{6}$. This $U_{6}$ would then be cleaned up by passing through salt beds and by distillation. The $\mathrm{Pu}$ would be purified by ion exchange.

Results of preoperational tests with nonradioactive simulated spent fuels revealed technical difficulties with these remote'operations. It was judged too risky to attempt the high temperature calcining and fluorination steps as a completely remote operation. Anticipated maintenance and safety problems would have led to low productivity and high unit operating costs.

These difficulties might have been circumvented if one additonal solvent extraction cycle had been incorporated into the flow scheme. However, the Morris Operation was designed as a very closely coupled plant, with no holdup tanks between processes. This design has the advantage of a minimum inventory of radionuclides in the process stream, but reduced flexibility in operation. If one unit has to be shut down, it becomes necessary to shut down the entire plant. Also, the close coupling leaves little room for design changes.

Concern was also expressed about the ion exchange technology for purifying $\mathrm{Pu}$; since the plant was never operated hot, this aspect was never tested.

Criticality was to be controlled largely via geometry constraints. Gaseous effluents would be purified by an iodine scrubber and a sand filter bed, and then vented from a 91-meter stack. HLW would be converted to solid on site and stored under water in containers pending shipment to a Federal repository. Other liquid wastes would be retained on site in a waste vault as immobile salt cake. Solid wastes would be stored on site in an underground lined vault.

\section{D2c. Experience}

Experience with the Morris Operation has been limited to cold testing. However, problems with these preoperational tests were of such a magnitude that the management concluded some of the remote operations could not be performed satisfactorily. As a result General Electric has opted not to commission the plant for radionuclides.

The concept of the Morris Operation, namely a small reprocessing plant colocated with a number of nuclear power reactors, has not been evaluated with this trial. Neither this aqua-fluor nor a full volatility 
process appear to be promising technologies for the reprocessing industry. Difficulties can be anticipated with the remote, high temperature operations.

\section{D3. The Allied-General Nuclear Services Plant (AGNS) - Barnwell,} S.C.

D3a. General

The AGNS plant at Barnwell, S.C. was meant to be a commercial venture to reprocess light-water reactor spent fuels. The plant is larger than any commercial reprocessing plant heretofore built in the United States. In that sense, it is an important new step in the application of nuclear fuel reprocessing technology.

The Barnwell Nuclear Fuel Plant is owned privately by the AlliedGeneral Nuclear Services Company, which is in turn jointly owned by the Allied Chemical Corporation and the Gulf-General Atomics Company (USAEC, 1970, 1973). It is located on a 1700-acre site on the Savannah River near Barnwell, South Carolina. Aiken, S.C., and Augusta, Georgia are 26 and 33 miles away, respectively. Its design capacity is five metric tons of uranium per day or $1500 \mathrm{Mg} / \mathrm{yr}$ Designed by the Bechtel Corporation of San Francisco, California, and built by the Daniel Construction Company of Greenville, S.C., the plant uses an improved version of the Purex process. The principa variation from the conventional Purex system is electrolytic reduction of $\mathrm{Pu}^{+4}$ to $\mathrm{Pu}^{+3}$ in the partitioning step. This eliminates the need for a chemical reducing agent and the consequent disposal problems.

The plant is unique in that there are no contaminated liquid effluents. Any water introduced into the process with reagents such as nitric acid is either recirculated or discharged through a 100-meter stack as water vapor. Cooling water, which is obtained from deep wells, does not come into contact with process fluids in the plant.

Before entering the stack, gaseous effluents are sent through a mercuric nitrate liquid scrubber and silver-zeolite adsorbers to remove iodine, and efficient filters to remove particulates. No provisions were made for the removal of ${ }^{85} \mathrm{Kr},{ }^{3} \mathrm{H}$, or ${ }^{14} \mathrm{C}$ from the gaseous effluent streams because of the estimated low concentrations of these materials after dilution and release to the atmosphere.

Two 300,000 gallon stainless steel tanks, continuously cooled, are available for temporary storage of highly radioactive fission-product waste. Solid wastes, including the washed zirconium hulls, are stored on-site in steel drums. Low-level liquid wastes are stored on-site in tanks inside a vault. The plant includes facilities for converting recovered uranium into $\mathrm{UF}_{6}$ needed by enrichment plants.
Although plant construction was completed over a year ago and the plant facilities have undergone prestart-up tests, no license to receive and process spent fuel elements has yet been issued by the Federal Government. Facilities are not yet available at Barnwell for the conversion of plutonium nitrate solution into solid $\mathrm{PuO}_{2}$ or for the solidification of high-level waste. Design of such facilities is not yet complete and probably will have to await resolution of the national energy policy concerning use of mixed-oxide fuel in power reactors.

The original cost of the Barnwell plant is estimated to have been about $\$ 250$ million, including the cost of a UF facility, but excluding that of $\mathrm{PuO}_{2}$ conversion and high level liquid. waste solidification facilities; at 1976 prices the cost would have been about $\$ 600$ million. AGNS has proposed Federal funding for the remainder of the facilities. Tables 3 and 4 show the similarities and differences between the Barnwell plant and the older ones at NFS and MFRP.

\section{D3b. Provisions for Safety in the Barnwell Separation Facility}

The process for spent fuel handling at Barnwell is quite unlike the process of generating electric power from reactors. Temperatures are much lower and, rather than being high, pressure in process equipment is kept below atmospheric pressure. Moreover, the plant and operations are designed to prevent criticality accidents.

Nevertheless, because of the presence of fission products and fissile materials in process fluids and in storage, very careful attention has been given to safe design and safe plans for operation. A few provisions for the Barnwell plant are worth noting:

a) Use of only a few intra-cycle process vessels and employment of efficient, low-volume pulse-column extractors limits the in-process inventory of hazardous materials.

b) Process controls are of the conventional reliable type, but double protection is always provided. No single credible equipment failure or human error can cause criticality, which is prevented by geometric control, fissile material mass or concentration control, fixed and soluble neutron absorbers, or a combination of two or more of these methods. Both geometry and concentration control of moderators are used where needed for safety. Criticality control in the dissolver is provided by adding gadolinium to the solution, and the rate of dissolution is controlled to limit release of gases to the purification system.

c) Chemical damage to the solvent, and generation of the explosive "red oil" complexes of TBP, $\mathrm{HNO}_{3}$, and uranium in the

TABLE $3 .^{\text {a }}$ Principal similarities of the AGNS separations facility compared to other commercial reprocessing facilities (USAEC, 1973).

\begin{tabular}{llll}
\hline \hline Comparison Item & \multicolumn{1}{c}{ AGNS } & NFS & MFBR \\
\hline $\begin{array}{l}\text { Fuel Unloading and } \\
\text { Storage }\end{array}$ & Underwater & Underwater & Underwater \\
Headend Process & Chop-Leach & Chop-Leach & Chop-Leach \\
$\begin{array}{l}\text { Stored Fuel } \\
\text { Criticality Safety }\end{array}$ & Spacing & Spacing & Spacing \\
Fuel Chopping & Mechanical Shear & Mechanical Shear & Mechanical Shear \\
$\begin{array}{l}\text { Fuel Dissolution } \\
\text { Material }\end{array}$ & Nitric Acid & Nitric Acid & Nitric Acid \\
$\begin{array}{l}\text { Fuel Dissolution } \\
\text { Technique }\end{array}$ & Semi.ontinuous & (No Similarity) & Semicontinuous \\
Fuel Dissolution & Baskets in Dissolvers & Baskets in Dissolvers & (No Similarity) \\
Solvent Cleanup & Alternate Contact with & Alternate Contact with & Alternate Contact with \\
& Sodium Carbonate and & Sodium Carbonate and & Sodium Carbonate and \\
Final Exhaust & Roughing and HEPA & Nitric Acid Solutions & Roughing and HEPA \\
\hline \hline
\end{tabular}

a Final Generic Environmental Statement of the Use of Recycle Plutonium in Mixed Oxide Fuel in Light Water Cooled Reactors, NUREG 0002, Vol. 3 , Chapter IV, C-42. 
TABLE 4. ${ }^{\text {a }}$ Principal differences of the AGNS separations facility compared to other commercial reprocessing plants (USAEC, 1973).

\begin{tabular}{|c|c|c|c|}
\hline Comparison Item & $\begin{array}{l}\text { AGNS Separations } \\
\text { Facility }\end{array}$ & NFS & MFRP \\
\hline Location & South Carolina & New York & Illinois \\
\hline Design Capacity & $5 \mathrm{MTU} /$ day & $1 \mathrm{MTU} / \mathrm{day}$ & $1 \mathrm{MTU} /$ day \\
\hline Shearing & $\begin{array}{l}\text { Entire fuel elements } \\
\text { including end fittings }\end{array}$ & $\begin{array}{l}\text { End fittings removed } \\
\text { before shearing }\end{array}$ & $\begin{array}{l}\text { Pins removed from } \\
\text { fuel elements before } \\
\text { shearing pins only }\end{array}$ \\
\hline $\begin{array}{l}\text { Criticality Control } \\
\text { in Dissolver }\end{array}$ & Soluble poison & Geometric limitations & Geometric limitations \\
\hline $\begin{array}{l}\text { Fuel Dissolution } \\
\text { Technique }\end{array}$ & Semicontinuous & Batch & Semicontinuous \\
\hline $\begin{array}{l}\text { Fuel Dissolution } \\
\text { Equipment }\end{array}$ & Baskets in Dissolvers & Baskets in Dissolvers & Vibratory Leacher Tray \\
\hline HA Contactor & Centrifugal Contactor & Pulse Column & Pulse Column \\
\hline $\begin{array}{l}\text { Partitioning } \\
\text { Technique }\end{array}$ & Electropulse column & $\begin{array}{l}\text { Solvent extraction with } \\
\text { chemical valence adjustment }\end{array}$ & Ion exchange \\
\hline $\begin{array}{l}\text { Interim High-Level } \\
\text { Waste Storage Form }\end{array}$ & $\begin{array}{l}\text { Acidic solution } \\
(1-5 \text { molar })\end{array}$ & Basic solution & Calcined solid \\
\hline $\begin{array}{l}\text { Iodine Removal From } \\
\text { Process Off-gas }\end{array}$ & $\begin{array}{l}\text { Mercuric Nitrate } \\
\text { Iodine Scrubbers plus } \\
\text { lodine Silver Zeolite } \\
\text { Adsorption Beds }\end{array}$ & Silver Reactor & $\begin{array}{l}\text { Sodium Hydroxide } \\
\text { scrubbers. Heated } \\
\text { Silver Zeolite }\end{array}$ \\
\hline Process Vent Filters & Roughing and HEPA & HEPA & Packed Fiberglass Filter \\
\hline Final Exhaust Filters & Roughing and HEPA & Roughing and HEPA & Sand Filter \\
\hline Feed Clarification & Centrifuge & None & None \\
\hline Tritium Disposal & As vapor up stack & As water to creek & As vapor up stack \\
\hline Uranium Product Form & Nitrate solution & Nitrate solution & Hexafluoride \\
\hline Liquid Effluent & Noncontaminated & Contaminated & Noncontaminated \\
\hline
\end{tabular}

a Final Generic Environmental Statement of the Use of Recycle Plutonium in Mixed Oxide Fuel in Light Water Cooled Reactors, NUREG 0002, Vol. 3, Chapter IV, C-42.

evaporators are avoided by keeping boiling temperatures below $135^{\circ} \mathrm{C}$. The solvent is always below its flash point in air. It is continuously purified by washing with acid and alkali, and filtering.

d) Tritium accumulates in process solutions and is eventually released to the stack. Concentration is monitored and dilution or delay of release can be accomplished if needed.

D3c. Radiological Environmental Effects of Plant Gaseous Effluents and Their Possible Removal Before Discharge

The principal sources of radioactivity in the gases leaving the plant through the 100 -meter exhaust stack are the isotopes ${ }^{14} \mathrm{C},{ }^{85} \mathrm{Kr}$, ${ }^{129} \mathrm{I},{ }^{3} \mathrm{H}$, and a small fraction of the ${ }^{103} \mathrm{Ru}$ and ${ }^{106} \mathrm{Ru}$. Of these, tritium is the most difficult to deal with because it cannot be separated from the very much larger quantities of water vapor also leaving. However, the quantity of tritium released from the operation of this plant would not contribute significantly to the global environmental dose rate. (See Chapter V.)

${ }^{14} \mathrm{C}$ as $\mathrm{CO}_{2}$ could be easily removed by absorption into alkaline aqueous solutions using proven technology. ${ }^{131} I$ and ${ }^{129} I$ are released from the spent fuel during dissolution. At least $90 \%$ of the ${ }^{131} I$ and ${ }^{129} \mathrm{I}$ present is volatilized during fuel dissolution and is almost quantitatively removed by two $\mathrm{Hg}\left(\mathrm{NO}_{3}\right)_{2}$ scrubbers and one silver zeolite absorber. About $10 \%$ enters the process streams and eventually leaves through the stack because there are no radioactive liquid effluents. After absorption into $\mathrm{Hg}\left(\mathrm{NO}_{3}\right)_{2}$ solution and deposition on silver zeolite, the residue presents a potential thyroid dose of only 4 $\mathrm{mrem} / \mathrm{yr}$ from ingestion of contaminated milk plus the whole-body exposure. This amounts to 0.3 percent of the individual limit of 1500 $\mathrm{rem} / \mathrm{yr}$.
${ }^{85} \mathrm{Kr}$ is difficult to remove because of its chemical inertness, but it can be adsorbed preferentially on porous solids or it can be condensed cryogenically. Processes for accomplishing this have been tested on small scales, but operating experience does not yet exist. If recovered and concentrated, the gaseous ${ }^{85} \mathrm{Kr}$ would have to be stored under pressure. Provisions for adding ${ }^{85} \mathrm{Kr}$ removal capability exist at AGNS.

Ruthenium goes eventually into the high-level acid waste in various chemical states. Some may escape as the waste is ventilated but, fortunately, reduction of the acid solution with sugar, used to reduce acidity and corrosion of the storage vessels, makes the ruthenium less volatile.

\section{D4. The EXXON Reprocessing Plant Proposal}

The Exxon Nuclear company has announced (Exxon Nuclear Co., 1976a, Wall Street Journal, 1977) its intention to construct a nuclear fuel storage and reprocessing facility near Oak Ridge. Tennessee at a projected cost of $\$ 1$ billion. Initially a permit is being sought from NRC to construct a $\$ 300-$ million storage facility for spent fuel, but the long range plans include chemical plants for separating uranium, plutionium, and fission products and for producing $\mathrm{UF}_{6}$ and $\mathrm{PuO}_{2}$. The capacity of the integrated unit is expected to be $2100 \mathrm{Mg}$ of spent fuel per year. Design calculations are based on $8 \mathrm{Mg} /$ day ${ }^{235} \mathrm{U}$ enriched up to 8.5 percent or $8 \mathrm{Mg} /$ day of heavy metal as mixed oxide fuel containing up to 3.5 percent. $\mathrm{Pu}$ admixed with $U$.

Exxon plans to use the Purex process. The first operation in the chemical plant would be chopping of the spent fuel rods into small pieces about $1 \mathrm{~cm}$. long, followed by dissolution in - nitric acid. Addition of small amounts of $\mathrm{Ce}^{+4}$ ion is mentioned as a possible aid 
to the dissolution of $\mathrm{PuO}_{2}$ particles. Such an operation would require a tantalum or a titanium vessel. Evolved gases will be treated for recovery of $\mathrm{I}, \mathrm{Kr}$, and $\mathrm{Ru}$. Undissolved solids, including $\mathrm{Ru}$, are expected to amount to about $15 \mathrm{~kg}$ per $\mathrm{Mg}$ of heavy metal processed. They will be separated from the aqueous phase by centrifuging.

The solvent extraction plant will employ pulse-column extractors. Reduction of plutonium will be acomplished chemically rather than electrolytically, by addition of hydroxylamine nitrate. Four different extraction steps are incorporated in the design. One is separation of $U$ and $\mathrm{Pu}$ from fission products, another for separation of $\mathrm{U}$ and $\mathrm{Pu}$ from each other, and two for purification and decontamination of the plutonium stream. Solvent treatment will occur by washing with sodium carbonate followed by an ion exchange. Recovery of nitric acid and nitrogen oxides will take place by conventional means.

Plutonium nitrate will be converted into the oxide by heating it to 400 to $450^{\circ}$ in a horizontal-trough screw calciner, rather than through the wet oxalate precipitation process. It is expected that the oxide can be milled to produce a oxide having the required characteristics. We note that thermal denitration is a new process, not tested beyond the laboratory scale.

Uranium nitrate hexahydrate will be converted to $\mathrm{UO}_{3}$ by calcining in a fluidized-bed reactor. The reaction with fluorine will take place in a second fluidized bed. The UF 6 product is condensed on cold surfaces and can be purified by selective solid adsorbents and by distillation.

\section{D5. The DuPont Design and Engineering Study:}

At the request of ERDA the Atomic Energy Division of the DuPont Company's Polymer Intermediates Department at Savannah River, S.C. is engaged in an engineering study of an integrated plant to receive spent LWR fuel, reprocess it, store and solidify high level waste, produce decontaminated $\mathrm{UF}_{6}$ and $\mathrm{PuO}_{2}$, and manufacture MOX fuel rods. (E. I. DuPont de Nemours \& Co., 1976a) A conceptual design, to be completed in 1980, is being done by the Company's Engineering Department. (E. I. DuPont de Nemours \& Co., 1976b)

The plant is being designed for a capacity of $3000 \mathrm{Mg} / \mathrm{yr}$ or 10 $\mathrm{Mg} /$ day, corresponding to about 80 percent overall operating attainment. The feed material will be spent LWR fuel which has received reactor exposure of $33 \mathrm{MWday} / \mathrm{kg}$ and has been cooled for more than one year. It will contain no more than 1.6 percent ${ }^{235} \mathrm{U}$ and 1.0 percent plutonium ( $75 \%$ fissile). Feed material will have met dissolvability standards before irradiation in reactors. Fluoride will be used only rarely if necessary to assist solubility in the reprocessing plant. Over short periods feed material containing as much as 4 percent plutonium can be processed if the ${ }^{235} \mathrm{U}$ content is below 0.7 percent. Recovery of uranium and plutonium will be about 99 percent. The plant will not recover neptunium, americium or curium; they will go to the high-level waste.

Reduction of fuel rods to small pieces will be accomplished in two steps. The end pieces will be removed first in a "wet cell"; this will be followed by shearing in a "dry cell" having an argon atmosphere to minimize fire hazards from zirconium chips. The "Voloxidation" process is being evaiuated for releasing ${ }^{14} \mathrm{C}$ and ${ }^{3} \mathrm{H}$ as gases before dissolving the pieces, but DuPont has concluded that further development work is needed to make the process reliable for remote operation and remote maintenance.

As in the AGNS plant at Barnwell, the flowsheet provides for zero emission of liquid waste to nearby streams. High level liquid waste, intermediate level liquid waste, and new mixed-oxide fuel rods exit in solid form, zirconium hulls and other solid wastes are encapsulated and stored temporarily, and radioactive gaseous effluents are collected and stored again temporarily. Only very small concentrations of radioactive substances should appear in the large volume of air, about $4 \times 10^{8} \mathrm{cu} \mathrm{ft} / \mathrm{day}$, emerging from the tall stack.

It seems likely that the plant will be designed around the successful experience in ERDA operation of the existing Savannah River Plant, as described by Bebbington (Bebbington, 1976). The operations involving radioactive substances will be conducted remotely in two parallel concrete canyons within which maintenance will be carried out remotely. This is in sharp contrast to the Barnwell plant of AGNS, where the Purex purification steps after removal of fission products will be operated remotely but maintained directly. Thus, the estimated plant cost of the DuPont design will very likely be much greater than that of AGNS, per unit of capacity. (See Section E.)

The six major areas of the plant, fuel storage, reprocessing, $U$ conversion, $\mathrm{Pu}$ conversion, MOX plant, and waste handling will be decoupled from each other with lag storage between them for ease of reliable operation. However, the six units will be located on the same site to avoid shipment of hazardous materials except solid waste and new MOX fuel.

The separation steps in the reprocessing plant will be those of the Purex process with which considerable experience has been attained. A chemical reducing agent, hydroxylamine nitrate, will be employed to change the valence of $\mathrm{Pu}$ before recovering it from the U/Pu mixture and a series of mixer-settler stages may be used for the solvent extraction steps. The high-level waste solution will be stored in six 600,000 -gallon stainless steel tanks. One tank provides enough space for one year's operation. After storage the solution will be reduced to solid form in spray calciners and incorporation in glass matrices inside containers. Close coupled waste solidification without interim tank storage is also being investigated.

\section{D6. Foreign Experience with Power Reactor Fuel Reprocessing}

All plants now in operation or under construction use variants of the Purex prócess. Substantial foreign plants exist at Marcoule, France (900-1200 Mg/yr), La Hague, France ( $800 \mathrm{Mg} / \mathrm{yr}$ ), Windscale, U.K. (2500 Mg/yr), Tokai-Mura, Japan (250 Mg/yr), and Tarapur, India. With the expanding nuclear power capacity, the construction of many additional reprocessing plants can be anticipated. West Germany, Italy, and Belgium all have limited experience with pilot plants. The Belgian plant was shut down in 1974.

\section{E. The Cost of Commercial Fuel Reprocessing}

\section{E1. Introduction}

The economics of commercial fuel reprocessing can not be estimated reliably at this time. Any estimates are subject to considerable uncertainties because an entire commercial-scale reprocessing facility has not yet been constructed and because many of the regulations relevant to the licensing of such a facility are still not completely formulated. Constraints from domestic and international safeguards are not yet elucidated clearly.

Also, there are two different approaches in designing commercial fuel reprocessing plants which can significantly affect the construction cost. One approach involves completely remote maintenance, as is used in the present ERDA facilities at Hanford and Savannah River and is reflected in the recently initiated ERDA-sponsored design studies by E.I. duPont de Nemours and Company for a commercial reprocessing facility (E. I. DuPont de Nemours and Co., 1975; ERDA, 1976). The other approach involves remote maintenance for feed preparation, first-cycle decontamination, and high-level waste treatment, with remaining portions of the plant being designed for direct maintenance (Kupp, 1977). The AGNS plant (Cholister, et al., 1976) at Barnwell, S.C., the proposed Exxon project (Exxon Nuclear Co., 1976a; Wall Street Journal, 1977) at Oak Ridge, Tennessee, and the Western Reprocessing Group Study (Stoller, 1976), are current examples of the semi-direct maintenance approach.

A principal factor affecting the cost of fuel reprocessing is the niethod of financing. The variables here include the relative proportions of debt and equity financing and the necessary rate of return on equity to attract the investment capital. The latter is logically affected by uncertainties to the investor, such as the costs of many yet undefined regulations which will govern reprocessing operations and which can cause delays in project completion, as well as the possibility that a project once underway into construction or operation could be aborted by Federal action.

Data and estimates which illustrate the cost of commercial fuel reprocessing, expressed as the cost per unit amount of fuel reprocessed, are analyzed in the following sections. Then, in Section IV.G we analyze the effect of these various estimates of the unit reprocessing costs on the overall economic incentive for fuel reprocessing. 
TABLE 5. Reprocessing plant estimate-Western Reprocessing Group Study (Stoller, 1976) $1500 / \mathrm{Mg} / \mathrm{yr}, 1986$ completion.

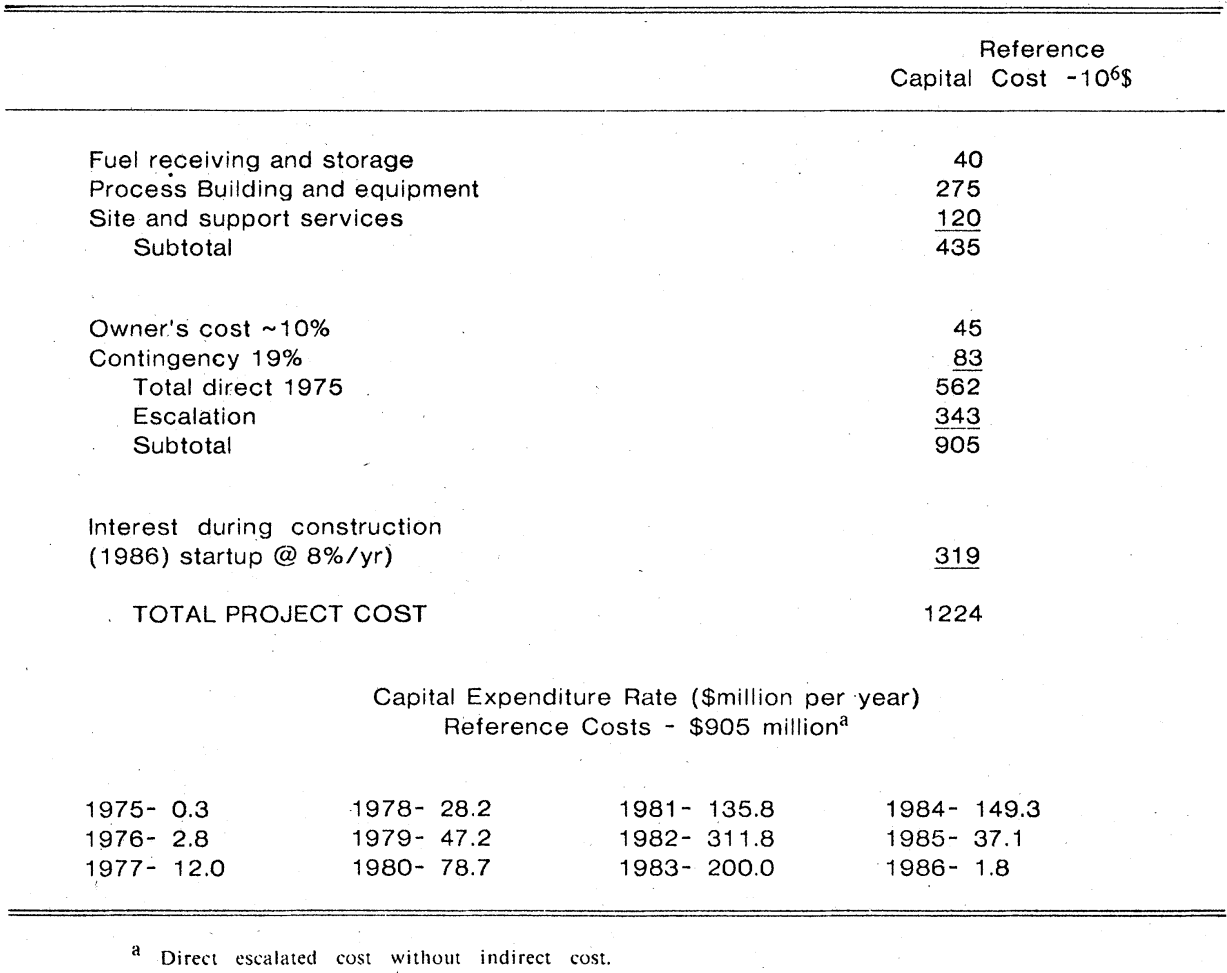

\section{E2. The Cost of Financing - The Western Reprocessing Group Study}

The recent analysis by S.M. Stoller (Stoller, 1976) of "The Morphology of Reprocessing: A Case for Regional Unility Planning" provides useful insight into the effect of financing variables on the cost of fuel reprocessing. This study was based upon a $1500 \mathrm{Mg} / \mathrm{yr}$ commercial reprocessing facility designed for the Western Reprocessing Group Study. $1500 \mathrm{Mg} / \mathrm{yr}$ is calculated on the basis of the quantity of actinides in the fuel prior to reactor irradiation. For the purpose of this study the project was assumed to begin in 1975 and to be completed and operable in 1987. As shown in Table 5, the estimated direct cost of construction is $\$ 562$ million, in 1975 dollars. Escalation through 1986 adds $61 \%$ more, or $\$ 343$ million. Interest on the increasing capital investment prior to completion of construction, calculated at the rate of $8 \%$ yr, adds $\$ 319$ million, bringing the total project cost to $\$ 1,224$ million. This total is properly interpreted as the cumulative cash flow to project completion, so it is no longer references to a constant-dollar uninflated cost. For simplicity, it is here referred to as cost in "1986 dollars".

Stoller also estimates costs of operating and maintenance and costs from local taxes, for 1986 operation and in "1986 dollars", in Table 6.
The second column is obtained by dividing the yearly cost by the yearly throughput of $1.5 \times 10^{6} \mathrm{~kg}$.

To determine the contribution of the capital investment to the unit reprocessing cost the method of project financing must be considered. This depends mainly upon:

1. The extent to which the project is debt financed and the cost of debt financing, and

2. the expected yearly return on the equity-financed portion of the capital investment.

The effect of these variables is illustrated in Figure 4 , where the unit reprocessing cost (i.e., "the service charge") in "1986 dollars", has been plotted against the percent of the total project cost which has been debt financed. There has also been included in this figure the contribution from off-site waste management at a constant value of $\$ 30 / \mathrm{kg}$., but the off-site waste management cost is not relevant to the present discussion.

Two regions are shown in Figure 4. The upper one is for a private industrial entrepreneur with a high component of equity and a high

TABLE 6. Yearly costs, for operation, maintenance, local taxes, and insurance, Western Reprocessing Group Study (Stoller, 1976).

\begin{tabular}{lcc}
\hline & $\begin{array}{c}10^{6} \$ / \mathrm{yr} \\
(1986 \text { dollars })\end{array}$ & $\begin{array}{c}\$ / \mathrm{kg} \text { of fue } 1 \\
\text { reprocessed }\end{array}$ \\
\hline Operation and maintenance & 25.5 & 17 \\
Local taxes and insurance & 19.5 & 13 \\
\hline
\end{tabular}




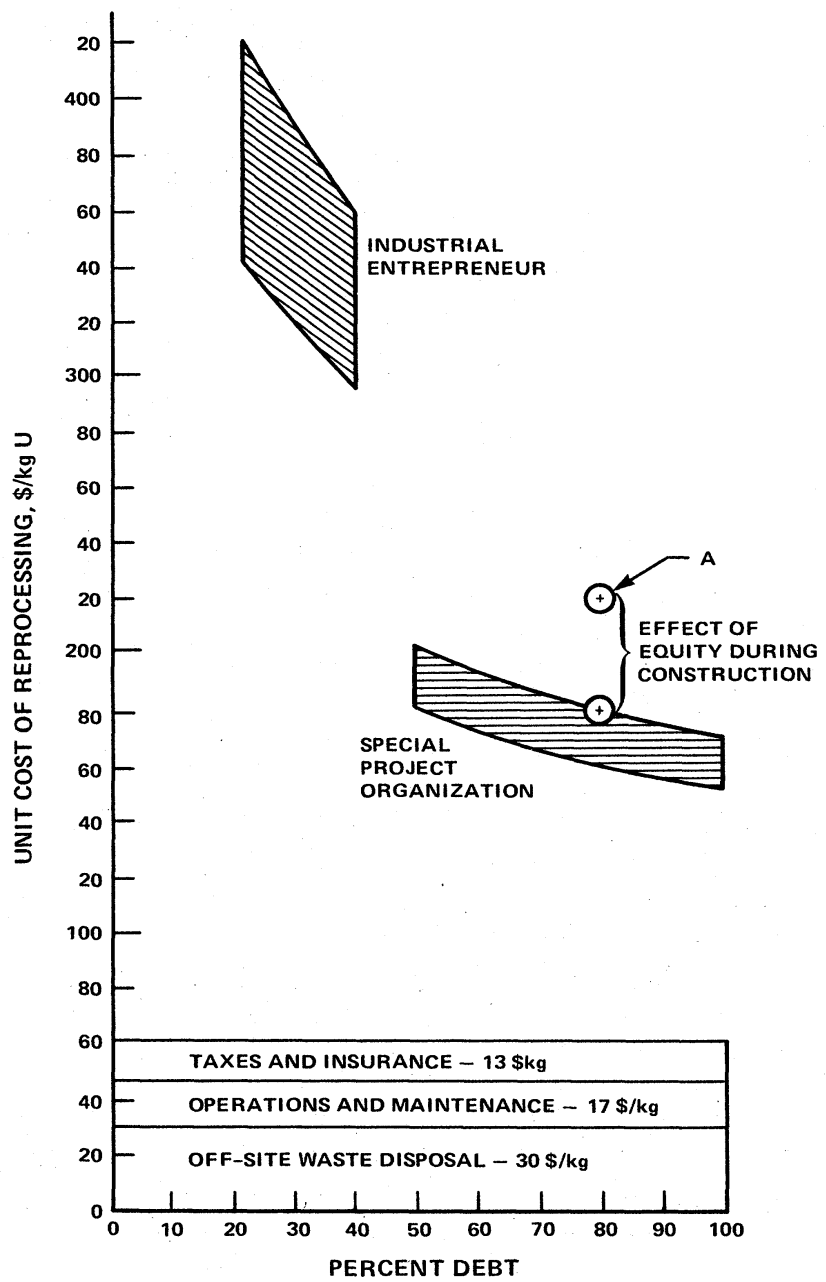

FIG. 4. Unit cost of fuel reprocessing-Western reprocessing group study.

after-tax return on equity investment, consistent with a venture of substantial risk. The other region is for a special project organization under a utility-type ownership with a higher percentage of debt financing. For each region the lower bound corresponds to an assumed continuation of present regulatory criteria, with little expectation for future changes affecting costs. The upper bound allows for possible additional regulatory requirements which can increase the capital and operating costs. The magnitude of this upper bound is necessarily of greater uncertainty because the extent and complexity of some of the regulatory requirements, such as safeguards requirements, are not now known.

The reference estimates in Table 5 of the total project cost result in a unit reprocessing cost which are the median of the upper and lower bounds of Figure 4. For example, if $80 \%$ debt financing were possible, though not characteristic of normal industrial operations, the effective annual charge on total capital investment could be as low as $13-1 / 2 \% / \mathrm{yr}$, resulting in a unit reprocessing of about $\$ 170 / \mathrm{kg}$. ${ }^{*}$ This falls within the lower band of Figure 4. Alternatively, assuming that the project could be financed by a group of utilities, with normal utility-type financing of about $50 \%$ debt, the yearly charge on total capital investment of about 16 to $17 \% / \mathrm{yr}$ for utilities (Kupp, 1977) would result in a unit reprocessing cost of about $\$ 195 / \mathrm{kg}^{*}$. The data within this lower band were calculated with the assumption of full debt financing during construction, with a debt service charge of $8 \% / y r$. However, if equity financing is involved at the same proportion during construction, the total project cost increases considerably. For the example of $80 \%$ debt financing, the effect of equity during construction is to increase the unit reprocessing cost to about $\$ 205 / \mathrm{kg}^{*}$ as shown by point "A" in Figure 4.

The estimates in Figure 4 for an industrial entrepreneur with a relatively large component of equity financing include allowance for return on equity during construction. The lower and upper bounds of this region correspond to $18 \% / \mathrm{yr}$ and $22 \% / \mathrm{yr}$, respectively, for the discounted after-tax return on investment (Kupp, 1977). For example, such an industrial project at $25 \%$ debt might cost from $\$ 335$ to 405 $\$ / \mathrm{kg}^{*}$, with an average of about $\$ 370 / \mathrm{kg}^{*}$. A government-financed project would result in a yearly charge on total capital cost of about $10 \% / y r$, assuming a federal bond rate of $7-1 / 2 \% / \mathrm{yr}$ and a plant life of 15 to 20 years (Kupp, 1977). This value also adjusts for the savings due to less interest during construction. Government payments in lieu of local taxes are not included. The resulting unit cost of reprocessing is then $\$ 142 / \mathrm{kg}^{*}$.

The Nuclear Regulatory Commission's GESMO Final Environmental Impact Statement (USNRC, 1976) considers 22.5 to $36.0 \% / y r$ to represent the appropriate range of yearly charge rates for industrial investment in fuel reprocessing. These yearly charges would yield unit reprocessing costs falling within the range of the Western Reprocessing Group Sudy. The large differences in the unit cost of reprocessing resulting from this large range of financing strategy are illustrated in Table 7.

To compare estimated reprocessing costs obtained from different sources, it is necessary to correct for escalation between different time periods of the estimates. Indices provided by R.W. Kupp (Kupp, 1977) allow the de-escalation of data in Table 7 to 1976 dollars. The total project cost, including $8 \%$ interest during construction, translates to $\$ 760$ million in 1976 dollars. Deleting costs of off-site management, we obtain the unit reprocessing costs listed in Table 8.

\section{E3. Cost of the AGNS Plant}

Allied-General Nuclear Services has designed and partly constructed a $1500 \mathrm{Mg} / \mathrm{yr}$ reprocessing facility at Barnwell, S.C (Cholister, et al., 1976). The construction costa and estimated operating cost of the now-completed separation facility, as well as the estimated costs for the facilities for $\mathrm{PuO}_{2}$ conversion and storage and for high-level waste soldification, are shown in Table 9.

The estimated total AGNS project cost is $22 \%$ less than the estimated 1976-dollar project cost for the Western Reprocessing Study. AGNS estimates a net unit reprocessing cost of $\$ 140$ per kilogram of heavy metal (actinides) processed $\dagger$, calculated in 1976 dollars and assuming that processing began in 1976. Assuming that the AGNS data for a capital cost of $\$ 595$ million include interest during construction, and using the operating cost of $\$ 40.8 \mathrm{million} / \mathrm{yr}$., we calculate that the net yearly charge on capital investment used by AGNS is $31 \% / y r$. This is consistent with the after-tax return on investment of $15 \% / y r$. quoted by AGNS. AGNS estimates an uncertainty of $\pm 20 \%$ in the capital cost of the $\mathrm{PuO}_{2}$ conversion and storage facility and $\pm 30 \%$ for the on-site waste management facility, resulting in reprocessing costs varying from 127 to $171 \$ / \mathrm{kg}$.

The AGNS plant at Barnwell had the technical capability of beginning reprocessing in 1976 and either shipping the recovered plutonium to the fabricator as liquid $\mathrm{Pu}\left(\mathrm{N}_{3} \mathrm{O}_{3}\right)_{4}$ or storing the liquid $\mathrm{Pu}\left(\mathrm{NO}_{3}\right)_{4}$ at Barnwell. However, there are several reasons why the schedule for beginning reprocessing at Barnwell has become increasingly uncertain:

1. The Barnwell storage capacity for liquid $\mathrm{Pu}\left(\mathrm{NO}_{3}\right)_{4}$ can accommodate the plutonium produced from full-capacity operation for about 15 months.

2. Federal regulations now require that; after June 1978 , plutonium be converted from a liquid to a solid before off-site shipment. This would require operation of a new $\mathrm{PuO}_{2}$ conversion facility, which AGNS estimates will not be completed until about four years after funding is committed and after design and construction are initiated.

* "1986 dollars"

$\dagger$ Does not include cost of off-site waste management. 
TABLE 7. Effect of financing on unit reprocessing costs from Western Reprocessing Group Study.

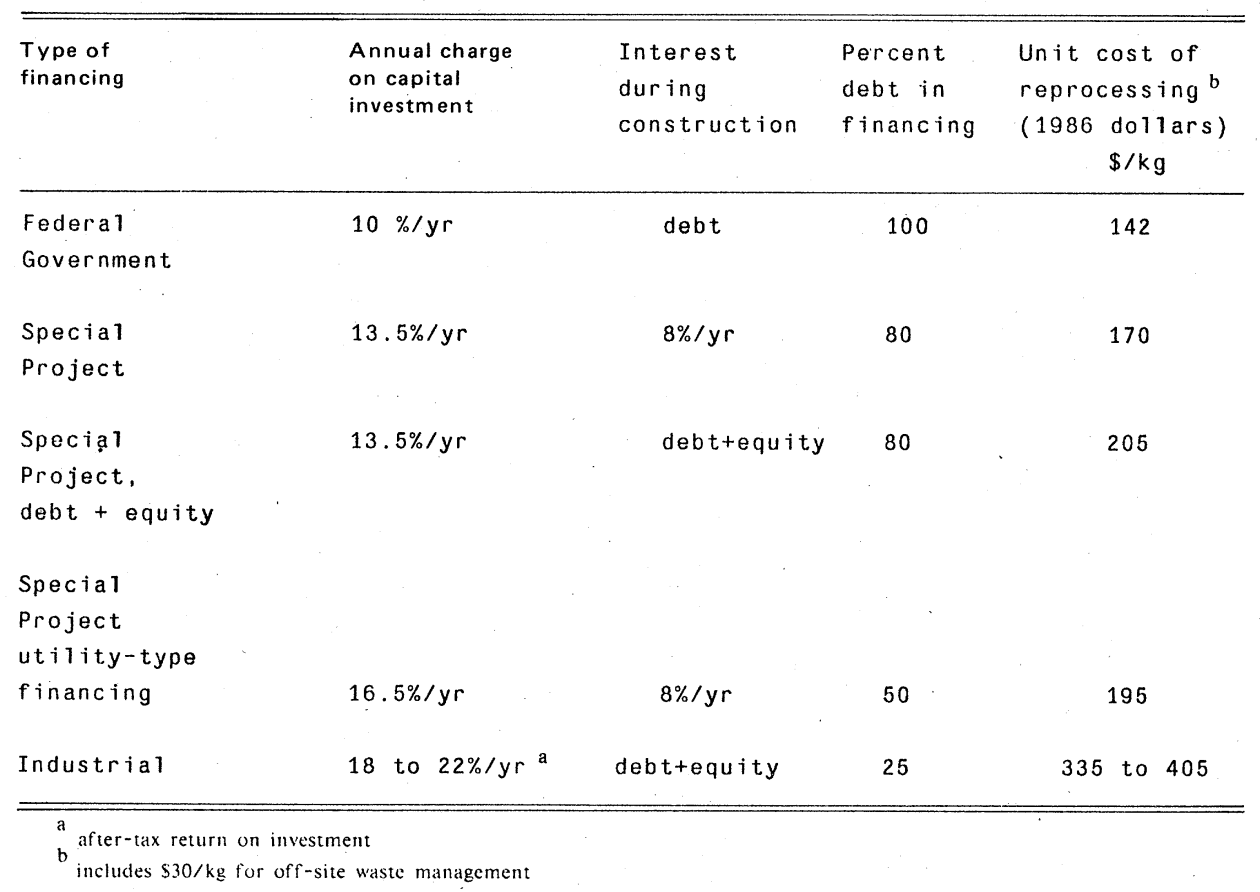

3. Recent legal decisions indicate that it might not be possible to license Barnwell even for uranium recovery alone until the hearing and decision on The Matter of Generic Environmental Statement of Mixed Oxide (USNRC, 1976) Fuel (Docket No. Rm-50-5) is completed. The hearing is not likely to be completed until mid-1977 or later. (In a recent policy decision, the President has requested that the GESMO hearing be terminated.)
4. The safeguards supplement to the NRC Environmental Impact Statement for the GESMO proceeding has not been issued. It is not known what new requirements for safeguards may be placed upon the Barnwell operation.

5. Presidential orders have deferred commercial fuel reprocessing for an indefinite period. The impact of the presidential order on the

TABLE 8. Unit reprocessing costs from Western Reprocessing Study translated to 1976 dollars (1500) $\mathrm{Mg} / \mathrm{yr}$.

\begin{tabular}{|c|c|c|c|c|c|}
\hline \multirow{2}{*}{$\begin{array}{l}\text { Type of } \\
\text { financing }\end{array}$} & \multirow{2}{*}{$\begin{array}{l}\text { Annual charge } \\
\text { on capital } \\
\text { investment }\end{array}$} & \multirow[t]{2}{*}{$\begin{array}{l}\text { Interest } \\
\text { during } \\
\text { construction }\end{array}$} & \multirow[t]{2}{*}{$\begin{array}{l}\text { Percent } \\
\text { debt in } \\
\text { financing }\end{array}$} & \multicolumn{2}{|c|}{$\begin{array}{c}\text { Unit cost of } \\
\text { reprocessing b } \\
\text { (1976 dollars) } \\
\$ / \mathrm{kg}\end{array}$} \\
\hline & & & & & \\
\hline Government & $10 \% / \mathrm{yr}$ & debt & 100 & ' & 73 \\
\hline \multicolumn{6}{|l|}{ Special } \\
\hline Project & $13.5 \% / y r$ & $8 \% / y r$ & 80 & & 92 \\
\hline Special & & & & & \\
\hline Project & $13.5 \% / y r$ & debt + equity & 80 & & 114 \\
\hline \multicolumn{6}{|l|}{ Special } \\
\hline \multicolumn{6}{|l|}{$\begin{array}{l}\text { Project, } \\
\text { utility-type }\end{array}$} \\
\hline financing & $16.5 \% / y r$ & $8 \% / y r$ & 50 & & 108 \\
\hline Industrial & 18 to $22 \% / y r^{a}$ & debt+equity & 25 & 200 & to 250 \\
\hline
\end{tabular}

a After-tax return on investment

b Includes $\$ 11 / \mathrm{kg}$ for operation and maintenance and $\$ 8 / \mathrm{kg}$ for local taxes and insurance. 
TABLE 9. Allied-General Nuclear Services estimates of cost of fuel reprocessing facilities at Barnwell, S.C. (Cholister et al ., 1976). (Capacity $=1500$ metric tons/yr. Costs ${ }^{\text {a }}$ are in 1976 dollars. Reprocessing was assumed to begin in late 1976.)

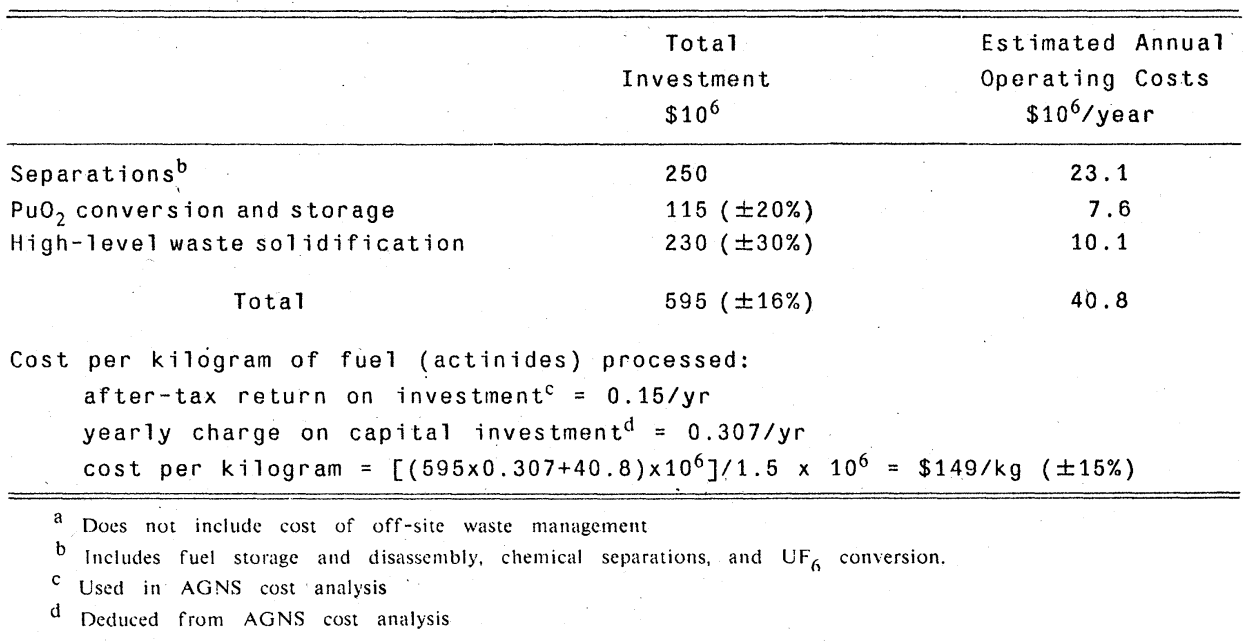

timing of the GESMO proceeding and upon the financial feasibility of the Barnwell operation has not been assessed. These presidential orders do not preclude the ultimate utilization and recovery of plutonium.

\section{E4. Cost of the Exxon Plant}

The next project for which there is an announced construction commitment is a $2100 \mathrm{Mg} / \mathrm{yr}$ reprocessing plant to be built at Oak Ridge, Tennessee by the Exxon Corporation (Wall Street Journal, 1977). The initial construction phase of the project, expected to begin in 1979 , will be a $\$ 300$ million storage facility for discharge fuel followed by a fuel-reprocessing center. The Exxon facility is being designed by the Bechtel Corp., which also designed the Barnwell facility. The AGNS and Exxon facilities are understood to incorporate a similar approach towards direct and remote maintenance, one of the key factors affecting capital costs. The recent published announcement of the Exxon facility does not explain whether the $\$ 1$ billion estimate of plant cost includes interest during construction and whether it is based upon actual dollars expended through project completion. However, a 1976 letter (Exxon Nuclear Co., 1976b) from Exxon Nuclear Corp., in connection with its PSAR application, quotes a construction cost of $\$ 600$ million in 1975 dollars, not including escalation and interest during construction. Therefore, a total project cost of $\$ 1$ billion in 1975 dollars will be assumed for the purpose of this analysis. We then estimate the capital cost per unit of throughput to be $\$ 500$ per $\mathrm{kg} / \mathrm{yr}$, which compares with $\$ 540$ per $\mathrm{kg} / \mathrm{yr}$ for the Western Reprocessing Plant Study and $\$ 400$ per $\mathrm{kg} / \mathrm{yr}$ for the AGNS estimates, all in 1976 dollars.

\section{E5. Cost from the DuPont - ERDA Design Study}

The DuPont Co., at the Savannah River Laboratory, is developing designs and cost estimates for a representative commercial reprocessing plant that could be completed with an eight-year lead time. We have received some of their preliminary estimates directly for our present study (O'Rear, 1976), and other duPont data are reported by ERDA in "Benefit Analysis of Reprocessing and Recycling Light Water Reactor Fuel" (ERDA, 1976). Although ERDA does not quote the cost of interest during construction, we deduce from their other data a completed project cost of $\$ 1.44$ billion, in 1977 dollars, for a $1500 \mathrm{Mg} / \mathrm{yr}$ reprocessing plant. This agrees with data supplied to us by duPont (O'Rear, 1976). This is $128 \%$ greater than the AGNS estimates and $68 \%$ greater than the Stoller estimates, all brought to the same basis of 1977 dollars. One reason for the higher costs of the duPont design is the adoption of completely remote maintenance in that design. This reflects the design and operating experience from the AEC reprocessing plants at Hanford and Savannah River, which were designed and built by duPont on an emergency basis during the 1940's and early 1950's for the weapons program. For this purpose of the present analysis, we assume that differences in maintenance philosophy account for the higher costs of the duPont design.

The effect of scale upon plant cost is indicated by ERDA's (Tooper, 1977; ERDA, 1976) estimates of the construction costs, excluding interest during construction, for reprocessing plants of two different capacities, as shown in Table 10.

These data follow the familiar empirical rule that plant cost is proportional to the capacity raised to some power near 0.6. Also DuPont (Tooper, 1977; ERDA, 1976) estimates a $3 \%$ saving in total plant cost if the plant is designed to reprocess spent fuel that has been cooled for 5 years instead of 1 year.

\section{E6. Costs Estimated by NRC}

In its cost-benefit analysis of reprocessing for the purpose of the GESMO Final Environmental Impact Statement, the Nuclear Regulatory Commission (USNRC, 1976) has used data identical with the AGNS estimates (Cholister, et al., 1976) for the plant capital costs. The AGNS cost estimates are for first cycle fuel, although the Barnwell plant was designed and built to process MOX fuels fabricated from uranium and recycle plutonium. The additional technical problems associated with MOX reprocessing are discussed elsewhere. NRC (USNRC, 1976) estimates that the cost of reprocessing MOX fuel will be $20 \%$ greater than for $\mathrm{UO}_{2}$, but there is too little experience to make accurate projections at this time.

\section{E7. Other Estimates of Reprocessing Plant Costs}

Other data and estimates of reprocessing plant costs, including the cost of the 1963 Nuclear Fuel Services plant, are summarized in Table 11. Rodger's (Rodger, et al., 1975) analysis of the evolution of

TABLE 10. Effect of plant capacity on construction cost (ERDA, 1976).

\begin{tabular}{cc} 
plant capacity & $\begin{array}{c}\text { construction cost } \\
\text { (excluding interest during } \\
\text { construction) } \\
\text { \$ billions }\end{array}$ \\
\hline 1500 & 1 \\
3000 & 1.5 \\
\hline
\end{tabular}


TABLE 11. Comparison with previous estimates for fuel reprocessing costs.

\begin{tabular}{|c|c|c|c|}
\hline $\begin{array}{l}\text { Source and Date } \\
\text { of Estimate }\end{array}$ & $\begin{array}{c}\text { Plant } \\
\text { capacity } \\
\text { metric tons } \\
\text { per year }\end{array}$ & $\begin{array}{l}\text { yearly charge } \\
\text { on capital } \\
\text { investment } \\
\% \text { per year }\end{array}$ & $\begin{array}{l}\text { reprocessing } \\
\text { cost } \\
\$ / \mathrm{kg}\end{array}$ \\
\hline $\begin{array}{r}\text { A1lied General Nuclear } \\
\text { Services, }(1976): \\
\text { start late }(1976)\end{array}$ & 1500 & 30.7 & 121 to 171 \\
\hline $\begin{array}{l}\text { Nuclear Fuel Services, (1963) } \\
\text { Wolfe and Lambert (1975) }\end{array}$ & 300 & $25^{\mathrm{a}}$ & $\begin{array}{r}45^{\mathrm{a}} \\
126^{\mathrm{b}}\end{array}$ \\
\hline $\begin{array}{l}\text { Wolfe, et al. (1976) } \\
\text { Schwoerer }(1975)\end{array}$ & & & $\begin{array}{l}126 \text { to } 290 \\
150\end{array}$ \\
\hline Rodger, et al. (1976) & 1500 & 25 & 170 \\
\hline Nuclear Fuel Services, (1976) & 600 & & $\sim 1000^{\mathrm{c}}$ \\
\hline
\end{tabular}

a Estimated by Rodger (Rodger, et al., 1975) for 1963 operations.

b Does not include conversion to $\mathrm{PuO}_{2}$

c Estimates by Nuclear Fuel Services of possible cost if NFS plant scale-up to $600 \mathrm{MT} / \mathrm{yr}$ is completed to new NRC regulatory requirements, including new seismic specifications for West Valley, N.Y.

commercial reprocessing plant costs reveals the effect of uncertainties which can be expected for future commercial ventures into fuel reprocessing. The $300 \mathrm{Mg} / \mathrm{yr}$ NFS plant at West Valley, N.Y. cost about $\$ 35$ million in 1963 dollars, whereas the $1500 \mathrm{Mg} / \mathrm{yr}$ AGNS plant will cost about $\$ 600$ million in 1976 dollars, if completed. The twenty-fold increase in cost is in part attributable to the larger capacity. Assuming costs scale as the 0.6 power of capacity, the fivefold greater capacity of AGNS would result in a cost greater by a factor of

$$
5^{0.6}=2.6
$$

Much of the increase is also due to inflation, which occured at 6\%/yr from 1963 through 1969 and 9\%/yr thereafter, resulting in an overall factor of

$$
(1.06)^{6}(1.09)^{7}=2.6
$$

Other influences on the cost must account for the remaining factor of

$$
20 /(2.6 \times 2.6)=3
$$

Rodger attributes these to the following:

1. Regulatory rules have changed

2. Partly because of changing regulations, the Barnwell plant contains equipment capable of carrying out functions not included in the NFS plant, e.g., conversion of UNH to UF, conversion of $\mathrm{Pu}\left(\mathrm{NO}_{3}\right)_{4}$ to $\mathrm{PuO}_{2}$ and $\mathrm{PuO}_{2}$ storage, and waste solificication.

3. Regulations required for orderly design and construction have not been made on a timely basis; consequently design and construction delays have resulted in large cost overruns. To illustrate the last point, if such factors result in a two-year delay in the construction of a committed and funded $\$ 600$ million project, the increased cost during a period of $9 \% / \mathrm{yr}$ inflation is $\$ 100$ million.

The other cost estimates in Table 11 are within the range of the more recent estimates already summarized in Tables 8 and 9 .

\section{E8. Operating Costs}

Because the capital cost dominates the unit cost of reprocessing variations in operating cost are of lesser importance at this time in the consideration of the economics of fuel reprocessing. Operating costs are compared in Table 12. It is not clear why the operating costs of DuPont's design of a remote-maintenance plant should be greater than for the two estimates for direct-maintenance plants. However, it is possible that this higher operating cost, which was quoted by ERDA, results because this is a more recent estimate and is assumed to include the more recent cost effects of appropriate safety, environmental, and safeguards criteria. It is these criteria which are still not well defined for reprocessing plants and are still subject to considerable uncertainty.

TABLE 12. Estimated operating costs of a reprocessing plant.

\begin{tabular}{llc}
\hline \hline & $\begin{array}{l}\text { Plant Capacity } \\
\mathrm{Mg} / \mathrm{yr}\end{array}$ & $\begin{array}{c}\text { Operating Cost } \\
(1976 \text { dollars) } \\
10^{6} \$ / \mathrm{yr}\end{array}$ \\
\hline $\begin{array}{l}\text { Western Reprocessing Group Study } \\
\text { Allied General Nuclear Services } \\
\text { (Cholister, et al., 1976) }\end{array}$ & 1500 & 28.5 \\
$\begin{array}{l}\text { DuPont (Savannah River Lab) } \\
\text { (C) }\end{array}$ & 1500 & 40.8 \\
\hline
\end{tabular}

a Estimated from data in Table 4

b $\$ 60 \times 10^{6} / \mathrm{yr}$ in 1977 dollars, de-escalated by $6 \% / \mathrm{yr}$ (ERDA, 1976). 
TABLE 13. Illustrative costs of fuel reprocessing $(1500 \mathrm{Mg} / \mathrm{yr}, 1976$ dollars $)$.

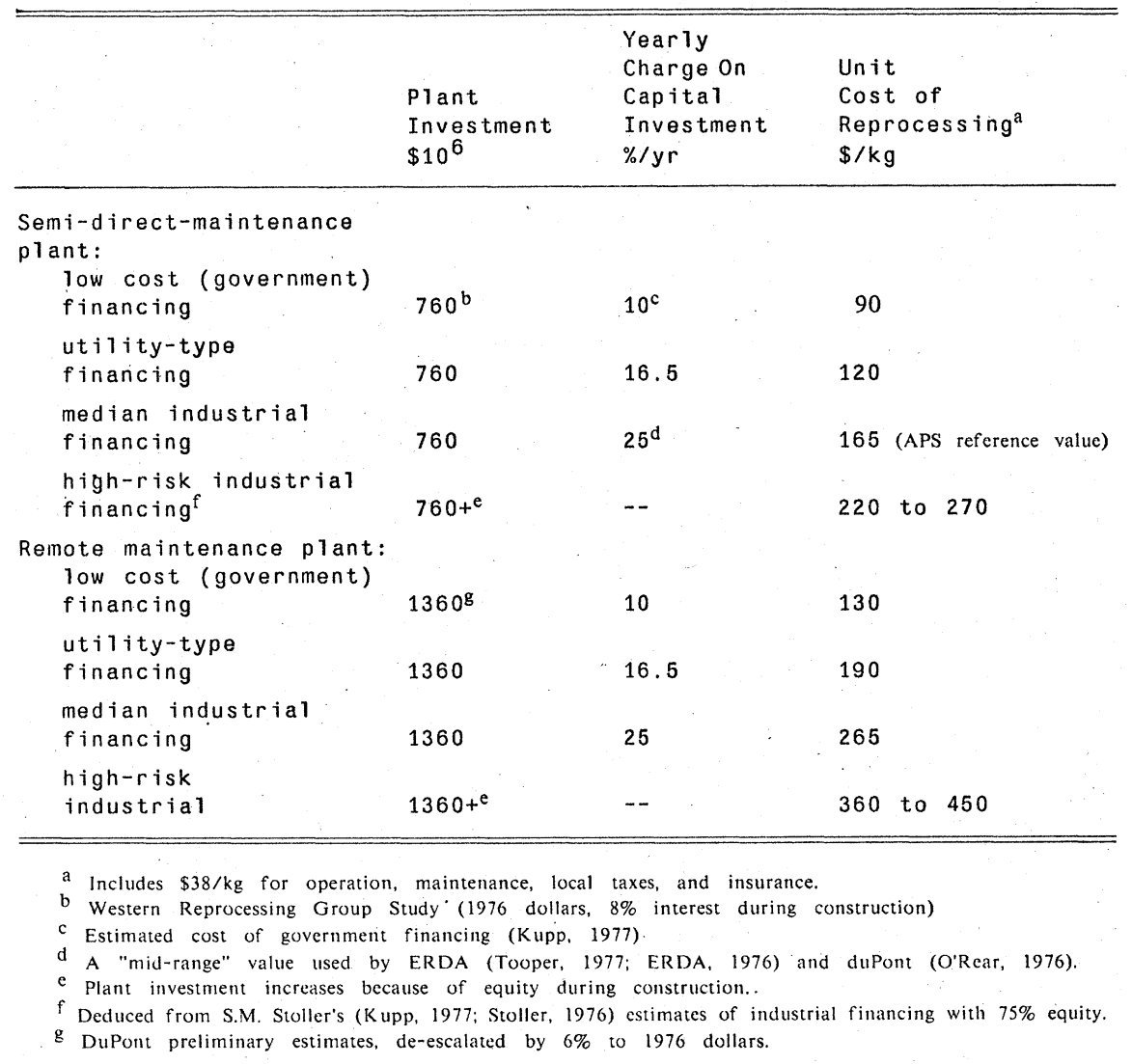

Any operational difficulties which might contribute to down-time and loss of plant capacity directly affect the capital cost contribution per unit of product. There are insufficient data available at present to determine whether there will be important differences in the capacity factors for direct-maintenance and remote-maintenance reprocessing plants.

\section{E9. Estimates of Unit Reprocessing Costs Used in This Study}

For the purpose of illustrating the effect of reprocessing cost on the incentive to reprocess discharge fuel from light water reactors, we will adopt the unit reprocessing cost estimates summarized in Table 13. The cost of the semi-direct maintenance plant is based upon the Western Reprocessirg Group Study. The cost of the remote maintenance plant is based upon the DuPont design study (ERDA, 1976). The operating cost from the DuPont study is assumed to apply to all cases. All costs are translated to constant-value 1976 dollars The methods of financing include the full range from government debt financing to high-equity industrial financing, using indices developed in Section E. The median-range industrial financing is that adopted by ERDA (ERDA, 1976), which assumes a yearly charge on total capital investment of $25 \% / \mathrm{yr}$. This is consistent with an aftertax return of $13 \% / y r$, which is representative of the required return on capital for the chemical industry (Tooper, 1977). For the same reprocessing plant with a given direct cost of construction, these different methods of financing the project result in a three-fold variation in the unit cost of fuel reprocessing at that plant. Return on investment, tax structure, debt/equity ratio, equity during construction, competing demands for capital, and risk factors to allow for uncertainties in federal action are the most important set of parameters that affect the cost of fuel reprocessing in the U.S.

For our subsequent fuel cycle cost analyses in Section IV.G we will adopt as the reference case a semi-direct-maintenance plant with median industrial financing, resulting in a unit reprocessing cost of
$\$ 165 / \mathrm{kg}$. We will later calculate the break-even reprocessing costs for various fuel cycle options, and these break-even costs will be compared with the other cases in Table 13.

\section{F. Mixed Oxide Fuel Fabrication}

\section{F1. Background}

Mixed oxide fuel elements have been fabricated in the United States and in France and England for several years. The principal U.S. Fabricators have been the Kerr McGee Plant in Oklahoma and the Babcock and Wilcox Nuclear Materials Division Plant in Pennsylvania. Flowsheets at these plants are similar, the main difference being in the headend treatment. The Kerr McGee plant used co-precipitation to form the mixed oxides whereas the Babcock and Wilcox plant prepares the oxides of $U$ and Pu separatsly and then mechanically blends the two oxides prior to calcining, slugging, and pellet pressing.

Each method has advantages, but the 1974 decision to ban shipments of plutonium in solution form after June 17, 1978 leads fuel fabricators towards admixing the two oxides rather than coprecipitation if the fabrication plant site is remote from the reprocessing plant. If the reprocessing plant and the fuel fabrication plant were located on the same site, then co-precipitation could be used. Also co-precipitation at the reprocessing plant to a plutonium rich MOX powder is viable, to be followed by final blending at the fuel fabrication plant.

The Kerr McGee plant is no longer in operation and the only mixed oxide fuel fabrication plant presently in operation is that of Babcock and Wilcox in Pennsylvania. The Babcock and Wilcox plant uses the admixing flowsheet which will not be affected when the ban on shipment of plutonium nitrate solution goes into effect. This plant 
is a glovebox operation with a throughput of about 1000 fuel pins per month for the Fast Flux Test Facility (FFTF) in Richland, Washington. It is basically a hand operation that is operable, from an occupational exposure viewpoint, only when the plutonium isotopic blend is 12 percent ${ }^{240} \mathrm{Pu}$ or less.

A second generation fuel fabrication plant has been proposed and designed by Westinghouse to be built at Anderson, South Carolina. They have chosen to use the admixture flowsheet, which will be compatible with receiving solidified plutonium. If a fuel fabricator opts for the co-precipitation method to make powdered fuel with the final fuel composition, then he must include a $\mathrm{PuO}_{2}$ dissolution step in his plant, thus increasing the size and cost of plant and the cost of operations. Since reactor designers will accept fuel made by the admixture route, the choice of the admixing flowsheet offers economic advantages.

The proposed Westinghouse Recycle Fuels Plant (WRFP) is representative of mixed oxide fuel fabrication plants for the 1980 time period. Such a plant could fabricate either an L.MFBR or a recycle LWR fuel. The plant has several automated features to reduce occupational exposure as well as cost of fuel fabrication. The major design features of the plant are given in Table 14, which was reproduced from Chapter IV, Volume 3 of GESMO (USNRC, 1976).

\section{F2. The Westinghouse Recycle Fuels Plant (WRFP)}

The process used in the Westinghouse Recycle Fuels Plant (WRFP) for fabrication of fuel rods is a dry system including powder blending, pelletizing, sintering, and encapsulation of the sintered pellets into stainless steel or zircalloy cladding. For production of thermal fuel rods to be loaded into Light Water Reactors (LWRs), natural $\mathrm{UO}_{2}$ would be blended with recycle $\mathrm{PuO}_{2}$ mixture. The technology of all unit operations is well established and safe operation of such systems has been demonstrated in gloveboxes on laboratory and pilot plant scales. The WRFP design has adapted these pieces of equipment to remote and semi-remote control on "cell" and "canyon" design concepts and on a much larger production-type scale.

\section{F2a. Feed Material Receiving and Storage}

The feed material for the WRFP will be $\mathrm{PuO}_{2}$ and $\mathrm{UO}_{2}$ powders packaged for transportation in containers approved by ERDA and DOT. The dock, the materials receiving area, and the $\mathrm{UO}_{2}$ storage area are outside the Class 1 manufacturing building since the material will remain in the DOT-approved container until needed in the process. The $\mathrm{PuO}_{2}$ containers are brought into the manufacturing building for storage in a shielded area to minimize personnel exposure.

\section{F2b. Powder Unloading, Blending, and Storage}

Containers of $\mathrm{PuO}_{2}$ are taken from storage to an unloading area where the pressure vessel is vented. After working through a series of compartments to remove the outer containers, the container of oxide is opened and pneumatically transferred to a critically safe storage tank within the Restricted Access Area (RAA). Drums of $\mathrm{UO}_{2}$ are connected to a drum-dumper mechanism and the contents are transferred into a storage hopper. The transfer system is confined within a special barrier and vent system to contain any $\mathrm{UO}_{2}$ that might become airborne.

The blending process consists of computerized, programmed transfer of the $\mathrm{PuO}_{2}, \mathrm{UO}_{2}$, and recycle mixed oxide (MOX) in to feed hoppers, then into their respective weight hoppers, and then into the mechanical batch-type blender. Both mechanical auger-type transfer and pneumatic transfers are used during this series of manipulations. Plugging or blockage of pneumatic transfer lines is one of the dominant operational problems which could have adverse safety implications. The design provides for all transfer lines to be in restricted access areas. Malfunction of screw feeders and blender connectors requiring maintenance will be correctable through remote manipulators. All hoppers and vessels are equipped with level alarms. Program sequence includes interlocks to prevent double batching as well as logic to prevent valves from opening at the wrong time. A system upset in this area would shut down the process and necessitate corrective measures by operating personnel to prevent release of material to the environment.

\section{F2c. MOX Conditioning and Pressing}

Analyzed powder that meets specifications is transferred pneumatically from one of the nine MOX silos to the feed hopper of the slug press. The powder is pressed into short cylindrical wafers, or slugs, which are discharged onto a convertor that feeds a granulator. The resulting granules pass through a set of classifier screens and acceptable material is pneumatically transferred to the feed hopper of a pellet press. The oversize granules are recycled to the granulator feed or to scrap and the undersize granules are recycled to the slug press feed hopper. Press feed is pressed into circular cylinders (green pellets) which are loaded into molybdenum sintering boats. Loaded boats are transferred to green pellet storage to await programmed demand for sintering furnace feed. This complete system operates continuously with the master control centered in the high/low level controls of the pellet press feed hopper. System interlocks at both ends of the system can stop all operations if no certified powder is available to feed the slug press, or if no empty boat is available to accept green pellets.

The complex instrumentation and control systems require many penetrations of the confinement barrier between the process enclosure (Restricted Access Area - RAA) and the gallery (Limited Access Area - LAA). In order to meet the stringent criteria for confinement, final design will incorporate the necessary HEPA filtration, backflow preventors, or breaks in instrument air supply systems to maintain positive control and to eliminate the possiblity of back-migration into normal access areas (NAA).

Another area requiring special consideration is the treatment and control of lubricants. Non-flammable lubricants will be used wherever possible, but the special lubricant used to assure smooth release of pellets from the die cavity must be a low residue material that will burn off in the sintering operation. Only about $0.1 \%$ will be used, which will be about one liter per press per 24 hours of operation. Most of this amount will be carried by the pellets, but an overflow collector will catch any excess from the press. Transfer of all spent lubricants or solvents to the waste treatment area will be on a daily basis to keep the inventory of flammable materials in the RAA to a minimum.

\section{F2d. Pellet Sintering, Grinding, and Inspection}

Green pellets are taken from storage and transferred by shuttle car to the entrance conveyor of one of several sintering furnaces. Sintered pellets coming out of the furnaces onto exit conveyors are transferred by another shuttle car to a sampling station for process control testing of density and other physical characteristics. Low density pellets can be routed for re-sintering, rejects can be sent to scrap recycle, and acceptable pellets are moved into storage to wait for grinding. Pellets are transferred from the boats onto a conveyor feeding a centerless grinder using coolant. The pellets are dried of surface moisture as they leave the grinder, and move single file through final inspection for diameter. Acceptable pellets are loaded onto storage trays and are then dried, sampled, and placed into pellet storage to await certification for fuel rod loading.

Furnace operation, grinding, inspection are done remotely. Provisions have been made in the design for manual calibration and adjustments of certain inspection equipment. Safety-related instrumentations and controls are adequate to assure containment of the material within the RAA.

\section{F2e. Fuel Rod Loading, Welding, and Inspection}

Fuel rod tubing, end plugs and springs are inspected, cleaned, and fed into the system, as required for production. One end plug is welded, $x$-rayed, and the subassembly and spring are transferred to fuel rod loading. Pellets are taken from storage, loaded into the tube, the spring is inserted, the tube end is decontaminated as required, and the final end plug is inserted. Loaded fuel rods are transferred by shielded carrier to the welding station where the plug is girth welded, the fuel is purged with helium, and the tip of the plug is seal welded. Completed fuel rods are transferred through an airlock from the manufacturing building into the rod inspection building. Fuel rod inspection includes mass spectrometer helium leak check, weld $x$-ray, gamma scan for fuel placement, and dimensional checks. Contamination control of the unsealed fuel rod will require constant 
attention of well trained operators. The potential for alpha contamination spread exists until after fuel rods are leak checked and given a final smear count as they go into inspection. The only place where flammable solvents will be used is at the hardware cleaning station. The standard safety can for solvents will be used, airflow throughhoods will be controlled, and the inventory of solvents will be limited so the anticipated evaporation rate in the minimum airflow of the hood will not be a flammable mixture.

\section{F2f. Scrap Recycle and Storage}

From the powder and pellet handling areas there will be physical and chemical reject material, spillage, and certain process residues that must be handled. Clean MOX will be returned to a set of reaction vessels designed to recondition the material by successive oxidationreduction cycles and return acceptable product to the blending area. Pellets and powder that are chemically unacceptable are packaged and stored for future recovery.

Clean scrap is transferred from the various areas to a receiving station where batching is done for the oxidation-reduction reactors. Storage of the scrap before and after reconditioning is in critically safe arrays. Operation of the reactors will be interlocked to preclude the operation of one unit on reduction while one or more of the others are on the oxidation cycle, which could result in a flammable mixture of gases in the exhaust header.

\section{F2g. Product Handling, Storage, and Shipping}

Fuel rods passing all final inspection and certification requirements are placed in shielded storage to await shipment. Shielded carriers, dollies, the storage components, and mechanisms for loading fuel rods from storage into trucks for transport to the assembly plant are all well designed, standard mechanical concepts and are readily available for adjustment or repair. Radiation exposure from an array of fuel rods can easily be controlled.

\section{F3. Alternatives in Technology}

The use of $\mathrm{UO}_{2}$ and $\mathrm{PuO}_{2}$ as starting feed material for a mixed oxide fuel fabrication plant seems to be the favored process, and assumes that the reprocessing plant will carry out the purficiation and conversion of both streams to usable $\mathrm{UO}_{2}$ and $\mathrm{PuO}_{2}$. This scheme also assumes that virgin or recycle uranium coming from an enrichment plant must be converted from $\mathrm{UF}_{6}$ to $\mathrm{UO}_{2}$ before being transferred to the fuel fabrication plant.

The fuel fabrication plant described does not contain, a scrap recovery or recycle capability where chemically impure MOX in various process residues can be purified and converted to a re-usable MOX which can be blended with virgin $\mathrm{UO}_{2}$ and $\mathrm{PuO}_{2}$ at the headend of the plant. If a fabrication plant were to be co-located with a reprocessing plant, scrap recovery could be accomplished in the Purex system along with the irradiated feed. The economics would have to be evaluated, since the MOX containing no fission products could be handled in a less elaborate plant.

TABLE 14. Commercial scale model mixed oxide fuel fabrication plant characteristics (ERDA, 1976).

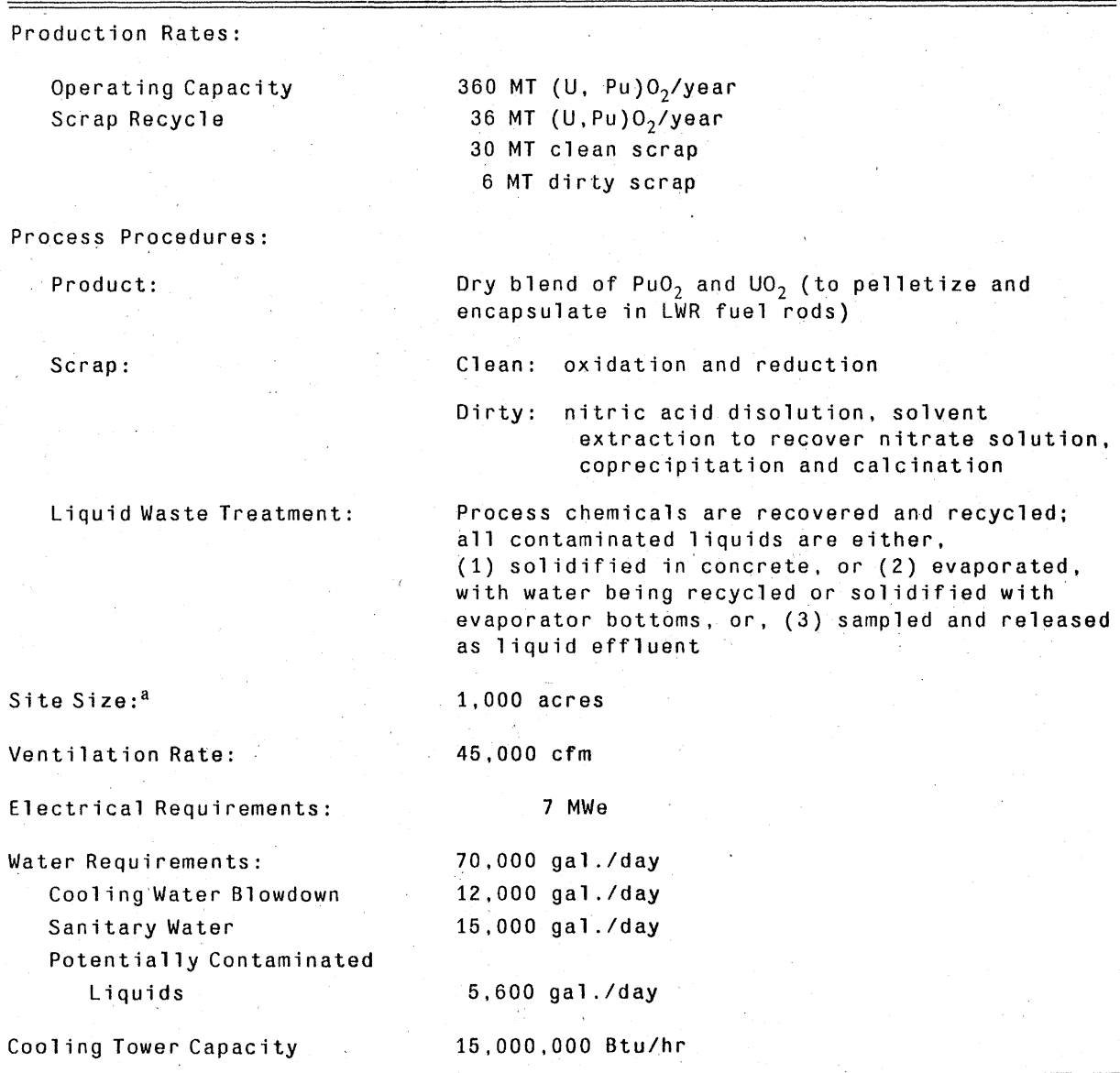

a Although mixed oxide fuel fabrication plants may be colocated with reprocessing or uranium fuel fabrication plants, the model MOX plant has been assumed to be located on a separate site. 
FIG. 5. Material flowsheet for reprocessing. pressurized water reactor, no

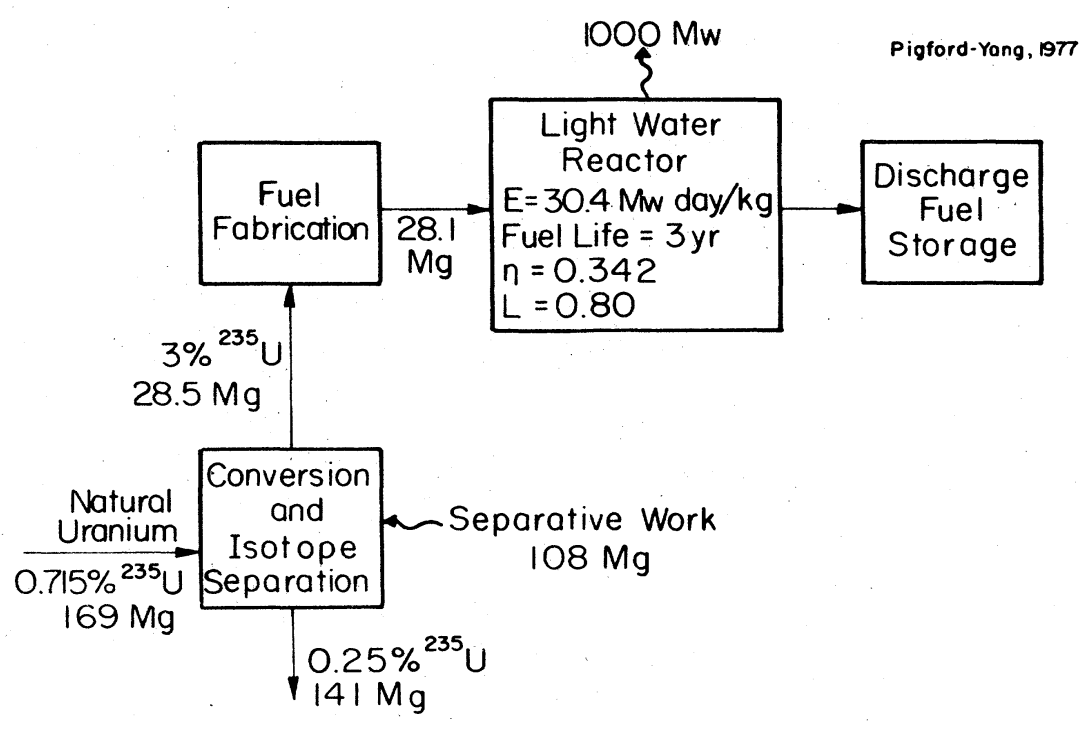

An alternate to dry blending that has been proven on a pilot scale is the co-precipitation process. Mixed nitrate of $\mathrm{U}$, and $\mathrm{Pu}$ are blended, precipitated with $\mathrm{NH}_{3}$ or $\mathrm{NH}_{4} \mathrm{OH}$, and the ammonium diuranate and plutonium hydroxide mixture (ADU- $\mathrm{Pu}(\mathrm{OH})_{4}$ ) is filtered. The filter cake is calcined in air to produce $\mathrm{UO}_{3}$ or $\mathrm{U}_{3} \mathrm{O}_{8}$ and $\mathrm{PuO}_{2}$, or can be calcined in a reducing atmosphere to produce $\mathrm{UO}_{2}$ and $\mathrm{PuO}_{2}$. This $(\mathrm{U}, \mathrm{Pu}) \mathrm{O}_{2}$ then becomes the feed to the pelletizing process. Proponents of the co-precipitaiton process emphasize the advantage of being able to recycle recovered mixed nitrates to the solution blend stage and producing press feed with more predictable pressing and sintering characteristics.

The principal differences in fabricating mixed oxide fuel for the LMFBR instead of the LWR appears to be personnel exposure and criticality considerations because of handling more concentrated materials and different plutonium isotopic compositions (Smith, et al., 1973). Since the use of recycle plutonium in either.case will necessitate remote operation and adequate shielding, it would appear that the incremental increase in handling costs would be overshadowed by other considerations.

\section{G. Fuel Cycle Economics and Resource Utilization}

\section{G1. Fuel Cycle Descriptions and Flowsheets}

The current and near term fuel-cycle aiternatives available to the U.S. power industry are those resulting from uranium fueling in light- water reactors (Pigford and Ang, 1975). These alternative fuel cycles are illustrated for pressurized water reactors, which constitute about two thirds of the U.S. reactors. The general conclusions reached in this study are also applicable to boiling water reactors. At present the only available alternative is the light water reactor fueled with slightly enriched uranium $\left(\mathrm{UO}_{2}\right)$ with no fuel reprocessing, as illustrated by the material flowsheet shown in Figure 5. The material quantities shown are the annual quantities, averaged over the 30-year reactor lifetime. These were calculated from detailed data (Shapiro, 1977) for the initial reactor loading and for each annual refueling, when onethird of the core fuel is removed and replaced with a new fuel reload. The quantities shown in these simplified flowsheets are the result of more detailed flowsheet calculations wherein the effects of process losses and scrap recycle have been taken into account.

The second alternative involves reprocessing the discharge fuel to recover and recycle uranium, containing about $0.8 \%$ enrichment of ${ }^{235} \mathrm{U}$ (See Figure 6.). The effect of uranium recovery and recycle is to reduce the equilibrium consumption of natural uranium by $18 \%$ and the separative work by $2 \%$. This altenative could be implemented now with operation of the Allied-General Nuclear Services reprocessing plant at Barnwell, S.C., with liquid storage of the separated $\mathrm{Pu}\left(\mathrm{NO}_{3}\right)_{4}$ and the high level wastes. However, the present storage capacity for $\mathrm{Pu}\left(\mathrm{NO}_{3}\right)_{4}$ at Barnwell, would limit full scale (1500 metric tons/yr) operation on this fuel cycle to about fifteen months.

The third alternative fuel cycle involves the recovery and recycle of
FIG. 6. Material flowsheet for pressurized reactor with uranium recycle and plutonium storage.

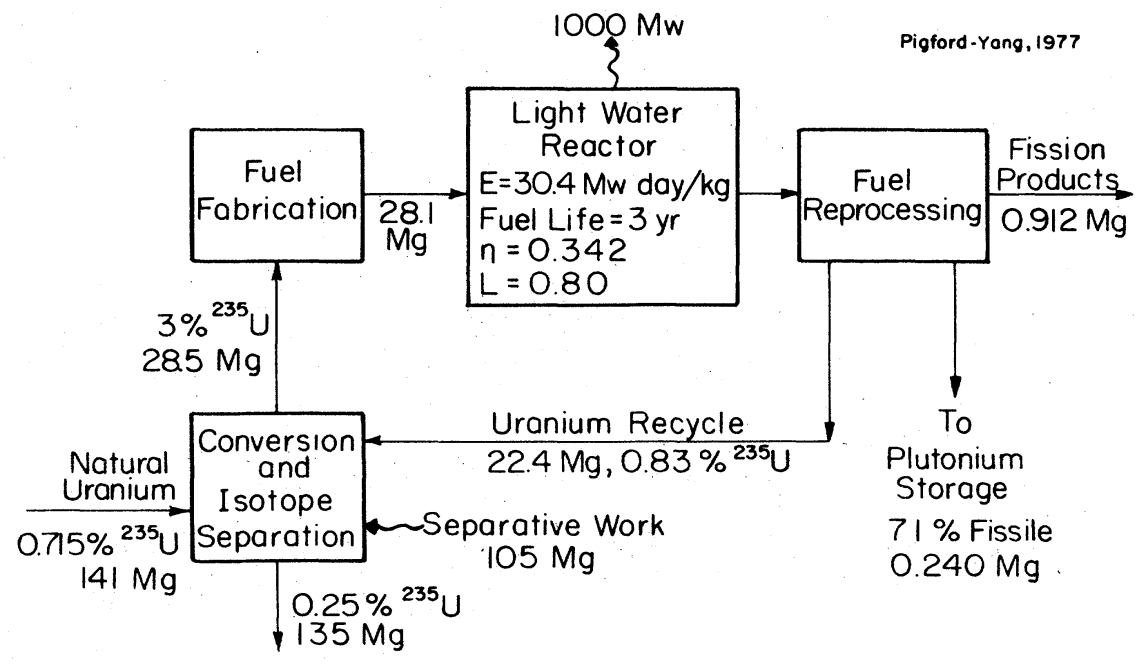




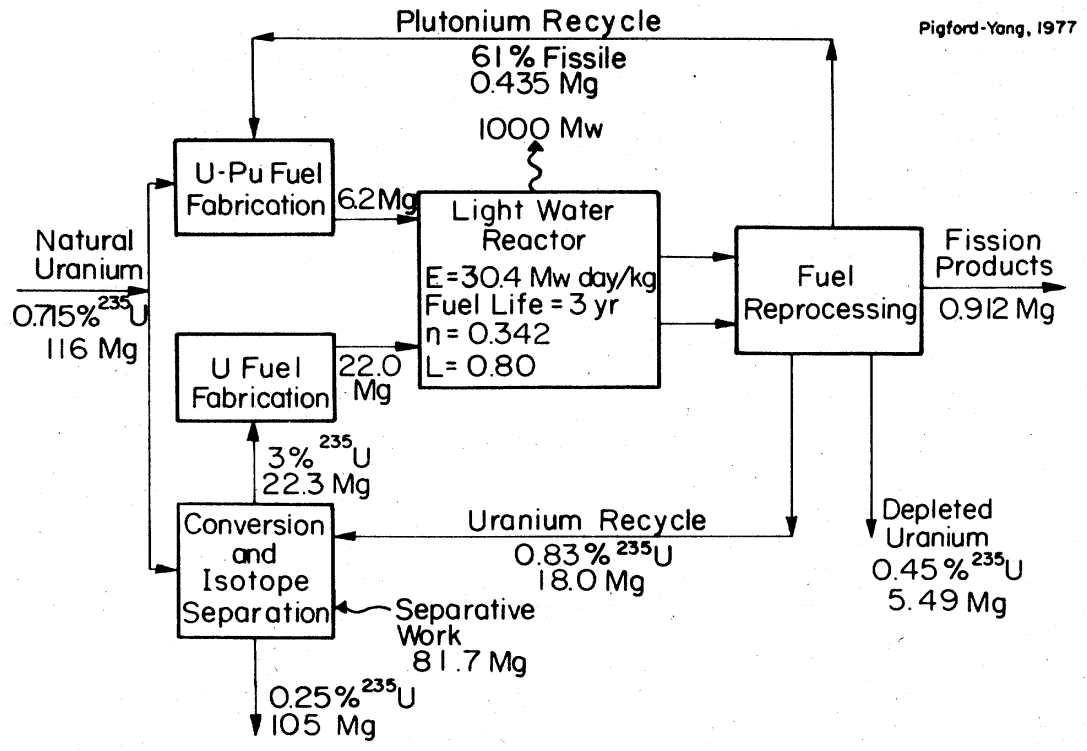

FIG. 7. Material flowsheet for pressurized water reactor with self-generated U-Pu recycle. both uranium and plutonium as illustrated in Figure 7. This is the cycle planned by the nuclear power industry and for which the U.S commercial fuel reprocessing plants have been designed. Here the flowsheet illustrates "self-generated plutonium recycle", whereby the plutonium recovered from discharge fuel is recycled to the same reactor in which it was produced. The recycled plutonium is blended with natural uranium as a $\mathrm{PuO}_{2}-\mathrm{UO}_{2}$ mixture, referred to as "MOX" fuel, to fuel a portion of the reactor. The remaining fuel rods in the reactor are formulated from the same slightly enriched $\mathrm{UO}_{2}$ fuel as in the previous flowsheets. Mixing the discharge $\mathrm{UO}_{2}$, fuel and MOX fuel in reprocessing would reduce the enrichment of the recovered uranium to $0.75 \%$. Alternatively, $\mathrm{UO}_{2}$ fuel elements and MOX fuel elements could be reprocessed separately, resulting in an enrichment of about $0.83 \%$ in the recovered and recycled uranium. As compared with the non-reprocessing cycle of Figure 5, the effect of recycling both plutonium and uranium is to reduce the lifetime average consumption of natural uranium by $32 \%$ and the separative work by 24\%. Extensive studies and designs have been made on techniques to fuel water reactors with combinations of $\mathrm{UO}_{2}$ fuel and MOX fuel so that the reactor characteristics are essentially the same as with $\mathrm{UO}_{2}$ fuel. Water reactors operating with plutonium loadings in MOX fuel $15 \%$ greater than the amount of self generated recycle have been considered in the recent GESMO study (USNRC, 1976). The extra $15 \%$ plutonium would be obtained from reprocessing the fuel discharged from uranium-fueled water reactors not utilizing plutonium recycle.

An alternative fuel cycle which is described in some cinalyses, but not considered here to be realisitic, is that of reprocessing for uranium recovery only and allowing the plutonium to follow the high-level fission-product wastes. Although technically possible, allowing plutonium to follow the wastes would make later recovery of the plutonium for its fuel value difficult and expensive. Better options are 1) to recycle both uranium and plutonium or 2) to store the unreprocessed fuel pending the development of alternate energy sources.

Similarly, the alternative of treating the discharged fuel as disposable waste, while described in some analyses of alternative fuel cycles, is not considered here to be realistic for the present or near future. As will be shown later, the non-reprocessing storage option of Figure 5 places no immediate strain upon our uranium resources. If, for possible reasons discussed elsewhere in this report, fuel reprocessing is delayed so that discharged fuel is stored, this stored fuel remains an already-mined resource that should be preserved for future use, pending the development of alternate sources of energy.

The above three fuel-cycle alternatives are all technically feasible with the existing water reactors. Once plutonium recycle is established commercially, there will arise some incentive to increase further the plutonium loadings in some reactors, while leaving other reactors to be fueled with uranium. As the amount of plutonium fuel in the reactor increases, the relatively high neutron-absorption cross section of plutonium reduces the effectiveness of the boron control absorbers, and more absorbers are required to maintain reactivity control. Some of the newer pressurized-water reactors have been designed with the flexibility of adding sufficient control absorbers so that these reactors can be fueled entirely with natural uranium and plutonium. The flowsheet of such a U-Pu fueled reactor, also referred to as a "plutonium burner" reactor, is shown in Figure 8. The plutonium

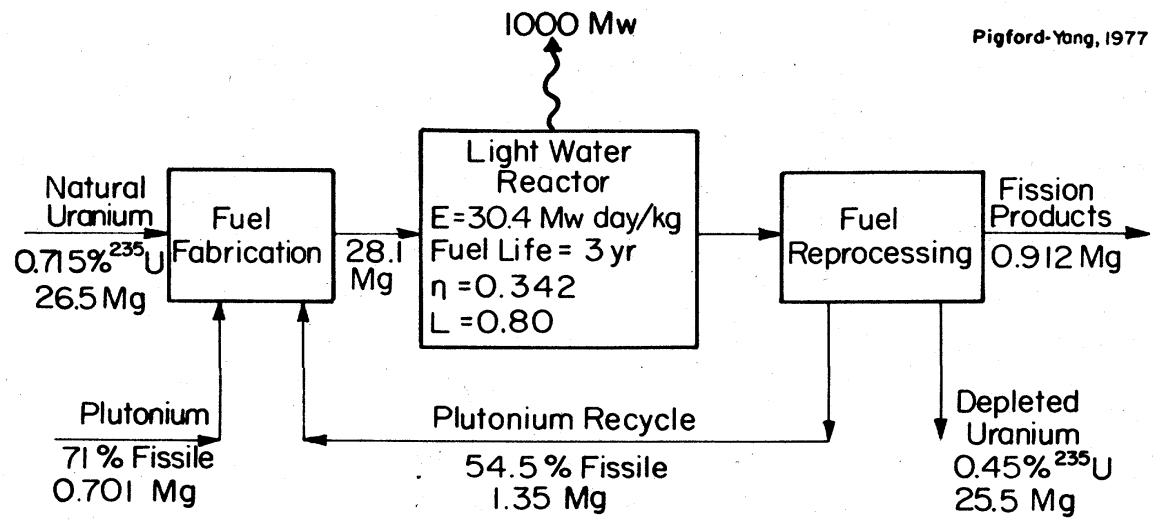

FIG. 8. Material flowsheet for $\mathrm{U}-\mathrm{Pu}$ fueled pressurized water reactor.

Rev. Mod. Phys., Vol. 50, No. 1, Part II, January 1978 
recovered from the discharge fuel is recycled, and make-up plutonium is obtained from reprocessing discharge fuel from uranium-fueled water reactors.

The plutonium burner water reactor is adaptable to those concepts wherein it is desirable to localize the issues connected with utilization of plutonium fuel. Such a reactor could be located at a nuclear energy center, where are also co-located facilities for fuel reprocessing and MOX fuel fabrication. Discharged fuel from off-site uranium-fueled reactors could be shipped to such an energy center, and the plutonium recovered from this fuel could be utilized on site as fuel for the plutonium-burner reactor. An overall system flowsheet for this concept is discussed under "Low Enrichment (Denatured) Safeguards Fuel Cycles" in Section VIII-F. For similar reasons, a utility company may find it desirable to centralize plutonium-burner reactors on some of its sites, to be fueled with make-up plutonium produced in its uranium-fueled reactors at other sites.

If plutonium recycle in water reactors is authorized, it is likely that the recycle plutonium will initially be utilized as a partial loading in uranium-fueled water reactors, with more interest in higher plutonium loadings developing as the plutonium recycle industry grows and matures. However, the utilization of plutonium in water reactors may give way to the competitive demand for this plutonium as start-up fuel for the fast breeder. If commercialization of the breeders were to begin in the 1990's, as had been expected by ERDA, the first-generation breeders would require several core loadings of plutonium recovered from water reactor discharge fuel for start-up. The present administration, however, has deferred fast breeder commercialization for an indefinite period and has similarly deferred reprocessing of spent LWR fuel. The amount of plutonium required for breeder start up is discussed in Chapter VIII. If breeders are introduced at a significant rate in the 1990 's, it will be necessary to discontinue plutonium recycle in water reactors to furnish enough plutonium to start the breeders. Given the previous ERDA schedule of introduction and growth of the breeder program, plutonium recycle in water reactors might be limited to the 1980's. Present policies, however, leave breeder commercialization uncertain and make it impossible to forecast accurately the time frame in which recycle might be viable.

Regardless of plutonium recycle in water reactors, introduction of fast breeders requires the capability of reprocessing uranium fuel from water reactors to obtain the discharge plutonium. Breeder introduction in the early 1990's would require that this capability exist in the latter half of the 1980's. The breeder program also requires the capability of reprocessing discharge core and blanket fuel from breeders for plutonium recovery and recycle. Although founded upon the same basic chemical technology, breeder reprocessing requires a different facility than that used in water reactor fuel reprocessing. Therefore, breeder introduction at a given time would require the existence of two different fuel reprocessing technologies; i.e., water reactor fuel for plutonium production reprocessing at least a few years earlier than breeder commercialization, and breeder fuel reprocessing within a few years afterward.

\section{G2. Uranium Ore Requirements}

The yearly requirements of natural uranium for the fuel cycles are shown on the flowsheets. The total ore required by a given reactor over its operating life must include the ore to supply the start-up fuel inventory as well as the cumulative replacement loadings over the operating life. With recycle operation the equilibrium condition may not be reached for a decade or more, and the additional ore requirements during this transient period must be included. The lifetime ore requirements calculated (Pigford \& Yang, 1977) for the light-water reactor fuel cycles appear in Table 15 . Ore requirements for these same light water reactors operating on the thorium cycle are presented in Chapter VIII.

An estimate of the total uranium consumed and committed for the U.S. electric power industry through the year 2000 can be made on the basis of the assumed nuclear power growth curve of Figure 9. Shown are the ERDA 1976 high growth to $625 \mathrm{GWe}$ and the low growth to $300 \mathrm{GWe}$ by the year 2000 , assumed to be accomplished entirely by light water reactors. Assuming that plutonium recycle from commercial fuel reprocessing begins in 1981, and taking into account
TABLE 15. 30-Year lifetime ore requirements for pressurizedwater reactors (1000 MW electrical power, $80 \%$ capacity factor).

\begin{tabular}{|c|c|c|}
\hline & \multicolumn{2}{|c|}{$\begin{array}{c}\mathrm{U}_{3}{ }^{0} 8 \\
\text { Short Tons }\end{array}$} \\
\hline & $\begin{array}{c}0.2 \% \\
\text { Depleted U }\end{array}$ & $\begin{array}{c}0.25 \% \\
\text { Depleted U } \\
\end{array}$ \\
\hline $\begin{array}{l}\text { No Recycle } \\
U \text { recycle } \\
\text { U-Pu self-generated recycle }\end{array}$ & $\begin{array}{l}6410 \\
5280 \\
4340\end{array}$ & $\begin{array}{l}6970 \\
5820 \\
4770\end{array}$ \\
\hline
\end{tabular}

time delays between new start-up and reprocessing-recycle of discharge fuel, we obtain the estimated cumulative consumption of uranium ore for the high growth case, as shown in Figure 10. The net benefit of uranium-plutonium recycle is to reduce the cumulative ore consumption through the year 2000 by $25 \%$. Because of the time delays and the effect of the increasing number of new start-ups, the cumulative ore saving is considerably less than the $32 \%$ saving derived from the lifetime ore commitments (Table 15 ). The $32 \%$ saving can eventually be realized over the lifetime of each reactor, however. The calculated lifetime commitments of uranium ore for the high growth case, with and without $\mathrm{U}-\mathrm{Pu}$ recycle, are also shown in Figure 10 More advanced fuel cycles with greater savings are discussed in Chapter VIII.

The ERDA-USGS estimates. (ERDA, 1975; USNRC, 1976) of the U.S. uranium resources at a 1976 cost up to $\$ 30 / \mathrm{lb}$ of $\mathrm{U}_{3} \mathrm{O}_{8}$ are also shown in Figure 10. The identified and probable resources within this cost are sufficient to supply the high growth cumulative ore requirments through the year 2000. However, the reserves and probable resources are clearly insufficient to supply the high growth lifetime commitments. Resources of even higher cost should be considered, since 1976 cost data for power plants to be completed in 1985 indicate that uranium ore can be purchased at prices well over $\$ 100 / \mathrm{lb}$, in 1976 dollars, without destroying the economic viability of nuclear power.

Also shown in Figure 10 are the ERDA-USGS estimates of the potential - - but not proved -- U.S. resources of ore up to $\$ 30 / 1 \mathrm{~b}$ $\mathrm{U}_{3} \mathrm{O}_{8}$. A more recent government estimate (Shapiro, et al., 1977)

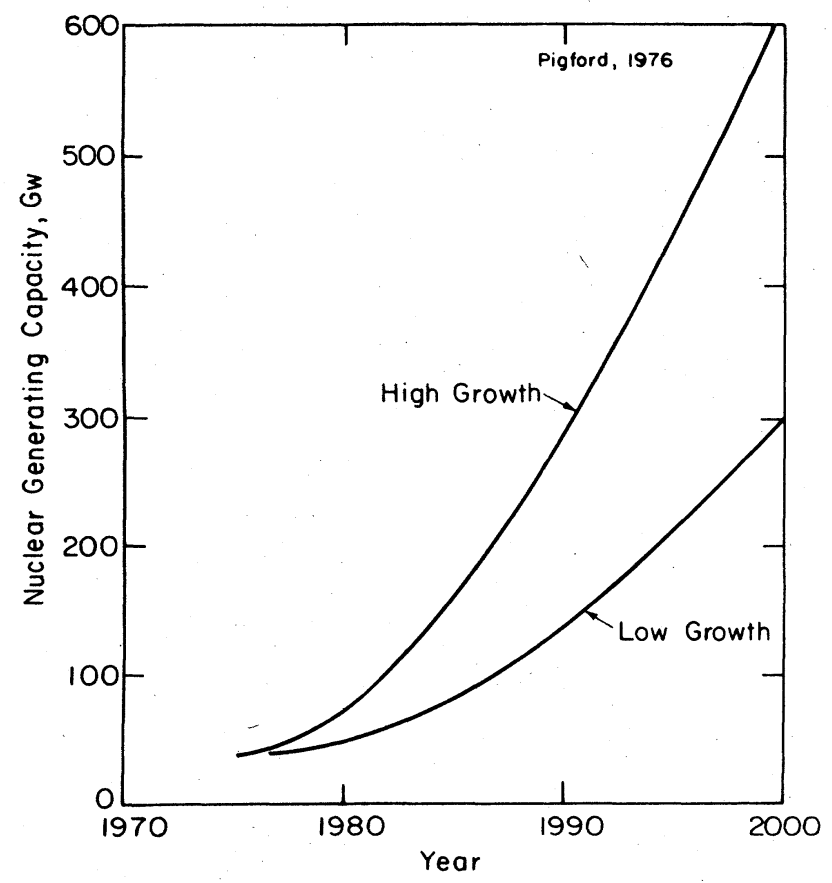

FIG. 9. Growth of U.S. nuclear electric generating capacity (ERDA 1976 estimates). 


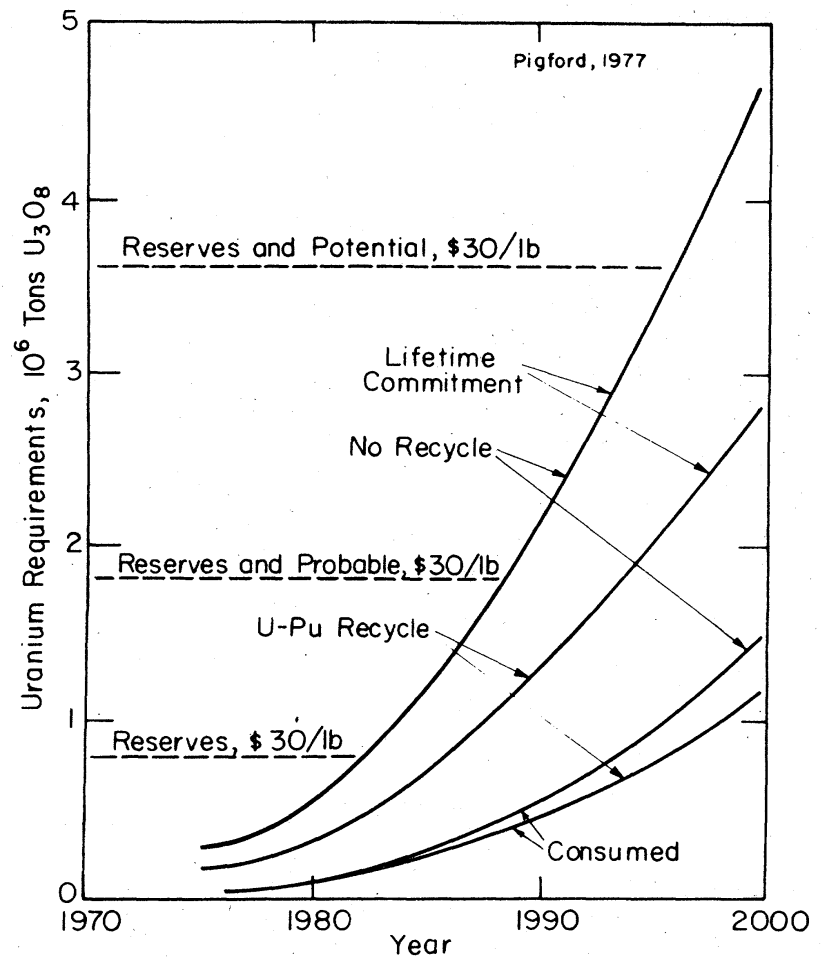

FIG. 10. Ore requirements for U.S. nuclear power industry for the high growth case $\left(0.25 \%{ }^{235} \mathrm{U}\right.$ in depleted uranium).

indicates potential U.S. resources of $8,710,000$ tons at a cost up to $\$ 50 / 1 \mathrm{~b} \mathrm{U}_{3} \mathrm{O}_{8}$, including resources anticipated with additional geologic data. Searl (Searl, 1974) has estimated a median resource of about 13.2 million tons recoverable at a cost of less than $\$ 100 / 1 \mathrm{~b} \mathrm{U}_{3} \mathrm{O}_{8}$ (1974 dollars). However, since these are not proven reserves, it is uncertain whether the U.S. resources will be sufficient for the lifetime ore commitments of these reactors if uranium and plutonium are not recycled. The uncertainty is less if both are recycled.

The cumulative and lifetime ore requirements for the low growth case are half those shown in Figure 10. The present reserves are sufficient for the low growth cumulative requiremenis, with or without recycling. The potential resources, if realized, would be sufficient even for the lifetime requirement, with or without recycle, but again the certainty of adequate supply is greater with recycle.

With uranium resources of the magnitude indicated above, the fast breeder, if successfully introduced, would make it possible to continue nuclear fission power at a steady or growing level for many centuries. The depleted uranium stock-piled from isotope separation will be sufficient to fuel the breeders for a very long time. The only ore requirement attributable to the breeder is that associated with the production of plutonium for start-up loadings of the first-generation breeders. This plutonium must be obtained from light-water reactors, and these reactors will then require more ore because they are thereby deprived of the benefits of plutonium recycle. If the doubling time for subsequent growth in breeder capacity is no shorter than the doubling time for the breeder to produce excess plutonium, no ore is required for subsequent breeders. For each GWe of first-generation breeder capacity installed in the 1990's, 44 GW-yr of light water reactors would be operated without plutonium recycle during the 1980 's and early 1990's to furnish the start-up plutonium.

\section{G3. Economics of Fuel Cycle Options}

The costs of the various fuel-cycle options for a given pressurized water reactor are analyzed here to present some of the incentives for fuel-cycle choices as they might appear to an electrical utility. The comparative economics of the fuel cycles have been analyzed on the basis of the cash-flow model (Pigford, 1976). The unit costs, recoveries, and transaction times for the fuel-cycle operations, adopted here as reference-case values, are given in Table 16 . The costs are quoted in 1976 dollars, and they are considered representative of noninflated costs appropriate for nuclear power plants beginning operation in the mid-1980's. The discount factor used in calculating the carrying charges for advanced or deferred payments and credits is $7.55 \% / y r$ (Pigford, 1976), typical of that appropriate for investorowned electrical utilities.

For each of the fuel cycle options data were developed (Pigford \& Choi, 1977; Pigford and Yang, 1977; Shapiro, et al., 1977) for the initial loading of fuel in the reactor and for the charge and discharge quantities and compositions of each replacement loading throughout the reactor life. The cost of electrical energy generated by each batch of fuel was determined, and the fuel cycle cost levelized over the 30yr. plant life was calculated.

\section{G3a. Costs of the Non-Reprocessing ("Stowaway") Fuel Cycle}

For the non-reprocessing fuel cycle it is assumed that the discharge fuel is stored in a water canal, either at the reactor site or elsewhere, until its heat generation rate by fission-product decay is at the same level as in high-level reprocessing wastes which could be shipped to a federal repository. It is assumed that the discharge fuel is then shipped to a federal repository, where the fuel is stored on a retrievable basis and is available, if needed, for later reprocessing to recover the uranium and plutonium. No credit for future reprocessing is considered.

The quantities of $\mathrm{U}_{3} \mathrm{O}_{8}$ required for each yearly fuel loading are shown in Figure 11. The initial fuel loading consists of three fuel batches. Each year thereafter one third of the core is replaced with a new fuel reload, i.e., a replacement "fuel batch". Although each fuel batch contains essentially the same mass of uranium, the enrichment varies from one batch to another, especially for the three initial-load batches and the next few replacement batches. The large first-year ore requirement in Figure 11 is a result of purchasing the entire initial load. The smaller amount of ore required for the second and third years reflects the lower enrichment required for these reload batches. Thereafter each annual reload is of constant enrichment and requires the same amount of ore, except for the last two reloads which are of lower enrichment and require less ore.

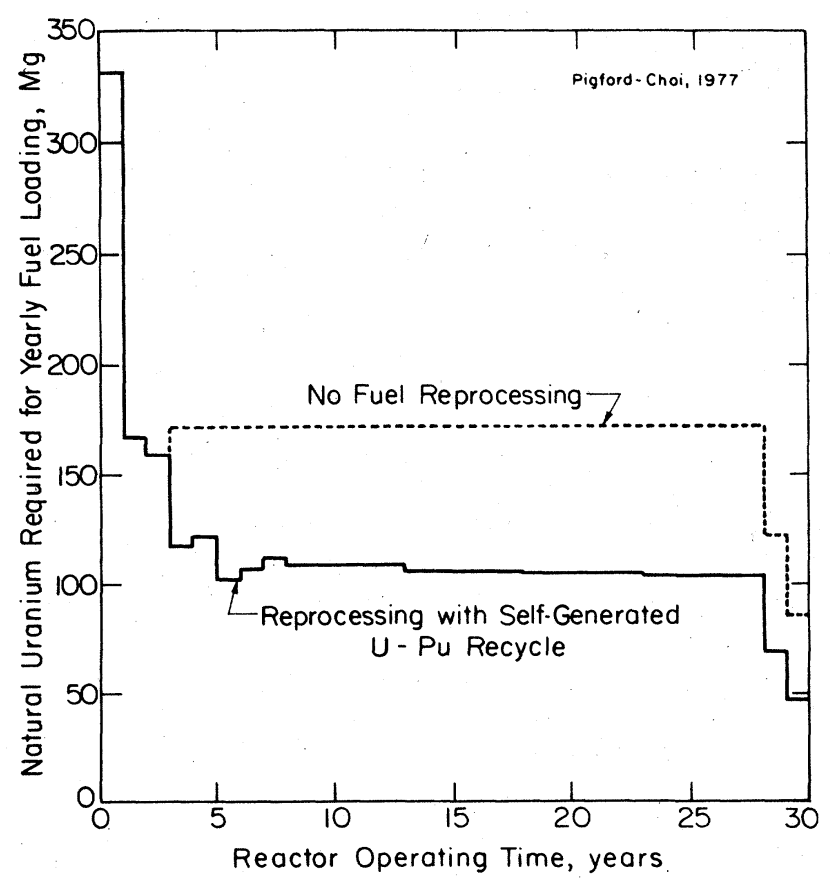

FIG. 11. Natural uranium required for yearly fuel loading of a 1000-MW pressurized-water reactor. 
TABLE 16. Unit costs, recoveries, process times and fuel cycle operations.

\begin{tabular}{|c|c|c|c|c|}
\hline Operation & $\begin{array}{l}\text { Unit Cost } \\
\text { in } 1976 \\
\text { do } 11 \mathrm{ars}^{\mathrm{a}} \\
\$ / \mathrm{kg}\end{array}$ & $\begin{array}{l}\text { ecovery } \\
\text { actor in } \\
\text { peration }\end{array}$ & $\begin{array}{l}\text { Relative Time } \\
\text { beginning of } \\
\text { fuel operation } \\
\text { yr }\end{array}$ & $\begin{array}{l}\text { of Expenditure } \\
\text { Relative to } \\
\text { fuel discharge } \\
\text { yr }\end{array}$ \\
\hline $\begin{array}{l}\mathrm{U}_{3} \mathrm{O}_{8} \text { purchase } \\
(\$ 28 \text { per pound } \\
\left.\text { of } \mathrm{U}_{3} \mathrm{O}_{8}\right)\end{array}$ & $\$ 72.64 / \mathrm{kg} \mathrm{U}$ & 1.00 & -2 & \\
\hline $\begin{array}{l}\mathrm{U}_{3} \mathrm{O}_{8} \text { to } \mathrm{UF}_{6} \\
\text { conversion }\end{array}$ & $\$ 3.50 / \mathrm{kg} \mathrm{U}$ & 0.995 & -1.5 & \\
\hline $\begin{array}{l}\text { Isotope } \\
\text { Separation } \\
\mathrm{UO}_{2} \text { conversion } \\
\text { and fabrication }\end{array}$ & $\begin{array}{l}\$ 75.00 / \mathrm{kg} \mathrm{SWU} \\
\$ 95.00 / \mathrm{kg} \mathrm{U}\end{array}$ & $\begin{array}{l}1.00 \\
0.99\end{array}$ & $\begin{array}{l}-1.0 \\
-0.5\end{array}$ & \\
\hline $\begin{array}{l}\text { Shipment of } \\
\text { discharge fuel }\end{array}$ & $\$ 15.00 / \mathrm{kg} \mathrm{HM}$ & 1.00 & & +0.75 \\
\hline $\begin{array}{l}\text { Fuel } \\
\text { processing }\end{array}$ & $\$ 165.00 / \mathrm{kg} \mathrm{HM}$ & $0.99 \mathrm{Pu}, \mathrm{U}$ & & +1 \\
\hline $\begin{array}{l}\text { Waste management- } \\
\text { federal repository }\end{array}$ & $\$ 50.00 / \mathrm{kg} \mathrm{HM}$ & 1.00 & & +11 \\
\hline $\begin{array}{l}\mathrm{PuO}_{2}-\mathrm{UO}_{2} \\
\text { conversion } \\
\text { fabrication }\end{array}$ & $\$ 198.00 / \mathrm{kg} \mathrm{HM}$ & 0.99 & -0.5 & \\
\hline $\begin{array}{l}\text { Shipment of } \\
\text { fissile } \mathrm{Pu} \\
\left(\text { as } \mathrm{PuO}_{2}\right)\end{array}$ & $\$ 40.00 / \mathrm{kg} \mathrm{Pu}$ & 1.00 & -0.75 & \\
\hline $\begin{array}{l}\text { Canal storage of } \\
\text { discharge fuel }\end{array}$ & $\$ 5.00 / \mathrm{yr} \mathrm{kg} \mathrm{HM}$ & 1.00 & & $+1,2, \ldots$ \\
\hline $\begin{array}{l}\text { Long-term storage } \\
\text { of discharge fuel } \\
\text { in repository }\end{array}$ & $\$ 100.00 / \mathrm{kg} \mathrm{HM}$ & 1.00 & & +11 \\
\hline
\end{tabular}

a "HM" denotes heavy metal, i.e., total actinides charged to the reactor. All unit cost data are from NRC (USNRC, 1976), except the cost of fuel reprocessing. The reference cost of UO $\mathrm{O}_{2}$ fuel reprocessing was derived in Section IV-E9. (See Table 13.) MOX fuel reprocessing is assumed to cost $20 \%$ more than $\mathrm{UO}_{2}$ reprocessing.

For each fuel batch the electrical energy generated is calculated and a cash-flow account for that batch includes all advanced payments for preparing that fuel and all later payments for storing and shipping that fuel when discharged. Thus, an apparent fuel-cycle cost for the complete handling of the fuel involved in each year of reactor operation is determined, and the results are plotted in Figure 12. The first-year cost reflects the large purchase of ore for the initial core loading, balanced in part by the savings from the lower enrichments

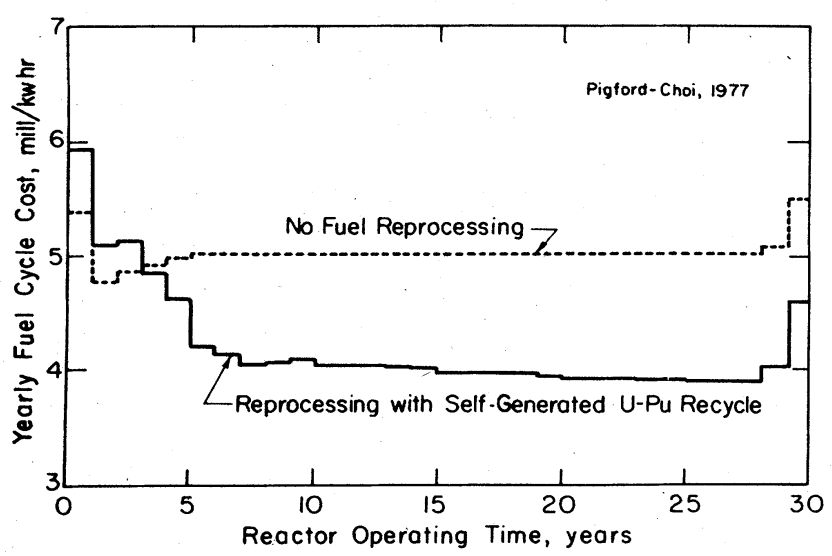

FIG. 12. Yearly fuel cycle cost for pressurized-water reactor (reference costs in 1976 dollars). of the first fuel batches. "Savings from lower enrichments of the first two replacement loadings continue up to the fifth year. The higher costs for the last two cycles occur because of the lower burnup of fuel purchased for these cycles.

The contributions to the 30-year levelized fuel cycle costs of stowaway cycle are shown in Table 17. The total fuel cycle cost is greater than the arithmetic mean of the yearly costs shown in Figure 12 because the levelizing calculation weights the costs occuring earlier in the reactor lifetime.

The unit yearly costs of canal storage of discharge fuel are such that an estimated fuel cycle cost with continued long-term canal storage is less than the cost shown here with 10-year canal storage followed by long-term storage in a geologic repository.

G3b. Costs of the Fuel Cycle with Reprocessing and UraniumPlutoniuin Recycle

The fuel cycle costs for reprocessing with uranium-plutonium recycle can be calculated directly from the cycle-by-cycle data on the fuel material quantities charged and discharged to the reactor. For self-generated recycle no plutonium or uranium is sold or purchased. Instead, the recovered plutonium is recycled, when available, thereby

* Although the present analysis assumes that for the reprocessing case all fuel batches are reprocessed for $\mathrm{U}-\mathrm{Pu}$ recycle, it would be more economical not to reprocess the first batch, discharged after the first year of operation. 
TABLE 17. Fuel cycle cost for a pressurized water reactor with no reprocessing of the discharge fuel (30-year levelized cost in 1976 dollars with unit costs from Table XVI.

\begin{tabular}{lc}
\hline \hline & $\begin{array}{c}\text { Fue1 Cycle Cost } \\
\text { mill/kwh }\end{array}$ \\
\hline $\mathrm{U}_{3} \mathrm{O}_{8}$ purchase & 2.72 \\
$\mathrm{U}_{3} \mathrm{O}_{8}$ to UF 6 conversion & 0.12 \\
Isotope Separation & 1.51 \\
Fue conversion and fabrication & $\underline{0.55}$ \\
$\quad$ Total cost of fuel charged to reactor & 4.90 \\
Storage of discharge fue1 for 11 years & 0.13 \\
Ship to federal repository & 0.02 \\
Store in federal repository & $\underline{0.17}$ \\
$\quad$ Total Fuel Cycle Cost & 5.22 \\
\hline \hline
\end{tabular}

reducing the amount of natural uranium and of enrichment service which must be purchased for subsequent cycles. The plutonim is followed through the five successive recycles during the $30-y r$. period, and it is assumed that the plutonium in a given batch of discharge fuel is the same as that later recovered from reprocessing, i.e., crossover between fuel batches in reprocessing is neglected. In this way the continued build-up of the higher-mass isotopes of plutonium, e.g., ${ }^{242} \mathrm{Pu}$, and their effect upon reactivity and burnup are properly taken into account.

Similarly, the uranium recovered from fuel reprocessing is recycled for isotopic enrichment to $3 \%{ }^{235} \mathrm{U}$, further reducing the amount of natural uranium and enrichment which must be purchased for later replacement fuel loadings. It is assumed that discharge $\mathrm{UO}_{2}$ fuel is reprocessed separately from discharge MOX fuel, to avoid the degradation in isotopic concentration of ${ }^{235} \mathrm{U}$ that would otherwise occur if the two types of discharge fuel were reprocessed togethr. The uranium recovered from the discharge MOX fuel is store?

The effect of ${ }^{236} \mathrm{U}$ in the recycled uranium is taken into accorric by calculating (de la Garza, 1977) the amount of ${ }^{236} \mathrm{U}$ which would follow the ${ }^{235} \mathrm{U}$ in the isotope enrichment process. It is assumed that a hypothetical captive isotope separation facility exists which operates only on the natural uranium and recycle uranium needed for this particular reactor. Thereby, the actual dilution of ${ }^{236} \mathrm{U}$ by the large amount of first-cycle uranium in the single U.S. enrichment system is neglected. The slightly increased enrichment of ${ }^{235} \mathrm{U}$ necessary to compensate for neutron absorption in the recycled ${ }^{236} \mathrm{U}$ from the recycled uranium is then calculated. The increased $235 \mathrm{U}$ enrichment requires greater amounts of natural uranium and of separative work. The presence of ${ }^{236} \mathrm{U}$ also slightly increases the separative work to produce a given ${ }^{235} \mathrm{U}$ enrichment. These perturbations in the composition of the fresh fuel are followed through the entire fuel cycle, and equilibrium perturbations in the concentrations of $235 \mathrm{U}$, ${ }^{236} \mathrm{U}$, and $\mathrm{Pu}$ in the discharge fuel are calculated. The corrections due to ${ }^{236} \mathrm{U}$ recycle with this model are found to be very small, resulting in an increase in fuel cycle cost of less than $2 \%$. This is in agreement with other published work on the effect of ${ }^{236} \mathrm{U}$ in recycle fuel (Colglazier \& Weatherwax, 1976; de la Garza, 1977; Geller and Gueron, 1969: Goellner, et al. 1967; Peak, 1975; Sprauge, 1974). Separate corections are not made for the build-up of ${ }^{242} \mathrm{Pu}$ when plutonium is recycled because this build-up is already taken into account in the cycle-by-cycle calculations (Shapiro, et al., 1977) for plutonium recycle used in this study.

The quantities of $\mathrm{U}_{3} \mathrm{O}_{8}$ required for each yearly fuel loading (Pigford and Choi, 1977), are shown in Figure 11. The initial loading and the first two replacement loadings are identical with those for the non-reprocessing cycle, because during this period no plutonium and uranium have been returned for fuel reprocessing. Thereafter the yearly requirement is smaller for the reprocessing cycle. The fluctuations in the yearly quantities from the third through the eighth year reflect small adjustments in the ${ }^{235} \mathrm{U}$ enrichment to finally achieve an equilibrium cycle of constant uranium enrichment in the
TABLE 18. Fuel cycle costs for pressurized water reactor with self-generated recycle of uranium and plutonium (Pigford and Choi, 1977) (30-year levelized cost in 1976 dollars with unit costs from Table 16).

\begin{tabular}{|c|c|}
\hline & $\begin{array}{c}\text { Fuel Cycle Cost } \\
(\mathrm{mill} / \mathrm{kwh})\end{array}$ \\
\hline $\mathrm{U}_{3} \mathrm{O}_{8}$ purchase & 2.06 \\
\hline $\mathrm{U}_{3} \mathrm{O}_{8}$ to $\mathrm{UF}_{6}$ conversion & 0.09 \\
\hline Isotope Separation & 1.24 \\
\hline \multicolumn{2}{|l|}{ Fuel converstion and fabrication: } \\
\hline $\mathrm{UO}_{2}$ & 0.47 \\
\hline MOX & $\underline{0.16}$ \\
\hline Total Cost of Fuel Charged to Reactor & 4.02 \\
\hline \multicolumn{2}{|l|}{ Ship discharge fuel: } \\
\hline $\mathrm{UO}_{2}$ & 0.05 \\
\hline $\operatorname{mox}$ & 0.01 \\
\hline \multicolumn{2}{|l|}{ Fuel reprocessing: } \\
\hline $\mathrm{UO}_{2}$ & 0.51 \\
\hline MOX & 0.09 \\
\hline Waste Management in federal repository & $\underline{0.08}$ \\
\hline TOTAL FUEL CYCLE COST & 4.76 \\
\hline
\end{tabular}

fuel charged to the reactor. The ore requirements for the last two reloadings are less than those of the equilibrium reloadings because of the lower burnups and lower enrichment requirements for these last reloads.

The apparent fuel-cycle costs for each year for this fuel cycle (Pigford and Choi, 1977) are shown in Figure 12. During the first three years the fuel cycle cost is higher than for the non-reprocessing cycle. This results because during the first three years the fuel cycle is being charged with the eventual cost of reprocessing these batches, the burnup of the first two discharge fuel batches is relatively small, and no reduction in ore and enrichment purchases due to recycle have yet occurred. The yearly fuel cycle costs are essentially constant after the beginning of the seventh year, except for the higher costs in the last two years due to the lower burnup of the last two reloads.

The contributions to the $30-y r$ levelized fuel cycle costs with reprocessing and recycle are shown in Table 18. The total fuel cycle cost for our reference case is $9 \%$ less than that for the stowaway cycle of Table 17.

G3c. Costs of the Fuel Cycle with Uranium Recycle and Plutonium Storage

Here it is assumed that the discharge fuel is reprocessed and uranium is recycled. The plutonium is to be stored for some arbitrary period, such as ten years, after which it, too, is recycled as waterreactor fuel or used for breeder start-up. To establish the plutonium value, a cash flow account was calculated over the lifetime of two identical reactors: one operating in self-generated $\mathrm{U}-\mathrm{Pu}$ recycle, as in F $3 \mathrm{~b}$, and the other operating with uranium recycle and the sale of its plutonium at the time produced. Equating the lifetime levelized fuel cycle costs of these two concepts, the value of plutonium as a waterreactor recycle fuel was established. Since all unit costs in the present model are assumed to remain constant with time, when expressed in constant-value dollars, the value of plutonium thus calculated applies as well when the plutonium is sold ten years later. However, the 
TABLE 19. Fuel cycle costs for a pressurized water reactor with uranium recycle and 10-year plutonium storate (Pigford and Choi, 1977) (30-year levelized cost in 1976 dollars, with unit costs from Table 16).

\begin{tabular}{|c|c|}
\hline & $\begin{array}{c}\text { Fuel Cycle Cost } \\
(\mathrm{mi} 11 / \mathrm{kwh})\end{array}$ \\
\hline $\mathrm{U}_{3} \mathrm{O}_{8}$ purchase & 2.26 \\
\hline $\mathrm{U}_{3} \mathrm{O}_{8}$ to $\mathrm{UF}_{6}$ conversion & 0.11 \\
\hline Isotope Separation & 1.48 \\
\hline Fuel converstion and fabrication & 0.55 \\
\hline Total Cost of Fuel Charged to Reactor & 4.40 \\
\hline Ship discharge fuel & 0.05 \\
\hline Fuel reprocessing & 0.58 \\
\hline Waste Management in federal repository & 0.08 \\
\hline Plutonium storage & 0.38 \\
\hline Plutonium credit & -0.16 \\
\hline TOTAL FUEL CYCLE COST & 5.33 \\
\hline
\end{tabular}

present value of that deferred sale of plutonium is less than if the plutonium had been sold immediately.

The contributions to the 30-year levelized fuel cycle costs (Pigford and Choi, 1977) are shown in Table 19. The total of $5.33 \mathrm{mill} / \mathrm{kwh}$ is $2 \%$ greater than that of the stowaway cycle. However, if plutonium were not stored and later sold but instead allowed to go to the waste, the fuel cycle cost would become $3 \%$ less than that of the stowaway cycle. As discussed earlier, degradation of plutonium to a nuclear waste seems undesirable pending a resolution of the longer-term issues affecting utilization of plutonium as a nuclear fuel.

It is conceivable that the possibly higher value of plutonium at some future date would reduce the economic penalty for delaying plutonium utilization. For this option of a 10-year delay in plutonium use to cost no more than the non-reprocessing option of Table 17, the plutonium value would have to increase by about $70 \%$ over the ten-year period, all other unit costs remaining constant. Possibly higher values of plutonium as fuel for start-up of future breeders, when and if they exist, are not likely to be realized until much later, when there is cost experience from commercial breeder construction and operation. However, a rise in the constant-dollar price of uranium during the reactor life is a real possibility, and this will affect both the plutonium value and the financial incentive for this fuel cycle. The effect of increases in uranium price on fuel cycle costs are discussed in Section G3e.

\section{G3d. Costs of the Fuel Cycle with Delayed Fuel Reprocessing}

Here we assume a 10-year delay in fuel reprocessing, during which discharge fuel is stored. The fuel is then shipped and reprocessed for recovery and recycle of uranium and plutonium. The non-inflated unit prices of uranium and plutonium and of all the fuel cycle operations are assumed to remain constant. The credit for recovered plutonium is calculated using the plutonium fuel value described in Section G3c. By a similar technique, the fuel value of recovered uranium levelized over the 30-year reactor lifetime is calculated and used to establish the credit for the uranium recovered after delayed
TABLE 20. Fuel cycle costs for a pressurized water reactor with 10-year delay in fuel reprocessing (Pigford and Choi, 1977) (30-year levelized cost in 1976 dollars with unit costs from Table 16).

\begin{tabular}{lc}
\hline & $\begin{array}{c}\text { Fue1 Cycle Cost } \\
\text { (mil1/kwh) }\end{array}$ \\
\hline $\mathrm{U}_{3} \mathrm{O}_{8}$ purchase & 2.72 \\
$\mathrm{U}_{3} \mathrm{O}_{8}$ to UF ${ }_{6}$ conversion & 0.12 \\
Isotope Separation & 1.51 \\
Fuel converstion and fatrication & 0.55 \\
Storage of discharge fuel for 10-years & 0.13 \\
Total Cost of Fuel Charged to Reactor & 4.90 \\
Ship discharge fuel & 0.02 \\
Fue 1 reprocessing & 0.28 \\
Waste Management in federal repository & 0.04 \\
Credit for recovered uranium and plutonium & -0.42 \\
& \\
\hline
\end{tabular}

reprocessing. The contributions to the 30-year levelized fuel cycle costs are shown in Table 20. The cost of this fuel cycle is $5 \%$ less than that of the stowaway cycle. Further, concerning the results in Table 19 and 20 , the cost of immediate reprocessing with uranium recycle and 10-year delay in plutonium recycle is greater than the cost of 10-year delay in reprocessing the discharge fuel. This difference results because the cost of storing separated plutonium for 10 years is much greater than the cost of storing discharge fuel for the same period.

\section{G3e. Effect of Price of Uranium Ore on Fuel Cycle Costs}

Table 17 through 20 show that the greatest single contributor to the fuel cycle costs is the purchase of natural uranium. The future price of natural uranium is one of the greatest uncertainties affecting analysis of fuel cycle options. The reference price of $\$ 28 / \mathrm{lb} \mathrm{U}_{3} \mathrm{O}_{8}$ is understood to be representative of many contracts which extend well along the operating life of reactors started in the mid 1980's, provided these uranium prices are expressed in terms of constant dollars. However, further contracts must be made. Even if de-esclated to 1976 dollars, the price of future contracts may increase because of (a) the depletion of the more readily available uranium resources and (b) the rise in the frie-market price of alternative fuels.

To illustrate the effect of the price of uranium ore, we consider two additional cases:

(1) The constant-dollar price of $\mathrm{U}_{3} \mathrm{O}_{8}$ increases linearly from the initial reference cost. The annual increase is $5 \%$ of the reference cost.

(2) The $\mathrm{U}_{3} \mathrm{O}_{8}$ price is doubled.

The results of calculations for the 30-year levelized costs for the different fuel cycles with the three different assumptions of uranium price are shown in Table 21 . For those options involving 10-year deals and credit for recovered uranium and/or plutonium, the credits were established by recalculating the fuel value of plutonium and recovered uranium by the model described earlier. The values of the recovered uranium and plutonium increase with the price of natural uranium.

The assumed linear increase in the price of natural uranium results in an increase of about $18 \%$ in the fuel cycle costs for both the stowaway cycle and for reprocessing with recycle. The $9 \%$ benefit of recycle is affected little by this assumed linear increase in uranium price, because the early part of the operating life is weighted when calculating the 30-year levelized cost of the fuel cycle. However, a two-fold increase in the uranium price over the entire operating life 
TABLE 21. Costs of various fuel cycle options as affected by the cost of natural uranium (1976 Dollars) (Pigford and Choi, 1977). (All unit costs are the same as in Table 16 except for the indicated increase in $\mathrm{U}_{3} \mathrm{O}_{8}$ cost.)

\begin{tabular}{|c|c|c|c|}
\hline & $\begin{array}{l}\mathrm{U}_{3} \mathrm{O}_{8} \cos \mathrm{t} \\
\text { constant } \\
\text { at } \$ 28 / 1 \mathrm{~b}\end{array}$ & $\begin{array}{l}\mathrm{U}_{3} \mathrm{O}_{8} \cos \mathrm{t} \\
\text { increase } \\
5 \% / \mathrm{yr}\end{array}$ & $\begin{array}{l}\mathrm{U}_{3} \mathrm{O}_{8} \cos \mathrm{t} \\
\text { constant } \\
\text { at } \$ 56 / 1 \mathrm{~b}\end{array}$ \\
\hline \multicolumn{4}{|l|}{ 1. Stowaway Cycle } \\
\hline $\mathrm{U}_{3} \mathrm{O}_{8}$ purchase, mill/kwh & 2.72 & 3.65 & 5.44 \\
\hline other costs, millkwh & 2.51 & 2.51 & 2.51 \\
\hline Total fuel cycle cost, mill/kwh & 5.23 & 6.16 & 7.95 \\
\hline \multicolumn{4}{|l|}{ 2. $U+P u R e c y c l e$} \\
\hline $\mathrm{U}_{3} \mathrm{O}_{8}$ purchase, mil1/kwh & 2.06 & 2.89 & 4.12 \\
\hline other costs, mill/kwh & 2.70 & 2.70 & 2.70 \\
\hline Total fuel cycle cost, mil1/kwh & 4.76 & 5.59 & 6.82 \\
\hline Change from stowaway cycle & $-9 \%$ & $-9 \%$ & $-14 \%$ \\
\hline \multicolumn{4}{|l|}{ 3. U Recycle 10-yr Pu Storage } \\
\hline $\mathrm{U}_{3} \mathrm{O}_{8}$ purchase, mill/kwh & 2.26 & 3.26 & 4.52 \\
\hline other costs, mill/kwh & 3.23 & 3.23 & 3.23 \\
\hline Pucredit, mill/kwh & -0.16 & -0.23 & -0.24 \\
\hline Total fue 1 cycle cost, mill/kwh & 5.33 & 6.26 & 7.51 \\
\hline Change from stowaway cycle & $+2 \%$ & $+2 \%$ & $-6 \%$ \\
\hline \multicolumn{4}{|c|}{ 4. 10-yr Storage Followed by U-Pu Recycle } \\
\hline $\mathrm{U}_{3} \mathrm{O}_{8}$ purchase, mill/kwh & 2.72 & 3.65 & 5.44 \\
\hline Other costs, mill/kwh & 2.66 & 2.66 & 2.66 \\
\hline U-Pu credit, mill/kwh & -0.43 & -0.65 & -0.75 \\
\hline Total fuel cycle cost, mill/kwh & 4.95 & 5.66 & 7.35 \\
\hline Change from stowaway cycle & $-5 \%$ & $-8 \%$ & $-8 \%$ \\
\hline
\end{tabular}

a A linear increase, beginning with $\$ 28 / 1 \mathrm{~b}$

increases the fuel cycle costs by about $50 \%$. The reprocessing benefit, defined as the percentage difference between the cost for the stowaway fuel cycle and the cost for this cycle of reprocessing and recycle, increases from $9 \%$ to $14 \%$ as the uranium price is doubled. Similar increases in fuel cycle costs and in benefits relative to the stowaway cycle occur with the other two cycles, as shown in Table 21 . With a two-fold increase in price of natural uranium, even reprocessing for uranium recycle and plutonium storage results in a $6 \%$ benefit over the stowaway cycle. Ultimately, at even higher uranium prices, the benefit from this fuel cycle will become as great as that for 10-year storage prior to reprocessing.

\section{G3f. Effect of Changes in Unit Costs of Fuel Cycle Operations}

The effect of an arbitrarily assumed two-fold increase in the unit cost of each of the fuel cycle operations is shown in Table 22. Each change is assumed to occur while all other unit costs are held at the reference values of Table 16. Although the cost (or price) of natural uranium is the most important individual contribution to the total fuel cycle cost, as demonstrated by the previous tables, the same percentage change in the cost of fuel reprocessing has more effect upon the reprocessing benefit than the same percentage change of any of the other parameters. For the reference case, a two-fold increase in the cost of fuel reprocessing would result in no benefit from reprocessing, i.e., the stowaway cycle would be the more economical. The possibilities of such a change in the reprocessing unit cost were discussed in Section IV.E and will be reviewed in the next section of this chapter.

The effect of uranium cost (or price) on the reprocessing benefit has already been demonstrated in Section G3e. The effects of twofold increases in the other parameters are relatively small.

The only other parameters whose increase would reduce the reprocessing benefit are the cost of MOX fabrication, $\mathrm{UO}_{2}$ and MOX shipment, spent fuel storage, and the ratio of the cost of geologic disposal of radioactive wastes to the cost of geologic storage-disposal of discharge fuel. However, it would require a very large increase in any one of these parameters, with no compensating increase in the others, to result in no reprocessing benefit. In its analysis of the possible variation of the unit costs, NRC (USNRC, 1976) estimates a possible $50 \%$ increase in the cost of MOX fabrication and a possible two-fold increase in the costs of fuel storage and transportation.

It is clear that the reprocessing benefit is most sensitive to the cost of fuel reprocessing. It is also sensitive to the cost of natural uranium but is relatively insensitive to the cost of the other fuel cycle operations. We turn in section $G 3 \mathrm{~h}$ to the evaluation of the reprocessing benefit in terms of the parameters which are expected to affect the unit cost of reprocessing.

G3g. Effect of Concentration of Depleted Uranium From Isotope Separation

The fuel cycle costs for the four different fuel cycle options in Sections G3a through G3f were calculated on the assumption of $0.25 \%$ ${ }^{235} \mathrm{U}$ in the depleted uranium from isotope separation. For the unit costs listed in Table 16 the economic optimum concentration of ${ }^{235} \mathrm{U}$ in the depleted uranium is nearer to $0.2 \%$. Operation at this lower concentration reduces the fuel cycle costs by 2 to $3 \%$. The reprocessing benefit remains at about $9 \%$ of the fuel cycle cost. 
TABLE 22. Effect of unit cost parameters on the benefit from reprocessing with U-Pu recycle (Pigford and Choi, 1977).

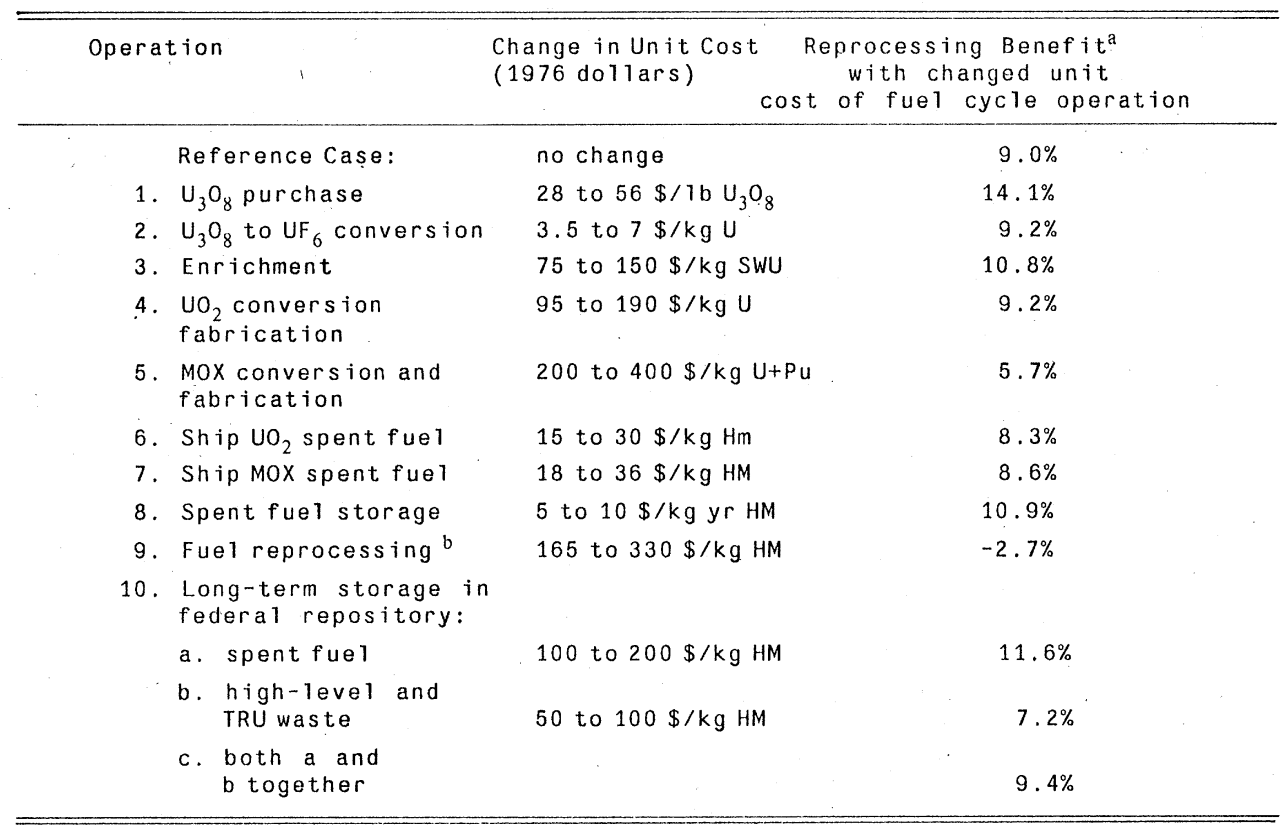

\footnotetext{
a "Reprocessing benefit" is the percentage difference between the fuel cycle cost for the stowaway fuel cycle and the fuel cycle cost with reprocessing and U-Pu recycle. For each change listed all other unit costs are held constant at the reference values given in Table 16 where a discount factor of $7.55 \% / y$ ear has been used.

${ }^{\mathrm{b}} \mathrm{MOX}$ reprocessing unit cost $=1.2 \times \mathrm{UO}_{2}$ reprocessing unit cost.
}

G3h. The Fue! Cycle Reprocessing Benefits for Different Reprocessing Plants

We define the reprocessing benefits as the percentage difference between the fuel cycle costs for the non-reprocessing stowaway cycle and the fuel cycle cost for reprocessing with $\mathrm{U}-\mathrm{Pu}$ recycle, i.e., the negative of the percentage changes from the stowaway cycle listed in Table 21. The data in Table 21 were calculated for a constant unit cost of $\mathrm{UO}_{2}$ reprocessing of $165 \$ / \mathrm{kg}$, which is the reference case for a $1500 \mathrm{Mg} / \mathrm{yr}$ reprocessing plant with semi-direct maintenance and with median industrial financing as developed in Section E. Similarly, the reprocessing benefits for other methods of financing the reprocessing plant and for remote-maintenance plants can be estimated, using the other unit reprocessing costs in Table 13 of Section E. The results are shown in Table 23, where the effect of different prices or uranium is also shown. For the reference case of $28 \$ / \mathrm{lb} \mathrm{U}_{3} \mathrm{O}_{8}$ and for the case of a linear increase from this reference price, the reprocessing benefit varies from a high of about $14 \%$ for the semi-direct-maintenance plant with government fiancing to about 2 to $3 \%$ for the same plant with high-risk industrial financing. For these same uranium prices, the reprocessing benefit for the remote-maintenance plant is about $11 \%$ for government financing, 2 to $3 \%$ for median industrial financing, and is negative for high-risk industrial financing. For a constant uranium price of $56 \$ / 1 \mathrm{~b} \mathrm{U} \mathrm{U}_{3} \mathrm{O}_{8}$ the reprocessing benefit ranges from $17.6 \%$ to $9.4 \%$ for the semi-direct maintenance plant and from $15.8 \%$ to $1.2 \%$ for the remote-maintenance plant.

No reprocessing benefit is predicted for the remote-maintenance plant with high-risk industrial financing and with a uranium price of $28 \$ / 1 b \quad \mathrm{U}_{3} \mathrm{O}_{8}$ or linearly increasing from that price.

It is apparent that within the context of these calculations there is a likely reprocessing benefit for the semi-direct-maintenance plant with any of the indicated methods of financing. Similarly, there is a likely benefit of the remote-maintenance plant for any but the highrisk-high-equity methods of financing.

\section{G3i. The Break-Even Unit Costs of UO, Fuel Reprocessing}

We define the break-even unit cost of $\mathrm{UO}_{2}$ reprocessing as that cost which results in zero benefit from reprocessing, i.e., the cost of the stowaway fuel cycle becomes identical to the cost of the fuel cycle with uranium-plutonium recycle. The break-even reprocessing costs for the three different assumptions of uranium price, holding all other unit costs at the values in Table 16, are shown in Table 24

Because all of the reprocessing fuel cycles except the one involving 10 -year plutonium storage result in net costs less than that of nonreprocessing storage of discharge fuel, the break-even $\mathrm{UO}_{2}$ reprocessing costs of 292 to $319 \$ / \mathrm{kg}$ for these cycles are greater than the presently estimated reference cost of $165 \$ / \mathrm{kg}$ for directmaintenance $\mathrm{UO}_{2}$ reprocessing. These break-even costs are also greater than the upper estimate of $270 \$ / \mathrm{kg}$ for semi-directmaintenance reprocessing with high-risk industrial financing, as shown in Table 13 of Section E. Therefore, there is a financial incentive for fuel reprocessing over the estimated range of costs of direct-maintenance reprocessing, assuming other unit costs are at the reference values.

Our estimates shown in Table 13 of Section $\mathrm{E}$ indicate that for the $1500 \mathrm{Mg} / \mathrm{yr}$ remote-maintenance plant with high-risk industrial financing, the unit cost of reprocessing is greater than the break-even cost unless the uranium price is increased to about $56 \$ / 1 b \quad \mathrm{U}_{3} \mathrm{O}_{8}$.

For the cycle involving reprocessing with uranium recycle, with plutonium recycle deferred for 10 years, to be more economical than non-reprocessing storage the unit reprocessing cost must be less than 131 to $134 \$ / \mathrm{kg}$, assuming a uranium price of $28 \$ / 1 \mathrm{~b} \mathrm{U}_{3} \mathrm{O}_{8}$, or linearly increasing therefrom, and assuming all other costs are at the reference values. According to Table 13 of Section E, this could be obtained with a semi-direct-maintenance plant with government financing. If the uranium price is $56 \$ / 1 \mathrm{~b} \mathrm{U} \mathrm{U}_{3} \mathrm{O}_{8}$ many other options of financing the reprocessing plant become viable for uranium recycle. 
TABLE 23. Reduction in fuel cycle cost by reprocessing and recycle as affected by reprocessing plant design and financing and by uranium cost.

\begin{tabular}{|c|c|c|c|c|}
\hline \multirow[b]{2}{*}{$\begin{array}{l}\text { Reprocessing } \\
\text { plant } \\
\text { and method of } \\
\text { financing }\end{array}$} & \multirow[b]{2}{*}{$\begin{array}{l}\text { Unit } \\
\text { reprocessing } \\
\text { cost }^{\mathrm{a}} \\
\$ / \mathrm{kg}\end{array}$} & \multirow[b]{2}{*}{$288 / 1 \mathrm{~b} \mathrm{U} \mathrm{O}_{8}$} & \multicolumn{2}{|c|}{$\begin{array}{l}\text { Reprocessing benefit }{ }^{\mathrm{b}} \text { as a } \\
\text { function of uranium cost }\end{array}$} \\
\hline & & & $\begin{array}{l}28 \$ / 16 \mathrm{U}_{3} \mathrm{O}_{8}+ \\
1.40 \mathrm{~s} / 1 \mathrm{~b} \text { yr } \\
\text { increase }\end{array}$ & $56 \$ / 1 \mathrm{~b} \quad \mathrm{U}_{3} \mathrm{O}_{8}$ \\
\hline \multicolumn{5}{|c|}{ Semi-direct-maintenance plant } \\
\hline \multicolumn{5}{|l|}{$\begin{array}{l}\text { low-cost } \\
\text { (government) }\end{array}$} \\
\hline $\begin{array}{l}\text { utility-type } \\
\text { financing }\end{array}$ & 120 & 12.1 & 11.9 & 16.3 \\
\hline $\begin{array}{l}\text { median industrial } \\
\text { financing }\end{array}$ & 165 & 9.0 & 9.2 & 14.2 \\
\hline $\begin{array}{l}\text { high-risk } \\
\text { industrial } \\
\text { financing }\end{array}$ & $\begin{array}{l}220 \\
\text { to } \\
270\end{array}$ & 1.7 & 3.1 & 9.4 \\
\hline \multicolumn{5}{|c|}{ Remote-maintenance plant } \\
\hline $\begin{array}{l}\text { low-cost } \\
\text { (government) } \\
\text { financing }\end{array}$ & 130 & 11.4 & 11.3 & 15.8 \\
\hline $\begin{array}{l}\text { utility-type } \\
\text { financing }\end{array}$ & 190 & 7.2 & 7.8 & 13.1 \\
\hline $\begin{array}{l}\text { median industrial } \\
\text { financing }\end{array}$ & 265 & 2.1 & 3.4 & 9.6 \\
\hline $\begin{array}{l}\text { ligh-risk } \\
\text { industrial }\end{array}$ & $\begin{array}{l}360 \\
\text { to }\end{array}$ & -4.5 & -2.2 & 5.3 \\
\hline financing & 450 & -10.7 & -7.5 & 1.2 \\
\hline
\end{tabular}

a From Scction E Table 13. Costs are in 1976 dollars.

b The reprocessing benefit is the percentage difference between the fuel cycle cost for the stowaway cycle and the fuel cycle cost for reprocessing with $U$-Pu recycle. All other unit costs of fuel cycle operations are the same as the values in Table 16, except for the indicated increases in the costs of $\cup_{3} \mathrm{O}_{8}$ :

G3j. Fuel Cycles Involving Disposal of Nuclear Fuel as Waste

An alternative fuel cycle involving treating discharge fuel as radioactive waste by emplacing it in essentially non-retrievable storage has been discussed (USNRC, 1976). Discharge fuel is an already- mined, valuable energy resource. If not reprocessed discharge fuel should be stored in retrievable form pending the development of alternate sources of energy. Similarly, if uranium were to be reprocessed without plutonium recycle, the plutonium should be stored for future fuel value rather than allowing it to follow the high level wastes into non-retrievable disposal.

TABLE 24. Break-even costs of fuel reprocessing by fuel cycle options as affected by cost of natural uranium (1976 dollars) (Pigford and Choi, 1977). (For each fuel cycle option the breakeven reprocessing cost results in the same fuel cycle cost as for the stowaway cycle. All unit costs are the same as those in Table 16 except for the indicated increase in the cost of $\mathrm{U}_{3} \mathrm{O}_{8}$. Cost of MOX reprocessing remains $20 \%$ greater than for $\mathrm{UO}_{2}$ reprocessing.)

\begin{tabular}{|c|c|c|c|}
\hline & $\begin{array}{l}\text { Break-even } \mathrm{UO}_{2} \\
\mathrm{U}_{3} \mathrm{O}_{8} \\
\text { constant } \\
\text { at } 28 \mathrm{~S} / 1 \mathrm{~b}\end{array}$ & $\begin{array}{l}\text { Reprocessing Cost, } \\
\mathrm{U}_{3} \mathrm{O}_{8} \\
\text { increase }^{\mathrm{a}} \\
5 \% / \mathrm{yr}\end{array}$ & $\begin{array}{l}\$ / \mathrm{kg} \\
\mathrm{U}_{3} \mathrm{O}_{8} \\
\text { constant } \\
\text { at } 56 \$ / 1 \mathrm{~b}\end{array}$ \\
\hline $\mathrm{U}+\mathrm{Pu}$ recycle & 292 & 320 & 474 \\
\hline U-Recycle, 10-yr Pu storage & 131 & 134 & 287 \\
\hline $\begin{array}{l}10-y r \text { storage, followed by } \\
\mathrm{U}-\mathrm{Pu} \text { recycle }\end{array}$ & 319 & 449 & 514 \\
\hline
\end{tabular}

a A linear increase, beginning with $28 \$ / 1 b$. 
TABLE 25. Total cost of electrical energy from pressurized-water reactor (70\% load factor, yearly charge on capital investment $=16 \% / \mathrm{yr}$, , reference unit costs of fuel cycle operations).

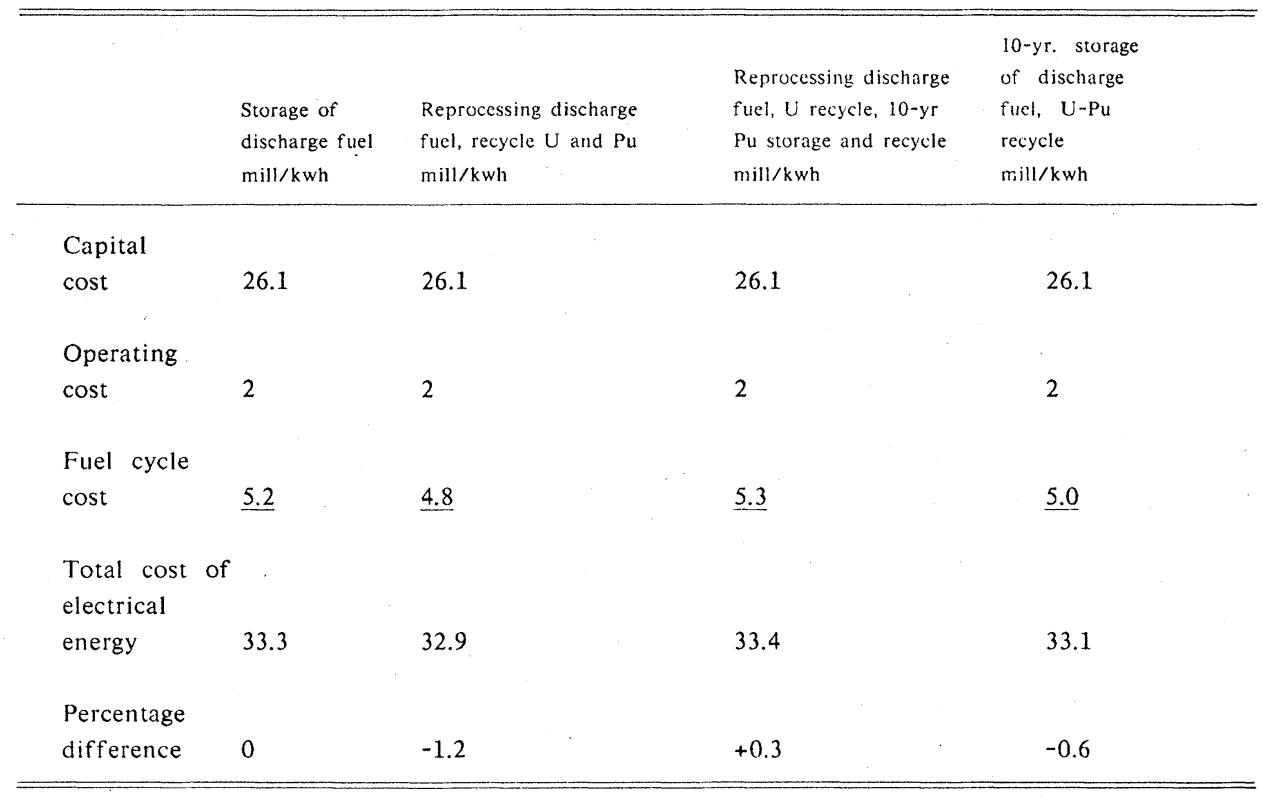

TABLE 26. Unit costs of fuel cycle operations used in ERDA analysis (ERDA, 1976) of reprocessing benefits.

\begin{tabular}{|c|c|c|c|}
\hline & $\begin{array}{l}\text { This study } \\
\text { ( Table } 16 \\
1976 \text { do } 1 \text { lars) }\end{array}$ & $\begin{array}{l}\text { ERDA }(1977 \text { do11a } \\
\text { base case } \\
\text { (ERDA, 1976) }\end{array}$ & $\begin{array}{l}\text { ars) } \\
\text { range }\end{array}$ \\
\hline $\mathrm{U}_{3} \mathrm{O}_{8}, \$ / 1 \mathrm{~b}$ & 28 & 34 and highera see & Fig. 13 \\
\hline $\mathrm{U}_{3} \mathrm{O}_{8}$ to $\mathrm{UF}_{6}$ conversion, $\$ / \mathrm{kgU}$ & 3.50 & 4.40 & 4.40 \\
\hline Is otope separation, $\$ / \mathrm{kg}$ SWU & 75 & 100 & $75-125$ \\
\hline $\begin{array}{l}\mathrm{UO}_{2} \text { conversion and } \\
\text { fabrication, } \$ / \mathrm{kg} U\end{array}$ & 95 & 90 & 90 \\
\hline $\begin{array}{l}\text { Shipment of discharge } \\
\text { fue } 1, \$ / \mathrm{kg} \mathrm{HM}\end{array}$ & 15 & 6 & \\
\hline $\begin{array}{l}\mathrm{UO}_{2} \text { fuel reprocessing, } \\
\$ / \mathrm{kg} \mathrm{HM}\end{array}$ & 165 & $280^{b}$ & $220-340$ \\
\hline $\begin{array}{l}\text { MOX fuel reprocessing, } \\
\$ / \mathrm{kg} \mathrm{HM}\end{array}$ & 198 & not specified & \\
\hline $\begin{array}{l}\text { Waste management-federal } \\
\text { repository, } \$ / \mathrm{kg} \mathrm{HM}\end{array}$ & 50 & 25 & $25-50$ \\
\hline $\begin{array}{l}\text { MOX conversion and fabricati } \\
\$ / \mathrm{kg} \mathrm{HM}\end{array}$ & 200 & 260 & 260 \\
\hline $\begin{array}{l}\text { Shipment of fissile } \\
\text { plutonium, } \$ / \mathrm{kg} \mathrm{Pu}\end{array}$ & 40 & & \\
\hline $\begin{array}{l}\text { Canal storage of discharge } \\
\text { fue } 1, \$ / \mathrm{kg} \mathrm{HM} \text { yr }\end{array}$ & 5 & 2 & \\
\hline $\begin{array}{l}\text { Long-term storage of dischar } \\
\text { fuel in federal } \\
\text { repository, } \$ / \mathrm{kg} \mathrm{HM}\end{array}$ & ge & 70 & $60-200$ \\
\hline $\begin{array}{l}\text { Plutonium storage, } \\
\$ / \mathrm{kg} \mathrm{fissile} \mathrm{Pu} \mathrm{yr.}\end{array}$ & 2000 & 300 & 300 \\
\hline Discount factor. $\% / y r$ & 7.55 & 10 & 10 \\
\hline
\end{tabular}

Rev. Mod. Phys., Vol. 50, No. 1, Part II, January 1978 


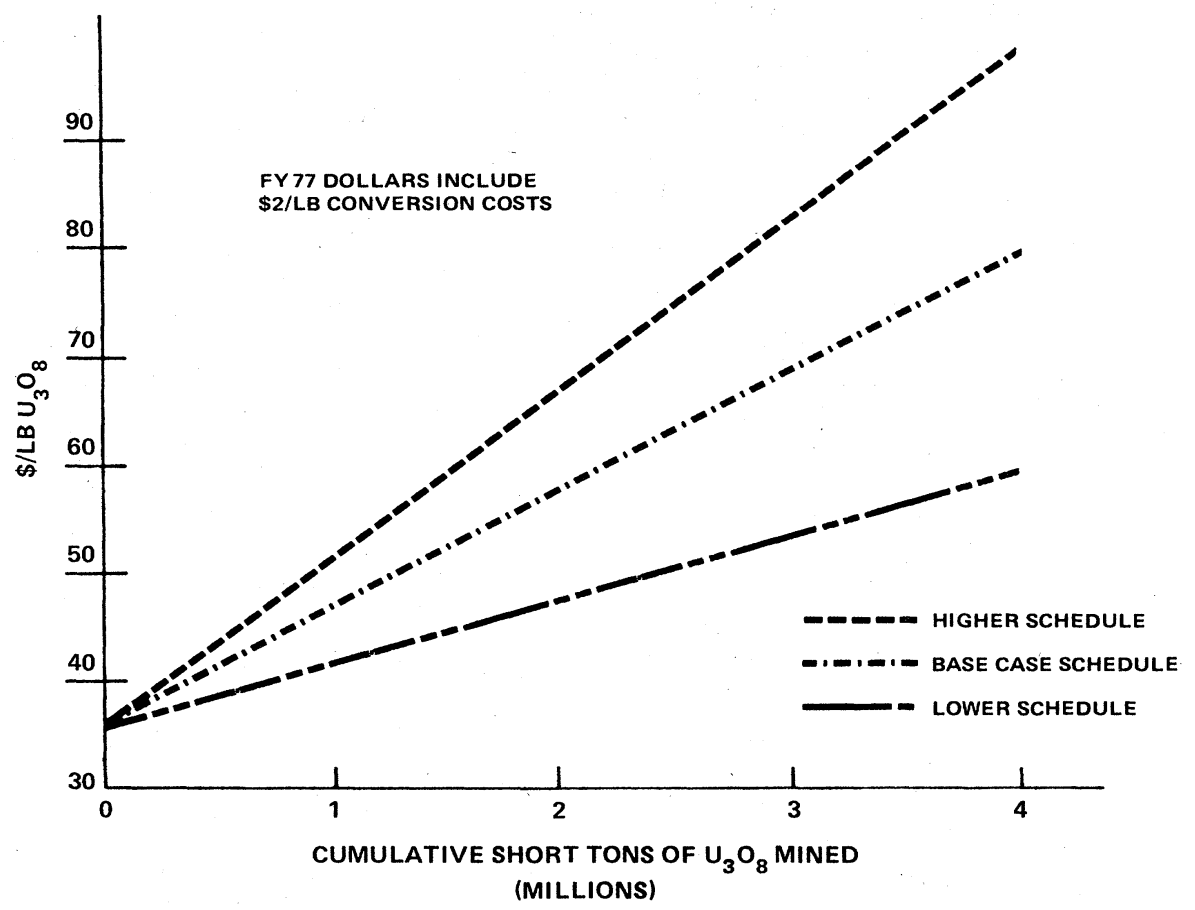

FIG. 13. ERDA projections of uranium price schedules.

\section{G3k. Total Costs of Nuclear Generated Electrical Energy}

The total costs of nuclear generated electrical energy can now be estimated, using a capital cost of $\$ 1000 / \mathrm{kW}$ for the power station and assuming $2 \mathrm{mill} / \mathrm{kWh}$ for the operating cost, as shown in Table 25 . Closing the fuel cycle by reprocessing to recycle uranium and plutonium decreases the total generating cost by $1.2 \%$. The uncertainties in the unit costs of many of the fuel cycle operations are large. Nevertheless, the variations considered herein, including uncertainties in construction costs and financing costs of reprocessing plants, indicate that with direct-maintenance reprocessing and median industrial financing, the savings in fuel costs are about 9 to $14 \%$ and those in generating costs are about 1 to $3 \%$ over the range of uranium prices considered. Similar savings are possible with a remotemaintenance reprocessing plant if financed with a sufficiently low yearly charge on capital investment or if operating in a period of higher uranium costs. Clearly, the matter can only be resolved when policics are clarified that affect the plant construction costs and methods of financing.

A reprocessing plant capable of handling $1500 \mathrm{Mg}$ of uranium and mixed-oxide fuel per year, and waste-management facilities necessary to complete the fuel cycle, could service 53 reactors operating in selfgenerated plutonium recycle (Figure 7 ), resulting in a saving under the base case assumptions of $\$ 150$ million/yr. for full uranium-plutonium recycle. For a mature nuclear power industry of $600 \mathrm{Gw}$ by the year 2000 , the annual saving is $\$ 1.7$ billion, which is $1.2 \%$ of the total electrical generating cost for that year. For $300 \mathrm{Gwe}$ in that year, the annual dollar savings are half of those indicated above.

G.31. ERDA Analysis aof the Benefits of Reprocessing and Recycling Light Water Reactor Fuel

The unit costs used by ERDA in its analysis (ERDA, 1976) of the benefits of reprocessing and recycle are listed in Table 26. Alto listed, for comparison, are the reference unit costs used in our present study (from Table 16). The most important difference is in the unit cost of reprocessing.

The ERDA unit cost of $280 \$ / \mathrm{kg}$ is based upon a preliminary forecast for the duPont design of a $1500 \mathrm{Mg} / \mathrm{yr}$ remote-maintenance plant costing $\$ 1.224$ billion, in 1977 dollars, financed with median $(25 \% / y r)$ industrial financing. This reprocessing cost has been included in our present analysis, where the ERDA-duPont estimate of
$280 \$ / \mathrm{kg}$ has been translateld to $265 \$ / \mathrm{kg}$ in 1976 dollars and appears in Table 23.

ERDA uses a schedule of uranim price as a function of the cumulative ore consumption as shown in Figure 13. The cost of conversion of $U_{6}$ is included in the uranium price. ERDA's analysis is concerned with the total cost of fuel cycle operations for a U.S. nuclear power industry growing, for the base case, to 500 Gwe of light-water-reactor capacity by the year 2000 . They calculate the total and discounted cumulative cash flow for the case of no fuel reprocessing and compare with similar cumulative costs for fuel reprocessing with uranium-plutonium recycle. The fuel reprocessing is assumed to begin in 1981 , with plants operating at $40 \%$ capacity the first year, $67 \%$ the second, and $100 \%$ thereafter. In the reprocessing case the plutonium is assumed to be recycled only in light water reactors; possible introduction of breeders is not included. The results for the 500 Gwe base case are summarized in Table 27.

ERDA estimates of the range of variations in the unit costs of fuel cycle operations are shown in Table 26 and 27 . The resulting changes in the cummulative reprocessing benefit are shown in Table 28.

The data in Table 27 correspond to an average price of uranium, averaged over the period from 1976 to 2000 , of $42 \$ / 1 b \mathrm{U}_{3} \mathrm{O}_{8}$ for the non-reprocessing cycle and $40 \$ / 1 b$ for reprocessing and recycle, with a beginning price of $34 \$ / 1 \mathrm{~b}$. For these uranium prices and for the ERDA-estimated reprocessing unit cost, the calculated percentage benefit from reprocessing is in general agreement with that predicted in our analysis (see Table 23), although our analysis is for a single plant and extends over the entire plant lifetime.

We note, however, that the ERDA analysis does not reflect the very large range of reprocessing costs that seem possible, and even realistic, depending upon the method of financing and upon the design approach towards direct or remote maintenance. As shown in Table 23, the method of financing remains the most. important single variable. A high-risk approach towards industrial financing, which might result from the considerations discussed in Section E, would lead to such high unit costs for a remote-maintenance plant that no reprocessing benefit would occur under the range of $\mathrm{U}_{3} \mathrm{O}_{8}$ prices considered in the ERDA analysis. Conversely, there seem to be many financing approaches, beyond the one median-industrial financing rate $(25 \% / \mathrm{yr})$ assumed by ERDA, which would significantly increase 
TABLE 27. ERDA analysis (ERDA, 1976) of effects of fuel reprocessing on recycle in LWR's from 1976 through 2000. Base case $=500$ Gwe of LWR in the year 2000. Costs are in 1976 dollars.

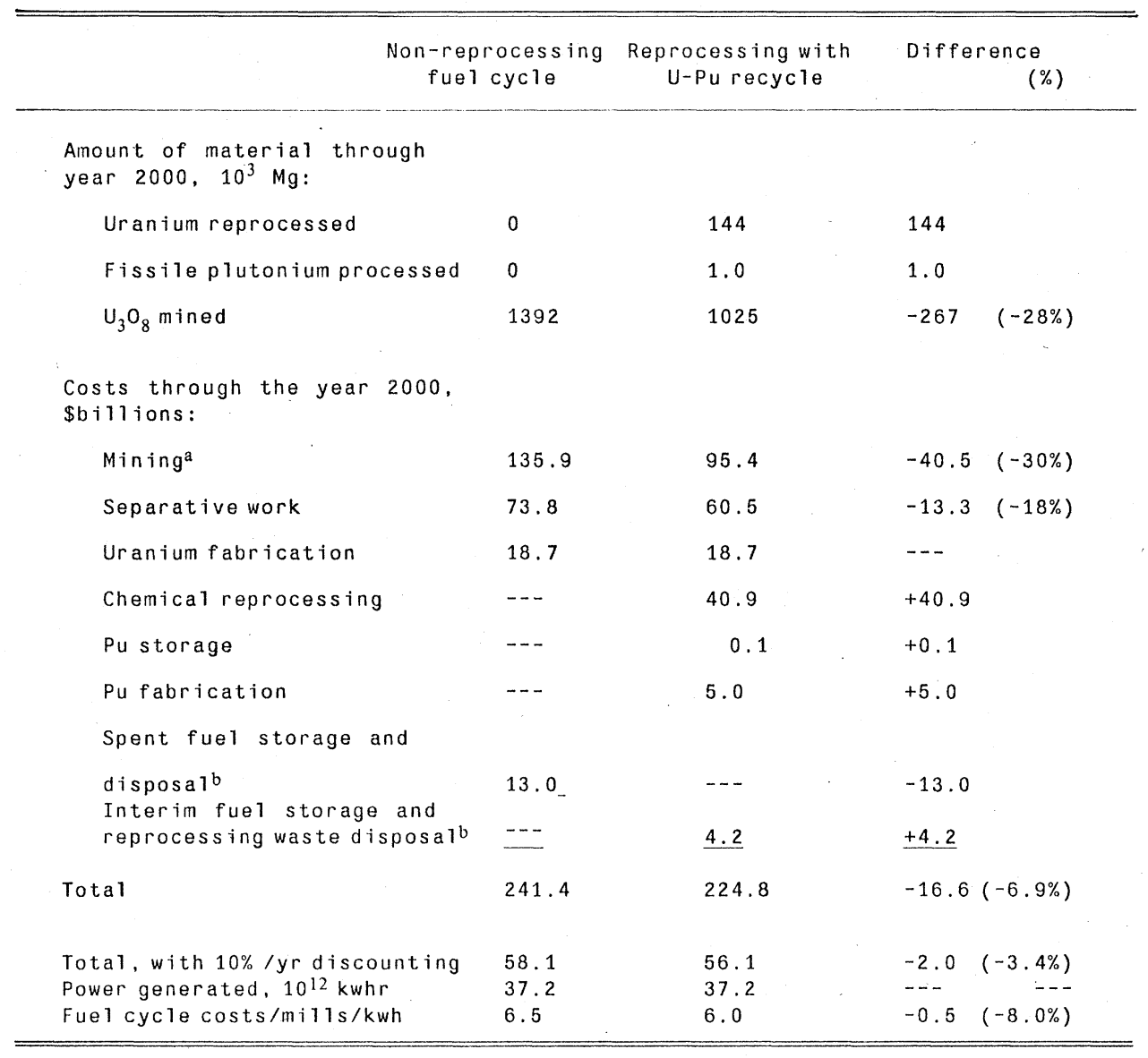

a Includes conversion to $\mathrm{UF}_{6}$. The average unit price of $\mathrm{U}_{3} \mathrm{O}_{8}$ is $4.7 \%$ less with reprocessing because of less cumulative ore requirement.

b Some fuel irradiated before the year 2000 has remaining in pool storage and as disposal liability at the year 2000. Also, some fuel reprocessed before the year 2000 generates waste which is disposed of after the year 2000. These costs are included in the total.

the reprocessing benefits, such as government financing or a special industrial project financed with a high ratio of debt to equity. Also, data from three recent U.S. industrial designs of plants with semidirect maintenance indicate that these designs, if successful, could lead to even further reductions in unit reprocessing cost than those considered in the ERDA study.

TABLE 28. ERDA (ERDA, 1976) analysis of the effect of uncertainties in the unit costs of $\mathrm{U}_{3} \mathrm{O}_{8}$ and of fuel cycle operations.

\begin{tabular}{ll}
\hline & $\begin{array}{l}\text { Cumulative fuel } \\
\text { cycle savings } \\
\text { with recycle, } \\
\text { \$bil1ions }\end{array}$ \\
\hline Base case & 16.6 \\
A11 cost parameters increase & 28.9 \\
Most favorab1e cost assumptions for recyc1e & 47.1 \\
Least favorable cost assumptions for recyc1e & -4.9 \\
\hline \hline a Cumulative savings through the year 2000 . 1977 dollars, not discounted
\end{tabular}

\section{References}

1. Baumgartner, 1976

Baumgartner, F., "The Purex Process for the Reprocessing of Nuclear Fuels with High $\mathrm{Pu}$ Content and High Burn-up", Kerntechnik, 18 (6) 245-52, June, 1976.

2. Bebbington, 1976

Bebbington, W. P., "The Reprocessing of Nuclear Fuels", Scientific American, Dec., 1976, pp. 30-41.

3. Bohnenstingle, et al., 1976

Bohnenstingle, J., et al., International Symposium on the Management of Radioactive Waste from the Nuclear Fuel Cycle" Vienna 22-26 March 1976, IAEA-SM-207/20.

4. Bonka, et al., 1974

Bonka, H., K. Brusserman, and G. Schwarz, "Umweltbelastund durch Radiokohlenstoff aus Kerntechnischen Anlagen", Reaktortagun Berline 2-5 April 1974.

5. Broothaerts, et al.

Broothaerts, J., et al., "Treatment and Control of Gaseous Effluents from Light Water Reactors and Reprocessing Plants".

Rev. Mod. Phys., Vol. 50, No. 1, Part II, January 1978 
6. Burkhart, et al., 1977

Burkhart, L., E. B. McCutcheon, and C. C. Conitz, "Simulation and Control of Nuclear Fuel Reprocessing Operations," B. F. Ruth Chem. Eng. Res. Symposium, Dept. of Chem. Eng., Iowa State Univ., March 10, 1977.

7. Cholister, et al., 1976

Cholister, R. J., W. A. Rodger, R. L. Frendberg, W.L. Godfrey, W. Knox, and W. B. Summer, "Nuclear Fuel Cycle Closure Alternatives", Allied-General Nuclear Services, Barnwell, S. C., April 1976

8. Cleveland, 1970

Cleveland, J. M., "The Chemistry of Plutonium", Gordon and Breach, New York (1970).

9. Colglazier and Weatherwax, 1976

Colglazier, E. W. and R. K. Weatherwax, "The ${ }^{236} \mathrm{U}$ Penalty for Recycled Uranium", COO 2220-81 (Princeton), (Sept., 1976).

10. de la Garza, 1977

de la Garza, A., "Uranium-236 in Light Water Reactor Spent Fuel Recycled to an Enriching Plant", Nucl. Tech. 32, 176-185 (Feb. 1977).

11. Draft Environmental Impact Statement for LMFBR Appendix, Chapt. IV.

12. E. I. DuPont de Nemours and Co., 1976a

E. I. DuPont de Nemours and Co., Composite Quarterly Report, Light Water Reactor Fuel Cycle, Savannah River Laboratory, Aiken, S. C.; DPST-LWR-76-2-1, Jan.-Mar., 1976;-2, April-June, 1976;-3, July-Sept., 1976.

13. E. I. DuPont de Nemours and Co., 1976b

E. I. DuPont de Nemours and Co., LWR Assistance Task Force Monthly Report, Savannah River Laboratory, Aiken, S. C.; DPST-LWR-76--13-8, Aug., 1976;-9, Sept., 1976;-10, Oct., 1976;-11, Nov., 1976;-12, Dec., 1976.

14. E. I. DuPont de Nemours and Co., 1975

E. I. DuPont de Nemours and Co., "Assessment of Nuclear Fuel Reprocessing", Savannah River Laboratory, DPST-75-488 Draft, September 1975.

15. ERDA, 1975

U.S. Energy Research and Development Administration, "A Subjective Probability Appraisal of Uranium Resources in New Mexico", GJ00110 (76), December, 1975.

16. ERDA, 1976

U.S. Energy Research and Development Administration, "Benefit Analysis of Reprocessing and Recycling Light Water Reactor Fuel", December 1976.

17. ERDA-43, 1976

U.S. Energy Research and Development Administration, Report ERDA-76-43, Alternatives for Managing Wastes from Reactors and Post Fission Operations in the LWR Fuel Cycle, (1976)

18. Exxon Nuclear Co., 1976a

Exxon Nuclear Co., Preliminary Safety Analysis Report, NTIS No. 50-564, 1976.

19. Exxon Nuclear Co., 1976b

PSAR Application Letter, Project No. 565, Feb. 14, 1976 (ERDA Public Document Room).

20. Geller and Gueron, 1969

Geller, L. and H. M. Gueron, "Effect of Uranium-236 on the Value of the Spent Fuel from Light Water Reactors", S. M. Stoller Assoc. SMSA-AH/1, Feb. 1969.
21. Glass, et al., 1974

Glass, R. W., et al., "Development of the Krypton Absorption in Liquid Carbon Dioxide (KALC) Process for HTGR Off-gas Reprocessing", Proceedings of the Thrirteenth AEC Air Cleaning Conference, August 13-15, 1974, Conf-740807.

22. Goellner, et al., 1967

Goellner, D. A., M. Benedict, and E. A. Mason, "The Effect of Uranium-236 and Neptunium-237 on the Value of Uranium as Feed for Pressurized Water Power Reactors", MIT-2073-6, MITNE-90, Dec. 1967.

23. Kupp, 1977

Kupp, R. W., S. M. Stoller Corp., Private Communication, March 1977.

24. Lerch, 1972

Lerch, R. E., "Dissolution of Unirradiated Mechanically Blended, Sol, Gel, and Co-precipitated Oxide Fuel", HEDLTME-72-67, June, 1972.

25. ORNL-TM-3534, 1971

Oak Ridge National Laboratory, LMFBR Fuel Cycle Studies Progress Report for July 1971, No. 29, ORNL-TM-3534, August, 1971

26. O'Rear, 1976

O'Rear, S. W., E. I. DuPont, Savannah River Lab., Private Communication, July 1976.

27. Peak, 1975

Peak, J. C., General Atomic Co., Testimony, In the Matter of Allied-General Nuclear Services, et al. (Barnwell Nuclear Fuel Plant), Docket No. 50-332, Sept., 1975.

28. Pigford, 1976

Pigford, T. H., "The Analysis of the Cost of Electrical Energy from Nuclear Power Plants", UCB-NE 3008 (September, 1976).

29. Pigford and Ang, 1975

Pigford, T. H. and K. P. Ang, "The Plutonium Fuel Cycles", Health Physics, 29, 451 (1975).

30. Pigford and Choi, 1977

Pigford, T. H. and J. S. Choi, "Analysis of the Cost of FuelCycle Options in Pressurized Water Reactors", UCB-NE3243 (April, 1977).

31. Pigford and Yang, 1977

Pigford, T. H. and C. S. Yang, "Thorium Fuel Cycles", UCBNE 3227, EPA 68-01-1962 (June 1977).

32. Rodger, et al., 1975

Rodger, W. A., J. K. Pickard, and J. M. Vallance, "The Throwaway Fuel Cycle", AIF Fuel Cycle Conference, Atlanta, GA, March 1975.

33. Schiefelbein and Lerch, 1971

Schiefelbein, G. F. and R. E. Lerch, "Dissolution Properties of $\mathrm{UO}_{2}-\mathrm{PuO}_{2}$ Thermal Reactor Fuels", BNWL-1581, June 1971.

34. Schwoerer, 1975

Schwoerer, F., "Fuel Cycle Costs in the 1980's: A Forecast", AIF Fuel Cycle Conference, Atlanta, GA, March 1975.

35. Searl, 1974

Searl, M. F., "Uranium Resources to Meet Long-Term Uranium Requirements", EPRI Special Reports, No. 5, November 1974.

36. Shapiro, et al., 1977

Shapiro, N. L., R. R. Rec, and R. A. Matzie, "Assessment of Thorium Fuel Cycles in Pressurized Water Reactors", EPRI NP-359 (Feb. 1977). 
37. Smith, et al., 1973

Smith, R. C., L. G. Faust and Brackenbush, "Radiation Exposure from Plutonium in LWR Fuel Manufacture", Batelle Pacific Northwest Laboratory, Richland, Washington, Nuclear Technology, 18 (2), pp. 79-108 (May 1973).

38. Stoller, 1976

Stoller, S. M., "The Morphology of Reprocessing: A Case for Regional Utility Planning", Proc. Pac. Basin Conf. on Nuclear Power Development and the Fuel Cycle, Hawaii, October 1976.

39. Sprauge, 1974

Sprauge, H. O. "Fuel Cycle Effect of U-236 in Recycled Uranium", Trans. ANS, 1974.

40. Tennery, 1976

Tennery, V. J., ORNL-TM-5538, June 1976

41. Tooper, 1977

Tooper, F. E., U.S. Energy Research and Development Administration, Private Communication, March 1977.

42. Trotter, et al., 1958

Trotter, J. R., M. R. Zelle, and H. Hollister, Science 128, 14990 (1958).

43. UNSCEAR, 1972

Ionizing Radiation: Levels and Effects, UNSCEAR, 1972, Vol.
44. USAEC, 1970

Allied Chemical Nuclear Products, Inc. and Gulf Energy and Environmental Systems, Inc., Barnwell Nuclear Fuel Plant, Safety Evaluation, U.S. Atomic Energy Commission, Docket No. 50-332, Sept. 18, 1970.

45. USAEC, 1973

Allied-Gulf Nuclear Services, Barnwell, S.C., "Final Safety Analysis Report", Barnwell Nuclear Fuel Plant Separations Facility, U.S. Atomic Energy Commission, Docket No. 50-332, Oct. 10, 1973.

46. USEPA, 1973

U.S. Environmental Protection Agency, EPA 520/4-73-002, "Environmental Radiation Dose Commitment", (1973).

47. USNRC, 1976

U.S. Nuclear Regulatory Commission, "Final Generic Environmental Statement on the Use of Recycle Plutonium in Mixed Oxide Fuel in Light Water Cooled Reactors", NUREG00002, vol. 4, p. XI-22, August 1976.

48. Wall Street Journal, 1977

Wall Street Journal, February 14, 1977.

49. Wolfe and Lambert, 1975

Wolfe, B. and R. W. Lambert, "The Back End of the Fuel Cycle", AIF Fuel Cycle Conference, Atlanta, GA, March 1975.

50. Wolfe, et al., 1976

Wclfe, B., R. W. Lambert, and G. F. Melde, "Will There be Fnough Plutonium?", ANS First Pacific Basin Conference, Honolulu, October 1976. 


\section{CHAPTER V. LWR Fuel Cycle -- Assessment of Radiation Exposure}

\section{A. Issues, Conclusions, and Recommendations}

\section{A1. Issues in Potential Radiation Exposure}

In this section we address the issue of the radiation exposure associated with the complete Light Water Reactor fuel cycle and examine radiation exposure to man as the most significant part of this problem. Specific issues considered include: what are the principal sources of potential exposure to radiation; how do the various fuel cycle contributions compare; what are the magnitudes of exposure to the general public and to those employed in the nuclear facilities; and what is the significance of the potential exposure to this and to future generations?

In particular we wish to address the question of the relative importance of several specific effluents and wastes: the gaseous effluents (which result from fuel reprocessing and produce global doses), mill tailings (which produce regional and local doses and potentially long-term buildup) and the fission products and long-lived actinides in high-level and transuranic wastes. As described in detail in Chapter VII, current plans are to place this latter waste in geologic repositories where it woúld produce public exposure only under conditions of unanticipated movement of the emplaced high-level wastes. Final!y, we consider changes in exposure that occur with various fuel cycles and the nature of the effluent or waste control procedures that are of interest in assessing risk-benefits of advanced fuel cycles.

The total fuel cycle is considered since analysis of reprocessing and waste management alone would not provide an adequate perspective. We discuss the radiation doses expected to be delivered to the general public on a national and global scale and to occupationally exposed populations. We have not analyzed local doses or even regional doses in detail since these two categories of exposure seem to be well treated elsewhere. Similarly we have not examined in detail the consequences of reactor accidents or of non-transuranic low-level wastes, in any detail; these too have been treated in other studies. However, in order to study the basis for possible future controls, we have analyzed the consequences of release of gaseous radionuclides from the reprocessing plant.

The significance of the radiation doses is explored from three points of view: by comparison with background, by calculation of health effects assuming a linear dose-health effect relation, and by examination of hazard indices. We analyze the extent of buildup of radiation exposure associated with the irretrievable release into the environment of radioactive material which would be associated with a worldwide nuclear power industry operating for a long but finite time into the future.

\section{A2. Conclusions}

a. With engineered effluent control for ${ }^{129} I$, the major gaseous effluents released from a reprocessing plant are ${ }^{14} \mathrm{C},{ }^{85} \mathrm{Kr}$, and ${ }^{3} \mathrm{H}$ in decreasing order of delivered whole body dose. The average global public whole body dose rates due to released radionuclides are greater and persist for longer periods of time for ${ }^{14} \mathrm{C}$ than for ${ }^{85} \mathrm{Kr}$ and ${ }^{3} \mathrm{H}$.

The technologies for control of these effluents are discussed in Chapter IV. Because the gaseous effluents of fuel reprocessing plants would be distributed over the entire globe, a unilateral decision by one nation to control effluent releases would not be completely effective without similar actions by other nations. In the long-term, international agreements are necessary in order to establish timing and control strategy.

b. We find that the most meaningful reference for assessing the global public radiation dose caused by the nuclear industry is the natural radiation background. The radiations for both are similar in nature, and the increments to background dose rates due to the normal operation of the industry should be small compared to fluctuations in background, even with a large and developed industry.
Especially useful are comparisons with natural spatial and temporal fluctuations in background that occur in a typical person's lifetime or that occur over the same time scale as increments from the fuel cycle.

c. Standards for acceptibility of operation should be based on the limitation of dose rate increment to any future generation as well as to the present generation.

The dose rate increment resulting from the release of $Q$ units of radioactivity per year for $T$ years is just the dose commitment from the release of $\mathrm{Q}$ units of radioactivity evaluated to year $\mathrm{T}$. At subsequent times, that increment will change according to the usual decay laws for the radionuclides released.

d. The average global public whole body radiation dose rates from the operation of $L W R$ fuel cycle with reprocessing do not limit substantial national and international development of nuclear electric power.

We conclude that even without engineered retention of ${ }^{14} \mathrm{C},{ }^{85} \mathrm{Kr}$, or ${ }^{3} \mathrm{H}$ at the reprocessing plants or the reactors, the continuous operation for 500 years of 600 large I.WR electric generating plants (each with 1,000 MWe installed capacity) and the reprocessing of their fuel would increase the average global whole body radiation dose rate about 0.1 percent above natural background (taken as $100 \mathrm{mrem} / \mathrm{y}$ ). The corresponding skin dose rate would be increased roughly 1 percent above background. With engineered retention of the indicated gaseous effluents, the corresponding radiation levels could be reduced further.

e. For regional and local population exposure, radionuclides in uranium mill tailings are potentially at least as important as the actinide chain elements in high-level waste; the relative accessibility of mill tailings contrasts with the isolation proposed for other actinide-containing wastes.

f. We have examined and concur with the position of the National Council on Radiation Protection and Measurcments, that current estimates of the probability for the induction of radiogenic cancer by low LET radiations, based on linear, no-threshold extrapolations from information collected at high doses and dose rates, provide an upper limit and not a best estimate to the health effects ascribed to exposures at rates comparable to background.

g. Estimates based on current operating experience indicate that the collective occupational doses may be up to four times larger than the collective global public doses. Our evaluation indicates that because the collective occupational dose is larger and delivered at higher dose rates than the global public dose, it is likely to be more important in producing biological effects in humans.

The collective occupational doses, ranging from 1 to 4 personrems/MWe-yr, may be delivered at dose rates up to 10 's of rem per hour. Individual occupational doses are limited by regulation to a long-term average dose rate to $5 \mathrm{rem} / \mathrm{yr}$ with no more than $12 \mathrm{rem}$ in a single year. Accordingly the dose and "instantaneous" dose rate delivered to occupationally exposed individuals may be greater by factors of roughly 100 and $10^{6}$, respectively, than those associated with average background radiation.

Operating experience shows that the major contributions to collective occupational dose in the LWR fuel cycle come from reactor operation, from reprocessing, and from mining. The reprocessing plant exposure is sensitive to the plant design and operating procedures. Estimates of the collective dose experienced at reprocessing plants (contact maintenance type) range from 0.6 to 2 person-rem/MWe-yr. Estimates for presently planned state-of-theart contact maintenance plants are as low as 0.01 person-rem/MWe$y \mathrm{r}$. Whether this is a realistic estimate can be determined conclusively only by examining the occupational exposure experience during operation of such plants.

h. With fuel reprocessing and recycle, there are potential improvements in overall health and safety in the fuel cycle 
associated with the decrease in uranium mining and milling. These decreases outweigh, on balance, the additional occupational and environmental exposure attendant 10 fuel reprocessing and refabrication.

However, there are sufficient uncertainties in the assessment of biological effects in both modes that no clear difference in risk of one mode of operation versus the other are demonstrable.

$i$. The utility of simplistic hazard indices for decision making is limited and may even obscure a realistic understanding and assessment of hazard.

Simplistic hazard indices involving dilution are widely used in waste management decision processes. A proper hazard index should include not only source magnitude but release terms, pathways to the biosphere, pathways to man, dose to man, an evaluation of dosimetric consequences, and a proper treatment of the set of probabilities involved.

$j$. For nuclear wastes the hazard associated with Ra appears to be inherenily greater than that for Pu. The differences are due to greater environmental mobility, the existence of a gaseous daughter product, and greater uptake across the mammalian gut.

$k$ : We have examined and concur with the conclusions of recent examinations that reject major alteration of existing plutonium inhalation concentration limits based on the "hot particle" hypothesis.

\section{A3. Recommendations}

a. The major sources of collective occupational dose in the entire fuel cycle should be identified, and design effort should be directed to collective dose minimization. Regulatory guides should be developed by NRC for the collective occupational exposure limits. This is not a recommendation on individual occupational exposure limits.

b. Practices for the disposition of uranium mill tailings require re-examination with respect to limiting the dispersion of radionuclides in the tailings piles, either by human intervention or by natural processes.

c. Steps should be taken towards development of international agreements for the degree and timing of control of gaseous effluents from reprocessing plants, principally ${ }^{14} \mathrm{C},{ }^{129} \mathrm{I},{ }^{85} \mathrm{Kr}$.

d. The chemical forms and sites of release of ${ }^{14} \mathrm{C}$ in the fuel cycle should be determined so that effective strategies for its control may be designed.

e. lodine treatment units in reprocessing plants should be operated for ${ }^{129}$ I removal.

f. Methods and technologies should be developed to sequester radionuclides which have been removed from gaseous fuel cycle effluent, in order to isolate the radioactivity from the biosphere.

g. High importance should be given in the design of fuel cycle facilities processing $\mathrm{Pu}$ (i.e., reprocessing plants, nitrate to oxide conversion facilities, and mixed-oxide fuel fabrication plants) to limit the internal occupational exposures.

h. Present research on the effects of long-lived actinides in animals should be continued and expanded to include ${ }^{232} \mathrm{U},{ }^{233} \mathrm{U}, \mathrm{Th}$, $\mathrm{Am}$ and $\mathrm{Cm}$.

i. Study should be continued toward a better understanding of the radiogenic induction of lung cancer in animals and man. Further epidemiological study of uranium miners, particularly the dosimetric aspects, should be included.

\section{B. Assessment of Risk Associated with Radiation Exposure}

\section{B1. Introduction}

A variety of techniques can be used to assess the degree of risk associated with a given operation, a generic type of operation, or a collective fuel cycle. This chapter will review various forms of risk assessment commonly used in connection with ionizing radiation exposure. We will select among them the ones we judge most appropriate for the purpose of providing perspective and reaching judgements.

The most simplistic type of risk estimate is based on a hazard index taken as the ratio of some amount of material to some measure of its potential for producing biclogical effects. One such estimator has been the amount of radioactivity associated with a practice divided by the Radioactivity Concentration Guide (RCG) in air or water. The RCG is not really a true measure of biological effect but rather is associated with the calculated dose rate to man, given longterm ingestion of air or water at the Radioactivity Concentration Guide. ${ }^{1 .}$ This estimator is quite incomplete and limited in applicability.

A second type of assessment is based on the "health effect estimate" in which a direct proportion is postulated to exist between the radiation dose and the likelihood of inducing an effect. This type of estimate, has been popularized by the Biological Effects of Ionizing Radiation (BEIR) Committee of the National Academy of Sciences (BEIR, 1972), and is widely used in effects assessment analyses. There is an implicit assumption that biological effects are independent of dose rate, that no repair of damage occurs and that consequently, all damage is cumulative. However, it is now well established, especially for low LET (i.e., beta and gamma) radiations, that dose rate exerts an influence (NCRP, 1975). The literature abounds with investigations of the time constants and mechanisms of repair of radiation damage in bacterial and cell culture systems as well as mammals. For low LET radiation, at low dose or low dose rate therefore, this estimate gives a measure of upper value rather than of expected value to risk.

A third approach characterizes the risk in terms of those from other exposures with similar characteristics. A natural background exists with radiation characteristics similar to those from the LWR fuel cycle and serves as the most reasonable basis for comparison. To extract an absolute risk from this assessment would require a knowledge of the absolute detriment associated with natural background exposures. We have noted above that such an estimate would require a variety of assumptions any of which might be over simplifications. As we discuss below in Section B3, an alternative approach toward determining absolute risk is obtained by noting that fluctuations in background exist in time and in space that may be comparable in magnitude with background itself (Beninson, 1977a). Since, in the course of human existence no statistically discernible effects have been associated with such fluctuations, ${ }^{2}$ it follows that the effects of increments in dose and dose rate small compared to background fluctuations will be small compared to an already undetectable level of effects.

\section{B2. Hazard Indices}

A variety of hazard indices have been used to assess the risks in nuclear waste management. To the extent that specific hazard indices are incomplete indicators of the real hazard involved in a total process, they are inadequate and may even tend to distort perceptions about the absolute and relative risks involved. As a result, in our opinion, they tend to divert both attention and efforts from analyses which should be undertaken. The problem is not that the concept of the hazard index is inappropriate, but rather that the manner in which it has been applied in the waste management field is so limited that the adjective hazard, is inapt. Most indices which have been applied are not indicators of hazard but instead measures of such things as the time required for an arbitrary amount of radioactive decay to 0.1 percent (10 half-lives) of its initial value or the amount of water or air required to dilute a given amount of activity to its RCG.

Hazard indices for waste management must ultimately be characterized in terms of dose to or effects on humans. It is our judgement that hazard indices are precise only if they take into account the amount of activity at issue, the radiological and chemical nature of the material, the degree to which the material is confined and handled, the degree to which material may escape, the degree to which material will become available to various parts of the environment (i.e., the geosphere and the biosphere), the degree to which the material will be reaccumulated by man and biota, the dose resulting to man, [also as a function of time with emphasis on the 
TABLE 5B-1. Summary of average dose equivalent rates (mrem/yr) from various sources of natural background radiation for the United States (NCRP, 1975c) and for the world (Beninson, 1977a).

\begin{tabular}{|c|c|c|c|c|c|}
\hline \multirow{2}{*}{$\begin{array}{c}\text { United States } \\
\text { Source }\end{array}$} & \multirow[b]{2}{*}{ Gonads } & \multirow[b]{2}{*}{ Lungs } & \multicolumn{2}{|c|}{ Bone } & \multirow{2}{*}{$\begin{array}{l}\text { G.I. } \\
\text { Tract }\end{array}$} \\
\hline & & & Surfaces & Marrow & \\
\hline Cosmic Radiation & 28 & 28 & 28 & 28 & 28 \\
\hline Cosmogenic Radionuclides & $0: 7$ & 0.7 & 0.8 & 0.7 & 0.7 \\
\hline External Terrestrial & 26 & 26 & 26 & 26 & 26 \\
\hline Inhaled Radionuclides & -- & 100 & -- & -- & -- \\
\hline Radionuclides in the Body & 27 & 24 & 60 & 24 & 24 \\
\hline U.S. Tota1s (rounded) & 80 & 180 & 120 & 80 & 80 \\
\hline \multicolumn{6}{|l|}{ Average Wor 1d } \\
\hline Totals (mrem/yr) & 91 & 539 & 112 & 86 & \\
\hline
\end{tabular}

maximum doses or dose rates that may occur in time], and estimates of the biological effects either by comparison with background or by absolute means if warranted. These calculations should be carried out as realistically as possible for each nuclide involved so that comparisons may be made between the nuclides and also between alternative schemes of handling. One should compare the best estimates of risk engendered by alternative schemes as opposed to comparing arbitrary indices with unspecified and undetermined relationships to hazards. We do not intend to develop such indices here, but note that examples of a preliminary approach to this are cited in Chapter VII, Section F.

\section{B3. Background Radiation}

All persons are exposed to background radiation that consists primarily of cosmic radiation, external terrestrial beta and gamma radiation from the naturally radioactive elements in soil and rocks, internally deposited radioactive material, artificial radionuclides made in nuclear tests, as well as from a variety of man-made radiation emitting sources such as X-ray machines. These contributions are described in Chapter III.

The background dose rate varies with altitude, with geographic location, and differs from organ to organ in the human body depending on such things as self-shielding, the degree of uptake of radionuclides by different organs, and even the living habits of people (NCRP, 1975c; Oakley, 1972). For example, internal ${ }^{40} \mathrm{~K}$ levels vary slightly from person to person according to sex and age and can introduce changes amounting to $\sim 5 \%$ of the $80 \mathrm{mrem} / \mathrm{y}$ average; changes in altitude can give rise to much larger variations in dose rate

TABLE 5B-2. Natural collective (population) doses (personrems) from natural background radiation.

\begin{tabular}{lcc}
\hline Somatic: 70 years & U.S. & World \\
& $2 \times 10^{8}$ people & $4 \times 10^{9}$ people \\
Gonads, GI tract, & $1.1 \times 10^{9}$ & $2.5 \times 10^{10}$ \\
bone marrow & $2.5 \times 10^{9}$ & $7.5 \times 10^{10}$ \\
Lung & $1.7 \times 10^{9}$ & $3.1 \times 10^{10}$ \\
Bone surface & & $1.1 \times 10^{10}$ \\
Genetic: 30 years & $4.8 \times 10^{8}$ & \\
Gonads &
\end{tabular}

comparable to the average annual background dose rate itself. Within the continental U.S. variations as large as a factor of two occur. Alternatively, one might focus on the time variability at a fixed location. There are three characteristic time periods: seasonal, in which snow cover and soil moisture content changes result in changes in shielding of soil activities; the solar activity cycle, which results in an eleven year variations in cosmic ray intensity and hence in natural background dose rate; the time variation of the earth's magnetic field, which changes over a 9000 year time scale and again influences cosmic ray intensity and background dose. The amplitude of variation from solar activity is several $\mathrm{mrem} / \mathrm{yr}$ while the amplitude from variations in the earth's field may be up to $1 \mathrm{mrem} / \mathrm{yr}$. (O'Brien, 1977). The latter two changes can be larger at higher altitudes and/or higher latitudes. In addition there have been irregular changes in the concentration of cosmogenically produced ${ }^{14} \mathrm{C}$. Thus over a typical lifetime interval of 70 years the variations in background dose rate even at a fixed location are at least of order several mrem/yr. If that individual should move about, spatial variations as large as 100 mrem/yr. may be encountered. In addition, the act of moving should it involve high altitude flight; would impose doses on the order of 1 mrem/2 hours of flight.

For purposes of this analysis we will use average values. The dose may be expressed both in terms of the per capita averages and in ternis of the cumulative population or collective dose, with one year's background dose taken to express the dose rate and periods of a year to a generation for collective doses. Table 5.B-1 shows that the average dose equivalent rate (here rad has been converted into rem by multiplying by the appropriate quality factor) for the gonads is about $80 \mathrm{mrem} / \mathrm{yr}$; for lungs, 180; for bone surfaces, about 120; for bone marrow, about 80; and for the GI tract, about 80 . No value for the whole body is given; however the gonadal value here will be taken as roughly r"presentative of the whole body average.

The higher value for the lungs obtains because of the contribution of radon daughters in surface air to irradiation of the lung (Eisenbud, 1973). Higher values to bone surfaces reflect the contribution of internally deposited naturally occurring radionuclides of the uranium and thorium series particularly ${ }^{210} \mathrm{~Pb}\left({ }^{210} \mathrm{Po}\right),{ }^{226} \mathrm{Ra}$, and ${ }^{228} \mathrm{Ra}$ (NCRP, 1975c). Accordingly an average per capita figure for the whole body can be taken as about $80 \mathrm{mrem} / \mathrm{yr}$ or over a reproductive lifetime of 30 years or 2.4 rems per generation. This would roughly be the genetically significant dose on a per capita basis from natural background. For a life span of 70 years the dose would be 5.6 rems.

For a reference U.S. population of 200 million people the annual collective dose commitment to the whole population due to natural background is about 16 million person-rem/yr, over a generation (30 years) the genetically significant dose is 480 million person-rem, and over a 70-year life span about 1,100 million persun-rem per generation. Corresponding values for a reference world population of 4 billion people, would be about 20 -fold those for the U.S. The collective (population) dose for the U.S. and world are summarized in Table 5.B-2. 
TABLE 5B-3. Naturally occurring inventories of ${ }^{14} \mathrm{C},{ }^{40} \mathrm{~K},{ }^{226} \mathrm{Ra}$, and ${ }^{222} \mathrm{Rn}$ in arbitrarily selected reservoirs.

\begin{tabular}{|c|c|c|c|c|c|}
\hline Nuclide & Half-Life & Medium & $\begin{array}{l}\text { Environmental } \\
\text { Inventory }(\mathrm{C} i)\end{array}$ & $\begin{array}{l}\text { Average or } \\
\text { a) } \\
\text { i) }{ }^{\text {a }} \text { Concentratic } \\
\end{array}$ & mrem/year \\
\hline${ }^{14} \mathrm{C}$ & $5,730 y$ & $\begin{array}{l}\text { Biosphere } \\
\text { and oceans }\end{array}$ & $2.8 \times 10^{8}$ & $6 \mathrm{pCi} / \mathrm{gC}$ & $\begin{array}{l}0.7 \text { to soft } \\
\text { tissues }\end{array}$ \\
\hline $40_{K}$ & $\begin{array}{l}1.26 x \\
10^{9} y\end{array}$ & soil & $9 \times 10^{7}$ & $12 \mathrm{pCi} / \mathrm{g}$ & $\begin{array}{r}19 \text { internal } \\
8 \text { external }\end{array}$ \\
\hline $226 \mathrm{Ra}$ & $1,600 \mathrm{y}$ & soil & $5 \times 10^{6}$ & $0.6 \mathrm{pCi} / \mathrm{g}$ & 6.6 interna $^{\mathrm{b}}$ \\
\hline $222 \mathrm{Rn}$ & $3.85 \mathrm{~d}$ & atmosphere & $1.5 \times 10^{6}$ & $0.1 \mathrm{pCi} / l$ iter & $\begin{array}{l}100 \text { to } 500 \\
\text { to lungs }\end{array}$ \\
\hline
\end{tabular}

a For ${ }^{14} \mathrm{C}$ the distribution is taken as global.

For ${ }^{40} \mathrm{~K}$ and ${ }^{226} \mathrm{Ra}$ in soils down to $100 \mathrm{Cm}$, assuming a soil density of $1.6 \mathrm{~g} / \mathrm{cc}$, the U.S. continental area is assumed to be $7.7 \times 10^{12} \mathrm{~m}^{2}$. Basic data taken from NCRP-1975c.

For ${ }^{222} \mathrm{Rn}$ the equilibrium amount in the atmosphere from the continental U.S. evolution is given.

$b$ This is the dose to bone surfaces (NCRP-45).

B4. Internal Emitter Exposure and Environmental Radionuclide Inventory, Natural and Man-Made

The naturally occuring inventories of ${ }^{14} \mathrm{C},{ }^{40} \mathrm{~K} ;{ }^{226} \mathrm{Ra}$ and ${ }^{222} \mathrm{Rn}$ in arbitrarily selected reservoirs are listed in Table 5.B-3. These nuclides and their daughters are considered to be among the major contributors of radiation dose to man from among the primordial and cosmogenically produced radionuclides. The reservoirs are: for ${ }^{14} \mathrm{C}-$ the global carbon pool; for ${ }^{40} \mathrm{~K}$ and ${ }^{226} \mathrm{Ra}$ - soil in the continental U.S. to a depth of 1 meter; for ${ }^{222} \mathrm{Rn}$ - the atmosphere above the continental U.S.

The inventories vary from $10^{6}$ to $10^{8} \mathrm{Ci}$. However, the inventory in the soil is approximately proportional to the depth considered so that there are about $10^{11} \mathrm{Ci}$ in the earth's crust to a depth of $10000 \mathrm{~m}$, the approximate depth of deepest drilled wells.

\section{B5. Health Effect Estimates}

Other groups have assessed the likelihood and total number of effects that might be induced from the radiation exposure of a person or population. For completeness these estimates will be described here although we have chosen to make the primary comparison of the impact of the LWR fuel cycle in terms of its addition to background rates.

Important factors influencing the likelihood of inducing biological effects are the types of radiations, the rate of delivery, the fractionation (i.e., periods of intermittent nonexposure), and the total amount of radiation delivered. The types of radiations may be categorized by their linear energy transfer (LET). Low LET radiations include primarily $X$-ray, gamma, and beta radiations, and high LET radiations, primarily alpha particles and neutrons. Rates of exposure can be characterized as environmental level (circa 0.1 $\mathrm{rem} /$ year) and occupational level (up to 10's of rem per hour). It is important to relate these to doses and rates that are characteristic of the conditions and exposures that underlie the basis for our understanding of biological effects in man and animals. Background dose rates are about $0.1 \mathrm{rem}$ per year and global exposures for the nuclear fuel cycle would add only a small fraction to this. Thus the total dose experienced by non-occupationally involved individuals would be delivered at rates characteristic of background. External occupational exposures, such as those to maintenance personnel at a reactor or fuel processing plant, might be at instantaneous rates as high as $10 \mathrm{rem} / \mathrm{hr}$ for an average accumulation of $5 \mathrm{rem} / \mathrm{y}$. Most exposures of animals in experiments to study health effects are at rates approaching $100 \mathrm{rem} / \mathrm{min}$, although some chronic long-term exposure experiments have been carried out with radioisotopic irradiators at dose rates as low as $0.3 \mathrm{rem} /$ day. Below $0.3 \mathrm{rem} /$ day, biological effects become infrequent and difficult to observe in mammals, and accordingly most experimental reports deal with dose rates above 0.3 $\mathrm{rem} /$ day (Cassarett, 1977).

It is generally conceded that cancer is likely to be the most important somatic effect. For this reason many of the analyses of health effects per unit of radiation exposures focus on an estimate of the carcinogenic potential. Equally important however are the mutagenic effects of radiation, which have been recognized since 1927. Present standards take into account mutagenic as well as somatic aspects. In this report we are not interested in assessing the radiation protection standards but instead are assessing the relative detriment that might be associated with the nuclear fuel cycle and in particular the waste management and reprocessing aspects. ${ }^{3}$

\section{B5a. Types of Studies Used As the Basis for Estimating Effects}

\section{Mutagenesis}

There is very little information on man that would allow one to estimate the mutagenic effects of ionizing radiation. The only mammalian. work with sufficient number of animals for valid statistics is that on the mouse by Russell (Russell, 1970) and Searle (Luning, 1971). Based partly on Russell's work BEIR (BEIR, 1972), UNSCEAR (UNSCEAR, 1972), and the ICRP (ICRP-14, 1969) have all made estimates of the frequency of induced mutations per rad again with the assumption that a linear relationship between dose and effect obtains. In the case of mutagenic effects, dose rate is accepted as important and the effect of dose rate on specific locus mutations observed in the mouse has been taken into account (BEIR, 1972). The dose rate to mice upon which this experimental evidence is based was in the range of $1 \mathrm{rad} /$ day in the case of female mice and slightly lower for male mice.

\section{Carcinogenesis}

Most quantitative estimates of carcinogenic effect in man come from epidemiological studies in which populations exposed either occupationally, purposefully in medical treatment, or in war (as in the bombing of Hiroshima and Nagasaki) have been studied for biological effects. Care has been taken to estimate the doses received; however, the degree of uncertainty in the dosimetric estimates depends on the study and conditions of exposure, (high, low or mixed LET, rates, etc.). Accordingly, to relate these observed effects to exposures out of the range of doses delivered to the exposed populations requires additional assumptions.

\section{B5b. The "Linear" Hypothesis for Carcinogenesis}

The question of the form of the dose-effect relation has been the subject of a number of studies and reports over the past several years. While all were based on the same set of data, there has not been unanimity of the assessments and conclusions especially for the 
important case of low LET radiations delivered at low dose rates that would characterize the radiation exposure to the general public. To apply existing data, extrapolations were required from environments in which there were mixed high and low LET radiations. In addition the doses were greater by factors of 1,000 and dose rates greater by factors up to $10^{8}-10^{9}$ than those for which estimates of effect is desired here. ${ }^{4}$ It is essentially impossible to make these extrapolations without adopting a mathematical form for the relationship. Typical of the reasoning used is that stated by the BEIR group who state "In view of the gaps in our understanding of radiation carcinogenesis in man and in view of its more conservative implications, the linear nonthreshold hypothesis warrants use in determining public policy in radiation protection; however, explicit explanation and qualifications of the assumptions and procedures involved in such estimates are called for to prevent their acceptance as scientific dogma [italics ours]. Futhermore, the linear hypothesis is the only one which permits the selection of the mean accumulated tissue dose to characterize the radiation exposure of a group under conditions of nonuniform exposure rate. The mean accumulated tissue dose is the only practical quantity that can be used to estimate the risk of cancer in such populations until the influence of the many interacting variables can be better specified."

It is generally agreed that at sufficiently low doses or dose rates any effects would be due principally to "single-track" interactions and so a linear dose-effect relation is expected on theoretical grounds. However, should "multi-track" interactions become important, nonlinear dependence on dose and on dose-rate would be expected for low LET radiations at higher doses. Hence, even if a linear hypothesis is adopted, the question of determining the slope appropriate for low level dose conditions remains. In the body of the report, the BEIR group states (page 99) "Estimates of risk are of course not scientifically reliable except in the range of observations from which they are derived and under corresponding conditions of exposure". In the summary of the BEIR report, the high rate-high dose data is used to evaluate the parameters of the linear, no threshold hypothesis to calculate the number of health effects due to background. It is clear that this is a conservative process intended to establish limits for radiation exposure guidelines. Yet, despite the pragmatic motivation and the clear caveat, many individuals have seized upon the linear no-threshold hypothesis as scientific dogma. ${ }^{5}$

UNSCEAR 1972 (page 403) states "Estimates of the risk per unit dose derived from the epidemiological investigations are valid only for rates at which they have been estimated, and they can be applied to a range of doses only if there is a linear relation between dose and effect since extrapolation beyond that range may lead to gross errors."

It is the general consensus of NCRP that linear estimates at low dose rates using slopes derived from high dose rates provide unrealistic overestimates of risk (for low LET radiations) rather than expected estimates of risk. Accordingly these estimates are useful only to the extent that they are used as upper limits. If they are misused as measures of expected risk, they tend to distort perceptions both among technical people and particularly the lay public. It is interesting that the BEIR Committee indicated that "furthermore it is becoming increasingly important that society not expend enormously large resources to reduce very small risks still further at the expense of greater risks (NCRP, 1975a) that go unattended (BEIR, 1972)." Accordingly, the manner in which the risk estimates are used, as upper limits or expected effects, is important. The EPA, for instance, tends to treat these estimates as expected (USEPA, 1973). ${ }^{6}$

The cumulative addition to the cancer risk is usually treated in terms of its cumulative frequency; there is a wealth of experimental literature both with ionizing radiation and other agents that shows this to be an oversimplistic manner of estimating carcinogenic risks, since not only is the frequency of occurrence important, but also the time of occurrence. It is generally found that with decreasing dose and dose rates, not only for radiation but for many substances, there is an increasing time delay in the appearance of tumors. Accordingly, sophisticated analyses of the effects on life shortening would tend to show lower effects at lower doses and lower dose rates. In general, however, this approach has not received sufficient attention and is a biomedical area deserving of further research.

The quantitative estimates of carcinogenic risk fall into two categories, absolute estimates of risk (i.e., frequency per unit radiation exposure assuming the linear hypothesis and independence of dose rate), and relative risk estimates. The latter comes about from expressing the experimental information in terms of an increase in the natural incidence of a cancer, whereas the former comes about from expressing the total excess risk in terms of the radiation dose. It is not definitively established which of the semiempirical procedures has the most validity. The relative risk method may, under certain circumstances, produce higher estimates of risk. For instance, we note that the presence of carcinogens and promoters other than radiation in the environment may make the interaction of other substances with radiation an important effect. Situations where this appears to be the case should be studied. A striking example is provided by experimental animal work which shows that irradiation by radon and its daughters alone at some levels is not an effective carcinogen, yet radon and daughters along with diesel exhaust or cigarette smoke may be.

The general approach has been to use the absolute risk method. The absolute risk of all cancer mortality as estimated by BEIR and by UNSCEAR is about $10^{-4}$ per person per rem (BEIR, 1972; UNSCEAR, 1972). Note however, that BEIR applies this estimator at all doses and dose rates while UNSCEAR applies it only to rates and dose rates comparable to those existent when effects were observed. Leukemia mortality from the Hiroshima and Nagasaki data is approximately $2 \mathrm{x}$ $10^{-5}$ per rem. Information to date would suggest that all other cancers, although arising later than the leukemias, may be four times more abundant. In short, about $10^{-4}$ total malignancy related fatalities per rem, for whole body irradiation is assumed.

Genetic defects are normally divided into two components, specific genetic defects (i.e., recessive lethal genes, for example) or defects with a complex etiology. The latter is more difficult to predict, and the scientific consensus is that each of these two categories is on the order of magnitude of $2 \times 10^{-4}$ per person-rem. However, much of this would be expressed in the first generation, and accordingly carcinogenic and mutagenic expressions of effect would be roughly equal in the exposed generation and first-generation progeny, respectively.

One can note the high dose and dose rates inherent in the data base: tens to hundreds of rem $/ \mathrm{min}$, relative to the much lower rates at or near background desired for our analysis (about $0.1 \mathrm{rem} / \mathrm{year}$ including natural background). Some improvement might be made if analysis were carried out with a statistically large enough population undergoing exposure at lower radiation levels than the above (although well above background). We identify the population of radiation workers in the U.S. as a group that may satisfy these criteria marginally.

We note the recommendations of the BEIR Committee with respect to assessing genetic effects, which is (page 52), "Our first recommendation is that the natural background radiation be used as a standard for comparison. If the genetically significant exposure is kept well below this amount, we are sure that the additional consequences will neither differ in kind from those which we have experienced throughout human history nor exceed them in quantity."

We concur with the NCRP position that current estimates of the probability for the induction of radiogenic cancer based on the linear, no-threshold extrapolations from information collected at high doses and dose rates provide only an upper limit (for low LET radiations) to the health effects that may be ascribed to exposures at rates comparable to background (NCRP, 1975a). For high LET radiations, the linear, no-threshold extrapolations may in fact give expected rates of health effect incidence.

\section{B5c. Recommendation}

Attention should be given to the possibility of an epidemiological study of radiation workers in the nuclear industries for the purpose of testing the validity of the linear no-threshold hypothesis for low LET indications at dose rates down to perhaps $5 R /$ year, still a factor of 50 above background but much less than that characteristic of the existing data from which the linear dose-effect relationship was parametized. The highly-exposed uranium miners represent the human population with the largest number of observed cancers from 
high LET radiation available for study, and yet have received relatively meager attention compared, for example, to the radium dial painters. The opportunity to carefully study this population intensively will exist only for the next several years. It is recommended that further studies on this group be conducted.

C. Environmental, Biological, and Dosimetric Aspects of Specific Radionuclides

C1. The Concept of Dose Commitment As Applied to the Assessment of Radiation Doses Resulting from Environmental Contamination

The concept of Dose Commitment was introduced many years ago by Lindell (Lindell 1960, 1976) and adopted in the UNSCEAR reports (UNSCEAR, 1964) to take account of future doses from radioactive materials already introduced into the environment from weapons testing. The dose commitment to a given tissue was defined as (UNSCEAR, 1966):

"The integral over infinite time of the average dose rates delivered to the world's population as a result of a specific practice..." of release of activity to the environment.

A complete mathematical description of the concept is given in the 1969 UNSCEAR report (UNSCEAR, 1969). This definition has since been modified to include calculation of doses into the future for finite times. The resulting dose is called the Truncated or Incomplete Dose Commitment (Beninson, et al., 1977).

We show in Appendix IV that the dose commitment to time $\mathrm{T}$ per unit release is identical to the dose rate per unit release rate at the end of a period of $T$ years during which the release was maintained at constant rate. Therefore, the usual dose commitment is the increase in dose rate which would be reached at a time far in the future if the release practice were to continue forever. If the release terminates, the associated dose rate at time of termination is called the Dose Rate Increment. The maximum dose rate as a result of that release is simply determined by considering the decay scheme for the activity. In particular, for the very important cases: radionuclide with stable daughter; release of all members of a decay chain in equilibrium, the Dose Rate Increment is the maximum dose rate that will occur as a result of the release. The significance of this interpretation is that it is potentially a basis for management programs. It is possible to determine a limit for release of radioactivity such that any limit to the increment of environmental dose rate to be experienced by the present or any future generation will not be exceeded as a result of the release.
In Figure 5.C-1, the envelope of the series of curves is the truncated dose commitment for $1 \mathrm{Ci}$ of activity irretrievably released. Alternatively, the individual curves represent the dose rate which would build up as a function of time if $1 \mathrm{Ci} / y e a r$ were released on a continuous basis. To illustrate the concept in a general way the horizontal axis is plotted in units of the number of effective halflives of the radionuclide(s) with respect to its potential to itradiate humans. If the practice is continued for a time period approaching several half-lives, the dose rate established in the environment will reach an asymptotic value proportional to the release rate. If however, the practice of release is short compared to the half-life then the situation is represented by the lower left portion and there will tend to be a linear buildup of dose rate with time. The exponential decreases shown labeled $T=1$, etc. refer to the rate at which dose is delivered after the practice of release is terminated at time $T=1$, etc. The situation with respect to the important nuclides considered in the LWR fuel cycle are discussed nuclide by nuclide in Appendix IV.

Figure 5.C-2 shows the fraction of the equilibrium or asymptotic dose rate increment which would be established for a nuclear fission economy with assumed finite durations of 50 to 500 years during which radionuclides are assumed to be released as effluents or into waste at a constant rate. The dose rate for ${ }^{3} \mathrm{H}$ and ${ }^{85} \mathrm{Kr}$ reaches an equilibrium value in a few decades. Subsequently it will depend primarily on the rate of generation of energy (power level) and not on how long the release practice has continued. For the long-lived nuclides in long-lived environmental reservoirs, the fraction of equilibrium established depends primarily on the duration of the nuclear industry relative to the half-life of the radionuclide. It is readily seen that the most persistent radionuclides (such as ${ }^{230} \mathrm{Th}$, parent of ${ }^{226} \mathrm{Ra}$ and ${ }^{222} \mathrm{Rn}$ in tailings) build only to a small fraction of the equilibrium or asymptotic value. The degree to which environmental dose rates for these are increased depends on additional factors, such as the magnitude of the source term, management practices, future remedial actions and the long-term environmental behavior of the nuclide.

In the short term, the accumulation of long-lived radionuclides in the environment will build up in proportion to the time that a given release is practiced. These include ${ }^{14} \mathrm{C}$ (the long-lived pool of the deep oceans), ${ }^{239} \mathrm{Pu},{ }^{240} \mathrm{Pu},{ }^{230} \mathrm{Th},{ }^{129} \mathrm{I}$, etc. We believe that the assessment of the buildup of dose rate is therefore preferable from the point of view of rational planning, since it is conceptually meaningful. The alternative, assessment of the dose to individuals with arbitrary lifetimes (as long as infinite) is not conceptually appealing nor readily dealt with in our normal experience.

The parameter of duration of the LWR industry can also be dealt
FIG. 5C-1. Identity of the truncated dost commitment for a single release integrated over finite times (envelope of curves) and the dost rate increment for for continuous release for the same times (individual components).

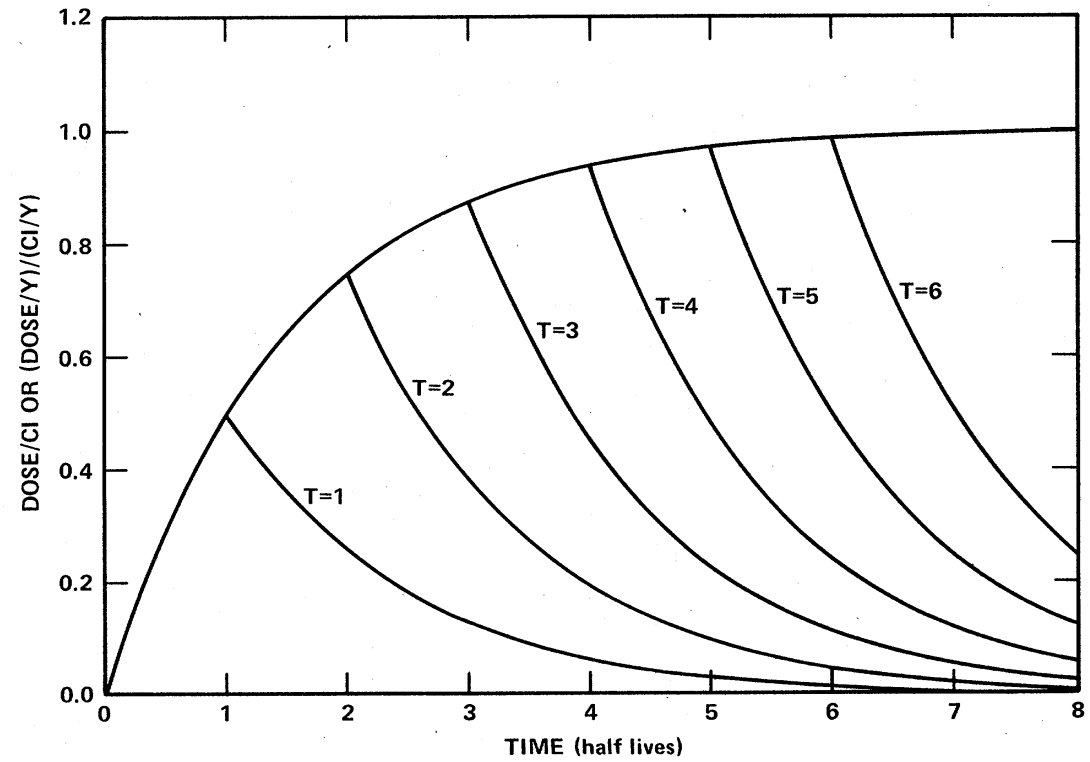




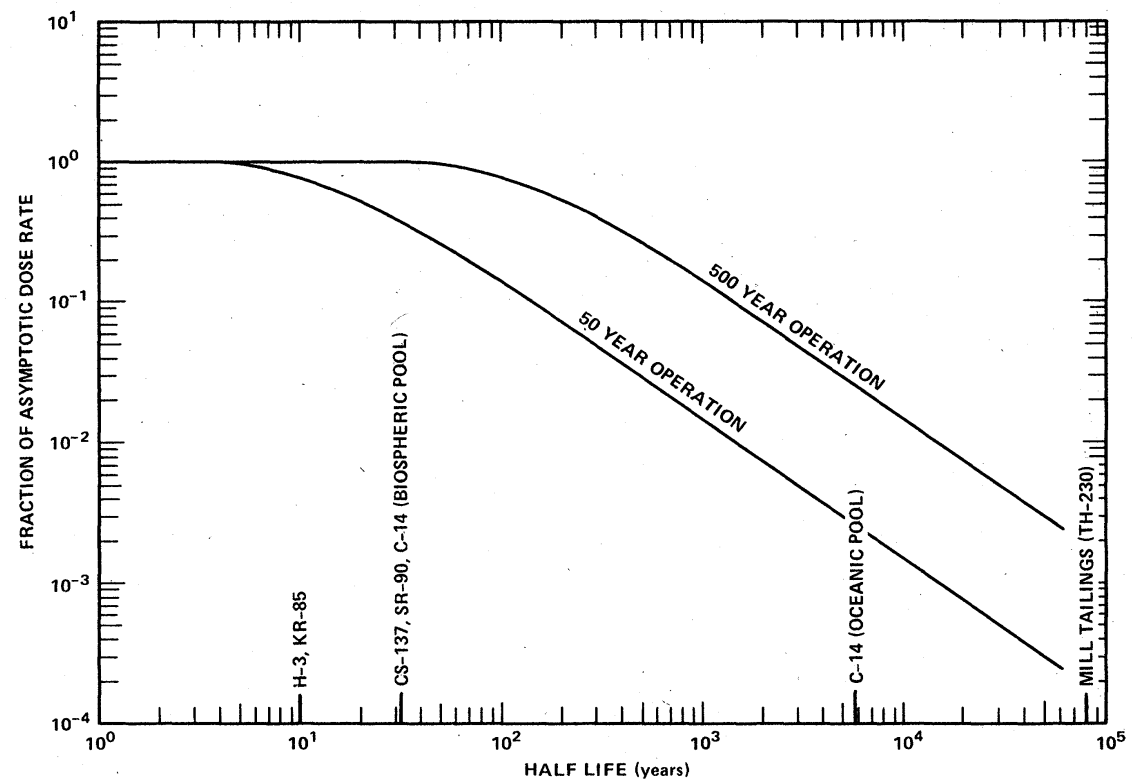

FIG. 5C-2. Fraction of the asymptotic dose rate increment attained for released radioactive effluents for two different periods of operation and release.

with conceptually from the point of view of duration imposed by resource limits. One may then examine the upper limit of increase of dose rate above background given certain emission or control strategies implemented over long time periods and beginning at arbitrary times.

C2. Summary of Dose Commitments/Ci Released for Individual Nuclides

Table 5.C-1 summarizes the results of calculations of the incomplete and complete dose commitments for $1 \mathrm{Ci} /$ year releases of ${ }^{85} \mathrm{Kr},{ }^{14} \mathrm{C},{ }^{3} \mathrm{H}$, and ${ }^{129} \mathrm{I}$ for times of $1,10,50,100$, and 500 years and to time infinity. In section $5-\mathrm{D}$, we synthesize the dose commitments or dose rate increments for any combination of releases of ${ }^{3} \mathrm{H},{ }^{85} \mathrm{Kr}$ and ${ }^{14} \mathrm{C}$, from such data for the overall LWR fuel cycle.

This approach allows the design of management programs which would have the objective that no future generation will be exposed in excess of some predetermined incremental dose rate. In short, rather than trying to focus on the integrated risk over all time $(T=\infty)$ the approach would be to limit the risk for any future generation. Perspective is gained by such an analysis in judging the relative importance of various nuclides. From Table $5 . \mathrm{C}-1,{ }^{14} \mathrm{C}$ is seen to be over 3 orders of magnitude more important per Curie released than is
${ }^{3} \mathrm{H}$ for time periods exceeding 50 years (i.e., if the practice of release were to continue for that long).

Use of this table allows one to synthesize the global dose rate buildup from the existence of any combination of sources of globally distributed ${ }^{14} \mathrm{C},{ }^{85} \mathrm{Kr},{ }^{3} \mathrm{H}$ (whole body), and ${ }^{129} \mathrm{I}$ (thyroid).

\section{Exposure and Source Terms for Specific Fuel Cycle Facilities}

\section{D1. Mining}

The mining process exposes previously inaccessible uranium so that the daughter activities, particularly radium and radon, have a greatly increased probability of transport to the general environment.

\section{Dla. Occupational Exposure}

Exposure to radon daughters is considered the most important occupational hazard in uranium mining. Following inhalation of radon which has diffused into the mine air from the ore body, the alpha-emitting daughters irradiate the tissues and cells of the lung and respiratory tract. The unit of exposure, the working level month (WLM) is discussed in Appendix IV, section 5.3. Present standards

TABLE 5C-1. Global per capita dose rate (rem/y) at the end of $T$ years from a continuous release of $1 \mathrm{Ci} / \mathrm{y}$ for $T$ years.

\begin{tabular}{|c|c|c|c|c|c|}
\hline$I(y)$ & $\mathrm{Kr}-85$ & $c-14$ & ${\underline{H}-3^{a}}^{a}$ & ${\underline{H}-3^{b}}^{b}$ & I-129 (Thyroid) \\
\hline 1 & $0.5(-14)$ & $9(-11)$ & $0.2(-12)$ & $0.1(-13)$ & $0.3(-8)$ \\
\hline 10 & 4. $(-14)$ & $8(-10)$ & $0.8(-12)$ & $0.7(-13)$ & 2. $(-8)$ \\
\hline 50 & 8. $(-14)$ & $2(-9)$ & 1. $(-12)$ & $1 \cdot 5(-13)$ & 7. $(-8)$ \\
\hline 100 & 9. $(-14)$ & $3(-9)$ & 1. $(-12)$ & $1.6(-13)$ & 8. $(-8)$ \\
\hline 500 & 9. $(-14)$ & $5(-9)$ & 1. $(-1<)$ & $1.6(-13)$ & 10. $(-8)$ \\
\hline$\infty$ & 9. $(-14)$ & $3.5(-8)$ & 1. $(-12)$ & $1.6(-13)$ & 1. $2(-4)$ \\
\hline
\end{tabular}

a Atmosphere release.

b Release to marine environment. 
allow 4 WLM exposure per miner per year (FRC, 1967, 1976). Since the particular health effect is due to exposure to part of an organ, no simple conversion to doses expressed in rem exists. However, we note that increased incidence of lung cancer has been observed in uranium miners exposed to more than $400 \mathrm{WLM}$, and possibly observed at 120 WLM.

\section{DIb. Population Exposure}

NRC has estimated that the release of radon in mine exhaust, as a result of the ievel of mining required to supply a nuclear electric power system, could inject as much as $2.4 \times 10^{7} \mathrm{Ci}$ of ${ }^{222} \mathrm{Rn}$ to the atmosphere between 1975 and 2000 (NUREG, 1976). However because of its short half-life, the equilibrium amount of radon present would be much less than this and depend on the instantaneous rate of release. On the average, over the 25 year period, the equilibrium amount present would be about $1.5 \times 10^{4} \mathrm{Ci}$, about $1 \%$ of the continental U.S. average radon background.

\section{D2. Milling and Tailings}

The separation of uranium from its ores and decay products increases the accessibility of the decay products substantially beyond that in ore. Ultimately, these radionuclides are left in the residues from chemical processing. The process waste products have a bulk approximately equal to that of the original ore and it has been common practice to dump them into tailing piles near the mills. Subsequent contamination of the environment has been observed including leaching of $226 \mathrm{Ra}$ into ground water and $222 \mathrm{Rn}$ into the atmosphere. The history in this regard has been recently reviewed by Siek (Siek, 1977).

\section{D2a. Occupational Exposure}

The relevant radionuclides for occupational exposure in milling include the radon daughters and in addition uranium and $230 \mathrm{Th}$ Although Stuart, et al. (Stuart and Beasley, 1967; Stuart and Jackson, 1975) have shown that for ore deposited in the lungs of animals, ${ }^{230} \mathrm{Th}$ fractionates from the uranium and remains in the lung, limited attempts to measure in vivo in millers have failed to detect ${ }^{230} \mathrm{Th}$. The levels of radon are much lower in the mills than in the mines. Accordingly, we will assume that the occupational population dose from milling is insignificant with respect to that from mining and will treat it no further.

\section{D2b. Environmental Impact of Mill Tailings}

An assessment of the hazard of uranium mill tailings has two distinct relations to policy in the fuel cycle and waste management area. Most obviously, it is a hazard that is reduced somewhat by implementation of plutonium or uranium recycle, simply because both of these require less ore to be mined for a given nuclear electricity production in the absence of recycle. Less directly, consideration of mill tailings lends perspective on waste management policy, as the long-range hazard of the surface-stored tailings can be compared to that of both the geologically confined high level wastes and the natural leaching of radium. The mass, area, and volume of mill tailings generated in the production of $1 \mathrm{GWe}-\mathrm{yr}$ of electricity from light water reactors with plutonium and uranium recycle are listed in Table 5.D-1.

TABLE 5D-1. Physical impact and characteristics of mill tailings generated in the production $1 \mathrm{GWe}-\mathrm{yr}$ of electricity from light water reactors with $\mathrm{U}$ and $\mathrm{Pu}$ recycle.

\begin{tabular}{ll}
\hline \hline Characteristic & Quantitative Impact \\
\hline Oremined & $1.5 \times 10^{5}$ metric tons \\
Mill tailing area & 32 acres, $1.6 \times 10^{5} \mathrm{~m}^{2}$ \\
Mill tailing mass & $1.5 \times 10^{11} \mathrm{grams}$ \\
Mill tailing volume & $2.1 \times 10^{5} \mathrm{~m}^{3}$ \\
\hline \hline
\end{tabular}

For no recycle case multiply values by 1.4 (GESMO, 1976).

For $\mathrm{U}$ recycle only multiply values by 1.2 .
TABLE 5D-2. Isotopic composition and activity ${ }^{a}$ of mill tailings generated in the production of $1 \mathrm{GWe}-\mathrm{yr}$ of electricity from light water reactors with $U$ and $P u$ recycle. ${ }^{b}$

\begin{tabular}{lc}
\hline \hline Radionuclide & Activity $(\mathrm{C} i)$ \\
\hline $\mathrm{U}-\mathrm{natura} \mathrm{f}^{\mathrm{c}}$ & 4 \\
${ }^{234} \mathrm{Th}$ & 4 \\
${ }^{230} \mathrm{Th}$ & 63 \\
${ }^{226} \mathrm{Ra}$ & 63 \\
${ }^{222} \mathrm{Rn}{ }^{\mathrm{d}}$ & 12.6 (in environinent) \\
& 50.4 (in pile) \\
${ }^{210} \mathrm{~Pb}$ & 63 \\
$210 \mathrm{Bi}$ & 63 \\
${ }^{210} \mathrm{Po}$ & 63 \\
\hline \hline
\end{tabular}

${ }^{\mathrm{a}}$ For $0.1 \% \mathrm{U}_{3} \mathrm{O}_{8}$ content in ore there will be $280 \mathrm{pCi}$ of daughter product activity per gm of ore assuming secular equilibrium (Swift, 1974). If ore is $0.15 \%$ $\mathrm{U}_{3} \mathrm{O}_{8}$ by weight, daughter activity will be $420 \mathrm{pCi} / \mathrm{gm}$. For $1.5 \times 10^{5}$ metric tons of ore there will be a total of $63 \mathrm{Ci} / \mathrm{GWe}-\mathrm{yr}$ activity for each daughter.

${ }^{b}$ For no recycle case multiply values by 1.4 . For U recycle only multiply values by $1: 2$.

${ }^{c}$ A ssuming $95 \% \mathrm{U}_{3} \mathrm{O}_{8}$ extraction in mill processing.

${ }^{\mathrm{d}}$ Assuming $20 \%$ emanation of ${ }^{222} \mathrm{Rn}$ from pile (Culot, 1973).

The radionuclides are more likely to impact the biosphere from tailings than from their original location in ore bodies. Radionuclide emissions from that fraction of the mill tailings attributable to the nuclear production of $1 \mathrm{GWe}$-year of electricity are given in Table 5.D-2. These are the result of the ${ }^{230} \mathrm{Th}$ decay chain after 95 percent of the uranium has been removed in the milling process. The thorium half-life is $7.6 \times 10^{4}$ years, so that the rate of radon production will be little diminished over this time span. After times long compared to the thorium half-life, there will still be a residual radon production rate at 5 percent of the original, governed by the remaining ${ }^{238} \mathrm{U}$ decaying to ${ }^{230} \mathrm{Th}$ at a half life of $4.5 \times 10^{9}$ years. Continued physical stability and integrity of tailings piles is unlikely over these long time periods, and accordingly the emission rate to the atmosphere is not accurately predictable. The emission rate depends on the amount of overburden, moisture content of the pile, temperature, meteorology, etc.

Possible problems with tailings are in several categorties:

1. Long-term emission of ${ }^{222} \mathrm{Rn}$ from piles and the cumulative buildup of a radon source with cumulative mining and milling.

2. Dispersal of the radionuclides in tailings into the environment by either natural forces or human intervention.

To put the above in perspective, a key comparison is the natural average background radon emission rate from the earth's surface, which is approximately $0.42 \times 10^{-12} \mathrm{Ci} / \mathrm{m}^{2} / \mathrm{sec}$ (Wilkening, 1975). A previous calculation estimating the ${ }^{222} \mathrm{Rn}$ emission rate, from a model inactive stabilized tailings pile at $480 \times 10^{-12} \mathrm{Ci} / \mathrm{m}^{2} / \mathrm{sec}$ (Swift, 1974), is about $10^{3}$ times greater per unit area than the average natural evolution rate of radon (see Table 5.D-3). Assuming an atmospheric inventory of $12.6 \mathrm{Ci}$ of ${ }^{222} \mathrm{Rn}$ per GWe-yr evolved from the tailings as presented in Table 5.D-2, a radon emanation rate from the model tailings pile area of $188 \times 10^{-12} \mathrm{Ci} / \mathrm{m}^{2}-\mathrm{sec}$ can be calculated. The calculation is presented in Table 5.D-4. This emanation rate is a factor of 2.5 times lower than that calculated by Swift, and is based on an assumption of $20 \%$ escape of radon from the pile.

Though the tailings pile emission rate is much higher per unit area than the continental average background, it obviously emanates from an area trivial in comparison with the continental land mass. The contribution to the natural radon background at large distances from a typical tailings pile is only $10^{-15}$ to $10^{-16} \mathrm{Ci} / \mathrm{m}^{3}$ as compared to the national average concentration of $10^{-10} \mathrm{Ci} / \mathrm{m}^{3}$ (Harley, 1975). Table 5.D-3 gives the calculation showing that the total ${ }^{222} \mathrm{Rn}$ emanation rate from a reference tailings pile is $2 \times 10^{-5}$ that from the continental land mass. 
TABLE 5D-3. Relationship between continental and reference tailings pile emission of ${ }^{222} \mathrm{Rn}$.

\begin{tabular}{lll}
\hline Factor & Tailings Pile & Continent \\
\hline Area & $1.53 \times 10^{5} \mathrm{~m}^{2} \mathrm{a}$ & $7.7 \times 10^{12} \mathrm{~m}^{2}$ \\
${ }^{222} \mathrm{Rn} \mathrm{F1ux} \mathrm{Rate}$ & $480 \times 10^{-12} \mathrm{Ci} / \mathrm{m}^{2} / \mathrm{sec}^{\mathrm{a}}$ & $0.42 \times 10^{-12} \mathrm{Ci} / \mathrm{m}^{2} / \mathrm{sec}^{\mathrm{b}}$ \\
Total ${ }^{222} \mathrm{Rn}$ Emanation & $7.34 \times 10^{-5} \mathrm{Ci} / \mathrm{sec}$ & $3.23 \mathrm{Ci} / \mathrm{sec}$ \\
Radon Emission Ratio & & \\
Tailings Pile/Continent $=$ & $2.3 \times 10^{-5}$ & \\
\hline
\end{tabular}

a Swift, 1974

b Wilkening, 1975

A reference source term for ${ }^{222} \mathrm{Rn}$ emission from tailings piles can be calculated by multiplying the radon emanation rate $(2.27 \mathrm{Ci} /$ day per GWe-yr) by an attenuation factor. The rate at which radon gas leaves the tailings pile decreases with increasing the earth cover depth over the pile and increasing moisture content of the cover (ORNL$\mathrm{TM}-4903,1975)$. Very substantial reduction of radon emission has theoretically been shown to occur when a mud cover $(37 \%$ moisture content) is maintained over the tailings pile. A mud cover as little as 6 inches in depth over the pile was calculated to result in an attenuation coefficient of approximately $10^{-4}$. In general past practice employed the use of a light earth cover, which did not minimize the escape of radon gas from the pile. Past practices and their domestic consequences have recently been described (Kennedy, et al., 1977; and Siek, 1977).

Using a typical attenuation coefficient of 0.2 , a reference source term for radon emission from tailings piles of $2.27 \mathrm{Ci} /$ day $(0.2)=0.5$ $\mathrm{Ci} / \mathrm{day}$ per 1 GWe-yr would be expected if long term tailings management were no better in the future than it has been in the past. Using this term the U.S. radon evolution would be doubled with the generation of $6 \times 10^{5} \mathrm{GWe}-y \mathrm{r}$ from the I.WR cycle if future practices of tailings management do not result in a change in emission rate relative to past practices. As will be shown, relatively simple techniques can be used to reduce this source term by factors up to 100 (see lable 5.D-5) over that characteristic of past practices.

The potential long-term buildup of a radon source in tailings requires better evaluation than it has had in the past. There was an extensive environmental evaluation program in the vicinity of 4 large tailings piles. Local doses even near large piles were not significantly elevated above background at distances greater than $1 / 2$ mile from the pile (Shearer and Sill, 1969; USPHS, 1969).

The highest exposures to the general public from tailings have come from the inappropriate practice of using mill tailings as fill for foundation materials for buildings, including homes, schools, and stores. The most obvious example of this was found in Grand Junction, Colorado, where such practice was extensive (Siek, 1977).
The resulting indoor radon concentrations ranged from background ( $0.1 \mathrm{pCi} / 1$ ) to 100 's of $\mathrm{pCi} /$ liter (Wrenn and Spitz 1975; Siek, 1977) and based on criteria suggested by the Surgeon General of the U.S. an extensive remedial program had to be undertaken. This seems clear evidence that the relative accessibility of tailings is the most hazardous aspect of past practice.

Despite the uncertainties in these conversion quantities, a significant question to be resolved in judging health implications is the familiar one of how to total up collective doses and effects at a very low rate over the full lifetime of the relevant isotope. This requires more attention, and the dose rate increment approach developed in Section V.C is suggested as an important analytical tool. Pohl has calculated that 400 "deaths" would be committed over the thorium decay span per GWe year electricity. These should be compared to a similarly calculated 400,000 deaths, which would be predicted over the same period due to natural background radon concentrations; we note that statistical variations in the estimated "deaths" from variations in background alone would be greater than the estimate from tailings.

The second major environmental and health impact of the tailings concerns the isotopes remaining in them and their proximity to the biosphere. Table 5.D-2 lists the isotopic composition of the tailings. As with radon, the other tailings isotopes will be in equilibrium with the ${ }^{230} \mathrm{Th}$ parent, so that the activity in the pile will not diminish by a factor of 2 over 80,000 years. Though the pile can be constructed and managed for minimal erosion over a conventional engineering time span, it certainly must be considered as no more secure than any other surface feature over a period of several tens of thousands of years.

With this in mind, it is illuminating to compare the hazard of the mill tailings with that of the high level wastes from nuclear fuel reprocessing. The $63 \mathrm{Ci} / \mathrm{Gwe}-\mathrm{yr}$ of ${ }^{226} \mathrm{Ra}$ compares with roughly $10^{7}$ $\mathrm{Ci} / \mathrm{Gwe}-\mathrm{yr}$ of fission products (ERDA-76-43, 1976). The mill tailings are comparable to the high level wastes as a potential health hazard after a few hundred years. When we consider the relative likelihood of biospheric contact with geologically buried plutonium as

TABLE 5D-4. ${ }^{222} \mathrm{Rn}$ emanation from reference tailings pile produced in the generation of 1 GWe-yr electricity.

\begin{tabular}{ll}
\hline \hline $\begin{array}{l}\text { Factor } \\
\left({ }^{222} \mathrm{Rn}\right)\end{array}$ & Value \\
\hline decay constant $(\lambda)$ & $0.18 \mathrm{day}^{-1}$ \\
mean 1ife $=$ & $5.56 \mathrm{day}$ \\
rate of ${ }^{222} \mathrm{Rn}$ production ${ }^{\mathrm{a}}=12.6 \mathrm{Ci} / 5.56=$ & $2.27 \mathrm{Ci} / \mathrm{day}$ \\
reference area & $1.4 \times 10^{5} \mathrm{~m}^{2}$. \\
${ }^{222} \mathrm{Rn}$ emanation $=$ & $2.27 \mathrm{Ci} / \mathrm{day} \mathrm{divided}$ by \\
& $\left(1.4 \times 10^{5} \mathrm{~m}^{2} \times 8.64\right.$ \\
& $\left.\times 10^{4} \mathrm{sec} / \mathrm{day}\right)=$ \\
& $1.88 \times 10^{-10 \mathrm{Ci} / \mathrm{m}^{2} \mathrm{sec}}$ \\
\hline
\end{tabular}

a This is the equilibrium amount produced in the tailings pile environment per day due to the production of 1 GWe-yr electricity. 
TABLE 5D-5. Possible alternatives to reduce uranium mill tailings emissions (NUREG0129, 1977).

\begin{tabular}{|c|c|c|c|}
\hline Alternative & Comments & $\begin{array}{l}\text { Radon } \\
\text { Reduction }\end{array}$ & $\begin{array}{c}\text { Cost } \\
\text { (mi11ions) }\end{array}$ \\
\hline I & $\begin{array}{l}\text { Tailings pond with scarified, } \\
\text { compacted basin floor; } 6 \mathrm{ft} \text {. } \\
\text { tailings cover including } \\
\text { topsoil; vegetation }\end{array}$ & $90 \%$ & 3.3 \\
\hline I I & $\begin{array}{l}\text { Same as I with the addition } \\
\text { of a clay } 1 \text { iner to reduce } \\
\text { ground water seepage }\end{array}$ & $90 \%$ & 3.4 \\
\hline I I I & $\begin{array}{l}\text { Same as II with the addition } \\
\text { of a clay cap }\end{array}$ & $\sim 98.6 \%$ & 3.6 \\
\hline IV & $\begin{array}{l}\text { Same as I with the addition of } \\
\text { an artificial Tiner to halt } \\
\text { seepage into ground waters }\end{array}$ & $90 \%$ & 5.9 \\
\hline V & $\begin{array}{l}\text { Same as IV with the addition of } \\
\text { an artificial (impervious cap) }\end{array}$ & $100 \%$ a & 8 \\
\hline VI & $\begin{array}{l}\text { Pipeline transport of slurry } \\
\text { tailings to remote mine pit } \\
\text { or to } 1 \text { ined pond (Same as I) }\end{array}$ & $90 \%$ & $3.9-10.9$ \\
\hline VII & $\begin{array}{l}\text { Temporary, holding area then } \\
\text { dry transport to pit }\end{array}$ & $\sim 90 \%$ & $6.6-15.5$ \\
\hline VII I & $\begin{array}{l}\text { Slurry pipeline to pit and } \\
\text { stabilization of tailings } \\
\text { with asphalt or concrete }\end{array}$ & $100 \%$ & $34.3-42.3$ \\
\hline
\end{tabular}

a $100 \%$ reduction connotes radon emanation identical to background.

opposed to surface piled ${ }^{226}$ Ra, the mill tailings may well be more important for the long-term.

Another useful comparison is that with the natural runoff of ${ }^{226} \mathrm{Ra}$ in rivers and streams. Approximately $150 \mathrm{Ci} / \mathrm{yr}$ of ${ }^{226} \mathrm{Ra}$ are found in the U.S. watershed runoff due to natural leaching. The tailings pile generated by the production of only 1 Gwe-year of nuclear generated electricity contains an amount comparable to this in a very precarious and erodible form. Thus, by a measure of potential perturbation of background radiation in water supplies, past practices for storage of the mill tailings appear rather precarious by comparison to plans for the emplacement of high level waste.

Following the implementation of strong guidelines to curtail the easy accessibility of tailings materials for constructional or other unintended purposes, serious efforts can be made in the direction of reducing radon emissions, gamma emissions and seepage into ground and surface water supplies from tailings piles.

Possible alternatives to reduce uranium mill tailings emissions utilizing practical available technology have been summarized in the ORNL-TM-4903, Vol. 1, 1975 and in the Environmental Statement related to the operation of the Rock Mountain Energy Company's Bear Creek Project (NUREG-0129, 1977) and appear in Table 5.D-5. Of the eight alternatives listed in Table 5.D-5 number III, the utilization of a tailings pond with a compacted clay bottom liner as well as a 10-inch clay cover was thought of as being the most environmentally sound, reliable, and reasonable method of tailings management using existing, available technology. A radon emission reduction of $98.6 \%$ (i.e., ensueing radon emission from the pile would be approximately twice background) was envisioned using this alternative. In any case, the subject of mill tailings management warrants attention.

\section{D3. Nuclear Power Reactors}

Electricity is generated at nuclear power stations using energy derived from fission. In addition to radiations directly connected with the fission process, there is possible atmospheric or liquid releases of radionuclides produced as fission products or by neutron activation.

\section{D3a. Occupational Exposures at the Power Plant}

Occupational radiation exposure at LWRs is due primarily to external exposure to low LET radiations, mainly gamma radiations from fission and activation products. Less information is available than desirable on the breakdown of the exposure by type of operation, but much of it appears to be associated with refueling, maintenance, inspection, and waste treatment operations. The size of plants on-line in the U.S. is increasing, and most new plants are in the 1,100 to 1,400 MWe range. There is insufficient operating experience with these larger plants as yet to accurately predict the collective doses required to operate them during their total design lives. The best estimate of expected performance may be taken from the record of current operational experience.

The exposures for 1973 to 1975 from the collective U.S. licensed plants are presented in Table 5.D-3. At this time there seems to be no clear trend of increase or decrease in exposure with size or age of the units. During 1973-1975 it required an occupational average population dose of about 400 to 500 person-rem to operate a unit for a year. Assuming the ratio of generated power to rated capacity was 0.7 for each year leads to an estimate of average occupational exposures of 1.2 person-rem/MWe(y) for the period 1973-1975 (see Table 5.D-3). This differs from the NRC estimate of 0.6 in the GESMO for the future. 
TABLE 5D-6. Reactor experience: Occupational exposure of LWR operating and maintenance personnel (NUREG 75/032, 75/108, 1975; Brooke, 1976).

\begin{tabular}{|c|c|c|c|c|c|c|c|c|}
\hline Year & $\begin{array}{l}\text { No. } \\
\text { BWRs }\end{array}$ & $\begin{array}{l}\text { No. } \\
\text { PWRS }\end{array}$ & $\begin{array}{l}\text { No. } \\
\text { LWRs }\end{array}$ & $\begin{array}{l}\text { Avg. Rated } \\
\text { Capacity } \\
\text { (MWe) }\end{array}$ & $\begin{array}{l}\text { Annuala } \\
\text { Electric } \\
\text { Energy } \\
\text { Produced }\end{array}$ & $\begin{array}{l}\text { Man-rem/ } \\
\text { unit/yr }\end{array}$ & $\begin{array}{l}\text { Man-rem/ } \\
\text { MWy (e) }\end{array}$ & $\begin{array}{l}\text { Total } \\
\text { man-rem } \\
\text { in year }\end{array}$ \\
\hline 1973 & 14 & 12 & 26 & 546 & 9900 & 542 & 1.4 & 14083 \\
\hline 1974 & 14 & 18 & 32 & 581 & 13000 & 448 & 1.1 & 14337 \\
\hline 1975 & & & 44 & 640 & 19700 & 482 & 1.1 & 21223 \\
\hline
\end{tabular}

a Estimated using 0.7 capacity factor on the average.

Exposures to individuals at reactor plants are generally kept below 5 rem/y with exposures exceeding that level rare. Since the usual practice in quoting average dose is to include many workers receiving $<5 \mathrm{rem} / \mathrm{y}$, an estimate of the average occupational dose is not significant without information on the dose frequency distribution in the population of workers.

\section{D3b. Population Exposures}

Fxternal exposure to low LFT radiations appears to be the dominant mode of exposure for the general public. Dose rates to local residents near I.WR reactors are generally $<10$ mrem/yr in the U.S. and in fact, the design dose rates from their effluents must be below this value as a condition of licensing. Regional population doses from fission product noble gases range between 10 to several hundred person-rem per larger plant, depending on the reactor sice, type, location, waste treatment plant, and geographical distribution of the regional population.

Contribution of ${ }^{85} \mathrm{Kr}$ from reactors to the global dose may be considered negligible relative to those from reprocessing since less than $1 \%$ of ${ }^{85} \mathrm{Kr}$ escapes at the reactor. An additional source of very local exposure is turbine shine (from the ${ }^{16} \mathrm{~N}$ decay) with BWRs. Radiation exposure at a given site depends on the power level, turbine design, incorporated shielding, and the site size and layout, as well as the distance to the nearest exposure location. Individual exposures up to $0.02 \mathrm{rem} / \mathrm{yr}$ have been reported. The population dose, however, is very small (typically less than 1 person-rem/yr) because the radiation intensity falls off rapidly with distance.

The nuclide, ${ }^{14} \mathrm{C}$ is also produced at LWRs. However, the amounts and forms and routes of release are not well established. This potential source should be studied carefully, since ${ }^{14} \mathrm{C}$ releases at reactors give a global dose that may not be insignificant relative to ${ }^{14} \mathrm{C}$ releases at reprocessing plants. Releases may be in the several $\mathrm{Ci} / \mathrm{y}$ range. (NRC uses $8 \mathrm{Ci} / \mathrm{y}$ in GESMO.) The recognition of the importance of ${ }^{14} \mathrm{C}$ in the collective doses is relatively recent. As a result, no mention of ${ }^{14} \mathrm{C}$ was made in the EPA environmental study of the fuel cycle (USEPA520/9-1973) nor in the ERDA study on alternatives for waste management (ERDA-76-43, 1976).

\section{D4. Fuel Reprocessing Plants}

At the reprocessing plant, spent fuel is chopped and dissolved in acid. Chemical separation procedures produce separated streams of $\mathrm{Pu}, \mathrm{U}$ and fission products.

\section{D4a. Occupational Experience}

Occupational Exposure at Commercial Fuel Reprocessing Plants

Prior to 1969, there were no regulations requiring detailed reporting of occupational exposure. Nuclear Fuel Services (NFS, West Valley, New York), the only commercial fuel reprocessing plant ever in operation in the U.S. carried out most of its reprocessing prior to 1969. As a result, the available records for occupational exposure at NFS are not complete. We estimate the exposure in the following manner. Prior to 1970 , about 530 tons of fuel were processed. A $1,000 \mathrm{MWe}$ plant has a fuel load of about 100 tons, of which roughly 30 tons per year are replaced. Accordingly this represented roughly the equivalent of 18 LWR reactor operating years. No acocunt was made for the degree of burnup of the reprocessed fuel which was less than that which would be characteristic of present LWR's. Based on reports filed with NRC on employees of NFS (including contractors) terminating employment between 1969 and June 1975, a collective dose of 7,300 person-rem was delivered. The total dose associated with operating the plant must exceet this number, and the degree to which the trite collective dose exceeds it is unknown. For purposes of this analysis it will be assumed that this (7,300 person-rem) is not a substantial underestimate. Accordingly the person-rem per $\mathrm{MWe}$ year, is about $7,300 /(18 \times 1,000$ MWe-y $\times 0.7 \approx 0.6$ person$\mathrm{rem} / \mathrm{MWe}-\mathrm{y}$. We have examined information furnished by NRC on the distribution of individual exposures for NFS by annual dose level (NRC, personal communication, 1976). Exposures in excess of 5 rem per year were not uncommon, but the records we examined showed all exposures less than $12 \mathrm{rem} /$ year, the upper limit allowable in any one year under federal regulations (USNRC, 10CFR20).

Allied-General Nuclear Services (AGNS, Barnwell, S.C.) has estimated that about 300 person-rem/yr will be sufficierit to operate the Barnwell plant if operation is in accord with design aims. In that plant mechanical operations associated with preparation of the fuel for dissolution including the chop-leach dissolver can be serviced remotely. At NFS, a much more complicated procedure was followed. Operations in which the exposure would be incurred are estimated as: production (45 percent), maintenance ( 27 percent), health physics (16 percent) and analytical (12 percent). This exposure, servicing 50 large reactors, would be equivalent to roughly: $300 /(50,000 \mathrm{MWe} \times 0.7)=$ 0.008 person-rem/MWe-yr. In evaluating the occupational experience at reprocessing plants $\mathrm{AEC}$ came to the conclusion that 0.045 personrem/MWe-yr was a reasonable estimate for a modern generic Fuel Reprocessing Plant (FRP) (WASH, 1974). The NRC generic estimate in GESMO is 0.025 person-rem/MWe-yr for a modern plant (NUREG-0002, 1976). UNSCEAR, citing operating practice in the U.K. (unpublished) suggests that the experience is 2 personrem/MWe-yr, 2 orders of magnitude higher (UNSCEAR, 1972; Beninson, 1977b).

D4b. Environmental: Population Exposure from Effluents from a $1,500 \mathrm{Mg} / \mathrm{y}$ Reprocessing Plant

The effluents of the fuel reprocessing plants account for most of the public population dose in the fuel cycle, and the most important nuclides are ${ }^{14} \mathrm{C},{ }^{85} \mathrm{Kr},{ }^{129} 1$, and ${ }^{3} \mathrm{H}$ (half lives $=5,700,10,1.6 \times 10^{7}$ and $12 \mathrm{y}$, respectively). Typically, all or most of the ${ }^{3} \mathrm{H},{ }^{85} \mathrm{Kr}$ and some of the ${ }^{129} 1$ and ${ }^{14} \mathrm{C}$ produced during reactor operation is released from the fuel at the reprocessing plant during shearing and dissolution. That which is released as gaseous effluent is quickly dispersed. ${ }^{85} \mathrm{Kr}$ mixes rapidly with the troposphere in its hemisphere of release and more gradually with the global atmosphere. ${ }^{3} \mathrm{H}$ and ${ }^{129} \mathrm{I}$ transfer to the surface waters and eventually to the deep oceans. For ${ }^{14} \mathrm{C}$, neither the production rate in the fuel cycle nor the fraction released is well known. Magno, et al. estimate that $30 \mathrm{Ci}$ are produced per 1,000 MWe-yr and most of this is released at the fuel reprocessing step (Magno, 1974). It may be both possible and relatively easy to prevent release of most of the ${ }^{1{ }^{4}} \mathrm{C}$, but for purposes of this analysis it will be assumed that it is all released. A detailed description of the dispersal and resultant doses following releases of these various effluents is given in Table 5-C.1 and Appendix IV.

In this section we calculate the dose rate increment which would result from the release of various effluents from a reprocessing plant. We assume as a reference reprocessing plant, a level of operation of 
FIG. 5D-1. Global whole body dose rate increment due to $T$ years of operation of a reference $(1500 \mathrm{Mg} / \mathrm{y})$ LWR reprocessing plant with no engineered retention of effluents.

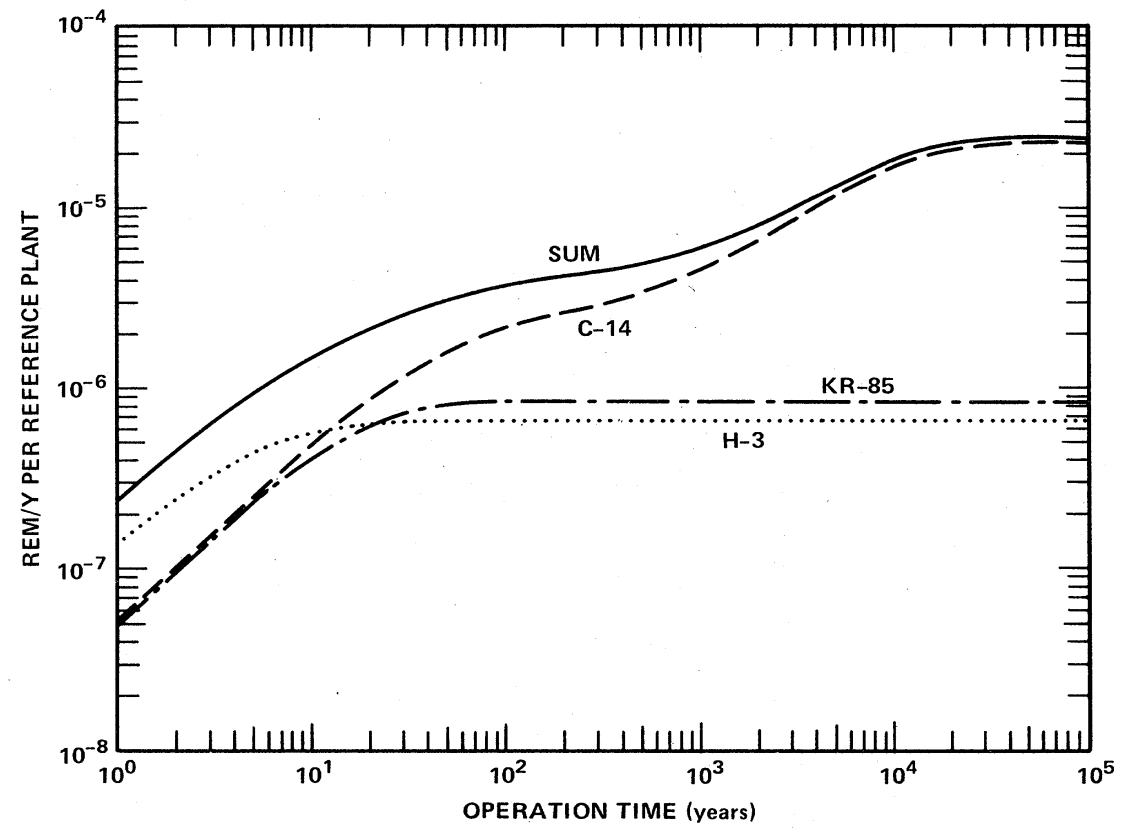

$1,500 \mathrm{Mg} / \mathrm{y}$. We assume that engineered effluent systems normally present for iodine retention are operated to prevent the release of ${ }^{129} \mathrm{I}$. Although technologies for the retention of ${ }^{3} \mathrm{H},{ }^{85} \mathrm{Kr}$, and ${ }^{14} \mathrm{C}$ exist in varying states of technological readiness, we assume that they are not used. In all cases, sufficient "cooling" of the fuel is assumed so that no other gaseous effluents need to be considered. The production and release of ${ }^{85} \mathrm{Kr}$ from the reference reprocessing plant is taken as $9.8 \mathrm{x}$ $10^{6} \mathrm{Ci} / \mathrm{y}$. We assume that $\mathrm{N}$ impurity at $25 \mathrm{ppm}$ is present in the irradiated fuel. All ${ }^{14} \mathrm{C}$ produced is assumed to escape as $\mathrm{CO}_{2}$. Under these conditions the release would be $\sim 660 \mathrm{Ci} / \mathrm{y}$. This estimate is in the range reported by several authors for ${ }^{14} \mathrm{C}$ in LWR fuel (Chapter IV). It is assumed that the plant releases no liquid effluent and that 75 percent of ${ }^{3} \mathrm{H}$ generated is released as gaseous effluent at a level of $8 \times 10^{5} \mathrm{Ci} / \mathrm{y}$ with the remainder being occluded in various metallic wastes.

The $1,500 \mathrm{Mg}$ of LWR fuel contains $62 \mathrm{Ci}{ }^{129} \mathrm{I}$. The amount released to the air depends on the efficiency of the engineered retention systems. Typical values for retention factors vary from a factor of 10 to 1,000 . We assume arbitrarily that the ${ }^{129} \mathrm{I}$ release will be $1 \mathrm{Ci} / \mathrm{y}$.

The problem of estimating the cumulative global public dose to all future generations, resulting from current or planned release of radioactivity is difficult (generally insoluble) and worst of all, of uncertain significance. The difficulties include incomplete knowledge of the population sizes and living habits far into the future. The uncertainty is in assigning significance to the numeric value of an integral obtained by integrating a nearly zero rate over an infinite interval. This problem has generally been dealt with by analyzing the dose commitment for a finite time into the future. Integration times chosen have been 50 years (NUREG-0002, 1976), 100 years (USEPA, 1977), and 500 years (Beninson, 1977b; Pochin, 1976). The rationale has been best expressed by Beninson, et al., who suggest that 500 years is the length of time a nuclear fission economy might exist. We have chosen to avoid both the difficulties and uncertainties by calculating the Dose Rate Increment for operation of a reference reprocessing plant for an arbirtrary but finite time $\mathrm{T}$ at constant effluent release rates corresponding to our predictions for production and release. The finite integration times chosen by others correspond to our operating times. Figure 5.D-1 shows the whole-body Dose Rate Increment after $T$ years of continued operation at constant release. In addition, the results are given in Table 5.D-4 for $\mathrm{T}=1,10,50,100$, 500 , and $\infty$ years.

Note that ${ }^{14} \mathrm{C}$ delivers the largest part of the dose commitment and that the ratio of ${ }^{14} \mathrm{C}$ dose rate to that from ${ }^{85} \mathrm{Kr}$ and ${ }^{3} \mathrm{H}$ increases steadily after several years. There is a reasonably flat portion of the curve between 50 and 500 years, indicating little buildup of dose rate (about $50 \%$ increase) in this time period. Hence, if a nuclear economy were to last 50 or 500 years the dose rate increment would not differ greatly.

After 50 years of operation of a single Reference Fuel Reprocessing Plant the global whole-body dose rate would be $3 \times 10^{-3}$ mrem/y, about half from ${ }^{14} \mathrm{C}$ and the remainder disiributed between ${ }^{3} \mathrm{H}$, and ${ }^{85} \mathrm{Kr}^{7}$ Operation at the same level for 500 years (i.e., operation of $\mathrm{N}$ reference plants in tandem, each for $500 / \mathrm{N}$ years) would increase the dose rate by only about 50 percent. The operation of 100 reference plants would increase the global whole-body dose rate by $0.3 \mathrm{mrem} / \mathrm{yr}$ after 50 years of operation $\left(5 / 8\right.$ is due to $\left.{ }^{14} \mathrm{C}\right)$ and to $0.4 \mathrm{mrem} / \mathrm{y}$ after 500 years $\left(3 / 4\right.$ due to $\left.{ }^{14} \mathrm{C}\right)$.

We note that the important gaseous effluents can be classified by their characteristic times. The nuclides, ${ }^{3} \mathrm{H},{ }^{85} \mathrm{Kr}$, and ${ }^{14} \mathrm{C}$ in the tropospheric pool have relatively short $(10$ to $30 \mathrm{y})$ characteristic times, while ${ }^{14} \mathrm{C}$ in the global pool and ${ }^{129} \mathrm{I}$ have very long characteristic times $\left(5 \times 10^{3}-16 \times 10^{6} \mathrm{y}\right)$. Thus, those in the first group will reach an asymptotic dose rate contribution in several decades at a level depending only on the number of reprocessing plants. If control is then exercised, the contributions will decay according to their characteristic time periods.

The dose rates for the second group will depend on the number of plants and the time over which they have operated. When control is exercised, the incremental dose rates will remain nearly constant for very long periods of time. at the levels existing at the time control was exercised. (See Figure 5.C-2.)

The EPA has issued general environmental standards (USEPA, 1977) which will limit, after 1983, the allowable emission of ${ }^{85} \mathrm{Kr}$ to $50,000 \mathrm{Ci} / \mathrm{GWe}$-year. The expected emission (Chapter IV) from LWR reprocessing per $\mathrm{GWe}-\mathrm{yr}$ is $372,000 \mathrm{Ci}$. If control facilities operate to limit the discharge to the standard, then the ${ }^{85} \mathrm{Kr}$ dose rate will be reduced to about $1 / 7$ that without controls. At 50 and 500 years the whole body dose rates would be reduced by $22 \%$ and $17 \%$ respectively. However the skin dose will be reduced to roughly $1 / 7$ that without controls. The corresponding limit for ${ }^{129} \mathrm{l}$ will be $5 \mathrm{mCi} / \mathrm{GWe}-\mathrm{yr}$ corresponding to about $0.2 \mathrm{Ci}$ for a $1500 \mathrm{Mg} / \mathrm{yr} \mathrm{FRP}$. Under those conditions the ${ }^{129} \mathrm{I}$ values in table $5 . \mathrm{D}-7$ would be reduced by a factor of $\sim 50$. 
TABLE 5D-7. Per capita global dose rate (rem/y) after $T$ years of operation from a continuous release from a reference $1500 \mathrm{Mg}$ LWR fuel-reprocessing plant with no engineered retention of ${ }^{85} \mathrm{Kr},{ }^{14} \mathrm{C},{ }^{3} \mathrm{H}$.

\begin{tabular}{|c|c|c|c|c|c|c|c|}
\hline$T(y)$ & ${ }^{85} \mathrm{Kr}$ & ${ }^{14} \mathrm{C}$ & ${ }^{3} \mathrm{H}^{\mathrm{a}}$ & SUM $^{b}$ & ${ }^{3} \mathrm{H}^{\mathrm{c}}$ & SUM $^{d}$ & $\begin{array}{c}{ }^{129} \mathrm{I} \\
\text { (Thyroid) }\end{array}$ \\
\hline 1 & $0.5(-7)$ & 6. $(-9)$ & $1.3(-7)$ & $1.8(-7)$ & $0.1(-7)$ & $0.6(-7)$ & $0.3(-8)$ \\
\hline 10 & 4. $(-7)$ & $5 \cdot(-7)$ & $6 \cdot(-7)$ & $1.5(-6)$ & $0.6(-7)$. & $1.0(-6)$ & 2. $(-8)$ \\
\hline 50 & 8. $(-7)$ & $1.5(-6)$ & 8. $(-7)$ & $3.1(-6)$ & $1.2(-7)$ & $2.4(-6)$ & $7 \cdot(-8)$ \\
\hline 100 & 9. $(-7)$ & $2 \cdot(-6)$ & 8. $(-7)$ & $3.7(-6)$ & $1.3(-7)$ & $2.8(-6)$ & $8 \cdot(-8)$ \\
\hline 500 & $9 \cdot(-7)$ & $3 \cdot(-6)$ & 8. $(-7)$ & $4.7(-6)$ & $1 \cdot 3(-7)$ & 4. $(-6)$ & $10 \cdot(-8)$ \\
\hline$\infty$ & $9 \cdot(-7)$ & $2.3(-5)$ & 8. $(-7)$ & $2.5(-5)$ & $1.3(-7)$ & 2. $4(-5)$ & $1.2(-4)$ \\
\hline
\end{tabular}

a indicates atmospheric release of ${ }^{3} \mathrm{H}$

$\mathrm{b}$ refers to the sum of the dose rates from the individual nuclides if ${ }^{3} \mathrm{H}$ is released to the atmosphere

c indicates ocean release of ${ }^{3} \mathrm{H}$.

d refers to the sum of the dose rates from the individual nuclides if ${ }^{3} \mathrm{H}$ is released to the marine environment.

D4c. Consequences of Accidents Involving Gaseous Effluent Release at Fuel Reprocessing Plants

The calculations presented in this section assume nearly complete release of all gaseous effluents. Accordingly they correspond on a global basis to maximum accident conditions.

If control were exercised over effluent release, the major accident would then be instantaneous release of the sequestered radionuclides. The global public environmental dose rate would approach the level it would have reached had no control been exercised over the period during which the nuclides were collected. On the other hand, the local dose rate in the vicinity of the storage facility would be very much higher.

Accordingly we recommend that if release of effluent radionuclides is to be controlled, appropriate storage technologies should be developed and attention directed to minimize the local consequences of accidental releases.

\section{D5. Mixed-Oxide Fabrication and Nitrate-to-Oxide Conversion Plants for Plutonium}

Following its separation as plutonium nitrate, the plutonium is converted into oxide form. Subsequently, mixed plutonium and uranium oxide fuel is fabricated for use in appropriate power reactors. The plutonium is potentially toxic because of its alpha activity and biological behavior. In addition neutrons are emitted both as a result of induced $(\alpha, n)$ reactions and from spontaneous fission.

Background and experience with plutonium appropriate to the fabrication of mixed-oxide fuels for plutonium recycle in light-water reactors is discussed in Chapter II of GESMO (NUREG, 1976). There is broad interest in this subject around the world, and there are and have been a number of pilot facilities around the world that are able to produce limited amounts of mixed-oxide fuel, including facilities in the United States, Belgium, Canada, Germany, India, Japan, the Netherlands, the United Kingdom, and Sweden. In the United States, Westinghouse at Cheswick, Pennsylvania, has made mixed-oxide fuels for reactors operating in Europe while Kerr-McGee and Numec have made mixed-oxide fuel for developmental work on the U.S. breeder reactor. There are, however, no large-scale commercial level fuel fabrication production plants.

Westinghouse currently has an application pending for a construction permit for a commercial level plant at Anderson, South Carolina. The design of this plant is different from past designs in that much of the work would be done remotely; gloveboxes, which were so widely used for handling military grade plutonium, would not be a major part of the environmental control. Indeed the high specific activity of the plutonium produced in light-water reactors as well as the neutron background engendered by the high alpha activity makes the questions of radiological protection from the point of view of occupational exposure more difficult. We note that fuel fabrication appears to be a point in the fuel cycle for which neutron exposures may be important. Accordingly the Westinghouse design that incoporates both neutron shielding and remote operation appears to be a logical and reasonable design step for the fabrication of mixed-oxide fuel.

Future fuel fabrication plants should minimize contact and opportunities for inhalation and should make maximum use of remote handling to avoid neutron exposures (IAEA, 1969; ICRP, 1969). The design of the Westinghouse plant would seem to take the latter into consideration and perhaps the former. Most projections of collective dose as a result of occupational exposure at mixed-oxide fuel fabrication plants is, of necessity, hypothetical because no industrialscale plant has operated.

The question of environmental releases is also important. Past experience with $\mathrm{Pu}$ fabricated in the military program shows that releases to the environnment from a routine operation can be held to a very low level. Aerosol release factors at least as low as $10^{-7}$ to $10^{-9}$ have been attained (Comar, 1976). Since well developed technologies exist, there is little reason to doubt that such containment is possible. Special efforts to preclude accidental releases will be necessary lest they become the major source of release. The fabrication of mixedoxide fuel for the breeder or for recycle plutonium involves handling plutonium with roughly similar radiological hazards. Both are high burnup plutonium, with high specific alpha activity and the ability to produce neutrons by $(\alpha, n)$ reactions with light elements. In addition external gamma exposure may result from the ${ }^{241} \mathrm{Am}$ daughter of ${ }^{241} \mathrm{Pu}$, which grows in with the decay of ${ }^{241} \mathrm{Pu}$.

We note that the fabrication of mixed-oxide fuel in particular and operation of the nuclear fuel cycle in general would be strongly influenced by hypotheses reasserted a few years ago concerning the possible effects of small "hot particles" of plutonium as an inhalation hazard. If it were true that such hot particles would be orders of magnitude more carcinogenic than a similar amount of plutonium uniformly distributed over the lung, the result could have an important effect on the whole fuel cycle. We have reviewed the available data and calculations concerning such effects and describe the details in Appendix IV. We find no substantial evidence that supports the hot particle hypothesis and, consequently, support the recent reexaminations by NCRP and others that reject proposals for a major reduction in plutonium inhalation concentration limits (NCRP46, 1975; NAS/NRC, 1974; Bair, 1977) based on the hypothesis.

GESMO describes the fabrication of mixed-oxide fuel to be expected in the year 2000. NRC suggests that as many as eight mixedoxide fabrication facilities might be required, each with a capacity of 360 MGHM/yr. By comparison the five pilot scale plants now licensed to fabricate mixed-oxide fuel have a total estimated capacity of 50 to $70 \mathrm{MGHM} / \mathrm{yr}$. The proposed Westinghouse plant to manufacture mixed-oxide fuel rods would have a nominal capacity of $200 \mathrm{MGHM} / \mathrm{yr}$ with eventual expansion to 400 . Because these plants would be large, the incremental costs associated with the use of the 
most modern engineering and design features to optimize containment during normal operation can be minimized although the capital requirements for safety, safeguards, and protection of the environment will be substantial for each plant. For purposes of comparison it is noted that possibly five model fuel reprocessing plants would be requried to support the input to eight such fuel fabrication plants. The isotopic composition of plutonium in the model reactor (recycle) would var: somewhat as to specific activity, primarily because the ${ }^{238} \mathrm{Pu}$ content would vary from 1.9 to 4.9 percent, depending on the number of recycles. The total alpha specific activity of the plutonium would range between a specific activity of 0.5 and $1 \mathrm{Ci} / \mathrm{gm} ; 1 \mathrm{Ci} / \mathrm{gm}$ is about 16 times higher than ${ }^{239} \mathrm{Pu}$ handled in the weapons program and roughly 16 times lower than ${ }^{38} \mathrm{Pu}$ handled in the space nuclear program. Chapter IV, Section G of GESMO describes the mixedoxide fuel fabrication and its environmental impact, with respect to light water reactors. NRC concludes that the releases to the environment from 1981 to 2000 would be less than $1 \mathrm{Ci}$ total of plutonium alpha activity, with 83 percent being released to the atmosphere, and the remainder to liquid effluents.

NRC has estimated that the largest dose that might be received by a resident $500 \mathrm{~m}$ downwind from such a mixed-oxide fabrication plant would be on the order of $170 \mathrm{mrem}$ to bone, which is equivalent to two years of average natural background exposure. NRC suggests that individual occupational doses may be about 1.2 rem from external radiation and 0.2 rem from internal radiation, implying that the neutron and gamma exposures are more important than internal exposure to plutonium. This is an interesting prediction because the handling of plutonium inevitably is associated with the opportunity for internal exposure. Experience with the operation of these plants will be required to demonstrate conclusively whether neutron and gamma exposures are more important than internal exposure. For planned occupational exposure the control of internal exposure is easier than the control of external exposure. With respect to accidental exposures this may not be the case, and the ratio of internal to external exposure in occupationally exposed personnel will likely depend on the accidental exposure experience in such facilities. We note that the NRC analysis of the total body collective dose commitment shows that the fabrication of MOX fuel contributes less than 0.005 person-rem/MWe-yr. Most of the exposure is occupational, and accordingly, if the NRC analysis is correct, MOX fuel fabrication would not appear to be a dominant contributor to the total fuel cycle exposure.

\section{Nitrate to Oxide Conversion Plant}

This is not explicitly analyzed in the GESMO statement. It would be, of necessity, located at the fuel reprocessing plant, since present NRC regulations prohibit transportation of the plutonium nitrate solution. There is no existing commercial plant and therefore no experience yet with large-scale commercial operations. When in operation substantial potential exposure to plutonium would exist; careful attention must be paid to health and safety in the design of such a facility (IAEA, 1969; IAEA, 1974).

\section{D6. High Level Waste}

High level waste (HLW) is defined as the waste solution from the first dissolution of spent fuel rods in a reprocessing plant (Fry, 1976; CFR 1050-F)); in case a "throw-away" fuel cycle was introduced, spent fuel would be considered as HLW. Present regulations require that HLW be converted to solid form, shipped to and emplaced in a geologic repository.

The intense radiation from HLW requires remote operation for all operations involved in its handling. We believe that sufficient experience exists for such handling to allow the operations of solidificaiton, transportation and emplacement to be carried out with occupational exposure held within statuatory dose limits. Since the number of individuals involved would be small, therefore, the collective occupational dose from high level-waste management is expected to be small compared to that incurred in the rest of the fuel cycle.

Because the waste is in solid form, there is no mechanism for widespread transport of the entrapped radionuclides (Bradshaw, 1970). The more local environmental aspects of HLW storage are treated in
Chapter VII. Hazards associated with these operations ultimately should be evaluated in terms of incremental dose rate to humans.

\section{D7. Low-Level and Transuranic Waste}

There have been several practices employed for the disposal of low level wastes including the burial of both packaged and unpackaged waste in surface trenches and the percolation of liquid wastes into the earth. Definitions of "low-level" have undergone dramatic changes in the last five years and still are not entirely unambiguous. Until 1974, broad categories of waste could be disposed of as "low level" waste. At that time a rule was proposed to bar all transuranic contaminated material, (TRU), for this treatment. Instead, it has implied that all material with alpha activity greater than $10 \mathrm{nCi} / \mathrm{g}$ be buried in a geologic repository as for HLW (USAEC, 1974). For our purposes, we will define all waste not already defined as HLW to be low level waste. Within our class, all material with alpha activity greater than $10 \mathrm{nCi} / \mathrm{g}$ will be classified as TRU waste.

Table 5.D-5 gives the activities in three classes in the "non-highlevel" wastes from each fuel cycle step. These are expressed as $\mathrm{Ci}$ generated per GWe-yr of electricity generated. Important assumptions include: fuel burnup of $25 \mathrm{GWe}$-days per MGHM, and a 0.5 percent $\mathrm{Pu}$ loss in mixed oxide fuel fabrication. Entries missing in the table indicate a contribution at least two orders of magnitude less than those that do appear in the same row. The key observations to be made from the table are that reactors and reprocessing plants contribute comparable waste activities of fission and activation products. However, these levels are nearly three orders of magnitude less than the activity of similar nuclides in the high-level reprocessing waste stream. For plutonium waste, the mixed oxide fuel fabrication plant is a major contributor. Even with optimistic plutonium containment estimates, the quantity of plutonium in the fuel fabrication waste stream would be roughly equal in magnitude to that in high level waste of the fuel reprocessing plant.

The policy implications are clear. If containment of actinides in HI.W for many thousands of years after emplacement is important, it is equally important to assure the same degree of containment for plutonium-bearing fuel fabrication wastes. The ERDA plan for HLW containment (ERDA, 1976) does not associate specific barriers (i.e., waste form, packaging, geologic site) with control of specific isotopes or time periods. The analysis presented in Chatper VII of this report suggests that at present only the geologic barrier can be relied upon as a prime barrier for the very long time period we are considering. Clearly, plans for actinide containment must be applied to fuel fabrication wastes as well as to HLW.

Another policy implication concerns waste volumes. Needed geologic storage capacity cannot be calculated from that of the classical "high-level" waste form. The much larger volume waste streams of other forms must be included. Similarly, though the treatment and packaging of the non-high-level waste streams is not yet defined, design plans must include the capability for treatment and packaging.

A perspective on waste treatment plans in general can be gained by considering torical treatment of the "non-high-level" material. Waste coni. ing plutonium equivalent to the "high-level" waste stream of 20 GWe-yr of nuclear electricity production with reprocessing has been intentionally percolated into shallow ground disposal sites on the Hanford reservation. Sixteen GWe-yr equivalent plutonium waste has been buried in groundwater inundated surface trenches at Misey flats and at Oak Ridge. While such practices have not been demonstrably detrimental to public health, they would not be permitted under present regulations and so inferences for the future operation of waste disposal repositories should not be derived from the examples just given. We believe that attention to nuclear power waste control should not be focussed on HLW alone.

\section{D8. Qualitative Treatment for Advanced Fuel Cycles}

All fission cycles produce the same fission products although the absolute yields of particular nuclides depend somewhat on the particular fissioning nuclear species. On the other hand, the production of actinides is quite different for varying fissioning species. We will show in Section V-E that the average background dose rate to the public and the whole-body occupational dose 
TABLE 5D-8. (ERDA, 1976). Non-high-level process waste activity. ${ }^{\text {a }}$

\begin{tabular}{|c|c|c|c|c|c|c|}
\hline & Mine & Mi11 & Reactor & $\begin{array}{l}\text { Spent Fuel } \\
\text { Storage }\end{array}$ & Reprocess ${ }^{d}$ & $\begin{array}{l}\text { Mox } \\
\text { Fabrication }\end{array}$ \\
\hline $\begin{array}{l}\text { Fission and } \\
\text { Activation } \\
\text { Products }\end{array}$ & -- & $-\sim$ & $5.5 \times 10^{3} \mathrm{~b}$ & 8.8 & $2.6 \times 10^{4}$ & -- \\
\hline Plutonium & -- & -- & -- & -- & $2.7 \times 10^{3}$ & $\begin{array}{c}5.6 \times 10^{4} \\
\left(71 \mathrm{~m}^{3} \text { volume }\right)\end{array}$ \\
\hline Total Actinides ${ }^{c}$ & -- & 70 & -- & -- & -- & -- \\
\hline
\end{tabular}

commitment for the nuclear industry do not change substantially if the recycling of plutonium is begun in present reactors. It should be noted that the reduced ore requirement then resulting would lead to a reduction in mining with a concomitant reduction in collective lung dose for uranium miners. In the present section we will qualitatively examine the extent to which changes in radiation doses might be expected if any of the advanced fuel cycles discussed in Chapter VIII were implemented.

\section{DSa. Introduction of Thorium Cycles}

To the extent that the introduction of any advanced cycle reduces the need for further mining and milling of uranium, radiation exposures associated with those practices will be reduced. The longterm tailings problem is much less for thorium milling than for uranium milling since the longest lived ${ }^{232} \mathrm{Th}$ decay product, ${ }^{228} \mathrm{Ra}$ has a half life of $5.7 \mathrm{yr}$.

Use of thorium in a reactor leads to several changes in the nature and amounts of resulting radionuclides. The magnitude of release of gaseous effluents in reprocessing is changed (Appendix IV) with about double the ${ }^{85} \mathrm{Kr}$ production and half the ${ }^{3} \mathrm{H}$ production. If ${ }^{233} \mathrm{U}$ were to be recycled even higher ${ }^{85} \mathrm{Kr}$ production might be expected (Appendix IV). As a result, the skin dose rate could be higher for a thorium cycle industry than for a U-Pu industry. Substantial quantities of ${ }^{232} \mathrm{U}$ and ${ }^{234} \mathrm{U}$ are formed in the fuel. $1.9 \mathrm{yr}{ }^{228} \mathrm{Th}$, a decay product of ${ }^{232} \mathrm{U}$ quickly builds up an intense, high energy gamma source. Thus there would be an incentive to minimize the time between reprocessing and fuel refabrication to minimize occupational exposure at the fabricating plant. In addition special handling procedure would have to be instituted during transport and at reactors to minimize exposure during fresh core loading. The consequences of the accidental release of high specific activity uranium isotopes is likely to be much more severe than for plutonium because of its high uptake at the mammalian gut (Durbin, 1973). It should be noted that the RCG for high specific activity uranium isotopes such as ${ }^{232} U$ and ${ }^{233} U$ are likely to be dec eased by about an order of magnitude in the near future.

Although the production of heavy actinides is insignficicant in a thorium fuel cycle, the presence of large amounts of ${ }^{233} \mathrm{U}$ and ${ }^{234} \mathrm{U}$ assures that at secular equilibrium there will be amounts of radium in the HLW quite comparable to those from a U-Pu cycle (Chapter VIIFigure 7B2). Accordingly, any long term HLW problem associated with the U-Pu cycle would not be alleviated by the use of a thorium cycle.

\section{D8b. Heavy Water and High Temperature Gas-Cooled Reactors}

Neutron activation of ${ }^{2} \mathrm{H}$ in the moderator of HWR leads to large ${ }^{3} \mathrm{H}$ inventories. This could lead to a somewhat higher collective occupational dose at HWR reactors and may modify the dose commitment ascribeable to accidents. The large amounts of carbon present in an HTGR core results in an increase of ${ }^{14} \mathrm{C}$ in the reprocessing streams by about an order of magnitude relative to that for water reactors. In this case the form $\left(\mathrm{CO}_{2}\right)$ and site of major release (chopping) of the nuclide are known so that effluent control should be feasible.

\section{D8c. Liquid Metal Cooled Fast Breeder Reactor}

The change in gaseous effluent production from I.MFBR fuel will depend on the mix of thorium and uranium in the fertile material. This production should be within a factor of 2 of those for the LWR cases. The expected increase in ${ }^{238} \mathrm{Pu}$ and ${ }^{242} \mathrm{Cm}$ does lead to eventual higher concentrations of ${ }^{226} \mathrm{Ra}$ in $\mathrm{HLW} /$ unit energy generation than would be true for LWR fuel. The use of sodium coolant introduces a new source of potential occupational exposure at the reactor resulting from $15 \mathrm{hr}{ }^{24} \mathrm{Na}$

\section{D8d. Summary}

In general, the environmental and occupational exposures attributable to the ${ }^{233} \mathrm{U}$-thorium fuel cycle are less well investigated than those for the Pu-U cycles. The biological effects of the external radiations are comparable. There may be differences in the amounts and likelihood of exposure to the internal emitters, particularly the actinides and alkaline earths (i.e., ${ }^{232} \mathrm{U},{ }^{233} \mathrm{U},{ }^{226} \mathrm{Ra},{ }^{210} \mathrm{~Pb},{ }^{228} \mathrm{Ra}$, etc.). Accordingly additional research in animals and humans may be required to adequately assess the risks in the U-Th cycles.

\section{E. Summary of Occupational and Public Exposure From the Fuel Cycle}

\section{E1. Introduction}

Occupational and public exposures from a variety of nuclear fuel cycles have been analyzed by several organizations and individuals. Most important among these analyses are those conducted by UNSCEAR (UNSCEAR, 1972, presently being updated for a 1977 version; also see the study by Beninson, et al., 1977), the Nuclear Regulatory Commission (NUREG-0002, 1976), and Pochin (Pochin, 1976). The summary that follows is based partly on these and partly on our own analysis. The types of exposure that may occur either to the public or to occupationally exposed groups are identified in Chapter III, Section C of this report, which divides the radiation into high LET (alpha and neutron) and low LET (beta and gamma). The public exposures are no different in kind, quality, or dose rate than those from natural background.

Tables 5.E-1 and 5.E-2 list estimates of collective whole-body occupational and population dose commitments from fuel cycle facilities expressed in terms of the number of person-rem/MWe-yr of power generated by nuclear means. Important exposures to other organs are discussed in the sections related to specific facilities in the fuel cycle or to the specific nuclide. We particularly note that contributions to dose commitment from mines occur only while the mines operate, with magnitude depending on the level of operation. Mill tailings, on the other hand, provide a release that depends on the total amount of processed ore to that point in time and may continue at a constant rate from that time on. 
TABLE 5E-1. World population dose commitment from fuel cycle facilities person-rem/MWe$\mathrm{yr}^{\mathrm{a}}$ (whole body or gonadal).

\begin{tabular}{|c|c|c|c|}
\hline & $\begin{array}{c}\text { Pochin } \\
(500 \text { years ) } \\
(\text { Pochin, 1976) }\end{array}$ & \multirow{2}{*}{ 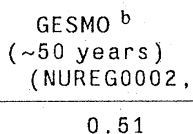 } & \multirow{2}{*}{$\begin{array}{c}\text { APS Study } \\
(500 \text { years }) \\
1976)\end{array}$} \\
\hline Mines & & & \\
\hline Mi11s & & 0.10 & $<0.06$ \\
\hline $\begin{array}{l}\text { Fuel fab., enrichment } \\
\text { conversion }\end{array}$ & & 0.01 & -- \\
\hline Reactors & 0.1 & 0.077 & 0.1 \\
\hline $\begin{array}{l}\text { Reprocessing Plants } \\
\text { (U or Pu recycle }\end{array}$ & 1.35 & 0.35 & $0.4^{\mathrm{d}}$ \\
\hline & & $2 \times 10^{-6}$ & \\
\hline Waste management & & & -- \\
\hline Transportation & 0.004 & 0.0002 & - - \\
\hline $\begin{array}{l}\text { Reactor Accidents } \\
\text { Other }\end{array}$ & 0.05 & & $\sim 0.1{ }^{\mathrm{e}}$ \\
\hline Total & 1.5 & 1.05 & $\sim 0.6$ \\
\hline
\end{tabular}

a This is numerically equal to the incremental per capita annual dose rate in mrem for a world installed capacity of $1 \mathrm{~kW}$ per capita, after many years of operation. For column 3 this time is taken as 500 years.

b $\mathrm{Pu}+\mathrm{U}$ recycle option.

c The contribution from mines continues only during period of their operation.

d This estimate is made assuming no engineered retention of gaseous fission products.

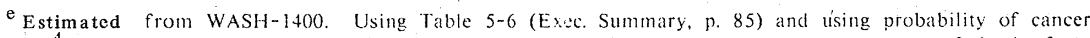
death $=10^{-4}$ per person-rem (Exec. Summary, pp. 85, 74) we deduce that the contral average rate of deaths from long term effects is approximatley 0.02 per reference reactor year and the equivalent Population Does Commitmen is 0.2 person-rem/MWe-yr. This number must be regarded as quite uncertain, and we quote the order of magnitude for reference purposes only.

\section{E2. Public Population Doses}

Table 5.E-1 lists the public population dose commitment from the operation of the fuel cycle, as projected by Pochin, by NRC (GESMO Report) and by this APS study group. In preceding sections we have signified that we prefer to interpret the dose commitment to be the increase in annual dose rate if the practices of release assumed in the estimates were continued for a very long time. We note that for a world in which there is a generated demand of $C \mathrm{kwe} / \mathrm{capita}$ and a population dose commitment $D$ person-rem/MWe-yr, the asymptotic increase in global public dose rate would be $C \cdot D$ mrem/yr. Aiso shown in Table $5 \mathrm{E}-1$, for reference purposes, is an estimate of the equivalent long-term average contribution inferred from estimates of latent cancer fatalities from reactor accidents, as reported in WASH1400. The reader should regard this estimate as quite uncertain.

The entries in the table suggest quite different sources for the contributions to the collective dose commitment. The analysis by
Pochin states that effluents from the reprocessing plant are expected to make the dominant contribution. On the other hand, the NRC analysis attributes most of the whole body dose to radon emissions in mining. The present analysis concurs with the assertion by Pochin that reprocessing effluents are responsible for the most important portion of the population dose. ${ }^{14} \mathrm{C}$ and ${ }^{3} \mathrm{H}$ produce a relative uniform whole body dose with no specific organ receiving an absorbed dose significantly different from the average. For ${ }^{129}$ I, the thyroid receives the bulk of the dose while the whole-body component is negligible. For ${ }^{85} \mathrm{Kr}$, the largest dose is delivered to the skin. Although the skin is considered more tolerant to radiation than many other organs, the skin dose should not be ignored.

\section{E2a. Discussion of the GESMO Report}

The population doses estimated by NRC for mining and milling are surprising and, at first glance, seem to be at a variance with our knowledge about the contribution of radon and its decay products to

TABLE 5E-2. Collective occupational dose from fuel cycle operations person-rem/MWe-yr (whole body or gonadal).

\begin{tabular}{|c|c|c|c|}
\hline & $\begin{array}{c}\text { Pochin } \\
\text { (Pochin, 1976) }\end{array}$ & $\begin{array}{l}\text { Gesmo b } \\
\text { (NUREG0002, 1976) }\end{array}$ & APS Study \\
\hline Mines & 0.1 & 0.26 & 0.1 \\
\hline Mills & $<0.1$ & 0.12 & 0.1 \\
\hline Fue1 Fab. \& enrichment & -- & 0.01 & $--a$ \\
\hline Reactors & 2.0 & $0.56^{\mathrm{b}}$ & $1.1^{\mathrm{b}}$ \\
\hline Reprocessing Plants & 2.0 & $0.025^{\mathrm{c}}$ & 0.008 to 0.6 \\
\hline Other: & & & \\
\hline Waste management & .03 & 0.002 & -- \\
\hline Transportation & .005 & 0.0004 & -- \\
\hline Total & 4.2 & 0.95 & 1 to 2 \\
\hline
\end{tabular}

a Entries marked -- were estimated to give negligible contribution.

b NRC and APS consider only reactors in the U.S.

c $U$ or $U+P u$ recycle. 
the whole body dose. The situation may be an artifact of the calculational models that were used. Mine effluents, solely ${ }^{222} \mathrm{Rn}$, are identified as having a normalized public whole body commitment of 0.62 person-rem/MWe-yr. This value is not only in excess of that due to other mine effluents but is over three times as large as the normalized public lung commitment from the same source. The combination appears inconsistent with behavior expected for radon in air (Table 5B-1).

We have discussed the NRC estimate with their staff and offer the following comments. The NRC estimate is for an industry that operates for 40 years with a dose calculated 50 years further into the future. Thus, their whole body dose must be due to the $210 \mathrm{Po}$ daughter of $22 y^{210} \mathrm{~Pb}$, the only long-lived daughter of ${ }^{222} \mathrm{Rn}$ which distributes in soft tissue. The result of this calculation, with such a non-standard choice of times, is particularly sensitive to the half life of the activity involved. We conclude that while the calculation quoted by NRC may be correct for their conditions, the results do not correspond to the usual meaning of dose commitment and so may be misunderstood. Further it is an example of distortions that occur because of the ambiguities possible in the usual dose commitment concept. The source term used by NRC also seems high. For a large uranium mine with a ventilation of $300,000 \mathrm{cfm}$, a concentration of ${ }^{222} \mathrm{Rn}$ of $300 \mathrm{pCi} / 1$ (the NRC limit) would emit about $3 \mathrm{Ci} / \mathrm{day}$, or roughly $10^{3} / \mathrm{Ci} / \mathrm{yr}$. Using the source term of $2.4 \times 10^{7} \mathrm{Ci}$ emitted from 1975 to $2000 \mathrm{implies}$ about $24,000 \mathrm{mine}-y e a r s$, or an average of about 1,000 mines operating per year for 24 years.

We will use the NRC source term to calculate the dose commitment from mines in a more standard manner. NRC assumes a release rate of $2.4 \times 10^{7} \mathrm{Ci} / \mathrm{yr}$ of ${ }^{2.22} \mathrm{Rn}$ while mining sufficient uranium to fuel reactors generating 4732 GWe-yr. We state in Section DIb of this chapter that the NRC assumed release rate would increase the concentration of ${ }^{222} \mathrm{Rn}$ in air about $1 \%$ of U.S. continental average. Such an increase will lead, with conservative assumptions, to an estimated asymptotic increase in the background due to radon and its daughters $(\sim 0.8 \mathrm{mrem} / \mathrm{yr})$ of about $1 \%$. Thus we estimate the normalized public whole body commitment from mines will reach an asymptotic value (i.e., after 100 years) of 0.08 person-rem/MWe-yr. The actual value will also depend on the duration of mine operation so that for 25 year operation the estimate would be 0.04 personrem/MWe-yr. For mill tailings the source term, corresponding to processing of ore for $4732 \mathrm{GWe}$-yr operation, is estimated to be $0.8 \%$ of background radon concentration in air in continental U.S. In a manner analogous to the preceeding analysis of mines, noting the mill tailing source emits indefinitely, this leads to an estimated normalized public whole body commitment of 0.06 person-rem/MWe-yr, as shown in Table 5E-1. As we have shown in the section on mill tailings, future tailings management practices could reduce the source term from tailings piles several orders of magnitude to small multiples of background Radon evolution per unit area.

\section{E2b. Summary}

We concluded that the operation of reprocessing plants would become a major source for the fuel cycle contribution to the public whole body dose commitment unless controls on effluents are eventually introduced. After 500 years of operation at constant level, $70 \%$ of the contribution comes from ${ }^{14} \mathrm{C}, 18 \%$ from ${ }^{85} \mathrm{Kr}$, and $12 \%$ from ${ }^{3} \mathrm{H}$. If operation ceased, the contributions from ${ }^{85} \mathrm{Kr}$ and ${ }^{3} \mathrm{H}$ would decay to negligible levels in several decades. The degree to which ${ }^{14} \mathrm{C}$ is released at reactors themselves is not yet well established. It would certainly appear possible to maintain the normalized public whole body dose commitment well below 1 person rem/y/MWe-yr by the identification of ${ }^{14} \mathrm{C}$ release sites and forms for both reprocessing plant and reactors, followed by the introduction of carbon capture technology with subsequent sequestration from the biosphere. Removal of ${ }^{85} \mathrm{Kr}$ then would be most important in reducing dose rate followed in turn by ${ }^{3} \mathrm{H}$ and ${ }^{129} \mathrm{I}$.

The infinite time population dose commitment due to ${ }^{129} \mathrm{I}$ appears very large. However, the associated rate is very small because the time scales of interest are much smaller than its half life of 16 million years. We focus our attention on a period of 500 years. Of the effluents released, the dose rate/MWe-y for ${ }^{129} I$ is less than that for the other isotopes. We note that, ${ }^{129} \mathrm{I}$ and, almost certainly, ${ }^{14} \mathrm{C}$ appear to be effluents whose control is possible and probably much less costly than those for ${ }^{85} \mathrm{Kr}$ or ${ }^{3} \mathrm{H}$. It is desirable that demonstration at industrial-scale of the capability for removal of these two nuclides from the effluents of reprocessing plants (and ${ }^{1+} \mathrm{C}$ from reactors) be undertaken. We note that attention must be given to the manner in which the controlled nuclides are to be secquestered, since this problem does not yet appear to have been adequately addressed. ${ }^{85} \mathrm{Kr}$ control is also desirable, especially if the ${ }^{2.3 .} \mathrm{U}-$ Thorium cycle were to be used in the fulure, because of the higher skin dose it delivers.

Except for the NRC analysis there appears to be only a rather small global or national public whole body commitment generated by the operation of mills or mines. The possibility of local and regional exposure does exist, particularly with respect to tailings from mills. We note once more that the hazard indices for high-level wastes decrease radically within the thousand or so years during which the bulk of fission product decay takes place. The magnitude of an appropriate hazard index must be very low because the HLW waste is buried underground with negligible access to the biosphere. Given the practice of retaining the mill tailings on or near the surface, accessible to human intrusion, it would appear that the potential for future population exposure exists. We urge a reexamination of procedures for handling mill tailings, to make their treatment more consistent with other waste management practices.

\section{E3. Collective Occupational Dose}

A summary of the collective occupational exposure per MWe-y is presented in Table 5.E-2. It includes estimates by Pochin (Pochin, 1976), NRC (GESMO) (NUREG-0002, 1976) and this APS study group. Both Pochin and NRC identify the reactor plant as a major contributor to the collective occupational dose from nuclear fuel cycle operations. There is a difference by a factor of 4 between their estimates for that contribution and an even greator factor between their estimates for the contribution of the reprocessing plant. The APS group also identifies the reactor plant as the major source of occupational exposure. We have examined NRC reports on operating experience and conclude that the expected value for collective occupational dose, based on current operating history, lies between the estimates of the NRC and Pochin. The APS estimates are based on U.S. experience alone.

Pochin identifies occupational exposure at reprocessing plants to be as important as that at reactors. Although a few reprocessing plants have operated for many years, there is scant information on their occupational exposure history and it is not easily accessible. The Pochin estimate is based on private communications and so cannot be checked. It appears likely that his estimate is based on experience at plants that have operated for a number of years. NRC on the other hand has used estimates based on a modern, next-generation plant such as the AGNS plant. We have examined the operating records from the NFS plant and the analyses by the designers of the AGNS plant. We have concluded that, in a modern plant such as the AGNS plant, it should be possible to control the collective occupational dose/MWe-y at reprocessing plants to a level below that typical of reactor plants. Thus the collective occupational dose estimates are 4 person-rem/ MWe-y (Pochin), 0.95 person-rem/ MWe-y (NRC) and 1-2 person-rem/ MWe-y (APS Study Group). We have not included the category of HLW management because there is no operating experience with the system. We note, however, for collective dose it should be possible to keep the waste management contribution low compared to that at the reactor and reprocessing plant.

All of these estimates of collective occupational dose are equal to or greater than the population dose commitment with or without reprocessing. Not only are the collective occupational doses greater, but in addition the individual exposures are much larger and take place at much higher rates. Estimates for the incidence of health effects based on linear, no-threshold extrapolation of high dose-high dose rate data may be more appropriate for occupation:ll exposure than for public environmental exposure. We identify occupational exposure as the most significant external exposure in the fuel cycle and the occupational group as that most affected.

We conclude that opportunity may exist for exposure reduction at the reprocessing plant, but the degree cannot be well ascertained at this moment because of lack of operating information at a industrial- 
FIG. $5 E-1$. Contributions to U.S. Average annual whole body dose rate $(\mathrm{mrem} / \mathrm{yr})$.

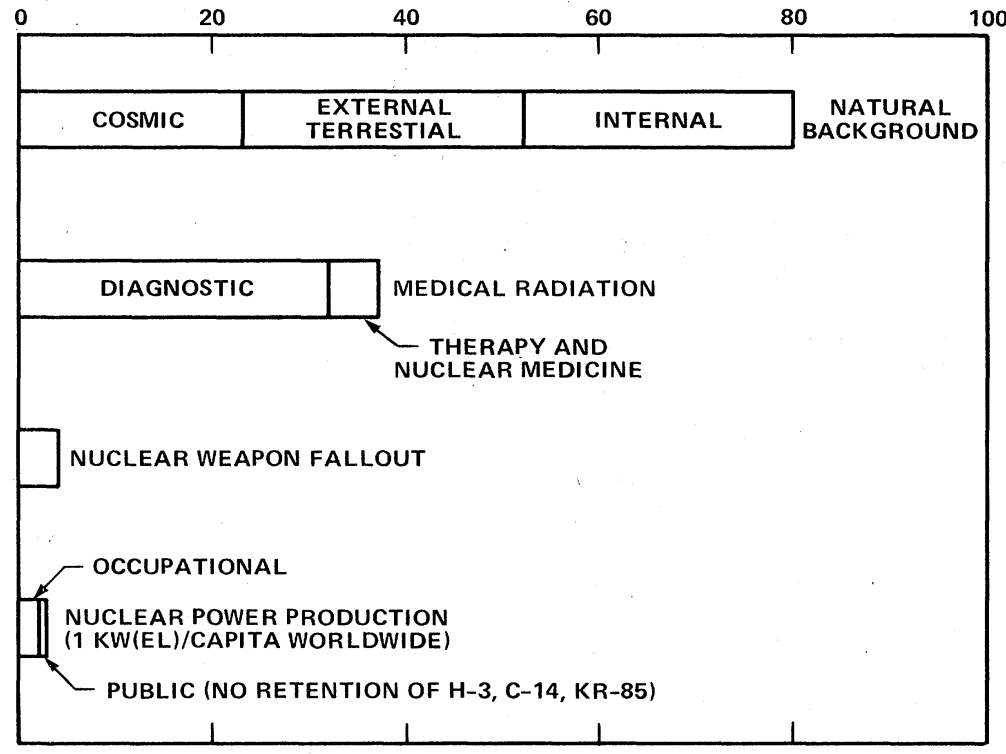

scale reprocessing plant. Accordingly, it would be useful to know whether the estimate of occupational exposure for a next generation plant will indeed turn out to be realistic. In any event, the reprocessing plant occupational exposure should not provide the largest fraction of the total fuel cycle collective occupational radiation dose, given present LWR design and operating experience. Operating experience is required, however, to know whether this conclusion is correct.

A summary of the various contributions to the average annual ionizing radiation background is presented in Fig. 5E-1. Naturally occurring background is presently the largest component. Radiation from medical and dental diagnostic radiology is next most important. The potential for increased examinaton rate means that, unless technical improvements are made to reduce the exposure per examination, this component may increase with time. The contribution from the nuclear power industry will not be as large as that from nuclear weapon fallout for many decades and then only if the worldwide level of nuclear electric power production reaches the level of electric power production currently prevalent in the U.S.

\section{Footnotes}

1 An earlier synonym for RCG was Maximum Permissible Concentration (MPC). The term MPC has fallen into disuse, and is commonly replaced with terms like "concentration limit," or "derived air concentration (DAC)," or annual limit of intake.

2 As a matter of curiosity, the life expectancy in the U. S. is lowest for those states for which natural background is lowest and no decrease in life expectancy for those states with highest background. See also Frigerio (Beninson, 1977a).

3 It has been pointed out by BEIR that "no radiation injuries have been documented in man or other mammals under exposure conditions compatible with existing radiation protection guides" (BEIR, 1972). The current dose limit recommendations of the International Commission on Radiological Protection (ICRP, 1965) and National Council on Radiological Protection and Measurements (NCRP, 1971) are given in Appendices A and B.

4 Thus a given body site might be traversed by ionizing radiation several times per day under background conditions but several thousand times/sec under these high dose rate conditions.

5 The Advisory Committee on Reactor Safety has criticized (ACRS, 1976a) the NRC for failing to adequately identify and express the assumptions inherent in this approach, which was taken in the GESMO (NUREG-0002, 1976) statement.
6 See also EPA policy statement on the use of linearity in dose effects estimates (USEPA, 1976).

7 The dose rate $3 \times 10^{-3} \mathrm{mrem} / \mathrm{y}$ is approximately $4 \times 10^{-5}$ of average background, or expressed-in another way, 20 additional minutes of background exposure/y. In still other terms, it is equivalent to flight at high altitude in a jet aircraft for about 20 seconds.

\section{References}

1. ACRS, 1976a

Advisory Committee on Reactor Safeguards (ACRS). Letter of Nov. 17, 1976: Subject: Report on the Final Generic Environmental Statement on the Use of Recycle Plutonium in Mixed Oxide Fuel in Light Water Cooled Reactors - Health, Safety, and Environment.

2. ACRS, $1976 \mathrm{~b}$

Advisory Committee on Reactor Safeguards (ACRS). Letter of Dec. 20, 1976: Subject: Report on the Management of High Level Radioactive Wastes.

3. Archer, 1973

Archer, V. E., J. K. Wagoner and F. E. Lundin, Jr., Lung Cancer Among Uranium Miners in the United States. Health Phys. 25:351-371 (1973).

4. Bair, 1977

Bair, W. "Review of Studies of the Hot Particle Problem", Proceedings of the International Radiation Protection Accociation, Paris, 1977.

5. Bair, 1777

Bair, W. J., Current Status of the Hot Particle Issue (A Review of Relevant Experimental and Theoretical Approaches), Proc. of the Fourth Congress of the International Radiation Protection Association, pp. 703-710 (1977).

6. Bair, 1977

Bair, W. J. "Toxicology of Plutonium." In: "Advances in Radiation Biology," vol. 4, New York: Academic Press (in press).

7. BEIR, 1972

U.S. National Academy of Sciences - National Research Council. "The Effects on Populations of Exposure to Low 
Levels of Ionizing Radiation." Report of the Advisory Committee on the Biological Effects of Ionizing Radiation (BE:IR Report), Washington, D.C. 1972.

8. Beninson, 1977a

Beninson, D. J., A. Bouville, B. J. O'Brien, and J. O. Sniths. "Dosimetric Implications of the Natural Sources of Iradiation." In: International Symposium on Areas of High Naltural Radioactivity." Brazilian Academy of Sciences, Rio de Janeiro (1977).

9. Beninson, $1.977 \mathrm{~b}$

Beninson, D., B. Bennett, and G. Webb. "Collective Dose Commitments from Nuclear Power Programs." Proceedings of International Conference on the Nuclear Fuel Cycle, IAEA$\mathrm{CN}-36 / 9.1977$

10. Bennett, 1975

Bennett, B. G. "Fallout ${ }^{239} \mathrm{Pu}$ Dose to Man." Fallout Program Quarterly Summary Report, HASL-278. 1975.

11. Bradshaw, 1970

Bradshaw, R. L., F. M. Empson, W. C. McClain and B. L. Houser. Results of a demonstration and other studies on the disposal of high level solidified, radioactive wastes in a salt mine. Health Phys. 18:63 (1970).

12. Brooke, 1976

Brooke, B. personal communication, 1976

13. Campbell, 1973

Campbell, E. E., M. G. Milligan, W. D. Moss, H. F. Schulte and J. F. Mclnray. "Plutonium in Autopsy Tissue." Los Alamos Sceintific Lab. Doc. LA-4875. 1973.

14. Casarett, 1977

Casarett, G. W. Radiobiologic Effects at Low Radiation Levels, International Symposium on Areas of High Natural Radioactivity, Pocos de Caldas, Brazil, pp. 159-165 (1977).

15. CFR -1020

"Standards for Protection Against Radiation." Code of Federal Regulations Part 20.

16. CFR-1050-F

"Standards for Protection Against Radiation." Code of Federal Regulations Part 50, Appendix F.

17. Cohen, et al., 1973

Cohen, N., T. Jaakkola, and M. E. Wresin. Lead-210 Concentration in the Bone, Blood and Excreta of a Former Uranium Miner, Healih Physics 24: 601-609. (1973).

18. Cohen and Kneip, 1969

Cohen, N. and T. J. Kneip. A Method for the Analysis of Lead-210 in the Urine of Uranium Miners, Health Physics 17: 125 (1969)

19. Comar, 1976

Comar, C. L., Ed., et al., Plutonium: Facts and Inferences, EPRI EA-43-SR (August 1976).

\section{Culot, 1973}

Culot, M.V.J., H. G. Olson, K. J. and Schiager. "Radon Progeny Control in Buildings", Uranium Mill Tailings, Colorado State University, Fort Collins, Colorado (1973).

21. Durbin, 1973

Durbin, P. W. "Metabolism and Biological Effects of the Transplutonium Elements." Uranium, Plutonium, Transplutonic Elements. (H. C. Hodge, J. N. Stannard, and J. B. Hursh, eds.), New York: Springer-Verlag, pp. 739-896. 1973.
22. Durbin, 1974

Durbin, P. W. "Transfer of Plutonium from Soil to Plants: A Review of the Problem." Berkeley, University of California, Document UCID-3689. 1974.

23. Eisenbud, 1973

Eisenbud, M. "Environmental Radioactivity." 2nd ed. Academic Press, New York. 1973.

24. Eisenbud, et al., 1969

Eisenbud, M., G. R. Laurer, J. C. Rosen, N. Cohen, and J. Thomas. In-Vivo Measurement of Lead-210 as an Indicator of Cumulative Radon Daughter Exposures in Uranium Miners, Health Physics 16: 637-646 (1969).

25. Eisenbud, et al., 1969

Eisenbud, M., G. R. Laurer, J. C. Rosen, N. Cohen, J. Thomas, and A. J. Hazle. In-Vivo Measurement of Lead-210 as an Indicator of Cumulative Radon Daughter Exposure in Uranium Miners, Health Physics 16: 637 (1969).

26. Fllett and Richardson, 1976

Ellett, W. H. M. and A. C. B. Richardson. Estimates of the Cancer Risk due to Nuclear-Electric Power Generation, U. S. Environmental Protection Agency, Technical Note ORP/CSD76-2 (Oclober 1976).

27. EPA-5209, 1973

Environmental Protection Agency (EPA). ENvironmental Analysis of the Uranium Fuel Cycle, Part 1 - Fuel Supply, USE:PA, Office of Radiation Programs, Washington, D.C. 20460 (FPA-5209-73-003-B) (1973).

28. ERDA-76-43, 1976

U.S. Energy Research and Development Administration, 1976, Report ERDA--76-43, Alternative for Managing Wastes from Reactors and Post Fission Operations in the LWR Fuel Cycle.

29. Evans, et al., 1969

Evans, R. D., A. T. Keane, R. J. Kolenhow, R. W. Neal and M. $M$. Shanahan. Radiogenic tumors in the radium and mesothorium case studies at MIT. In: "Delayed Effects of Bone Seeking Radionuclides" (C. W. Mays, W. S. S. Jee, and R. D. Lloyd, eds.), pp. 157-194. Univ. of Utah Press, Salt Lake City. 1969.

30. FRC, 1967

Federal Radiation Council. "Guidance for the Control of Radiation Hazards in Uranium Mining" (Staff Report of FRC). Rep. No. 8, revised. FRC, Washington, D.C. 1967.

31. FR, 1976

Federal Register. "Uranium Milling: Intent to Prepare a Generic Environmental Impact Statement." Washington: 41 FR 22430, June 3, 1976.

32. Fry, 1976

Fry, R. M. and J. E. Cook. "Comment to Health Effects of Radon-222 from Uranium Mining." Search 7:350 (1976).

33. Harley, 1975

Harley, J. H. "Environmental Radon." In: The Noble Gasses, Moghissi, A. A. and R. E. Stanley, Eds. (U.S. Government Printing Office) Washington, D.C. (1975).

34. Hemplemann, 1973

Hemplemann, L. H., W. H. Langham, C. R. Richmond and G. L. Voelz. "A Twenty-seven Year Study of Selected Los Alamos Plutonium Workers." Health Phys. 25:461-479 (1.973).

35. Hodge, et al., 1973

Hodge, H. C., J. N. Stannard, and J. B. Hursh. "UraniumPlutonium, Transplutonic Elements," In: "Handbook of 
Fxperimental Pharmacology," vol. 36, New York: Springer Verlag (1973).

36. IAEA, 1969

International Atomic tinergy Agency. "Manual on Safety Aspects of the Design and Equipment of Hot Laboratories." IAt:A, Viemna (1969).

37. IAEA, 1974

International Atomic Energy Agency. "Safe Handling of Plutonium." STI/PUB/358. IAEA, Saf. Ser. 39 (1974).

38. ICRP, 1969

International Commission on Radiological Protection (ICRP) "General Principles of Monitoring for Radiation Protection of Workers." ICRP Publication No. 12, Pergamon Press, Oxford. 1969.

39. ICRP, 1959

International Commission on Radiological Protection (ICRP) "Report of Committee II on Permissible Dose for Internal Radiation." ICRP Publication No. 2, Pergamon Press, Oxford. 1959.

40. ICRP, 1965

International Commission on Radiological Protection (ICRP) "Radiation, Protection and Recommendations of the International Commisison on Radiological Protection." ICRP Publication No. 9, Pergamon Press, Oxford. 1965

41. ICRP, 1969

ICRP 14. Radiosensitivity and Spatial Distribution of Dose, Pergamon Press, New York (1969).

42. ICRP, 1972

International Commission on Radiation Protection (ICRP). "The Metabolism of Compounds of Plutonium and Other Actinides." ICRP Publication No. 19. Pergamon Press, Oxford. 1972.

43. Jee, 1976

Jee, W. S. S., (Ed.) The Health Effects of Plutonium and Radium. The J. W. Press, Salt Lake City (1976).

44. Kennedy, et al., 1977

Kennedy, R. H., L. J. Deal, F. F. Haywood, W. A. Goldsmith. Management and Control of Radioactive Wastes from Uranium-Milling Operations, IAEA Conference on Nuclear Power and Its Fuel Cycle, IAE.A-CN-36/479, 1977.

45. Lindell, 1960

Lindell, B. An Approach to the Question of Computing. Doses and Effects from Fallout, Health Physics 2: 341-365 (1960).

46. Lindell, 1976

Lindell, B. Radiation and Man, Health Physics 31: 265 (1976)

47. Lundin, 1969

Lundin, F. E., Jr., J. W. Lloyd, E. M. Smith, V. E. Archer and D. A. Holaday. Mortality of uranium miners in relation to radiation exposure, hard rock mining and cigarette smoking, 1950 through Sept., 1967. Health Phys. 16:571 (1969).

48. Lundin, 1971

Lundin, F. E., Jr., J. K. Wagoner and V. E. Archer. "Radon Daughter Exposure and Respiratory Cancer: Quantitative and Temporal Aspects." NIEHS-NIOSH Joint Monograph No. 1. Public Health Service, U.S. Dept. of Health, Education and Welfare, Washington, D.C. 1971.

49. Luning, 1971

Luning, K. G. and A. G. Searle. "Estimation of genetic risks from ionizing radiation." Mut. Res. 12:291-304 (1971).
50. Magno, et al., 1974

Magno, P. J., C. B. Nelson, and W. H. Ellett. Proc. of the 13th Air Cleaning Conference, USAEC, San Francisco, Calif. (1974).

51. Mays, et al., 1975

Mays, C. W., R. D. Lloyd, and J. H. Marshall. Malignancy Risk to Humans from Total-body Gamma-Ray Irradiation, CONF-730907, pp. 417-428, Proc. of the 3rd International Congress, International Radiation Protection Association, Washington, D.C., 1973, available as U.S. Atomic Energy Commission, Oak Ridge, Tennessee (1975).

52. MRC, 1975

Medical Research Council. "The Toxicity of Plutonium." Medical Research Council Committee on Portection against Ionizing Radiations, Her Majesty's Stationery Office, 1975.

53. Mills, 1977

Mills. 1977, in press, Atomic Industrial Forum

54. NAS/NRC, 1974

NAS/NRC. Health Effects of Alpha Fmitting Particles in the Respiratory Tract, Report of the Ad Hoc Committee on Hot Particles of the Advisory Committee on the Biological Fffects of lonizing Radiations, Washington, D. C. (1974).

55. NCRP, 1971

National Council on Radiation Protection and Measurements. Basic Radiation Protection Criteria, Rep. No. 39, NCRP, Washington, D.C. (1971).

56. NCRP, 1975a

National Council on Radiation Protection and Measurement. "Review of the Current State of Radiation Protection Philosophy." NCRP Rep. No. 43, Washington, D.C. 1975.

57. NCRP, 1975b

National Council on Radiation Protection and Measurement. "Krypton-85 In the Atmosphere - Accumulation, Biological Significance, and Control Technology." NCRP Rep. No. 44, Washington, D.C. 1975.

58. NCRP, $1975 \mathrm{c}$

National Council on Radiation Protection and Measurement. "Natural Background Radiation in the United States." NCRP Rep. No. 45, Washington, D.C. 1975.

59. NCRP, 1975d

National Council on Radiation Protection and Measurement. "Alpha Emitting Particles in Lungs." NCRP Rep. No. 46, Washington, D.C. 1975.

60. Nelson, 1969

Nelson, N. Some biological effects of radiation in relation to other environmental agents. AEC Symp. Ser. 16 (1969).

61. NIOSH, 1971

National Institute for Occupational Health and Safety (NIOSH). Radon Daughter Exposure and Respiratory Cancer: Quantitative and Temporal Aspects, National Institute of Environmental Health Science, Joint Monograph No. 1 (1971).

\section{NUREG 75/032, 1975}

U.S. Nuclear Regulatory Commission. "Occupational Radiation Exposure at Light Water Cooled Power Reactors (1969-1974)." Washington, D.C., June, 1975.

63. NUREG75/108, 1975

U.S Nuclear Regulatory Commission. "7th Annual Radiation Exposure Report (1974)." Washington, D.C., Nov., 1975. 
64. NUREG-0002, 1976

U.S. Nuciear Regulatory Commission (NUREG-0002). Final Generic Environmental Statement on the Use of Recycle Plutonium in Mixed Oxide Fuel in Light Water Cooled Reactors; Ch. II. "Background and Experience with Plutonium," Ch. III. "Projected Plutonium Recycle Industry," and Ch. IV. "Environmental Impact Due to the Implementation of Plutonium Recycle." August, 1976 (GESMO).

65. NUREG-0129, 1977

U.S. Nuclear Regulatory Commission (NUREG-0129). "Bear Creek Project, Rocky Mountain Energy Company", 1977.

66. Oakley, 1972

Oakley, D. T. "Natural Radiation Exposure in the United States." U.S. Environmental Protection Agency, Report ORP/SID 72-1, Washington, D.C. 1972.

67. O'Brien, 1977

O'Brien, K. private communication, April 1977.

68. ORNL-TM-4903, Vol. 1, 1975

ORNL-TM-4903, Vol. 1, M. B. Sears, R. E. Blanco, R. C. Dahlman, G. S. Hill, A. D. Ryon, J. T. Witherspoon, Correlation of Radioactive Waste Treatment Cost and the Environmental Impact of Waste Effluents in the Nuclear Fuel Cycle for Use in Establishing "As Low as Practicable" Guides--Milling of Uranium Ores, (1975).

69. Pochin, 1976

Pochin, E. E. "Estimated Population Exposure from Nuclear Power Production and Other Radiation Sources." Nuclear Energy Agency, Organization for Economic Co-operation and Development, Paris. 1976

70. Pohl, 1976

Pohl, R. O. "Health Effects of Radon-222 from Uranium Mining." Search 7:350 (1976).

71. Price, 1973

Price, K. R. "A Review of Transuranic Elements in Solids, Plants, and Animials." J. Environ. Quality 21:62-66 (1973).

72. Richmond, 1970

Richmond, C. R., J. Langham and R. S. Stone. Biological response to small discrete highly radioactive sources. Health Phys. 18:401 (1970).

73. Russell, 1970

Russell, W. L. "Mutagenesis in the mouse and its application to the estimation of the genetic hazards of radiation." Proc. IVth International Congress of Radiation Research, Evan, France, June 29-July 4 (1970).

74. Ryon and Blanco, 1975

Ryon, A. D. and R. E. Blanco. Correlation of Radioactive Waste Treatment Costs and the Environmental Impact of Waste Effluents in the Nuclear Fuel Cycle for Use in Establishing "As Low as Practicable" Guides--A ppendix A. Preparation of Cost Estimates for Volume I, Milling of Uranium Ores, ORNL-TM-4903, Vol. 2 (May 1975).

75. Saccomonno, 1969

Saccomonno, G. "Uranium Miners Health in Radiation Standards for Uranium Mining," Hearings before the Subcommittee on Research, Development and Radiation of the JCAE Congress at U.S. Part I., pp. 301-315, 1969.

\section{Sears, 1975}

Sears, M.D., et al. Correlation of Radioactive Waste Treatment Costs and the Environmental Impact of Waste Effluents in the Nuclear Fuel Cycle for Use in Establishing "as Low as Practicable" Guides - Milling of Uranium Ores,
Oak Ridge National Laboratory, Oak Ridge, Tennessee, ORNL-TM-4903, Vol. 1 (1975).

77. Shearer, 1969

Shearer, S. D., Jr., and C. W. Sill. Health Phys, 17:77-88 (1969) and USPHS DER 69-1, "Evaluation of Radon-222 Near Uranium Tailings Piles." (1969).

78. Siek, 1977

Siek, R. D. "Review of Environmental Aspects of Uranium Mill Operations", in Workshop on Methods for Measuring Radiation in and Around Uranium, 1977.

79. Spitz, 1974

Spitz, H. B. and M. E. Wrenn. "The Diurnal Variation of the Radon-222 Concentration in Residential Structures in Grand Junction, Colorado", In: Health and Safety Laboratory Second Workshop on the Natural Radiation Environment, HASL-287 (1974).

80. Staff, 1977

Staff. Environmental Statement related to the operation of Bear Creek Project, Rocky Mountain Energy Company (Draft), NUREG-0129, Docket No. 40-8452, USNRC (1977).

81. Staff, 1969

Staff. USPHS Report (1969).

82. Stannard, 1973

Stannard, J. N. Toxicology of Radionuclides. Annu. Rev. Pharmacol. 13:325-357 (1973).

83. Stannard, 1975

Stannard, J. N. Toxicity of Transuranic Elements with Special Emphasis on Pu, HASL-291, ERDA's Health and Safety Laboratory, New York (1975).

84. Stover, et al., 1972

Stover, B. J. and W. S. Jee, eds., Radiobiology of Piutonium, J. W. Press, Univ. of Utah, Salt Lake City (1972).

85. Stuart and Beasley, 1967

Stuart, B. O. and T. M. Beasley. Non-Equilibrium Tissue Distribrtions of Uranium and Thorium Following Inhalation of Uranium Ores by Rats, In: Inhaled Particles and Vapours II, C. N. Davies, Ed., Pergamon Press, Oxford, pp. 291-197 (1967).

86. Stuart and Jackson, 1975

Stuart, B. O. and P. O. Jackson. The Inhalation of Uranium Ores, ERDA-93, Conf. on Occupationa! Health Experience with Uranium (1975).

\section{Swift, 1974}

Swift, J. J., J. M. Hardin, and H. W. Calley. "Assessment of Potential Radiological Impact of Airborne Releases and Direct Gamma Radiation from Inactive Uranium Mill Tailings Piles", USEPA, Office of Radiation Programs, Washington, D.C. (Oct. 1974).

88. Tamplin, 1974

Tamplin, A. P. and T. B. Cochran. "Radiation Standards for Ho: Particles." National Resources Defense Council, February 1974.

89. UNSCEAR, 1969

UNSCEAR, Annex A, Section III, A.2, Definition of the Dose Commitment, (para. 40-89) (1969).

90. UNSCEAR, 1972

United Nations Scientific Committee on the Effects of Atomic Radiation (UNSCEAR). "Ionizing Radiation Levels and Effects," Vol. 1, Levels. United Nations, New York (1972); also, reports for years 1962, 1964, 1969. 
91. USAEC, 1974

USAEC. "Transuranic Waste Disposal." Proposed Amendments to 10 CFR 20, Federal Register, 39, 173, Sept. 12, 1974 (39 FR 132921).

92. USEPA， 1973

U.S. Environmental Protection Agency, Environmental Analysis of the Uranium Fuel Cycle, Part II -- Nuclear Power Reactors, (November 1973).

93. USEPA, 1976

U.S. Environmental Protection Agency. "Final Environmental Statement (40 CFR 190), Environmental Radiation Protection Requirement for Normal Operation of Activities in the Uranium Fuel Cycle, Volume, 1, page B1, 1976

94. USEPA, 1977

USEPA. "Environmental Radiation Protection Standards for Nuclear Power Operations" 40 CFR Part 190, Federal Register, 42, 9, January 13, 1977 (42 FR 2858)

95. USPHS, 1957

U.S. Public Health Service. Control of radon daughters in uranium mines and calculations on biologic effects. U.S. Pub. Health Serv., Publ. 494 (1957).

96. WASH1224, 1974

U.S. Atomic Energy Commission. "Comparative Risks, Costs Benefit Studies of Alternative Sources of Electrical Energy." USAEC Report WASH-1224, Washington, D.C. December 1974.

97. WASH1359, 1974

U.S. Atomic Energy Commission. "Plutonium and Other Transuranium Elements: Sources, Environmental Distribution and Biomedical Effects." USAEC Report WASH-1359, Washington, D.C. December 1974.

98. WASH-1400, 1975 (NUREG-75/014)

U.S. Nuclear Regulatory Commission. Reactor Safety Study.
99. WASH1535, 1974

U.S. Atomic Energy Commission. "Proposed Final Environmental Statement-Liquid Metal Fast Breeder Reactor Program." USAEC Report WASH-1535, Washington, D.C. December 1974.

100. Wilkening, 1975

Wilkening, M.H., W. E. Clements, and D. Stanley. "Radon-222 Flux Measurements in Widely Separated Regions", In: The Natural Radiation Environment II, (Adams, J.A.S., Lowder, W.M. and Gesell, T., Eds.), USAEC, Oak Ridge, Tenn. (1975).

101. Wilson, 1974

Wilson, R. and W. Jones. Energy, Ecology, and the Environment, Academic Press, p. 351 (1974).

102. Wrenn, 1977

Wrenn, M. E. Internal Dose Estimates, International Symposium on Areas of High Natural Radioactivity, Pocos de Caldas, Brazil, pp. 131-157 (1977).

103. Wrenn, 1977

Wrenn, M. E. Relationship of Hazard to Toxicity for Pu and Other Substances, Draft Electric Power Research Institute report, M. E. Wrenn, Scientific Editor, available late 1977.

104. Wrenn, et al.

Wrenn, M. E., N. Cohen, J. C. Rosen, M. Eisenbud, and R. L. Blanchard. In-Vivo Measurements of Lead-210 in Man, Proc. Assessment of Radioactive Contamination in Man, International Atomic Energy Agency, Vienna, IAEA-SM150/36, pp. 129-146.

105. Wrenn and Schulte, 1975

Wrenn, M. E. and H. F. Schulte. "Ionizing Radiation", In: Industrial Environmental Health, 2nd Edition, pp. 179-217, Academic Press, Inc., New York (1975). 


\section{A. Issues, Conclusions and Recommendations}

\section{A1. Issues}

Safeguards in the nuclear fuel cycle are extremely important in reducing the risks to society associated with possible theft or diversion of fissionable nuclear materials. As outlined in Chapter III, it is accepted by many (Foster, 1973; OTA, 1977; Willrich and Taylor, 1974) that the most difficult step in building a nuclear device is acquisition of strategic quantities of enriched uranium or plutonium. There are two main safeguards concerns with an expanding commercial nuclear industry. First, the commercial fuel cycle may directly or indirectly lead to increased proliferation of nuclear weapons states. Of course, nations which wish to devote the resources can obtain nuclear weapons materials through isotope separation or production reactors, completely independent of commercial facilities. Nevertheless, the concern is that commercial facilities may provide additional impetus and possible sources from which nations might divert nuclear materials into a weapons program. Second, commercial nuclear materials or facilities may become targets for sub-national groups desiring to steal fissionable materials. Given the difficulties presented to sub-national groups by isotope enrichment and by chemical reprocessing of intensely radioactive spent fuel, commercial plutonium recycle introduces a safeguards problem: fresh fuel would contain large quantities of plutonium which would circulate in commercial traffic in a form obtainable by comparatively straightforward chemical separation. The highest standards of security must therefore prevail over the lifetime of the nuclear industry.

When the study began, we anticipated that a comprehensive review of safeguards would be available in the Safeguards Supplement to the GESMO (NUREG-0002, 1976) and would form the basis for our considerations. However, this supplement has not been released as yet although information on its scope of work is available (NUREG$75 / 060,1975)$. In its absence, we have not attempted a broad and definitive analysis of the many physical and technical measures which may contribute to safeguards. Neither do we analyze the many institutional, political and legal issues that arise, with their economic consequences as well as potential social costs. Rather, we focus our work upon several technological approaches which are especially pertinent to the control of nuclear materials. Where necessary, we also delineate some of the broader non-technical matters that form the essential context in which the various technical safeguards measures would be utilized, but we make no attempt to analyze the non-technical matters in detail. We examine to what extent selected technical measures can verify that the nuclear materials have been contained in authorized channels and, in the event that a theft has occured, maximize the time and talents needed for converting the material into a form useful as a nuclear explosive. We also center on certain technical aspects of so-called denatured fuel cycles and examine to what extent their technical features may permit them to contribute to international safeguards. Based on this primarily technical analysis, we reach a number of conclusions and recommendations.

\section{A2. Conclusions}

1. The heart of an effective safeguards system must consist of a set of conventional physical security measures as barriers to the theft and subsequent misure of fissile material, which are institutionalized through regulations, standards and other procedures. There are a variety of "standard" measures, similar to those used in protecting any valuable and/or dangerous material, available to help accomplish the safeguards objective, e.g., guard forces and specially designed containers. However, the degree to which these measures will be put into effect rests upon a complicated social/political decision, which must balance the risks, costs and benefits offered by nuclear power against those offered by other energy strategies. Further, complex international arrangements and agreements must be reached.

2. Technical measures do not, themselves, provide sufficient safeguards protection. They can play important roles in complementing and reinforcing conventional physical security measures. Such complementary barriers are especia!ly pertinent to the problem of maintaining effectiveness over a substantial time period, which is a concern of equal importance to that of initially establishing and codifying fuel cycle safeguards.

3. Technical safeguards measures fall into two broad categories: modification of the fuel form itself and fissile material accountability or. measurement systems.

3a. For safeguards considerations, it would be preferable to ship $\mathrm{PuO}_{2}$ only when diluted with $\mathrm{UO}_{2}$. The purpose is to necessitate chemical processing for stolen material to be put into a form usable in a nuclear explosive. Such dilution can be accomplished without essential complication either by admixing $\mathrm{PuO}_{2}$ and $\mathrm{UO}_{2}$ or by coprecipitating $\mathrm{U}$ and $\mathrm{Pu}$ at the separation facility to reach a $\mathrm{Pu} / \mathrm{U}$ ratio below that required for fast critical assembly but compatible with the specification needed for MOX fuel rods. The coprecipitation approach has the important safeguards advantage that $\mathrm{Pu}$ never appears separately in the entire fuel cycle; this may be especially important in addressing the problem of maintaining effective safeguards for the entire life of a nuclear reprocessing plant. However, utilization of the coprecipitated mixed oxides involves economic penalties in increased ${ }^{236} \mathrm{U}$ recycle and in complications for the fuel fabrication because of varying uranium enrichment.

3b. Modifications of the fuel form by incorporation of neutron emitters or absorbers, gamma emitters, or radioactive tracers to lead to recovery of stolen material do not appear very promising.

3c. Strict accountability of fissile material cannot be achieved practically throughout the entire fuel cycle. Nevertheless, an automated nondestructive-assay, real-time accountability system, after separation of plutonium from the fission products at the reprocessing plant and at the MOX fabrication plant, is feasible and would be very valuable for safeguards. Real-time methods could be an effective complement to standard assay-accountability methods. An added benefit would be enhanced process control. The elements of such a system, based upon the unit process concept, ais largely at hand. These elements must be integrated, and necessary measurements must be performed at or in the process streams of commercial scale plants, with proper sampling of the various process and waste streams. Different signal attenuations in the widely varying chemical and physical forms present in the fuel cycle contribute uncertainties to all measurements.

4. Isotopically denatured fuel cycles -- involving "national" reactors operating on low-enriched uranium, and plutonium-burner reactors, reprocessing and enrichment restricted to "international" sites -- may diminish the threat of sub-national theft and impede the use of fuel cycle facilities and materials for weapons production. Even so, nuclear weapons capability can be attained independent of the spread of fuel cycle facilities. The lead time required for weapons construction invariably will be shortened as nuclear technology becomes more widespread. Without the constraints of large throughput or competitive economics, many nations can reprocess spent fuel from a commercial or research reactor and/or enrich natural or low-enriched uranium to weapons grade material. This may become increasingly easier as the number of trained personnel increases. Political agreements and international inspection are still necessary to deter misuse of commercial facilities and also construction of non-safeguarded facilities.

5. We have considered various denatured LWR fuel cycles for international safeguards. Further information on advanced denatured fuel cycles is contained in Chapter VIII.

5a. Plutonium inevitably is produced in all denatured fuel cycles. Thorium cycles reduce such plutonium production but do not eliminate it.

5b. The international "stowaway" fuel cycle with low-enriched LWR fuel is an immediate option. For safeguards purposes, the plutonium-bearing spent fuel should be stored under international control. 
5c. There are no technical barriers to near term implementation of an isolopically denatured fuel cycle in which national reactors are fueled with low-enriched uranium while international fuel cycle centers contain reprocessing and plutonium-burner reactors. However, the associated political agreements and institutional arrangements are complex, and economic burdens are largely unevaluated.

5d. Denatured thorium cycles, in which national reactors are fueled with thorium and uranium with ${ }^{233} U$ isotopic content less than 20\%, offer a long-term alternative. However, such cycles have both advantages and disadvantages with respect to the denatured uranium cycle. An attractive feature for safeguards is that the associated plutonium production may be reduced by as much as a factor of seven, with a corresponding increase in the number of denatured national reactors per reactor at each international site. The plutonium must be stored or fissioned at the international facility, and the safeguards considerations are not qualitatively different from those in the denatured uranium cycle. Disadvantages lie in the increased complication of the associated reprocessing - refabrication operations, in the possible safeguards problem of introducing highly-enriched uranium as make-up, and in the utilization in national reactors of denatured fresh fuel which can be enriched further to weapon grade with comparatively little work.

\section{A3. Recommendations}

The following principal recommendations are based on our examination of several selected technical measures for safeguards. We recognize that these recommendations should be reevaluated when a broader study is completed of the many physical security and technical safeguards measures that might be utilized.

1. We urge evaluation of "safeguards advantageous" fuel cycles using low-enrichment uranium fuel, especially the economic, institutional and technical arrangements associated with co-located reprocessing, refabrication, and plutonium-fueled reactors. (Sec Chapter VIII)

2. Real-time, nondestructive-assay accountability systems should be developed further for possible use for control of special nuclear materials in industrial-scale reprocessing and refabrication plants.

3. The economic impact on the fuel cycle of coprecipitating to a fixed $\mathrm{Pu} / \mathrm{U}$ ratio should be evaluated. Evaluation should include the penalty associated with increased ${ }^{236} \mathrm{U}$ recycle and the consequences to the MOX fuel fabrication arising from varying uranium enrichments. Data are needed on the conditions under which a solid solution of $\mathrm{PuO}_{2}$ and $\mathrm{UO}_{2}$ can be achieved reliably in coprecipitation.

\section{B. Perspective on Nuclear Saf eguards}

It is far from trivial to implement an acceptable and effective system for safeguarding strategically significant quantities of the special nuclear materials $(\mathrm{SNM})^{1}$ present at various stages of the nuclear fuel cycle. The functions of a safeguards system are clear: first, to deter unauthorized access to SNM by either unauthorized persons or "insiders"; second, to reduce the probability that any such attempt will be successful; and finally to reduce the probability that illegally acquired SNM will be used successfully against the public. It is also clear that such a system is needed. For example, possession of significant amounts of plutonium by terrorist groups would render the public susceptible to the real or threatened use of the material as the core of a nuclear explosive weapon, or possibly in a radiological dispersal device.

Many proposals, focusing upon combinations of nuclear materials accountability and physical containment, have been advocated as providing adequate safeguards protection. Nevertheless, definition of the words "adequate" or "acceptable" or "effective" in the context of the nuclear safeguards issue involves a perception of the societal risk that the public is willing to accept in return for the benefits of nuclear-generated electricity. The social decision rests upon a comparison of the risks, benefits, and availability of alternate energyproducing technologies, the comparison involving public health and safety, environmental impacts, the approach to "energy independence", etc.
A further dimension of the safeguards problem concerns the possible impact of the commercial nuclear fuel cycle upon the proliferation of nuclear weapons throughout the world. The concern here is that the availability of nuclear materials and technology, especially spent fuel reprocessing facilities, would allow non-nuclear weapons states either to construct weapons or at least to shorten substantially the lead time needed to construct weapons (Lamarsh, 1976; Keeny, et al., 1977; OTA, 1977). Consequently, American nuclear policy must be formulated with regard both to the export of nuclear fuels and technology and to the implications of a domestic plutonium recycle decision for proliferation.

The issues outlined above will ultimately require national decisions based upon a complicated set of technical, economic, social and political considerations which will involve economic costs, both direct and indirect, as well as potential social costs, arising from effect of institutions, procedures and laws on our way of life. Obviously, we cannot give the ultimate answer to such problems in this report. Rather, our goal in this chapter will be to define somewhat more explicitly the basic problem, outline various proposed safeguards approaches, and evaluate the technical base relevant to fulfillment of the essential safeguards functions.

\section{B1. What is the problem?}

An inescapable consequence of the generation of electric power by nuclear fission is that large amounts of fissionable uranium and/or plutonium, potentially useful in the manufacture of nuclear weapons, are present throughout the nuclear fuel cycle. Furthermore, the amounts required for nuclear violence, e.g., several kilograms of plutonium, are insignificant compared to the amounts needed in a nuclear-fueled economy, so that substantial measures of vigilance and security are required to prevent thef $t$ of nuclear materials by subnational groups. Other commercial activities involving extremely valuable commodities, for example banks, require extreme security and real-time accountability to guard against theft of very small fractions of their inventories. However, the combination of extraordinary security measures and the potential for unacceptable social harm resulting from any failure probably is unparalleled in other commercial ventures. It is important to remember that we are discussing the problem in the context of a commercial enterprise, since the safeguarding of SNM has been an AEC-ERDA function and that of safeguarding assembled nuclear weapons obviously has been a government function for many years.

We shall now attempt to narrow the problem somewhat. First, we shall make the assumption that the technology for isotope separation is not available to subnational groups from whom the SNM is being protected. $\mathrm{Tr}_{\mathrm{i}}$ is assumption should be valid both for technological reasons and because of resource requirements. The means for isotope separation presently used are based upon slight differences in the mechanical motions of different mass isotopes. The American system hinges upon the slightly different diffusion rates of gaseous ${ }^{235} \mathrm{UF}_{6}$ and ${ }^{238} \mathrm{UF}_{6}$ through porous barriers. To achieve significant mass flows, an advanced technology is involved, and enormous capital expenditures and power requirements must be met. This capability is beyond that of sub-national groups and, in fact, is presently beyond that of most non-nuclear nations for commercial scale operation. Futhermore, the chance of performing the enrichment clandestinely on the scale necessary for producing nuclear weapons is very small. However, it must be kept in mind that this assumption is crucial to all that follows, and the emergence of a cheaper, simpler isotope separation scheme would necessitate a complete re-evaluation of the safeguards question.

In contrast to the difficulty in separating isotopes, the separation of different elements by chemical means is comparatively inexpensive and straightforward. This, of course, is what provides the option of plutonium recycle. However, it also leads immediately to a basic safeguards conclusion, namely, that the only "sure" way to denature fissile isotopes is by diluting them with non-fissile isotopes of the same element. For example, uranium with a composition of less than ${ }^{20 \%}{ }^{235} \mathrm{U}$ plus ${ }^{23 .} \mathrm{U}$ is considered denatured (Willrich and Taylor, 1974); for uranium, the dilutent is ${ }^{238} \mathrm{U}$, the most abundant isotope found commonly in nature. As discussed in Chapter III, such isotopic denaturing is not possible for plutonium, ${ }^{2}$ so that plutonium-bearing 
materials will always lead to a safeguards problem after chemical separation. Indeed, since ${ }^{239} \mathrm{Pu}$ is bred by transmutation of ${ }^{238} \mathrm{U}$ in the reactor, the implication for the safeguards question is that all nuclear fuel cycles must involve fuel assemblies (fresh and/or spent) that contain potentially dangerous SNM in forms obtainable by chemical separation. We stress that equally stringent safeguards measures are required for fuel containing ${ }^{233} \mathrm{U}$ (if not diluted with ${ }^{238} \mathrm{U}$ ) and plutonium.

To a large degree, the SNM in spent fuel is self-protecting, since the fuel is then intensely radioactive and the chemical separations work must be carried out remotely and in a heavily shielded environment. Consequently, we shall assume that the safeguards problem for spent fuel assemblies is manageable, since such irradiated fuel assemblies do not represent a very desirable target for theft by subnational groups. We certainly do not mean to imply that no security problem exists in the transport of irradiated fuel rods but only that thieves would be confronted by a formidable problem in processing clandestinely the spent fuel; nevertheless, stringent security measures are required. Therefore, with this assumption, the lowenriched uranium "stowaway" fuel cycle presently in use in the United States does not represent a great safeguards risk. The situation would, of course, be quite different with the advent of plutonium recycle, since then the plutonium would be separated from the irradiated fuel, transported to a fuel fabrication facility, and incorporated into fresh fuel assemblies. One $1000 \mathrm{MWe}$ reactor typically produces 200 to 250 $\mathrm{kg}$ of $\mathrm{Pu}$ per year contained in the spent fuel. Consequently, with recycle large amounts of plutonium would circulate through the economy in forms requiring tight physical security. Therefore, the decisons involving acceptable safeguards and plutonium recycle are in fact intimately connected.

Some qualifications should be added to this last statement. First, the dangers of sabotage and of theft of spent fuel are present in any fuel cycle, with or without plutonium recycle. For example, cooling ponds filled with spent fuel rods could present a target for conventional explosives, the aim being to disperse the radioactive actinides and fission products in the atmosphere. This is a security question that we shall not address. Second, we have been concerned implicitly with the theft of SNM in amounts sufficient to construct a nuclear explosive device. Considerably smaller quantities might suffice for possible blackmail schemes or for radiological dispersal devices. However, it is difficult to believe that overt thefts of heavily guarded SNM would be attempted for either of these purposes, since potentially more dangerous chemical agents could be obtained with less difficulty. This is not to say that the problem is trivial. There is still the possibility of covert theft of small amounts of SNM at various commercial fuel cycle facilities and, perhaps even of radioisotopes from commercial or research activites totally outside the commercial nuclear power industry. Needless to say, such activities are widespread and rapidly expanding, and the control of these nonstrategic quantities of SNM presents a serious safeguards problem. Nevertheless, our concern in this report will be primarily with the protection of strategic amounts of SNM in the nuclear fuel cycle.

A final qualification concerning the causal dependence of the safeguards problem on domestic plutonium recycle is that the international proliferation problem may prove insensitive to our own national decision. Other countries are proceeding witl: plutonium recycle, and the separations technology is available in the open literature. The possible domestic benefits must be weighed against the projected international political consequences (Keeny, et al., 1977). Our approach in this chapter will be to examine the safeguards problem for the domestic LWR fuel cycle (with plutonium recycle) and then to discuss briefly (in section E) some of the technical information needed in considering the proliferation problem.

\section{B2. Safeguards in the LWR Fuel Cycle}

In the LWR fuel cycle with plutonium recycle, safeguards must be imposed effectively both at fixed-site nuclear facilities and during transportation of SNM between sites. As described above, the fuel is self-protecting from the time it is inserted into the reactor until the chemical separations are performed. The product of the separations process is plutonium nitrate solution, which according to current regulations will be converted to solid plutonium oxide at the fuel reprocessing plant and temporarily stored. The plutonium oxide may be shipped to a fuel fabrication facility, where it would be mixed with uranium oxide and incorporated into MOX fuel assemblies. Actually, mixed oxide rods may be sent to another facility for bundling into fuel assemblies, but this does not qualitatively change the safeguards problem. The fuel assemblies would finally be transported to the power reactor, where the fuel again becomes self-protecting. During the transportation and storage periods, the plutonium would appear only in sealed integral containers. Thus, it would be impossible to divert systematically small amounts of material; i.e., accountability reduces in this case to a simple counting problem. On the other hand, tight security is necessary to prevent overt theft of the containers.

However, while resident in the process streams at the separations and fuel fabrication facilities, the plutonium would not be in a form amenable to completely accurate accounting procedures, thereby raising the possibility of long-term systematic diversion. This possibility is reduced somewhat by the fact that, almost by definition, the SNM is least accessible to the thief when the accounting procedures are most difficult. Nevertheless, long-term undetected diversion is a particularly unsettling problem because the chances for recovery of diverted materials before their use are considerably less than chances of recovery following overt theft. It is clear that an effective safeguards system must include reinforcing elements of physical security and accountability; that is, the accountability system should reduce the rate of possible undetected diversion, which is limited by the intrinsic uncertainty of specific physical measurements, to a level requiring such a large number of thef ts for acquisition of strategic quantities of SNM that the probability of escaping the physical security system would be negligible. It is also clear that an effective accountability system should detect any flow of SNM outside the normal channels; that is, a periodic inventory is not sufficient if substantial quantities of SNM were unaccounted for during the intervening period.

Table VI.1 summarizes the nuclear safeguards concepts which have been proposed. The various entries in the table are grouped according to safeguards function and according to the nature of the barrier presented to theft and subsequent use of SNM. The safeguards functions have already been mentioned in the introduction: deterrence indicates the ability of a safeguards measure to discourage would-be thieves; containment indicates the ability of a safeguards measure to reduce the probability that an attempted theft will succeed; and recoverability indicates effectiveness for preventing misuse of stolen SNM either by leading to recovery of the material itself or by degrading the materials with respect to unauthorized use. Furthermore, physical security barriers refer to safeguards measures taken with respect to the material, planning, and personnel intended to contain the SNM, while technical barriers refer to measurement schemes intended to provide accurate materials accountability and to modification of the fuel form itself to render it more difficult to steal and/or use. Technical barriers depend on the specific nature of the material being safeguarded. It is obvious that there are several multiple entries in the table, since particular safeguards measures may contribute to the fulfillment of more than one function. For example, any physical barrier highly effective in enhancing containment and recoverability will also act as a deterrent.

Most of the concepts listed in the table are easily understood. The physical barriers center upon designing containers and upon establishing the guard and response forces needed to defeat the "maximum credible threat" for overt theft. With regard to covert theft, the need is to design a plant surveillance system which prevents the removal of SNM. In addition, there are personnel considerations, e.g., required security clearances and/or psychological profile testing and organizational decisions, e.g., the forced co-location of fuel cycle facilities so as to minimize transportation of SNM, which may accomplish safeguards goals. An important technical barrier is imposition of a real-time accountability system to monitor flows of SNM through fuel cycle facilities, thereby establishing that other protective safeguards systems are in fact working. It should be noted that all of these measures basically are designed to keep track of SNM at all times and, in doing so, they fulfill additional roles in assuring occupational health and safety. For example, they would certainly help in preventing a nuclear criticality accident. Other concepts listed deal with the direct modfication of the fuel form itself to accomplish one of several goals: to maximize the time and talents needed for 
TABLE VI.1. Proposed nuclear safeguards concepts, grouped according to safeguards function and according to nature of barrier presented to theft and subsequent use of SNM.

\begin{tabular}{|c|c|c|c|}
\hline & Deterrence & Containment & Recoverability \\
\hline $\begin{array}{l}\text { Physical } \\
\text { security } \\
\text { barriers }\end{array}$ & $\begin{array}{l}\text { Special1y designed } \\
\text { containers. } \\
\text { Guard forces. } \\
\text { Surveitlance, alarins } \\
\text { Special communi- } \\
\text { cations. } \\
\text { Personne1 consid- } \\
\text { erations. } \\
\text { Co-location of fuel } \\
\text { cycle facilities. }\end{array}$ & $\begin{array}{l}\text { Specially designed } \\
\text { containers. } \\
\text { Guard forces. } \\
\text { Surveillance, a } 1 \text { arms } \\
\text { Special communi- } \\
\text { cations. }\end{array}$ & $\begin{array}{l}\text { Specially designed } \\
\text { containers. } \\
\text { Homing devices. }\end{array}$ \\
\hline $\begin{array}{l}\text { Technical } \\
\text { barriers }\end{array}$ & $\begin{array}{l}\text { Materials account- } \\
\text { ability. } \\
\text { Restricted fuel } \\
\text { forms. } \\
\text { Dilution. } \\
\text { Protective radi- } \\
\text { ation hazard. }\end{array}$ & $\begin{array}{l}\text { Dilution } \\
\text { Protective radi- } \\
\text { ation hazard. }\end{array}$ & $\begin{array}{l}\text { Degraded SNM } \\
\text { Chemically released } \\
\text { tracers. } \\
\text { Dilution. } \\
\text { Protective radi- } \\
\text { ation hazard. }\end{array}$ \\
\hline
\end{tabular}

converting stolen SNM to "useful" forms (restricted fuel forms); to increase the amount of material which must be stolen to achieve a strategic value of plutonium (dilution); to increase the hazards of stealing and processing the SNM (protective radiation hazards); to diminish the value of the stolen material for use in a nuclear explosive device (degraded SNM); to aid directly in the recovery of the stolen material (chemically released tracers). An optimization must be performed since some of the modification concepts will very clearly lead to increased difficulty with accounting measurements and to possible occupational exposures.

The limitations on the extent and nature of physical security measures quite possibly may be determined by social and political considerations rather than by technical and economic ones. For example, if a Federal security force were established, the latter considerations might constrain the size of the force, but the scope of activities and the jurisdiction and authority of the force would most likely be fixed by political considerations. Concerns over the civil liberties implications of a security force with broad investigative powers either for preventive measures or for action in time of crisis, have been expressed already (Ayres, 1975). Analysis of broader social costs has also been presented (Keeny, et al., 1977).

Before proceeding to a discussion of various safeguards concepts, we must note that our information is drawn entirely from sources available to the public. The decision as to how much of an in-place safeguards system is to be made public is a delicate one. Complete revelation would presumably have the greatest deterrent value but might seriously compromise the containment and recoverability functions. We do not attempt to resolve this question.

\section{Physical Security Measures as Safeguards Barriers}

In the absence of the special Safeguards Supplement to the GESMO, we have not performed a detailed analysis of physical safeguards measures, since these generally do not involve new or unique technological approaches. Physical barriers are obviously crucial in containing SNM, and many measures and degrees of implementation are possible. A detailed evaluation, presumably including a social/political decision defining an "acceptable" level of safeguards protection, is forthcoming in the GESMO supplement. To provide a background for our subsequent discussion of technical barriers, we shall discuss briefly some of the more obvious physical safeguards measures. It is important to note that, with the exception of co-location of fuel cycle facilities, the safeguards measures listed in Table VI.1 as physical barriers are not peculiar to the task of containing SNM. Similar precautions are taken in the protection of any valuable and/or dangerous commodity.

We start by describing some of the measures possible for protection of the SNM during transportation. Each shipment could have a separate armed escort vehicle which would maintain constant visual and communications contact with the transport vehicle. Furthermore, since the routes travelled will probably be standardized, frequent periodic contact could be maintained with appropriate response forces. The transportation cask itself could be massive, as they must be for spent-fuel assemblies; giant cranes would be needed to remove it from the vehicle. The cask could require special tools to be opened, and, of course, these would exist only at fixed-site nuclear facilities. In fact, the last two possibilities could be combined by requiring that the cask be lif ted by crane to allow access to the inside of the cask. The transportation vehicle could have independent immobilization mechanisms, operable from inside, from the escort vehicle, and perhaps remotely as well. Homing devices could be attached to the cask to facilitate recovery. At some level of implementation, a combination of these and possibly other steps can presumably be made effective. The question is one of setting that level. One estimate of the personnel costs (Willrich and Taylor, 1974) required for implementation yields an increase in the cost of nucleargenerated electricity of less than 1\%; other costs, such as those for providing sophisticated communications systems and transport vehicles, cannot be estimated reliably prior to specification of safeguards performance criteria.

At fixed-site facilities, the problem reduces to one of surveillance and of limited access. Presumably, these facilities should be designed so as to allow minimum personnel access to SNM, especially in storage areas. In these areas, each container could have a separate alarm system, for example, by remotely storing the cannisters on load cells in fairly tight-fitting compartments. Access to the process streams cannot be avoided entirely, since sample points are necessary for controlling the product quality. However, such access can be strictly controlled (e.g., by having fingerprint-reading devices grant access) and could be done mechanically (e.g., to avoid hostage scenarios). Also, doorway neutron and/or gamma monitors, perhaps together with metal detectors, could prevent unauthorized removal of SNM from the access area, and complete changes of clothing could be required of workers for both occupational health and safeguards reasons.

It is clear that, in the normal course of operations, certain personnel must have access to and control over the flow of SNM, for example, technicians sampling a process stream or drivers of transport 
vehicles. Consequently, the requirement of ERDA security clearances and of various psychological reliability assessments has been proposed. The effectiveness of this as a key element in a multi-barrier safeguards approach is suspect; the important considerations remain limited access and surveillance. With an effective safeguards system, a possible situation for an attempted theft of strategic quantities of SNM from fuel cycle facilities would be one of abnormal operation. That is, during an emergency, whether caused by accident or by sabotage, rapid evacuation might preclude application of normal safeguards precautions. For this eventuality, an additional outer security ring could be activated.

One regulatory decision having a direct impact on safeguards would be to require co-location of reprocessing, plutonium storage, and MOX fuel fabrication facilities. This would eliminate the problem of safeguarding during shipment material directly usable in a nuclear explosive. Such regulation might well create commercial problems, in that different companies performing different fuel cycle functions would have to operate as closely-coupled partners. It might also entail increased costs to one or more of these companies. We see no insurmountable obstacle to overcoming the interfacing problems, especially since, at least for the near future, industry coupling would be dictated by the small number of facilities in operation. The question as to balancing the value of eliminating one fuel cycle link requiring safeguards versus the economic costs and institutional problems is not one that industry should resolve. Such decisions, involving the distributed social benef it of safeguards versus the cost to industry, are properly adjudicated by the approriate Federal agency (i.e., NRC). Additional data which would be useful in reaching the co-location decision are a comparison of the environmental impacts resulting from co-location versus dispersed siting and a study of possible cost reduction from integration of conversion and MOX fuel fabrication plants.

In summarizing this section, it appears quite possible that effective physical security can be imposed throughout the fuel cycle at an economic cost small compared to that of nuclear-generated electricity (Willrich and Taylor, 1974). The measures described above are all extensions of those already used in other social activities requiring security and it must be accepted that physical security measures for safeguards are essential in any $\mathrm{Pu}$ recycle power economy. Presumably, the GESMO Safeguards Supplement will include a detailed analysis both of the level of protection required and of the associated social and economic costs.

\section{Technical Measures for Safeguards Barriers}

Physical security lies at the heart of any system designed to confine SNM to authorized channels. Technical measures for safeguards are important in complementing and reinforcing conventional physical security barriers, especially with regard to the problem of maintaining effectiveness over a substantial time period. As already remarked, technical measures fall into two broad categories: modification of the fuel form itself, especially during transportation, and accurate SNM accountability systems at fuel cycle facilities. These measures aim primarily at preventing overt theft and covert diversion, respectively. As in the last section, we are concerned primarily in this section with the national safeguards problem.

\section{D1. Modified Fuel Form}

\section{Dla. Dilution}

In the event of successful overt theft of SNM, an important factor in determining the likelihood of successful recovery of the materials is the time necessary for converting the material into a form useful for constructing a nuclear explosive device. This is especially so if highly refined technical and industrial skills are needed and if special equipment, such as heavy cranes, must be employed. Consequently, it is preferable for safeguards that SNM not be shipped in a form directly usable in nuclear weapons and therefore that the transportation of pure $\mathrm{PuO}_{2}$ in strategic quantities not be allowed. Practically, such a regulatory decision would allow two options: either co-location of the spent fuel reprocessing and MOX fabrication plants or shipment of $\mathrm{PuO}_{2}$ diluted by depleted, natural, or low-enriched $\mathrm{UO}_{2}$. In this case, a large amount of material would require chemical processing to achieve a critical mass of plutonium. For example, about 2000 liters of concentrated nitric acid are needed to dissolve a mixed oxide fuel with $5 \%$ plutonium to obtain $50 \mathrm{~kg} \mathrm{PuO}_{2}$. To process several kilograms of the mixed oxide fuel per day, a substantial glove box facility would be needed for the dissolution, precipitation, filtration and calcining steps. Approximately 4000 liters of liquid effluent would be generated. Alternative processing with additives such as fluoride or ceric ions to enhance the solvent activity would decrease the volumes to be handled. However, the difficulty in handling these extremely corrosive solvents would be so enhanced as to more than offset any advaritage gained by the volume reduction. Thus, in the absence of quite substantial and sophisticated reprocessing facilities, months might be required to complete the operation, and this would greatly aid local and Federal response forces in their recovery operations.

The co-location option has been discussed already, but the dilution approach itself offers two choices, admixing or coprecipitation. As presently envisioned, MOX fuel fabrication plants will receive separately $\mathrm{PuO}_{2}$ and natural or depleted $\mathrm{UO}_{2}$ powder and then admix these to a Pu concentration of about 5\%. It must be noted that, since shipment of plutonium nitrate solution will not be allowed and since the fuel fabricator justifiably does not want to redissolve the $\mathrm{PuO}_{2}$ powder, ${ }^{3}$ the fuel fabricator is in any case dependent upon the conversion plant for the quality of his feed product. Therefore, it would not be unreasonable to require that the $\mathrm{PuO}_{2}$ be admixed with $\mathrm{UO}_{2}$ before leaving the conversion plant to a $\mathrm{Pu}$ concentration somewhat above that to be used in MOX fuel rods, for example, in the 5 to $15 \%$ range. This amounts to co-locating part of the blending operation envisioned at the fabrication plant with the reprocessing plant.

A question arises concerning the effectiveness of admixing in retarding conversion of stolen SNM to weapons material. Since the crystal densities of $\mathrm{PuO}_{2}$ and $\mathrm{UO}_{2}\left(11.46 \mathrm{gm} / \mathrm{cm}^{3}\right.$ and $10.96 \mathrm{gm} / \mathrm{cm}^{3}$, respectively) are slightly different, physical separation of the oxides (e.g., by elutriation) may seem possible. Nevertheless, many factors besides the nominal crystal densities enter in determining the feasibility of such approaches, and, to the best of our knowledge, such separations have not been performed. For example, the oxide particles, as produced, generally have considerable porosity, so that the effective densities may not be very different. Therefore, while theoretically possible, elutriation is almost certainly more difficult than dissolution. This is especially so for the admixed oxides since 3$4 \mathrm{M}$ nitric acid would dissolve most of the $\mathrm{UO}_{2}$ but only little of the $\mathrm{PuO}_{2}$, assuming it is calcined at $750-900^{\circ} \mathrm{C}$. While the residue would not be pure $\mathrm{PuO}_{2}$, it would probably be usable weapons material for a low yield device, and the separations step could be avoided. Therefore admixture would require steps at least as complicated as chemical dissolution, but a solid solution of the oxides, as might emerge from coprecipitation, would require the somewhat lengthier procedure of dissolution plus chemical separation.

Coprecipitation of $\mathrm{Pu}$ and $\mathrm{U}$ at the separations plant of fers the additional safeguards advantage that the $P u$ never appears undiluted throughout the entire fuel cycle. We shall discuss coprecipitation in some detail but similar remarks apply to other nitrate to oxide conversion methods. For example, direct co-calcining could be used (see the discussion of the proposed EXXON plant in section D4 of Chapter IV). Coprecipitation is effective in addressing both the transportation problem and the problem of theft or diversion at the reprocessing plant, especially from Pu storage areas. This feature may be especially important with regard to the problem of maintaining the necessary vigilance for the entire lifetime of the plant. With regard to diversion, the increased amount of material which must be stolen to achieve the same $P u$ value is a significant benefit. The extreme approach (Puechl, 1975) is to coprecipitate all $\mathrm{Pu}$ and $\mathrm{U}$, thus lowering the $\mathrm{Pu} / \mathrm{U}$ ratio for recycle fuel to about $1 / 100$. There are several problems with this approach. The most obvious economic penalty is that, assuming the oxide is not redissolved, the remote expensive fabrication steps designed for MOX fuel would have to be used for a substantially larger number of fuel rods. Second, all of the ${ }^{236} \mathrm{U}$ in spent fuel is returned to the fuel cycle with this approach, rather than discharging almost half of it to the tails during enrichment. Further, the reactivity of the coprecipitated fuel would be so low that more highly enriched uranium would be needed as make-up, thereby introducing another safeguards problem. It should be remembered 
that highly enriched uranium presents the least difficulty in weapons fabrication (i.e., in handling and design).

A better approach might be coprecipitating directly to a $\mathrm{Pu} / \mathrm{U}$ ratio of about 5-15 parts per hundred. Basically, one would like to achieve the lowest $\mathrm{Pu} / \mathrm{U}$ ratio compatible with the specifications needed for MOX fuel rods while not imposing a sizable economic penalty on the industry. With this scheme, there is still an increase in ${ }^{236} \mathrm{U}$ recycle but it is considerably less than in the complete coprecipitation approach. However, the fabricator may still be confronted with receiving uranium of greatly varying enrichments. For example, the enrichment in discharge fuel during reactor start-up varies by about 50\% (Shapiro, et al., 1977) while discharges from low burnup uranium loadings or from MOX fuel loadings would have higher and lower enrichments, respectively. A detailed analysis of the economic impact in the fuel cycle of coprecipitation to a specified $\mathrm{Pu} / \mathrm{U}$ ratio should be carried out for the various fuel cycles of interest.

"Partial" coprecipitation can be accomplished in several ways. For example, in the Purex flowsheet, after solvent extraction of $\mathrm{U}$ and $\mathrm{Pu}$, reduction of $\mathrm{Pu}^{+4}$ to $\mathrm{Pu}^{+3}$ returns the plutonium to aqueous solution in the partitioning column. This step also reduces some $\mathrm{U}^{+6}$ to $\mathrm{U}^{+4}$, both of which still complex strongly with TBP at high acidity. Therefore, the partitioning column is operated at high acidity in order to keep the $U$ in organic solution and thereby effect the partitioning. Lowering the acidity favors movement of $\mathrm{U}$ from the organic to the aqueous phase and thus one could control the $\mathrm{Pu} / \mathrm{U}$ ratio extracted in the aqueous phase. The uranium and plutonium in aqueous solution would then be coprecipitated. It should be noted that the precipitation process will increase the $\mathrm{Pu} / \mathrm{U}$ ratio with regard to that in the aqueous solution, since the $\mathrm{Pu}$ precipitates more quantitatively. This can be used to advantage. For example, the partitioning column instead could be used as a stripping column by operating at low acidity; ${ }^{4}$ all the $\mathrm{Pu}$ and most of the $\mathrm{U}$ could be extracted in aqueous solution, and the final $\mathrm{Pu} / \mathrm{U}$ ratio could be controlled entirely at the conversion step, e.g. some oxidation of the $U$ by increasing the molarity of the nitric acid solution would increase the $\mathrm{Pu} / \mathrm{U}$ ratio in the oxalate precipitate. An objection to this proposal might be that the oxalate supernatant would require further reprocessing in order to extract the uranium value; there will also be a small, perhaps 50 mg/liter, concentration of Pu. However, this additional step is not a serious requirement, since a simple solvent extraction or ion exchange process could be used in an unshielded facility. Obviously, this would be a factor in the economic study advocated above.

A question which remains concerns the accuracy and reliability with which specific $\mathrm{Pu} / \mathrm{U}$ ratios can be obtained with the coprecipitation scheme. The data for this can be obtained easily, and one would expect that reliability can be achieved. However, there is no need for very high accuracy, since the isotopic composition of the product will be measured accurately in any case and since further admixing will allow flexibility in changing the $\mathrm{Pu} / \mathrm{U}$ ratio somewhat. We repeat that the advantage of this approach is that plutonium appears only in dilute form. Nevertheless, this certainiy does not "solve" the safeguards problem and strict physical security must be maintained.

Another question concerns the reliability with which a solid solution can be formed by coprecipitation. Presently available evidence indicates that solid solution is not achieved consistently (Walling, 1977), although consistency might be achieved with further research and experience. Sintering the calcined product at high temperatures $\left(\sim 1600^{\circ} \mathrm{C}\right)$ would lead to a solid solution but is likely to make subsequent pressing into pellets very difficult. On the other hand, there is evidence (Walling, 1977) that, for plutonium concentrations less than $15 \%$, nitric acid does not preferentially dissolve the uranium oxide even if a true solid solution is not formed. Detailed investigations are needed in these areas.

\section{D1b. Gamma Emitters}

Another fuel modification that has received serious consideration is the incorporation of gamma emitting isotopes in the fuel or adjacent to it (i.e., attached to the fuel rods, fuel assemblies, or inner surfaces of the transportation casks). It is clear that such concepts must be evaluated carefully with regard to public and occupational safety and with respect to increased costs to the fuel cycle resulting from increased handling problems. To be effective, spiking must lead to disabling doses (on the order of at least several thousand rems). Incorporation of such large amounts of gamma activity into the fuel itself is impractical, since then all steps in the entire fuel cycle would have to be carried out remotely behind heavy shielding and occupational risks would be great. The only feasible alternative ${ }^{5}$ (IRT, 1975 ) is attachment of intense sources, presumably ${ }^{60} \mathrm{Co}$, to the transportation casks, thus requiring only that the loading and unloading of casks be handled remotely. The price of ${ }^{60} \mathrm{Co}(\$ 0.50$ per curie of $25 \mathrm{Ci} /$ gram $\left.{ }^{60} \mathrm{Co}\right)$ is sufficiently small so that the direct economic penalty is not prohibitive. Nevertheless, we believe that this is not a very attractive concept. Assuming that a group capable of the theft of an entire transport venicle also has substantial resources, then the additional problem posed by the ${ }^{60} \mathrm{Co}$ source is minimal; the cask simply must be unloaded behind heavy shielding. Having accomplished that, the massive ${ }^{60} \mathrm{Co}$ source itself becomes a possible threat in a radiological dispersal device. Furthermore, an accident in the normal handling of the source might easily lead to occupational or even public exposure.

\section{D1c. Neutron Emitters and Absorbers}

Other fuel modification possibilities focus upon degrading the SNM for use in nuclear explosives by altering the fast neutron spectrum. Appropriate fast neutron absorbers do not exist in nature (IRT, 1975). 5 Another approach is offered by the fact that a significant increase in the neutron background would make plutonium weapon design more difficult. The problem posed by the neutron background is that a neutron might initiate the explosion when the device just reaches criticality, rather than when the desired degree of supercriticality is achieved. Then, the device will disassemble prematurely, thereby preventing further fissioning of the SNM and resulting in a comparatively low yield. The probability for predetonation clearly increases with higher neutron background and, for comparable reliability and efficiency, places greater demands on the designer for short assembly times and sophisticated initiation. The so-called "fizzle" yield is still significant (perhaps tens of tons of TNT) but small on the scale of attainable yields. A possible candidate for this scheme is ${ }^{252} \mathrm{Cf}$, with a spontaneous fission half-life of only 85 years. Reactor grade plutonium emits approximately 400 neutrons/gm/sec from the spontaneous fission of ${ }^{238} \mathrm{Pu}$ and ${ }^{240} \mathrm{Pu}$, with spontaneous fission half-lives of $5 \times 10^{10}$ years and $10^{11}$ years, respectively, plus a comparable amount from $(\alpha, n)$ reactions on oxygen. A ${ }^{252} \mathrm{Cf} / \mathrm{Pu}$ ratio of $10^{-9}$ would increase the spontaneous fission neutron background by roughly an order of magnitude. With this ratio, and at a cost of $\$ 10 / \mu \mathrm{g}{ }^{6}$ (Crandall, private communication, 1977) of ${ }^{252} \mathrm{Cf}$, the incremental direct increase of the cost of nucleargenerated electricity would be about $0.3 \%$. Furthermore, both the incremental occupational hazards and the modification of reactor operating characteristics would appear to be manageable.

The remaining question is the difficulty required to purify the plutonium by removing at least $90 \%$ of the added ${ }^{252} \mathrm{Cf}$. The chemistry of $\mathrm{Cf}$ is very similar to that of dysprosium, a rare earth present in the fission product yield; its only common valence istate is +3 although both +2 and +4 are known, whereas it is the +4 states that form ion complexes allowing separation of plutonium from dissolved mixed oxides. However, in chemical extraction of individual rare earth elements from $\mathrm{Th}, \mathrm{U} ;$ and $\mathrm{Pu}, 10^{-8}$ seems to be a "limiting concentration" (Ryabohikov, 1970); for example, with $10^{-6}$ to $10^{-7}$ parts Dy/Th, TBP extraction is only $85 \%$ efficient, and a complex series of extractions increases this efficiency to $93-95 \%$ (i.e., still in the $10^{-8}$ range) (Cleveland, 1970; Keller, 1971). Similar remarks apply to other possible purification schemes, such as electrochemical refining of plutonium metal. Therefore, the best estimate seems to be that such trace amounts of $\mathrm{Cf}$ can be separated from $\mathrm{Pu}$ but only by those having very high sophistication technology, (R. Penneman, private communication, 1977) such as a Purex separatory facility or the technology for plutonium metal production and purification. For those having to rely on a battery of separatory funnels and lacking access to anhydrous glove box facilities able to operate under corrosive conditions at high temperatures, the problem is formidable, requiring several steps to go from spiked to clean plutonium. Thus the goal of significantly retarding conversion of stolen SNM to useful form may be accomplished, and this alternative should receive more detailed 
consideration. In particular, little knowledge exists concerning the separation of rare earths at the $10^{-8}-10^{-9}$ level from uranium and plutonium, and such data should be obtained.

\section{D1d. Chemically Released Tracers}

As a last fuel modification possibility, we consider the idea of including a radioactive element in the fuel which will act as a tracer leading to recovery of the stolen material. Elements which are easily entrapped in the chemical separations process can be shielded so that the signal outside the clandestine laboratory will be small compared to background. Consequently, we examined the idea of including in the fuel a radioactive noble gas, ${ }^{85} \mathrm{Kr}$, which would be released upon chemical processing and present a significant problem for entrapment. Evaluation of this concept is difficult, since it depends upon local terrain and meteorological conditions. Using NCRP "standard" meteorological conditions (NCRP, 1975) we find that this spiking approach is not effective with presently available detection schemes and with reasonable amounts of ${ }^{85} \mathrm{Kr}$ in the fuel. Reevaluation may be warranted if meaningful advances in detection technology occur.

In concluding this section, it appears that the only modification concept that emerges as an economically feasible, safe and effective technical safeguard is that of dilution. Admixing $\mathrm{PuO}_{2}$ and $\mathrm{UO}_{2}$ at the conversion plant eases the problem of safeguards during transportation; coprecipitation (or cocalcining) additionally reduces the problem of diversion of $\mathrm{PuO}_{2}$ from the reprocessing plant, and this may be important in addressing the problem of maintaining effective safeguards for the life of the plant. Detailed economic studies are called for. The ${ }^{252} \mathrm{Cf}$ spiking concept also seems possibly useful, but it should be recognized that the higher neutron background would not present an insurmountable obstacle to construction of a reliable device if the designer has access to advanced and sophisticated (and classified) design information and to the resources and high technical skills needed to realize the design. Finally, the utility of tracers seems questionable.

We stress that none of these approaches is intended to be a "solution" of the safeguards problem. Rather, the aim is to complicate the process by which stolen SNM can be converted into material usable in a high-yield nuclear explosive device; it is assumed that the increased time and talents needed by thieves significantly enhances the opportunities for recovery by appropriate security forces. This is clearly a case where a political decision must be made balancing increased fuel cycle costs and potentially increased occupational exposures against less quantifiable factors, such as confidence in the primary physical barriers. To the extent that physical safeguards measures are deemed completely reliable, these fuel modification approaches are unnecessary.

\section{D2. Accountability}

A central ingredient in the overall multi-barrier safeguards scheme is an effective accountability system capable of measuring accurately the amounts of SNM resident in or flowing through various process steps in fuel cycle facilities, e.g., in MOX fuel fabrication or in irradiated fuel separations facilities. Accounting is complementary to physical security measures and is an independent measure of the effectiveness of the latter. Present-day methods emphasize periodic inventory with chemical and mass spectrometric assay. To be most effective, the accountability system should also provide "real-time" information on flows of SNM as it leaves normal chanuels and on inventories of SNM in each unit process in the facility. Real time information is especially important during or immediately after periods of abnormal operations, e.g., during an industrial accident at the facility. In principle, the process flowsheet should be broken down into as many measurement units as possible, e.g., a dissolving tank at the separations facility or a pellet press at the fuel fabrication plant. This is significant for several reasons. First, any diversion of SNM by a plant employee may focus upon a particular unit and an accountability system which isolates that unit clearly supplies the most sensitive signal that diversion is taking place. Effectively, a much smaller amount of material is involved in such a measurement as compared to net balance of total plant input and output. Second, accountability measurements at a specific unit process is likely to be most accurate when the SNM is in its purest form and is most accessible; consequently the measurements will be most effective precisely when the material represents the most desirable target for theft. This increased effectiveness would be weakened if a material balance were drawn only about the entire plant because of the inherent difficulty of making completely accurate measurements, as discussed below. Finally, such a system enhances plant and quality control, including criticality control, since the goal of the accountability system is to keep track continuously of the SNM. We shall attempt to summarize present capabilities for providing such a system. It should be clear that, in the absence of the GESMO supplement, we are not attempting a comprehensive review of the many proposed accountability schemes; in particular, we focus upon realtime systems which have the greatest potential for improvement, rather than upon present systems relying on periodic inventory.

Proposed accountability systems generally combine non-destructive assay (NDA) techniques with advanced computer technology, the NDA measurements providing the computer with data for real-time analysis. Development of such a system is complicated by the fact that SNM occurs in many different chemical and physical forms throughout the fuel cycle, so that a variety of measurement lechniques are needed. For example, plutonium will appear in acid solution at the reprocessing plant, in pellets at the fuel fabrication plant, and, in small amounts, in waste streams throughout the fuel cycle. Nevertheless, the measurements will involve some combination of weight measurements (to determine the total amount of material present), chemical analysis (to determine the amounts of various elements) and gamma ray and neutron measurements (to determine the amount of various isotopes present). We shall concentrate for the most part on the isotopic measurements. The fissionable isotopes of greatest interest have well defined gamma signatures resulting from their own decay or from the decay of a daughter isotope; for example, ${ }^{239} \mathrm{Pu}$ emits a moderately intense $\left(3.5 \times 10^{4} \mathrm{sec}^{-1} \mathrm{gm}^{-1}\right)$, interferencefree line at $413.7 \mathrm{keV}$. Consequently, high resolution $\mathrm{Ge}(\mathrm{Li}) \gamma-$ spectrometers offer a powerful technique for isotopic measurements. Passive neutron detectors are useful when the isotopic distribution of a sample is already well known. Most actinides decay primarily by alpha emission and these produce neutrons in interaction with light materials in the matrix. Coincidence counting of spontaneous fission neutrons yields a quantitative measurement of the amount of emitting isotopes. Thermally-fissile isotopic content of a sample can be determined by active neutron interrogation in which the sample is irradiated and the resultant fission neutrons detected in coincidence. This technique can achieve high precision only for well characterized materials.

It is clear that there are certain generic difficulties with the gamma ray and neutron measurements. First, the signal must be reasonably strong compared to the background. This generally implies that high resolution detectors are needed, such as $\mathrm{Ge}(\mathrm{Li})$ detectors for gammas versus the higher efficiency, lower resolution $\mathrm{NaI}$ crystals, and neutron coincidence counters for neutrons. Second, lack of precise knowledge about the composition and distribution of matrix material in the sample can lead to significant error, since gamma ray atteriuation by the matrix very of ten requires a substantial correction factor. Furthermore, self-attenuation will be signficant if the particles containing the gamma emitting isotope are too large. The attenuation problem is mitigated somewhat by concentration on high energy gamma rays $(>120 \mathrm{keV})$, where absorption is less dependent upon the composition of the material. Also, attenuation can be checked by measuring transmission strength through the sample of appropriate gamma rays from a calibrated source. Nevertheless, gamma attenuation remains a source of significant uncertainty in interpreting the results of NDA measurements.

Of course, measurement of any given radioactive isotope involves errors. The problems just outlined produce uncertainties and, in addition, factors such as detector efficiency and geometry of the measurement must be folded in. This uncertainty leads to the concept of MUF (material unaccounted for), defined as the difference between the amount of SNM which should be in the plant and that which is measured to be there. It must be stressed that existence of a MUF does not necessarily mean that SNM is missing, and the term might appropriately be replaced by MID (material inventory difference) or BPID (book-physical inventory difference). For example, selfattenuation effects lead to a MUF by producing a counting rate lower than expected for the quantity of SNM actually present. In general, 
FIG. VI.1. Accumulated MUF for a unit process as a function of successive batches. Data were generated using a simple Monte Carlo model. The upper curve is for uniform diversion of $10 \mathrm{~g}$ from each $1-\mathrm{kg}$ batch, while the lower curve is for the same throughout but with no diversion.

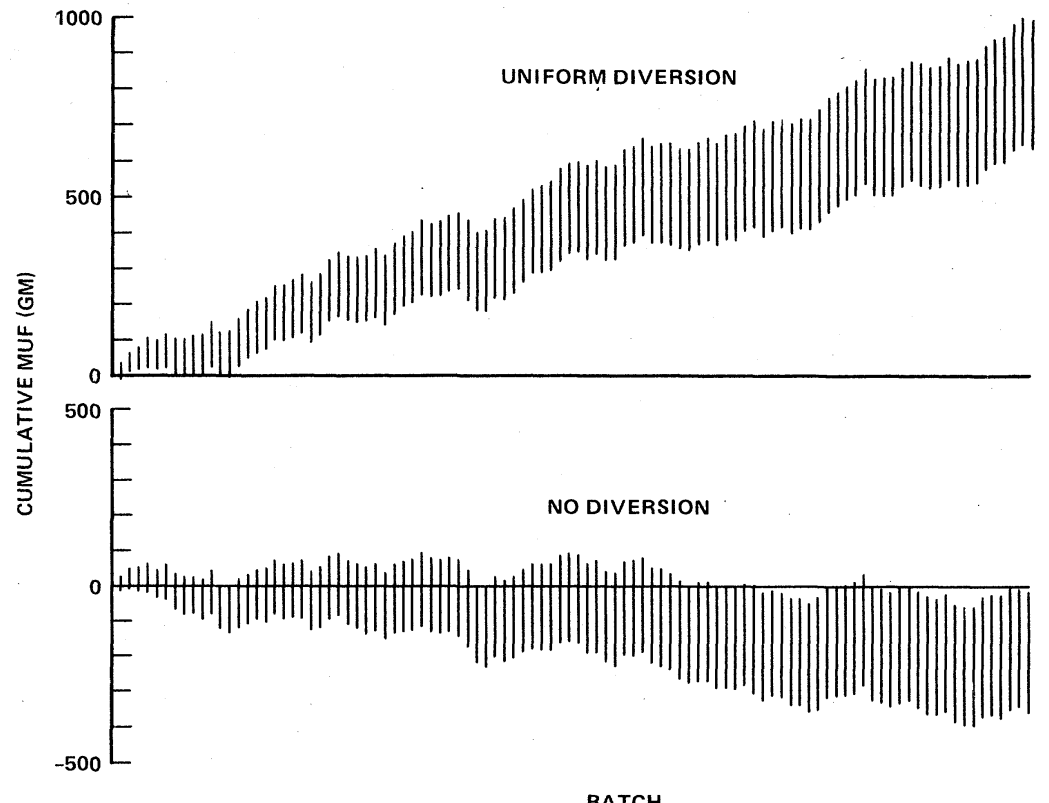

BATCH there can be several contributions to MUF, including the inherent measurement uncertainties, statistical and/or systematic, unmeasured in-plant holdup, unmeasured losses to waste streams, bookkeeping errors, as well as diversion or theft of SNM from the plant. The last component is the object of concern with regard to safeguards, but a meaningful bound on this can be attained only by strictly limiting the remaining MUF contributions.

Real-time systems are being studied at several laboratories. As one example, we have examined in detail the DYMAC (Dynamic Materials Control) system being developed at Los Alamos. This accountability system is based upon automated measurement devices at each unit process in the plant, each device interactively coupled to a central computer. First, the improved plant control resulting from the comprehensive unit process approach will appreciably reduce MUF contribution from unmeasured hold-ups and losses and will lend confidence to the attenuation correction factors applied to measurements. In addition, this approach will minimize the effects of a fixed fractional measurement uncertainty by greatly reducing the amount of SNM involved in a measurement "batch". Finally, the computer will both eliminate many possible bookkeeping errors and statistically analyze the measurements from every unit process and keep a record of the accumulated MUF between complete plant inventories. Consider a simple example, namely a Monte Carlo simulation of the unit process taken from Los Alamos Report LAUE-75-1817. The model assumes that measurements of input, product and residue are distributed normally about the true (representative) values $J_{0}=1000 \mathrm{~g}, P_{0}=900 \mathrm{~g}$ and $R_{0}=100 \mathrm{~g}$, respectively, for each batch passing through the unit process. The associated standard deviation is assumed to be $10 \mathrm{~g}$ for each measurement with the residue measurements comparatively less precise, so that each MUF value has a standard deviation of $17.3 \mathrm{~g}$. Assuming that 100 batches pass through the unit process between plant inventories, after which we start from zero again, the total process throughput would be $100 \mathrm{~kg}$ of SNM.

In order to illustrate the effectiveness of the DYMAC approach, consider the Monte Carlo simulation results shown in Figure VI.1 for the cases of no diversion and uniform diversion. We repeat that "diversion" here would include any unmeasured hold-ups or losses. For the uniform diversion, the diverter was assumed to have taken $10 \mathrm{~g}$ from each of the 100 batches, so that a total of $1 \mathrm{~kg}$ of SNM would have been diverted without any one diversion being detectable, i,e., the $10 \mathrm{~g}$ increment is less than one standard deviation of the MUF for a single batch. The figure shows the accumulated MUF, the midpoint of each bar representing the sum of the MUF values and the length of the bar, the standard deviation of the sum. The value of the cumulative MUF at the end of the 100 batch run corresponds to the MUF value that would have been obtained at physical inventory. The interesting result is that the uniform diversion case displays a clear signal that material is missing after only a few batches. Consequently if a diverter is to achieve some target quantity of SNM, the DYMAC system will force a considerably greater number of thef ts of smaller amounts, i.e., of amounts very small compared to the uncertainty of any one batch measurement. Thus, such trend analysis greatly increases the time required for significant theft and consequently the probability that other safeguards measures will lead to detection.

The system must also be tamper proof. The NDA instruments will probably have to be designed with sealed, internal calibration sources that can be assayed (looking at a slightly different, but significant, gamma energy level) in conjunction with the assay of the $\mathrm{Pu}$ and/or $\mathrm{U}$ with all signals fed to a central computer for analyses to detect any attempt at instrument modification, accidental or deliberate. In addition, having the NDA instrument output sent automatically to the accountability system computer would remove an opportunity for someone to modify records to hide a diversion. Access to the data in the computer file could be limited via remote terminals in the plant. Identity cards, fingerprint readers or some other method must be developed to allow only authorized personnel to activate the terminal. This procedure would still allow someone to enter falsified data, but diagnostics in the computer as well as procedural requirements could be developed to minimize if not eliminate the possibility of such an entry going undetected. For example, it might be a requirement that two people act in unison to make data entries to the accountability computer via remote terminals. Equipment and proecedures must be developed and demonstrated in an operating plant to show that the accountability system is not susceptible to covert manipulation.

The question remains as to the present status of dynamic accountability systems effective for monitoring flows of SNM through MOX fuel fabrication facilities and spent fuel separations facilities. Fully integrated data acquisition and computer systems do not presently exist. Various NDA instruments alluded to above, especially those based upon gamma assay, have proved highly reliable for measuring SNM in specific forms. In the absence of high background fission product activity and in a well characterized matrix, measurements of various important isotopes, such as ${ }^{239} \mathrm{Pu}$, can be made to accuracies of a few percent. However, these measurements have been carried out in what are essentially laboratory conditions and it remains to be seen how reliably such measurements can be carried out remotely in or at the process stream of a commercial scale fuel cycle facility. Considerable thought must be given to the mechanics of obtaining representative measurement samples at each unit process. In 
addition, the considerable electronic interfacing of the output from each SNM measurement device to a central data processor utilizing a uniform accounting format remains to be worked out. The newly constructed Los Alamos plutonium processing facility (TA-55 site) is fully equipped to perform unit process accountability measurements. The cost of the DYMAC system was approximately $2 \%$ of the cost of the plant, and this might be a representative number for commercial plants. However, the "complete" costs will become known only with specification of safeguards criteria and with operation of a commercial scale facility, since there can be savings due to increased process control and less frequent complete plant inventory or losses due to increased maintenance demands, for example, for the cryogenic system needed for $\mathrm{Ge}(\mathrm{Li})$ detectors.

In considering the possible application of a real-time system in a conmercial reprocessing plant, it must be stressed that the idea of applying complete accountability on the entire fuel cycle is probably unrealistic. There is a significant uncertainty in not knowing how much plutonium has been produced in the reactor. Direct measurements are not feasible because the plutonium is produced very unevenly throughout the core, so burnup codes must be used to predict the amount. However, the amount produced depends upon the detailed operating history of the specific core and the prediction cannot be relied upon for high accuracy. The first point at which accurate measurements can be made is after dissolution of the spen fuel. However, several waste streams will have been created by this point, and these do not lend themselves to highly accurate measurement; e.g., the small plutonium value remaining with the hulls cannot be measured accurately without complete dissoluton of the hulls themselves. Also, accurate measurement of the plutonium content in the dissolved fuel or in the HLW stream would require a series of separation steps on an appropriate sample. ${ }^{7}$ In fact, the first point at which accurate measurements are both practical and fairly easily made is after the separation column, when the plutonium is free of the fission product and other transuranic activity. Prior to this, the intense radioactivity and consequent inaccessibility make the plutonium-bearing material difficult and unattractive to steal Therefore, the goal of an accountability system should be to monitor accurately the flow of plutonium from the separations step until MOX fuel is inserted into a reactor.

After separations, solution assay systems (SAS) can be used to follow the ${ }^{239} \mathrm{Pu}$, which is always in liquid form. The SAS would consist of $\mathrm{Ge}(\mathrm{Li})$ detectors, in some cases coupled to flowmeters measuring total flow through pipes. Presently, measurement accuracies of $1-2 \%$ are obtained with $50 \mathrm{~g} /$ litre solutions with residence times of about one minute in a holding tank; a $50 \mathrm{mg} / \mathrm{litre}$ concentration, as might typically be present in an oxalate supernatant, would require at least an hour residence time for reasonably accurate measurement. Consequently, some engineering is needed to insure that either the necessary residence time or sample homogeneity is provided.

Various solid waste streams present a problem." These generally contain small amounts of plutonium in a variety of matrices (hulls, rubber gloves, filters, sludges, etc). Measurements of these streams can be expected to be far more inaccurate. One problem is that, with high $-Z$ elements in the matrix, the gamma ray measurements must be replaced with coincidence neutron counters and for the latter to be effective, the sample composition must be well known. Consequently, determination of the $\mathrm{Pu}$ isotopics after the separation column (with gamma and neutron counters) would aid later in measuring $\mathrm{Pu}$ in waste streams. Also one must consider the conversion plant which takes the plutonium nitrate solution from the separations plant and produces solid $\mathrm{PuO}_{2}$ powder. The calcination/filtration process by nature is very dusty and will lead to some loss of $\mathrm{Pu}$ into waste, e.g., in ventilation filters. Again, these waste streams are not accurately measured. In general, very accurate measurements in these various waste streams will be possible only if the plutonium is separated from the considerably larger amount of diverse matrix materials, e.g., by complete dissolution followed by solvent extraction. However, it is quite possible that such a process, besides being time consuming and costly, would both increase the total waste volume and further aggravate the safeguards problem. The small amount of plutonium with the solid wastes is not easily accessible. Obviously, the separation process would result in having the plutonium readily accessible in many "batches" at points outside the main process stream. This creates a new safeguards problem, namely, strict physical safeguard requirements at the analytical services facility. Consequently, it seems better to monitor the solid waste streams directly, simply to affirm that a large amount of plutonium is not going out with the waste. Research to lower the uncertainty in these measurements should be continued.

The situation is quite different for a MOX fuel fabrication plant. The input to the plant, $\mathrm{PuO}_{2}$ and $\mathrm{UO}_{2}$ powder, is well characterized both chemically and isotopically, and these remain in solid form throughout the fabrication process. Consequently, accurate weight measurements should suffice for the main process stream. Again, the solid waste streams require a different approach; gamma scans and coincidence neutron measurements will be needed for low-and high-Z matrix material, respectively. We stress that at both the reprocessing and fuel fabrication facilities, the accountability measurements can probably be made with high accuracy in the process streams but with considerably lower accuracy in the waste streams, which presumably contain only a few percent of the plutonium. This emphasizes the important complementarity between unit process accountability measurements and good process control. Control must be enforced over the lifetime of the plant, overcoming any problems of plant aging, of personnel turnover, and of simple carelessness.

In concluding this section, we repeat that the elements of a realtime NDA unit process accountability system are largely at hand and can augment the present non-real-time methods. Such a system is highly desirable for safeguards considerations as well as process control at the "back-end" of reprocessing plants and at MOX fuel fabrication plants. A realistic goal of such a system for overall fuel cycle accountability might be an uncertainty of a few percent of the plutonium. Such an uncertainty obviously represents a great deal of plutonium. Nevertheless, the safeguards value of such a system is significant because the unit process approach isolates and, in general, provides the most accurate measurements for units in which the $\mathrm{Pu}$ represents the most desirable target for theft; thus it can greatly complicate diversion by "insiders" and enhance the deterrent value of the overall safeguards system. Integration of the various elements remains to be done and demonstration of effectiveness and reliability in remote operation at industrial scale plants remains to be proved. While the parts largely exist and several systems have been under development for several years at different laboratories, the engineering of a complete, integrated, accurate system remains to be completed. We recommend that real-time, nondestructive-assay accountability systems be developed further for possible use for control of special nuclear materials in industrial-scale reprocessing and refabrication plants.

\section{E. International Safeguards--Denatured LWR Fuel Cycles}

International safeguards issues involve both certification of national safeguards programs in countries generating nuclear power and prevention of the use of commercial nuclear fuels and technology in advancing nuclear weapons programs. Denatured fuel cycles, meaning fuel cycles in which strategic quantities of weapons grade material appear separate from radioactive fission products only in secure, internationally controlled fuel cycle centers, have been proposed as an effective means to accomplish the safeguards objectives. In this section, we shall discuss denatured LWR fuel cycles $^{8}$ within the context of the technical information, expertise and facilities needed for nuclear weapons capability.

The aim of national safeguards programs is, in principle, identical to that already discussed for the United States; i.e., nuclear materials are to be protected from sub-national groups. The problem deserves special attention in the event that plutonium is recycled. It is expected that nuclear power reactors will be operating or under construction in about fifty nations within the next decade. If plutonium is to be an important energy resource of the future, then all countries will want a secure source of supply plus the knowledge and facilities to use it. International control must deal with all three factors.

Several governments have expressed the desire to develop spent fuel reprocessing technology in order to supplement their fuel supply. Doubts have been expressed ${ }^{9}$ (Wohlstetter, et al., 1976) that small 
and/or developing nations can institute safeguards programs as effective as those in advanced industrial nations. This problem is clearly one of international concern, since it is not difficult to move stolen materials across national borders.

The possibility of national diversion presents a considerably different problem. States have significant resources at their disposal. Further, physical security measures alone would not prevent governmental diversion from nationally-owned and controlled facilities. Even with international safeguards supervision of national facilities, the safeguards system may detect but not prevent overt governmental diversion. The safeguards system must also guard against covert use of fuel cycle facilities.

The deployment of denatured fuel cycles alleviates both of these problens in restricting the availability of weapons grade material. The presently used "stowaway" LWR fuel cycle fits our definition of a denatured cycle, although it would be preferable for safeguards to have spent fuel returned to a fuel supplier state or sent to an international facility for storage. With the advent of plutonium recycle, the denatured I.WR fuel cycle involves an extension of the co-location concept discussed in Section C. A detailed flow sheet is given in Chapter Vill, Figure 11. Basically, "national" reactors consume low-enriched uranium, and the spent fuel is returned to an internationally operated fuel cycle facility, where it is reprocessed to extract the plutonium and uranium. The plutonium is consumed in "international" reactors located at the facility, and low-enriched uranium fuel is returned to the national reactor. Obviously political and economic agreements are needed to fix the values of the nuclear materials and services involved. With the LWR U/Pu cycle, the ratio of "national" to "international" reactors is only about 2.5 consequently, economy of scale implies that clusters of "Pu-burners" would enhance the attractiveness of this cycle with regard to economics. ${ }^{10}$ The problems of having large concentration of power production capacity at international sites could be avoided if nuclear supplier countries, under IAEA supervision, executed the function of the international site. These countries could utilize the plutonium domestically without qualitatively altering the safeguards situation, assuming they are prepared to use MOX fuel. Therefore, to the extent that plutonium recycle proceeds in nuclear supplier states, there are no technical barriers to implementing such a denatured LWR fuel cycle. However, the political obstacles may be significant, since such a cycle may both restrict the options of and impose an economic penalty upon a participating nation. Implementation can be expected only with a clear consensus that such a cycle effectively reduces proliferation of nuclear weapons and, in fact, that such a reduction is indeed beneficial to all.

It must be remembered that with any denatured fuel cycle, international inspection is still essential in guaranteeing that fresh fuel and/or natural uranium are not being diverted to small enrichment facilities, that reactors are not being misused to produce plutonium and that small non-safeguarded separation facilities are not operated in addition to those at international centers. Such inspection goes beyond present IAEA agreements but must become part of any future political arrangements which institutionalize denatured fuel cycles.

An alternative advanced LWR denatured fuel cycle involves ${ }^{233} \mathrm{U} / \mathrm{Th}$ fueling (Feiveson and Taylor, 1976). The attraction here is that ${ }^{233} \mathrm{U}$, which is formed in the reactor by neutron capture in ${ }^{232} \mathrm{Th}$, can be denatured by dilution with ${ }^{238} \mathrm{U}$. A detailed flow sheet for the denatured fuel cycle is shown in Figure 12 of Chapter VilI. As discussed in Chapters $I I I$ and IV, reprocessing-refabrication are both essential and more complicated for this fuel cycle. Note that we have analyzed in detail only one example of a denatured thermal-reactor thorium cycle; this involves light-water reactors at both national and international sites. Also, we have insisted that the amount of plutonium outside the international site be minimized by working at the highest ${ }^{233} \mathrm{U} /{ }^{238} \mathrm{U}$ ratio consistent with the definition of denatured fuel."

Inspection of the denatured U/Th flow sheet reveals advantages as well as several problems for safeguards. First, the ${ }^{238} \mathrm{U}$ denaturant leads to the production of plutonium. For comparable power generation in national reactors, the amount of plutonium produced is reduced by about a factor of seven compared to that in the U/Pu cycle (See Chapter VIII); this is primarily a reflection of the comparative
${ }^{238} \mathrm{U}$ initial loadings in the two cases. Nevertheless, the amount is still large compared to that needed for a weapon and still represents a potentially signficiant energy resource. Consequently the same safeguards considerations that apply to the low enriched uranium cycle apply here as well: Assuming that the energy potential of the bred plutonium is to be realized, an internationally operated plutoniumburner reactor is needed. However, the ratio of national to international power generation is approximately fifteen, so that the international fuel cycle center could serve a comparatively large number of national reactors. This is a signficant improvement relative to the previously discussed LWR-uranium-plutonium cycles. On the other hand, if the plutonium were intentionally left with the wastes during reprocessing, then the wastes themselves become a safeguards issue (as are the spent fuel rods in the uranium stowaway option), and should be stored under international control.

A second problem is that highly enriched urnaium is needed as make-up to maintain reactivity of the recycled fuel. While this enrichment would go on under international control, the special attractiveness of high enriched uranium for both handling and weapons design makes this a potentially significant safeguards problem. This requirement could be eased by working with more dilute ${ }^{233} \mathrm{U}$ in the fresh denatured fuel, but more plutonium would be produced in the national reactors as a result.

Another safeguards issue is raised by the use of $20 \%{ }^{233} \mathrm{U}$-content fuel. The work required to enrich this further to weapons grade material $^{12}$ is comparatively small. This can be illustrated quantitatively for ${ }^{235} \mathrm{U}$; in raising ${ }^{235} \mathrm{U}$ enrichment from $0.7 \%$ to $93 \%$, approximately $90 \%$ of the work is done in reaching $20 \%$ enrichment. Furthermore, the substantially larger mass difference between ${ }^{233} \mathrm{U}$ and ${ }^{238} \mathrm{U}$ reduces the relative work needed to enrich denatured ${ }^{233} \mathrm{U}$ to weapons grade. Although the radioactivity of recycled uranium containing ${ }^{233} \mathrm{U}$ and ${ }^{232} \mathrm{U}$ presents difficulties for commercial scale isotope separation, the technology to carry out such enrichment on non-economical, non-commercial scale is available in the open literature. This requires further evaluation.

Therefore, denatured LWR thorium cycles have both safeguards advantages and disadvantages with respect to the uranium cycle. Less plutonium is produced, but the safeguards considerations are not qualitatively altered. The ratio of national-to-international power, assuming plutonium-burner reactors are used at the international site, is increased signficantly. If the plutonium is not utilized, safeguards must still be applied to the stored plutonium. Disadvantages lie in the increased complication of the associated reprocessing-refabrication and in the possible safeguards problems of introducing high-enriched uranium make-up and of utilizing fuel which can be enriched to weapons grade with comparatively little work. In any case, the thorium denatured cycle does not represent a near-term alternative because of the technical developments still needed (see Chapter IV).

Whether safeguards on nationally located isotopic enrichment plants are considered adequate depends upon the technology employed. For example, gaseous diffusion plants designed to produce only $3 \%$ uranium would not be readily convertible to weapons production. If appropriately constructed, it would be necessary to take over the facility in open violation of safeguards agreements and largely to rebuild the plant. A centrifuge facility, on the other hand, is more suited to the clandestine production of weapons and, in such a situation, these facilities should be placed at international facilities.

In summarizing the role of denatured fuel cycles, it must be remembered that these options may be very important not only with regard to proliferation but also with regard to the national safeguards problem which would exist when many nations have significant nuclear power generation. Denatured fuel cycles have the effect of restricting easily accessible weapons material to a comparatively small number of internationally controlled sites. Consequently, the safeguards measures could be enforced uniformly and stringently, as might not always be the case with a multitude of individual national programs. The thorium cycle has additional beneits with regard to reinforcing national safeguards in that the spent fuel is less attractive for theft. This follows because ${ }^{232} \mathrm{U}$ in the fuel appreciably complicates the handling problems, after separation, for subnational groups and because the lowered concentration of plutonium in the 
fuel implies that a correspondingly greater amount of intensely radioactive fuel must be processed to obtain weapons material.

Furthermore, we have discussed LWR denatured fuel cycles in order of decreasing near-term availability. It is clear that the nature of the international institutional/political agreements needed to implement any of the cycles is essentially the same and that the technical development needed to implement the more advanced resource-efficient denatured fuel cycles applies most directly to the fuel cycle facilities at the international site. Therefore, if the energy potential of plutonium bred in thermal reactors is to be realized, a "natural" evolution of denatured fuel cycles can be envisioned in which the international site progresses from a stowaway site to a U/Pu reprocessing/refabrication center to a U/Th fuel cycle center. Simultaneously, the transition from the U/Pu to the U/Th cycle would mark a decrease in the ratio of international to national reactor power; consequently, it might be natural that the first international centers be located in present nuclear supplier states, which already have access to plutonium and which presently have most of the world's nuclear generating capacity, while the later more advanced centers could be located elsewhere as part of a regional multinational fuel cycle. In any case, as implied above, the most difficult problems reside in formulation and ratification of the necessary international agreements, which are equally important for near-term denatured fuel cycle options as for those offered by the U/Th cycle.

In evaluating the effectiveness of denatured fuel cycles for slowing nuclear weapons proliferation, it is important to examine the technology needed for assembling a weapon. There are several avenues toward nuclear weapons capability, entailing either completely indigenous efforts or misuse of commercial fuels. ${ }^{13}$. Proof that developing nations can construct nuclear explosives without the benefit of a nuclear power industry is offered by the Indian test of such a device, fueled by plutonium bred in a research reactor and separated in a small-scale non-commercial reprocessing facility.

For the purposes of this discussion, it is useful to adopt Wohlstetter, et al. (Wohlstetter, 1976) classification of countries according to competence for nuclear weapons construction. These are:

Category 0: Nuclear weapons built and tested.
Category 0: Nuclear explosion detonated, but no weapons claimed.

Category 1: Well-developed nuclear technological base and access to fissile material.

Category 2: $\quad$ Operating reactor (a power or research reactor) and some technological base.

Category 3: None of the above.

The majority of nations are still in Categories 2 and 3 . A concern is that, even without an explicit decision to acquire nuclear weapons, non-nuclear weapons states may "drift" toward the acquisition of such weapons as the necessary materials and technological expertise grow; i.e., with the development of nuclear power, Category 2 and 3 nations will rise to Category 1. For nations possessing this level of nuclear technology and experience, there is no technical barrier to constructing a weapon. Wohlstetter, et al. (Wohlstetter, 1976) estimate that this could be done within a year at a cost of less than a million dollars; in turn, the shortened lead time could itself be a factor in the decision as to whether or not to build a device. For our purposes, it is not necessary to accept all the details of the argument leading to this estimate. It is clear that some drift may occur with or without denatured fuel cycles. The aim of the denatured fuel cycle is to limit and/or complicate access to weapons grade material.

For governments consciously embarking upon a nuclear weapons development program, several possibilities exist even with denatured fitel cycles. Possible routes ${ }^{14}$ for Category 2 or 3 nations are summarized in Table VI.2, together with a very general characterization of the availability of the requisite knowledge, experience and facilities and of the design effort required for a modest weapons program. The first two approaches, using plutonium production reactors or isotope separation, of fer completely indigenous approaches; the last involves misuse of commercial spent fuel. We shall discuss these approaches below because they form the context in which denatured fuel cycles must be evaluated. It is worth noting that development of commercial nuclear power is likely to have the indirect effect of making generally available the experienced personnel and facilities (research reactors and small-scale non-commercial

TABLE VI.2. Possible routes to nuclear weapons capability for small or developing nations (assuming access to low-enriched or natural uranium).

\begin{tabular}{|c|c|c|c|}
\hline & $\begin{array}{l}\text { Production } \\
\text { Reactors }\end{array}$ & $\begin{array}{l}\text { Isotope } \\
\text { Separation }\end{array}$ & $\begin{array}{l}\text { Commercial } \\
\text { Reprocessing }\end{array}$ \\
\hline \multicolumn{2}{|l|}{ i } & . & . \\
\hline Technical & & \multicolumn{2}{|l|}{ Limited } \\
\hline Information & Available & Availability & Avai1ab1e \\
\hline Experienced & \multicolumn{3}{|l|}{ Genera11y } \\
\hline Personne 1 & Availab $1 e$ & Limited & Limitod \\
\hline Facilities & Limited & Unavaitab1e & Unavai1able \\
\hline \multicolumn{4}{|l|}{ Fissile } \\
\hline Material & Weapons grade & \multirow{3}{*}{ Enriched ${ }^{235} U$} & \multirow{3}{*}{$\begin{array}{l}\text { Reactor grade } \\
\text { plutonium }\end{array}$} \\
\hline \multirow[t]{2}{*}{ Produced } & plutonium & & \\
\hline & & & \\
\hline \multirow{4}{*}{$\begin{array}{l}\text { Weapons } \\
\text { Design } \\
\text { Sophistication }\end{array}$} & Medium & & \multirow{4}{*}{$\begin{array}{l}\text { Medium to high } \\
\text { (implosion weapon; } \\
\text { high neutron } \\
\text { background) }\end{array}$} \\
\hline & (implosion & Low (gun- & \\
\hline & weapon ) & type weapon) & \\
\hline & & & \\
\hline
\end{tabular}


reprocessing plants) for the production reactor and reprocessing routes. Further developments in isotope separation are likely to do the same for the enrichment route. Needless to say, a significant pool of personnel experienced in fuel cycle activities is an important asset in a developing weapons program.

Lamarsh (Lamarsh, 1976) studied the possibility that a small and/or developing nation might design and construct its own plutonium production reactor. He concludes that, except for natural uranium, all design information and components are openly available; furthermore, the necessary natural uranium resources exist in many nations and can be fairly easily purchased in international trade. As an example, the reactor analyzed was a natural-uranium-fueled, graphite-moderated, air-cooled nuclear reactor, basically a simplifed version of the Brookhaven Graphite Research Reactor, for which all design and performance parameters have been published. This is a simple reactor to design, build, and operate, involving neither enrichment nor production of heavy water. A $30 \mathrm{MW}$ reactor would take about four years to design and build, assuming only moderate personnel requirements, and would produce about $10 \mathrm{~kg} \mathrm{Pu} /$ year. For low burnup, the separations would be comparatively easy and the plutonium of better quality for explosives design (i.e., almost pure ${ }^{239} \mathrm{Pu}$ ) than is commercially produced plutonium. Furthermore, virtually all the information needed to construct a low to moderateyield implosion type weapon is available in the open literature, ${ }^{15}$ including neutron transport theory and fast critical assembly, critical mass tables, high explosive design and performance, etc.; an obstacle in constructing reliable, high-yield devices might be in initiator design. Therefore, although the result will most likely be something less than the reliable, high-yield, compact explosives tested by the nuclear powers, it is clear that indigenous nuclear weapons programs are possible even for countries with modest industrial bases.

Another indigenous method for acquiring weapons would be through enrichment of natural uranium. This approach has the advantage of yielding high-enriched ${ }^{235} \mathrm{U}$, with which the simple guntype weapon is possible. Commercial-scale isotope separation plants, such as the American gaseous diffusion plants, require enormous capital expenditures and power requirements. However, the boundary conditions for a small weapons program are very different, not requiring large throughputs or competitive economics. Several separation technologies, such as those using nozzles, thermal diffusion, gas centrifuges or, eventually, lasers, may be suitable for these purposes. For example, Wohlstetter, et al. (Wohlstetter, 1976) estimate that an adequate centrifuge facility could be established in approximately five years at a cost on the order of one to two hundred million dollars.

Other routes to nuclear weapons might employ either spent or fresh fuel from commercial reactors. In effect, neither the selfprotection nor the enrichment assumptions used in discussing national safeguards may be valid in reference to small-scale governmental efforts. The technology for separating plutonium from high-burnup commercial fuel (reviewed in Chapter IV) is well known, although experience is not yet widespread. However, without the constraints of high throughput, economics, and low occupational exposures, this technology is within the grasp of most nations. An even simpler variant might be to insert natural uranium in the core of a power reactor with the purpose of breeding in short irradiations ${ }^{16}$ enough plutonium for a weapon.

With regard to fresh, low-enrichment fuel, it is important to note that more than half the separative work necessary to produce highly enriched ${ }^{235} \mathrm{U}$ has already been done in producing low-enrichment ( 3 to $3.5 \%)$ LWR fuel. Consequently, a nation may be willing to invest the time and money to "top off" the enrichment of a small fraction of supplied LWR fuel using a technique which does not have a sufficiently great mass flow to be practical commercially. Centrif uge technology has been mentioned already. A technically simpler example would be calutron enrichment. The Calutron program at Oak Ridge achieved currents of $0.1 \mathrm{Amps}$ with a high resolution beam $(0.8$ Amps with low resolution); higher currents are difficult to obtain because of space charge limitations. Starting with 20\% enriched uranium, which was fixed by the AEC as the enrichment below which nuclear weapons construction becomes impractical, such a device could produce a critical mass of weapons grade uranium in about ten years; obviously, this time could be reduced by the construction of several such cyclotrons. In any case, misuse of fresh and/or spent fuel in denatured fuel cycles is within the technological capacity of a large number of countries.

In summary, international safeguards problems in the LWR U/Pu fuel cycle involke both certification of national safeguards programs and avoidance of nuclear weapons proliferation. With regard to the latter, it is important to distinguish between a commitment to nuclear weapons and a drift towards acquisition of such weapons as the nuclear technology and materials base grows. Denatured fuel cycles could contribute substantively in diminishing the threat of subnational theft and in limiting access to weapons grade material. On the other hand, there are no insurmountable technical barriers preventing a small or developing nation, committed to a nuclear weapons program, from successfully constructing a nuclear explosive. The lead time required will invariably be shortened as nuclear technology becomes more widely available; ultimately, political restraints against proliferation must be relied upon.

Footnotes

1 The term special nuclear materials (SNM) was defined in the Atomic Energy Act of 1954 to include enriched uranium, plutonium, and ${ }^{233} \mathrm{U}$. However, we shall apply the term SNM to a slightly more restricted set of materials, namely, materials usable in nuclear weapons without isotopic separation, i.e., low enriched uranium is excluded.

$2{ }^{238} \mathrm{Pu}$ could be used as a denaturant in the sense that large amounts of this isotope would lead to such a significant self-heating that the nuclear device could not be assembled, but it would be impractical to incorproate such amounts in commercial nuclear fuel.

${ }^{3}$ The fabrication plant flowsheet does not involve liquid streams in the main process stream. However, chemical operations are involved in scrap recovery.

${ }^{4}$ Very low acidities $\left({ }_{\sim} 0.5 \mathrm{M}\right)$ must be avoided so that the $\mathrm{Pu}$ does not polymerize.

5 This represents an extensive examination of fuel "spiking" concepts, namely, incorporation of gamma emitters and of neutron emitters and absorbers in or near the fuel.

6 This could probably be lowered to $\$ 6-7 / \mathrm{mg}$ if the market were to grow.

7 A direct solvent extraction process would $n \circlearrowleft t$ be adequate, since there could be non-extractable species in the sample.

8 A more general discussion of the technical aspects of denatured fuel cycles, involving both $\mathrm{Pu}$ and ${ }^{233} \mathrm{U}$ recycle in LWR and breeder reactors, is given in the discussion of advanced fuel cycle alternatives (Chapter VIII)

9 For example, see Wohlstetter, et al., 1976.

${ }^{10}$ Such a denatured LWR U/Pu fuel cycle can, of course, be applied also to the domestic power economy. However, the large clusters of nuclear reactors required in such a plan do not appear likely in the near future. See the Nuclear Energy Center Site Survey, NUREG-0001.

${ }^{11} \mathrm{By}$ lowering the ${ }^{233} \mathrm{U} /{ }^{238} \mathrm{U}$ ratio, the enrichment of the uranium makeup fuel could be lowered at the expense of having more plutonium produced in national reactors. Since the make-up fuel would be generated inside the international site, we have assumed minimization of the plutonium production is the controlling consideration.

12 It must be remembered that the $20 \%$ cutoff in enrichment is somewhat arbitrary (see Table 3D)-1 in Chapter III). It is still conceivable that weapons could be made with such material, although large masses would have to be assembled in short times for non-negligible yield.

${ }^{13} \mathrm{We}$ are not concerned with other possible routes which do not use natural or low-enriched uranium as the starting material (e.g., purchase of a fabricated weapon from another country).

${ }^{14}$ It should be noted that less "conventional" approaches are also possible. For example, a possible safeguards problem in the use of protactinium in a weapon is discussed in Appendix VI.1.

${ }^{15}$ For example, see J. Foster, Encyclopedia Americana.

${ }^{16}$ With the CANDU reactor, this can be done with minimal impact on the commercial operation. 


\section{References}

1. Ayers, 1975

Ayers, R. W. 1975, Harvard Civil Rights--Civil Liberties Law Review 10, 369, Policing Plutonium: The Civil Liberties Fallout.

2. Cleveland, 1970

Cleveland, J. M. "The Chemistry of Plutonium", (Gordon and Beach, Inc., New York, 1970)

3. Crandall, 1977

Crandall, J. 1977, private communication.

4. Feiveson and Taylor, 1976

Feiveson, H. A., and T. B. Taylor 1976, Bulletin of the Atomic Scientists, page 14.

5. Foster, 1973

Foster, J. S. 1973, Encyclopedia Americana 20, "Nuclear Weapons (New York).

6. IRT-378-R, 1975

International Research and Technology Corporation Report IRT-378-R 1975, Modification of Strategic Special Nuclear Materials to Deter Their Theft or Unauthorized Use.

7. Keeny, et al, 1977

Keeny, S. M., Jr., et al. "Nuclear Power Issues and Choices", Report of the Nuclear Energy Policy Study Group (Ballinger, 1977)

8. Keller, 1971

Keller, C. "The Chemistry of the Transuranic Elements", (Verlag Chemic Gmbh., 1971).

9. Lamarsh, 1976

Lamarsh, J. R. 1976, Library of Congress, Congressional Research Service QC 170 Gen.

10. NCRP-44, 1975

NCRP Report No. 44. 1975, Krypton-85 in the Atmosphere-Accumulation, Biological Significance, and Control Technology.
11. NUREG-0002

NUREG-0002. Final GESMO.

12. NUREG-75/060, 1975

NUREG-75/060. Special Safeguards Study, Scopes of Work, U.S. Nuclear Regulatory Commission, June 1975; See also WASH-1327, Draft GESMO, August, 1974, Volume 4, pp. V39 to $\mathrm{V}-45$.

13. OTA, 1977

Office of Technology Assessment. United States Congress, "Nuclear Proliferation and Safeguards", (Prepublication Draft, April 1977)

14. Penneman, 1977

Penneman, R. 1977, private communication.

15. Puechl, 1975

Puechl, K. H. 1975, Nuclear Engineering International 20, 687.

16. Ryabohikov and Ryabukhin, 1970

Ryabohikov, D. I. and Ryabukhin. "Analytical Chemistry of Yttrium and the Lanthanide Elements", (Humphrey Science Publishers, Ann Arbor, Mich., 1970).

17. Shapiro, et al., 1977

Shapiro, J. R. Rec and R. A. Matzie. 1977, EPRI Report NP359, Assessment of Thorium Fuel Cycles in Pressurized Water Reactors.

18. Walling, 1977

Walling, M. 1977, ORNL, private communication.

19. Willrich and Taylor, 1974

Willrich, M. and T. B. Taylor. 1974, "Nuclear Theft: Risks and Safeguards, Ballinger Publishing Company (Cambridge, Mass.).

20. Wohlstetter, 1976

Wohlstetter, A., et al. 1976, U.S. Arms Control and Disarmanent Agency Report ACDA/PAB - 263, Moving Toward Life in a Nuclear Armed Crowd? 
CHAPTER VII. High-Level and Transuranic Waste Management

A. Issues, Conclusions and Recommendations

\section{A1. Issues}

The source of the largest potential radiological hazard associated with wastes from the nuclear fuel cycle is the high level waste (HLW) stream from fuel reprocessing, or the spent fuel assemblies in the absence of reprocessing. The viability of a nuclear energy industry depends upon the ability to dispose of this material reliably and safely. A second category of solid wastes, which includes a wide variety of materials such as cladding hulls and miscellaneous wastes from fuel reprocessing and refabrication steps, and which will be referred to as transuranic (TRU) wastes, represents a problem comparable in importance to that posed by isolation of the actinide component of the HLW. The first set of conclusions in this section of the report addresses the question of the feasibility of long term isolation from the biosphere of high level and transuranic waste.

This report considers plans and options relevant to the future development of the nuclear energy industry and therefore does not address two additional problems of HLW management. One is what to do with the stored HLW currently stored at the non-operating the Nuclear Fuel Services (NFS) Plant in West Valley, New York (NUREG 004?). The other concerns plans for the disposal of the wastes generated by the AEC-ERDA weapons program. The fission product activity $(\sim 600 \mathrm{MCi})$ of these weapons associated wastes is comparable with that of commercial spent fuel currently in storage pools (Willrich, et al., 1976). The magnitude of the disposal problem may also be put in perspective by noting that the current volume of stored defense wastes is comparable with (Rowe and Holcomb, 1974) or greater than (Willich, et al., 1976) that projected to be generated by the commercial industry to the year 2000, assuming reprocessing of commercial spent fuel. The difficulties of disposal of the NFS and the military program waste inventories are exacerbated by problems of older technologies involving both the chemical composition and the details of physical storage of the waste, which preclude straightforward application of the techniques developed for treatment of future commercial wastes. We emphasize the need for careful evaluation and solution of these problems but confine the present discussion to the problem of future commercial HLW.

Federal regulations, $10 \mathrm{CFR} 50$, require that the high level liquid wastes from fuel reprocessing plants be converted to solid form within five years after reprocessing and that this solid waste be delivered to a federal waste repository within ten years after reprocessing. The design and operation of this repository is the responsibility of ERDA. ERDA is also actively engaged in development and demonstration projects to show the feasibility of one or several solidification processes.

ERDA 76-43, Technical Alternatives Document or TAD (ERDA$43,1976)$ is a five volume document summarizing the state of development as of September 1975 of many aspects of the waste management program and includes descriptive summaries of work both in the U.S. and Europe. It includes discussion of treatment methods appropriate to non-high level wastes as well as high level wastes, of "main line" processes, which could form the basis of a waste management technology within a few years, and of more sophisticated concepts which could not be brought to fruition until 10 or more years in the future. Estimates of the environmental impact of the waste management portion of the fuel cycle, based in large part on the GESMO (NUREG-0002, 1976), have recently appeared in NUREG0116. The discussion in this chapter is based principally upon information available as of the early winter, 1976, but is occasionally tempered by more recent developments.

A likely sequence for handling HLW from fuel reprocessing involves the calcining (high temperature drying and conversion dominantly to the oxides) of the liquid wastes, mixing of the resulting powdered calcine with powdered glass of appropriate composition, melting of the mixture, $\sim 1 / 4$ wastes and $\sim 3 / 4$ glass, and casting and sealing in canisters, possibly stainless steel, about $0.3 \mathrm{~m}$ in diameter and $3 \mathrm{~m}$ long. These canisters would then be shipped to a federal nuclear waste repository for first temporary or "retrievable" and then "terminal" storage. "Retrievability" implies the ability to remove the waste from storage with essentially the same techniques and equipment as were required for emplacement. "Terminal" storage would be a second phase in which the need for recovery was no longer anticipated. In the event of an extended deferral of reprocessing, spent fuel may be stored for possible future reprocessing, either in surface facilities or in geologic repositories, or may be treated as a waste and isolated much as HLW produced in fuel reprocessing.

Several time scales are of relevance in the characterization of encapsulated wastes or spent fuel. Tens of years or less represent typical times anticipated for the operational phase of a waste repository during which one might wish to handle waste canisters without concern over loss of mechanical integrity. In a thousand years the activity of the wastes or spent fuel decays by three or more orders of magnitude because of decay of the fission products. Residual activity of the actinides may be of concern for as long as $10^{5}$ to $10^{6}$ years.

Because of the extensive data base and experience gained with salt over the past twenty years, the first waste repository test facility probably will be mined caverns in deep bedded salt deposits. The HLW would be stored retrievably for a period of two to five years while tests are carried out to verify that the predicted behavior -thermal, chemical, and mechanical -- of the surrounding salt formation is indeed observed and that no unforeseen difficulties arise. The data and experience gained in this stage would then add to the basis for a decision whether to convert the facility to operate in a non-retrievable or terminal storage mode. A second group of conclusions concerns ERDA's program to cope with waste management needs in a timely manner. Problems of technological readiness as well as regulatory and licensing delays are involved, and strategies depend upon choices among recycle and non-recycle options for the nuclear fuel cycle.

The TAD describes additional options in waste management techniques including alternate geological waste isolation modes, more sophisticated wasteforms, and the partitioning of HLW into fission product and actinide components. A final group of conclusions comments on some of these advanced waste management concepts.

\section{A2. Conclusions}

\section{A2a. Feasibility of Waste Isolation}

1. The geologic and hydrogeologic conditions that can provide for satisfactory isolation of radioactive waste exist in a sufficient number of places that we anticipate no difficulty in locating several suitable sites in different geologic media within the immediate future. (Section F6)

2. Considerations of the management of $H L W, T R U$ waste and spent fuel do not put significant constraints upon choices among the various fuel cycles discussed in this report. In particular, arguments concerning such waste management are not important in deciding between recycle and non-recycle fuel cycle options. (Section B3)

3. Current knowledge and technology are adequate to design and locate a suitable waste repository of the conventional mined type, if utilized with appropriate site selection criteria. (See recommendation A 3-3 and Section E.) In particular,

a. the repository would not be breached instantaneously as a consequence of either surface nuclear explosions or meteorite impact and it would not be directly compromised by erosion;

b. it is highly unlikely that the repository would be compromised by tectonic or volcanic activity;

c. modifications of the geologic environment related to emplacement of the waste would not compromise the waste isolation.

4. Non-random drilling or mining by a future generation 
searching for natural resources may represent a threat to the integrity of a repository. This threat would likely be more serious for a repository in salt than in other recommended media; however, we conclude that a repository could be located so as to minimize this possibility. (Section E3)

5. Ground water is the only transport medium of importance for radioactive waste emplaced in a repository. A favorable groundwater regime can provide isolation as stable and as effective as that provided by the physical integrity of the geologic unit in which the waste is emplaced, in that the radionuclides would be transported so slowly that they would not reach the biosphere during the desired period of isolation. (Section F6)

6. Our confidence in the ability to isolate HLW, TRU waste and spent fuel from the biosphere for periods greater than 30 years is based upon estimates of the efficacy of geologic isolation, irrespective of wasteform solubility. Leachability data characterizing currently available glasses suggest that immobilization of HLW in glass may provide substantial protection against dissolution and release of radionuclides to a transport medium on time scales of $10^{3}$ years or more. However, there are uncertainties in the extrapolation of these data to the behavior, in geologic environments and for long times, of actual waste-glasses produced in routine industrial-scale processing. These uncertainties preclude reliance on the durability of glass as the principal barrier, in itself, to the release of radioactive species. (Section G3)

\section{A2b. Current Program}

1. We expect that a repository site in bedded salt with suitable hydrogeology can be found, although an adequate data base does not yet exist to permit completion of the recommended analysis of groundwater flow and mass transport. We foresee no difficulty in obtaining the required data for a specific site and in completing such an analysis within the next few years. We conclude that at least one test facility should be developed in a medium other than salt, preferably granite, and thoroughly evaluated before the decision is made to develop a facility into a full repository. (Section $\mathrm{H} 3 \mathrm{a}$ )

2. We foresee no important technical barrier to the implementation by 1985 of the technology for the solidification, encapsulation, transport and emplacement of commercial HLW into mined salt caverns. Retrievability would require improved design of protective sheathing and of emplacement details to protect the canisters from corrosive attack by occluded brine in the salt and from mechanical entrapment by creep deformation of the salt. The absence of appropriate licensing regulations and the need for NEPA procedures may introduce a substantial time delay that could prevent operation of a waste repository by 1985. (Sections C1, C2 and C3)

3. Spent muclear fuel which has not been reprocessed should be stored in such a manner as to allow recovery of the fuel with minimal economic penalties. Several technical options are available including pool storage, air cooled surface storage and geologic storage. Geologic interim storage, particularly in salt, may require special care to avoid damage to the fuel by corrosion but is easily converted to terminal storage if that is desired at a later time. In any event, whatever time scale is adopted regarding a decision on reprocessing, we conclude that two waste repository test facilities, in different geologic media, should be completed in an orderly and timely manner and one should be demonstrated to be licensable. (Section 3C)

4. A greater and more clearly defined effort directed toward improved understanding of waste-rock interactions is required that would include experimental evaluation in a waste repository test facility. (Section $\mathrm{H} 3 \mathrm{a}$ )

5. Modeling techniques under current development, which include consideration of transport processes and concentration attenuation mechanisms as well as some direct measure of the potential hazard to man of a particular radionuclide, are essential to provide a sound basis for waste management planning. In contrast, arguments for waste management decisions based on simple hazard indices or rules of thumb concerning the number of half lives of decay of a particular radionuclide, can be very misleading. (Section B2 and F7)

\section{A2c. Future Alternatives}

1. We expect that increased confidence in our ability to handle $H L W$ and TRU wastes will lead to removal of the current emphasis on retrievability. Of various disposal concepts for deep continental geologic isolation that could then be considered, we conclude that a rock melting technique and waste canister emplacement utilizing superdeep drilling may provide the best alternative. (Section D3c)

2. We consider seabed isolation an unacceptable alternative at this time, because of uncertainties about the reliability of fabricated containment and about the migration of radionuclides within ocean sediments and in the underlying rock once waste containers are breached. Work in progress may remove these uncertainties. (Section D3a)

3. Uncertainties about climatic factors and ice sheet stability and about residence times of both ice and glacier-derived water within ice sheets make ice sheet isolation an unacceptable alternative. (Section D3b)

4. The development of wasteforms that provide a reliable barrier 10 long-term radionuclide release, and/or increased understanding of radionuclide transport, could open additional options for the isolation of $H I . W$. For the U.S. these options might be more desirable for a variety of reasons, including geographic diversity and cost effectiveness. For some other countries they may open important alternatives for isolation. Developing a highly durable wasteform should be possible; establishing confidence in its long-term durability will be more difficult. (Section G1 and G3)

5. Transmutation of partitioned actinide waste is not a sensible procedure for disposal of actinides without the development of highly efficient chemical partitioning of the wastes and of dramatic reductions in the magnitude of the TRU waste generated in refabrication and other steps of the fuel cycle. Such chemical processing is unlikely to be available before the next century and clear incentives to pursue partitioning and transmutation of actinides have not been established. Analyses of the incentives for reactor transmutation of partitioned wastes must include consider tition of the consequences of the resultant much larger actinide inventories in reactors and reprocessing plants. (Section D2)

\section{A3. Recommendations}

1. High priority should be given to the timely completion and continued refinement of Federal regulations and standards concerning required solidification, processing, transport and subsequent storage or isolation of high-level and transuranic wastes and spent fuel elements. (Section B2)

2. We recommend the orderly development and construction of two repository test facilities, each in different geologic media. Procedures should be initiated for licensing of the more favorable of the two, and the needs for storage should dictate when that facility should be developed into a full repository. Depending upon projected waste volumes, desired redundancy, and other factors, the second test facility may or may not be developed as a full-scale repository. Although we see nothing wrong with developing a test facility in salt, we recommend that, because of unresolved factors such as future anthropogenic activity that may most affect salt, a test facility also be developed in at least one other medium, preferably granite, before the decision is made to complete a full-scale repository. Whatever time scale is adopted with regard to reprocessing, we strongly recommend that development proceed until licensability can be demonstrated for one facility. (Section H3c).

3. For selection of sites for geologic waste repositories, we recommend the following criteria which we regard as necessary and sufficient to define the basic geologic integrity of the repository for the long term:

a. Appropriate depth and location. Site selection criteria should include specifications on the depth of a repository which must be met to minimize the possibility of breaching by surface explosions, meteorite impact, or erosion, as well as specifications on the probability of breaching by faulting or volcanism. (Section E) 
b. Suitable medium. A waste repository should be placed in a geologic medium that is sufficiently devoid of mineral or element concentrations or that is found in a sufficient number of places that it would not be inadvertently breached by a future generation seeking mineral resources. (Section E3)

c. Satisfactory hydrogeology. Criteria for selection of a waste repository site should include specifications of appropriate hydrogeologic parameters. These specifications should be satisfied by the present-day hydrogeology and by the projected bounds of the future hydrogeology of any specific site. (Section F7)

We recognize that additional criteria will be needed to define economic, general environmental and other factors such as exploration and engineering procedures employed in the location, development and operation of any particular site; such factors must not be allowed to compromise the criteria listed above, which are central to safe longterm nuclear waste management.

4. Because unreprocessed spent fuel is a potentially valuable resource, the waste management program should develop the capability to store L.WR spent fuel for several decades if required. Storage, either in surface or geologic facilities, should allow later recovery of fuel without substantial economic penalties. (Section C3)

5. Further engineering development should be carried out to assure protection of waste canisters from corrosive deterioration and mechanical entrapment during the period of retrievable storage. Detailed studies are needed of the effects of the possible generation of hydrogen and other gases of radiolytic or corrosive origin. (Section $\mathrm{C} 3$ and $\mathrm{H} 3 \mathrm{e}$ )

6. Modeling techniques should be further developed for improved assessment of both generic and site specific waste isolation options. Such studies should also be used to provide definition of quantitative goals for improved wasteform development programs. (Section B2, F7 and G2)

7. The relative magnitudes of the actinide activity in mill tailings, TRU wastes and HLW or spent fuel, per unit of energy generated, suggest that all three waste streams may present problems of comparable magnitude for the very long term (i.e., greater than a thousand years). Careful evaluation of the relative hazards to present and future generations associated with current and proposed trealments of all waste streams is needed to provide a necessary perspective to decisions concerning waste management. (Section B2 and $G 5)$

8. We recommend active research on rock melting concepts of waste disposal that would be carried out in parallel with current programs related to conventional mined cavities. Because salt limestone, and shale may not be appropriate host media for emplacement that involves rock melt, we further recommend the immediate development of a test facility in granite, which would be appropriate for this purpose. In addition to being a highly acceptable rock type for retrievable storage in conventional mined cavities, development of a repository in granite could lead logically and systematically to a superior future disposal alternative involving rock melt by perhaps the mid 1990's. (Section D3c and H3b)

9. We recommend evaluation of the technology for emplacemen of waste canisters litilizing superdeep drilling. (Section D3c)

\section{B. Alternate Fuel Cycles and Waste Management}

\section{B1. Fuel Cycle Comparisons}

The nuclear fuel cycle alternatives considered here are:

1. Pressurized water reactor (PWR) with no fuel reprocessing;

2. PWR with reprocessing for uranium recycle and plutonium storage;

3. PWR with reprocessing for uranium recycle and self-generated plutonium recycle;
4. High-temperature gas-cooled reactor (HTGR) with uranium recycle.

The high-level wastes from these fuel cycles are compared in terms of a hazard index, defined as

hazard index ${ }^{a}=\sum_{i} \lambda_{i} N_{i} /(R C G)_{i}$

where $\lambda_{i}=$ Radioactive decay constant of nuclide $\underline{i}$

$N_{i}=$ number of atoms of nuclide $i$

$(\mathrm{RCG})_{\mathrm{i}}=$ radioactivity concentration guide for nuclide $\underline{\mathrm{i}}$ for public consumption

a Also denoted as "toxicity index" in some references. Even though referred to herein as a "hazards index", a true and proper measure of hazards must include other factors not incorporated in the presently defined index.

For geologically implanted wastes the ingestion hazard index is a more meaningful measure than the inhalation hazard index (Cohen, 1977). Using the water RCG for public drinking water, the ingestion hazard index is then the total volume of water required to dilute the wastes to public drinking water standards. This calculated hazard index is thus a crude measure of the potential danger of radioactive material. However, it is quite limited in validity as a true measure of hazard because it takes no account of the pathways, and barriers thereto, for a radionuclide in a solid waste material to reach the biosphere and to be assimilated by mankind.

The ingestion hazard indices for the high-level wastes from reprocessing non-recycled PWR fuel are shown in Figure 7B1, calculated on the basis of wastes produced from one gigawatt year of reactor operation (Pigford and Choi, 1976). After a fission-product decay period of about 600 yeers, the hazard index is governed by $2+1.243 \mathrm{Am}$, followed successively by ${ }^{239} \mathrm{Pu},{ }^{226} \mathrm{Ra},{ }^{225} \mathrm{Ra},{ }^{129} \mathrm{I}$, and finally by decay daughters of residual ${ }^{238} \mathrm{U}$. However, these are the important radionuclides during each time period only if all nuclides have the same probability of being ingested by the same number of people, and that is unlikely to be the case.

Hazard indices of high-level wastes from the four alternate fuel cycles (Pigford and Choi, 1976) are compared in Figure 7B2. Relative to the example of Figure $7 \mathrm{~B} 1$, recycling plutonium increases the production of americium and curium, whose radioactivity and decay daughters increase the ingestion hazard index by about an order of magnitude during the period governed by actinides and ${ }^{226} \mathrm{Ra}$. The hazard index of unreprocessed fuel, which contains all of the plutonium discharged from the reactor, is about thirty times higher than the hazard index of wastes from reprocessing non-recycled fuel. The hazard index of HTGR high-level wastes is low after the fissionproduct period because there is relatively little ${ }^{238} \mathrm{U}$ in the fuel to form ${ }^{239} \mathrm{Pu}$; however large quantities of ${ }^{226} \mathrm{Ra}$ and ${ }^{225} \mathrm{Ra}$ appear during the interval from a few hundred thousand to a few million years. ${ }^{1}$

Also to be considered are the low-gamma-level and intermediategamma-level transuranic wastes from reprocessing and similar wastes from fabricating recycled fuel. Although the actinides are at relatively low concentrations ${ }^{2}$ in these wastes, the total amount of plutonium is within the same order of magnitude as that ultimately appearing in the high-level wastes. The sources of waste plutonium and radium for self-generated plutonium recycle are compared in Table 7B1. The hazard indices of these transuranic wastes are about $14 \%$ of those of the high-level wastes after fission products and americium have decayed.

The ingestion hazard indices for the high-level wastes from reprocessing non-recycle fuel are compared with those of other residuals from this same fuel cycle in Figure $7 \mathrm{B3}$ (Pigford and Choi, 
TABLE 7B1. Amount of ${ }^{239} \mathrm{Pu},{ }^{240} \mathrm{Pu}$, and ${ }^{226} \mathrm{Ra}$ potentailly present in wastes from pressurized water reactor with self-generated $\mathrm{Pu}$ recycle. ${ }^{\mathrm{a}}$

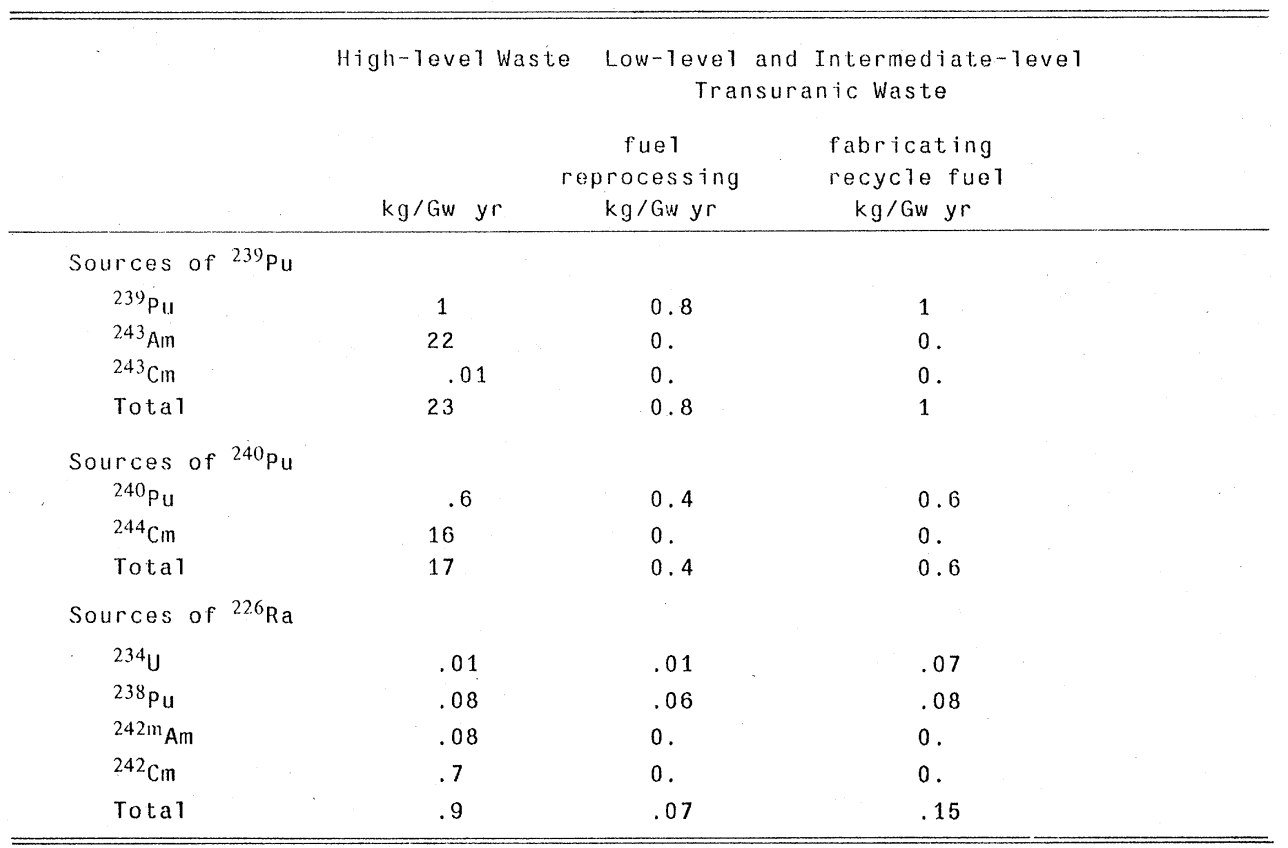

a Quantities are calculated for waste formed after $1 \mathrm{Gw}$ yr of operation +150 days pre-processing cooling (Pigford and Ang, 1975). Losses to wastes (Kee, et al., 1976): 0.5\% of U and Pu and $100 \%$ of Am and $\mathrm{Cm}$ to high-level wastes, $0.38 \%$ of $U$ and $P u$ to intermediate and low-level reprocessing waste, and $0.5 \%$ of $U$ and $P u$ to

1976). These indices are normalized to that of the uranium ore mined for one gigawatt year of reactor operation. The ore hazard is due mainly to ${ }^{226} \mathrm{Ra}$, which is in secular equilibrium in the ${ }^{238} \mathrm{U}$ decay chain. In the processes of milling and concentrating uranium ore ${ }^{226} \mathrm{Ra}$ and its precursor 80,000 yr ${ }^{230} \mathrm{Th}$ follow the tailings, so the ore ingestion hazard is preserved there for a few hundred thousand years until ${ }^{230}$ Th decays. Thereafter the tailing hazard continues at a lower level determined by the residual uranium in the tailings, assumed here to be $5 \%$ of the uranium processed. If the depleted uranium from

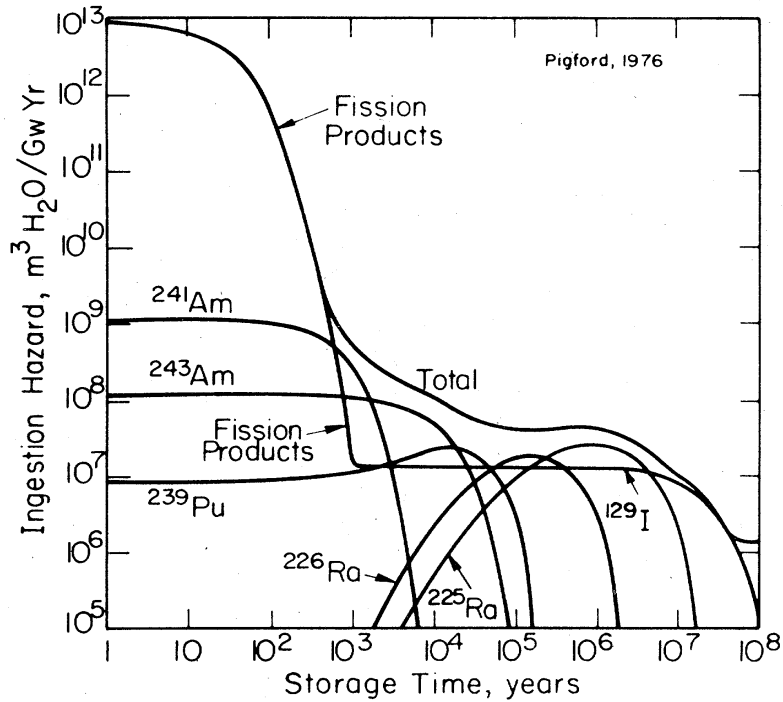

FIG. 7B1. Principal contributions to the ingestion hazard index at HLW from the reprocessing of a uranium fueled LWR as a function of time. isotope separation is never used for breeder fuel, the uranium daughters, particularly ${ }^{226} \mathrm{Ra}$, in this stored $\mathrm{UF}_{6}$ will be eventually restored to a hazard index level within a few percent of that of the original ore.

The hazard index of the high-level wastes falls below that of the original ore after a period of about 600 years. The total hazard index of all residuals falls below that of the original uranium ore after a

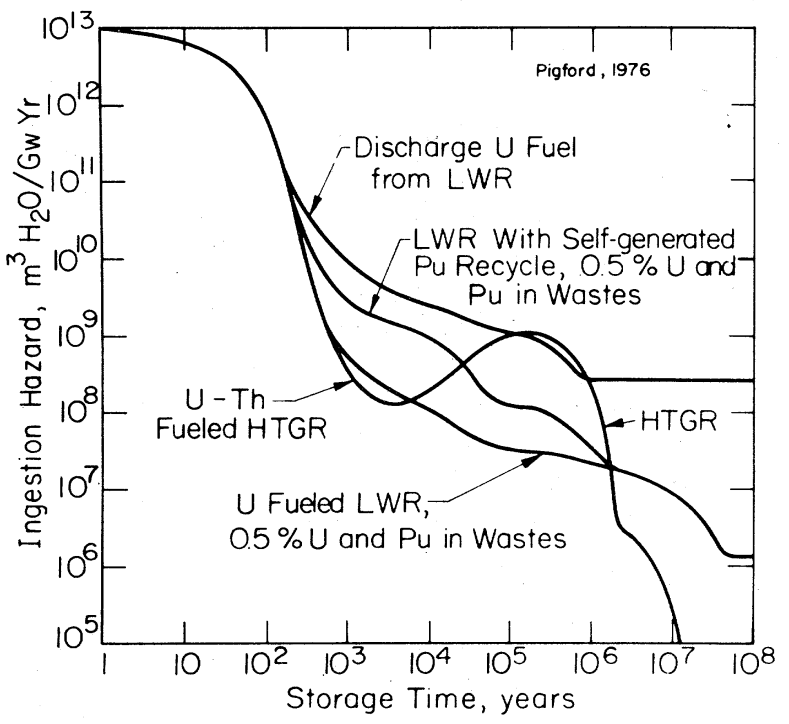

FIG. 7B2. Ingestion hazard index of high-level wastes from LWR, with and without reprocessing, and from HTGR (LWR = Light Water Reactor, HTGR = High-Temperature Gas-Cooled Reactor). 


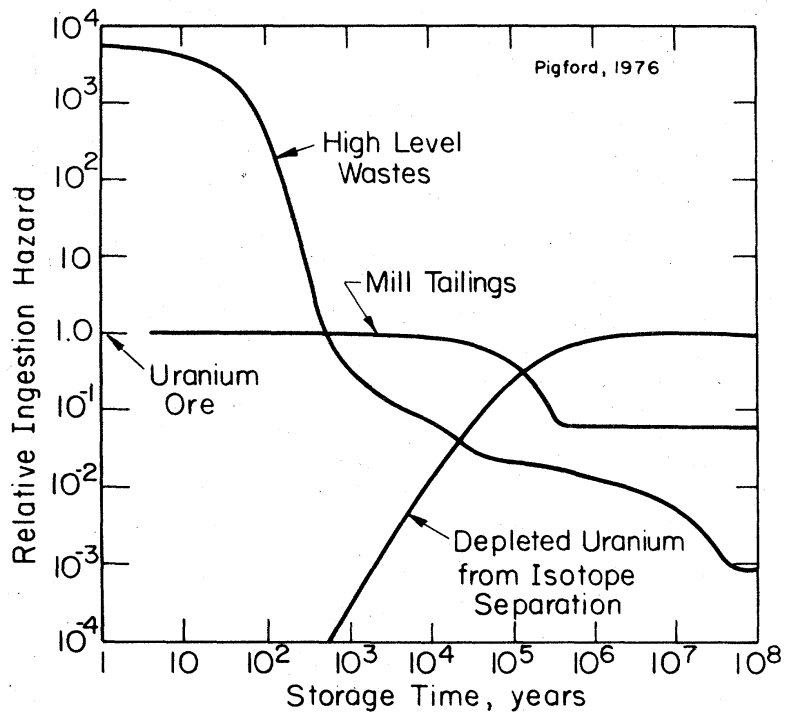

FIG. 7B3. Relative ingestion hazard of solid residuals from light-water reactor fuel cycle (U Fuel, $0.5 \%$ of $\mathrm{U}$ and $\mathrm{Pu}$ in high-level waste).

decay time of about 140.000 years. This reduction results from the enrichment of natural ${ }^{234} \mathrm{U}$ in isotope separation and its destruction in the reactor by neutron absorption, thereby depleting one of the sources of ${ }^{226} \mathrm{Ra}$.

\section{B2. Hazard Indices and Criteria}

It is misleading, however, simply to make comparisons of this hazard index in evaluatin'g alternative waste management options. For example, for a given radioactive waste the relative value of the ingestion hazard index is a measure of the relative hazard at a given decay time only if the radionuclides principally contributing to the hazards at this and other decay times all have the same probability of reaching mankind. This is unlikely to be the case. For example, Figure 7B1 indicates that for the high-level wastes from a uraniumfueled PWR the ingestion hazard index of ${ }^{239} \mathrm{Pu}$ at 10,000 years is about the same as the hazard index of ${ }^{226} \mathrm{Ra}$ at $200,000 \mathrm{yr}$. However, these relative hazard indices do not reflect meaningfully the relative hazards if there are appreciable time delays involved in radionuclide transport. Assuming geologically isolated wastes reach ground water at the same rate during each of these decay periods, and assuming that the ground water moves a considerable distance through earth before surfacing, more of the radium would be expected to reach potable water because of less adsorption and ion exchange of radium with the soil and because of the longer half life of the radium parent, $80,000-$ yr ${ }^{230} \mathrm{Th}$. Therefore, it would be expected that the radium would ultimately, at some later time period, result in a greater dose rate and a greater individual hazard than the ${ }^{239} \mathrm{Pu}$. This is borne out by the calculations of Burkholder, et al. (Burkholder, et al. 1975) for transport of radioactive nuclides through desert soil. Only in the case of some postulated accident, which might intrude upon the geologic isolation and bring the wastes relatively quickly to a source of potable water, do the theoretical ingestion hazards appear to have much direct relation to real hazards.

Another index frequently quoted in discussing hazards of radioactive waste is the ingestion hazard per unit volume of material. This is illustrated in Figure 7B4, where the high-level waste hazard indices of Figure 7B1 have been divided by the volume of high-level wastes produced per gigawatt year. This volumetric hazard index is frequently compared with the hazard per unit volume of natural uranium ores, as indicated in Figure 7B4. This comparison has led to the proposition that the waste hazard index per unit volume must be reduced to that of pitchblende or of some lower-grade ore to achieve an acceptable solution to the problem of radioactive waste

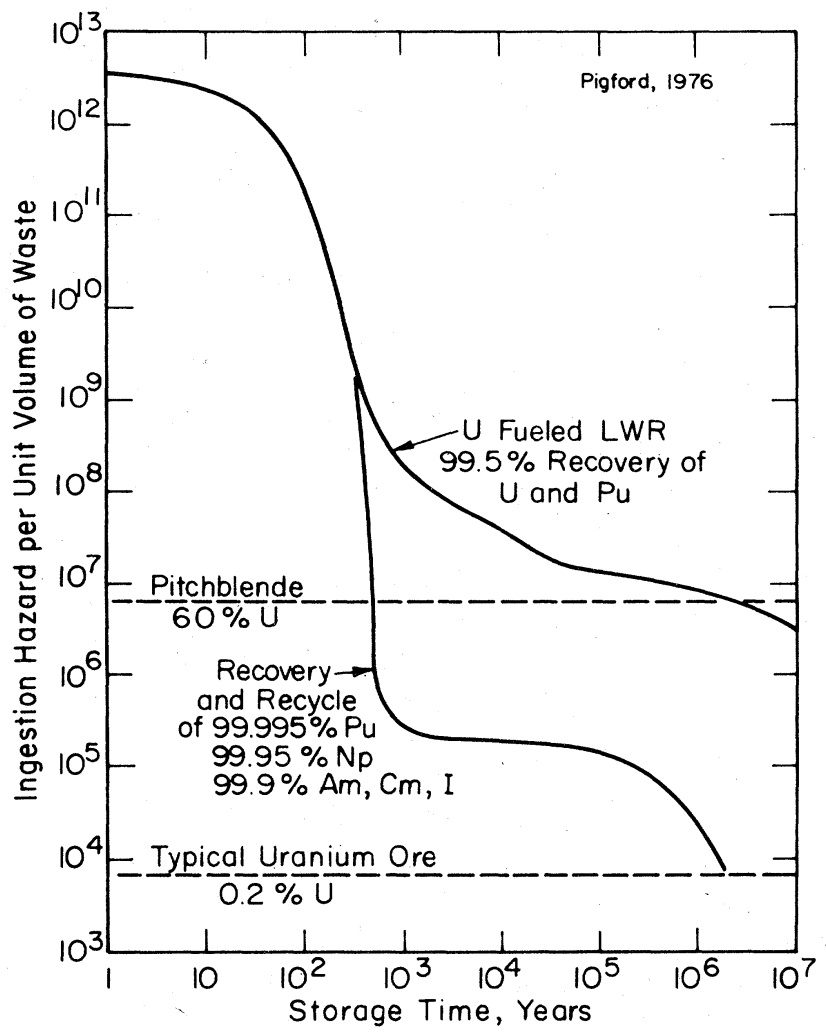

FIG. 7B4. Ingestion hazard index per unit volume of waste from the reprocessing of uranium fueled LWR with and without partitioning compared with the index for pitchblende and a typical uranium ore.

management. From the same comparison it has been proposed that because the waste hazard index does not fall below that of pitchblende until about one million years, these wastes constitute a radiological hazard and must be contained and isolated for as long as one million years (Willrich, et al., 1976). This same comparison with the volumetric hazard index of ore has led to the proposal that if the actinide hazard index per unit volume could be reduced about a hundred fold, as shown by the suggested recovery parameters for actinide partitioning and recycle indicated in Figure 7B4, the volumetric hazard index would fall below that of natural ore and the time period of concern over the hazards of radioactive wastes could be reduced to the several hundred years of fission product decay (Claiborne, 1972). It is obvious that regardless of whether the hazard is presented per gigawatt year or per unit volume, a hundred-fold reduction in actinides results in a hundred-fold reduction in the hazard index. What is not demonstrated, however, is that comparison with the hazard index per unit volume of pitchblende or of other ore is a meaningful criterion for assessing the hazards of radioactive waste management.

It is not contemplated that these wastes are to be distributed throughout the earth's crust in a manner simulating nutural ores. Assessment of hazards from a waste isolation facility must instead take into account the effectiveness of the many barriers that isolate the wastes from the biosphere. Instead of relating hazards on the basis of a hazard index per unit volume, it would seem more logical to credit the potential benefits from the highly concentrated forms of wastes. The smaller volumes to be handled should more easily result in practicable technology for reliable geologic emplacement and isolation. If improperly used, the measure of hazard index per unit volume of wastes may obfuscate the potential hazards of the lowconcentration transuranic wastes, which have already been discussed above. Indeed, if low hazard per unit volume were a principal measure of our objectives in waste isolation, it would be far easier to 
obtain the goal of low volumetric hazard by dilution than by such sophisticated and expensive schemes as actinide partioning and recycle schemes requiring very high chemical recoveries. However, the index of hazard per unit volume could be meaningful in relation to anthropogenic issues. In considering the possibility of later inadvertent mining of wastes implanted in a geologic repository, highly diluted wastes would remove the chance of striking concentrated radioactive material.

Another criterion frequently assumed for suggesting the time span over which radioactive wastes are a significant hazard to the public is the time for the decay of a particularly toxic radionuclide to a trivial level. Adopting the usual rule that a radionuclide is of no real concern after it has decayed for ten half lives, i.e., its amount has been reduced by a factor of about $10^{3}$, we find the frequent assumption (Willrich, 1976) that the geologic waste isolation facility must provide special protection to the public for ten half lives of ${ }^{239} \mathrm{Pu}$, i.e., 244,000 years. Evidently this is based upon the recognition that ${ }^{239} \mathrm{Pu}$ is one of the radionuclides of potential hazard, as shown in Figure 7B1. Considering the other nuclides which are controlling at later periods, one could just as well demand ten half lives of the ${ }^{226} \mathrm{Ra}$ precursor ${ }^{230}$ Th or $8 \times 10^{5}$ years, ten half lives of 129 I or $1.7 \times 10^{8}$ years, or even ten half lives of ${ }^{238} \mathrm{U}$ or $4.5 \times 10^{10}$ years. The ${ }^{2,38} \mathrm{U}$ criterion is obviously without merit, because it seems likely that any geologic waste isolation facility reasonably selected will provide a greater protection and lower hazard to mankind from the ${ }^{238} \mathrm{U}$ decay chain than the hazards already experienced from natural uranium ores--not because the wastes contain a higher or lower concentration than the natural ores but because they are in geological formations selected for the purpose of isolation. Similarly, physical phenomena, such as insolubility, ion exchange-adsorption and slow migration, can yield a low estimated hazard for plutonium in a properly selected geologic isolation facility. Therefore, there is no basis now for assuming that isolation for ten half lives of any of these radionuclides represents any meaningful criterion for facility design.

It is apparent that there is a growing need for more realistic analyses of the hazards from geologically isolated radioactive wastes. The problem differs from that of evaluating hazards over the lifetimes of reactors or of reprocessing facilities where, by suitable decommissioning the hazards from those facilities disappear at the end of the operating life. Here we do not know over what length of time hazards may exist. However, the basic criteria as to reasonable and allowable radiation exposures for normal releases, if they occur, and for accidental releases could be reasonably adopted from those criteria developed for other licensed operations. One of the first tasks is to apply such criteria in analyses which have the purpose of defining the time span over which the hazards are non-trivial.

As a first step the hazards could be reasonably assessed in terms of individual dose rates, calculated over a time span sufficient to encompass dosi rates in the ranges considered significant to nuclear facility licensing, i.e., as specified in 10 CFR 20 regulations. Calculating population exposures is more nebulous because of the difficulty of making any meaningful estimates of population densities and locations so far in the future. However, there do not now appear to be specified criteria in nuclear facility licensing in terms of population exposures, and there is no apparent reason for burdening the designers of the geologic disposal facility with making such an estimate if we have no yardstick for its evaluation in present licensing practice.

Estimates of enormously large population doses result from integrating what may be very small dose rates over large populations and very long periods of time, as illustrated by Comey (Comey, 1975) in his calculation of population exposures from mill tailings over a 80,000 -yr. period. Such estimates can be put in perspective by comparison with reference estimates of exposure of the same population for the same time period to other sources such as background radiation. (See Chapters III and V.)

Claiborne and Gera (Claiborne and Gera, 1974) and Burkholder, et al. (Burkholder, et al., 1975) have illustrated the kinds of methodology that can be applied towards the evaluation of hazards from geologically isolated radioactive wastes. The fact that the hazards from mill tailings may also be important over a similar time span is all the more reason for proceeding with this kind of hazards analysis. Hopefully, we will begin to learn more about the hazards than what is so vaguely implied by the hazard indices now used.

\section{B3. Implications}

In the absence of more refined measures of the relative hazards of different radioisotopes, the hazard index may be used as a crude measure of the potential waste problems associated with the different fuel cycles. Figure 7B2 indicates that there are no dramatic advantages of one fuel cycle as compared with another in terms of the radiological hazards :ssociated with isolated HLW. On the 300 year time scale, the fission products dominate and are essentially the same for all cycles. On the longer time scale there is a range of about thirty in the ingestion hazard. The most favorable case, however, is for uranium only recycle with plutonium storage, which cannot be compared with the others until the ultimate fate of the plutonium is decided. In view of the anticipated efficacy of geologic isolation, the range of 30 or less in hazard of the wastes after 1000 years does not give a strong incentive for choosing one alternative fuel cycle over another.

The magnitude of the TRU waste problem, as indicated by Table $7 \mathrm{~B} 1$, is made substantially worse by reprocessing and fuel recycle. This is a consequence of the creation of new low and intermediate level waste streams associated with the reprosessing and fuel refabrication which were obviously not present in the stowaway or throwaway cycles in which all wastes, except perhaps some volatiles associated with the spent fuel, are contained by the fuel cladding and subsequent encapsulation. Thus the advantage of the reduced actinide content of the HLW in the case of recycle, as compared with that in the spent fuel for non-recycle, is offset by the need to process the TRU waste streams. The uncertainties (discussed in detail above) implicit in the use of the hazard index further indicate that arguments such as these are hardly persuasive one way or another in deciding among alternative fuel cycles.

Other differences between fuel cycles may influence the waste management problem. For example, the thermal effects on long time scales $\left(>10^{3}\right.$ years) are greater by substantial factors $(\sim \times 3)$ for spent fuel than for HLW. Economic factors are unlikely to be important in view of the estimate (Section IVG) that waste management costs are about $1 \frac{112 \%}{2}$ of the total fuel cycle cost. Although influencing details of repository design, none of the factors we have identified concerning waste management are of determining importance in the choice among fuel cycles.

\section{Currently Available HLW Management Technology}

\section{C1. Solidification}

Calcination at full commercial scale and glassification at reduced scale have been demonstrated with both hot and cold simulated wastes and tests are in preparation for a full scale demonstration of glassification in a remote facility (ERDA-43, 1976). The adequacy of the resulting glass as a satisfactory wasteform is an important and complex question. Two rather distinct time scales are of importance. One concerns the thermal stability and mechanical strength and integrity of the material during the time of manufacture, handling, transport to the repository, emplacement and retrievability, namely the time in which the internally generated heat is high and during which an accident might expose the waste to man's immediate environment. The other concerns the stability and leachability during the time after conversion of the repository to a terminal storage mode when the wasteform durability offers a possible barrier to radionuclide release. This second question of the adequacy of the glass wasteform as a barrier to radionuclide release is discussed in Section G. Present ERDA planning relies on geologic isolation as the principal barrier to transport of radionuclides to the biosphere (Kuhlman, 1976), and current arguments for the safety of HLW management techniques do not rely on wasteform durability on time scales of 100 years or longer.

On the short time scale ( $\sim 30$ years) the glass encapsulation of the wastes gives a preferred solution to the handling problem, despite some advantages of the alternative of storage as powdered calcine. An argument in favor of storage as a powdered calcine is that it avoids the expense and the additional occupational exposure associated with operation and maintenance of the vitrification step in the 
solidification plant. The availability of a single step process (ERDA$43,1976)$ for conversion of liquid waste to a glass wasteform could remove this advantage. Also, the high residual canister stresses (BNWL-1932, 1975) produced by the differential contraction of metal and glass during cooling, may lead to problems of stress-induced corrosion during the period of retrievable storage which could be avoided by using the powdered calcine.

These possible advantages of the calcine, however, are outweighed by concerns over the problems associated with dispersal of the waste in case of an accident, either in handling or transport. With the calcine the waste is easily dispersable either by air transport or dissolution and water transport. A monolithic glass block, or even glass reduced to fragmented or granular form by impact associated with an accident or by devitrification (Smith and Ross, 1975), represents a much more satisfactory form to care for in case of a spill than a powdered calcine with particle size the order of microns (McElroy, et al., 1976). Similarly if, for any reason, it is necessary to recover stored waste from a waste repository after substantial deterioration of the encapsulating canister, the glass wasteform will make that task far simpler. In addition the higher thermal conductivity of the glass over the powder allows the use of larger diameter canisters without the development of an excessive centerline temperature which could lead to the release of volatile species from the waste. This affords some advantage in ease of handling simply by reducing the number of canisters involved. Finally the much higher durability (lower leachability) of the glass may provide an additional barrier to radionuclide release on the 1.00 year time scale or longer, although, for reasons discussed in Section G, currently available data do not warrant the extrapolation of experimentally determined leach rates of test glasses to longer times or to uncertain hydrochemical environments. There may be questions, as well, concerning the quality control that would be required to achieve these leach rates routinely in a commercial scale operation. Nevertheless, the choice of the more durable wasteform seems a prudent one, irrespective of one's ability to prove conclusively its adequacy on the longer time scale.

\section{C2. Transportation}

Concerning the transportation of solidified waste from reprocessing plant to repository, the extensive experience in the design and transport of spent fuel bundles provides the necessary design base. Risk analysis studies are in progress at Battelle Northwest Laboratory to develop material for the Environmental Impact Statement on waste management. Ne see no reason to suppose that the transport of the solidified wastes should represent a serious hazard.

\section{C3. Emplacement and Retrievability}

Emplacement. In the concept of conventional mined cavities, the waste canisters are to be stored in widely separated (e.g., 10m) holes in the floor of the mined cavern. Current concepts of equipment for handling and emplacing the canisters were developed by ORNL during the development of the Lyons, Kansas site for waste disposal and are described in Project Salt Vault (Bradshaw and McClain, 1971) and TAD (ERDA-43, 1976). As with the transportation, the extensive experience in the nuclear industry with remote handling equipment should allow the development of a satisfactory technology for the emplacement, and retrieval if necessary, of canisters which are in good mechanical condition.

Retrievability. There are several motivations for operating a waste-isolation repository in a retrievable mode, at least for an interim period. Experiments possible during a retrievability period will allow improved confidence in the estimates of the thermal and mechanical response of the geological environment to the heat load produced by the waste. Although one cannot "prove" the concept of geologic isolation, one can increase one's confidence by demonstrating that the short-time effects are consistent with predictions. A variety of experiments concerned with the interaction of the waste with the surrounding medium will also be possible, extending those already carried out in Project Salt Vault (Bradshaw and McClain, 1971). If improved waste treatment technologies are developed during the period of retrievability it may be desirable to retrieve the waste for further processing to a more durable form. Finally, the adoption of the retrievability concept, simply by keeping open a variety of options, seems a prudent decision in the early stages of technology evolution.
There are of course penalties resulting from the requirement of retrievability. The technical facilities must be designed to allow for this added option, ventilation must be maintained during the period of retrievability, and the encapsulation and emplacement of the canisters may become more complex as a consequence of the need for protective sleeves and/or overpacking. Monitoring of the stored canisters may imply higher occupational exposures. There may be problems associated with the creep of the surrounding rock and the need to maintain access to the storage vaults.

For the case of a repository in bedded salt the effects of the interaction of the salt environment with the waste canister during the proposed 2-5 year period of retrievability are of some concern. Current plans call for the insertion of a carbon steel liner in the emplacement holes to isolate the waste canister from direct contact with the salt and to allow easy removal of the canister for visual inspection and for overpacking if deterioration is observed.

The main threat to the concept of retrievability in this instance is the presence of small inclusions of brine, a fraction of a millimeter in size, typically found in bedded salts. In the presence of a thermal gradient these inclusions migrate toward the source of heat and as a consequence can deliver the brine into the cavity in which the waste canisters are emplaced. Estimates (Jenks, 1972) based on a concentration of trapped brine of $0.5 \%$ by volume and a welldeveloped theory of the migration mechanism suggest that a total of about 301 of brine will be delivered to the canister in the 50 year period during which the thermal gradients are of significance. This corresponds to roughly one $\mathrm{cm}^{3}$ of brine per $\mathrm{cm}^{2}$ of canister surface area. Two possible problems associated with the brine migration are the evolution of $\mathrm{H}_{2}$ and other gases, and the corrosion of the waste canister.

The rate of generation of $\mathrm{H}_{2}$ is calculated to be such that the normal ventilation provided in the storage caverns to keep heat levels tolerable will be more than adequate to prevent the build-up of an explosive mixture of $\mathrm{H}_{2}$ in air (Jenks, 1972). These estimates in fact indicate that explosive concentrations would build up only after a year with no ventilation whatsoever. Similarly any toxic gases released as a product of radiolytic action are released sufficiently slowly that ventilation adequate to maintain reasonable temperature for human access assures that there will be no significant accumulation of these gases. The effects of possible build up, after conversion to terminal storage, of $\mathrm{H}_{2}$ or other gases generated by the interaction of the waste canisters with the salt are unlikely to be important, but require more detailed study. In particular we would urge the direct emplacement of a few canisters, without concern for retrievability, during the test phase of a repository to gain additional information about corrosion and gas evolution which would be relevant to later development of the repository after the retrievability requirement is dropped.

The corrosion of the waste canister represents a more severe problem. Results of the Project Salt Vault experiment (Bradshaw and McClain, 1971) showed attack in the salt environment of heater shrouds and simulated waste canisters constructed of type 304L stainless steel, this attack presumably resulting from the release of the included brine. The surface of the simulated canister and heaters which were maintained at temperatures well above the boiling point of the brine, though superficially in good condition, showed extensive stress corrosion cracking to a depth of one-half of the wall thickness of about $6 \mathrm{~mm}$ in the case of a stainless steel heater shroud. Much more severe was the attack of the stainless steel conduit feeding this heater. This attack was at a position on the conduit surmised to be where condensation of the brine-generated steam was occurring. A similar corrosive attack of an unprotected HLW canister would seem likely; in view of the substantial variation in temperature along the canister resulting from the dead volume above the waste, condensation would be expected at some point on the canister. Clearly such attack must be prevented if the canister is to maintain the strength to allow removal up to 5 years after emplacement. A carbon steel shrouded heater showed minor scaling during the one year heater experiment but was not severely attacked either at the level of the heater or at the level where water condensation occurred. These results form the basis of the suggestion for protecting the waste canister from exposure to the brine vapors and salt with a carbon steel sleeve. Comparison of the brief testing time, $\sim 1$ year, during which severe corrosion occurred, with the target retrievability period of 5 years shows the 
need of careful testing and design of protective shields if easy retrievability is to be maintained.

An additional problem associated with maintaining retrievability of HLW or spent fuel emplaced in salt results from the gradual mechanical deformation or creep of the salt in response to thermal stresses and stresses induced by the mining excavation. This creep is likely to be accelerated by the high ambient temperatures associated with the storage of the HI.W or spent fuel. The engineering design must assure both that access to the emplaced waste can be maintained during the retrievability period, and that the canisters are not mechanically entrapped by the deformation of the surrounding salt. The proposed carbon steel sleeve thus serves a double function, protecting the canister against both corrosion and entrapment.

The concept of relrievability takes on added importance in the case of an extended or indefinite delay in reprocessing. What is to be the long term technique, in that case, used for the storage of spent fuel assemblies? In view of the uncertainties in current estimates of uranium reserves and in absence of the availability of a long term alternate energy source, solar or fusion, this spent fuel repesents a valuable resource and should be kept available, not treated as waste. ERDA (and AEC) planning seems to have been predicated on the assumption of the development of a commercial reprocessing industry and there has apparently been little or no planning for long-term storage of spent fuel. Such storage could be accomplished either in interim surface storage facilities or in geologic repositories. Surface storage avoids the problem of corrosive attack of fuel-containing canisters that may be present in a geologic repository, particularly in salt. The geologic storage has the advantage, however, that the conversion of the storage facility to a terminal storage mode would be easily accomplished were there an ultimate decision never to reprocess the stored fuel.

We are concerned about the suggestion to encapsulate spent fuel in an overpack equivalent to that anticipated for HLW and to store it "retrievably" using simply the technology developed for the geologic disposal of HLW (Kuhlman, 1976). The retrievability period anticipated for the test phase of a HLW repository is of the order of 5 years or less. It is easily conceivable that final decisions on the question of reprocessing of stored LWR fuel could be delayed as long as 10 to 30 years since they depend upon such factors as the better definition of uranium resources, breeder development, availability of alternative energy sources and establishment of international agreements. Retrievability on a time scale of 5 years does not assure retrievability after 30 years, particularly when two of the threats are corrosion by the migrating brine and mechanical entrapment by creep of the salt; both processes are expected to continue for a time the order of 50 years or more. Thus the emplacement design for testphase retrievable storage of HLW will differ from that for interim storage of spent fuel; although these are clearly related design problems, the solution of the easier should not be assumed adequate for the harder.

\section{C4. TRU Wastes}

Although this section of the report focuses on the problem of HLW, a closely related problem is the handling of TRU wastes. These are wastes involving a wide variety of materials slightly contaminated with $\alpha$-emitting actinide radionuclides. The low activity level of these wastes per unit volume greatly eases the problems of handling and treatment as compared with the HI.W; but the long range potential for contamination by these wastes is considerable. Table $7 \mathrm{Bl}$ indicates the total actinide content of the TRU wastes to be greater than $10 \%$ of the actinides in the HLW in the case of plutonium recycle, which suggests that the problem of disposal of the TRU wastes is of comparable magnitude to that of the HLW when talking of the time scales $10^{3}$ to $10^{6}$ years.

The absence of significant heat generation allows a wider variety of options for disposal of these wastes. These include encapsulation in bitumens, cements, grouts, glasses, or ceramics. The encapsulated TRU wastẹs could then be transferred to a geologic repository for retrievable, and then terminal, storage as is proposed for the HLW. Those of the options that involve no fire hazard seem entirely adequate in the context of the current waste management program.
Since the principal barrier to release is the geologic containment, the criteria for determining encapsulation techniques are reasonably based on factors such as volume reduction and ease of handling. Although (see Table $7 \mathrm{Bl}$ ) the TRU waste problem is made substantially more severe by the introduction of reprocessing and plutonium recycle, the total waste volumes involved are compatible with disposal in mined caverns and so this additional problem does not argue significantly against fuel cycles involving reprocessing.

\section{Alternative Options of Waste Isolation}

\section{D1. Preface}

The different options which have been proposed and seriously considered for isolation of radioactive waste can be separated into two general categories: (1) elimination of portions of the waste from existence on earth, and (2) storage or disposal of the wastes in various geologic media. The options have been rather extensively discussed in ERDA 76-43, Technical Alternatives Document (or TAD) and, earlier, in BNWL-1900, High-level Radioactive Waste Management Alternatives.

\section{D2. Elimination from Earth}

The observation that the ingestion hazard index of the waste after 1000 years is dominated by the actinides (see Figure 7B1) which are a minor chemical constituent, has led to the suggestion that the actinides be separated, or partitioned, from the fission-product and processingchemical wastes at the time of fuel reprocessing. The total quantities of the actinides involved would be sufficiently low that one might imagine such "total" disposal schemes as ejection from the earth by rocket or elimination by transmutation. The efficacy of any such technique relies not only upon finding a satisfactory scheme for rocket ejection or transmutation, but also upon reducing greatly the actinides lost to low level waste streams (TRU wastes) in comparison with current practice, and upon having an exceedingly efficient partitioning technology. The practicability of chemical processing with adequate partitioning efficiency is under current study. We do not anticipate the availability of such technology before the year 2000, if then, and hence these schemes are not near-term alternatives nor should they be relied upon as long-term options. The magnitude of fission-product wastes precludes the consideration of such a "total" disposal scheme for this component of the waste.

\section{D2a. Ejection by Rocket}

The technical feasibility of extraterrestrial disposal depends upon (1) providing a reliable space flight system for a solar escape mission with adequate payload, and (2) providing a high-integrity capsule to assure no breach of containment even for a worst case abort or launch pad explosion. Because activity and thermal power restrict payloadto-package ratios, the potential for permanent disposal of waste by this alternative depends critically upon partitioning efficiency. Although the basic engineering technology for transport exists, estimates regarding the development of a reliatle space shuttle system indicate the earliest likely date to be 1990 .

\section{D2b. Transmutation}

We concur with the conclusion reached in TAD that the concepts of transmuting actinides using direct bombardment by coulombexcitation-enhanced beta decay or by photon initiated transmutation are unfeasible on the basis of inapplicability or of power consumption. Transmutation by fusion reactors is hardly to be relied upon in the absence of fusion reactors; if they did exist, we would not be concerned with long-term incentives for actinide transmutation.

Another possibility to be considered is the use of neutrons produced by accelerator induced reactions. The intense flux of high energy neutrons produced by certain accelerators make them candidates for actinide transmutation facilities. We consider three such accelerators. (See Table 7D1) 
TABLE 7D1.

\begin{tabular}{|c|c|c|c|c|c|c|c|}
\hline & \multirow[t]{2}{*}{ Accelerator } & \multirow[t]{2}{*}{ Reaction } & \multicolumn{2}{|c|}{ Beam } & \multirow{2}{*}{$\begin{array}{l}\text { Neutron Yield } \\
n-\sec ^{-1}\end{array}$} & \multirow{2}{*}{$\begin{array}{l}\text { Specific Energy } \\
\text { watt-sec-n }-1\end{array}$} & \multirow{2}{*}{$\begin{array}{l}\text { Annual Yield } \\
n-y r^{-1} \quad(300 \mathrm{~d})\end{array}$} \\
\hline & & & $\mathrm{mA}$ & $\mathrm{MeV}$ & & & \\
\hline (A) & LASL & $d-T$ & 100 & .2 & $10^{15}$ & $2 \times 10^{-11}$ & $2.6 \times 10^{22}$ \\
\hline (B) & BNL & $d-L i$ & 100 & 30 & $2 \times 10^{1.6}$ & $1.5 \times 10^{-10}$ & $5 \times 10^{23}$ \\
\hline (C) & ING(AECL) & p-spall & 65 & 1000 & $8 \times 10^{18}$ & $8 \times 10^{-12}$ & $2 \times 10^{26}$ \\
\hline
\end{tabular}

The "specific energy", or energy required to produce each neutron, for the best case (C) may be compared with an estimate of the energy generated in a typical reactor per actinide nucleus in the waste of $7 \times$ $10^{-10}$ watt-sec/nuclide to show that at least $1 \%$ of the power generated would be required to operate the transmutation accelerator, assuming that all of the energy to operate the accelerator appears in the beam and that all of the neutrons produce transmutations. A comparison of the accelerator neutron annual yield, again for the best case (C), with an estimate of the annual actinide yield, in one $1100 \mathrm{MWe}$ reactor, of $4 \times 10^{25}$ actinide nuclei, shows the need of one accelerator to service each five reactors. The capital and operating costs of such a device are not known at present but it would appear that the incremental cost per reactor makes this concept unattractive.

The actinides ${ }^{3}$ separated by chemical partitioning may also be recycled and destroyed by transmutation in reactors. At the end of each reactor irradiation cycle the actinides in the reactor discharge are recovered in fuel reprocessing and fabricated into recycle target elements. Because of the low transmutation rate of these recycled actinides of about $7 \%$ per year, the total actinides inventory builds up in this recycling to a steady state value of roughly 14 times the yearly actinide production. The actinide wastes generated in each cycle are proportional to the inventory in the reactor and to the fractional losses to wastes in reprocessing and refabrication. After the reactor and fuel cycle inventory have reached equilibrium, the actinides accumulate in wastes linearly with time.

Claiborne (Claiborne, 1972) has calculated this rate of release of actinides to high-level wastes when partitioned with assumed recovery factors listed in the first two colunns of Table 7D2. Claiborne's calculated ingestion hazard index (Section B1,2) per unit volume of the high level wastes generated in these recycle operations is shown in Figure 7B4. After a decay period of about 1000 years, the hazard index of the high-level wastes with partitioning and recycle is about a hundred-fold less than that of the high-level wastes without partitioning. It is this reduction factor of about 100 in the amount of actinides in the wastes which illustrates the objectives of the ERDA partitioning program. A meaningful evaluation of the magnitude of this incentive for partitioning requires, among other things, an assessment of the hazards of geologic disposal of unpartitioned waste and of the occupational exposures associated with the more complex processing.

When the incentives of actinide partitioning and recycle are presented in terms of a hazard index per unit volume of waste, as in Figure 7B4, attention is focused on the high-level wastes, which are relatively concentrated. However, as has been discussed elsewhere in this report, actinides in low-level wastes, even though more dilute, probably contribute to the potential hazard in disposal to an extent comparable to the hazards from high-level wastes. Furthermore, when actinides are partitioned and recycled there are greater amounts of actinides to be processed and fabricated. Extreme care must be taken to maintain very low losses of these actinides to the low level wastes from reprocessing and fabrication of recycled fuel. It is obvious that if the partitioning program is to develop the degree of hazards reduction implied by the hazard comparisons of Figure 7B4, the total losses to the wastes from all process sources must be of the magnitude indicated in the above table.

Evaluation of the reduction in actinide residuals by partitioning and recycle must also include the inventory of actinides in the reactor and fuel cycle, on the grounds that upon the eventual termination of fission power this actinide inventory must also be disposed of Therefore, we will define here a more realistic time-dependent reduction factor as the ratio of accumulated actinides in wastes without recycling to the total inventory of actinides in the reactor, fuel cycle, and wastes with recycling. The results for a pressurized water reactor, calculated (Pigford and Choi, 1976) from Claiborne's cycle-by-cycle transmutation data, are shown in Figure 7D1. The asymptotic reduction factor, which is that calculated by Claiborne and others and illustrated in Figure 7D1, occurs at a time long enough that the actinides accumulated in wastes with recycle are of much greater quantity than those in the reactor and fuel cycle inventory. The time constant to reach the asymptotic value is roughly equal to the reciprocal of the fraction of actinides lost to the wastes per year. For $0.1 \%$ loss to wastes per cycle, normally quoted as a reasonable objective for actinide recycle, several thousand years are required to reach reduction factors approaching the asymptotic values. However, water reactors will probably be limited by fuel resources long before appreciable reduction factors are obtained.

A fast breeder reactor would be much more effective in transmuting the actinides, since all of these actinides fission in a fastneutron spectrum. Beaman and Aitken (Beaman and Aitken, 1976) have calculated the transmutation of actinides in a typical commercial LMFBR. However, they have assumed a relatively short time (1.1 yr.) for the actinide irradiation cycle, resulting in a large ratio of external inventory to in-core inventory. Using their data, we calculate timedependent reduction factors for LMFBR transmutation which are slightly lower than those shown in Fig. 7D1 for a pressurized water reactor. The breeder transmutation results could be improved with longer in-core time for actinide irradiation.

The asymptotic reduction factors of Figure 7D1 are the reduction

TABLE 7D2. Actinide losses to high-level wastes assumed to illustrate partitioning-recycle inventives.

\begin{tabular}{|c|c|c|c|}
\hline & $\begin{array}{l}\text { recovered from } \\
\text { wastes and } \\
\text { recycled, } \\
\text { with } \\
\text { partitioning }\end{array}$ & $\begin{array}{l}\text { lost to } \\
\text { high } 1 \text { evel wastes } \\
\text { per cycle, } \\
\text { with } \\
\text { partitioning }\end{array}$ & $\begin{array}{l}\text { lost to high } \\
\text { level waste per } \\
\text { cycle for normal } \\
\text { reprocessing, } \\
\text { without special } \\
\text { partitioning }\end{array}$ \\
\hline plutonium & $99.99 \%$ & $0.01 \%$ & $0.5 \%$ \\
\hline neptunium & $95 \%$ & $5 \%$ & $100 \%$ \\
\hline $\begin{array}{l}\text { uranium, americium, } \\
\text { curium, iodine }\end{array}$ & $99.9 \%$ & $0.1 \%$ & $100 \%$ \\
\hline
\end{tabular}




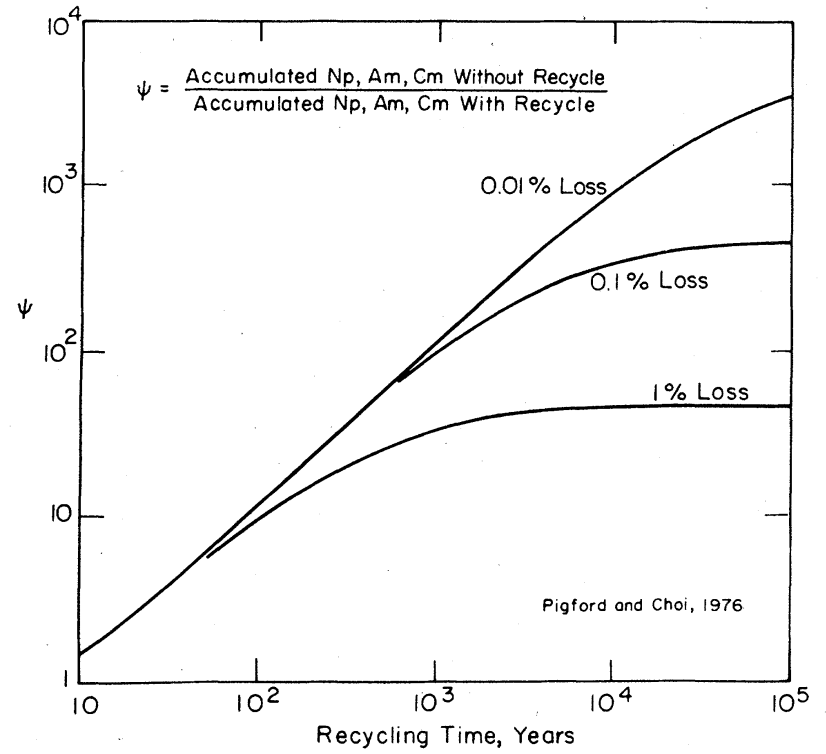

FIG. 7D1. Actinide reduction factor resulting from partitioning and recycle as a function of time for different values of actinide loss to the waste stream.

factors which are calculated when the only residuals considered are those in the radioactive wastes. These are the reduction factors which have been used to illustrate the incentives for actinide partitioning, recycle, and transmutation. For example, recovery and recycle of $99.5 \%$ of the $\mathrm{Np}, \mathrm{An}: \mathrm{Cm}$ reduces the amounts of these radionuclides in the actual wastes by about a factor of 100 . However, to illustrate the importance of also considering the recycled actinide inventory, we will postulate that breeders begin operating to transmute selfgenerated recycled actinides in the year 2000. At the end of a $30-y \mathrm{r}$. reactor lifetime, i.e., in the year 2030, the inventory of recycled actinides is $1 / 2.4$ of the total actinides produced during that period. The total actinide production is what would have gone to the wastes if there were no recycle. Assuming that very little of the actinides have actually been allowed to go to the wastes, the overall reduction factor by the year 2030 is still only 2.4 .

The actinide inventory from these retired breeders is then transferred to the replacement breeder and its fuel cycle, and partitioning and transmutation continue. Assumin such replacement of retired breeders by new breeders to maintain constant total power, after 100 years the actinide inventory in the reactor and fuel cycle is now only $1 / 8.1$ of the total actinides produced since the beginning of the original transmutation operation. Consequently, the overall reduction factor has been increased to 8.1 . With a continued progran of transferring actinide inventory from a decommissioned reactor to a replacement reactor, the actinide inventory in the wastes from recovery and recycle finally, after a few thousand years, becomes comparable to, or greater than, the inventory in the reactor and fuel cycle, and the overall reduction factor finally approaches the asymptotic value described earlier.

The U.S. uranium and thorium reserves are probably adequate to continue breeders for such a long period, assuming that the total power from fission is not increasing rapidly over this time. Furthermore, all during this long period of a few thousand years the actinides accumulated in the actual wastes are small. They are less, by a factor equal to the asymptotic reduction factor, than the total actinides produced. However, it is the prospect that fission power may possibly discontinue at some time during this long period of transmutation operation that argues for treating the actinide inventory in the reactor and fuel cycle as a potential waste, in which case the lower overall reduction factor of Figure $7 \mathrm{DI}$ is appropriate. We must allow for this possibility, since we have no way of knowing the criteria, constraints, and alternatives which may dictate the choice of power systems even during the next century.
Therefore, we assume an arbitrary reduction in the number of reactors at some arbitrary time, e.g., 100 yr., after the beginning of actinide recycle. The $\mathrm{Np}, \mathrm{Am}$, and $\mathrm{Cm}$ contained in these decommissioned reactors are now loaded into the remaining breeders. The overall reduction factor now increases more rapidly with time because these earlier reactors are no longer generating new $\mathrm{Np}$. Am, and $\mathrm{Cm}$.

Our calculated results (Pigford and Choi, 1977) are i'lustrated in Figure 7D2, for an assumed step reduction in the number of breeder reactors by a factor $\gamma$ at the end of the first 100 years. The remaining reactors continue at constant power. The effective reduction factor, normalized to the asymptotic value, is calculated as a function of the continued constant-power operating time for the remaining reactors, for various assumed values of the parameter $\gamma$. The overall reduction factor increases to the same asymptotic value at infinite time as that calculated for continuous self-generated recycle $(\gamma=1)$, but it approaches the asymptotic value more rapidly than in the case of selfgenerated actinide recycle. These results illustrate that transferring residual actinide inventories from terminated reactors to the fewer continuing reactors can be an effective way of handling the residual inventory.

The curves of Figure 7D2 have been calculated without constraints on the amount of actinides that can actually be loaded into the remaining reactors. Some such overloading should be possible, since in self generated recycle only a few percent of the core volume is occupied by the recycled actinides. In the neutron spectrum of a fast breeder core the actinides are roughly equivalent in reactivity and fission power density to the normal $\mathrm{PuO}_{2}-\mathrm{UO}_{2}$ fuel that they replace. Therefore, the continuing breeders should be able to take over the actinides from a large number of discontinued reactors and their fuel cycles.

The previous discussion and illustrations for simplicity have concentrated on reduction factors in terms of the masses of $\mathrm{Np}$. Am, and $\mathrm{Cm}$. However, it is the reduction in potential hazard from these radionuclides that is the incentive for actinide recycling and transmutation. Transmutation does build up relatively large activities of ${ }^{244} \mathrm{Cm}$, and when the hazard index of the total $\mathrm{Np}, \mathrm{Am}, \mathrm{Cm}$, group is examined at the time of waste generation, we find greater hazard associated with the smaller mass of recycle wastes than in the wastes without actinide partitioning and recycling. However, $17.6-y \mathrm{r}{ }^{244} \mathrm{Cm}$ decays over a few decades, and the long-term ingestion hazard of the wastes with partitioning and recycling is then reduced below that of the wastes without partitioning and recycling. The hazard reduction factor then becomes essentially the same as the mass reduction factor.

The above discussion has emphasized recovery and transmutation of $\mathrm{Np}, \mathrm{Am}$, and $\mathrm{Cm}$, to reduce the long-term ingestion hazard index of the wastes. However, to obtain a total reduction in the long-term hazard index of about two orders of magnitude, as has been the indicated incentive for partitioning and recycle, the losses of uranium and plutonium to wastes must be reduced considerably below the losses now considered to be typical of fuel reprocessing. Likewise, the fission product ${ }^{129} \mathrm{I}$ must be recovered and transmuted. Claiborne has assumed a reduction in plutonium loss from $0.5 \%$ to $0.005 \%$ and a reduction in iodine loss to $0.1 \%$; we have not determined whether such reduction factors are realistically achievable.

Also, if some breeders are eventually to be decommissioned withont replacement, as has been assumed in calculating Figure 7D2, the large inventory of plutonium in these decommissioned breeders must be consumed. This should be possible by operating the remaining breeders at a breeding ratio of less than unity long enough to consume the residual plutonium. The remaining uranium inventory can also be consumed by the continuing breeders.

In summary, actinide partitioning and recycle of fer possible longterm benefits to the waste management program; because no technology is expected to be available during this century, such schemes do not provide near-term options. It is apparent that the incentives for actinide partitioning, heretofore identified in terms of asymptotic reciction factors, may need re-evaluation, taking into account the increased actinide inventories. Much effort is appropriately directed towards the chemical technology of partitioning to obtain very low actinide losses to the high-level wastes. Reducing the losses to low-level and intermediate-level wastes from reprocessing 


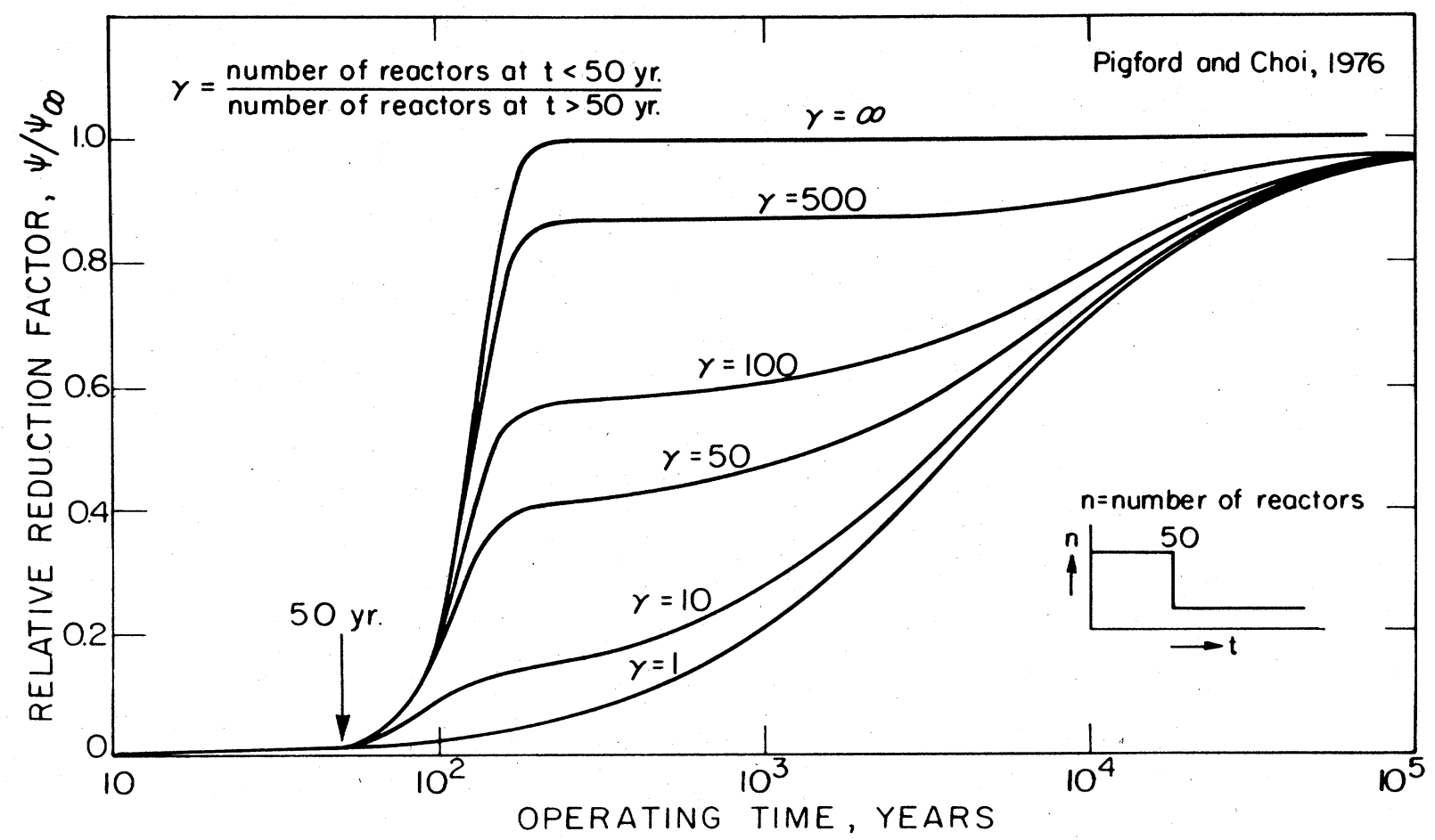

FIG. 7D2. Constant-power operation with self-generated actinide recycle for 50 years, followed by discontinuous decrease in nimber of reactors, with all remaining actinide inventory transferred to a remaining LMFBR reactors.

and refabrication operations is also important in achieving the longterm incentives for partitioning and recycle. However, the uncertainty of the future suggests that more emphasis be given to reducing the inventory of recycled actinides in the reactor and fuel cycle. When this inventory is considered as a potential residual, as in the overall reduction factor in Figure 7D1, during the first few hundred years of partitioning and recycle the overall reduction factor depends little upon attaining extremely low fractional losses to the wastes. Instead it is controlled more by the irradiation time and specific power for actinide transmutation and the external hold-up time in actinide reprocessing and refabrication. Means of improving these variables and the feasibility of high actinide loadings in breeder reactors as a means of transmuting the residual inventory of $\mathrm{Pu}, \mathrm{Np}, \mathrm{Am}$, and $\mathrm{Cm}$ from decommissioned reactors should be explored.

Other aspects of the actinide partitioning-recycle program which bear upon its significance 10 the future waste-management program are the technological problems, the timeliness, the cost and the health hazards associated with possible solutions to carry out low-loss separations and refabrication.

ERDA estimates that if the present program on actinide partitioning and recycle leads to successful and practicable technology, the technology could not be reasonably implemented prior to the year 2000. Consequently, the present waste management program for geologic waste isolation must proceed on the basis of solidifying and isolating radioactive wastes formed during this century. Actinide partitioning and recycle falls in the category of continued research and development which may result in process improvement for later waste-management processes in the next century.

\section{D3. Geologic isolation}

Seabed isolation, ice sheet isolation, and deep continental geologic isolation are the alternatives that exist for storage or disposal of radioactive waste on earth. Numerous concepts have been advanced for each of these alternatives, which are made specific by choice of site, wasteform, and emplacement medium and method.

\section{D3a. Seabed isolation}

This alternative would involve placement of solid wasteforms in sediments or in rocks under selected areas of the oceans. The transportation engineering, capability of locating and recovering lost waste canisters, and capability of emplacement and recovery of waste canisters at site locations all appear, in principle, to be achievable with straightforward extension of existing technology for working in the deep ocean environment. However, critical emplacement parameters and breaching modes remain to be identified and evaluated.

A continuing effort since 1973 to assess the oceans as potential sites for radioactive waste disposal has led to identification of the North Pacific .midplate/midgyre region as one appropriate for research and development feasibility studies. Various glacial cycles have had no obvious effect on the bottom environment in this region, as sediment cores reveal extreme uniformity of sedimentation during the last four million years. Studies of rock samples a few hundred meters below the sediment/rock interface in semirelated areas elsewhere suggest, however, that fluids may percolate through this upper layer of oceanic crust. Moreover, current-meter data indicate that even in such a supposedly "tranquil" ocean basin area, water is moving with sufficient advective energy that the ocean water itself would not represent any significant barrier to migration of radionuclides. Where "residence times" have been calculated for water in ocean basins, the "age" may be no greater than 1000 to 3000 years, and it is frequently much less. Radionuclides in solution in the deep ocean thus might be expected to reach the sea stirface in 3000 years or less.

Uncertainties exist with regard to the breaching of waste containers and the migration of radionuclides within ocean sediments once released from fabricated containment. Because of the typically high porosity of the sediments and their saturation with water, a thermal potential produced by emplaced waste could cause significant mass and energy transport through the typically thin $(20-40 \mathrm{~m})$ bottom sediment. It is clear that current understanding of ion transport phenomena in the marine environment lags far behind existing engineering capabilities of working in the deep ocean or ocean floor. 
Thus, at our present state of knowledge, seabed isolation is not an acceptable alternative for radioactive waste isolation. Continuing research on both wasteform and radionuclide migration in marine sediments seems appropriate and may remove the uncertainties that now exist with regard to this disposal alternative.

\section{D3b. Ice Sheet Isolation}

Radioactive waste could be placed on, within, or under existing glaciers such as those of Antarctica. The disposal of radioactive waste in Antarctica has appeal primarily in that it offers geographical isolation in the largest area on earth that is essentially devoid of any flora or fauna and it is the most remote region from normal human contact. All systems required for transportation, emplacement, and retrieval are available or could be developed through existing technology. Disposal in ice has the additional apparent advantage that, like salt, ice is impermeable to water, and fractures developed in it are self-healing through recrystallization and plastic flow. The thermal output of high-level waste would, however, result in a meltwater envelope around a waste canister and in descent through the ice sheet at a rate that would place the canister at the bottom of the typical ice sheet within about 10 years after emplacement near the surface. In that location the canister, and its contained radioactive waste, would be accessible to transport processes operative in the underlying bedrock as well as those within the ice sheet.

Current knowledge of ice sheet physics and history makes prediction of the stability of ice sheets for periods greater than a few thousand years uncertain. As quoted in the TAD from an international conference on this radioactive waste disposal alternative, "Even a complete understanding of the behavior of the ice sheet with respect to the present boundary conditions, including climate, geothermal flux and sea level is insufficient to allow the precise determination of the ice sheet's future. For that we need at least a corresponding knowledge of the future changes in the boundary conditions".

The observed presence of water at the ice-bedrock interface beneath $2 \mathrm{~km}$ of ice at the Byrd Station, the uncertainty regarditig residence times of both ice and glacier-derived water within the ice sheet with the possibility of rapid transfer of ice, basal meltwater, and any contained radionuclides to the world ocean (possibly within $10^{4}$ $10^{5}$ years), as well as uncertainties regarding climatic factors in the future and ice sheet stability make ice sheet isolation unacceptable.

\section{D3c. Deep Continental Geologic Isolation}

A variety of concepts for deep continental geologic isolation have been advanced and include (1) conventional mined cavities, (2) solution-mined cavities, (3) a matrix of drilled holes, (4) superdeep holes, (5) hydrof racture emplacement, (6) deep-well injection, and (7) various rock-melting concepts. These alternatives differ in the nature of the wasteform (solid or liquid), the manner of emplacement, and the geologic medium in which the waste is to be emplaced. With the exception of conventional mined cavities, the remaining alternatives are disposal concepts in that retrievability of the emplaced waste, although possible, would be beyond existing technology, significantly more expensive, or appreciably more difficult than emplacement of the waste. As long as retrievability of the emplaced waste remains a basic tenet of waste isolation philosophy, disposal alternatives cannot be employed. Several disposal alternatives could represent viable options for the future, however, and these deserve serious consideration and research support.

(1) Conventional mined cavities constitute the only deep continental geologic isolation concept based on a demonstrated technology for both the emplacement and the retrieval of radioactive waste. In this option solid radioactive wasteforms would be lowered through a vertical shaft to a mined cavity, located several hundred meters below ground level, and emplaced in a series of holes made in the floor of th: cavity to receive the waste containers. The depth of the mined cavity would be dependent, in part, on the mechanical properties of the rock; the depth would be limited for a rock such as salt which flows readily under moderate pressure, but less so for a rock such as granite. Spacing of the holes would depend upon thermal properties of the rock and on waste loading of the containers. Of primary concern are the potential modes of breaching the geologic confinement and possible modes of transport to the biosphere once the radionuclides are released from fabricated containment. These concerns are addressed in Sections $E$ and $F$.

Concepts utilizing (2) solution-mined cavities, (3) a matrix of drilled holes, and (4) superdeep holes all involve emplacement of canisters containing the radioactive wastes. The technology exists for the first two alternatives but not for large-diameter superdeep holes of 10 thousand or more meters. Inasmuch as the first two alternatives involve emplacement at depths not likely to exceed $2 \mathrm{~km}$, principal concerns must be the nature of the groundwater regime and the physical integrity of the geologic unit in which the waste is emplaced (see section F). Little is known at present about the stability of solution-mined cavities that have dried out, the methods of drying them out, or about the optimal size or depth of such cavities. Furthermore, this concept is necessarily restricted to emplacement in a geologic medium that is soluble--viz., rock salt. Although the matrix of drilled holes is not restricted to a specific geologic medium, it does require identification of a geologic unit with limited or no fractures or other features capable of transmitting ground water at significant rates. Moreover, the probability of compromising the integrity of containment is greatly increased by the multitude of holes that would penetrate the disposal formation and that would have to be sealed. Because of this we feel that this alternative is unacceptable. In both concepts of disposal the number of canisters that could be placed in a given cavity or drilled hole and/or the waste loading of canisters would have to be restricted in order to prevent the melting of the emplacement medium by heat generated by the high level waste-unless, of course, this were desired. Some promise of the possibility for superdeep emplacement is provided by the fact that large-diameter holes have been completed to several thousand meters; extension of existing technology and the development of associated techniques to permit emplacement of waste canisters through such holes to greater depths would make this a highly attractive future disposal alternative. Such a mode of emplacement would eliminate the need for conventional mining to the desired disposal medium and, thus, also eliminate certain costs and problems associated with conventional mining.

(5) Hydrofracture emplacement and (6) deep-well injection concepts both involve the pumping of liquid waste into deep geologic units. The technology for both alternatives is well established and routinely practiced by industry. We conclude that principal safety concerns prior to emplacement are containment during transportation and the integrity of all pipes and well casing. (It should be recalled that Federal regulations, $10 \mathrm{CFR} 50$, currently require that high-level liquid wastes be converted to solid form within five years.) The major safety consideration after emplacement is to insure the integrity of long-term isolation in the geologic medium. If the restriction of retrievability were removed and an appropriate matrix (cement, resin, etc.) were developed to provide initial confinement, we conclude that this alternative could be acceptable for disposal in favorable hydrogeologic regimes (see section F).

(7) Rock melting concepts involve various methods of emplacement, in a variety of geologic media, of both liquid and solid wasteforms that have sufficiently high thermal power to bring the host geologic medium to its melting range locally. Waste having a thermal power of $10 \mathrm{KW} / \mathrm{m}^{3}$, for example, would result in melting of typical basalt if emplaced in a cavity $1 \mathrm{~m}$ in diameter; some high-level wastes can generate up to $200 \mathrm{KW} / \mathrm{m}^{3}$. Several specific rock melting disposal schemes have been formally proposed; these are discussed in ERDA 76-43. Provided that an appropriate host medium is selected, such melting and subsequent crystallization of the melt upon cooling could provide an integral rock mass, with extremely low permeability, in which the radioactive waste is most nearly in chemical equilibrium with its environment. Certain rock types, such as shale and limestone, may not be suitable for such a disposal alternative because it is possible that vapors, particularly water and carbon dioxide, will be expelled due to decomposition of the rock. No disposal technique involving melting has yet been extensively investigated, but preliminary calculations and experiments suggest to us that one or another of the rock melting concepts may be a highly attractive radioactive waste disposal alternative for the future. 


\section{E. Potential Modes of Breaching Geologic Confinement}

\section{E1. Introductory Remarks}

For purposes of this section, the breaching of confinement refers to the inability of the geologic medium to prevent exposure of radioactive waste emplaced within it to a medium of transport--viz., air or water. Thus, breaching of the repository vault and exposure of its contents to either air or water would result in loss of confinement but, as discussed in section F, this need not imply the loss of geologic isolation -- i.e., the groundwater regime itself may represent an effective means of restricting transport to the biosphere.

Either natural or anthropogenic causes may result in the breaching of confinement by directly effecting a transfer or exposure of contaminant in the repository to the biosphere, or by indirectly effecting a transfer or exposure by altering the hydrogeologic regime.

\section{E2. Natural Causes of Breaching Geologic Confinement}

In principle, the integrity of a radioactive waste repository may be compromised by natural events such as tectonic or igneous activity, erosion, and meteorite impact. Several natural hazards (e.g., impact by a huge meteorite and volcanism) would, if they were to affect a repository, result in an essentially instantaneous breaching of the site and immediate dispersal of contaminants. Most geologic processes, however, operate at extremely slow rates and couid require tens of thousands of years to effect the breaching of confinement.

\section{E2a. Tectonic Activity}

The specific types of tectonic activity of greatest possible consequence to the integrity of a radioactive waste repository are fracturing, faulting, and diapirism. Fracturing is important primarily in regard to its possible effect on the hydrogeologic regime and subsequent transport of contaminants by ground water; the effects of fracturing will therefore be considered in section $F$ dealing with hydrogeologic transport. Faulting and diapirism both may effect contaminant transfer by large-scale movement of rock masses-faulting by relative displacement along a surface or zone, diapirism by the mass flow and penetration of overlying strata by a rock unit of high ductility. Transport to the biosphere would still require contact of the waste-bearing rock masses with surface or ground water. Faulting can, alternatively or concomitantly, affect the hydrogeologic regime and groundwater flow patterns, as well. The latter effect will also be treated in the section on hydrogeologic transport. This section deals only with the large-scale displacement of rock masses that could be effected by faulting and by diapirism, respectively.

In theory, either or both of two conjugate faults symmetrically oriented with respect to the maximum principal stress axis could develop in sufficiently stressed rocks. Each would typically make an angle of about $30^{\circ}$. with this axis and their line of intersection would be the direction of the intermediate principal stress. Thus, a vertical fault typically reflects a horizontal maximum principal stress and is characterized by horizontal displacement along it. Similarly, a fault dipping $60^{\circ}$ typically reflects a vertical maximum principal stress and is characterized by vertical displacement which is a component of slip parallel to the dip of the fault.

The rate at which relative offset occurs along a fault surface or fault zone can vary by several orders of magnitude for different faults. Available data indicate that the rate tends to be faster for nearly vertical faults along which the relative displacement is horizontal than for steeply dipping faults with large components of vertical displacement. The horizontal movement along the San Andreas fault in California, for example, is estimated to be about 4 $\mathrm{cm} / \mathrm{yr}$; that for vertical displacement on major faults in the Basin and Range Province of the western United States probably does not exceed a few $\mathrm{mm} / \mathrm{yr}$ and is more likely to be an order of magnitude slower than that. Horizontal displacement along a vertical fault would be of significance with regard to a waste repository site only insofar as it affects the hydrogeologic regime. Vertical displacement at a rate of 1 $\mathrm{mm} / \mathrm{yr}$ would indicate a realistic estimate of the maximum vertical displacement in 250,000 years to be about $250 \mathrm{~m}$. We conclude that a repository could be located at sufficient depth that faulting, if it were to occur at the site, would not transfer the rock mass containing radioactive waste to the biosphere during the period of concern.
For site specific studies, an estimate can be made of the probability of a fault intersecting the repository. To provide an estimate of what this might be for a specific site in SE New Mexico, Claiborne and Gera (Claiborne and Gera, 1974) calculated a probability of $4 \times 10^{-11}$ per year that a fault will intersect the $8 \mathrm{~km}^{2}$ area of the site. As they point out, it cannot be assumed that this probability is necessarily that of breaching the geologic confinement. The relationship between the two probabilities is dependent upon the probability that the fault would either provide for a hydraulic connection or for contact of the disposal horizon with an aquifer. Detailed geologic information that would permit an analysis of the mechanics of faulting would provide for the most reliable estimates of these probabilities. Because of the possible effect of a fault, even of limited displacement, on the hydrogeologic regime, we feel that such a site-specific fault analysis should be made part of the site selection criteria for any proposed repository site.

Diapirism is a phenomenon most common in salt and other evaporites, but diapirism can occur in shale and certain other rock types as well. Pressure disequilibrium and high ductility, both of which can be enhanced by high temperature, are the principal contributing factors to diapirism. Of printary concern with regard to the integrity of a repository are the ductility and rupture strength of rock units immediately overlying the disposal unit, and the pressureand time-dependent behavior of the mobilized unit (which may be the disposal unit). These factors control the mass flow of the involved units and the likelihood of diapiric penetration.

For conditions most likely to be encountered in radioactive waste disposal sites, rock salt is likely to display the greatest mobility of the geologic media being considered. Estimates of the rate of salt movement based on studies of the development of salt domes suggest a maximum of about $0.3 \mathrm{~mm} / \mathrm{yr}$. This rate would produce a total vertical displacement of less than $100 \mathrm{~m}$ in 250,000 years. We conclude that a repository can be located at sufficient depth that possible salt mobility would not be capable of transferring the wastecontaining salt to the biosphere during the period of concern.

\section{E2b. Igneous Activity}

Geologic processes that involve molten rock and associated gases constitute igneous activity; volcanism includes all such processes that occur at or near the earth's surface. Because the heat generation and fluids associated with volcanism can totally dominate transport within the vicinity of the activity, the probability of such activity occurring in the vicinity of a possible repository site must be evaluated. Volcanism is almost invariably associated with faulting or large-scale fracturing of the earth's crust, although many major faults and fractures commonly have no volcanic activity associated with them. As Claiborne and Gera (Claiborne and Gera, 1974) point out, the probability of volcanism being initiated in a tectonically stable area must therefore be significantly lower than the probability of formation of a great fault. The association of volcanism with major crustal features is well documented, and its distribution within recent geologic periods is reasonably well known. However, intrusive bodies such as dikes and sills are commonly observed in sedimentary sequences, and other evidence of igneous activity such as diatremes, which are breccia-filled volcanic pipes formed by gaseous explosion, are sometimes encountered in unexpected places. Nevertheless, information on past igneous activity and faulting as determined from geologic exploration can be used to identify areas with high and low probabilities of volcanic activity, and we conclude that numerous repository sites can be located for which the probability of breaching by faulting or by volcanism can be reduced to very low levels.

\section{E2c. Erosion}

The effects of erosion of possible consequence to a radioactive waste repository site are (1) lowering of the land surface and exposure of the waste to the agents of erosion and (2) changes in the hydraulic head distribution and significant alteration of the hydrogeologic flow regime.

Various geologic observations indicate that a general lowering of the land surface by erosion of more than $50 \mathrm{~m}$ in 250,000 years is unlikely. Where the principal agent of erosion is stream action the rate of erosion locally could possibly be several times greater. 
However, the limiting depth of erosion can be assessed in terms of the potential base level for the streams (e.g., sea level). Even if the most rapid known rates of erosion are used to predict the total depth of erosion over a significant length of geologic time, and the maximum likely regional uplift is assumed, we conclude that it would be possible to locate a repository site well below the depth of the projected erosion. Moreover, inasmuch as the maximum local relief would be associated with stream valleys, the probability of exposure by erosion would have to be coupled with the probability of a stream valley being developed directly above the repository.

For repository sites to be located in areas of possible future glaciation, an estimate of the maxium possible scour (erosion) by the glacier would need to be made. Estimates of glacial scour indicate that this could be as much as several hundred meters in some instances. A repository could be located at greater depths.

Although exposure of a deep repository by erosion is highly in probable, modification of the hydrogeologic flow regime by erosion is possible. The nature and extent of such modification would depend largely on the magnitude of the local relief but also on the areal distribution of this relief, insofar as these affect this configuration of the water table. The effect of the maximum probable erosion on the hydrogeologic regime at a specific site should be a required part of the hydrogeologic analysis, as will be discussed in section $F$ on hydrogeologic transport.

\section{E2d. Meteorite Impact}

Claiborne and Gera (Claiborne and Gera, 1974) have discussed at length the probability of meteoritic impact and its possible consequences. Two cases are identified with respect to a waste repository: (1) the depth of cratering reaches or exceeds the depth of burial; and (2) the depth of cratering is less than the depth of burial but fracturing associated with the impact extends to the burial horizon.

Craters produced by impact have a total depth about one-third of the diameter. For an assumed depth of burial of radioactive waste at $600 \mathrm{~m}$, for example, Claiborne and Gera point out that a crater nearly $2 \mathrm{~km}$ in diameter would be required to cause an instantaneous release of part of the radioactive material and that the probability of formation by meteoritic impact of a crater of this size or larger is

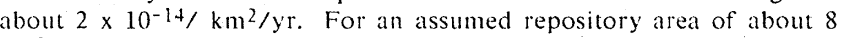
$\mathrm{km}^{2}$ the probability of a catastrophic hit on this area would thus be about $2 \times 10^{-7}$ in a period of $10^{6}$ years. We conclude that a repository could be located at sufficient depth that it would not be breached by meteorite impact.

\section{E3. Anthropogenic Causes of Breaching Geologic Confinement}

Possible causes of loss of confinement that may result from the activities of man include: sabotage, surface nuclear explosions, drilling, and modifications of the geologic environment related to emplacement of the waste. The first three of these have been discussed by Claiborne and Gera (Claiborne and Gera, 1974).

\section{E3a. Sabotage}

If the intent of sabotage were the release of large quantities of radioactive materials to the environment, this would be extremely difficult to impossible once the respository is sealed. Such actions would require utilization of machinery for drilling and excavating, and the protection for considerable periods of time by an armed force capable of resisting counter-measures by the authorities. Prior to sealing of the repository, appropriate protective measures against possible sabotage would need to be implemented, but we conclude that sabotage is not a likely mode of breaching the geologic confinement once the repository is sealed.

\section{E3b. Surface Nuclear Explosions}

According to Claiborne and Gera (Claiborne and Gera, 1974) a 50-megaton explosion would produce a potential crater depth in dry soil of $340 \mathrm{~m}$ and a fracture zone extending to $500 \mathrm{~m}$. We conclude that a repository site can be located at depths well below the direct or indirect effects of a surface burst of nuclear weapons of known or projected capacity.

\section{E3c. Drilling}

Inadvertent encounter with radioactive waste canisters in a repository by drilling from the surface would presumably occur only with the loss of records caused by war or political or social upheaval. Claiborne and Gera (Claiborne and Gera, 1974) conclude that, even if the complete collapse of our present civilization is postulated, the possibility of a random encounter with the radioactive waste by drilling is sufficiently small and the consequences, if random encounter were to occur, are so restricted that this is thought not to present a serious hazard.

Claiborne and Gera did not consider the possible effects on the hydrogeologic regime of drilling for water or the possibility of a subsequent civilization inadvertently encountering the disposal horizon in its attempt to exploit a mineral deposit. Ground water is a valuable resource and is extensively "mined" in areas that require irrigation for agricultural production. The withdrawal of large amounts of ground water can significantly alter the water table configuration and, as a consequence, change both groundwater flow velocities and directions. Factors that affect hydrogeologic transport will be discussed in section $F$ of this chapter.

Man commonly drills in an effort to uncover or exploit resources. It is conceivable that at some time in the future such activity may occur in the vicinity of a repository because of the possible association of the repository with a resource. When the resource being sought is salt, it is further possible that solution mining may be instituted and, as a result, radioactive material might be brought to the surface. We cannot evaluate this possibility quantitatively but believe it to be small. Because man has historically sought first deposits that are characterized by the highest concentration of desired material, we strongly recommend location of a repository in a geologic medium that is sufficiently devoid of mineral or element concentrations or that is found in a sufficient number of places that it. would likely be unattractive to a future generation seeking mineral resources.

\section{E3d. Modifications Related to Waste Emplacement}

The stability of the repository vault during the desired period of demonstrated retrievability is a short-term consideration; it is affected both by heat produced by the emplaced radioactive waste, and by altered stress distributions caused by excavation and related to the cavern geometry. The short-term effects can be evaluated with reasonable confidence by means of in situ testing and appropriate modeling. Mo:e difficult is the prediction of long-term consequences of thermal loading in the disposal unit owing to the continued heat generation of the contained radioactive waste. Specific effects will be dependent upon the rock type involved and upon temperature levels achieved. For salt, the effects may be predominantly mechanical; for shales and other rock types containing water or hydrous phases, the effects could be more complex, and could involve changes in both the mechanical and transport properties of the rock mass. It is clear that the nature of the wasteform could be quite important in this regard; for example, the thermal history after emplacement of spent fuel elements would be very different from that produced by waste derived from reprocessed fuels. We strongly support and encourage appropriate research on the thermal effects on the mechanical and chemical stability of candidate rock types and upon the groundwater regime so that specified thermal loadings for individual rock types can be made an integral part of the design basis for each proposed repository.

\section{F. Transport to the Biosphere}

\section{F1. Introductory Remarks}

For buried radioactive waste the only important medium of radionuclide transport -- i.e., capable of potential transfer of radionuclides from a repository to the biosphere -- is ground water. A favorable groundwater regime can by itself provide effective waste isolation in that radionuclides would be transported so slowly that they would not reach the biosphere during the desired period of isolation. Because of the extreme importance of hydrogeology to HLW management, the parameters that characterize the hydrogeologic system and an appropriate means of quantitatively analyzing the groundwater regime are discussed in some detail in this section. 


\section{F2. Contaminant Transport in Groundwater Systems}

Contaminants move in the subsurface as a result of two physical processes, convection and dispersion. Dispersion will be discussed in Section F5 along with other concentration attenuation mechanisms. With convective transport, the ground water carries the contaminant along in solution; the direction of convective contaminant transport therefore coincides with the direction of groundwater flow. As a first approximation, the rates of convective contaminant transport and groundwater flow of ten are assumed to be equal. However, it is well known that other phenomena that are related to the electrical properties of the porous medium can act to enhance or impede convective ion transport (Corey, et al., 1963; Krupp, et al., 1972; Merten, 1966).

The fact that ground water generally moves in the subsurface implies the existence of a three-dimensional potential field that provides the driving force. The configuration of the potential field depends on at least four factors:

1. The particular forces or potentials that act within the region (e.g., gravity, chemical or temperature potentials).

2. The geometry of the region in which the potential field exists.

3. The existing boundary conditions.

4. The nature and variation of properties that control the flow within the region.

When these factors are completely defined, the resulting potential distribution dictates the flow velocities throughout the field.

In shallow groundwater systems gravity is the dominant driving force moving water through the system, and the configuration of the water table and the geologic configuration -- i.e., the subsurface variation in hydraulic conductivity ${ }^{4}$-- are principal factors that determine the potential distribution. This particular case of groundwater flow will be used as the basis for analysis in section F6 In deep basins thermal gradients and other potentials may affect groundwater flow; such possible effects would have to be considered in the analysis of specific sites (cf. Olsen, 1972).

\section{F3. The Groundwater Basin}

The groundwater basin is a fundamental concept for the analysis of groundwater flow in shallow systems over extensive regions. (The term should not be confused with "sedimentary", "structural", or "topographic" basin.) In its simplest form, the groundwater basin is a closed three-dimensional region in that all waters that enter the groundwater systems within that region also exit within that same region. In other terms, the lateral and bottom boundaries of the region are no-flux boundaries (i.e., flow cannot pass through them). Water moves into and out of the region only through the upper boundary, which is the water table.

Field studies indicate that the extent of a groundwater basin is controlled by major topographic features -- i.e., major groundwater divides create the lateral boundaries of the region. These boundaries will be exactly vertical only if the water-table configuration is symmetric on opposite sides of the divide. Theoretical analysis demonstrates, however, that deviation from the vertical would generally not be large and the effect on the flow pattern of asymmetry across water-table divides is small (cf. Freeze, 1969). In practice, the lateral boundaries of the basin are placed at the major water table divides in recharge and discharge areas.

A groundwater basin is assumed to be bounded on the bottom by an impermeable surface that is typically nearly horizontal. In practice, the effective depth to the basal-impermeable boundary can be taken as the depth to the bottom surface of the lowermost high-permeability layer in the system. Although units below this boundary may not be actually impermeable, the equipotential lines there are usually vertical and the boundary will behave as though it were impermeable. This statement will be quantitatively more exact as the permeability contrasts increase (Freeze, 1969).

Tóth (Tóth, 1962, 1963) has emphasized that the configuration of the water table is often a subdued replica of the topographic configuration. Thus, this surface can be approximated from topographic relations. To measure the actual water-table configuration it is necessary to place observation wells in the basin.

\section{F4. The Hydraulic Potential Field}

The gravity potential field driving groundwater flow is dependent on the following three factors (Freeze, 1969):

1. The ratio of depth to lateral extent of the groundwater basin.

2. The configuration of the water table.

3. The geologic configuration controlling contrasts in hydraulic conductivity.

Depth/Lateral Extent Ratio. As the depth to lateral extent (D/L) ratio of the groundwater basin becomes small, the lateral extent of the flow field becomes greater than the depth of circulation, and an extensive area of lateral flow exists. As the $\mathrm{D} / \mathrm{L}$ ratio becomes large, the region of lateral flow vanishes, or shrinks to a line, with major components of flow being directed both upward and downward. In general, D/L ratios varying from $1: 12$ to $1: 4$ appear not to alter significantly the largely lateral flow that occurs in the lower third of the basin. However, if the water-table configuration (see below) is hummocky, one might expect significant vertical convection at several places throughout the basin, with specific effects being very much dependent upon the distribution of hydraulic conductivities (cf. Freeze, 1969).

Water-Table Configuration. The driving potential for isothermal groundwater flow in a region is related to the water-table configuration, and careful determination of the water-table configuration is essential to accurate definition of the groundwater flow system.

The general effects of water-table configuration have been evaluated by Freeze (Freeze, 1969) in several theoretical models in which the D/L ratio was varied from 1:4 to $1: 12$ and the total relief of the water table (difference in elevation between its highest and lowest points) to lateral extent $(\mathrm{R} / \mathrm{L})$ ratio was varied from $1: 20$ to 1:60 for several water-table configurations. His conclusions include the following:

1. A gentle, constant regional water-table slope over a homogeneous medium results in flow that is essentially horizontal.

2. The existence of a hummocky water-table configuration results in numerous sub-basins within the major groundwater basin; instead of moving laterally for large distances, ground waters tend to have significant upward and downward flow components.

3. Larger depth/lateral extent ratios result in a smaller influence on the total flow pattern by individual hummocks in the water-table configuration.

The latter two conclusions confirm the findings of Tóth (Tóth, 1963) that the influence of the hummocks increases as (a) their amplitude increases and (b) the depth/lateral extent ratio decreases.

Geologic Configuration. The water-table configuration and the geologic configuration are the principal governing factors that control groundwater flow in isothermal systems. Both can exist in infinite variety. However, as with the analysis of water-table configuration, the general influence of the geologic configuration can be assessed effectively by means of theoretical models. For each of two representative water-table configurations, Freeze (1969) has investigated a wide range of hydraulic conductivity configurations including 2-layer cases, 3-layer cases, partial aquifers, and sloping aquifers and aquicludes. Conclusions especially relevant to the subject of this report include:

1. The vertical flow through a low-permeability layer becomes more pronounced with an increase in the permeability ratio between it and an underlying layer of higher permeability.

2. The flow pattern resulting from a 2-layer case in which the upper layer has the higher permeability is almost identical to that of the homogeneous single-layer case. 
3. The presence of a partial basal aquifer in the recharge half of the basin results in a vertical upward flow in the middle of the basin, above the downstream termination of the aquifer.

\section{F5. Concentration Attenuation Mechanisms}

The primary mechanism of contaminant transfer through a subsurface flow system is convective transfer, by which contaminants travel at the same rate of flow and in the same direction as the carrier fluid. Other processes are secondary mechanisms and tend to produce a divergence from this idealized transport process. Both chemical and nuclear processes act in addition to the physical processes to affect radionuclide migration. Of greatest significance are sorption and radioactive decay. The former has the effect of reducing the flow velocity of contaminants relative to that of the groundwater; contaminant ions in solution are coupled via exchange reactions with the solid phase of the porous medium to bring about a reduction of contaminant concentrations in solution (cf. Robinson, 1962; Borg et al, 1976). Spontaneous radioactive decay of contaminants constitutes an important concentration attenuation mechanism that is independent of groundwater flow velocity and dispersion, the physical and chemical environment, and properties of the medium.

Dispersion. Mechanical dispersion is a mixing phenomenon. It is caused by variations in velocity which on a microscopic scale result from heterogeneity of the pore structure and on a macro- and megascopic scale from heterogeneity of the porous medium with respect to hydraulic conductivity. Differences in velocity between dissolved contaminants and the convective front will typically occur because of differences in length of flow paths for the transported ions. Dispersion causes a convective contaminant front moving through a porous medium to spread laterally and longitudinally and, hence, to dilute; it is therefore an important concentration attenuation mechanism. A secondary component of the dispersion mechanism -- mass flow in response to a concentration gradient, or diffusion -- can also contribute to dilution. However, diffusion is only important when the velocity of ground water is very small (Reddell and Sunada, 1970) and, hence, it is generally subordinate to convective mixing as a concentration attenuation mechanism. Freeze (Freeze, 19/2) comments that many authors have included molecular diffusion in their mathematical analyses, but all have concluded that its attenuating influence is unimportant in comparison with dispersion.

The magnitude of dispersion effects is generally considered to be directly proportional to the product of the velocity of groundwater flow and the dispersivity, a characteristic parameter of the porous medium (Bear, 1972). Porous medium dispersivity is difficult to measure because the magnitude of dispersion appears to be heavily dependent upon the scale of the field of measurement. As the scale increases, macroscopic and megascopic, as well as microscopic, heterogeneities affect the dispersion. Laboratory measurements have been shown to underestimate porous medium dispersivity of natural systems by as much as three orders of magnitude (Reddell and Sunada, 1970). Field measurements are most satisfactory, but may not be possible if flow velocities are very low. Some recent work suggests that independent estimates of porous medium dispersivity may be possible if sufficient data on the conductivity characteristics and spatial stıucture of the porous medium are available.

In media with low dispersivity, the extent of contaminant transport approximates that resulting entirely from forced convection. As the dispersivity increases, the contaminants are spread over a much larger area--in some instances, the area nearly doubles with each order-of-magnitude increase in the dispersivity. Moreover, the contaminant zone is enlarged significantly when a layer of higher dispersivity overlies a layer of lower dispersivity, than vice versa. And, the position and extent of a contaminant zone is highly dependent upon the contaminant inflow location relative to the groundwater flow pattern in the basin (cf. Schwartz, 1975).

Cation Exchange. Parameters representing the possible exchange between contaminant ions in solution and those in the solid phase of the porous medium include the distribution coefficient $\left(\mathrm{K}_{\mathrm{d}}\right)$, cation exchange capacity (CEC), and ion valence. The effects of these parameters on contaminant distribution by ground water are evaluated by Schwartz (Schwartz, 1975).
The distribution coefficient characterizes the degree of coupling between the sorbed phase and the contaminant in solution. It is of ten assumed that the movement of a contaminant species past an active exchange site is slow enough that pointwise equilibrium is obtained between solution and exchanger. The effect of this reaction is to partition the contaminant material between the pore solution and the solid phase. When the distribution coefficient has a high value, strong coupling occurs via the cation exchange reaction. In these instances, and if the solution is sufficiently dilute that the ion exchange sites are far from being saturated by the contaminant, the average velocity of the contaminant ion is reduced to the pore solution velocity times the fraction of the ion in solution. The fraction in solution is $\left(1+\mathrm{K}_{\mathrm{d}} \rho_{\mathrm{e}}\right)^{-1}$ where $\rho_{\mathrm{e}}$ is equal to the bulk density of the solid divided by its effective porosity.

The CEC measures the capacity of the sorptive reservoir provided by the porous medium, and it is primarily dependent on the abundance and the type of clay minerals present. Because of this, the CEC may vary considerably within a basin owing to the presence of geologic layering. As would be expected, the extent of the contaminant zone is reduced in direct proportion to the magnitude of the cation exchange capacity. It should be emphasized that the measured CEC is meaningful only when groundwater flow is intergranular; the CEC of a porous medium is reduced as an increasing proportion of transport occurs within a fracture network.

Ion valence is a third parameter controlling cation exchange. Contaminant spread is minimized when two monovalent contaminant species dissolved in the pore fluid are able to exchange with one adsorbed divalent ion in the solid phase; the least amount of confinement occurs when the dissolved contaminant is divalent and the adsorbed species is monovalent. The area of the contaminant zone is essentially quadrupled from the former to the latter situation.

The exchange process is influenced considerably by ion size, hydration, and seat of the exchange position on the clay mineral, as well as valence, concentration of competing ions in solution (especially $\mathrm{Ca}^{++}, \mathrm{Mg}^{++}$, and $\mathrm{Na}^{+}$), and $\mathrm{pH}$ of the pore solution. An excellent review of factors affecting cation exchange is provided by Robinson (Robinson, 1962).

Weathering (Leaching) Rate. The maximum rate at which leachates (contaminants) might enter the groundwater system is controlled by the chemical weathering (leaching) rate of the radioactive waste material. The effect of increasing the time required for all the waste to enter the groundwater system is to decrease the degree of contamination by increasing the area of the contaminated zone and by shifting the center of this zone toward the recharge area (cf. Schwartz, 1975). Although data are being accumulated regarding leaching rates of waste forms (cf. Sections G2 and G3), little is known about the weathering or leaching rates of fission products and actinides in natural materials.

Some insight in this regard has been provided by the OK1.O phenomenon in the Gabon Republic of West Africa (OKLO, 1975), where convincing and quantitative evidence exists that a natural reactor operated there approximately 1.8 billion years ago. Because of the obvious difficulty of devising experiments to evaluate the stability over $10^{-4}-10^{-6}$ years of ions included in or adsorbed on various mineral species, information regarding ion mobility at OKLO may have particular relevance to problems of radioactive waste management. At least half of the approximately 30 fission-product elements have remained immobilized; $\mathrm{Rb}, \mathrm{Cs}, \mathrm{Sr}$, and $\mathrm{Ba}$, and probably $\mathrm{Cd}$, all made in the reactor, have mostly disappeared. Yet, ${ }^{90} \mathrm{Zr}$ produced by the decay of ${ }^{90} \mathrm{Sr}$, is accounted for, indicating that little of the ${ }^{90} \mathrm{Sr}$ was transported from the vicinity of the reactor. Walton and Cowan (OKLO-75) concluded that the principal radioactive products that were released into the biosphere in measurable amounts at the time of the reaction were ${ }^{85} \mathrm{Kr}$ and possibly a little ${ }^{137} \mathrm{Cs}$ and ${ }^{90} \mathrm{Sr}$. Plutonium was efficiently confined.

\section{F6. Quantitative Analysis of Groundwater Flow and Mass Transport}

Of primary concern in the evaluation of a radioactive waste storage/disposal site are accurate determination of the expected degree of confinement in the subsurface and accurate prediction of the probable pattern of contaminant migration. The complexity of the 
processes involved in the transport of radioactive ions makes it impossible to evaluate simultaneously by empirical means all of the factors that control transport and attenuation. With the added complexity of a real geologic situation, the advantages of a predictive mathematical approach become evident (cf. Schwartz, 1975).

The contaminant transfer problem is formulated mathematically by a coupled pair of partial differential equations; one describing the flow of ground water and the other describing the flow of dissolved radioactive contaminant. The parameter that couples these two equations is the groundwater velocity, and a quantitative description of the velocity is a necessary requirement for a solution of the contaminant flow equation. The velocity distribution can be calculated from the temporal and spatial distribution of hydraulic head which, with specification of appropriate boundary conditions, is described by solution of the following equation, where $S_{s}$ equals specific storage, $\mathrm{L}^{-1} ; \mathrm{K}$ equals hydraulic conductivity, $\mathrm{LT}^{-1} ; \mathrm{h}$ equals head, L:

$$
\frac{\delta}{\delta \mathrm{X}_{\alpha}}\left(\frac{\mathrm{K} \frac{\delta \mathrm{h}}{\delta \mathrm{X}_{\alpha}}}{)}\right)=\mathrm{S}_{\mathrm{s}} \frac{\delta \mathrm{h}}{\delta \mathrm{t}} \quad, \quad \alpha=\mathrm{x}, \mathrm{y}
$$

The contaminant flow can be represented by the following form of the convection-dispersion equation that is obtained by combining the continuity equation for the $i$ th contaminant in a multicomponent system, a flux-force equation involving the concentration gradient and an equation of state:

$$
\begin{aligned}
& \frac{\delta}{\delta \mathrm{X}_{\alpha}}\left(\mathrm{D}_{\alpha \beta} \frac{\delta \mathrm{C}}{\delta \mathrm{x}_{\beta}}\right)-\underset{\delta \mathrm{x}_{\alpha}}{\left.-\frac{\delta}{\mathrm{C}} v_{\alpha}\right)+\sum_{k=1}^{\mathrm{n}} \mathrm{R}_{k}=\frac{\delta}{\delta \mathrm{t}}(\varepsilon \mathrm{C}),} \\
& \alpha, \beta=\mathrm{x}, \mathrm{y}, \mathrm{z}
\end{aligned}
$$

where $D_{\alpha \beta}=$ dispersion coefficient which is a second rank tensor, $\mathrm{L}^{2} \mathrm{~T}^{-1} ; \mathrm{C}=$ concentration of the dissolved form of the constituent, in mequiv. $/ \mathrm{ml} ; v_{\alpha}=$ velocity of the fluid mixture in the $\alpha$ direction; $\mathrm{R}_{k}$ $=$ rate of production of constituent in reaction $k$ from $n$ different chemical processes, $\mathrm{ML}^{-3} \mathrm{~T}^{-1} ; \varepsilon=$ porosity, dimensionless; $\chi_{\alpha}$ are the coordinates of a three dimensional cartesian system, and the summation convention has been used in both equations. This equation is based on the assumption that convective mixing or dispersion is describable in a mathematical form similar to Fick's diffusion equation and that single-phase, isothermal-flow conditions exist. Electrical forces and/or mechanical friction forces that might force a porous medium to behave as a semi-permeable membrane are not considered here. A complete description of the subsurface distribution of multicontaminants requires the solution of an array of mass-balance equations, one for each of the dissolved contaminant species in the system and one for each of the major ion constituents that react chemically with the contaminant species. If steady recharge conditions obtain and the fluid density is independent of the aqueous contaminant concentrations, it is sufficient to solve the flow problem once, independent of chemical considerations, and incorporate the steady velocity field into the mass-balance equation.

As Schwartz (Schwartz, 1977) points out, it is unreasonable to expect a model to yield a precise description of contaminant distributions in a real subsurface situation. Problems of accurately specifying the actual configurations and of determining appropriate values of transport parameters add uncertainties to the analysis. Nevertheless, the predictive methodology provides the only way to evaluate simultaneously all significant transport and attenuation processes. By selecting realistic and conservative parameter values the minimum and maximum extent of subsurface contamination can be obtained from two deterministic model trials. Taken together, the results yield a range in contaminant distributions that reflects the uncertainty of the input parameters.

F6a. General Groundwater Conditions Required for Site

Although a proposed site may at the present time be "dry" and seem free of the effects of ground water, it undoubtedly is, or at some time during the period of concern (possibly up to $10^{6}$ years) will be, in fact, located within an active groundwater flow system. Thus, careful consideration must be given to the factors that most affect transport and attenuation mechanisms associated with groundwater flow, and quantitative analysis of groundwater flow and mass transport should be an integral part of the site selection criteria for each proposed repository. In this regard the following are appropriate general conditions that could be expected to lead to acceptability if demonstrated for a specific site:

1. The direction of groundwater flow, if any, in the disposal unit is horizontal.

2. The waste can be placed at locations in the unit where upward vertical flow of ground water is not likely or possible.

3. The hydraulic conductivity in the disposal unit is extremely low.

The intent of the third condition is to insure that the quantity of water that moves through the unit is sufficiently small and is sufficiently slow that contaminants will not reach the biosphere with unacceptable activity during the desired period of isolation. This restricted transport is dictated largely by characteristics of the medium (e.g., permeability, effective porosity, sorption characteristics). The first and second conditions depend upon the configuration of the water table, on the geologic configuration of hydraulic conductivities, and on the influence of other potentials. The intent of these conditions is to increase the path length and, hence, increase the time required for transport to the biosphere.

Although the quantity of water transmitted through a geologic unit depends also on the hydraulic gradient, a hydraulic conductivity less than $10^{-8} \mathrm{~cm} / \mathrm{s}$ would result in a negligible amount of groundwater flow for typical gradients. Second, by selecting a geologic unit for the disposal site location which has a permeability one or more orders of magnitude lower than units overlying it, but higher than units underlying it, the direction of any groundwater flow at the site would be horizontal if gravity is the major driving force. Finally, the probability of upward vertical flow at the site produced by gravity flow can be reduced to a negligible level by locating the site in or adjacent to the stagnant zone beneath a recharge area of the groundwater basin. The rate and extent of contaminant distribution is especially sensitive to site location relative to the groundwater flow system. Although location in a stagnant zone may be most desirable, a site in the transient zone between recharge and discharge areas can be quite acceptable for sufficiently long path lengths to the biosphere. It is important also to evaluate the mechanism of discharge for the particular flow system -- i.e., whether discharge is into a closed basin or an open valley, into a lake or a river, or directly into the atmosphere by evaporation.

In addition to quantitative analysis of the groundwater flow and mass transport for existing conditions at a proposed site, an analysis should be made for extreme conditions that could possibly affect the site during the desired period of isolation. Thus, the effects on the water-table configuration of possible changes in climate, in topographic configuration, and in demands on water supply need to be evaluated, along with the effects on the geologic configuration (i.e., the permeability distributions) of possible faulting and fracturing, in order to determine that unacceptable variations in the groundwater velocity field are not likely to develop during the desirable period of isolation.

\section{F6b. Groundwater Modeling as a Predictive Methodology}

We have undertaken some modeling 5 of an isothermal groundwater system for which gravity is the most important driving force, and present the results here to iliustrate the essential characteristics of a predictive methodology. Two groundwater basin models are used for this purpose: the first illustrates groundwater flow and contaminant distribution in a layered sequence of homogeneous and isotropic geologic units of different permeabilities; the second illustrates the effects on the groundwater flow and contaminant distribution of a major high permeability (e.g., fault) zone that passes through the layered model. The geologic configurations of the two models are shown in Figures $7 F 1$ and $7 F 2$. 


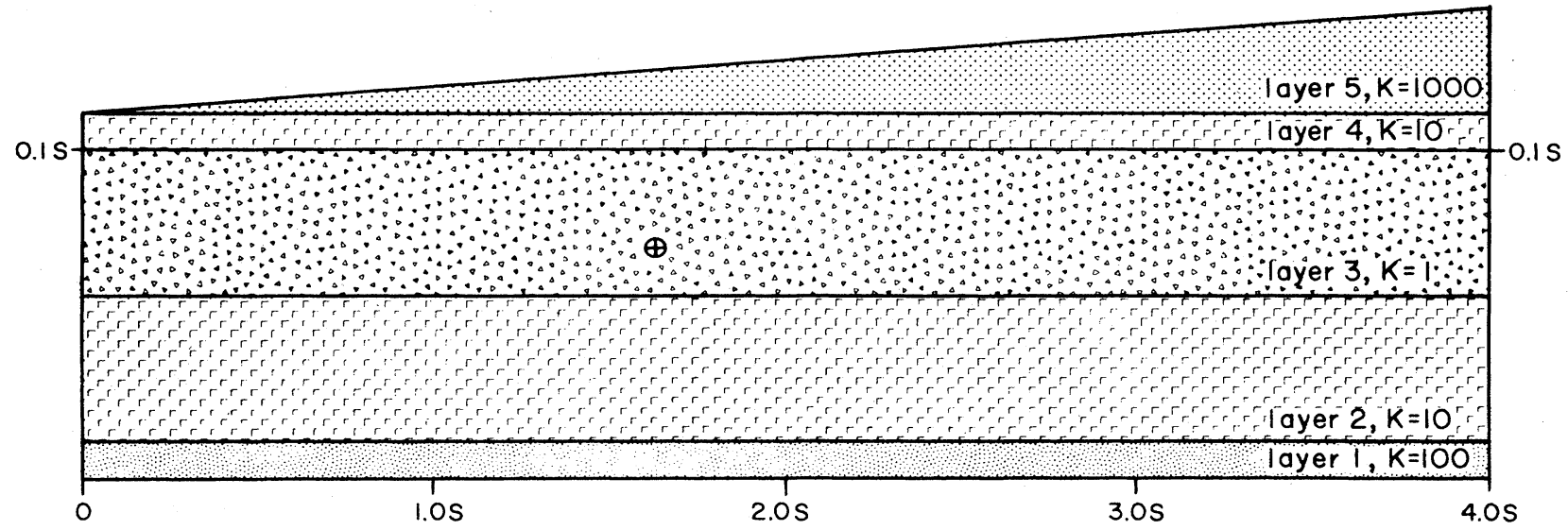

FIG. 7F1. Horizontally layered model with no-flow boundaries at sides and bottom; water-table gradient is $8.3 \times 10^{-3}$. Relative hydraulic conductivities of layers are indicated on figure; values assigned in the modeling results presented here are: layer $1=10^{-5}$; layer $2=10^{-6}$; layer $3=10^{-7}$; layer $4=10^{-6}$; and layer $5=10^{-4} \mathrm{~cm} / \mathrm{sec}$, respectively; and $s=11420 \mathrm{~m}$.

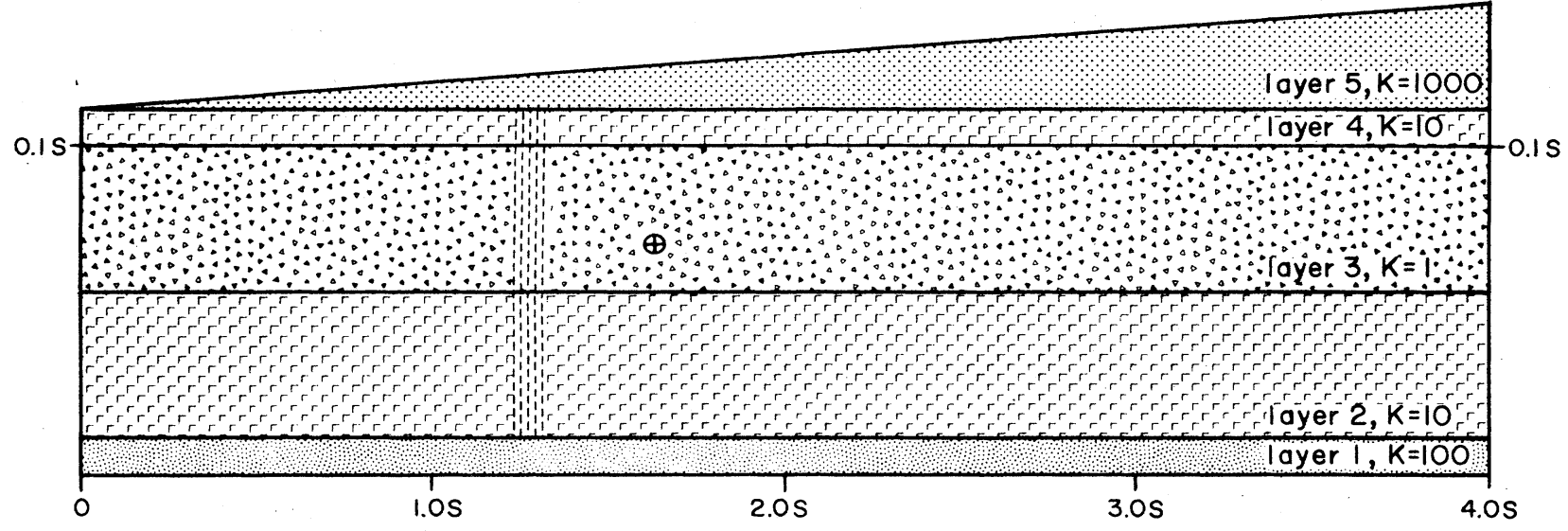

FIG. 7F2. Horizontally layered model identical to that of Fig. $7 \mathrm{~F} 1$ with the addition of a high permeability vertical zone having a relative hydraulic conductivity $K=100$. The assigned value for this zone in the modeling is $10^{-5} \mathrm{~cm} / \mathrm{sec}$.

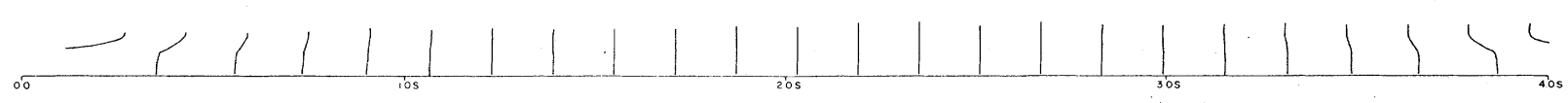

FIG. 7F3. Contoured hydraulic head distribution for configuration given in Fig. 7F1. Contour interval is $15 \mathrm{~m}$.

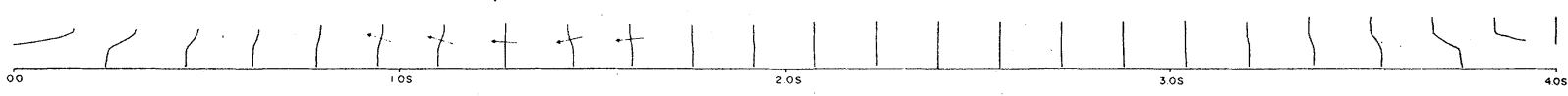

FIG. 7F4. Contoured hydraulic head distribution for configuration given in Fig. 7F2. Contour interval is $15 \mathrm{~m}$. 
The hydraulic conductivities assigned to the layers are given in the captions; these may be compared with typical measured values for different-geologic formations given in Table 7F1. The sides and the bottom of each model are no-flux boundaries, and the hydraulic head distributions used to determine the velocity fields solely reflect the water-table configuration which has a uniform gradient of $8.3 \times 10^{-3}$. In this modeling we have not considered the effects on hydraulic potential of sediment compaction, osmotic pressure, fluici density or temperature variation and other factors that may need to be considered in site-specific analyses. The contoured hydraulic head distributions thus derived for the two models are shown in Figures $7 \mathrm{~F} 3$ and $7 \mathrm{~F} 4$. Because these cross-sections are close to true scale and the geologic units are isotropic, flow directions will be perpendicular to the equipotential lines shown on the diagrams.

The respective velocity fields defined by the hydraulic head distributions are used to determine the effects of time, dispersivity, and contaminant source location on the distribution of a nondecaying contaminant that does not interact with the porous medium -- i.e., we do not incorporate here the effects of such nuclear and chemical processes as radioactive decay and sorption. In the modeling of contaminant distribution presented here a finite number of reference particles (e.g., 20) are supplied to the transport medium (ground water) during a fixed time step (e.g., $10^{4}$ years). Each model consists of a rectangular array of nodal points that define 13 horizontal rows and 36 vertical columns of cells, and the particles initially are randomly distributed along a horizontal line that extends \pm 0.05 one cell length from the center (indicated by a $\oplus$ in the figures) of a designated cell within the modeled disposal unit.

For each time step each particle moves in two displacement steps -- the first is deterministic, the second is random. The first displacement step is determined by the groundwater velocity vector.

TABLE 7F1. Hydraulic conductivities of geologic media.

\begin{tabular}{lc}
\hline \hline Material & Hydraulic Conductivity \\
\hline slate & $1.1 \times 10^{-9} \mathrm{~cm} / \mathrm{sec}$ \\
quartzite & $1.6 \times 10^{-9}$ \\
graywacke & $2.6 \times 10^{-9}$ \\
clay, montmorillonite & $4.7 \times 10^{-9}$ \\
till, clay-loam (intergranular) & $2.7-6.0 \times 10^{-9}$ \\
salt, bedded & $6.3 \times 10^{-9}$ \\
till, clay-loam (fractured) & $1.8 \times 10^{-7}$ \\
shaleb & $0.7-2.0 \times 10^{-7}$ \\
siltstone & $1.2 \times 10^{-7}$ \\
limestone, argillaceous & $0.9 \times 10^{-7}$ \\
dolomite & $0.9 \times 10^{-6}$ \\
sandstone, silty & $2.2 \times 10^{-6}$ \\
limestone & $6.6 \times 10^{-6}$ \\
silt & $1.0-7.8 \times 10^{-5}$ \\
graywacke (fractured) & $3.9 \times 10^{-5}$ \\
sandstone, coarse & $0.9 \times 10^{-3}$ \\
sand, river alluvial & $1.7 \times 10^{-2}$ \\
\hline \hline
\end{tabular}

a Measurements for some salts give values as high as $1.5 \times 10^{-4}$

b Measurements for some shales give valucs as low as $0.8 \times 10^{-10}$.
The groundwater velocity (hydraulic conductivity $\times$ hydraulic gradient/effective porosity) at each particle cooidinate position within a cell is calculated from the velocities at the four nodal points defining the cell. The character of the random displacement step is determined by a normal distribution for which one standard deviation in the longitudinal direction (parallel to flow) is $\sigma_{\mathrm{l}}=(2 \xi \mathrm{V} \triangle \mathrm{t})^{1 / 2}$ and in the transverse direction is $\sigma_{\mathrm{T}}=(0.4 \xi \vee \triangle \mathrm{t})^{1 / 2}$ where $\xi$ is the dispersivity, $V$ is the magnitude of groundwater velocity, and $\Delta t$ is the time step. The effect on contaminant distribution of the dispersivity can be appreciated from these relationships. For these models the longitudinal dispersivity is taken to be five times larger than the transverse, similar to what might be expected in a natural geologic situation.

The resultant particle density distribution after any specified period (number of time steps) can be used to determine concentrations by assigning mass values to each particle as it enters the transport (ground water) system. The concentration in a given cell is equal to the total mass of all particles contained in the cell divided by the volume of water in the cell (cell area $X$ porosity). The amount of mass supplied to the transport medium (ground water) is determined by the leaching rate of the contaminant at the source and the period of time during which leaching occurs.

The distribution of contaminant with increasing time is seen in Figure 7F5. For this and the other similar figures that follow, the number of particles found at any point within the intermediate contoured area would be between 2 and 10 percent of those in the system at that time; the outermost contour encloses all particles in the system. For the model configuration shown in Figure 7F5, 99.8 percent of the particles added to the system remain confined within a zone $3800 \mathrm{~m}$ lateral by $630 \mathrm{~m}$ vertical even after $0.8 \times 10^{6} \mathrm{yrs}$. The remaining 0.2 percent lie within an additional lateral distance of 1270

Figure $7 \mathrm{~F} 6$ illustrates the effect of dispersion length on the distribution of contaminant for the same model configuration after $0.8 \times 10^{6} \mathrm{yrs}$. A dispersion length of $15 \mathrm{~m}$ can be taken as typical. It is clear that longer dispersion lengths cause the contaminant to be more extensive:y distributed. Nevertheless, 98 percent of the particles added to the system during this period remain confined within a zone $3800 \mathrm{~m}$ lateral by $630 \mathrm{~m}$ vertical even with a dispersivity of $30 \mathrm{~m}$. Most of the remaining 2 percent is distributed in the layer overlying the disposal unit, with some particles having reached the uppermost aquifer by that length of time.

The effects of a major vertical zone of higher permeability, such as might characterize certain fault zones (it should be noted that other fault zones may represent effective barriers to flow), that passes through the disposal unit are illustrated in Figures $7 \mathrm{~F} 7$ and $7 \mathrm{~F} 8$. Comparison of the contoured hydraulic head distributions shown in Figures $7 \mathrm{~F} 3$ and $7 \mathrm{~F} 4$ shows that the presence of a vertical zone of high permeability alters the velocity field of groundwater flow. Figure 7F7 illustrates how this can affect contaminant distribution with increasing periods of time when the zone lies to the discharge side of the contaminant source location. As might be expected, the consequences of a zone of high permeability passing through the contaminant source location are serious. As suggested by Figure 7F8, significant losses of particles to the biosphere occur with increasing time - 9 percent after 10,000 yrs. and 25 percent after $15,000 \mathrm{y}$ 'rs. Fortunately, as previously stated, we have concluded that the probability of a fault intersecting a repository could be made very small, perhaps $10^{-11}$ per year or less.

The values of hydraulic conductivity and dispersion length used for these models are not extreme (see Table 7F1); indeed, the assigned values and the geologic situation represented by the uniformly layered model are sufficiently ordinary that we anticipate no difficulty in locating several sites with suitable hydrogeology within the immediate future. Although the layered model is characteristic of a sedimentary rock sequence--which would include salt, shale, and limestone, currently being considered by ERDA--a single-layer or more complex model can be developed for crystalline rock (e.g., granite), with or without overlying sedimentary layers. The values of hydraulic conductivity and dispersion length, and the number of layers (as well as their configurations) can be changed to simulate either an existing or a hypothetical situation. 

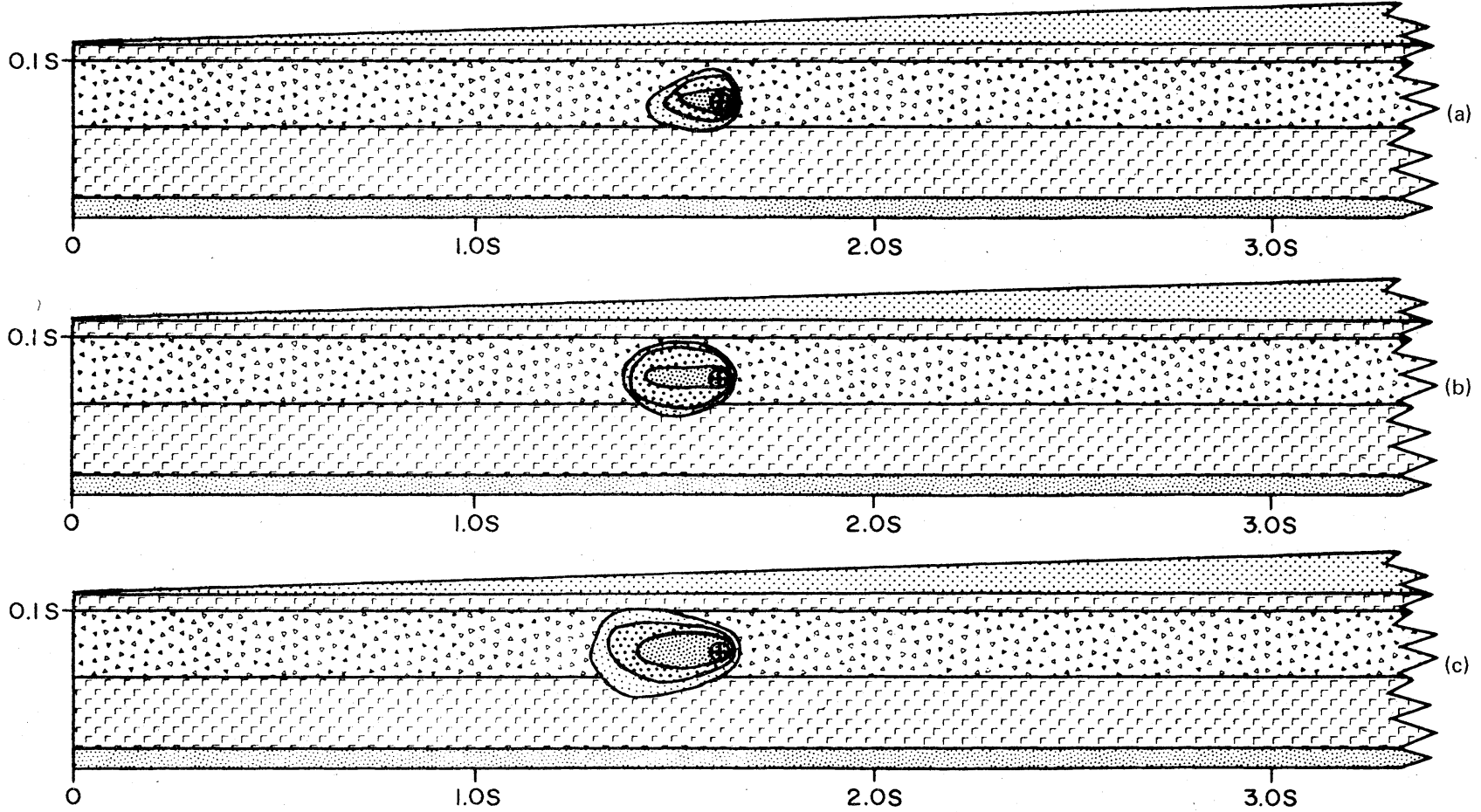

FIG. 7F5. The effect of increasing time on contaminant spread; (a) after 200,000 yrs; (b) after 400,000 yrs; (c) after 800,000 yrs. Hydraulic conductivities as given in Fig. $7 \mathrm{~F} 1$; dispersivity value assigned in all layers is $15 \mathrm{~m}$; effective porosity is 10 percent. Contaminant source location indicated by $\oplus$. Intermediate contoured area includes between 2 and $10 \%$ of particles in system; the outermost contour encloses all particles in the system.

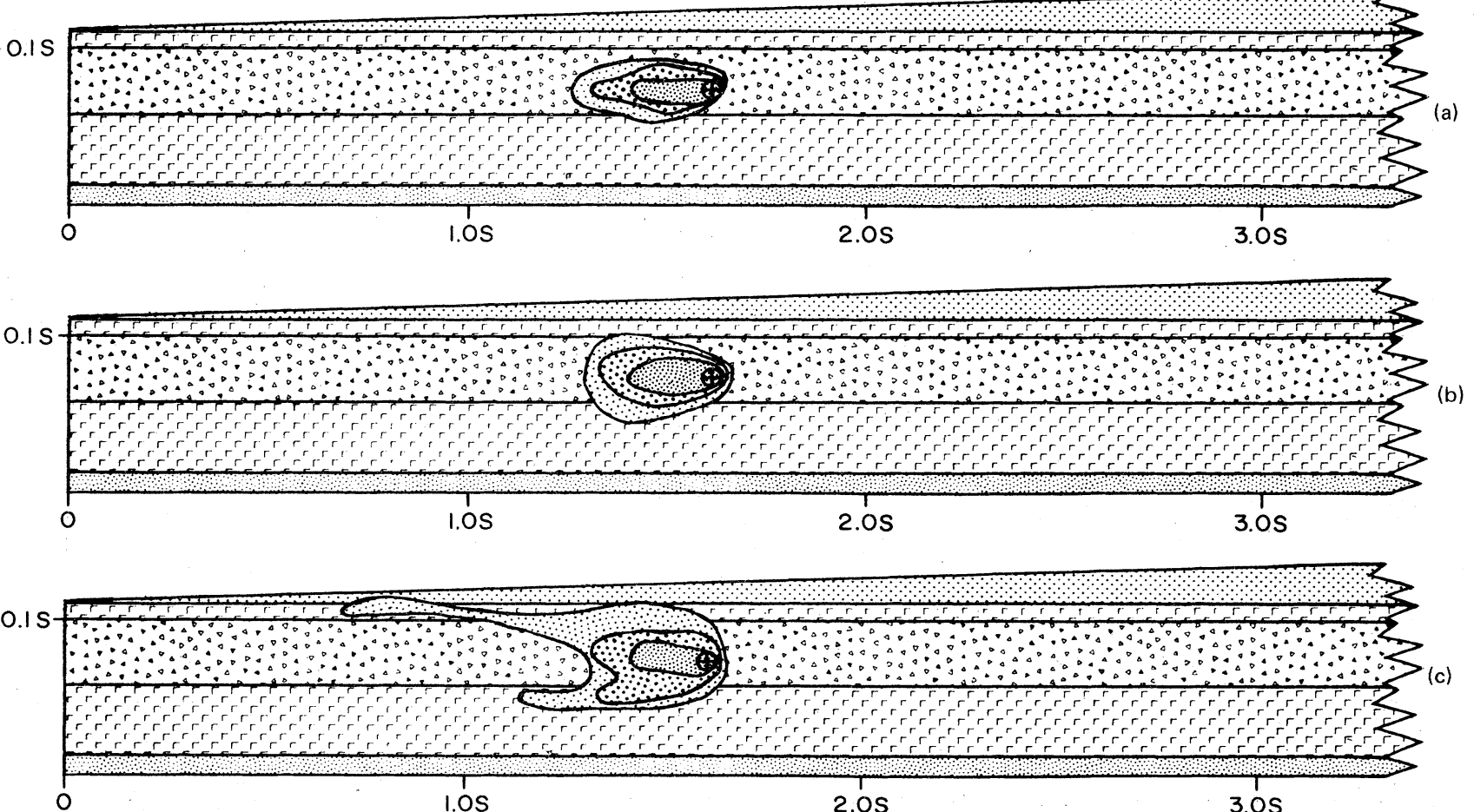

FIG. 7F6. The effect of dispersivity on contaminant spread after a period of $800,000 \mathrm{yrs}$; dispersion lengths assigned are: (a) $3 \mathrm{~m}$; (b) $15 \mathrm{~m}$; (c) $30 \mathrm{~m}$. Contaminant source location indicated by $\oplus$. 

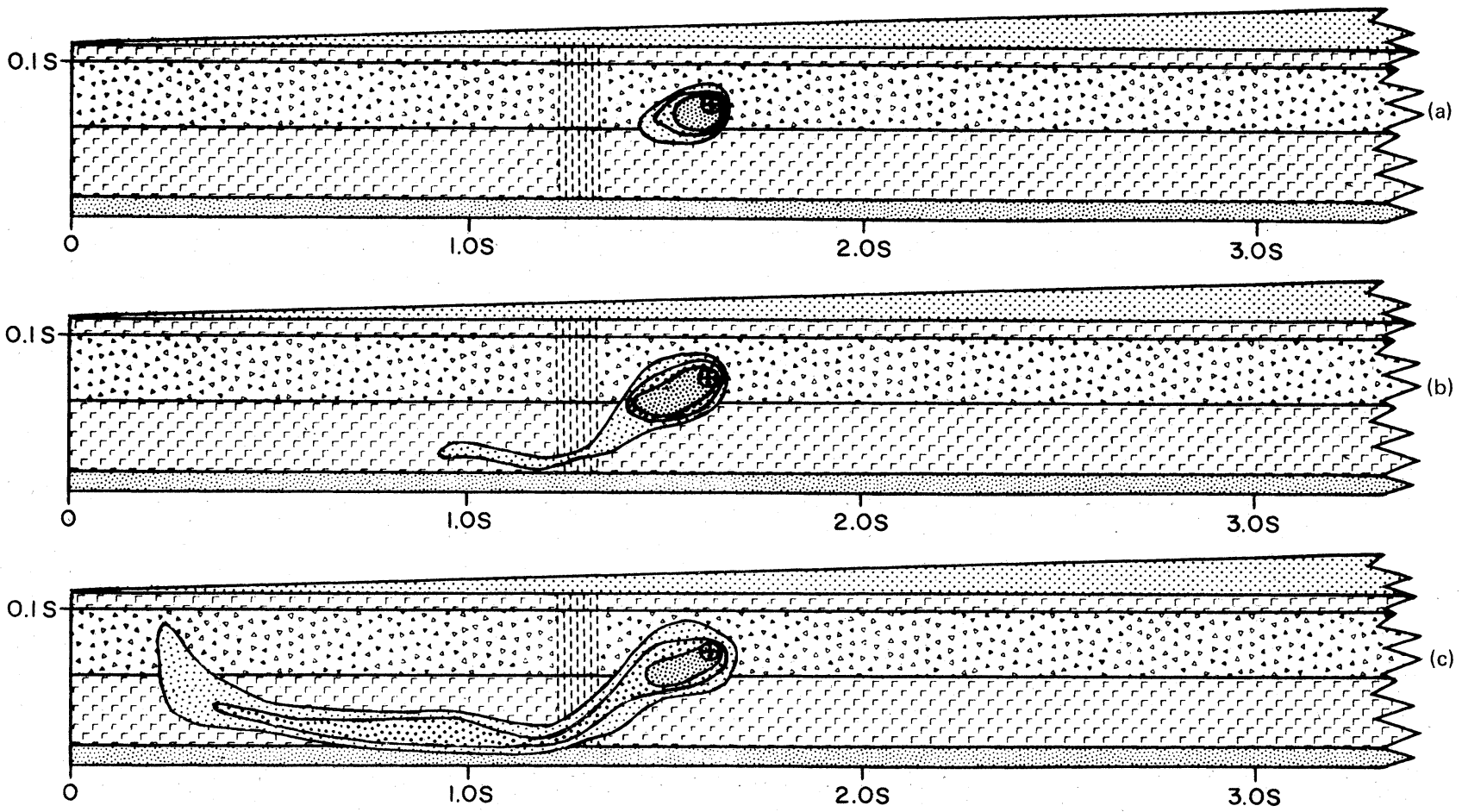

FIG. 7F7. The effect with time of a vertical zone of high permeability located between contaminant source and groundwater discharge area: (a) after $200,000 \mathrm{yrs}$; (b) after 400,000 yrs; (c) after $800,000 \mathrm{yrs}$, Hydraulic conductivities as given in Fig. 7F2. Contaminant source location indicated by $\oplus$.
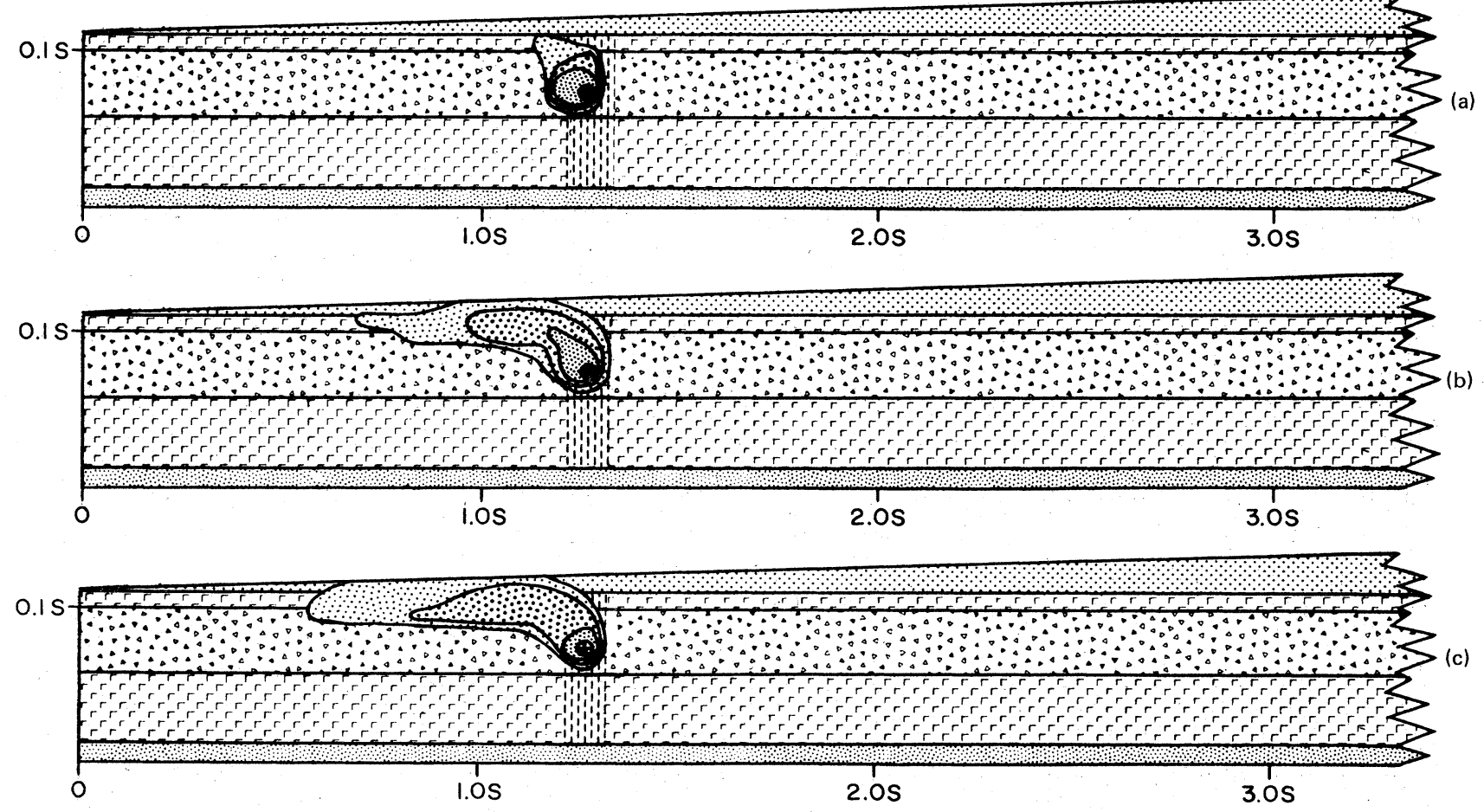

FIG. 7F8. The effect with time of a vertical zone of high permeability passing through a contaminant source: (a) after 5,000 yrs; (b) after 10,000 yrs; (c) after 15,000 yrs. Contaminant source location indicated by $\oplus$. 


\section{F7. Site Suitability Criteria}

The examples of groundwater modeling we have presented, as previously stated, are solely to illustrate the essential characteristics of a predictive methodology. Because the effects of concentration attenuation mechanisms, which most certainly would be operative in a natural environment, were not incorporated, the modeling is conservative. (Recall, however, that the effects of potentials other than gravity have also not been incorporated. In certain site-specific evaluations, these could have significant influence.) Conservative modeling of this type would be appropriate for establishing a site suitability criterion. Incorporation of the effects of various concentration attenuation mechanisms would lead to realistic modeling which could provide a quantitative basis for comparing the relative desirability of acceptable repository sites. We feel strongly that the criteria for suitability of a site for a repository should include specifications of appropriate hydrogeologic parameters, and that these specifications should have to be satisfied by the present-day hydrogeology and by the projected bounds of the future hydrogeology for the specific site. This, in addition to criteria for site selection that would minimize the possibility of the repository being breached during the period of desired isolation, either by natural or by anthropogenic processes, should constitute the necessary and sufficient conditions that would ensure safe and reliable isolation of radioactive waste from the biosphere. We recognize that additional criteria will be needed to define exploration and engineering procedures (as well as satisfy environmental and other factors) for any particular site, so as not to compromise the geologic factors given above.

In closing this section we should emphasize that any consideration of the "appropriate" geological conditions for waste emplacement should take into account not only the likelihood that wastes might move (i.e., be transported by ground water), but also the probability of these entering the biosphere and the dosimetric consequences if that were to occur. A means of evaluating the potential health effects of a release could be used to derive generic as well as site-specific criteria. A question that needs to be addressed is: What are acceptable consequences in terms of dose to a future exposed population? This question would not have to be answered prior to an analysis if a set of reference criteria were established. For example, it might be specified that a population living in the locale where the wastes might contact the biosphere should not be exposed to (a) levels above some percent of background; (b) levels greater than background (i.e., $0.1 \mathrm{rem} / \mathrm{yr}$.) (c) levels above present standards (i.e., $5 \mathrm{rem} /$ generation); (d) chronic exposures at levels at which biological effects might be manifested but are unlikely to be observed (i.e., $0.5 \mathrm{rem} /$ day); or (e) levels that would elicit acute biological effects.

We recommend that modeling of the process from emplacement to ultimate exposure by man be undertaken to determine the sensitivity of the dosimetric consequences to the parameters and assumptions used in the modeling. The modeling should include the effects of such processes as sorption and radioactive decay. Such modeling could assign different leaching and sorption rates to different radioactive species, which would affect their. mobility rates, as well as include the respective decay constants, which would determine levels of activity with time. The results could be used to define both research needs and the critical parameters that might be incorporated in criteria for site suitability; these could also be utilized in determining the period over which isolation is required.

\section{G. Improved Wasteforms}

\section{G1. Motivation}

In view of the conclusion that the emplacement of vitrified waste into a geologic repository is likely to provide an adequate solution to the problem of HLW management, is there motivation for further research and development on wasteforms and if so what should be the aims and goals of this development? The incentives for improved wasteform processing are several: technical improvements and modifications in existing processes can increase operating efficiency and reduce occupational radiation exposures associated with routine operation and maintenance; alternative wasteforms, e.g. metal-matrixencapsulated calcine pellets, may offer better solutions than vitrification to the problem of meeting design objectives of low dispersability in case of accident, and of retrievability, at minimum radiological hazard and economic cost; alternative wasteforms, e.g. insoluble ceramics, which allow for safe waste disposal despite continued contact with rapidly flowing groundwater would open a wider range of geological disposal possibilities, perhaps useful for the U.S., but most important for countries, e.g. Japan, with very limited options for waste disposal; and such a wasteform would offer the advantage of double protection if used in conjunction with a "safe" geological environment.

\section{G2. Criteria}

As in any engineering problem, the decisions concerning solidification technologies require trade-offs among a variety of objectives, and cannot be made without some definition of the costs or benefits associated with these various objectives. In the case of solidification technology, U.S. pilot plant and European full scale operations are providing a base for estimating the economic and radiological exposure costs of at least the calcination and glassification processes and reasonable estimates of similar costs are possible for various proposed alternatives. Similarly one may evaluate the advantages of the various options as related to the problems of transport to, and emplacement in, a federal waste repository and of retrievability. The difficulty in making reasonable overall assessments comes in defining criteria for wasteform performance on time scales longer than 100 years.

One measure of the long term radiological hazard associated with the HLWW is the ingestion hazard index defined in section Bl, and a variety of waste management strategies have been supported by consideration of this index. It should be noted that this harard index gives no indication of absolute hazards from radioactive wastes, since it takes no account of the mechanisms and pathways for the individual radionuclides to reach the biosphere and ultimately to reach mankind. Also, as discussed in section. B2, only in very limited cases is the hazard index a meaningful measure of even the relative hazards of radioactive wastes.

The establishment of well defined criteria for wasteform performance is essential to the orderly development of improved wasteforms. As indicated by the discussion of section B2, there will be no simple index which can be used to define the hazard associated with waste isolation. Sensible criteria can be developed on the basis of modeling calculations and associated sensitivity analyses in which wasteform leach rates are an input parameter. Development of modeling techniques and the collection and evaluation of appropriate geological input data are essential to the meaningful definition of these criteria.

The impact of such studies upon development of new wasteforms may be substantial. A possible conclusion is that the confidence in geologic isolation is sufficient that the prime wasteform criteria should relate only to short term safety, i.e., processing, handling and retrievability problems. Will modeling calculations show that actinide transport in relevant environments is negligible and that the concern is therefore only with behavior for 1000 years, not longer? Will the modeling show that there are indeed unlikely but conceivable circumstances where wasteform durability on a $10^{3}$ year or $10^{5}$ year time scale would of fer significant added protection? Answers to these sorts of questions and the development of more meaningful criteria than "as low a leach rate as possible" are necessary as a guide to a sensible researih program on future wasteforms.

One sees already the conflict between short and long term goals in the development of glassification technology. In improving the behavior of glasses with long term goals in mind, i.e., good homogeneity and low leachability, programs have shifted to higher melting point glasses to reduce leachability, and melting at as high a temperature as possible to improve, through lower viscosity, the mixing and the uniform dissolution of the HLW in the glass. The resultant short term penalties involve problems of ruthenium volatility, equipment maintenance, corrosion of the canister by the melt, and high residual canister stress resulting from the differential contraction of stainless steel and glass upon cooling of the canister from the higher temperatures. Without well defined development goals and criteria it is impossible to decide how to make the necessary design trade offs. 
Since the principal means of transport of radionuclides from the geological emplacement site to the biosphere is via aqueous transport, an obvious design objective is to develop a wasteform of sufficiently high resistance to dissolution by groundwater that whatever the rate of groundwater transport, the rate of release of harmful material to the aqueous medium would be sufficiently low 10 avoid excessive subsequent exposure of any individual. Is it reasonable to suppose that wasteforms of sufficient chemical durability are achievable? Are the leach rates in the range $10^{-4}-10^{-7} \mathrm{~g} / \mathrm{cm}^{2}$ day quoted in the TAD in a range which is useful?

Although meaningful predictions and design criteria will be obtained only by careful modeling of specific situations, a very simple example will serve to illustrate that a leaching rate of $10^{-7} \mathrm{~g} / \mathrm{cm}^{2}$ day, if reliably achievable, could be a meaningful barrier to radionuclide release. Consider the extreme case that a canister containing vitrified high level waste is left in the drinking water reservoir of a small city, without the protection of an outer can. Combining the leach rate above, with the canister dimensions, a reservoir volume of $10^{6} \mathrm{~m}^{3}$, and a flushing time of 100 days leads to concentrations of radionuclides below those specified as maximum permissible concentrations under the regulations 10 CFR 20 during the time in which the fission product activity dominates the hazard, that is for the order of 500 years after disposal. For the exposure to the transuranics, equal protection (concentrations below RCG's) would be obtained with leach rates $10^{3}$ times greater. We are well aware of many subtle difficulties with such arguments; we make it only to illustrate that low leach rates can give a very meaningful assist in the isolation of radioactive wastes. The effectiveness of a low leach rate is not predicated on assuring no release to the biosphere but on the knowledge that, in the event of the failure of complete confinement, the stability against leaching can limit the release rate to what may be deemed acceptable levels. This argument is modified if one anticipates a loss of mechanical integrity of the glass, perhaps due to devitrification, to give characteristic dimensions the order of millimeters instead of tens of centimeters, thus increasing the surface area exposed to the leachant. Such a granulation of the waste increases the steady state concentrations by a factor of one hundred and the previously "safe disposal" of the waste in the reservoir now gives rise to significant, though not disasterous, health effects. Clearly one needs not only a low estimated release rate, but a high degree of confidence in the reliability of that estimate.

Thus an important longer-term goal for the waste management program is the development of a wasteform with predictably low leachability. The development of criteria for "sufficiently low" obviously requires more sophisticated modeling than the previous argument and we have already commented on the need for such modeling. Our concerns here are with two other aspects of the problem. First, as noted above, some existing data indicate there already exist materials with attractively low leach rates. We wish to address the question of the extrapolation of these data to long times and to geologic environments. Second, we consider some alternate wasteform concepts which may prove more satisfactory in providing higher chemical durability than the borosilicate glasses.

\section{G3. Wasteform Durability}

Leachability. Confidence in the ability of a wasteform to prevent long-term radionuclide release to groundwater requires detailed characterization of the leachability of a variety of radionuclides from the wasteform, and the ability to extrapolate that characterization for long times to uncertain hydrochemical conditions.

Leach rates are measured by exposure of the wasteform to water and determining either the erosion rate of the matrix or the rate of leaching of individual chemical species from the matrix. Results are typically, though not invariably, expressed in units of $\mathrm{g} / \mathrm{cm}^{2}$ day. If the leach rate is determined by sample weight loss, the units are natural ones and the rate is calculated simply as the weight loss per day divided by the exposed area of the sample. If the rate is determined by radioactive or chemical assay of a particular species in the leachant the rate is quoted in terms of the mass of material that would have to be dissolved to release the measured amount of assayed species under the assumption of no internal diffusion of that species within the solid and no penetration of the leaching solution into micropores of the material. In many examples, leach rates so expressed are much larger than the actual dissolution rate of the material because of rapid solid state diffusion of the assayed species to the surface. Note that a dissolution rate measured by sample weight loss may grossly underestimate the release rate of a highly mobile species.

The difficulty of extrapolation of such test results can perhaps best be illustrated simply by listing some of the sources of complexity and uncertainty that arise.

1. Leach rates of different chemical species frequently differ by one or more orders of magnitude. Alkalis may leach by diffusion to, and dissolution at, an external surface or crack, while leaching of actinides, typically slower, may result from direct dissolution of the glass matrix.

2. Leach rates may be time dependent, raising questions about long term extrapolation, particularly if the relevant mechanisms are not understood. Diffusion of ions to the surface, for example, gives a leach rate decreasing with time (Godbee and Joy, 1974) as $\mathrm{t}^{-1 / 2}$.

3. $\mathrm{pH}$ of the leaching solution is important for some mechanisms. Cation diffusion to the surface can be markedly enhanced in low pH solutions (Elliot and Auty, 1968); the rapid diffusion of hydrogen ions into a glass can produce space charge electric fields which drive other cations out of the glass at a rate much higher then predicted from simple diffusion arguments. The breakdown of the glass matrix, possibly the rate limiting step for the release of actinides, is observed to increase with increasing $\mathrm{pH}$ in some glasses (Elliot and Auty, 1968).

4. Flow rate of the leaching solution is of ten an important parameter, and its effect may, be of either sign. For slow flow or nearly stagnant conditions the dissolution rate may be limited by the solubility of the ion in question or its rate of diffusion through a stagnant surface layer of water to flowing water beyond. Increasing flow rate will increase the dissolution rate. But the contrary is also possible (Kelley and Wallace, 1975). Stagnant conditions can lead to an increase in $\mathrm{pH}$ due to hydrogen-cation interchange; this increase in $\mathrm{pH}$ then enhances the attack of the glass matrix. In this case, increasing the flow rate can decrease the dissolution rate.

5. Concentrations of appropriate ions in the leaching solution may result in the precipitation of new minerals upon dissolution of particular cations from the wasteform, thus effectively preventing their release to aqueous transport. Other chemical species in the water might increase the concentration of low solubility ions through appropriate formation of chemical complexes.

6. Comparison of different glasses under a set of standard conditions, e.g., the IAEA standard leach test (Hespe, 1971), may give misleading implications concerning their relative performance under substantially different circumstances which might better approximate actual conditions of exposure.

7. Because of the likelihood of devitrification of glass cooled for tens of years in canisters with initial centerline temperatures as high as $800^{\circ} \mathrm{C}$, leaching tests made on the homogeneous glass may have little relevance to the long term behavior of the wastes.

8. Leach testing of inhomogeneous material, such as partially or completely devitrified glass, offers further problems of interpretation. Two examples might serve to illustrate. A wasteform consisting of highly soluble crystallites in an insoluble matrix could serve as quite a satisfactory waste form. A leach test in which the material was crushed to expose a large surface area, as is frequently the practice, would expose many of the unprotected soluble crystals and hence the material would appear to perform poorly in the test. In contrast consider a material in which the radionuclides are tied up in fairly low solubility crystals in a highly soluble matrix containing little radioactivity. A short term test on a monolithic sample, such as the IAEA test, might result primarily in dissolution of the matrix and a few exposed waste-containing crystals. Prolonged leaching, however, could destroy the matrix rather rapidly, exposing orders of magnitude more surface area of waste crystals to dissolution. Such a material would show a release rate of radioactivity which would increase with time. Such intergranular leaching has been observed (De, et al., 1976) in studies on a glass-ceramic wasteform. 
9. Devitrification of glass does not lead to a uniquely defined state, but rather to one of a number of states whose properties, including leachability, may depend upon the detailed thermal and stress history of the glass. Simulation of the devitrification anticipated in actual storage conditions may be difficult.

These ideas should indicate some of the difficulties inherent in trying to predict reliably the long term behavior of the glass wasteform. Establishing confidence in any such predictions will require a much broader empirical data base, such as is currently being obtained at BNWL, on a variety of materials and over a wide range of conditions. It will also require a deeper detailed understanding of the leaching mechanisms and the consequences of devitrification. We do not feel that the data base or the understanding currently justifies reliance completely upon low leach rates as the principal barrier to radionuclide release.

Mechanical Integrity. A second question of concern is that of mechanical integrity of the wasteform after devitrification. The devitrification of glasses can in some instances lead to a ceramic with mechanical properties superior to that of the parent glass; in other cases it may lead to a complete loss of mechanical integrity. The reduction of the waste from a monolith of characteristic dimensions 10 's of centimeters to a powder of characteristic grain size 100's of microns would naively be expected to increase the release rate to the environment by a factor of $10^{3}$. For a given glass composition there are a large number of stages involved in the devitrification process (Roy, 1962), and the state which obtains depends upon the detailed thermal (and adiation) history of the material. "The devitrified state" of a given glass composition is hardly a uniquely defined state of the material, raising the obvious question again of the relevance of the laboratory tests on material devitrified, using some particular thermal cycling, to the behavior of a real waste product which may experience quite a different thermal history.

Radioactivity. A final set of problems derives from the presence of radionuclides in the waste and the effects upon the wasteform of the radioactivity and transmutations associated with the decay of these radionuclides. The integrated radiation exposures during the decay of the waste are impressive. Figure 7G1 shows, as a function of storage time, the accumulated $\alpha$ dose for (1) $\mathrm{UO}_{2}$ fuel and (2) the additional dose associated with moving to mixed oxide fuel (Mendel, et al., 1976). Note that in the $\alpha$ decay, the recoil nucleus creates about 1500 atomic displacements before it comes to rest. At a dose of $2 \times 10^{19} \alpha$-decays/gram the number of atomic displacements is $3 \mathrm{x}$. $10^{22} /$ gram or roughly one displacement for each atom in the glass. In addition to the radiation damage one must be concerned with the possible mechanical degradation of the waste by the stresses associated with the precipitation of helium generated by the $\alpha$ decays; the chemical changes associated with the transmutation of the fission products, particularly the $\mathrm{Cs} \rightarrow \mathrm{Ba}$ and $\mathrm{Sr}-\rightarrow \mathrm{Zr}$ decays, present yet another problem.

These problems, with the exception of the transmutation chemistry, have been addressed experimentally, primarily with vitreous glasses and to some extent with devitrified material. The effect of ionizing radiation is simulated by exposure of experimental glasses to intense $\gamma$-radiation; the $\alpha$ damage is simulated by doping of glasses with ${ }^{238} \mathrm{Pu}$ or ${ }^{244} \mathrm{Cm}$ which, because their decay is faster than that of typical actinide components of the wastes, can deliver in a few years, doses comparable with those expected from high level wastes over thousands of years (Mendel, et al., 1976). Despite the extremely heavy doses, a few $x 10^{18} \alpha$ decays corresponding to several x $10^{21}$ atomic displacements, there seems remarkably little effect on the properties of the glasses, including the leach rates; presumably there is extensive anmealing, perhaps enhanced by the irradiation, at the ambient temperature.

Again the question arises as to the relevance of these results to the real problem. In devitrified material will the crystalline phases be as insensitive to the radiation damage as the vitreous phase appears to be? Will future, chemically more durable glasses show comparable insensitivity? The segregation of the actinides to specific components of the devitrified material implies a spatial inhomogeneity in the distribution of the $\alpha$-damage. The lattice expansion ( $\sim$ tenths $\%$ ) produced by the $\alpha$ damage will then cause local strains as grains containing. high actinide concentrations expand, but neighboring grains, depleted in actinides, do not. Do these strains represent a threat to the mechanical integrity of the devitrified material?

Helium generation by the $\alpha$ decay is also a threat to the mechanical integrity of the glass and canister. Whether or not the generated helium remains entrapped in the glass or is released to the dead space above the glass in the canister depends in detail on the temperature of the glass, which controls the diffusion constant of the helium, and the mechanical integrity of the glass, which determines the characteristic distance of diffusive transport required to release the gas to pores, or cracks, via which it is then easily released to the canister enclosure. With a dead volume of $20 \%$ of the glass volume the accumulated helium, if immediately released to the dead space, will produce one atmosphere excess pressure only after several thousand years of storage of uranium fuel waste. For waste from mixed oxide fuel, however, this time is reduced to less than ten years, and one must either rely on helium entrapment by the glass or design the waste canister to support moderate internal pressure (a few atmospheres) to maintain integrity for the 5 year time span of the retrievable storage. If helium release requires diffusion of the helium through a glass monolith of dimension 1 the order of $10 \mathrm{~cm}$, then the characteristic time for release $1^{2} / 1$ ), is about 300 years for the measured diffusion rate at $350^{\circ} \mathrm{C}$ (Mendel, et al., 1976). These numbers imply adequate protection, but only marginally so, and the argument relies both on the mechanical integrity of the glass and a low glass temperature. Fracturing which reduces the characteristic diffusion length to $1 \mathrm{~cm}$, reduces the release time to 3 years, and removes the barrier to helium release (at $350^{\circ} \mathrm{C}$ ).

Another point of concern is the possible accumulation of helium in existing or newly developed voids in the wasteform. Using the solubility of helium in waste glass at STP of $.6 \times 10^{-3} \mathrm{~cm}^{3}$ of $\mathrm{He} / \mathrm{cm}^{3}$ of glass (BNWL-1932, 1975), and assuming the equilibrium solute density proportional to the helium pressure in the void, indicates the possibility of establishing void pressures of 1.6 kilobars $\left(\sim 2.5 \times 10^{4}\right.$ psi) at an $\alpha$ dose of $10^{19}$ decays/gm $\left(10^{5}\right.$ years of uranium fuel waste but only $\sim 100$ years of MOX waste), if the fractional void volume remains small $\left(\sim 10^{-3}\right)$. This pressure may be of sufficient magnitude to threaten the mechanical integrity of the glass, altiough such factors as void size and the microstructure of the glass would be of crucial importance.

Finally the question of the stability of wasteforms against transmutation, although occasionally raised, seems not to have been seriously addressed. About $40 \%$ of the monovalent fission produce cesium decays to divalent barium after the decay of the ${ }^{137} \mathrm{Cs}$ with 30 year half life and $50 \%$ of the divalent strontium decays to tetravalent zirconium. Again there are the questions--what will be the long term consequences in terms of the chemical stability (leach rate) and mechanical integrity of the material, be it in a vitreous or crystalline form, of this substantial modification of chemical composition?

These arguments provide the basis of the statement in an earlier section of this report that our present knowledge of the properties of the borosilicate glass as a wasteform is inadequate to place reliance on the glass as the principal barrier to radionuclide release. On the other hand, if the currently measured leach rates of the more durable glasses could be relied upon for geologic environments and times, then the use of such a well characterized glass wasteform could open a variety of new disposal techniques or, alternatively, offer a reliable additonal barrier to radionuclide release using the current concept of disposal in conventional mined cavities. Thus current programs to improve the characterization of wasteforms, and to investigate in detail the effects of devitrification upon the glass durability are most appropriate. These programs must address, among others, the questions raised earlier in this section.

\section{G4. Alternative Forms}

A number of possible future wasteforms, alternatives to glass, are also described in the TAD (ERDA-43, 1976). There are two important concepts which, with differing weights and in different forms, underly these alternatives. The first is to develop a wasteform in which the waste ions are incorporated chemically not as an incidental ion, or solute, in a glassy structure, but as an essential ion in a stable crystalline form. The second is to embed particles or pellets of waste material in an inert and chemically durable matrix. 
One of the principal difficulties in the use of glass is the high probability of devitrification with the associated uncertainties in the mechanical integrity and chemical durability of the resultant partially crystalline state. Since the source of the difficulty is the metastability of the glassy state, why not prepare the material intentionally in a polycrystalline state in which each radionuclide is incorporated as an essential chemical component of one or another crystalline phase of high stability and low solubility? Having prepared the material in a mixture of thermodynamically stable phases the ambiguities of the effects of future phase changes are removed. If suitably insoluble phases can be found, it may be possible to tailor a material to have satisfactorily low leachability.

This concept is being pursued in several laboratories. At Sandia Laboratories (SAND-76-0105, 1976) a process is proposed in which the radioactive ions are removed from liquid wastes by ion exchange columns with $\mathrm{Na}\left[\mathrm{Ti}_{2} \mathrm{O}_{5} \mathrm{H}\right]$ as the ion exchange medium. Subsequent drying and treatment at $\sim 600^{\circ} \mathrm{C}$ leads to the formation of a calcine containing a number of fission product and transuranic titanates, species which are much less soluble than the various simple oxides resulting from the usual calcining processes. A group at Penn State (McCarthy, 1976) proposes to add appropriate species such as nitrates of $\mathrm{Ca}, \mathrm{Sr}, \mathrm{Al}$ plus colloidal $\mathrm{SiO}_{2}$ to the liquid wastes to promote, in a normal calcining process, formation of stable mineral forms. In both cases the powdered form needs further treatment, either to convert it to a ceramic furm via pressing and firing, or to consolidate it at least loosely for incorporation in pellet form into a metallic matrix. A less ambitious scheme, but one with similar objectives, is that being developed at the Hahn-Meitner Institute in Berlin (De, et al., 1976). Here the proposal is again to produce a mixture of stable crystalline phases, but now by the addition of chemical species to a glassy melt to promote crystallite nucleation, with subsequent controlled devitrification of the glass obtained by appropriate thermal cycling of the glass. By control of the devitrification one obtains a very fine grained glass ceramic, grain size $\sim 10 \mu$, as opposed to uncontrolled devitrification which can lead to grain sizes as large as millimeters. The most important features of the glass ceramic process are the good mechanical integrity of the product resulting from the fine scale of the grain structure, and some ability to control the phases precipitated.

It is too early to predict whether these processes will ultimately compete with the glassification technique. Although attractive in terms of the final anticipated product, there are difficulties. Realization of the advantages of the more sophisticated calcines still requires the development of techniques to produce low porosity ceramics from these calcines. Compatibility of these techniques with remote operation and minimal maintenance requirements for the associated equipment will be important requirements for acceptance of such technologies. It may be difficult to justify substantial increases in current occupational exposure risks in order to achieve an improved wasteform. The German proposal is compatible with the existing glassification programs, requiring only appropriate additions to the glass melt and control of an appropriate thermal cycle to establish the desired devitrification. It is therefore likely to be available as a workable technique long before the other two schemes.

Because of the necessary tailoring of the additives to the specific waste composition (although some latitude in composition is possible) there is concern over the possible lack of tolerance of these processes to variations in the waste composition at the input end of a solidification plant. Would extensive monitoring of the input waste composition be necessary in order to make appropriate adjustments in the subsequent treatment?

Given the final product, we are now back to most of the same questions that were raised about the glasses, with, of course, the exception. of the effects of devitrification. Again leaching mechanisms must be understood before empirical leach rates may be extrapolated for any substantial length of time, and the effects of radiation damage upon chemical dissolution rates and mechanical integrity must be established.

There is a point here of particular concern relating to the basic philosophy of the use of crystalline wasteforms as opposed to glasses. One of the principal arguments in favor of the crystalline form over the glass is its higher thermodynamic stability and hence predictable stability. However, the radiation damage from the activity in the waste, $\gamma$ 's and $\beta$ 's from the fission products and the $\alpha$ 's from the actinides, leaves considerable stored energy in the material, typical numbers for both $\gamma$ and $\alpha$ damage at doses relevant to our problem being tens of calories per gram (Mendel, et al., 1976). The heat of fusion of cristobalite, a form of crystalline quartz of 40 calories per gram gives a measure of the magnitude of the thermodynamic instability of the glass relative to the crystalline form. The fact that the radiation induced instability (stored energy) is comparable with the extra stability gained by transformation to the crystalline state must make one at least somewhat cautious about casual acceptance of the importance of the thermodynamic stability criterion (McCarthy, 1976). We not only need a chemically durable product, but we must have confidence in its long term durability. Similarly, one must be cautious in assuming microscopic integrity of the crystalline phases after $\alpha$ damage amounting to the order of one radiation induced displacement per atom of waste product.

The transmutation problem seems particularly troublesome for those wasteforms which depend upon the high stability of specific crystalline phases. Pollucite is the favored mineral phase for stabilization of the ${ }^{137} \mathrm{Cs}$. What will be the chemical stability of a crystallite of pollucite after the decay of $50 \%$ of the ${ }^{137} \mathrm{Cs}$, or $20 \%$ of the total monovalent $\mathrm{Cs}$ in the pollucite, to divalent Ba? One might expect a glass with a random network structure to be more forgiving of such a transmutation than a crystalline material with a highly ordered structure. Note that this problem cannot be simulated simply by studying crystalline phases with appropriate mixtures of $\mathrm{Cs}$ and $\mathrm{Ba}$, since in the transmutation problem the $\beta$-decay electron is maintaining charge neutrality, while in a mixed $\mathrm{Cs}-\mathrm{Ba}$ compound, that function would be carried out by an appropriate variation of anion stoichiometry. Unless there is a reasonable way to produce material heavily enriched in ${ }^{134} \mathrm{Cs}$ there seems to be no straightforward accelerated simulation of this problem, although an acceleration of a factor of $2-1 / 2$ could be obtained by using $100 \%$ ${ }^{137} \mathrm{Cs}$ in place of the $40 \%$ anticipated in the HLW. A similar problem exists, of course, for the ${ }^{90} \mathrm{Sr}$ which decays in two steps to tetravalent $\mathrm{Zr}$.

A possible solution to the transmutation problem is the incorporation of the waste atoms in a compound or mineral containing ambivalent elements which, on accepting the decay electrons from the fission product transmutation, could take on a different role in the crystal structure, but without destroying its stability (Matthias, 1976). The Sandia proposal to incorporate strontium in titanates or zirconates of the perovskite structure suggests some possible alternatives to the $\mathrm{Sr}-\rightarrow \mathrm{Zr}$ decay problem. $\mathrm{SrPbO}_{3}, \mathrm{PbZrO}_{3}$, and $\mathrm{SrZrO}_{3}$ crystallize in similar perovskite structures. The $\mathrm{Pb}$ in the first is tetravalent, but in the second is divalent. A mixed crystal $\mathrm{Sr}(\mathrm{Pb}, \mathrm{Zr}) \mathrm{O}_{3}$ could then sustain the $\mathrm{Sr} \rightarrow>$ $\mathrm{Zr}$ decay by conversion to the mixed crystal $(\mathrm{Sr}, \mathrm{Pb}) \mathrm{ZrO}_{3}$ with the initial $\mathrm{Pb}$ concentration being chosen equal to or greater than the concentration of radioactive strontium. "All that is required" is the interchange in the lattice of the newly formed $\mathrm{Zr}$ with the $\mathrm{Pb}$ and the appropriate change of $\mathrm{Pb}$ valence from $4+$ to $2+$. Is $\mathrm{TiZrO}_{3}$ a stable perovskite? If so, one might also expect $\mathrm{SrTiO}_{3}$ to be stable against transmutation, partial decay of the $\mathrm{Sr}^{-}$taking $\mathrm{SrTiO}_{3}$ to $(\mathrm{Sr}, \mathrm{Ti})(\mathrm{Ti}, \mathrm{Zr}) 0_{3}$. For the $\mathrm{Cs}$ incorporated in pollucite are there similar ways to get around the transmutation problem?

Although the transmutation problem is noted in a variety of reports (McCarthy, 1976; SAND-76-0105, 1976; De, et al., 1976), it seems not to be seriously addressed in current research efforts. For glasses it certainly raises questions which must be answered before one has confidence in the long term behavior of these materials. For the various super calcine and ceramic concepts, in which the aim is to incorporate the waste atoms in a highly specific chemical environment, the transmutation raises serious questions concerning the basic concept and needs to be addressed simultaneously with the development of the relevant mineral species. It is not a problem that can be postponed for consideration after other details of the process are worked out.

The alternate wasteform proposal of matrix isolation suggests the isolation of the waste by physical encapsulation in an inert matrix rather than chemical stabilization in a glass network or crystalline form. This separation of function allows the choice of the most 
suitable matrix from the point of view of stability, without concern about the chemical incorporation of waste ions, and the choice of the simplest possible stabilization process for the hot waste, without concern for leachability. If the waste is itself in a desirable form, one may of course have achieved a double barrier by this scheme.

The development of this idea has concerned itself primarily with the use of metallic matrixes, either melt casting, or sintering of a powdered metal in the U.S., and lead or aluminum casting in Europe, to give a couple of examples (ERDA-43, 1976). The high thermal conductivity of the metal matrix allows the use of canisters of relatively large diameter, and the metallic ductility eliminates the problem of fracture upon impact that would be associated with a handling or transportation accident. Considerable further development of this concept and the design and implementation of characterization studies will be required before one can evaluate the likelihood that the metallic matrix idea will contribute significantly as a long term (1000 year) barrier to radionuclide release.

\section{G5. TRU Wastes}

If the highly sophisticated wasteforms now being developed or considered for the disposal of HLW are to contribute meaningfully to the reduction of hazards of waste management on time scales longer than $10^{3}$ years, corresponding improvements are required in the disposal of TRU wastes. As noted earlier, recycling of nuclear fuel implies that the total actinide activity to be disposed of in these two forms, HLW and TRU wastes is, within about one order of magnitude, the same. Efforts are in progress to improve the characteristics of cements for encapsulation of TRU wastes, and polymer impregnated cements have been tested with leach rates the order of $10^{-6} \mathrm{~g} / \mathrm{cm}^{2}$ day, a value comparable with many waste glasses (Colombo and Nielsen, 1976). The problem of the TRU wastes has not however received the attention it merits and there is a significant imbalance implied by the relative support of the TRU and HLW encapsulation problems. There is little point in developing a highly sophisticated solution, such as a supercalcine ceramic, to the HLW problem without, in parallel, either developing an "unleachable cement" of predictable reliability or learning how to incorporate contaminated rubber gloves in the ceramic.

\section{G6. Improved Canisters}

The corrosive attack of stainless steel waste canisters by the salt environment as observed in Project Salt Vault (Bradshaw and McClain, 1971) can likely be avoided by appropriate protective

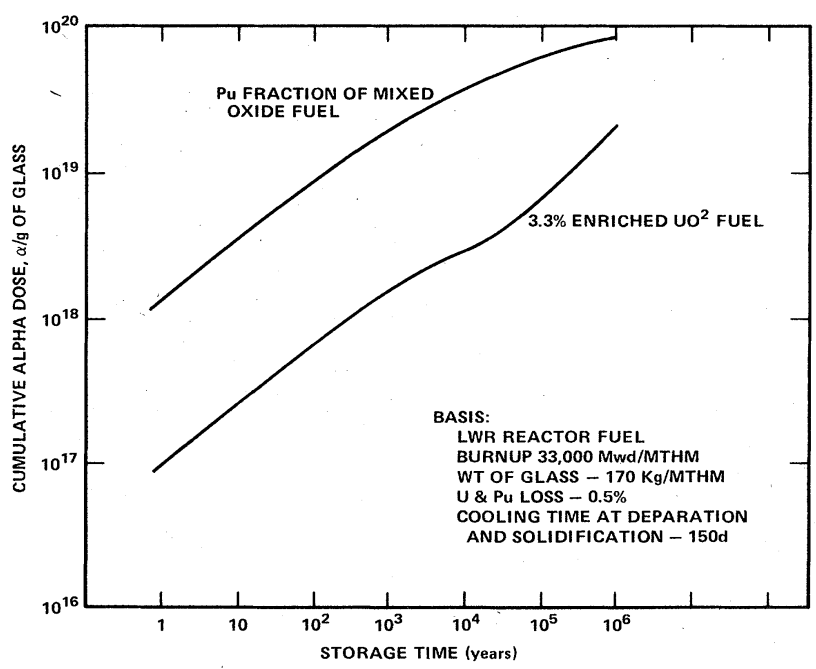

FIG. 7G1. Cumulative alpha dose of typical glass containing $\mathrm{HLW}$, as a function of storage time, for HLW from urania fuel and from mixed oxide fuel. sleeves. Obviously a simpler solution would be the development of alternate canister materials that would both be compatible with the ultimate solidification process and be sufficiently resistant to brine exposure. Note that once confidence is established in the concept of disposal in conventional mined cavities and the interim storage is converted to permanent storage this motivation for an improved canister disappears since further emplacement of new waste will presumably be done without concern for possible retrieval.

A more ambitious goal is to develop a canister of sufficient durability to provide a barrier to radionuclide release on time scales of thousands of years or more. There has been no. serious consideration by this group to such alternatives nor does it represent an area of development in the ERDA program. As in the development of durable waste forms, the very tough question to answer will be the predictability of the behavior of the "impregnable" container. To what stresses will such a canister be exposed as the surrounding medium responds to changing geologic conditions; can one guarantee that an apparently attractive alloy container will not become embrittled and be subject to fracture on the very long time scales; and what will be the corrosion rate of such a container?

\section{H. ERDA High-Level Waste (HLW) Management Program}

\section{H1. Program Rationale and General Description}

The conceptual framework for the present U.S. HLW management program was formulated in an April, 1957 report (NAS-I) by a National Academy of Sciences committee which was formed at the request of the USAEC to study the geologic aspects of radioactive waste disposal and to recommend appropriate solutions for the long term. The 1957 report, reconfirmed by a second National Academy review (NAS-II) in 1969, evaluated various environmental situations and geologic formations that seemed suitable and worthy of further detailed study. The Academy committee recommended burial of radioactive waste in underground salt beds to be the method of first choice. In addition, thick shales were deemed to be very good potential sites; low permeability, high-exchange capacity siltstones and sandstones were suggested as a third choice. The committee also recommended that the liquid HLW be put into a solid, stable form for transport and emplacement in the geological waste repository.

Our views on these matters have been described in the preceding sections of this report. We place more emphasis on modeling the containment and possible breaching modes of the HLW repository, both as an analytical tool and as a means of guiding the program. This section of our report is intended as a review and critique of ERDA's program. We first describe the program framework and timetable and then provide our evaluation and recommendations.

Because of the lack of commercial HLW in the late 1950's and early 1960's the AEC instituted a measured and relatively low level program to study the main features of geologic HLW storage as recommended by the Academy committee, culminating in an experimental program (Bradshaw and McClain, 1971) carried out in a bedded salt formation near Lyons Kansas. Although much knowledge was gained in the experiments conducted at the site, a viable waste repository was not established because of the presence of nearby commercial solution mining of salt in that particular location, as well as uncertainties about the ability to seal adequately old oil and gas exploration wells.

Following the creation of ERDA in 1974, that agency carried out an administrative review (ERDA, 1975) of the nuclear fuel cycle and focused major attention on the need for a viable demonstration of HLW management. A comprehensive technical evaluation of available waste technology also was carried out in 1975 and 1976 with the aid of most of the country's waste-management experts. This evaluation centered on the present state of the technology, identifying those areas where technology was missing or needed improving and evaluating the options available for terminal disposal. The Technical Alternatives Document (ERDA-43, 1976), published in May 1976, was the result. The agency also carried out a major reorganization and expansion of its waste-management program.

The revised and expanded ERDA commercial waste program has three parts differing somewhat in objective. First ERDA is obligated 
to develop, construct, and operate repositories for the high-level waste from the commercial industry. Such ERDA repositories are to be licensed by the Nuclear Regulatory Commission. A pending rulemaking by the Nuclear Regulatory Commission could extend this obligation to transuranic wastes.

Second, ERDA has an obligation to provide processes to convert wastes into forms in which they can safely be packaged, handled, and transported. The product of this phase of the ERDA program is technology that may be adopted by industry at its option for incorporation into a facility. The responsibility for selecting, constructing, licensing, and operating currently resides with industry.

Third, a generic environmental impact statement must be prepared, evaluating the impact of all viable alternative methods for dealing with each of the waste streams from the post-reactor fuel cycle. The technical basis for the alternatives to be treated has already been presented in the Technical Alternatives Document. The draft generic environmental impact statement is expected to be issued in 1977. Of course, specific environmental impact statements must be prepared and issued for each plant or repository.

Thus, the program expansion includes a geologic isolation program, with the target date for beginning the test phase of at least one repository 'with HLW by the end of FY85. The ERDA program also calls for the completion of research and development suitable for a full scale HLW solidification facility to operate by 1983 . In addition, target dates have been set for other associated features-improved methods for volume reduction and fixation of TRU wastes, conceptual designs for retrievable storage facilities, and the necessary environmental impact and safety studies. ERDA is also conducting an accelerated program of research and development for permanent disposal of its backlog of wastes from defense programs. The fiscal year 1977 budget numbers indicated a large increase in the program for radioactive wastes from the U.S. military program, with emphasis on the permanent disposition of the large backlog of the latter type of waste. ERDA expects that some of the developments originating in the defense waste programs will be applicable to commercial waste. Both programs are structured to exploit this possibility. ERDA is now evaluating a bedded salt formation in southeastern New Mexico as a promising site for a pilot repository looking toward permanent disposition of a large backlog of transuranium-contaminated solid waste from the military program. However, ERDA intends this repository to have experimental capability for studies on the suitability of that particular geological formation for high-level radioactive waste.

The review of available methods for the disposal of high-level waste and transuranic waste rather decisively settled on deep geologic disposal as the only potentially final solution that could reasonably be brought to reality in time to receive waste in the mid-1980's. Seabed and ice sheet disposal, transmutation, and extraterrestrial options were evaluated, and it was found that current technology has not progressed to the point where any of these options could be depended on in the next decade. The construction of a repository in a stable geologic formation, on the other hand, was deemed to be well within present state-of-the-art methods of geology, mining, engineering, and construction. Emplacement of waste in such a geologic repository provides a storage method that combines safety with a very minimum requirement for human surveillance.

The associated FY77 proposed budget request to Congress totaled $\$ 90$ million for commercial and defense waste compared to a fiscal year 1976 level of just under $\$ 31$ million--a threefold increase. The increased emphasis on commercial waste is even more dramatic--a fivefold increase from $\$ 12 \mathrm{M}$ to $\$ 60 \mathrm{M}$. On June 29,1976 , both houses of Congress voted for a budget that added $\$ 5.6$ million to the Administration's request. As further evidence of the importance attached to the commercial waste program by the Administration, a statement by the Energy Resources Council (ERC), entitled "Management of Commercial Radioactive Nuclear Wastes--A Status Report," was issued and discussed by the Chairman of the ERC. This document is a consolidated view of the Council on Environmental Quality, Department of Commerce, U.S. Geological Survey, Environmental Protection Agency, Federal Energy Administration, and ERDA. The document contains timetables for major ERDA tasks and also contains information about the roles of other agencies. A revised timetable was presented in October 1976 in the President's major statement on nuclear policy. The $\$ 60$ million Presidential 1977 commercial waste budget proposal provided for an increase in terminal-storage research and development from $\$ 4.6$ million in 1976 to $\$ 33.7$ million in 1977 and an increase in waste-processing research and development from $\$ 4.7$ million in 1976 to $\$ 19.9$ million in 1977.

ERDA is also the U.S. focal point for coordination with HI.W work that is being done in various European countries. The similarity of approach taken to both waste-processing problems and to terminal storage is striking. At present, ERDA is engaged in radioactive wastemanagement exchanges with several countries including Canada, the Federal Republic of Germany, the United Kingdom, Belgium, France, Italy, and Sweden. The participation involves various wastemanagement activities of the International Atomic Fnergy Agency, including service on the Steering Committee for High-level and Transuranic Waste. FRDA is also participating in the work of the Radioactive Waste Committees of the Nuclear Energy Agency (NEA) of the Organization for Economic Cooperation and Development. The NEA Radioactive Waste Committee is conducting the collaborative research and development program identified by the International Atomic Energy Agency.

\section{H2. National Waste Terminal Storage Program (NWTS)}

\section{H2a. Program Status And Logic}

The principal organizations involved in the overall management of the NWTS program (OWI, 1976) are ERDA's Division of Nuclear Fuel Cycle and Production, ERDA's Oak Ridge Operations Office, and the newly created Office of Waste Isolation under Union Carbide Corporation direction. The program responsibilities of each organization are as follows:

Organization

Program responsibility

ERDA's Division of Nuclear Fuel Cycle Production

Policy formulation

Program objectives and definition Overall direction for program management Approval of key technical decisions Final determination of budget

ERDA's Oak Ridge

Operations Office

Program cordination

Federal interagency agreement

Participation of ERDA field organizations Facility design and construction contracts Administration of UCC-ND contract

Office of Waste Isolation (OWI)

Program contracting and management Technical coordination of ERDA contractors Development and presentation of overall budget Accounting for all funds

Other management activities deemed necessary

The general plan for the NWTS program calls for the construction of facilities for terminal storage in various geologic formations at multiple locations in the United States. ERDA believes that this approach incorporates a number of advantages into the program. In their view the timely operation of a terminal storage facility becomes more feasible because of the multiplicity of facilities. A retrievability concept for stored waste becomes practical because other facilities are available to receive the waste should it become necessary to remove it from one of the facilities for any reason. It will make it possible for more than one site to serve the country as a terminal storage facility. Reduced waste-transportation costs are possible if more than one facility is used.

\section{H2b. Development Sequence}

Although the sequence for developing a terminal storage facility may differ slightly from one geologic formation to another, the generalized sequence can be characterized as having six distinct steps:

1. Identification of formations of interest

2. Reconnaissance surveys

3. Area studies

4. Detailed confirmation studies

5. In situ tests

6. Repository operations 
These steps not only imply a scientific program but also a decision process. It is important to emphasize that it is the intent of OWI and FRDA to conduct an extensive series of reviews by Federal, State, and local organizations at appropriate decision points in the development sequence for each formation. Although the review groups are still in the process of being established, it would be appropriate to indicate a few of them. First, a long-term geologic review group has been established. Reporting to the Director of OWI, it consists of senior geologists, hydrologists, and other appropriate experts who will review the entire geologic program on a continuing basis. Second, basin or regional review groups, consisting of experts knowledgeable about the particular basin or region, also will be created. Third, Federal and State review groups will be created. These will consist of representatives of agencies of the Federal and State governments and other organizations that would have responsibility for, or interest in, some aspect of the NWT'S program. A review group comprised of the Association of American State Geologists has already been established. In addition, to aid in the decision process, a draft environmental impact statement will be prepared for each facility site. These draft environmental impact statements will be circulated for comment by Federal, State, and local government agencies as well as by the public. A public hearing or hearings on the draft environmental impact statements will also be convened as appropriate. The final environmental impact statements will be issued and circulated with substantive comments attached. Although the six steps are distinct, one may anticipate that some of them will be carried out simultaneously. For example, the in situ tests could proceed in parallel with area studies.

Identification of Formations of Interest. The process of identifying geologic formations of interest begins with a review that is based solely on the general knowledge of the geologic properties and/or fundamental properties of the rock type involved. If the review is promising, a reconnaissance survey is undertaken.

Reconnaissance Surveys. The purpose of the reconnaissance survey is to collect all the available data on those properties and characteristics of the formation needed for waste-disposal considerations. This information provides a regional evaluation of the potential for using the formation for waste disposal and may include (1) structure, stratigraphy, depth, and thickness; (2) hydrology; (3) mineralogy and petrology; (4) natural resources; and (5) general surface characteristics. On the basis of the information compiled at this stage, the prospects for the formation are evaluated and reviewed, and a number of smaller, perhaps "county size". regions are identified for further geologic area studies.

Area Studies. The geologic area studies that follow the reconnaissance survey are designed to develop new and specific data on the areas of interest. These studies include (1) core drilling at a density of perhaps six to ten holes per 1000 square miles to obtain adequate definition of the important subsurface characteristics of the formation, (2) field geologic mapping, (3) hydrologic studies, (4) geophysical surveys, and (5) other geologic studies. On completion of these studies, the suitability of the area is again reviewed. If it still appears promising, one or more specific locations will be identified for even more detailed confirmation investigations. There will be extensive reviews at this point involving all review groups that have been established. The selection by OWI and ERDA of specific locations for more detailed confirmation investigations and the final selection of pilot-plant sites will be firmly based on all these reviews as well as on public comments obtained during public progress reports.

Detailed Confirmation Studies. The detailed confirmation studies are directed toward specific locations of perhaps 5 to 10 square miles each. The investigations involve primarily the drilling and testing of four or more core holes at each location as well as the continuation of any specific geologic studies for which the previous results are inadequate, to evaluate the suitability of the location. The results of this phase of the investigation would be to fully qualify locations for consideration as repository sites.

In Situ Tests. Once the formation is identified as a possible candidate for waste disposal, a set of in situ experiments will be conducted in parallel with the geologic area studies. These tests will range from the emplacement of simple electrical heaters in exposed surface outcrops of the formation to extensively instrumented vault tests in excavations especially constructed at the expected depth of the repository. In general, the vault tests are designed to permit tests with both electric heaters and canisters of simulated waste, which are removed at the conclusion of the experiment. They provide extensive information on the physical behavior of the rock and of the waste canister, as well as the stability of the underground layout.

Repository Operations. After adequate review, a repository site will be selected and a repository will be constructed to evaluate the handling and storage operations using actual canisters of high-level waste and to confirm all design calculations. This will be done with surface handling equipment and underground excavations that are portions of the full-scale facilities. Experiments will be performed to test retrievability devices and emplacement concepts, and to evaluate the stability of the underground operations.

The data obtained during the test phase of the repository development will be used to design the expanded facility, which will be operated until it has successfully demonstrated that (1) it can safely receive, emplace, and store high-level waste at the design rate; (2) all criteria for a Federal repository have been met; and (3) no anomalies in the geologic formation have been found

At some point in the development program, when all license requirements imposed by the U.S. Nuclear Regulatory Commission are met, the facility will be converted to a Federal repository. At that time, ERDA expects that the retrievability option will be dropped.

\section{H2c. Schedule for Facilities}

As a result of the early work performed by ORNI, the first two steps in the general development sequence for geologic disposal have been completed for sall formations. Still remaining are the area studies and the subsequent steps leading to an operating repository. A detailed planning schedule for the first two pilot plants is shown in Fig. $7 \mathrm{H1}$. This figure is applicable to both pilot plants because they are expected to be constructed in parallel at two different sites. Both of these facilities will be located in salt formations. What the planning schedule shows is that the identification of acceptable sites for the first two pilot plants will not occur until the end of fiscal year 1978 , and it will take a total of 9 years before the pilot plants go into operation.

It is now planned to construct six terminal storage facilities. ${ }^{6}$ The planning schedule for these facilities is shown in Fig. 7H2. In this figure the solid triangle indicates the time at which area studies are starter in the geologic formations of interest. Earlier work required for formations other than salt are not shown on this schedule. As already indicated, the first two pilot plants will be constructed in salt formations. The remaining pilot plants are expected to be constructed in other formations of interest. The startup for receiving waste in the first two plants is scheduled for the middle of 1985. The next set of two facilities will start operations simultaneously two years later. The fifth and sixth pilot plants will start at 2-year intervals after the second set of two.

Formations of Interest. At present a number of formations are of interest in the NWTS program. Many of these are shown in Figs. $7 \mathrm{H} 3,4$ and 5 . Figure $7 \mathrm{H} 3$ shows the rock salt formations in the United States. Of these, the Salina Formation, the interior province of the Gulf Coast dome region, and the Permian Basin are all considered to be formations of high potential for location of the first two pilot plants. Figure $7 \mathrm{H} 4$ shows the crystalline rock formations in the United States, and Fig. 7H5 shows the shale and clay formations in the United States. Considerable work has yet to be done in the formations other than salt because the reconnaissance surveys have not been initiated in many instances and the in situ tests are yet to be started.

Timetable. As established in the President's Nuclear Policy Statement of October 1976, the principal actions and dates for development, construction, EIS preparation, standards and licensing formulation are as follows:

1976 - ERDA issued for public review the Technical Alternatives Document which explains the current state of waste management technology. 


\begin{tabular}{|c|c|c|c|c|c|c|c|c|c|c|c|c|}
\hline & FY-77 & FY-78 & FY.79 & FY-80 & FY.81 & FY.82 & FY-83 & FY-84 & FY-85 & FY-86 & FY-87 & FY-88 \\
\hline \multicolumn{13}{|l|}{$\begin{array}{l}\text { REPOSITORY FEASIBILITY STUDIES } \\
\text { 1. Repository Site Selection } \\
\text { 2. Preliminary Design Studies }\end{array}$} \\
\hline & & & & & & & & & & & & \\
\hline \multirow{2}{*}{\multicolumn{13}{|c|}{$\begin{array}{l}\text { LAND ACQUISITION AND LONG-LEAD ITEMS } \\
\text { 1. Procure Long-Lead Items } \\
\text { 2. Purchase Land and Land Rights }\end{array}$}} \\
\hline & & & & & & & & & & & & \\
\hline \multirow{2}{*}{\multicolumn{13}{|c|}{$\begin{array}{l}\text { REPOSITORY DESIGN } \\
\text { 1. Title I and Partial Title } \| \text { Design }\end{array}$}} \\
\hline & & & & & & & & & & & & \\
\hline \\
\hline & & & & & & & & & & & & \\
\hline \multirow{2}{*}{\multicolumn{13}{|c|}{$\begin{array}{l}\text { 2. Cold Testing } \\
\text { 3. NRC License }\end{array}$}} \\
\hline & & & & & & & & & & & & \\
\hline \multirow{2}{*}{\multicolumn{13}{|c|}{ REPOSITORY HOT OPERATION }} \\
\hline & & & & & & & & & & & & \\
\hline \multicolumn{13}{|l|}{ SAFETY AND ENVIRONMENTAL STUDIES } \\
\hline \\
\hline \multirow{3}{*}{\multicolumn{13}{|c|}{$\begin{array}{l}\text { 2. Environmental Studies } \\
\text { a. Regional Study Plans } \\
\text { b. Regional Baseline Study }\end{array}$}} \\
\hline & & & & & & & & & & & & \\
\hline & & & & & & & & & & & & \\
\hline $\begin{array}{l}\text { d. Draft Environmental Impact } \\
\text { Statement }\end{array}$ & & & & & & & & & & & & \\
\hline
\end{tabular}

FIG. 7H1. Preliminary schedule for federal repositories 1 and 2 (initial phase).

1977 - ERDA issues draft generic environmental impact statement on waste management no later than the early part of the year and begins extensive program to identify, test and select a site.

- FPA proposes draft of generally applicable standards for permanent storage of high-level wastes.

- NRC publishes draft standards for solidified high-level wastes and draft siting, engineering and operating criteria for repositories for high-level wastes. Each element will include the appropriate draft environmental impact statements.
1978 - ERDA completes initial demonstration work on canister design, waste solidification, and preliminary repository design, and continues site selection process.

- NRC finalizes proposed site selection criteria, solidification criteria, waste definitions and operating criteria and regulations.

- EPA issues final general ambient standards for high level waste disposal.

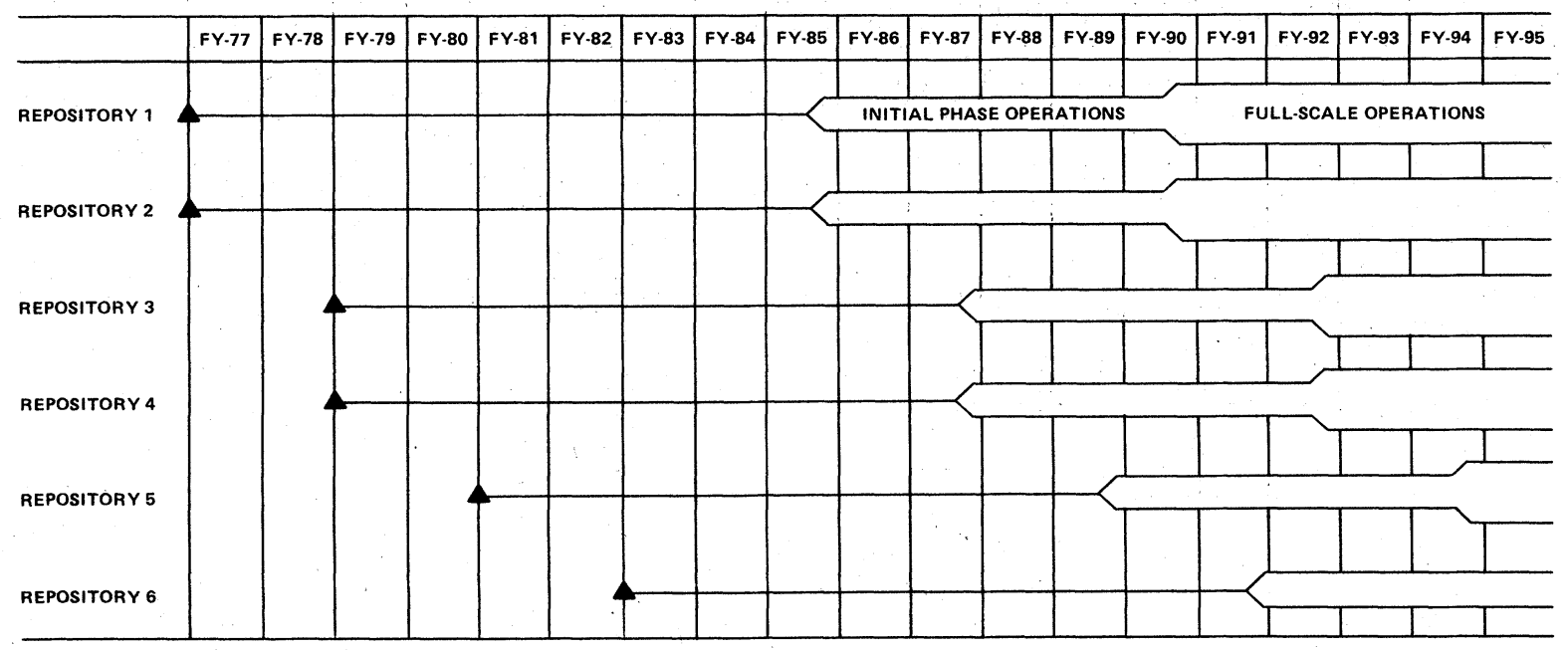

FIG. 7H2. General plan for geologic terminal storage. 


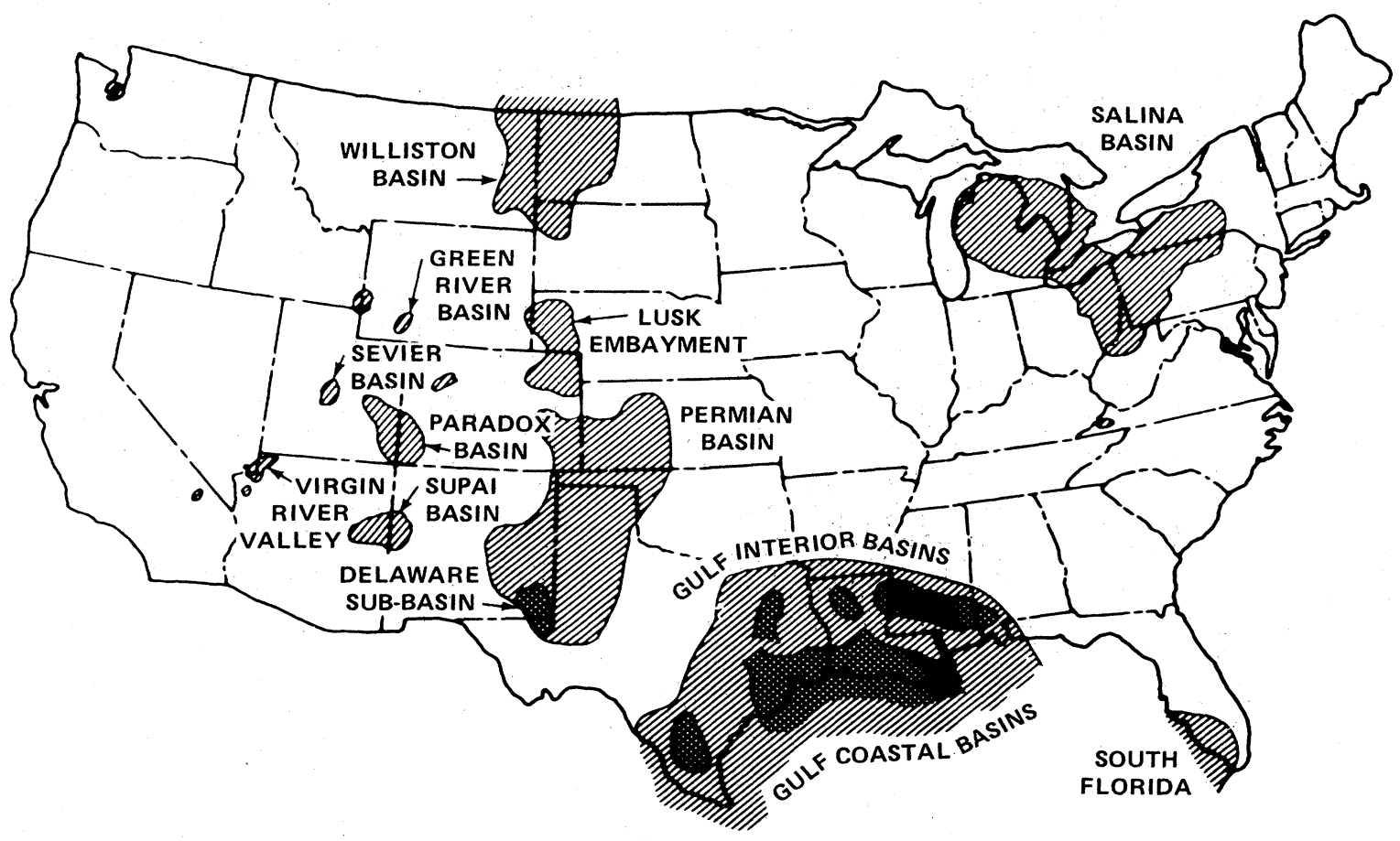

FIG. 7H3. Rock salt deposits in the United States (After Pierce and Rich, U.S. Geol. Surv. Bull.1148).

1979 - ERDA selects a particular repository site, issues a draft site specific EIS, and begins intensive site and design work.

- NRC performs early site review of ERDA repository; issues next phase of draft regulations for canister design, transportation, etc.
1980 - ERDA completes site and design studies, submits preliminary safety analysis and environmental report to NRC in support of construction permit.

1981 - ERDA begins construction with approval of NRC.

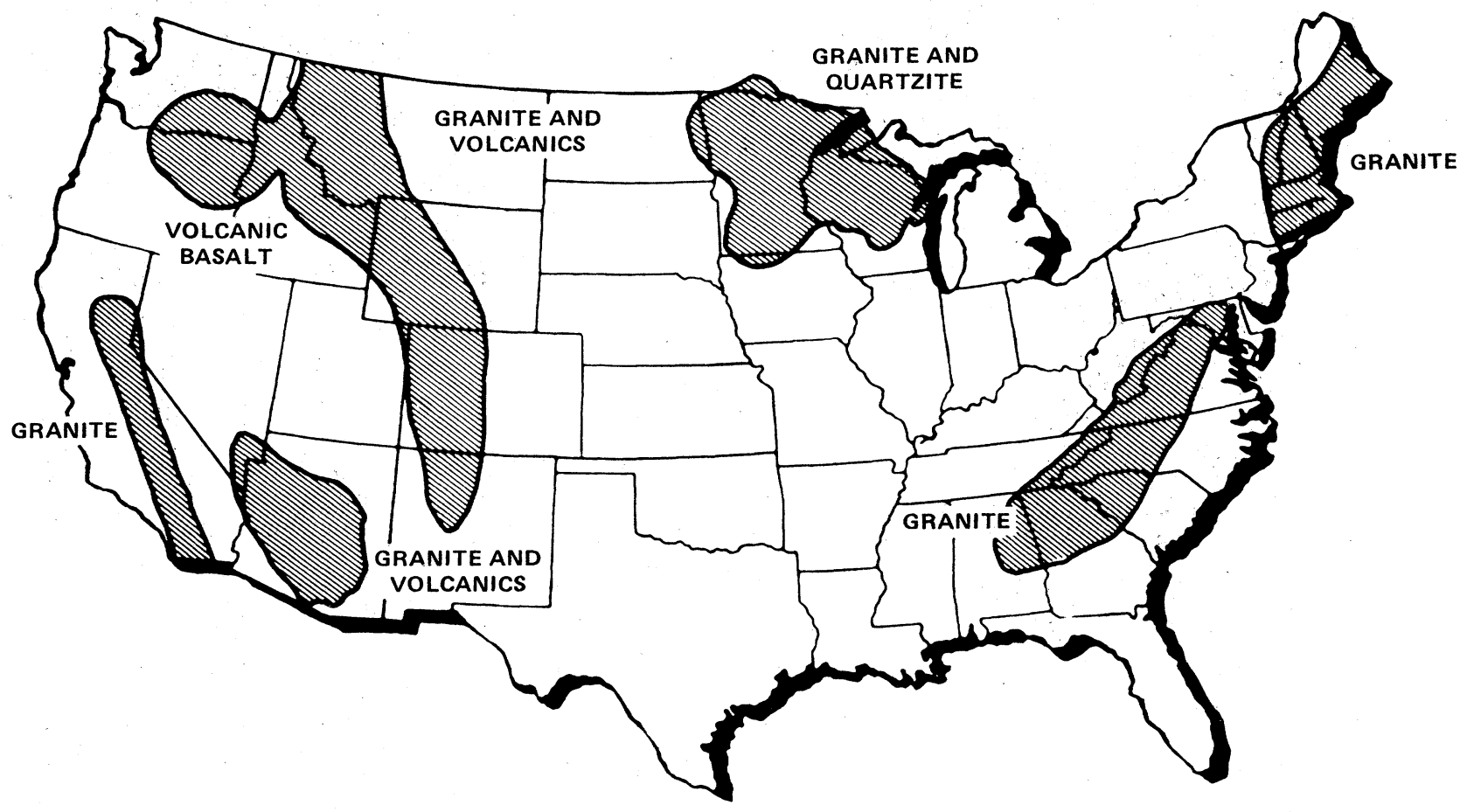

FIG. 7H4. Crystalline formations in the United States. 


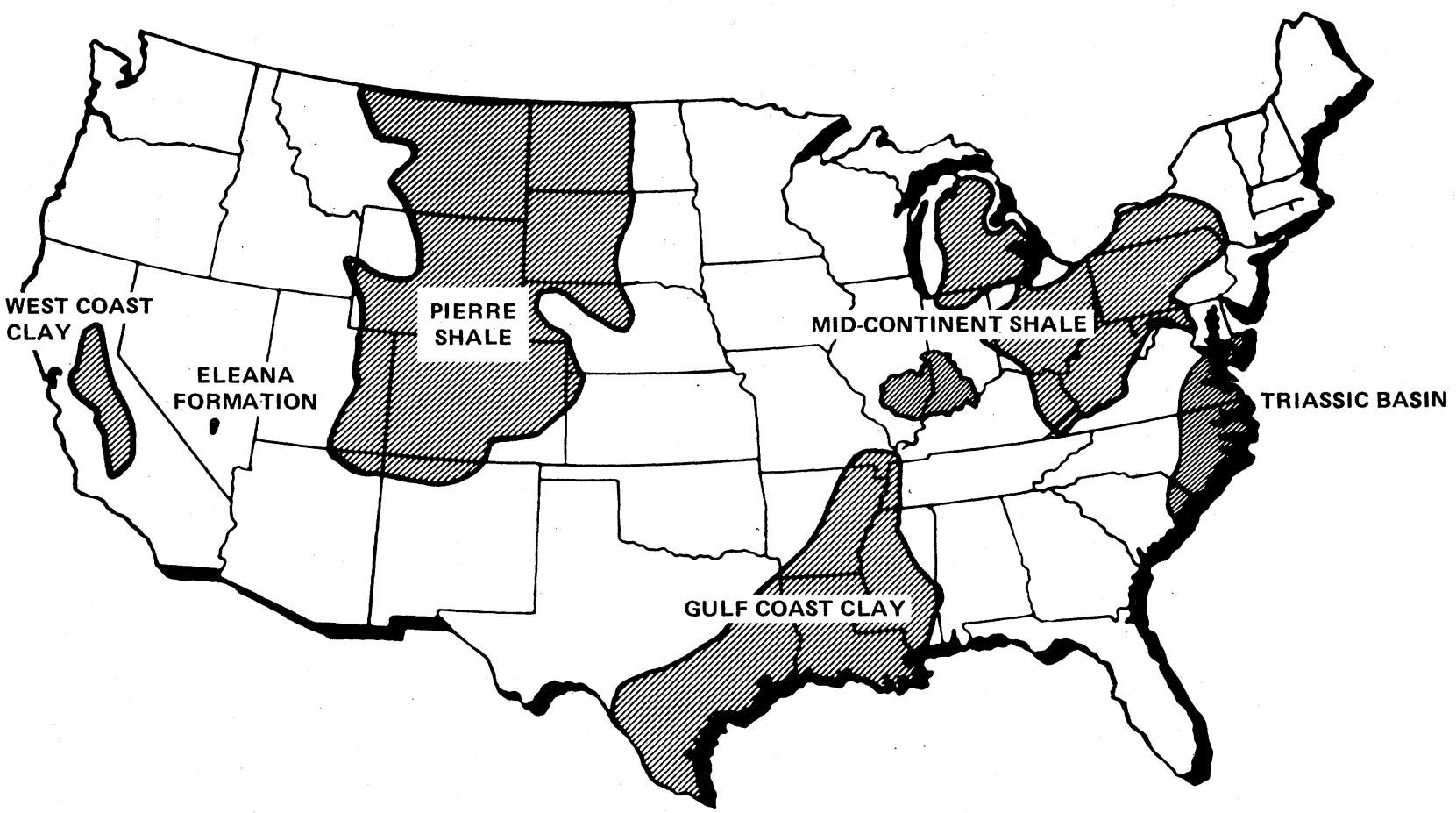

FIG. 7H5. Argillaceous formations in the United States.

1984 - Construction completed, repository tested with "cold" wastes.

1985 - NRC issues repository license.

- Repository begins initial commercial-scale operations.

\section{H2d. Regulation Of Waste Management}

The Nuclear Regulatory Commission has publicly stated its recognition of the need for a high-priority national effort on waste management. While NRC responsibilities are in the area of regulation, the Commission is necessarily working closely with other agencies to help assure the soundness of the programs for dealing with nuclear wastes. The overall national program is intended to assure that necessary facilities and arrangements will be in place when they are needed to deal with nuclear wastes in a safe and environmentally acceptable manner. The NRC believes that present licensing procedures and criteria are adequate for the short-term, and that priority attention must be given to the longer term, when the quantities of waste to be managed will be greater.

The Commission's nuclear waste management program has three major tasks:

--First, to develop objective performance goals (technical, social, economic, and environmental) against which nuclear waste management programs and strategies can be evaluated;

--Second, to devise an evaluation methodology (and the data base needed to make effective use of this methodology) for assessing the performance of proposed waste management programs and strategies against these goals;

--Finally, to establish a framework of regulations, standards, and guides for management of nuclear wastes within which NRC can effectively and efficiently carry out the functions dictated by its legislated role to protect the public health and safety. This framework will have to be supported by a comprehensive environmental impact statement (or series of statements).

The NRC has defined (NUREG-0116, 1976) the work that needs to be done within these tasks and has assigned priorities to the work.
They have prepared a schedule, and are adding key staff needed to meet that schedule. First, the NRC has established organizational capabilities to handle both the regulatory responsibilities directed by the Atomic Energy Act and inherited from the AEC, and the new responsibilities given the Commission by the Energy Reorganization Act. A first step in this development was the establishment of a Waste Management Branch, in the Office of Nuclear Material Safety and Safeguards, in June 1975. Initial staff has been brought on board; however, the staffing effort will continue until the program peaks sometime in the late 1970's. In addition, the NRC is developing an overall waste management program. Initial stages have been completed, regulatory needs have been tentatively established and a program is underway to meet these needs according to the target dates listed in the President's established timetable. The NRC is also establishing a confirmatory research program to support regulatory and licensing activities. A number of research needs have been identified and some projects are already underway.

\section{H2e. HLW Processing Technology}

Our review of the processing technology clearly identifies solidification of the high-level waste stream from reprocessing plants as by far the most critical requirement in process development. Basic technology was found satisfactory, but application development at commercial scale was deemed necessary. It is generally agreed that the product from a solidification process should be chemically and radiolytically stable and at least in its packaged form should retain a monolithic character on impact. The solid and its container clearly must last for the period of transportation and emplacement as well as for such time as the repository is operated as a pilot flant. More quantitative criteria must wait for guidance from the regulatory agencies and from a more detailed definiton of the operating mode of the repository during the pilot plant stage to accommodate retrievability. The conceptual design of the pilot repository required for the establishment of these criteria is a part of the ERDA program for FY77.

Considerable characterization of the various candidate solid-waste forms has been done. The data obtained must now be used for formal hazard analysis studies by both ERDA and the Nuclear Regulatory 
Commission. These analyses have been started by ERDA and will be continued in the fiscal year program. A variety of potential solidification processes are now available from former AEC work. A calcined product has been routinely produced for over 10 years at the ERDA Idaho site by fluidized-bed calcination of acidic high-level waste. At Battelle Pacific Northwest Laboratories an integrated spray calciner/in-can melter vitrification process has been operated at a 1 ton/day rate with simulated hot waste characteristic of a modern reprocessing plant. The spray-calciner part of the process has been operated at a 3-ton/day rate using cold simulated waste. Smaller scale work on other concepts such as a ceramic melter and the application of wiped-film evaporator technology is directed toward slightly longer range options. Scaleup and hot testing of both spray-calciner and fluidized-bed calcination units are now planned to provide commercially useful data. Though the borosilicate glass is receiving early prominence in hot testing of these units, stabilized-calcine, metal-matrix, ceramic, and concrete forms will continue to be investigated. Despite the prominence given to glass at this time, no decisive basis exists for ruling out other forms. ERDA expects that more than one waste form may meet the criteria eventually established for terminal storage.

ERDA's review of the technology for handling waste streams other than high-level generally showed acceptable hardware and process to be commercially procurable. Although there are economic incentives for improved processes in several cases, there are currently available methods that can safely handle the miscellaneous wastes. Two areas were identified in which the incentive for methods of greater efficiency was sufficiently great to receive special emphasis. These are (1) methods for handling the hulls from the head-end step of the reprocessing plant, and (2) methods for reducing the volume of the so-called combustible waste. Both wastes may contain sufficient plutonium and other transuranics to necessitate, under proposed U.S. regulations, disposal in a repository similar to that for high-level waste. The cost saving from volume reduction would be particularly important. The ERDA fiscal year 1977 program supports work on five improved methods for handling hulls and five methods for the "incineration" of combustible material. Processes suitable for industrial use are targeted to be available by the end of 1980 for hulls and 1979 for handling the combustible wastes.

Obviously, a decision not to reprocess fuel from reactors requires either the interim storage of fuel elements in anticipation of possible future reprocessing, or else the handling of spent fuel elements as waste. $^{6}$ A possible technique for emplacing fuel elements in a repository was demonstrated as long ago as 1965 (Bradshaw and McClain, 1971). However, ERDA acknowledges that more research and development on techniques and processing steps are required.

\section{H3. Evaluation of HLW Management Program}

As previously indicated, the general plan for the NWTS (OWI, 1976) program is based on the concept of multiple repository sites-i.e., the eventual construction of facilities for terminal storage in various geologic formations at multiple locations in the United States. The plan includes a development sequence, a facility schedule, and provision for review groups to help in the decision-making process leading to construction of the repositories. The repository design is expected to be similar for all types of geologic formations, with certain details to be dictated by the nature of the waste to be stored ERDA has the responsibility for development of waste form technology. In the sections following we will comment on these aspects of the program.

\section{H3a. Multiple Repository Sites} are:

The advantages cited by ERDA of having multiple repository sites

"1. Feasibility of a timely operation of a terminal storage facility is increased because of the simultaneous and parallel activities.

2. A retrievability concept for storage of waste becomes practical because other facilities are available to receive the waste should it be necessary to remove it from one of the facilities for any reason.
3. It will be possible for more than one site to serve the country as a terminal storage facility so that no one location need bear the burden for the entire United States.

4. Reduced waste transportation costs are possible if more than one facility is used since the facilities will be dispersed around the country.

5. It eliminates concern regarding possible federal government reluctance to abandon possible sites after significant expenditure since other facilities will be available."

We believe that the selection of sites for waste isolation will undoubtedly reflect a complicated combination of political, social, economic, and scientific factors. Because the relative influence of these factors cannot be predicted, a multiple-repository approach seems only prudent if an operating facility is to be available when needed.

The current ERDA HLW-Management Program Plan calls for the completion of two waste repositories by the end of FY84, with cold testing to be started during FY84 and testing with radioactive waste to begin late in FY85. Site selection for these two repositories is to be completed by the end of FY78 and repository design will continue through FY79 and FY80, with construction of the repositories to begin in FY81.

The severe constraints of the current schedule and the fact that retrievability is a basic tenet of current policy require that ERDA employ existing technology and demonstrated techniques for emplacement, storage and retrievability of the waste. (In this contex retrievability implies the ability to remove the waste from storage with the same techniques and equipment as were required for emplacement.) It is understandable, therefore, that the greatest attention would be given to a conventional mined cavity in bedded salt, inasmuch as 20 years of effort have been directed at the problems of emplacement of radioactive waste in this geologic medium. If the time constraint were to be eased, we believe it would be preferable to develop at least one of two test facilities -- i.e., pilot-scale operation -- in a medium other than salt, as will be discussed below.

As we have noted, the conceptual framework for burial of radioactive waste in underground salt beds was formulated in a 1957 report by a Committee of the National Academy of Sciences (NAS-1). This committee's recommendations have been reconfirmed on several occasions by subsequent reviews, with the following advantages of salt as a disposal medium usually being cited

1. Salt is plastic under moderate pressure and temperature; consequently, any fractures or openings in the salt will be self-sealing;

2. Salt has a good thermal conductivity, approximately twice that of other common rock types;

3. Salt is widespread geographically and inexpensive to mine.

The first is of importance with respect to long-term effects and the hydrogeologic regime, the second with respect to short-term effects and thermal loading in the repository, and the last with respect to choice of geographic location and ease of construction. As already noted, we consider hydrogeologic aspects to be of the greatest concern.

Based on our analysis of hydrogeologic transport we expect that the conditions that would provide for satisfactory geologic isolation of radioactive waste--i.e., a suitable groundwater environment--are present in a sufficient number of places that several acceptable sites in different geologic media can be located without difficulty within the immediate future. We expect that sites with adequate hydrogeology can be located in bedded salt formations and that ERDA's plans to locate several are likely to meet with success. Nevertheless, we are convinced that a broader program is appropriate when all factors are taken into account.

We consider it to be in the best interests of the United States to demonstrate in a timely way the technical base, on a commercial scale, for alternative solutions to radioactive waste disposal. Thus, it is highly desirable and appropriate that several geologic formations in addition to salt be evaluated fully for repository sites. This would serve not only to broaden our own options geologically and 
geographically, but would also help other nations avoid undesirable alternatives that could ultimately affect us adversely.

Present ERDA plans are to develop the first repository in bedded salt, and the second in domed salt. ${ }^{6}$ Four additional repositories are included in the general plan and these will be in other types of formations; tentatively being considered are shale, limestone, and granite. We find no compelling technical reason to place a second repository in salt; the reason most commonly advanced is that it is the only way to meet the constraints of the current schedule because it would allow utilization of the same technology and techniques to develop the first two repositories. However, for conventional mined cavities essentially the same techniques of mining, transport, and emplacement would be used for repositories developed in different geologic formations, and only modest design changes would be required to allow for the different thermal and mechanical properties of these formations. These properties need to be determined and evaluated for other rock types; this need will be addressed below.

We believe that it would be desirable to develop a test facility in a medium other than salt -- e.g. granite, or possibly shale. We call particular attention to the possibilities offered by Precambrian granites which are located in sparsely populated areas in the most stable parts of the earth's crust, are typically characterized by low permeability, and generally are unattractive with regard to mineral resources and agriculture. Granitic masses have the advantage-unlike sedimentary sequences, including bedded salt, shales, and limestones--(1) of being homogeneous and isotropic over extensive vertical as well as lateral distances (thus eliminating possible pathways along sedimentary features for groundwater movement), (2) of not being subject to compaction or other volume change owing to decomposition or loss of water (which could contribute to hydraulic pressure differentials), (3) of having substantial strength to resist any stress differences that may exist or develop in the rock, and (4) of not being part of a sedimentary basin (which typically can be expected to contain active groundwater systems).

Among sedimentary rocks other than salt, we consider certain types of shale to be possibly acceptable; however, we recognize that an appreciable amount of work would have to be done to evaluate the possible physical and chemical effects of heat generated by HLW or spent fuel elements emplaced in the rock. Would significant desiccation occur with concomitant fracturing? Would thermal gradients and chemical potentials in the shale induce significant hydraukic gradients? Would the normally high selectivity coefficients of shales be realized under such conditions? Clearly, the answers to these and related questions must be carefully evaluated. Because the excellent sorption characteristics and low permeabilities of certain shales, as well as their widespread distribution, make them highly attractive as a possible waste disposal medium, we would encourage and support an active and orderly research program on shales and other argillaceous rocks.

In regard to resources, concentrations of any element or mineral are invariably the most valuable and thus the first to be exploited, rather than dispersed or disseminated elements or minerals. Formations characterized by such concentrations should be avoided if possible. The purity of large salt formations makes these an important resource. Although salt is an abundant resource, it is, nevertheless, mined by man, of ten from considerable depth, rather than obtained from the processing of sea water or less pure sources. The deliberate solution mining of a salt deposit and inadvertent intrusion of a repository by some future generation represents an anthropogenic activity of possibly serious consequence to the integrity of a repository in salt. Although the likelihood of this happening is extremely low, and presumably would require the loss of records, we cannot dismiss the possibility. The consequences, if this were to happen, could be effectively evaluated through appropriate modeling of such an occurrence. If, indeed, the activity of a future uninformed generation were to lead to loss of geologic confinement, it should be noted that the probability of this occurring would increase significantly in the case of a salt dome, the ERDA choice for its second repository. The distinctive characteristics associated with salt domes could be expected to attract the attention and arouse the curiosity of an intelligent civilization. The probability of such an occurrence cannot be determined; the resolution of the associated anthropogenic concerns will have to be obtained via the licensing processes. It is not obvious to our group what the outcome of these processes will be, and therefore we consider it prudent to pursue the evaluation of other geological media in addition to salt formations.

The solubility of salt is a clear disadvantage if the medium is exposed to water. It has been argued that this susceptibility to solution demonstrates that ground water has not circulated at the levels where salt has been preserved for millions of years. Nevertheless, we would caution that man has the ability to alter significantly the groundwater regime and that analysis of possible anthropogenic effects must be made. As stated above, we foresee no difficulty in locating a repository site in salt with satisfactory hydrogeology, but cannot emphasize strongly enough our belief that quantitative analysis of groundwater flow should be required for the proposed site. Indeed, we recommend that such analysis be made part of the site selection criteria for all proposed repository sites.

\section{H3b. Development Sequence}

Although the NWTS program is in its earliest stages of (1) identifying formations of interest and (2) reconnaissance surveys, it is clear that a well defined decision process has been established that can be expected to result in the selection of several satirfactory repository sites. The search for suitable alternative rock types for radioactive waste disposal appears to be thorough and progressing well. However, because understanding of the characteristics and properties of other rock types for the most part lags far behind the research already completed on salt, we strongly support and encourage immediate and concentrated research on the appropriate hydrogeologic, timedependent, sorption, and thermal properties of alternative rock types.

In addition to the determination of basic mineralogic, hydrogeologic, and mechanical properties for candidate rock types, in situ tests and related laboratory experiments need to be undertaken as soon as practicable to evaluate the effects of specific thermal loadings on mineral stability, chemical changes, and fluid and mechanical properties of these rocks for possible site locations. In some instances it may be appropriate for such tests to be carried out in existing mines or in special excavations rather than in the actual excavation for a repository. When this can be done it would provide the additional benefit of permitting the results to be utilized in the decision process prior to site selection and excavation.

Further, we would encourage an active and intensive effort on rock melting concepts which might provide a superior disposal alternative by the mid 1990's. The possibility of combining a rock melting concept with standard emplacement techniques in a conventional mined cavity (e.g., in granite) should be evaluated, as this could provide the experience and confidence needed to proceed ultimately to less conventional emplacement techniques combined with rock melting. Upon cooling and re-solidifying; the crystallized rock selvage around the waste could provide a significant barrier, in and of itself, to radionuclide migration. Some aspects of the problem that require evaluation include determination of (1) waste loadings that would produce heating and cooling histories resulting in the greatest degree of physical integrity of the cooled mass, (2) observed melt radii relative to those predicted. (3) relative merits of thin and thick selvages of newly crystallized rock, and (4) distribution and characteristics of crystalline phases in the selvages.

\section{H3c. Facility Schedule}

We are not aware of either scientific or technologic barriers to the realization of an operating repository in the immediate future--i.e., by 1985. However, we are not completely confident that the current schedule will be met because of difficulties and delays that may be introduced by the licensing processes. The NRC is still developing its regulations, standards and guides in this field; even the methodology is not codified. Thus the impact of the licensing process and the NEPA hearings on the time schedule will only become apparent as the process unfolds.

We favor an orderly and measured program that would involve the development and construction of two test facilities in different geologic media. Procedures should be initiated for licensing the more favorable of the two, and the needs for storage should dictate when that facility should be developed into a full repository. The second test facility may or may not need to be developed into a full-scale 
repository. We recognize that the current schedule and other factors may lead to further redundancy. The number of repositories to be included in the schedule could be based on the projected growth of generated waste, or could reflect those needed to demonstrate conclusively our ability to develop acceptable repositories in a variety of geologic media. Two or three may be sufficient in the former instance, whereas three or four would be desirable in the latter, depending upon whether the first two are in salt. In either instance construction would not need to proceed to the level of a full-scale repository from each test facility. Current repository designs are such that the total projected volumes of high-level, intermediate-level, and low-level transuranic commercial waste through the year 2005 could be accommodaled in one repository.

The orderly program we advocate would include a quantitative analysis of groundwater flow and of possible thermal effects for each proposed repository site, in addition to in situ testing and other aspects of the development sequence already established. Proper attention to all aspects of the sequence could require a less compressed and more evenly spaced sequencing of facility development and construction than that represented by the current schedule. In this regard, we see no technical reason why a repository would have to be operative before 1988 at the earliest; a ten-year schedule ${ }^{6}$ is an arbitrary choice, and it could be extended a few years if the circumstances warranted taking longer.

\section{H3d. Review Groups}

In providing direction to the NWTS program and help in the decision-making process we consider the input from senior level review groups of appropriate experts to be essential, and note that establishment of a senior continuing review group and supplementary ad hoc workshop groups to review specific aspects of the program has already begun.

With reference to both reconnaissance surveys and area studies, we strongly encourage and support the establishment and utilization of the Basin/Regional Review and Federal/State Review Groups to assist in the location and expert assessment of potential repository locations. A large body of scientific and technical information exists for many areas, in some instances unpublished, that could be identified through the assistance of such groups. Moreover, these groups may provide valuable assistance in identifying and dealing with important social and political questions that will undoubtedly be raised.

We consider it imperative that such groups include individuals outside ERDA, that their recommendations be made part of the public record, and that OWI responses to these recommendations also be made public.

\section{H3e. Waste Form Technology}

The ERDA plan (ERDA, 1976a) in the area of solidification technology emphasizes, both by explicit statement and by budget allocations, the use of borosilicate glass to immobilize the HLW, and continues development of three different processes leading to this wasteform as likely technologies to be adopted in the first few solidification plants to be in operation in the U.S. This program seems more than adequate to assure the availability, for commercial solidification plants, of technologies to convert liquid HLW safely and economically to a solid wasteform of the low dispersability required to assure safety in handling and transport.

Longer term plans involve the development of alternative wasteforms and of improved methods of wasteform characterization. Apparently lacking in this program outline, however, is any focused effort to develop decision making criteria for evaluating various alternatives or to develop specific design goals for those involved in wasteform development. It is laudable to propose "improvements in both short-term and long-term durability of materials under interim and final storage conditions," and appropriate to have the "work....narrowed in scope to one alternate...starting in 1981". Will, however, the basis for this "narrowing" decision be available by 1981; which time scale, "interim" or "final", presents the more important problem? How would one decide, for example, which of the following two wasteforms would be preferred? The first, a metallic matrix form, has excellent properties on the 5 year time scale, is produced easily with no significant occupational exposure, but is of dubious stability over 1000 years or more. The second is a glass ceramic, requiring significant occupational exposure for the maintenance of the equipment, but which is effectively insoluble on the $10^{6}$ year time scale. Hopefully the section of the ERDA plan denoted "Supporting Studies and Evaluations" will be devoted in part to the definition of specific design objectives and decision criteria for waste form development. Certainly there are no such objectives or criteria at the present time to guide the development of improved wasteforms.

A casual reading of the ERDA 1976 Plan would suggest that the decision had already been made to start fuel reprocessing in the U.S. ${ }^{6}$ The whole plan seems predicated on the assumption of reprocessing, a reflection, of course, of the long-term thinking and planning in the AEC and ERDA. Little explicit planning is described in the event of a decision not to reprocess. "Preliminary evaluations do not indicate any major difficulties in developing repositories (either interim storage or geologic isolation) with enough flexibility for acceptance of either spent fuel or waste canisters from a spent fuel processing plant. Hence no funding is provided in the FY 77 commercial waste budget for the "throwaway" fuel cycle option." (ERDA, 1976b)

If a decision not to reprocess indeed means a firm commitment to "throwaway", then there is no quarrel with this point of view. As noted earlier in this report, however, in view of current uncertainties in natural uranium resources and the possibility of a reversal of a decision not to reprocess it seems desirable to interpret a negative reprocessing decision at this time as dictating a "stowaway" fuel cycle. Because the retrievability period defined by the stowaway concept, perhaps 30 years, is much longer than the 2-5 year test-phase retrievability period planned for the HLW repository, considerable engineering is required to allow the use of geologic repositories, particularly in salt, for interim storage of spent fuel. This development should be an important part of the ERDA program, ${ }^{6}$ and this program should not simply consider spent fuel to be treated as waste in the event of a decision not to reprocess.

One aspect of the section of the ERDA plan on "Supporting Studies and Evaluations" is a detailed evaluation of the advantages of the partitioning of HLW to allow for alternative disposal techniques for the long-lived actinide component of these wastes. We would emphasize again the large actinide content of the TRU wastes in the case of the recycle option, viz, greater than $10 \%$ of that of the HLW. There is no significant advantage in partitioning unless the losses of the actinides to the low level waste streams in both the reprocessing plant and the refabrication plant can be kept at least two orders of magnitude below current practice, or techniques can be found to concentrate the actinide component of the TRU waste sufficiently to combine it with the partitioned actinides from the HLW.

Finally, we are puzzled by apparent inconsistencies in the past AEC-ERDA program in waste management which suggest a lack of perspective. Why have TRU wastes and the disposition of mill tailings been treated so cavalierly when the magnitude of these problems is comparable with that of the problem of the actinide component of the HLW, a problem which is receiving such sophisticated attention? Part of the problem is the lack of design criteria; we simply do not know yet how severe a problem exists. Part of the difficulty may be one of compartmentalization of the research in the various aspects of waste management with a consequent loss of perspective on the relative magnitudes of the several problems. Private discussions have suggested that these inconsistencies are beginning to be recognized. Some organized efforts, either within ERDA or via an outside review group, to develop a more balanced perspective in this area would be welcome.

\section{Footnotes} ${ }^{238} \mathrm{U}$.

$1{ }^{226} \mathrm{Ra}$ exists in nature as a daughter in the radioactive decay chain of

2 A proposed regulation that transuranic wastes at concentrations as low as 10 nanocuries/gram or greater be subjected to geological isolation would, according to many in the nuclear industry, result in all radioactive wastes from commercial reprocessing and recycle-fuel fabrication being sent to geologic isolation as a less expensive option than performing the required radioassay to separate those wastes which would be below this limit. 
3 For the purposes of this section the actinides to be considered are $\mathrm{Np}$, $\mathrm{Am}$, and $\mathrm{Cm}$.

4 Hydraulic conductivity is a parameter that reflects properties of both the porous medium (i.e., permeability) and of the fluid (i.e., density and viscosity). Specific discharge has units of velocity and is defined as $q=\mathrm{K} i$, where $\mathrm{K}$ is hydraulic conductivity and $i$ is hydraulic gradient (dimensionless). The average velocity of groundwater flow is equal to the specific discharge divided by the effective porosity.

5 We are indebted to Dr. F. W. Schwartz of the University of Alberta for his invaluable assistance with the modeling.

6 The principal results of the APS Fuel Cycle Study were released last April 25, 1977 and the detailed text of this Chapter was completed in May and June. Since that time some events indicate tentative redirection of governmental programs addressing the main points at issue for waste management. (See, for example, minutes of the Meeting of The Advisory Committee on Reactor Safeguards at Richland, Washington, July 19, 1977.) ERDA has put renewed emphasis on dealing with spent fuel elements; a spent Unreprocessed Fuel Facility (SURFF) is being designed for interim storage The development of SURFF is apparently to proceed concurrently with the basic long-term geologic repository program. Changes in schedule of the later program will undoubtedly reflect a renewed emphasis on SURFF, but details are not yet clear at the time this study report is printed.

References

1. Beaman and Aitken, 1976

Beaman, S. and Aitken, A. E., 1976, Nuclear Regulatory Commission Report NR-CONF-001, Feasibility Studies of Actinide Recycle in LMFBR's as a Waste Management Alternative.

2. Bear, 1972

Bear, J., 1972, Dynamics of Fluids in Porous Media, Elsevier, New York, N.Y., 764.

3. BNWL-1932, 1975

Battelle Pacific Northwest Laboratory, 1975, Report BNWL-1932, Quarterly Progress Report, BNWL-1900, High-level Radioactive Waste Management Alternatives.

4. Borg, 1976

Borg, I. Y., et al., 1976, Information pertinent to the migration of radionuclides in ground water at the Nevada Test Site, Lawrence Livermore Laboratory Report UCRL-52078, 216.

5. Bradshaw and McClain, 1971

Bradshaw, R.L. and W.C. McClain, 1971, Report ORNL-4555, Project Salt Vault: A Demonstration of the Disposal of High-Activity Solidified Wastes in Underground Mines.

6. Burkholder, et al., 1975

Burkholder, H.C., M.O. Cloninger, D.A. Baker, and G. Jansen, 1975, Report BNWL-1927, Incentives for Partitioning High Level Waste.

7. Claiborne, 1972

Claiborne, H.C., 1972, Report ORNL-TM-3964, Neutron Induced Transmutation of High Level Radioactive Waste.

8. Claiborne and Gera, 1974

Claiborne, H.C. and F. Gera, 1974, Report ORNL-TM-4639, Potential Containment Failure Mechanisms and their Consequences at a Radioactive Waste Repository in Bedded Salt in New Mexico.

9. Cohen, 1977

Colen, B.L., 1977, Rev. Mod. Phys. 49, 1, High Level Waste from Light Water Reactors.

10. Colombo and Nielson, 1976

Colombo, P. and R. Nielson, 1976, Report BNL-21571, Some
Techniques for the Solidification of Radioactive Wasrtes in Concrete.

11. Comey, 1975

Comey, D. D., 1975, Bulletin of Atomic Scientist, 31, \#7, 43, The Legacy of Uranium Tailings.

12. Corey, et al., 1963

Corey, et al., 1963, Soil Sci. Soc. Am. Proc., 27, 258-262, Miscible Displacement in Saturated and Unsaturated Sandstone.

13. Dé, et al., 1976

Dé, A. K., B. Lucksheiter, W. Lutze, G. Malow, E. Schiewer, and S. Tymochowicz, 1976, International Symposium on the Management of Radioactive Wastes from the Nuclear Fuel Cycle, IAEA-SM-207/11, Fixation of Fission Products in Glass Ceramics.

14. Elliot and Auty, 1968

Elliot, M. N. and D. B. Auty, 1968, Glass Technology 9, 5, The Durability of Glass for the Disposal of Highly Radioactive Waste.

15. ERDA, 1975

U.S. Energy Research and Development Administration, March 1975, Report ERDA-33, Nuclear Fuel Cycle.

16. ERDA, 1976a

U.S. Energy Research and Development Administration, August 11, 1976, LWR Waste Management Program Plan, Draft III.

\section{ERDA, 1976 b}

U.S. Energy Research and Development Administration, May 7, 1976, ERDA's Program for Management of Radioactive Waste from Commercial Nuclear Power Reactors.

18. ERDA-43, 1976

U.S. Energy Research and Development Administration, 1976 , Report ERDA--76-43, Alternative for Managing Wastes from Reactors and Post Fission Operations in the L.WR Fuel Cycle.

19. Freeze, 1969

Freeze, R. A., 1969, Scientific Series No. 3, Inland Waters Branch, Dept. of Energy, Mines \& Energy, Ottawa, Ontario, Theoretical Analysis of Regional Groundwater Flow.

20. Freeze, 1972

Freeze, R. A., 1972, IBM Jour. Res. Develop., March, 117-129, Subsurface Hydrology at Waste disposal Sites.

21. Godbee and Joy, 1974

Godbee, H. W. and D. S. Joy; 1974, Report ORNL-TM-4333, Assessment of the Loss of Radioactive Isotopes from Waste Solids to the Environment. Part I: Background and Theory.

22. Hespe, 1971

Hespe, E. D., 1971, At. Energy Rev. 9, 195, Leach Testing of Immobilized Radioactive Waste Solids, a Proposal for a Standard Method.

23. Jenks, 1972

Jenks, G. H., 1972, Report ORNL-TM-3717, Radiolysis and Hydrolysis in Salt Mine Brines.

24. Kee, et al., 1976

Kee, C. W., A. G. Croff, and J. O. Blomeke, 1976, Report ORNL-TM-5427, Updated Projections of Radioactive Wastes to be Generated by the U.S. Nuclear Power Industry.

25. Kelley and Wallace, 1975

Kelley, J. A. and R. M. Wallace, 1975, Report DP-MS-7548, and Nuclear Technology 30, 47 (1976). Procedure for Determining Leachabilities of Radioactive Waste Forms. 
26. Krupp, et al., 1972

Krupp, et al., 1972, Soil Sci. Soc. Am. Proc., 36, 412-417, Relative Flow Rates of Salt and Water in Soil.

27. Kuhlman, 1976

Kuhlman, C. W., 1976, International Symposium on Management of Wastes from the LWR Fuel Cycle. Denver, Colorado, July 13, 1976, The Requirements and Schedule for Viable Solutions.

28. Matthias, 1976

Matchias, B., 1976, private communication

29. McCarthy, 1976

McCarthy, G. J., 1976, Report ERDA-COO02510-10, and Nuclear Technology (to be published), High Level Waste Ceramics: Materials Considerations, Process Simulation and Product Characterization.

30. McElroy, et al., 1976

McElroy, J.. L., W. F. Bonner and J. E. Mendel, 1976, Report BNWL-SA-5873, Waste Solidification U.S.A.

31. Mendel, et al., 1976

Mendel, J. E., W. A. Ross, F. P. Roberts, R. P. Turcotte, Y. B. Katayama, and J. H. Westsik, Jr., 1976, Report BNWL-SL-5534 and IAEA-SM-207/100, Thermal and Radiation Effects on Borosilicate Waste Glasses.

32. Merten, 1966

Merten, U., 1966 Desalination by Reverse Osmosis, U. Merten (Editor), M.I.T. Press, Cambridge, Mass., 15-24, Transport Properties of Osmotic Membranes.

33. NAS-I

National Academy of Sciences, National Research Council, 1957, The Disposal of Radioactive Waste On Land (Harry H. Hess, Chairman), Publication 519.

34. NAS-II

National Academy of Sciences, National Research Council, 1969, Report to the Division of Reactor Development and Technology, U.S. Atomic Energy Commission; National Academy of Sciences, National Research Council, Committee on Geologic Aspects of Radioactive Waste Disposal, May 1975. See also Disposal of Solid Radioactive Waste in Bedded Salt Deposits, National Academy of Sciences, National Research Council, Committee on Radioactive Waste Management, Panel on Disposal in Salt Mines, published November 1970.

35. NUREG-0002, 1976

U.S. Nuclear Regulatory Commission, 1976, Report NUREG-0002, Final Generic Environmental Statement on the Use of Recycle Plutonium in Mixed Oxide Fuel in Light Water Cooled Reactors (GESMO).

36. NUREG-0043, 1976

U.S. Nuclear Regulatory Commission, 1976, Report NUREG-0043. Alternative Processes for Managing Fxisting Commercial High-Level Radioactive Wastes.

37. NUREG-0116, 1976

U.S. Nuclear Regulatory Commission, 1976, Report NUREG-0116, Environmental Survey of the Reprocessing and Waste Management Portions of the LWR Fuel Cycle.

38. OKLO, 1975

The Oklo Phenomenon, IAEA-SM-204/1 (1975), contains a series of research articles. See especially the article by $R$. D. Walton and G. A. Cowan, "The Relevance of Nuclide Migration of Oklo to the Problem of Geologic Storage of Radioactive Wastes", pp. 499-507.

39. Olsen, 1972

Olsen, H. W., 1972, AAPG Bull., 56, 2022-2028, Liquid
Movement Through Kaolinite Under Hydrostatic, Electric and Osmotic Gradients.

40. OWI, 1976

Office of Waste Isolation, Oak Ridge, Tenn., National Waste Terminal Storage Program; Prospective Participants Conference, Y/OWI-4, Rev. (May 21, 1976). See also Progress Reports beginning with Y/OWI-8 (Period April 1, 1975 to September 30, 1976).

41. Pigford and Choi, 1976

Pigford, T. H. and J. Choi, 1976, "Effect of Fuel Cycle Alternatives on Nuclear Waste Management", Proc. of the Symposium on Waste Management, Tucson, Arizona, ERDA CONF-761020.

42. Pigford and Choi, 1977

Pigford, T. H. and J. Choi, 1977, UCB-NE 3241, Transmutation of Radionuclides in Power Reactors.

43. Pigford and Ang, 1975

Pigford, T. H., and K. P. Ang, 1975, Health Physics 29, 451, The Plutonium Fuel Cycles.

44. Reddell and Sunada, 1970

Reddell, D. L. and D. K. Sunada, 1970, Hydrol. Paper, 41, Colo. State Univ., 79, Numerical Simulation of Dispersion in Groundwater Aquifers.

45. Robinson, 1962

Robinson, B. P., 1962, U.S.G.S. Water Supply Paper 1616, 129, Ion-Exchange Minerals and Disposal of Radioactive Wastes--A Survey of Literature.

46. Rowe and Holcomb, 1974

Rowe, W. D. and W. F. Holcomb, 1974, Nuclear Technology 24, 286, The Hidden Commitment of Nuclear Wastes.

47. Roy, 1962

Roy, R., 1962, Symposium on Nucleation and Crystallization in Glasses and Melts, American Ceramic Society 39, Phase Equilibrium and Crystallization of Glass.

48. SAND-76-0105, 1976

Sandia Laboratories, 1976, Report SAND-76-0105, Sandia Solidification Process.

49. Schwartz, 1975

Schwartz, F. W., 1975, Jour. Hydrol., 27, 51-71, On Radioactive Waste Management: An Analysis of the Parameters Controlling Subsurface Contaminant Transfer.

50. Schwartz, 1977

Schwartz, F. W., 1977, Jour. Hydrol., 32, 257-277, On Radioactive Waste Management: Model Analysis of a Proposed Site.

51. Smith and Ross, 1975

Smith, T. H. and W. A. Ross, 1975, Report BNWL 1903, Impact Testing of Vitreous Simulated High-Level Waste in Canisters.

52. T6th, 1962

T6th, J., 1962, Jour. Geophys. Res., 67, 4375-4387, A Theory of Groundwater motion in Sinall Drainage Basins in Central Alberta, Canada.

53. Toth, 1963

T6th, J., 1963, Jour. Geophys. Res., 68, 4795-4812, A Theoretical Analysis of Groundwater Flow in Small Drainage Basins.

54. Willrich, et al., 1976

Willrich, M., S. C. Greenberg, R. K. Lester, H. C. Mitchell, and D. A. Walker, 1976, M.I.T, Energy Laboratory, Report MIT-EL-76-011, Radioactive Waste Management and Regulation. 


\section{A. Issues, Conclusions and Recommendations}

\section{A1. Issues}

The preceding chapters have centered on the normal LWR fue cycles which utilize low-enrichment uranium, with options to recycle or discard the unused ${ }^{235} \mathrm{U}$ and the ${ }^{239} \mathrm{Pu}$ produced. In this chapter we consider advanced fuel cycle alternatives to examine their possible contribution to more effective utilization of uranium ore resources. Attention is focused first on alternatives involving improvement of light water reactors, since these are the present reactors of the U.S. nuclear power industry. To the extent that significant resource extension can be obtained with modifications to LWR's, such options are more readily implemented than options involving the introduction of completely different reactor concepts.

However, continuation of nuclear power eventually will require the greatest resource utilization possible with other reactor concepts, such as the CANDU heavy water reactor operating with thorium fueling, the high-temperature gas-cooled reactor (HTGR), and the fast-breeder reactor. Since each of these other reactors concepts requires fissile material for operation and/or startup, use of LWR plutonium for these advanced reactors is a logical step and is included in our analysis. Alternative use of highly enriched uranium for these advanced reactors is also considered in this chapter.

We also examine some of the safeguard implications for uranium fuel cycles and thorium-uranium fuel cycles, including "nationalinternational" safeguards fuel cycles which involve fueling national reactors with denatured uranium and restricting operations involving plutonium or highly enriched uranium to special "international" sites.

\section{A2. Conclusions}

1. The fast breeder reactor offers the potentiality for large extensions in uranium resources and is the most resource efficient of all fission options.

1a. Plutonium is the most efficient choice for breeder. start-up and provides the best performance of any fissile material. From the point of view of technology and resource utilization, the plutoniumuranium fueled fast breeder is a logical follow-on to the uraniumfueled light water reactors. First-generation breeders can be fueled initially with plutonium recovered from LWR fuel. Reprocessing and refabricating water reactor fuel provide a useful technological base for designing equivalent facilities for breeder fuel cycles, but breeder fuel presents special problems requiring further development. (See Chapter IV.)

1b. Fast breeders can also be started on uranium enriched to $20 \%{ }^{235} \mathrm{U}$, but with significantly higher. first-generation fuel cycle costs than for plutonium start-up. If first-generation commercial breeders were started on ${ }^{235} \mathrm{U}$ instead of plutonium from LWR's, the start-up fissile requirement would be increased by a factor of about 1.5 to 2.4 , the total cost of the fissile material for start-up would be greater by a factor of about 2.3 to 3.7 , there would be a substantial loss in breeding gain over many refueling cycles, and the life-time levelized fuel-cycle cost would be increased by a factor of about 2.3 to 3.6.

1c. Fast breeders could also be started using ${ }^{233} U$ if thorium cycles were to precede the introduction of commercial fast breeders. ${ }^{233} \mathrm{U}$ is intermediate in start-up efficiency but supplies do not exist. Fast breeder fuel cycles breeding ${ }^{233} \mathrm{U}$ from thorium also may have safeguards advantages for the long term (See Conclusion 6).

2. Thorium cycles with thermal reactors would provide a number of advantages for the future. In particular:

2a. a significant resource extension if uranium supplies become short; such cycles may prove essential if the breeder is delayed significantly or if the breeder is never commercialized;

2b. the possibility of more economical power generation as uranium ore prices rise; 2c. another means of introducing denatured thermal-reactor fuel cycles with possible safeguards advantages (as summarized in Conclusion 6).

3. Improved light water reactors can provide significant benefits but would result in less resource extension than would heavy-water and HTGR thorium reactors. Light water reactors are the most direct means of introducing thorium fueling. Future modifications to light water reactors, such as "spectral shift reactors", may provide significantly improved conversion ratios and can be expected to result in significant resource savings with thorium fueling. Even if lightwater breeder reactors could be implemented in existing LWR's, they do not appear attractive relative to other thorium thermal reactor alternatives. Thorium fueling in L.WR's requires the development of industrial-scale technology for reprocessing and refabricating uraniathoria fuel and additional information is needed on irradiation exposure of urania-thoria fuel. (See Chapter IV.)

4. Heavy-water reactors offer as much resulurce extension as can be available from non-breeder reactors. A logical choice is a CANDU-type reactor with thorium fueling, using plutonium from water reactors or highly enriched uranium as make-up fissile material. However, before commercialization in the U.S., this cycle would require additional redesign and development of CANDU fuel for high burnup, development of industrial-scale thoria reprocessing and refabrication technology, and evaluation of acceptability under U.S. licensing criteria. The higher uranium costs which would justify thorium fueling will also justify redesign of the heavy water reactor to higher conversion ratios. With such redesign, the resource savings would be even greater than estimated in this report. However, operation at conversion ratios near unity, although possible, would require a large initial fuel commitment and would produce net uranium ore savings only after many years of operation.

5. The current HTGR-thorium reactor is intermediate in resource requirements between the $L W R$ and CANDU. The design flexibility of the HTGR offers the possibility of modifications which could result in conversion ratios and ore utilization comparable to that now predicted for the thorium-fueled CANDU reactor. The HTGR is not now commercialized, and the graphite-based fuel requires reprocessing and refabrication technology which is more complicated and not as well developed as for thoria fuel.

6. Several fuel cycles, proposed for international safeguards control, have been considered in this study. We also examine their "national" and "international" safeguards implications, especially the options for denatured fuel cycles. Denatured uranium cycles, using low-enriched fresh fuel, are constructive alternatives for the near term. However, plutonium inevitably is present in the spent fuel; denatured thorium cycles may reduce such plutonium production by a factor of about seven. Denatured thorium cycles can have long term safeguards benefits, albeit with uncertain costs; but they do not alter substantively the nature of the institutional and political safeguards arrangements required for denatured uranium cycles. We summarize these alternatives in order of their near-term significance.

6a. National reactors are fueled with low-enrichment uranium, and discharged fuel is stored under international control, i.e., the "international stowaway" cycle. This is the simplest cycle in terms of fuel-cycle operations. It could be implemented in the near term.

6b. National reactors are fueled with low-enrichment uranium, discharge fuel is reprocessed at international centers, and the plutonium is consumed in plutonium-burner reactors colocated at these international centers. This utilizes existing technology and could be implemented in the near future.

6c. National reactors are fueled with thorium and denatured uranium, i.e., uranium containing a fissile isotopic content of $20 \%$ or less. The discharge fuel is reprocessed at an international center. Recovered plutonium is consumed in a reactor colocated at the center, and fissile make-up for the national reactors is obtained from colocated enrichment facilities. Plutonium production is reduced below that of (b) by a factor of about seven. There is corresponding 
reduction in the ratio of power of the colocated plutonium-burner reactors to the power of the national reactors, i. e. approximately 17 national reactors can be supported by one reactor at the center. The fuel cycle operations are more complex. Relatively large and colocated isotope-separation capacity and colocated refabrication are required. We note that the $20 \%$ fissile uranium may be relatively easy to enrich surreptitiously to weapons-grade concentration in noncommercial facilities: If not consumed in a colocated reactor, the plutonium in high-level wastes could become a perpetual safeguards issue.

6d. National reactors are fueled with thorium and denatured uranium, as in (c), and the fissile make-up is supplied by thoriumblanketed fast breeders colocated at the international reprocessing center. This offers excellent long-term ore utilization. A number of breeder options appear possible with thorium in the breeder blanket and/or core. During the early generation of breeders, start-up with plutonium is likely, so complicated reprocessing and refabrication systems are required. Excess fissile production by international breeders would be quite limited and further breeder growth also would be limited. Therefore, for the same national reactor power, the power of such international breeder reactors would have to be much larger than in (b). Variations on this cycle could include national breeders with denatured uranium cores and uranium-plutonium-thorium fueled international breeders which might have been started up with fissile uranium produced in other breeders or in earlier thermal reactors. These concepts have not been evaluated completely, but they illustrate the many very long-range safeguards and resource alternatives which could involve thorium cycles and denatured uranium. These should be evaluated further as a means of identifying future long-range alternatives for the fast breeder program.

7. Any of the safeguards-motivated fuel cycles considered herein will impose some financial burden upon the participating countries, partly because each of these cycles involves operations not necessary with the unrestricted fuel cycles described earlier. These costs of complexity might be mitigated by economics of scale associated with the international operations. Nevertheless, these financial burdens may reduce the acceptability of these cycles. The financial burdens and institutional feasibility of the proposed safeguards-motivated fuel cycles needs evaluation.

8. There is no resource, economic, or safeguards benefit of the suggested "tandem" LWR-HWR cycle which cannot be obtained more easily, reliably, and economically with alternate technology.

9. Improvements in isotopic enrichment technology and the evolution of small scale and less expensive techniques of uranium isotope stparation could make fissile material readily available without regard to nuclear power fuel cycles. (See Chapter VI.) Such developments are also pertinent to the denatured thorium fuel cycles considered in this chapter, which have a development time scale of at least 10 to 20 years, and which use material that is already appreciably enriched.

\section{A3. Recommendations}

There is already an ongoing research and development program for fast breeder technology. With respect to the other advanced fuel cycles and within the context of the studies reported herein,' we recommend that:

1. Reprocessing and refabrication technology should be developed for mixed-oxide uranium-thorium fuels and for graphite-uraniumthorium fuels, carried to a pilot scale suitable for engineering scale-up to useful fuel cycle operations. The development program should include plutonium in the make-up and discharge fuel, and the modifications associated with denatured uranium should be considered. The program should provide information suitable for a periodic re-evaluation of the merits of these thorium-based fuel cycles. Carbide, nitride, and metallic fuels for fast-breeder alternatives also should receive attention.

2. We urge evaluation of the technical feasibility, safety, and environmental features of heavy water reactors, including the CANDU-type, under the U.S. licensing criteria; the evaluation should include the HWR's with modified fuel, involvine enrichment and recycle.
3. Relative feasibility should be evaluated for advanced HWR's HTGR's and spectral shift LWR's for achieving higher conversion ratios. The study should address effectiveness of ore utilization over the 30-year reactor lifetimes, practicality of the fuel technologies and reprocessing-refabrication requirements, relative development costs, and time scale of development and commercialization.

4. Necessary modifications should be studied for heavy-water reactors and their fuel assemblies to achieve the higher fuel burnups possible and desirable with enriched and recycle fueling, as well as the necessary modifications to obtain higher conversion ratios.

5. Fuel reprocessing-refabrication requirements should be studied for both national and international reactors utilizing safeguardmotivated fuel cycles. The study should also include isotope separation requirements, fuel availability, special reprocessing and fuel refabrication, approach to equilibrium, and analysis of financial burdens. The merits of such cycles should be examined with improved LWR's, HWR's and HTGR's as near-term and intermediate-term solutions while evaluating the breeder and other future long-term energy sources.

6. Safeguards considerations suggest that the relative requirements and merits (economics, resources, and safeguards) of various $\mathrm{Pu}-\mathrm{U}-\mathrm{Th}$ fast breeder fuel cycle options should be evaluated. The normal Pu-U breeder should continue to receive emphasis, but consideration of breeder fuel cycles should be broadened to include the "denatured" breeders as well as the possible synergy of mixes of $\mathrm{Pu}-\mathrm{U}$ and $\mathrm{Pu}-\mathrm{Th}$ $\left({ }^{23} \mathrm{U}\right)$ breeders in equilibrium with LWR's and HWR's utilizing denatured ${ }^{233} \mathrm{U}$

\section{B. LWR-Thorium and Advanced LWR Cycles}

The most straightforward manner for the United States to take advantage of improved fuel utilization capabilities of the thorium fuel cycle is to substitute thorium for ${ }^{238} \mathrm{U}$ in the conventional PWR and BWR technologies. Uranium-thorium mixed-oxide fuels have been fabricated, and satisfactory performance has already been demonstrated in LWR's on an experimental basis. Full-scale fuel fabrication and commercial-scale demonstration for extended time periods has not yet been completed. Similarly, thorium-oxide fuels can be reprocessed based on the existing thorex chemistry, as discussed in Chapter IV. Such spent fuel has been reprocessed in the U.S. at the Nuclear Fuel Services Plant in West Valley, New York. Thus, the research base for use of thorium oxide fuels in LWR's is largely completed, although process improvements would be necessary for a modern commercial thorex reprocessing plant in order to minimize the solids content in radioactive wastes and to reduce environmental releases of radionuclides. What remains is completion of development and pilot-scale demonstration of the complete LWR-thorium fuel recycle technology, including fuel refabrication. A demonstration of irradiation performance of urania-thoria fuel is: also needed.

The same type of pressurized water reactor considered in the uranium-plutonium flowsheets of Chapter III has been analyzed (Pigford and Yang, 1977) to illustrate the thorium fuel cycles in which natural ${ }^{232} \mathrm{Th}$ replaces a fraction of $238 \mathrm{U}$ isotope in the previous flowsheet. The make-up fissile material ${ }^{\mathrm{a}}$ is either ${ }^{235} \mathrm{U}$ or plutonium recovered from discharged fuel, as shown in Figures 1 and 2 . The ${ }^{23} \mathrm{U}$ resulting from neutron absorption in thorium is recycled with the other uranium isotopes. The recovered thorium is radioactive because of 1.9 year ${ }^{228} \mathrm{Th}$ and thus is discarded in our analysis. It could be recycled after being stored for about two decades. Referring to Figure 1, the natural uranium is enriched, converted to oxide, and mixed with the thorium oxide in the fuel fabrication. The uranium in the discharged fuel, which contains ${ }^{235} \mathrm{U}$ and ${ }^{233} \mathrm{U}$, is recovered and recycled. To simplify comparison with the earlier discussion on uranium-fueled LWR's, the same total heat generation per fuel rod for fueling with urania or thoria has been assumed. Because thoria is of lower density than urania, the average thermal exposure of $30.4 \mathrm{Mw}$

a Safeguards issues relevant to highly enriched $\left(93 \%{ }^{235} \mathrm{U}\right)$ uranium make-up are discussed in Section VIII.F. Actinide reactions in thorium fueling are discussed in Appendix III. 


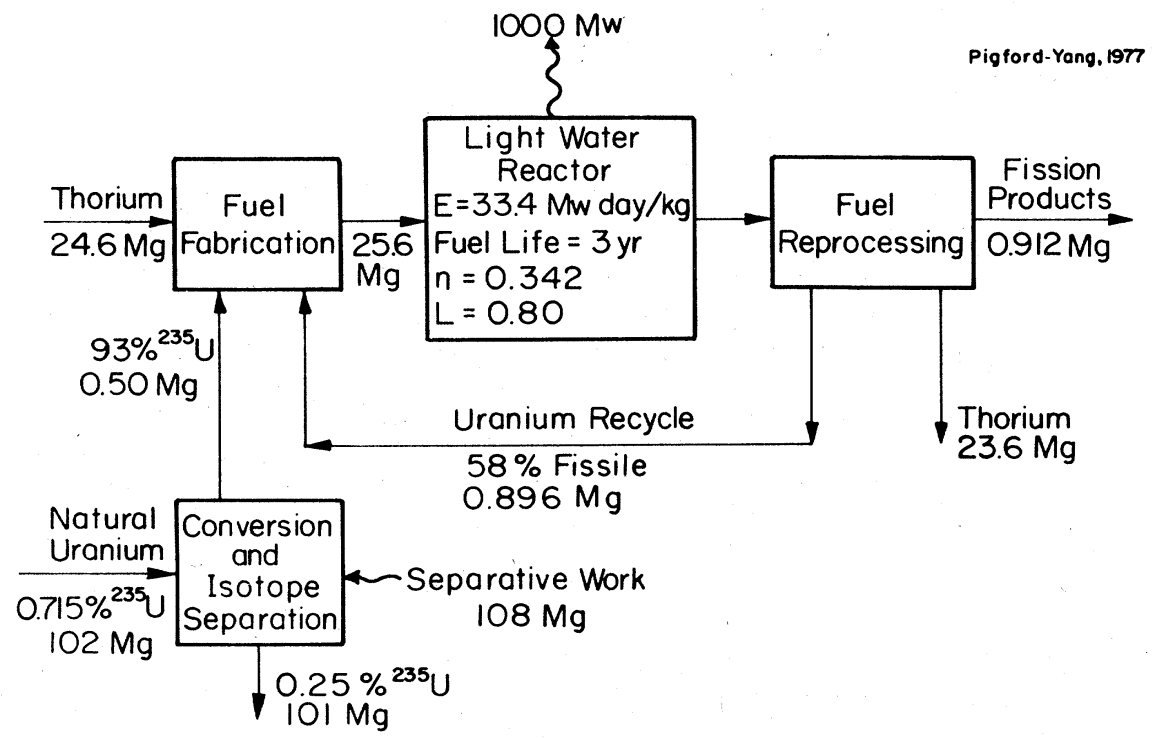

FIG. 1. Lifetime-average annual quantities for uraniumthorium fueled pressurized water reactor. day $/ \mathrm{kg}$ for urania is equivalent on this basis to $33.4 \mathrm{Mw}$ day $/ \mathrm{kg}$ for thoria fuel. Referring to Figure 2, $70 \%$ fissile plutonium is utilized as the oxide with thorium mixed oxide fuel. This plutonium must be supplied by another reactor, and our calculations of lifetime ore requirements will include such a uranium-fueled water reactor to supply this make-up in plutonium. As shown in the diagram, the remaining plutonium and the uranium produced from the thorium are both recycled, and the remaining thorium is stored.

The thirty year lifetime requirements for all of these cases is summarized in Table 1. The first case, a), is the normal uranium norecycle case, and b) and c) are the two previously discussed cases of uranium-only recycle and uranium-plutonium recycle. There is significant reduction in the 30 year lifetime ore requirements. The net lifetime gain from. U-Pu recycle of case c) versus case a) is approximately $32 \%$.

Cases d), e) and f) involve the use of thorium mixed oxide fuels; d) involves the flowsheet of Figure 1 and e) and f) the flowsheet of Figure 2. By comparing cases c) and d) we note a further saving in uranium ore of approximately $16 \%$ from the use of thorium. To fue the thorium reactor with plutonium one must consider two cases-Case e) is the first generation situation wherein a uranium-fueled water reactor must operate for a few cycles before plutonium is recovered from discharge fuel. This plutonium must supply several total fissile loadings for the thorium reactor, until uranium and plutonium recovered from discharge fuel from this reactor can be recycled. Additional ore is required to fuel the many cycles before equilibrium is reached; the limitation is avoided in subsequent generations of the same type of reactor. The new generation is started from the fuel inventory of the previous generation, so equilibrium can be reached almost immediately. The lifetime ore requirements of second and subsequent generations of identical reactors are shown as case (f) in Table 1 . The second-generation reactors (case f) require about $22 \%$ less, uranium ore than case $e$.

Recent estimates (Shapiro, et al., 1977) indicate about the same fuel-cycle costs with $\mathrm{Pu}$-Th fueling (case e) as for $\mathrm{U}$ fueling with U$\mathrm{Pu}$ recycle (case c). ${ }^{235} \mathrm{U}$ fueling (case d) is estimated to be more expensive, because of the relatively high costs of $93 \%{ }^{235} \mathrm{U}$. However, there is too much uncertainty in the cost of fuel reprocessing, particularly for thorium fuel reprocessing, for the small differences in the estimated costs for these fuel cycles to be significant. Also, the additional cost of research and development to advance Thorex reprocessing technology to a commercial scale was not considered in these estimates.

Assuming no significant cost advantage for thorium fueling in the near future, thorium fueling could become more attractive at a future time when uranium supplies are more limited and the cost of uranium is relatively high. Then reactors with less uranium consumption would have a greater economic advantage and would be more useful to the power economy. There would also be greater incentive to redesign light water reactors to higher conversion ratios for better ore utilization than is indicated in Table 1.

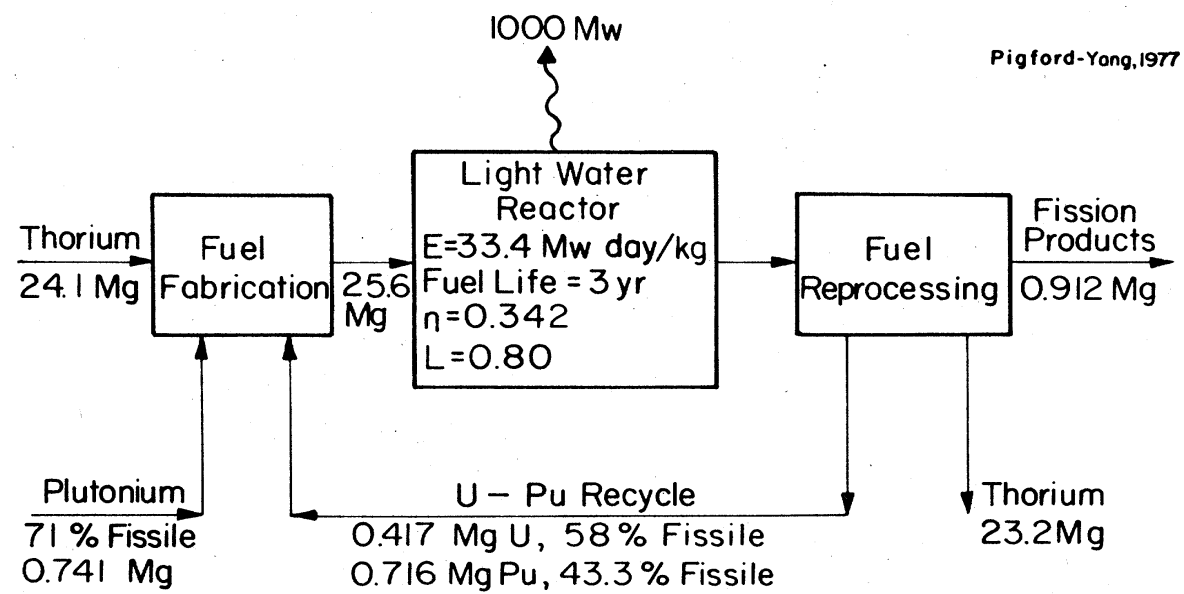

FIG. 2. Lifetime-average annual quantities for plutoniumthorium fueled pressurized water reactor. 
TABLE 1. 30-year lifetime ore requirements for pressurized-water reactors $(1000 \mathrm{Mw}$ electrical power, $80 \%$ capacity factor).

\begin{tabular}{|c|c|c|c|c|c|}
\hline & \multicolumn{5}{|c|}{$\mathrm{U}_{3} \mathrm{O}_{8}$} \\
\hline \multicolumn{6}{|c|}{ Short Tons } \\
\hline & & $\begin{array}{l}0.2 \% \\
\text { Depleted U }\end{array}$ & $\begin{array}{l}0.25 \% \\
\text { Depleted U }\end{array}$ & $\begin{array}{l}\text { Conversion } \\
\text { Ratio }\end{array}$ & $\begin{array}{l}\mathrm{ThO}_{2} \\
\text { Short Tons }\end{array}$ \\
\hline a) & No recyc1e & 6410 & 6970 & 0.60 & \\
\hline b) & U recycle & 5280 & 5820 & 0.60 & \\
\hline c) & U-Pu recycle & 4340 & 4770 & 0.613 & \\
\hline d) & ${ }^{235} \mathrm{U}-\mathrm{Th}$, uranium recycle & 3650 & 3970 & 0.665 & 969 \\
\hline e) & Pu-Th, U-Pu recycle $1 e^{a}$ & 4220 & 4650 & 0.611 & 197 \\
\hline f) & $\begin{array}{l}\text { 2nd Gen. Pu-Th, } \\
\text { U-Pu recycle }\end{array}$ & 3327 & 3619 & 0.611 & 276 \\
\hline
\end{tabular}

The proposed light-water breeder reactor is such a concept. It involves a higher ratio of fuel to water than in present reactors, separated and localized regions of fissile and fertile material, and the use of movable fuel for reactivity control. All of these modifications increase neutron absorption in thorium, resulting in higher conversion ratio. Thorium-cycle conversion ratios of near unity seem achievable. However, the breeding gain is very small and specific power is low, so pre-breeders of intermediate conversion ratios are proposed as a means of providing the start-up fissile inventory. A recent analysis (Kasten, et al., 1977) indicates an increased ore requirement during the period of introducing prebreeders and breeders, with net ore savings only after a very long period of operation. Thus its value is marginal relative to other alternatives.

A possibly more useful and realistic concept, to improve the conversion ratio and ore consumption with near-term light-water reactors, is to modify these reactors for "spectral shift" operation. The reactor coolant system would be modified so that heavy water $\left(\mathrm{D}_{2} \mathrm{O}\right)$ could be introduced into the coolant at controlled concentrations. After each refueling cycle the excess neutron production from fresh fuel would be controlled by replacing enough $\mathrm{H}_{2} \mathrm{O}$ with $\mathrm{D}_{2} \mathrm{O}$ for less efficient neutron moderation. This excess neutron production, normally absorbed in boron or other non-fertile absorbers, would be consumed by the absorption resonances of the fertile materials ${ }^{238} \mathrm{U}$ or ${ }^{232} \mathrm{Th}$, thereby increasing the fissile production and conversion ratio.

As fuel burnup proceeds the $\mathrm{D}_{2} \mathrm{O}$ is replaced by $\mathrm{H}_{2} \mathrm{O}$ to maintain reactivity, and the process is repeated for each refueling cycle. Typically, at the beginning of a refueling cycle the reactor coolant would consist of about $85 \% \quad \mathrm{D}_{2} \mathrm{O}$. During the cycle the coolant is diluted with normal water resulting in a concentration of less than about $25 \% \mathrm{D}_{2} \mathrm{O}$ at the end of the one-year cycle. A facility must be provided to reconcentrate the heavy water. The spectral shift water reactor received some attention over a decade ago, but it was not justified economically at that time. Preliminary estimates indicate significant improvement in conversion ratio over that of any of the light water cycles listed in Table 1, even using the lattice of presentday pressurized water reactors. For fuel burnups of 33.4 megawatt day per $\mathrm{kg}$ a ${ }^{235} \mathrm{U}$-Th loading was calculated to operate at an integral conversion ratio of 0.7 and a ${ }^{233} \mathrm{U}$-Th loading at a conversion ratio as high as 0.87 . This indicates the possibility of reducing the lifetimeore requirements well below $3000 \mathrm{Mg}$. Benefits from the higher conversion ratios of spectral shift LWR's must be balanced against increased costs resulting from the increased complexities of using heavy water in LWR's. Facilities must be provided to adjust $\mathrm{D}_{2} \mathrm{O}$ concentration in the LWR coolant and to re-enrich the $\mathrm{D}_{2} \mathrm{O}$ diluted by $\mathrm{H}_{2} \mathrm{O}$ during the fuel cycle. An on-site distillation system for heavy-water enrichment is a possibility.

The presence of concentrated deuterium in the coolant will increase the rate of production of tritium in the coolant. In the pressurized water reactor this increase in tritium production by neutron absorption in deuterium will be offset, in part, by the loss of tritium production from fast-neutron reactions in dissolved boron which will no longer be needed for reactivity control. A higher concentration of tritium in the coolant may complicate the open-core refueling techniques now used in LWR's because of the possibility of tritium escape during refueling. It may also require additional controls to minimize the environmental release of tritium via noncondensable off-gases during normal operation.

Control of burnup reactivity by spectral shift boiling-water reactors would eliminate the burnable-poison absorbers now incorporated in the fuel rods in these reactors. However, since the burnable absorbers also provide an effective means of adjusting the axial distribution of neutron flux and power density in these reactors, some other technique must be developed for power-density control in a spectral shift boiling water reactor. The larger negative void coefficient of reactivity in spectral shift operation would also be a problem.

Nevertheless, the spectral shift concept might be relatively easy to implement in some present LWR's and should be included in further evaluations of alternatives for improved resource utilization. Spectral shift operation with thorium fueling provides the greatest gain in resource utilization, but the improved utilization of uranium fuel with spectral shift operation may become justified when uranium ore prices increases.

There is another way in which thorium may be utilized in a present-day or spectral shift light-water reactor, not merely to extend resources but as a part of an overall approach to international safeguards. As is discussed more completely in Section $F$ of this chapter, the recycled uranium in a uranium-thorium cycle is diluted with $238 \mathrm{U}$ to about 15 to $20 \%$ fissile isotopic concentration. This results in somewhat less plutonium production than in a lowenrichment uranium cycle. Such a fuel cycle is completed by storing or reprocessing the discharge fuel to recover the uranium and plutonium.

Our overall conclusion is that the use of thorium in present light water reactors offers a real but marginal advantage for resource extension alone. Larger benefits are possible with redesign of the 


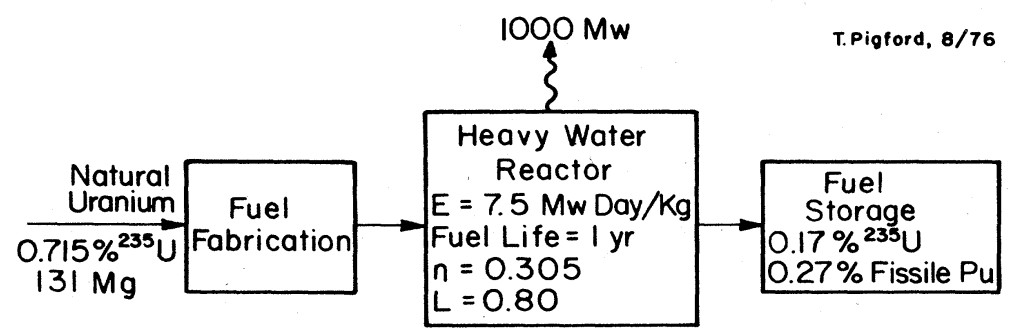

FIG. 3. Annual quantities for uranium-fueled CANDU reactor, no recycle. reactor core or of the moderator-coolant system. Were the fast breeder reactor to be delayed or eliminated altogether, it might be desirable to introduce the use of thorium in LWR's modified for higher conversion ratios, since the overall reduction in uranium ore demand for a large number of LWR's could be important.

\section{CANDU and Advanced Heavy Water Reactor Cycles}

The pressure tube heavy water reactor already commercialized in Canada is unique in that it can be fueled directly with natural uranium (Foster and Critoph, 1976; Meneley, 1976). This reactor was described in broad detail in Chapter III where several variations with different coolant were mentioned, all of them moderated with heavy water. As was pointed in Chapter III, ordinary water has a greater neutron absorption cross section than heavy water but is more effective in slowing down the neutrons quickly. Thus, a heavy water reactor must be larger to provide for neutron moderation, but the larger heavy water reactor with lower neutron losses can operate with lower isotopic enrichment. Because of the higher conversion ratio and shorter irradiation exposure typical of the CANDU reactor, greater quantities of fissile plutonium are produced per unit of thermal energy. However the short irradiation exposure and high fuel throughput result in plutonium in CANDU discharge fuel at relatively low concentration. Present costs of uranium and of fuel-cycle operations do not justify reprocessing now to recover the plutonium from the CANDU fuel, so the discharge fuel is put into long term storage. However, future higher costs of uranium ore may ultimately justify reprocessing the discharge fuel to recover and recycle plutonium. We consider here some variations of the present fuel cycle in which plutonium is utilized and also consider variations in which thorium is utilized rather than natural uranium.

The flowsheet (Pigford, 1977a) for a CANDU reactor fueled with natural uranium is shown in Figure 3, and the flowsheet (Pigford, 1977a) for natural uranium and recycle plutonium (Till, et al., 1977) is shown in Figure 4. The depleted uranium and a small amount of plutonium from fuel reprocessing are discarded. Recycling the plutonium makes a significant difference in the fuel burnup, which rises from the low value 7.5 megawatt days per kilogram, typical of natural uranium, to 16 megawatt days per kilogram as shown in the flow chart (Till, et al., 1977; Slater, 1975; Kasten, 1977). This is approximately half the burnup typical of light water reactor fuel. The data in this flowsheet are derived from calculations which assumed that the plutonia-urania fuel with the same fuel and cladding dimensions as present CANDU fuel can operate to the higher burnups without modification. This is an optimistic assumption, since the higher burnups will generate more fission gases. Fission-gas plenums and thicker fuel cladding may be required.

As shown in Table 2 the lifetime uranium ore requirements for the CANDU reactor with self-generated plutonium recycle, variation b), are about two-fold less than for the present non-recycle operation. The requirements are significantly less than the ore requirements for any of the light water fuel cycles shown in Table 1, indicative of the overall higher conversion ratio of the heavy water reactor. However, the burnup per cycle is still too low for this fuel cycle to be more economical than the CANDU non-reprocessing stowaway cycle, assuming the fuel-cycle cost parameters used in analyzing the LWR fuel-cycle econimics in Chapter IV. At some future higher price of uranium ore this fuel cycle could become economically attractive.

The same CANDU reactor can also be fueled with thorium and make-up fissile material derived from an external source (Till, et al, 1977; Critoph, et al., 1976; Hatcher, et al., 1975; Kasten, et al., 1977; Till and Chang, 1977; Banerjee, et al., 1977). Figure 5 is the flowsheet (Pigford and Yang, 1977) for the equilibrium cycle of the CANDU reactor fueled with $93.5 \%{ }^{235} \mathrm{U}$, thorium, and recycled uranium. The fuel burnup has been specified at 27 megawatt days per kilogram of heavy metal, near that typical of light-water fuel. As shown in Table 2 , uranium-thorium fueling increases the average conversion ratio to 0.92 , a result of the greater number of fission neutrons per absorption for the bred and recycled ${ }^{233} \mathrm{U}$. The uranium ore consumption is 39 to $45 \%$ less for this cycle than for uranium fueling with self-generated plutonium recycle. Alternatively, the make-up fissile material for the thorium-fueled CANDU reactor can be plutonium recovered from uranium fuel discharged from a CANDU reactor or from a light water reactor. The flowsheet (Pigford and Yang, 1977) for this cycle at equilibrium, utilizing plutonium produced in a natural uranium CANDU reactor, is shown in Figure 6. The lifetime ore requirements for the plutonium-thorium CANDU reactor, shown in Table 2, are calculated for 30 Gwe-yr of electrical energy from a reactor system consisting of a uranium-fueled CANDU reactor to provide the startup and make-up plutonium and a plutonium-thorium fueled CANDU to consume the plutonium. The uranium ore required for this system is $20 \%$ less than for the CANDU fueled with natural uranium and self-generated plutonium recycle, and it is 22 to $36 \%$ greater than for the ${ }^{235} \mathrm{U}-\mathrm{Th}$-fueled CANDU with uranium recycle.

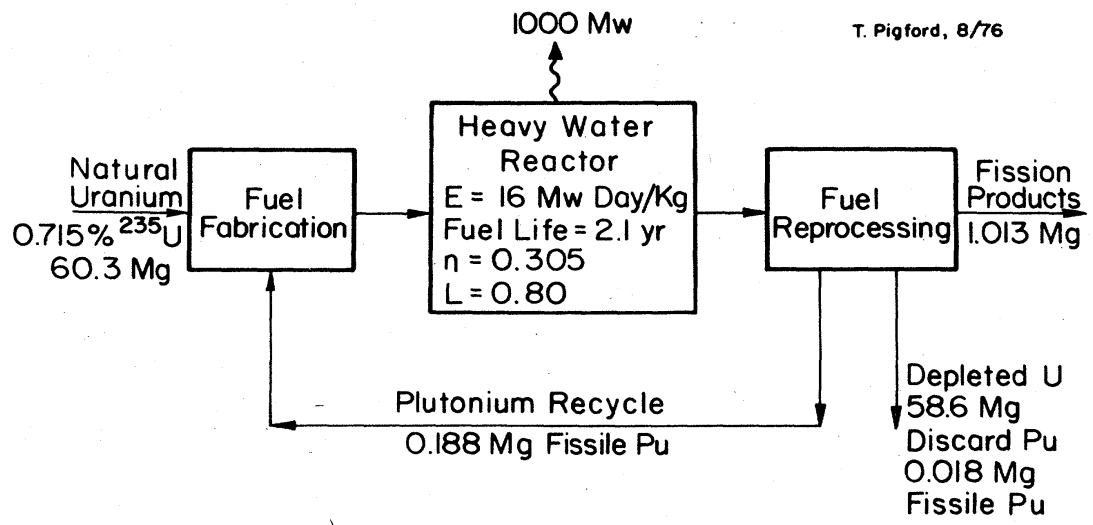

FIG. 4. Annual quantities for uranium-fueled CANDU reactor with self-generated plutonium recycle (equilibrium fuel cycle). 
TABLE 2. 30-year lifetime ore requirements for heavy water reactors (Pigford and Yang, 1977) (1000 Mw electrical power, $80 \%$ capacity factor).

\begin{tabular}{|c|c|c|c|c|}
\hline & & $\mathrm{U}_{3} \mathrm{O}_{8}$ & Conversion & $\mathrm{ThO}_{2}$ \\
\hline & & Short Tons & Ratio & Short Tons \\
\hline a) & Natural U fue1, no recycle & 5263 & 0.75 & \\
\hline b) & Natural U fue1, Pu recycle & 2861 & 0.74 & \\
\hline c) & $235 \mathrm{U}-\mathrm{Th}$ fue 1 , U recycle & & & \\
\hline & $0.20 \%$ dep 1 eted $U$ & 1690 & & \\
\hline & $0.25 \%$ dep 1 eted U & 1870 & 0.92 & 1410 \\
\hline d) & Pu-Th fue 1, U recycle $e^{a}$ & 2290 & 0.92 & 771 \\
\hline
\end{tabular}

ancludes U-fueled HWR to supply make-up Pu. Total system power $=1000 \mathrm{Mw}$.

In the present conceptual design of a thorium-fueled CANDU reactor, the lattice spacing and specific power have been kept the same as for the natural-uranium CANDU reactor. Because the fuel burnup chosen for these thorium cycles is 3.6 times greater than for present CANDU uranium fuel, the void volume in each fuel rod has been increased by $9 \%$ to provide for the accumulation of fission gases. The cladding dimensions have been kept the same, although the higher burnups may require thicker cladding. There are no published data on the performance of CANDU fuel elements at these high burnups.

A heavy water reactor lattice can be modified to operate at yet higher conversion ratios, even as a thermal breeder with thorium make-up and uranium recycle (Till, et al., 1977; Critoph, et al., 1976; Hatcher, et al., 1975; Kasten, et al., 1977). A conversion ratio of unity can theoretically be obtained on the present CANDU lattice if fueled with thorium and uranium of low fuel burnup per cycle. The conversion ratio can also be increased by increasing the thorium loading, operating at lower specific power and at lower burnup, and increasing the calandria lattice spacing. Although the fuel savings from higher conversion ratio are large, they cannot be realized at the beginning of reactor life time. In attaining the higher conversion ratios possible in advanced heavy water reactors, a larger initial fissile loading is required. The smaller cumulative ore requirements are realized only after a number of years of operation, as illustrated in
Figure 7. For the thorium fuel reactor having a conversion ratio approaching unity, the entire ore requirement is essentially that for the initial inventory and for the start-up fuel cycles before equilibrium is reached.

The CANDU reactor, optimized with respect to the variations described above, offers an attractive alternative for the future when uranium ore prices rise. If the fast breeder is significantly delayed or cancelled, a high-conversion-ratio reactor, such as the CANDU, may become necessary to conserve uranium resources.

As was pointed in Chapter III the CANDU reactor has not yet been licensed for operation in the United States. An analysis must be carried out before the possible problems of licensing this special reactor under the U.S. safety criteria can be ascertained. It is our recommendation, therefore, that the U.S. government (ERDA and/or NRC) carry out such an evaluation of the CANDU reactor, including not only the present design but also reasonable modifications necessary to accomplish these incentives for future U.S. application. The redesign of CANDU fuel rods for higher burnup, the failure modes of the CANDU coolant system, and the emergency cooling of CANDU fuel are all worthy of consideration. With interest in both countries concerning effective utilization of uranium ores, it is a matter of organizational coordination and cooperation to develop the desired information.
FIG. 5. Annual quantities for CANDU reactor fueled with ${ }^{235} \mathrm{U}$, thorium, and recycled uranium (equilibrium fuel cycle).

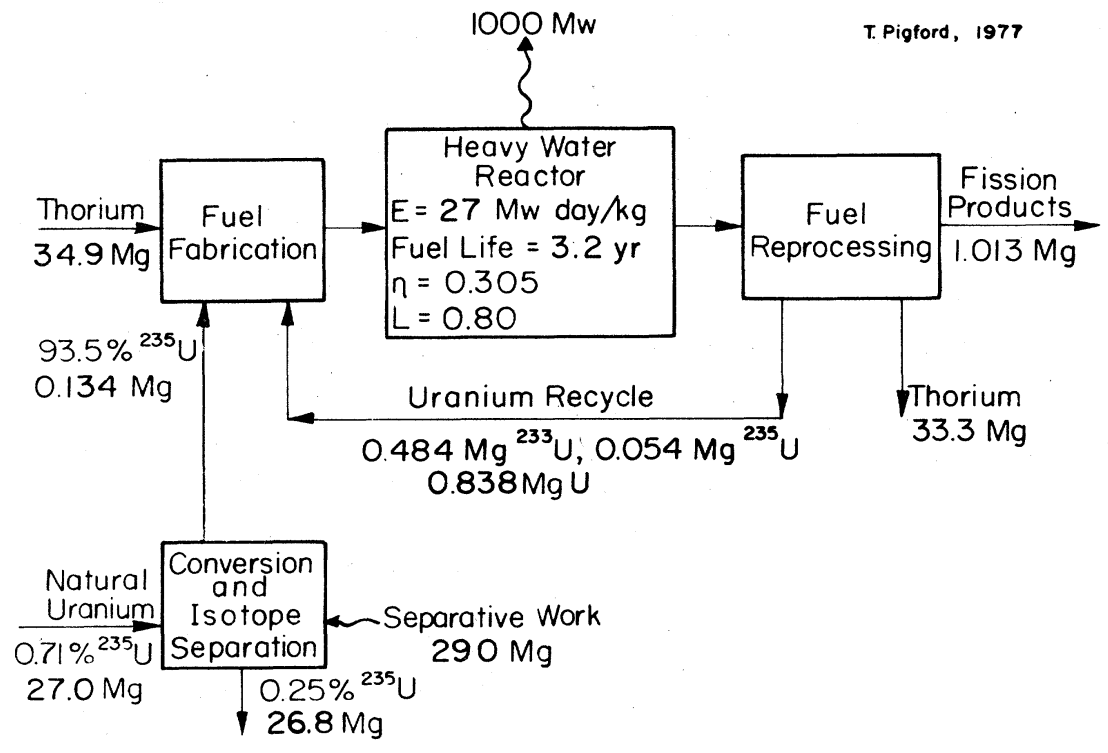




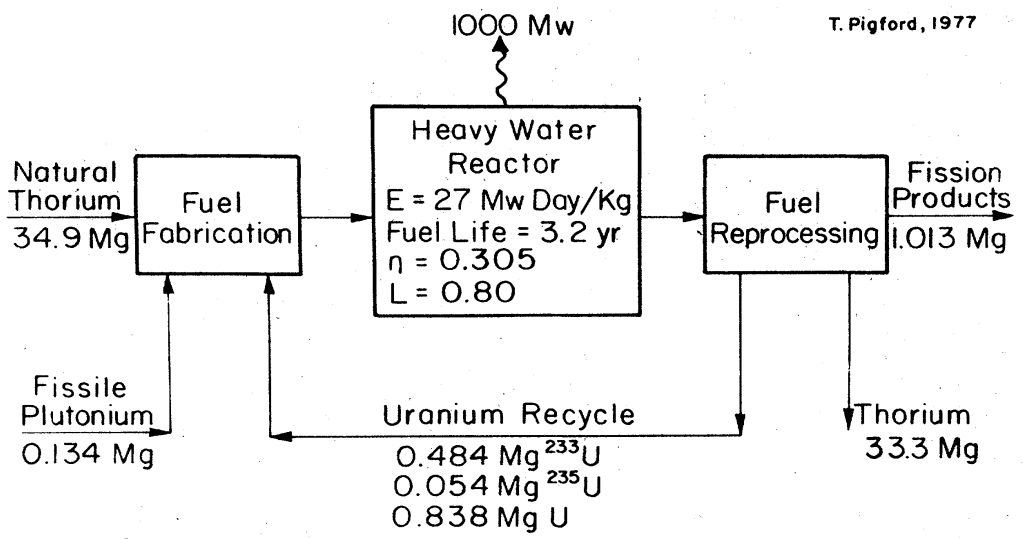

FIG. 6. Annual quantities for CANDU reactor fueled with plutonium, thorium, and recycled uranium (equilibrium fuel cycle).

\section{High Temperature Gas Reactor (HTGR)}

The high temperature gas reactor described in Chapter III is undergoing. demonstration tests as an alternative nuclear power plant of the future. It is a helium cooled graphite moderated reactor using natural ${ }^{232} \mathrm{Th}$ as a fertile material and highly enriched uranium as fissile material. As shown in the flowsheet (Pigford, 1977a) of Figure 8 , the reactor is fueled with thorium, make-up ${ }^{235} \mathrm{U}$, and uranium $\left.{ }^{233} \mathrm{U},{ }^{234} \mathrm{U},{ }^{235} \mathrm{U},{ }^{236} \mathrm{U}\right)$ recovered from the discharge fuel and recycled. The fuel consists of coated particles of uranium and thorium embedded in a prismatic graphite matrix. Helium coolant flows through holes in the graphite. The hexagonal cross-section fuel-moderator prisms are stacked to form the core structure. The graphite matrix provides a means of obtaining very high fuel burnup without loss of mechanical integrity. The design burnup is 94 megawatt days per kilogram of uranium and thorium, which is about 3 times that experienced in typical light water reactors. A commercial prototype is now operating at Fort St. Vrain in Colorado. However

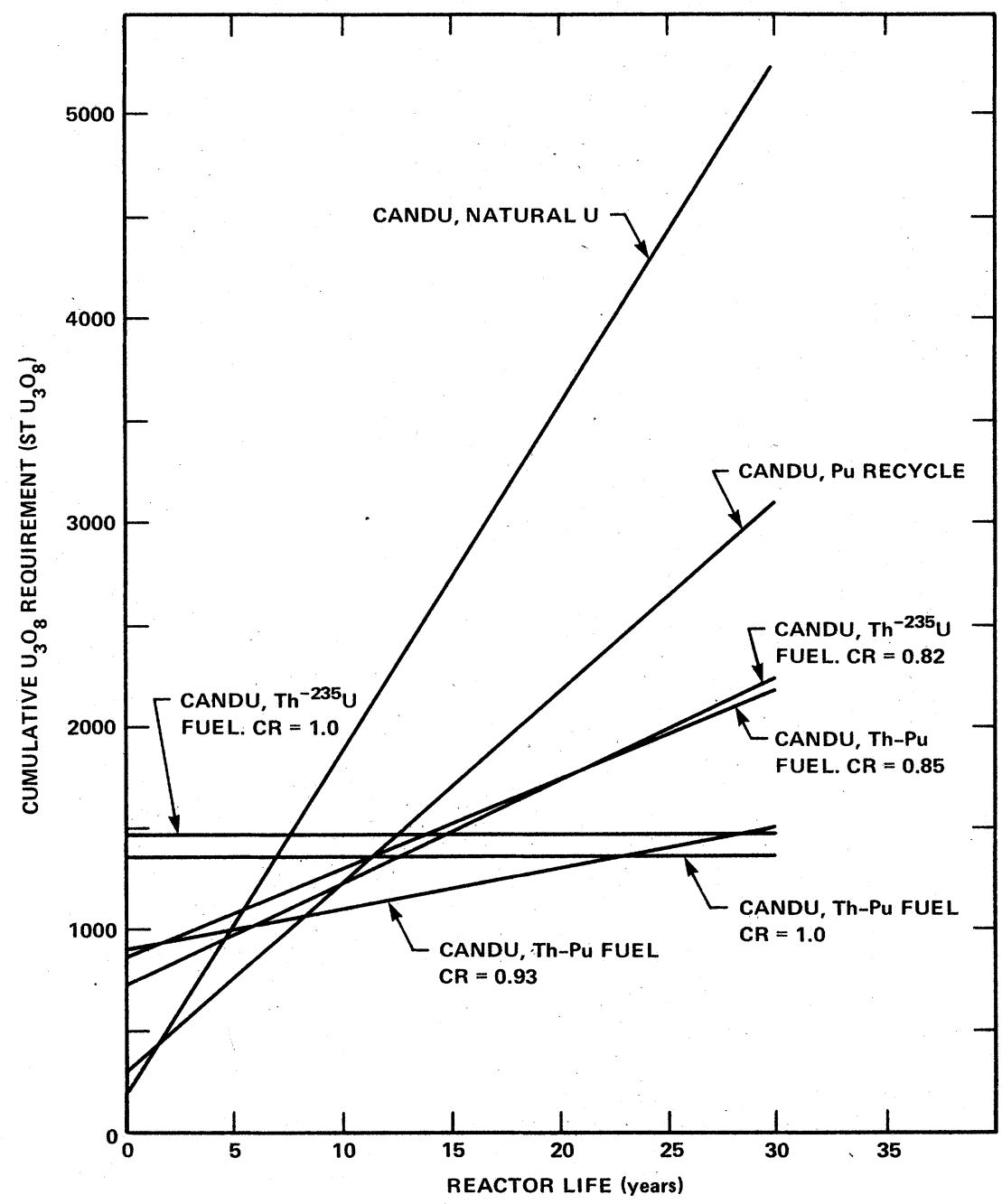

FIG. 7. Cumulative uranium ore requirements for the CANDU reactor (Kasten $e t$ al., 1977).

Rev. Mod. Phys., Vol. 50, No. 1, Part II, January 1978 
FIG. 8. Annual quantities for HTGR fueled with ${ }^{235} \mathrm{U}$, thorium, and recycled uranium (equilibrium fuel cycle).

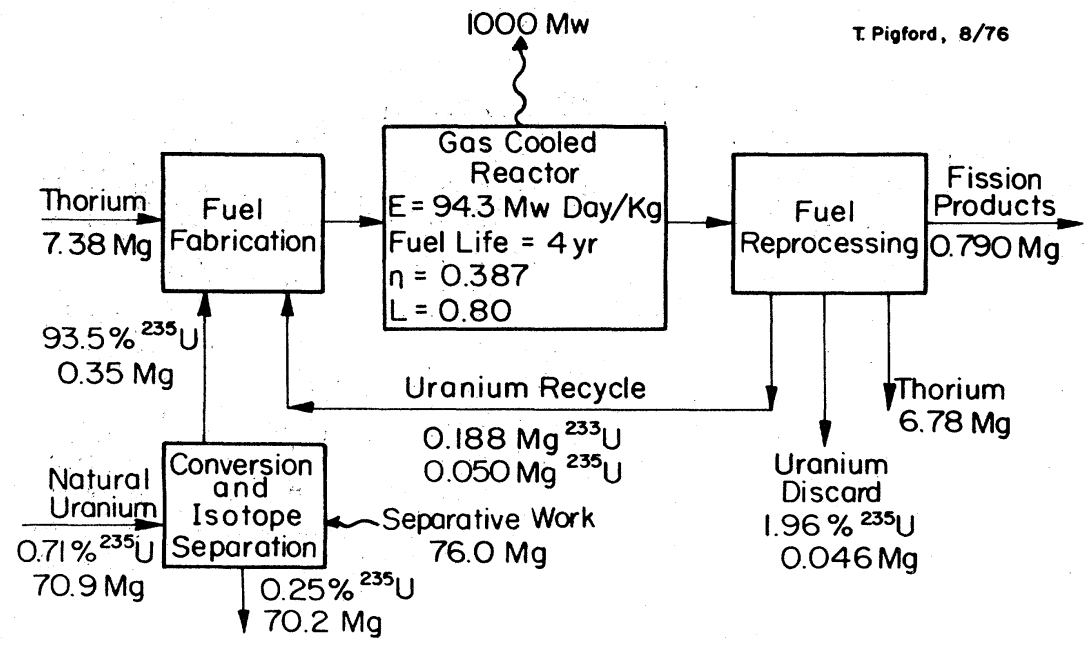

the U.S. manufacturer, General Atomic, has recently withdrawn its earlier sales of full-scale commercial plants. Development of HTGR fuel reprocessing and refabrication technology continues under ERDA sponsorship. It is uncertain when and if this reactor will return to the commercial U.S. market.

An alternative means of realizing the fuel value of plutonium recovered from discharged LWR fuel would be to recycle this plutonium in the HTGR in lieu of highly enriched ${ }^{235} \mathrm{U}$ make-up normally used (Pigford and Ang, 1977; Brogli, et al., 1975). The flow sheet (Pigford, 1977a) for this plutonium utilization is shown in Figure 9. The uranium produced as a result of neutron absorption in thorium is recycled, along with the plutonium remaining in the discharge fuel.

The thirty-year life-time ore requirements for the current design of the HTGR are shown in Table 3, where three cases are described. The first is the normal HTGR operation with ${ }^{235} \mathrm{U}$-thorium fuel. The second is the plutonium-thorium fueled reactor following the flowsheet of Figure 9. The ore requirements listed for this case include an ordinary LWR to supply the makeup plutonium. Much of the requirements in cases (a) and (b) are to supply the extra fissile material required during the many irradiation cycles before the equilibrium recycle shown in these flowsheets is attained. However a second-generation reactor can start-up with the fuel-cycle inventory left from a retired first-generation reactor. The lifetime ore requiremients for such second generation reactors are shown in cases (c) and (d). The HTGR can also be operated on low-enrichment uranium; with or without U-Pu recycle. Comparing the data in Table 3 with those in Table 2, it is apparent that the thorium-fueled CANDU reactor requires about 50 to $60 \%$ less uranium ore than does the current HTGR.
The current reference HTGR, which is a basis of the flowsheets of Figures 8 and 9 and of the ore requirements of Table 3 , is a design optimized for current or near-term fuel-cycle cost parameters, including uranium ore price. However, a feature of the HTGR fuel concept is the flexibility for making relatively large changes in the fuel loading without altering the fuel thermal performance of the overall mechanical design. For a future era of higher uranium ore prices and greater incentive to improve ore utilization, the conversion ratio of the HTGR can be increased by the following modifications:

1. Increase the thorium loading in the core, which increases neutron absorption in thorium relative to non-productive absorption and leakage. Decrease the core power density. For the same fissile concentration, this decreases the neutron flux and reduces the flux-dependent neutron absorption in ${ }^{135} \mathrm{Xe}$ and ${ }^{23}{ }^{3} \mathrm{~Pa}$.

2. The greater core volume, for the same thermal power, provides volume for further increases in the thorium loading.

3. Decrease the time interval between refueling, thereby decreasing the loss of neutrons to control absorbers.

4. Reduce the thickness of the coatings on the fuel particles, allowing greater thorium loading in the fuel holes in the graphite prisms, allowing greater thorium loading per prism.

5. Distribute the fuel particles uniformly throughout the graphite prisms.

Estimated improvements in the conversion ratio and ore requirements possiblc by such approaches are shown in Table 4 .
FIG. 9. Annual quantities for HTGR fueled with plutonium, thorium and recycled uranium (equilibirum fuel cycle).

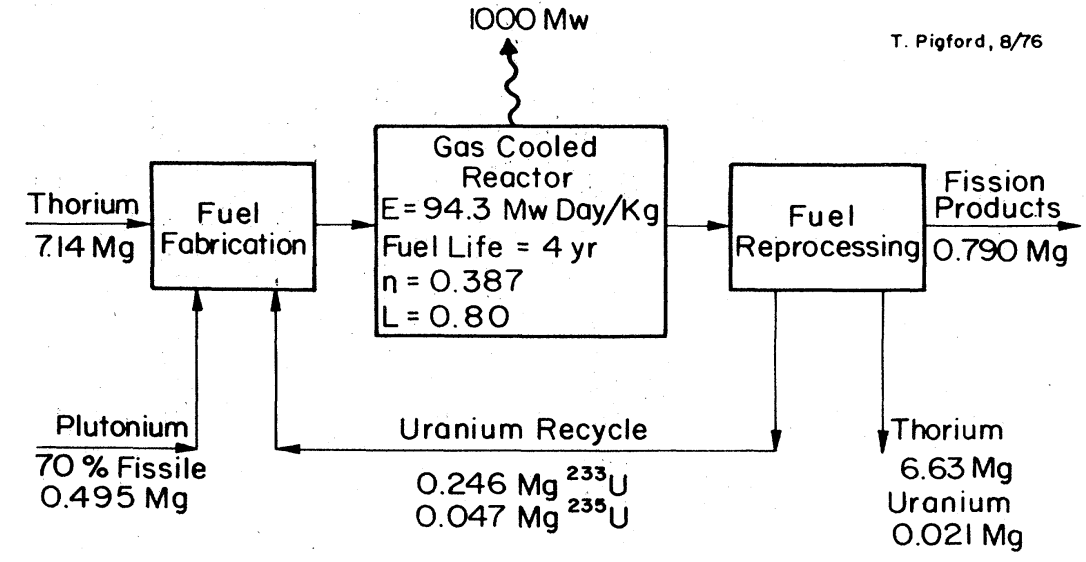


TABLE 3. 30-year lifetime ore requirements for high-temperature gas-cooled reactors (Pigford, 1977a) (1000 Mw electrical power, $80 \%$ capacity factor).

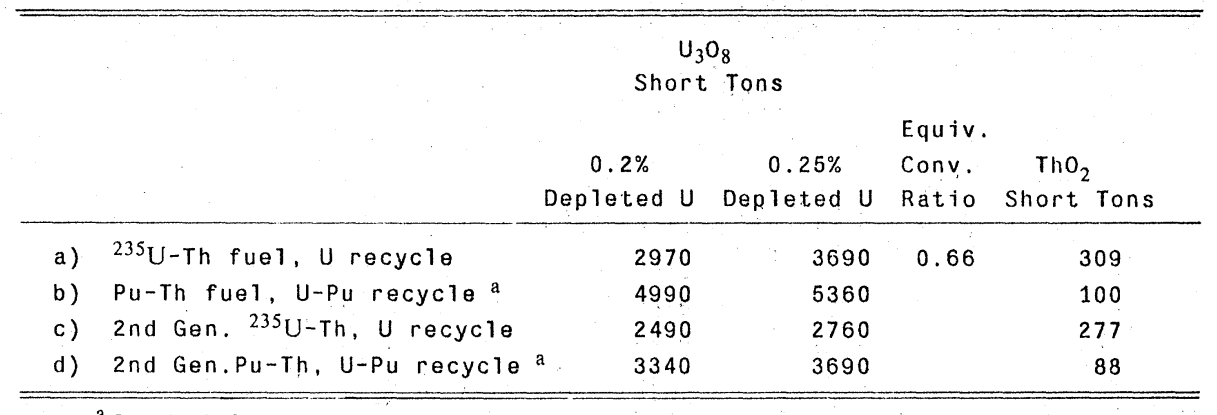

${ }^{a}$ Includes U-fueled water reactor to supply make-up $\mathrm{Pu}$. Total system power $=1000 \mathrm{Mw}$.

The first two modifications, which increase the conversion ratio to 0.76 , can be achieved with the current fuel element design. This reduces the life-time ore requirement to a level about $58 \%$ greater than that of the CANDU reactor operating on the same fuel cycle.

The most significant parameter in increasing the HTGR conversion ratio is the increased thorium loading. This requires corresponding increases in the initial and start-up loadings of fissile ${ }^{235} \mathrm{U}$, resulting in a greater investment in fuel early in the reactor life. The higher initial investment contributes to a higher levelized fuel cycle cost, but if the price of uranium increases more rapidly than does the effective discount factor during the plant life, the lower annual ore requirements for fuel reloads throughnut the plant life could possibly offset this higher initial fuel investment.

One feature of the HTGR which benefits its fuel cycle cost and its resource utilization is the very high fuel exposure of 94 Mwday per kilogram of heavy metal. This means that for fuel reprocessing and refabrication to make the same contribution to the cost of electrical energy, the unit costs of these operations, expressed in cost per unit amount of heavy metal processed, can be correspondingly greater for these operations in the HTGR fuel cycle. However, whether these HTGR operations, can be carried out within the greater allowable unit costs is uncertain at this time. As discussed in Chapter IV, the HTGR reprocessing operations are yet to be carried out on a pilot-plant scale, so the technological foundation for estimating the cost of commercial-scale operations is now quite limited.

The burnup advantage of HTGR fuel over LWR and CANDU fuels decreases, but does not disappear, as modifications are made to improve the HTGR conversion ratio. The improvements in conversion ratio and ore requirements listed in Table 4 were calculated on the assumption that the fuel would be irradiated for a constant time interval of four years, as in the present HTGR reference design. Therefore, as the thorium loading and fissile loading are increased to improve the conversion ratio, the burnup correspondingly decreases.

Although the HTGR reactor design is well founded and is readily adaptable to the modifications described herein, the technical complexities and lack of engineering-scale experience in the HTGR fuel cycle suggest caution in economic comparisons with other fuel cycles. Thorough and periodic engineering evaluation of the economics of the HTGR fuel cycle and of alternative thorium-based fuel cycles is important to the ERDA program.

Similar reactors are under development in Germany, where designs of the prismatic type as well as advanced pebble bed designs are being considered. Uncertainties in the large investment to commercialize this reactor and the lack of fuel reprocessing and refabrication operations even at pilot scale make it unlikely that this reactor will emerge during this century at a scale significant to the overall consumption and utilization of uranium ore.

\section{E. Fast Breeder Fuel Cycles}

\section{E1. The Normal Pu-U Fast Breeder}

The principles of fast breeder operation and a brief discussion of the LMFBR were provided in Chapter III. The discussion there centered on the key features of breeder operation, namely the production of ${ }^{239} \mathrm{Pu}$ from the fertile ${ }^{238} \mathrm{U}$ or ${ }^{233} \mathrm{U}$ from fertile ${ }^{232} \mathrm{Th}$

TABLE 4. Conversion ratio improvements possible for the HTGR fueled with ${ }^{235} \mathrm{U}$, Th, and recycled uranium.

\begin{tabular}{|c|c|c|c|}
\hline Modification ${ }^{a}$ & Conversion ratio & $\begin{array}{r}\text { Relative } 1 \text { ifetime } \\
\text { requirement } \\
\end{array}$ & or $e^{b}$ \\
\hline Reference HTGR & 0.66 & 1.0 & \\
\hline Increases thorium 10 ading by $25 \%$ & 0.71 & 0.89 & \\
\hline $\begin{array}{l}\text { Change from yearly fueling to } \\
\text { semiannual fueling }\end{array}$ & 0.76 & 0.80 & \\
\hline $\begin{array}{l}\text { Reduce core average power dens } \\
\text { from } 8.4 \text { to } 6.0 \text { watts } / \mathrm{cm}^{3}\end{array}$ & ity & 0.68 & \\
\hline $\begin{array}{l}\text { Use modified fuel elements } \\
\text { and/or improved fuel particles }\end{array}$ & $\begin{array}{l}0.90 \\
0.95\end{array}$ & 0.57 & \\
\hline
\end{tabular}

Rev. Mod. Phys., Vol. 50, No. 1, Part II, January 1978 
FIG. 10. Annual quantities for LMFBR fueled with natural or fuel cycle, Greebler, 1977). depleted uranium (equilibrium

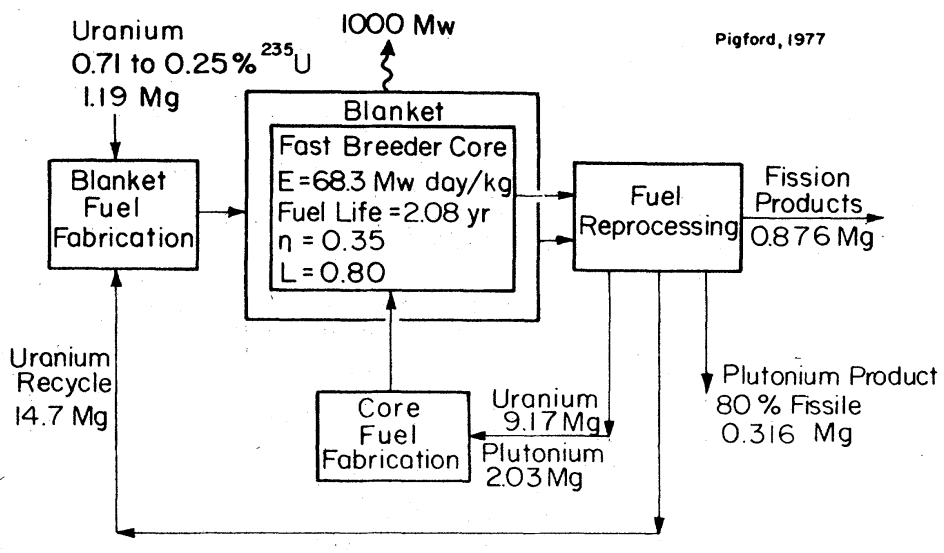

through the use of fast neutrons. Higher breeding ratios and shorter doubling times are possible with the uranium-plutonium cycle, and plutonium will be available from water-reactor fuel to start up the first generation breeders. Moreover, the Purex reprocessing technology is available for the uranium-plutonium cycle. Consequently, the LMFBR program is aimed towards the development of firstgeneration breeders fueled with uranium and plutonium. In the $\mathrm{Pu}-\mathrm{U}$ fast breeder the core consists of plutonium-uranium mixed oxide fuel pins. Surrounding the reactor core is a blanket of depleted uranium which absorbs neutrons leaking from the reactor core to produce additional plutonium. The blanket consists of two parts, both axial and radial portions. Cooling is accomplished by means of liquid sodium in the case of the LMFBR or helium gas in proposed gascooled designs. Figure 10 shows a flowsheet for a possible early LMFBR operating on an equilibrium fuel cycle (Greebler, 1977), fueled with natural or depleted uranium. The excess plutonium production from this breeder can be used to start-up subsequent breeders, provided that the doubling time for increasing breeder capacity is no shorter than the doubling time for excess fissile production by the breeder.

A large amount of depleted uranium from isotope separation will have been stockpiled by the time when present low-cost uranium resources are consumed by water reactors. Assuming that breeders replace the water reactors then being retired in the next century, and assuming that total fission power continues at a constant level, the stockpiled depleted uranium is an already-mined resource sufficient to fuel these breeders for hundreds of years. The fast breeder is the most efficient of all fission systems in terms of long-term ore utilization.

The only ore requirements attributable to the breeder is that associated with the production of plutonium for start-up loadings of the first-generation breeders. This plutonium must be obtained from light-water reactors, and these reactors will then require more ore because they are thereby deprived of the benefits of plutonium recycle. When operating without $P u$ recycle, the $1-G w$ L.WR (Figure 6 of Chapter IV) produces $171.4 \mathrm{~kg} / \mathrm{yr}$ of fissile Pu. LWR's must operate for $43.8 \mathrm{Gw}-\mathrm{yr}$ without $\mathrm{Pu}$ recycle in order to produce the $7500 \mathrm{~kg}$ of fissile Pu required to start-up a 1-Gw L.MFBR (Greebler, 1977; see also Table 5 of Chapter. VIII). The uranium ore attributable to $\mathrm{Pu}$ production is the difference between the ore required for operating LWR's with $U$ recycle only and that required with $U-P u$ recycle. Using the data of cases (b) and (c) of Table 1 and scaling to $43.8 \mathrm{Gw}$-yr., we estimate 1530 tons $\mathrm{U}_{3} \mathrm{O}_{8}$ for $0.25 \%$ depleted uranium tails, attributable to $\mathrm{Pu}$ start-up as shown in Table 5 .

If the doubling time for subsequent growth in breeder capacity is no greater than the doubling time for the breeder to produce excess plutonium, no ore is required for subsequent breeder generations. For each gigawatt of first-generation breeder capacity installed in the 1990 's, which was ERDA's projected era of breeder commercialization, $43.8 \mathrm{Gw}-\mathrm{yr}$ of light water reactors must be operated without plutonium recycle during the 1980's and early 1990's to furnish the start-up plutonium (Pigford, 1977b). Therefore, ERDA's schedule of breeder introduction would have required the existence of industrialscale LWR reprocessing before the 1990's. The present schedule for commercialization is uncertain.
The data in Table 5 indicate that over a 30 -year operating life, three uranium-fueled light water reactors could produce enough plutonium to start up two fast breeders, if no plutonium were to be recycled in water reactors. Alternatively, nine water reactors operating during their last ten years of life without plutonium recycle will generate enough plutonium to eventually start up two breeders. The 1974 ERDA projections of U.S. nuclear power growth indicated a growth to $124 \mathrm{GW}$ of fast breeder capacity by the end of the century, along with $644 \mathrm{GW}$ of light water reactors. Calculations (Pigford and Ang, 1975) of the amount of start-up plutonium required for such a large scale of breeder introduction showed that plutonium recycle in water reactors would have to be discontinued in the early 1990's to insure sufficient plutonium for breeder start-up. However, events since 1974 suggest that such a rapid introduction of breeders is not likely, and delays in LWR fuel reprocessing and in the construction of additional LWR fuel reprocessing facilities seem more likely to result in an over supply in the 1990's of plutonium which can be extracted from water reactor fuel.

From the above it is apparent that there are several situations, any one of which could warrant operating water reactors entirely with uranium fueling so that all of the plutonium produced would be available for breeder start up. Examples are:

TABLE 5. Fissile, ore, and enrichment requirements to start a first-generation fast breeder reactor with water-reactor plutonium (1000 Mw electrical power, $80 \%$ capacity factor).

Fissile Pu required for
$\quad$ fast breeder start-up a
Operation of U-Fueled
$\quad$ water reactor to generate
$\quad$ Pu start-up inventory
$\begin{aligned} & \text { U ore attributable to production of } \\ & \text { startup Pu } \\ & \quad 0.20 \% \text { depleted } U \\ & \quad 0.25 \% \text { dep leted } U\end{aligned}$

Additional separative work due

to loss of Pu-recycle in

water reactors:

$0.20 \%$ depleted uranium

$1200 \mathrm{Mg}$

$0.25 \%$ depleted uranium $\quad 1020 \mathrm{Mg}$ Example: To start up $1 \mathrm{GW}$ of FBR requires that $4.38 \mathrm{Gw}$ of $L W R$ be
operated for 10 yr. without Pu recycle. Total ore utilized $=8490$ short tons $\mathrm{U}_{3} \mathrm{O}_{8}(0.25 \%$ depleted $U)$. Total ore attributable to breeder start-up $=2980$
. The short tons:

${ }^{a}$ a ased upon $3000 \mathrm{~kg}$ fissile $P u$ for the initial core plus $4500 \mathrm{~kg}$ for replacement loadings before $\mathrm{Pu}$ in discharge fuel is recycled (Greebler, 1977). 
(a) a very limited supply of uranium ore

(b) a sufficiently large ratio of first-generation breeders to previously installed water reactors

(c) a desire to move as rapidly as possible into a breeder power systemr.

\section{E2. Fast Breeder Start-up with ${ }^{235} \mathrm{U}$}

Plutonium is the best of all the fissile isotopes in achieving high breeding ratios and low doubling times in fast breeders (Greebler, 1977; Pigford, 1977b; Baker and Ross, 1973; Yamashita, 1970; Yamashita, 1969). Although enriched (20 to $22 \%){ }^{235} \mathrm{U}$ from isotope separation could be used for breeder start-up, the relative penalties associated with ${ }^{235} \mathrm{U}$ start-up are large. The smaller number of neutrons per fission for ${ }^{235} \mathrm{U}$ results in larger fissile inventory and a lower breeding ratio than with plutonium fueling.

Calculations of the amount of uranium ore and separative work for ${ }^{235} \mathrm{U}$ start-up and of the cost penalties for ${ }^{235} \mathrm{U}$ start-up are presented in Appendix V. It is shown that for a commercial fast breeder optimized for an equilibrium plutonium-uranium fuel cycle, the amount of fissile uranium required for start-up is from 1.5 to 2.4 times as large as. the amount of fissile plutonium that would be required, depending upon the method of reprocessing the core fuel. ${ }^{235} \mathrm{U}$ start-up would consume greater quantities of uranium ore than that attributable to $\mathrm{Pu}$ start-up from LWR's, and also would require greater quantities of electrical energy for isotope separation. As shown in Appendix $\mathrm{V}$, the corresponding total cost of the fissile material for start-up would be greater by a factor of 2.3 to 3.7 for enriched uranium than for plutonium.

The breeding ratio is significantly lower during start-up cycles with ${ }^{235} \mathrm{U}$, and this effect persists for many subsequent reloads until most of the ${ }^{235} \mathrm{U}$ has been recycled and consumed. The new deficit in breeding-gain production of fissile plutonium due to ${ }^{235} \mathrm{U}$ start-up of a $1000 \mathrm{Mw}$ LMFBR is about $1700 \mathrm{~kg}$. This considerably increases the breeding-gain doubling time and will delay the start-up of secondgeneration breeders, assuming these are to be fueled initially with $\mathrm{Pu}$ from first generation breeders.

As has been shown in Chapter IV, for a given uranium enrichment capability and an existing base of uranium-fueled water reactors to produce plutonium, the greatest resource benefit before the breeder is introduced results from fissioning both uranium and plutonium in these water reactors. If there are commercial water reactors and firstgeneration fast breeders, it is best to fission uranium in the existing base of water reactors for the remainder of their useful life, and to fission plutonium in the breeders. The technical and fuel cycle cost considerations raised in this chapter indicate that start-up of breeders with ${ }^{235} \mathrm{U}$ is to be avoided once breeder commercialization has begun.

\section{E3. Fast Breeders with ${ }^{233} \mathrm{U}$ Cycles}

There is some interest in breeders fueled with thorium and recycled uranium. For example, if the breeder program were to be significantly delayed and if thorium fueling of thermal reactors were to be introduced, as discussed earlier, to conserve uranium resources, these thermal reactors would eventually become sources of ${ }^{233} \mathrm{U}$ instead of plutonium for breeder start up. Although ${ }^{233} \mathrm{U}$ is far better than ${ }^{235} \mathrm{U}$ for this purpose and results in reasonable breeding ratio, it is still inferior to plutonium.

When $\mathrm{ThO}_{2}$ is substituted for $\mathrm{UO}_{2}$ in the core fuel, case (b), the breeding ratio decreases. This is a result of the lower fast fission cross section of ${ }^{232} \mathrm{Th}$ and also from the partial replacement of ${ }^{239} \mathrm{Pu}$ by ${ }^{233} \mathrm{U}$ as the latter builds up and fissions during the irradiation cycle. (See Chapter III, section A for background information.) Substituting $\mathrm{ThO}_{2}$ for $\mathrm{UO}_{2}$ in the blanket only slightly decreases the breeding ratio because of the relatively few fissions in the blanket. A core fueled with ${ }^{233} \mathrm{UO}_{2}-\mathrm{ThO}_{2}$ results in an even lower breeding ratio. Operation is also possible with a core of ${ }^{233} \mathrm{UO}_{2}$ diluted to $20 \%$ or less with ${ }^{238} \mathrm{UO}_{2}$, a denatured fast breeder, as discussed in Section F2c).

Since the irradiation behavior of $\mathrm{UO}_{2}-\mathrm{ThO}_{2}$ fuel appears to be similar to that of $\mathrm{PuO}_{2}-\mathrm{UO}_{2}$ fuel, it is likely that LMFBR's designed and introduced with $\mathrm{PuO}_{2}-\mathrm{UO}_{2}$ fueling could be converted later to $\mathrm{UO}_{2}-\mathrm{ThO}_{2}$ fueling. A longer doubling time would result, but the extent to which this would be a problem would depend upon the desired rate of breeder introduction.

Uranium-thorium fueling in breeder cores may have some safety advantage because of its smaller increase in reactivity from sodium voiding than with plutonium-uranium fueling. However, there are other means of reducing the reactivity effects of sodium voiding, if this proves to be necessary in the LMFBR development program. Introducing thorium fueling in breeders would introduce many of the problems that would be encountered with thorium fueling in thermal reactors. The build-up of ${ }^{232} U$ in the irradiated fuel and the highenergy gammas of the ${ }^{232} \mathrm{U}$-decay daughters would require more shielding and remote handling in fabricating recycled fuel, and it complicates fuel reprocessing. The ${ }^{232} \mathrm{U}$ build-up in a thorium-fueled fast reactor is likely to be considerably greater than in thorium-fueled thermal reactors. Also, the reprocessing would have to be based upon Thorex technology, which is not as well developed as Purex reprocessing and is expected to be more difficult and expensive. The control of shut-down reactivity is more difficult with ${ }^{2.33} \mathrm{U}$ fuel because of the relatively long (27.4 day) half life of ${ }^{233} \mathrm{~Pa}$, the ${ }^{233} \mathrm{U}$ precursor. The long half life results in increased precursor concentrations during operation. Significant reactivity is added by ${ }^{23}{ }^{3} \mathrm{~Pa}$ decay after reactor shutdown, and more control absorbers are required with uranium-thorium fueling. Also, the delayed neutron fraction for ${ }^{233} \mathrm{U}$ is lower than that for ${ }^{239} \mathrm{Pu}$, so lower worth for individual control absorbers and slower withdrawal rates to avoid prompt criticality may be required. These operational problems can all be accommodated through proper design, but they can affect the economics of uranium-thorium fueling.

More advanced sodium-cooled breeders designed for higher breeding ratios and higher specific powers may be based upon fuel materials in the form of carbides, nitrides or metals. These advanced fuel materials offer better theoretical thermal and neutronic performance (see Table 6), but less is known about their irradiation behavior than is known about oxide fuels. Also, ${ }^{14} \mathrm{C}$ formation in nitride fuels may result in greater expense in environmental controls and in waste management. Although uranium-metal fuels have been considered unacceptable for the high burnups required for breeder cores, experience of the EBR-II experimental breeder now indicates that alloyed uranium metal may be suitable. Fuels of thorium-base alloy may be an even more attractive possibility. The isotropic facecentered-cubic structure of thorium metal is more stable than uranium to irradiation damage and swelling. Thorium undergoes its solidphase transformation at $1365^{\circ} \mathrm{C}$, which is much higher than the $660^{\circ} \mathrm{C}$ transformation temperature of uranium metal. Also, thorium melts at $1725^{\circ} \mathrm{C}$, as compared with $1132^{\circ} \mathrm{C}$ for uranium. However, because of the limited solubility of uranium and plutonium in thorium, the irradiation behavior of $\mathrm{U}-\mathrm{Th}$ and $\mathrm{U}-\mathrm{Pu}-\mathrm{Th}$ alloys for core fuel may not be as good as that expected for thorium metal. The irradiation behavior of such alloys at operating temperatures and design burnups is not sufficiently known.

The higher thermal conductivity of thorium-based alloys could result in higher specific power than with oxide fuel. Also, the higher atomic density of the metal should result in a breeding ratio higher than that attainable with oxides, as shown by cases (d) and (e) in Table 6. The higher specific power and breeding ratio both result in a lower doubling time for the thorium-alloy fuel. Also, with metal fuel the reactivity effects from sodium voiding are further reduced below those predicted for the oxides. These possible advantages from thorium-alloy fuel in breeders, as compared to thorium oxide fuel, must be weighed against the greater uncertainties in irradiation behavior and possibly more expensive fuel fabrication. Also, thorium alloy fuels will be subject to the same problems of thorium technology described above. Therefore, the present state of knowledge on thorium fueling in fast breeders does not suggest diversion from the $\mathrm{PuO}_{2}-\mathrm{UO}_{2}$ fuel now under development. Advanced carbide and thorium-alloy fuels do offer promise for longer-range improvements in advanced breeder designs.

Thorium-alloy fuels for breeder cores are not adaptable to the concept of a breeder fueled with a denatured uranium for safeguards fuel cycles. If the recycled ${ }^{233} \mathrm{U}$ were diluted by natural uranium to a 
TABLE 6. Comparison of Pu-U and U-Th fueling in LMFBR's ${ }^{a}$ (1000 Mwe, 0.8 load factor).

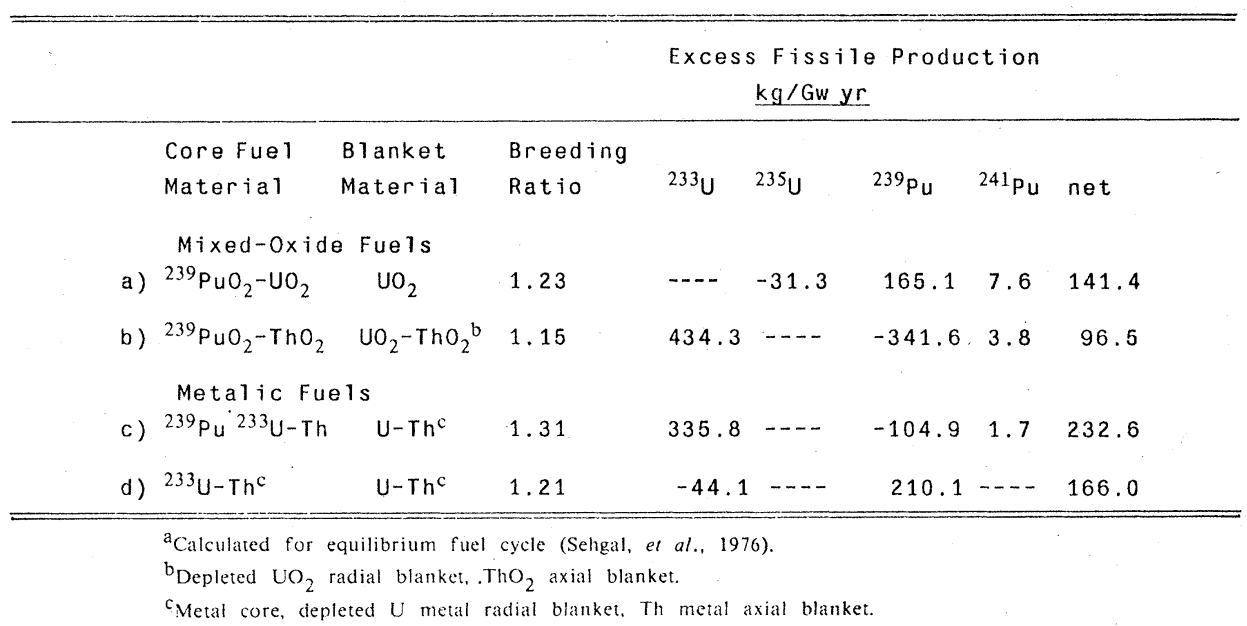

fissile content at $20 \%$ or less, as is proposed for the denatured uranium fuel cycles, core reactivity limitations do not allow further dilution with thorium.

Helium-cooled fast breeders have also been studied and are still receiving research and development support. Higher breeding ratios are theoretically possible. However, less is known about the structural stability of the fuel and the irradiation behavior of fuel cladding. Also, approaches to emergency cooling which differ from those designed for the LMFBR are necessary.

\section{F. Technical Safeguards Features of Advanced Fuel Cycles}

\section{F1. Safeguards in Normal Thorium Fueling}

Normal thorium fueling consists of thorium mixed with fissile make-up and recycled uranium. For the equilibrium fuel cycle of a uranium-thorium fueled light water reactor the recycled uranium typically contains about $55 \%{ }^{233} \mathrm{U}$ and $10 \%{ }^{235} \mathrm{U}$, which is a fissile content sufficient for nuclear explosives. However, the recycled uranium will contain several hundred parts per million of ${ }^{232} \mathrm{U}$. As illustrated in Figure 11, the gamma activity and external gamma dose rate due to ${ }^{232} \mathrm{U}$ daugiiters grows rapidly after fuel reprocessing. After 100 days a metallic uranium part as small as one kilogram and containing $100 \mathrm{ppm}{ }^{232} \mathrm{U}$ will produce a gamma dose rate as large as $0.1 \mathrm{rem} / \mathrm{hr}$ at one meter. Recycled uranium in a uranium cycle may contain about 200 to $1000 \mathrm{ppm}$ of ${ }^{232} \mathrm{U}$, depending in part upon the content of ${ }^{230} \mathrm{Th}$ in the make-up thorium and also upon the energy spectrum of the neutron flux. Consequently, the surface dose rate will be several times greater then shown in figure 11. Therefore, recycled uranium from thorium irradiation will require more shielding than reactor grade plutonium. This could affect the practical utility of ${ }^{233} \mathrm{U}$-rich recycled uranium for explosives.

The fissile make-up for thorium fueling consists either of highly enriched $(93 \%){ }^{235} \mathrm{U}$ or plutonium recovered from discharge fuel from uranium-fueled water reactors. Highly enriched ${ }^{235} \mathrm{U}$ is the least radioactive of all the separated fissile materials. It can be handled with relatively little hazard from its radioactivity. Although its fastassembly critical mass is greater than that of ${ }^{239} \mathrm{Pu},{ }^{235} \mathrm{U}$ has a relatively low neutron background from spontaneous fission and from $(\alpha, n)$ reactions. It can be assembled into simple gun-type devices. Uranium metal is less reactive chemically than plutonium metal. Therefore, the use of highly enriched ${ }^{235} \mathrm{U}$ introduces what may be the most significant of all the safeguards concerns in the various nuclear fuel cycles.

In reprocessing thorium fuels appreciable quantities of undecayed ${ }^{23 .} \mathrm{Pa}$ may be present. Chemical separation soon after discharge can yield pure protactinuum, which then decays to pure ${ }^{233} \mathrm{U}$, which would be an attractive material for explosives.

\section{F2. Fuel Cycles for Imternational Safeguards Control}

Various special fuel cycles have been suggested as technical means of making fissile material less accessible for diversion to nuclear weapons. These safeguards fuel cycles are associated with institutional and geographical arrangements such that those fuel cycle operations involving greatest accessibility to fissile material would be carried out in locations under special international control. They are intended to provide a means of locating power reactors in countries which would agree to surrender their discharge fuel to an international center, in exchanges for monetary value or for fresh fuel which contains fissionable material not easily separated into material suitable for weapons. However, it will be evident from the following discussion that some international control and inspection will still be necessary to avoid misuse of these national reactors. We have performed only a preliminary examination of the technical features of these safeguards

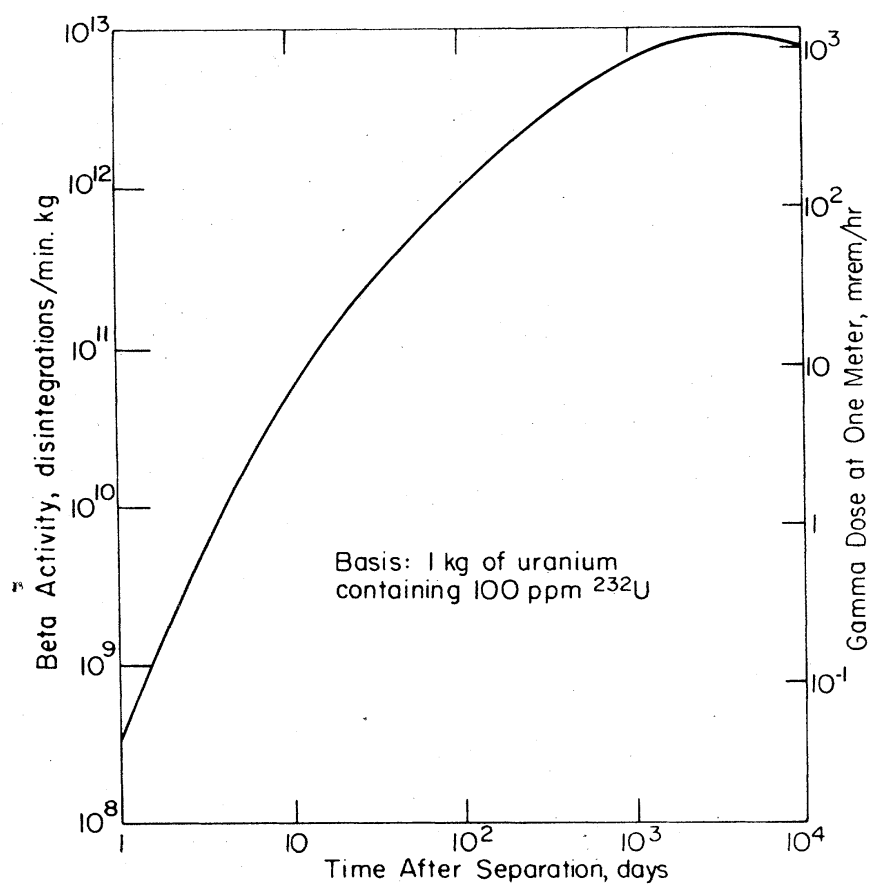

FIG. 11. Growth of beta activity and gamma dose rate due to ${ }^{232} \mathrm{U}$ in uranium from irradiated thorium. 
fuel cycles. It is important to evaluate financial burdens of these fuel cycles.

\section{F2a. Low-Enrichment (Denatured) Uranium Safeguards Cycles}

The non-reprocessing fuel cycle for a uranium-fueled light water reactor, as shown in Figure 5 of Chapter IV, could be adapted to an international safeguard fuel cycle. The discharge fuel, which contains significant quantities of fissionable plutonium, could be stored under international inspection or control (i.e., an international stowaway cycle). This cycle will ultimately entail higher costs, since it is the greatest consumer of natural uranium and requires a relatively large supply of slightly enriched uranium. The alternative of reprocessing the discharge fuel and storing the recovered plutonium under international inspection or control may impose additional safeguards financial burdens. The stored plutonium must be protected, and the cost of storing separated plutonium is high compared with the cost of storing discharge fuel. Nevertheless, the stowaway cycle represents the simplest of the alternatives discussed below and can be consistent with their later implementation. If such international safeguards fuel cycles are to be utilized, the stowaway version represents a possible first step that could be implemented with existing technology.

Another alternative is to fuel all such national reactors, to be under individual safeguard control, with slightly enriched uranium and to ship the discharge fuel to a centralized fuel reprocessing center under international control. The recovered plutonium would be consumed on-site in plutonium-burner reactors operating as in Figure 4 of Chapter IV-F. The electrical distribution system receiving the energy generated by plutonium-burner reactors would, require relatively little uranium ore. The uranium ore thus saved could then be used as feed to a centralized uranium-enrichment plant to supply the slightly enriched uranium fuel for the externally located uraniumfueled reactors. The total uranium ore consumption for the entire generating system would be the same as if all reactors were nationalized and operating with self-generated uranium-plutonium recycle. An overall flowsheet (Pigford and Yang, 1977) of this safeguards fuel cycle is shown in Figure 12. Since the fuel discharged from the uranium-fueled reactors would still contain plutonium, the storage and shipment of the discharge fuel would have to be under safeguard control. Again, this cycle represents a step based on an existing technology and could be implemented in the near future.

\section{F2b. Denatured-Uranium Thorium Safeguards Cycles}

An alternative to the uranium cycles is the thorium-uranium cycle, in which ${ }^{233} \mathrm{U}$ is formed by neutron absorption in ${ }^{232} \mathrm{Th}$. The ${ }^{233} \mathrm{U}$ can be isotopically denatured by dilution with natural uranium or enrichment tails to about $15-20 \%$ fissile content. At such concentrations critical mass for explosives becomes very large. Thorium is then added as fertile material so that the overall fissile concentration in the fuel is a few percent, typical of LWR fuel. This fresh fuel of denatured uranium and thorium is similar to low enrichment (i.e., "denatured") uranium fuel in that isotopic enrichment would be necessary in either case to produce uranium suitable for an explosive assembly. It differs in that much of the ${ }^{238} \mathrm{U}$ has been replaced by thorium, so that the production of chemically separable plutonium has been suppressed. However, appreciable quantities of plutonium are still present in the spent fuel, and the same set of issues as to its.disposition still arise.

To obtain such a safeguarded international fuel cycle, the uranium-thorium flowsheet of Figure 1 would be modified by diluting all uranium streams with natural uranium, so that the fissile isotopic content of uranium supplied to the reactor is no greater than about $20 \%$ as shown in the flowsheet (Pigford and Yang, 1977) of Figure 13. The increased ${ }^{238} \mathrm{U}$ concentration results in considerably more plutonium production than in the normal uranium-thorium cycles described earlier, so the discharge fuel will still require safeguards control. However, the amount of plutonium produced in this denatured uranium-thorium cycle is less than that in the lowenrichment uranium cycle by a factor of about seven. More practical designs, wherein no compensation is made for the decreased selfshielding of the ${ }^{238} \mathrm{U}$ resonances due to the lower concentration of ${ }^{238} \mathrm{U}$ in the fuel, may decrease this factor from seven to about three. Also, the assumption here of $20 \%$ fissile enrichment for denaturing is based upon criticality data for ${ }^{235} \mathrm{U}-{ }^{238} \mathrm{U}$ mixtures. An even lower fissile enrichment may be required for ${ }^{233} \mathrm{U}$ to give the same degree of denaturing as for ${ }^{235} \mathrm{U}-{ }^{238} \mathrm{U}$. Till and Chang (Till and Chang, 1977) have assumed that $12 \%{ }^{233} \mathrm{U}$ in ${ }^{233} \mathrm{U}-238 \mathrm{U}$ is the equivalent limit. This will further increase the plutonium production in national reactors, over and above that indicated in Figure 13 . The externally located reactors fueled with denatured uranium and thorium reactors could also be "spectral shift LWR's", gas-cooled, or heavy-water reactors.

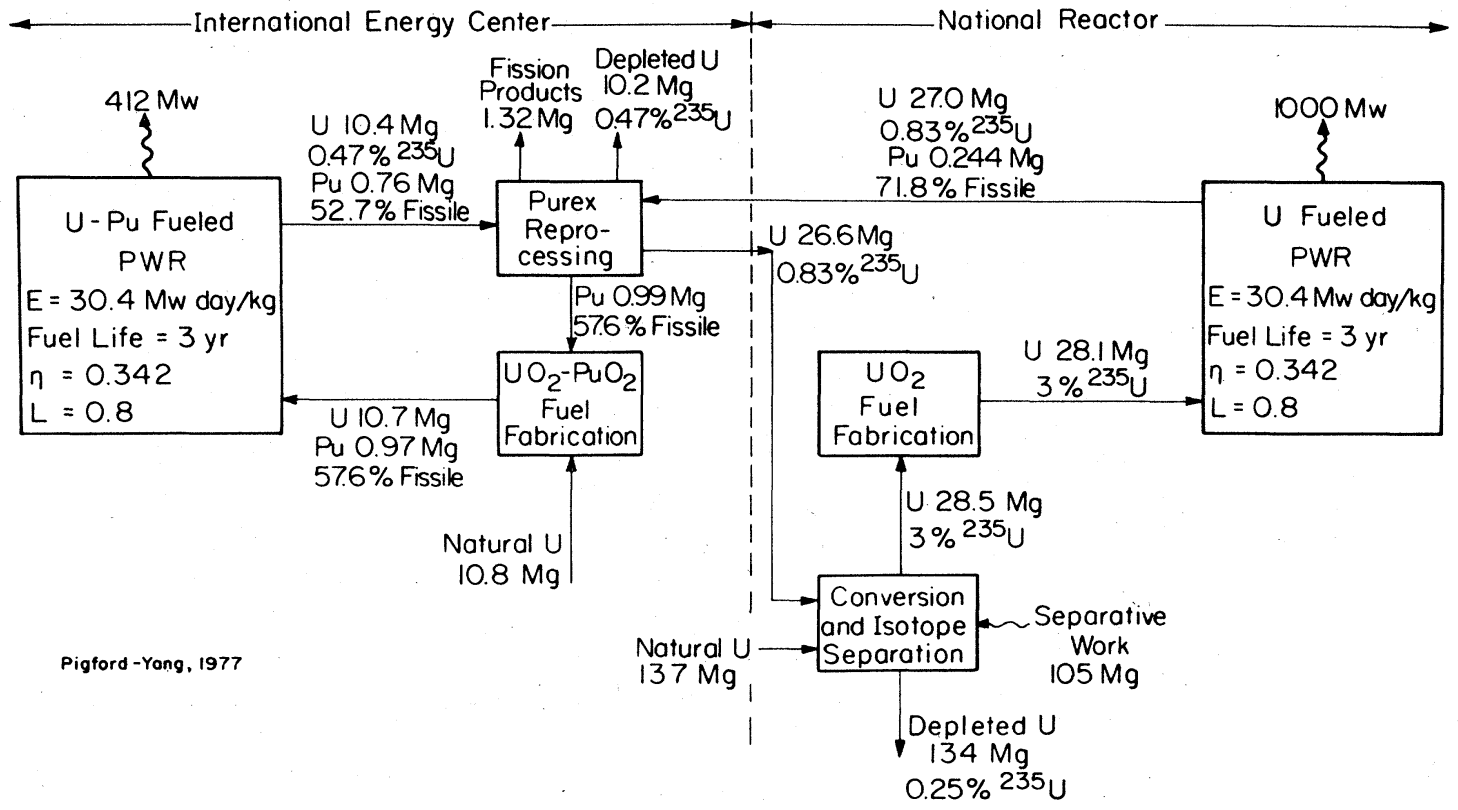

FIG. 12. Annual quantities for LWR cycle for international safeguards, national reactors fueled with low enrichment (denatured) uranium. 


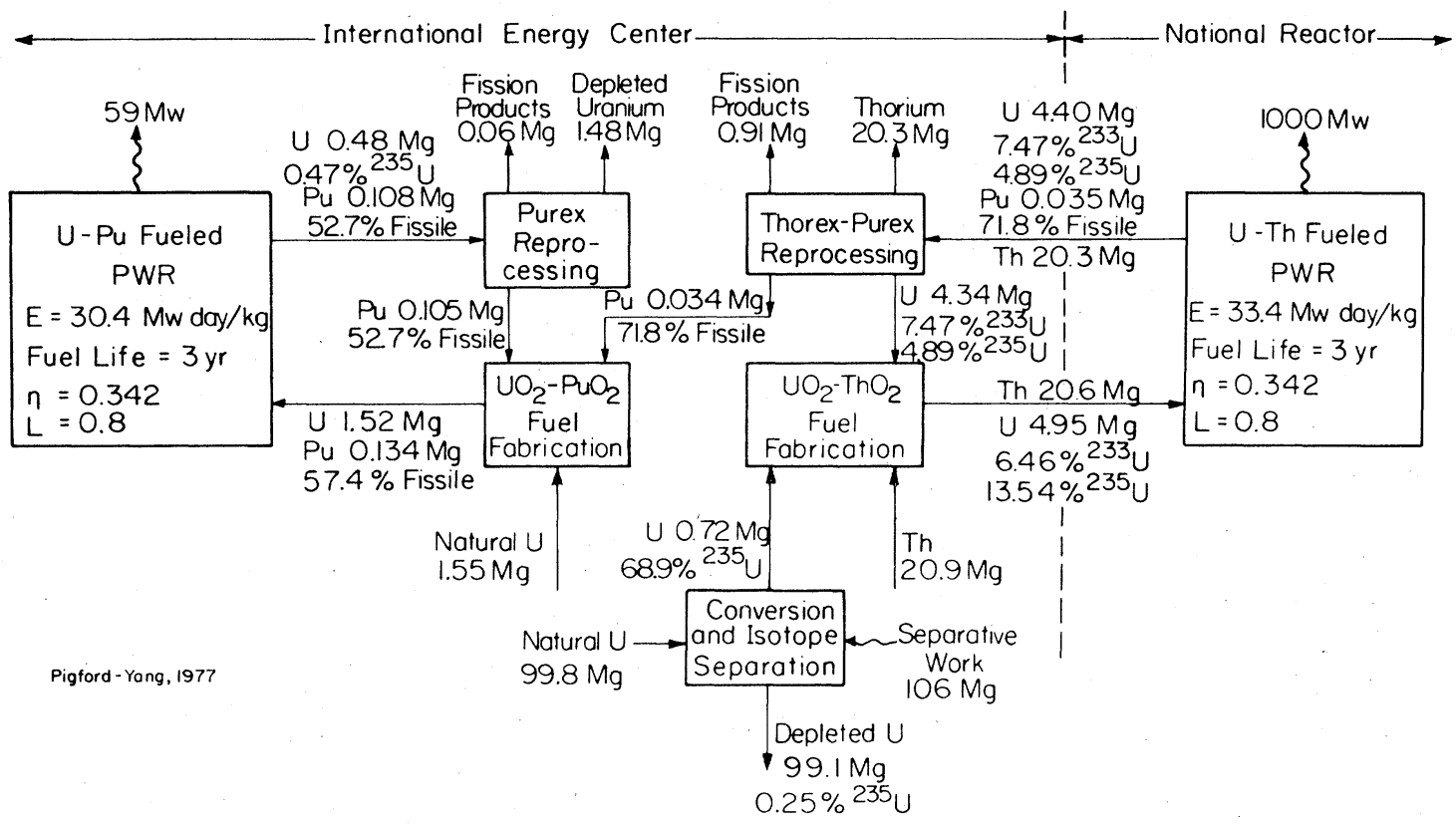

FIG. 13. Annual quantities for LWR cycle for international safeguards, national reactors fueled with thorium and denatured uranium

The discharge fuel could be shipped to an internationally controlled centralized reprocessing center. Although the plutonium could be allowed to follow the fission products to the high level wastes, the reprocessing chemistry is such that this would not materially simplify the separation operations. If the plutonium were to follow the high-level wastes, the fissile content of those wastes could be as high as about two weight percent, which is greater than that in discharge fuel from uranium-fueled water reactors. Thus, the high-level wastes would have to be safeguarded. Alternatively, the plutonium could be recovered and consumed in on-site plutoniumburner reactors, as shown in the overall flowsheet (Pigford and Yang, 1977) of Figure 13. Safeguards issues remain whether plutonium is allowed to follow the wastes or is consumed in a reactor at the international center.

Using denatured uranium with a fissile content in the range of 10 to $20 \%$ creates a new safeguards issue in that relatively little work of isotope separation would be required to isotopically enrich this uranium to the level of highly enriched material. This can be illustrated in terms of the ${ }^{235} \mathrm{U}$ equivalent. Highly enriched uranium is usually regarded to be $93 \%{ }^{235} \mathrm{U}$, which is made by isotopically enriching natural uranium. Of the total work required to enrich natural uranium to $93 \%{ }^{235} \mathrm{U}$, about $90 \%$ of the work is expended in enriching to $20 \%{ }^{235} \mathrm{U}$. Only $10 \%$ more work is required to further enrich to $93 \%{ }^{235} \mathrm{U}$. This illustrates the relative ease of making highly enriched uranium from uranium containing $20 \%$ fissile concentration. Because of the lower atomic mass of ${ }^{233} \mathrm{U}$, the relative work required to enrich ${ }^{233} \mathrm{U}-{ }^{238} \mathrm{U}$ denatured uranium to the high-enrichment level would be even less than estimated here. Technology for this is discussed in Chapter VI. Although recycled uranium containing ${ }^{233} \mathrm{U}$ and ${ }^{232} \mathrm{U}$ could not be enriched in commercial isotope separation plants because of the radioactivity, it is conceivable that a relatively small and not necessarily efficient isotope separation system could be devised to enrich this $20 \%$ fissile uranium. The technology to carry out such enrichment on non-economical, non-commercial scale is available in the open literature. This is another aspect of the denatured uranium-thorium cycle that requires further evaluation.

As compared to the low-enrichment uranium safeguards cycle described in Section F2a above, the denatured uranium-thorium cycle has the advantage that a single international reprocessing center could service a large number of national reactors, with only a relatively small total power of plutonium-burner reactors at the international center. However the required uranium enrichment capacity would be greater than in the case of the low-enrichment uranium cycle of Figure 12. The enriched product, containing about $69 \%{ }^{235} \mathrm{U}$ in uranium, would have to be safeguarded until it is diluted with the recycled uranium in the fuel fabrication facility. Also, the denatured U-Th cycle requires far more complicated reprocessing and fuel refabrication operations. The technology base for this would require further development and engineering scale-up before industrial-scale operations could begin.

\section{F2c. Fast-Breeder Safeguards Cycles}

Fast breeder reactors under international control could also be used as the source for the fissile uranium make-up for national denatured uranium-thorium reactors. Portions of the breeder blanket, such as part of the radial blanket, could contain thorium instead of depleted uranium. The thorium blanket would be reprocessed along with recycled uranium-thorium fuel from the national reactors. The recovered uranium would be diluted with natural or depleted uranium prior to off-site shipment as denatured uranium. It is likely that this concept would be technically possible by modifying the blanket loadings for even the first generation LMFBR's, which are expected to be started on plutonium.

A flowsheet (Pigford and Yang, 1977) of a safeguards fuel cycle involving international breeder reactors is shown in Figure 14. This has been calculated from the characteristics of commercial-scale LMFBR's designed for possible introduction in this century (Pigford and Ang, 1975). It has been assumed that all of the breeding-gain fissile production of the breeder is drawn off as ${ }^{233} \mathrm{U}$ to be used as fissile make-up for the denatured U-Th national reactors. As a result, no fissile breeding gain is available from this breeder to start up additional breeders, i.e., the effective doubling time for breeder fissile inventory becomes infinite. In principle additional breeder capacity could be introduced as needed, even when existing breeders operate at zero breeding gain, by starting the new breeders with isotopically enriched uranium. However, we have shown in Section $E$ that ${ }^{235} U$ start-up is not a good economical alternative for commercialized breeders. Therefore, operating an international breeder as shown in Figure 14 would be possible only after many decades when, even with an assumed zero growth of total fission electric power, the assumed breeders have finally been introduced to a level sufficient to replace the water reactors then being retired.

During the first few decades of breeder introduction the excess 


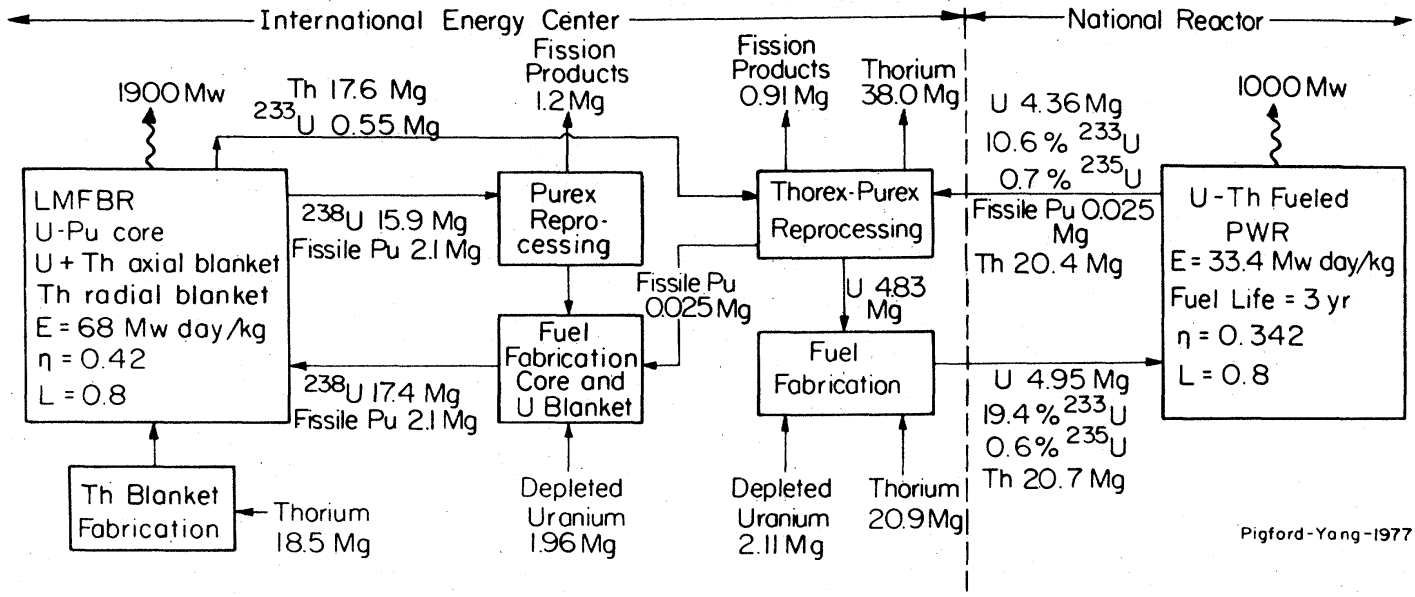

FIG. 14. Annual quantities for national PWR fueled with thorium and denatured uranium, international LMFBR produces make-up ${ }^{233} \mathrm{U}$.

fissile production from these breeders likely to be needed to start up new breeders, so less fissile production is available as make-up for ${ }^{233} \mathrm{U}$ for national reactors. Consequently, the relative power of the international breeder shown in Figure 14 is the minimum breeder power, relative to the power of the national reactors, to supply the fissile make-up for the national reactors. To maintain a finite breeder doubling time an even larger relative power of the breeder would be required. Howeyer, the necessary breeder power can be reduced somewhat if the more favorable breeding gains calculated for future advanced breeders are assumed.

The fuel cycle flowsheet of Figure 14 has been calculated on the assumption that thorium can be used in both radial and axial blankets. The radial blanket alone will not produce sufficient ${ }^{23.3} \mathrm{U}$ at this power level. However, thorium in the axial blanket requires that the axialblanket thorium pellets be segregated from the core pellets prior to reprocessing. Otherwise normal reprocessing of the entire fuel rod would dilute the ${ }^{233} \mathrm{U}$ with core uranium to the extent that it would be unsuitable for use in the denatured-uranium cycle. However, if the breeder power were increased to $3500 \mathrm{Mw}$, sufficient ${ }^{2.3 .3} \mathrm{U}$ would be produced in the separate radial blanket, and normal head-end reprocessing techniques for core fuel could be used.

Safeguards fuel cycles involving denatured thermal national reactors and international breeders can provide excellent long-term ore utilization, but they require the greatest total power and the greatest reprocessing-refabrication capacity at the international facility. Also, such cycles have all the complexity of reprocessing and refabrication facilities necessary for both uranium-plutonium fueling and uranium-thorium fueling. They appear to be the least realistic in terms of time schedule and availability of any of the cycles considered here. It is also technically possible for the national reactor to be a breeder with a denatured ${ }^{233} \mathrm{U}$ uranium core, with the fuel discharged from core and blanket sent to the international center for reprocessing. As illustrated in the flowsheet of Figure 14, the breeder at the international center would consume the plutonium produced in the national breeder, and ${ }^{233} \mathrm{U}$ produced in the international breeder would be denatured by ${ }^{238} \mathrm{U}$ dilution and exchanged for the plutonum produced in the national breeder. An example of such an international breeder is provided in case b) of Table 6. Although the breeding gain possible with ${ }^{233} \mathrm{U}$ fueling in the breeder core is less than for plutonium in the core, the international-national breeder system has more advantageous performance than a system with thermal reactors at national sites, and thus it remains a possibility for the very long-term.

In principle, the breeder could be operated entirely on the ${ }^{233} \mathrm{U}$ thorium cycle, eliminating the need for dual reprocessing facilities. However, the fast breeders being developed in the U.S. and Europe are planned to be introduced on the plutonium cycle, since plutonium is the most desirable start-up material and since plutonium is and will continue to be available from water-reactor uranium fuel. There is presently no thorium fueling in water reactors, thorium reprocessing technology is a very long way from being commercialized, and there are no plans for its commercialization. Therefore, the idea of introducing commercial breeders with anything other than plutonium seems unrealistic for the first generation. These breeders could, however, eventually be converted to full uranium-thorium fueling if there were some clear incentive to do so.

\section{F2d.' The Tandem Cycle}

A tandem cycle has been proposed as a means of obtaining additional energy from discharge fuel from light water reactors, thereby reducing the uranium ore requirements and reducing the amount of fuel reprocessing. It could have some safeguard advantage in that the additional energy would be obtained without reprocessing and without plutonium appearing in a separated form. The concept involves reconstituting discharged fuel from light water reactors into fresh fuel for heavy water reactors. Even though all fission products remain, the reactivity of the reconstituted fuel is roughly equivalent to that of natural uranium supplied to CANDU reactors. If additional burnup equivalent to that of natural uranium in the. CANDU is obtained, the uranium ore requirement decreases by about $20 \%$, as compared to the non-reprocessing cycle in water reactors.

However, considerable experience form earlier AEC programs, wherein attempts were made to fabricate recycle fuel from discharge fuel containing fission products, indicates that this concept will be so expensive as to be impractical. Fuel fabrication must be precise and reliable, and this could not be achieved at anything approaching an economical cost in the presence of the fission products. Such highlevel fabrications would likely be far more expensive than conventional fuel reprocessing. Also, the tandem cycle would result in increased quantities of plutonium in the final discharge fuel, which would have to be safeguarded.

The non-reprocessing incentives of this cycle can already be achieved with present LWR fueling. Greater ore saving can be obtained with many of the realistic near-term alternatives discussed in Chapter IV and earlier in this chapter. Given suitable incentive to do so, present LWR's can be redesigned to operate at greater burnup and greater ore saving without reprocessing, and this is also true of heavywater and gas-cooled reactors.

\section{F2e. Comparison of Safeguards Cycles}

It is not at all clear that any of these safeguards fuel cycles, by itself, solves the nuclear proliferation issue. We do not find a marked safeguards advantage of one over the other, except in terms of nearterm versus long-term availability. In all cases fissile content in the charged and/or discharged fuel still present safeguards issues. In all cases, political agreement supplemented possibly with inspection, must 
be relied upon to insure that the reactors are used in the way specified by these fuel cycles. Unfortunately, restricting commercial-scale reprocessing plants to international centers is no assurance that smallscale non-commercial reprocessing cannot and will not be carried on elsewhere; other non-proliferation agreements would be needed to limit this. Also, any of these safeguards fuel cycles may impose additional financial burdens upon the participating countries, because each of these cycles involves operations not necessary with unrestricted fuel cycles.

However, the denatured fuel cycles do have useful characteristics in that the fresh fuel requires isotopic enrichment capability to produce weapons-grade materiai, and the spent fuel is to some extent self protecting. If some form of safeguards fuel cycle were to be required, the stowaway fuel cycle is the simplest of all the alternatives considered here. Among those safeguards fuel cycles which seek to obtain greater utilization of uranium ore through reprocessing and recycle, the low-enrichment uranium cycle of Figure 13 is the simplest, requires the least amount of new technological development, and is the most likely to be available on a time scale useful to these issues. It also may be the most economical, in that it requires only uranium-plutonium reprocessing technology.

It may be possible to turn to advantage the fact that all the safeguards fuel cycles considered in our study require basically the same type of institutional and political safeguards agreements. In all cases the national reactors receive qualitatively similar denatured fresh fuel, and all discharge fuel contains unused energy resources including enough plutonium to require its safeguarding. What changes from one of the cycles to another are the detailed facilities at the international sites. Therefore, if appropriate institutional and political agreements can be negotiated to make possible even the simplest of the cycles, i.e., the international stowaway fuel cycle, then substantially the same agreements and arrangements can remain in effect as more and more resource-efficient fuel cycles are introduced in the course of time. Thus it is important to fully analyze such safeguards fuel cycles for their economic, social and political consequences as well as their technical viability so that the practical advantages and limitations of such an evolutionary strategy can be more widely appreciated.

\section{References}

1. Babcock and Wilcox, 1962

Babcock and Wilcox Co., "Spectral Shift Control Reactor". (1962)

2. Baker and Ross, 1973

Baker, A. R., and R. W. Ross, "Comparison of the Value of Plutonium and Uranium Isotopes in Fast Reactors", Proc. Conf. on Breeding, Economics, and Safety in Large Fast Power Reactors, Argonne, ANL-6792. (Oct. 1973)

3. Banerjee, et al., 1977

Banerjee, S., S. R. Hatcher, A. D. Lane, H. Tamm, J. I. Veeder, "Some Aspects of the Thorium Fuel Cycle in Heavy-Water Moderated Pressure Tube Reactors", Nucl. Tech. 34, 58-68. (June, 1977)

4. Benedict and Pigford, (in progress)

Benedict, M., and T.H. Pigford, "Nuclear Chemical Engineering", (2nd, Edition, McGraw Hill). (in progress)

5. Brogli, et al., 1975

Brogli, R. H., R. C. Dahlberg, C. H. George. Trans. ANS, 17, 298. (1975)

6. Brogli, et al., 1975

Brogli, R. H., M. L. Hays, R. K. Lane, and R. F. Turner, "The High Conversion HTGR For Resource Conservation", GAA13606. (October, 1975)

7. Critoph, et al., 1976

Critoph, E., S. Banerjee, F. W. Barclay, D. Hamel, S. M. Milgram, J. I. Veeder, "Prospects for Self-Sufficient
Equilibrium Thorium Cycles in Candu Reactors", AECL-5501. (1976)

8. Foster and Critoph, 1976

Foster, J. S., and E. Critoph, "The Status of the Canadian Nuclear Power Program and Possible Future Strategies", Wingspread Conference on Advanced Nuclear Coverters and Near Breeders. (May, 1976)

9. Greebler, 1977

Greebler, P., (General Electric Co.), Private Communication, (March, 1977)

10. Hatcher, et al., 1975

Hatcher, S. R., S. Banerjee, A. D. Lane, H. Tamm, J. I. Veeder, Trans. ANS, 22, 334. (1975)

11. Kasten, et al., 1977

Kasten, P. R., et al., "Assessment of the Thorium Fuel Cycle in Power Reactors", ORNL/TM-5565. (January, 1977)

12. Meneley, 1976

Meneley, D. A., "Candu Systems", ASEM-ANS International Conference on Advanced Nuclear Energy Systems. (March, 1976)

13. Pigford and Ang, 1975

Pigford, T. H., and K. P. Ang, "The Plutonium Fuel Cycles", Health Physics, 29, 451. (1975)

14. Pigford, $1977 \mathrm{a}$

Pigford, T. H., "Fuel Cycle Alternatives for Nuclear Power Reactors", IEC Fund., 16, 61. (Feb., 1977)

15. Pigford, $1977 \mathrm{~b}$

Pigford, T. H., "Start-up of First Generation Fast Breeders with Plutonium or Enriched Uranium", UCB-NE 3240. (March, 1977)

16. Pigford and Yang, 1977

Pigford, T. H., and C.S. Yang, "Thorium Fuel Cycles", UCBNE 3227, EPA 68-01-1962. (June, 1977)

17. Sehgal, et al., 1976

Sehgal, B. R., J. A. Naser, C. Lin, W. B. Loewenstein, "Thorium-Based Fuels in Fast Breeder Reactors", Nucl. Tech. 35, 635. (Oct., 1977)

18. Shapiro, et al., 1977

Shapiro, N. L., J. R. Rec, R. A. Matzie, "Assessment of Thorium Fuel Cycles in Pressurized Water Reactors", EPRI NP-359. (Feb., 1977)

19. Slater, 1975

Slater, J. B., "CANDU-BLW Reactor Design for Plutonium Recycle", Health Physics, 29, 451. (1975)

20. Till, et al., 1977

Till, C. E., et al., "A Brief Survey of Considerations Involved in Introducing CANDU Reactors into the U.S.", ANL-RSSTM-1. (Feb., 1977)

21. Till and Chang, 1977

Till, C. E., and Y. I. Chang, "CANDU Physics and Fuel Cycle Analysis", ANL-RSS-TM-2. (May, 1977)

22. Yamashita, 1969

Yamashita, S., "Variations in Neutronic Characteristics Accompanying Burnup in a Large Fast Breeder", Jour. $\mathrm{Nucl}$. Sci. Tech., Japan, 6, 573. (Oct., 1969)

23. Yamashita, 1970

Yamashita, S., "Variations in Neutronic Characteristics Accompanying Burnup in a Large Fast Converter", Jour. Nucl. Sci. Tech., Japan, 7, 341. (July, 1970) 


\section{CHAPTER IX. Institutional and Organizational Issues of the Nuclear Fuel Cycle and Waste Management Program}

\section{A. Issues and Conclusions}

Superficially, an analysis of the technical aspects of nuclear fuel cycle planning may not appear to involve the associated institutional and organizational relationships. However, public concern in nuclear matters has been so acute for so many years that procedural and organizational constraints have become very major factors in technology design and development. The viability of alternative strategies in fuel cycle and waste management technology cannot be assessed without considering these organizational matters. In the primer (Chapter III) we have catalogued the basic formal responsibilities for governmental and non-governmental organizations involved in the fuel cycle. In this Chapter we will examine the interfaces between organizations in areas of overlapping or unresolved jurisdiction, as well as problems, benefits, and implications of the existing set of relationships.

The organizational and institutional structure of the U.S. nuclear fuel cycle is marked by a high degree of decentralization of authority and responsibility. The decentralization has been increasing in recent years. Many factors have contributed to this trend, particularly the perceived need for independent regulatory autiority in nuclear development, and a generally widening public sensitivity to the broad impacts of technology and industrial development. An institutional framework has emerged, and continues to evolve, which strongly influences the technological directions and the public perception of present and projected technology. Principal federal government components, as outlined in the primer, are the Energy Research and Development Administration (ERDA), with responsibility for research, development and demonstration of nuclear technologies; the Nuclear Regulatory Commission (NRC), with authority over licensing and regulation of nuclear facilities and materials; and the Environmental Protection Agency (EPA), with jurisdiction over the ambient standards for radiological contaminants in the general environment. The goals and constraints of these governmental units are then juxtaposed on those of the private nuclear equipment manufacturers and operators of nuclear facilities.

These principal agencies are also affected by other organizations and influences. Individual state agencies use a variety of legal and political pressures to inject their own views into the system. Abundant opportunities for adjudication of regulatory and procedural steps provide a route for public and special interest group participation. International economic, environmental, and nuclear weapon proliferation considerations involve the State Department and the Arms Control and Disarmament Agency, along with that of international nuclear energy agencies. Relationships of civilian nuclear technology to that of the weapons program indirectly link the Defense Department and ERDA military programs to the civilian industry.

The diversity of these influences and the conflicting goals of the various organizations have a strong impact on the planning and operation of the nuclear fuel cycle, of ten overriding strictly technical considerations. Consideration of these interactions in the body of this Chapter has led to the following conclusions:

1. The decentralization has augmented our system of "checks and balances" and review procedures in nuclear fuel cycle decisionmaking. This review system has led in specific instances to stringent standards for safety and environmental protection in individual facilities of the overall nuclear system in the U.S. Public participation in licensing actions, judicial review through NEPA procedures, and the independent standards-setting role of EPA have all contributed to the "checks and balances" mechanism.

2. At the same time, the decentralization and review have made developments in U.S. nuclear technology slow and costly. Further, although. the decentralized review procedures may result in optimizing safety and environmental protection of individual facilities of the overall system, there may not be sufficient regard for the safety and reliability of the whole.
3. Decentralized policy-making and strong public and political input to decision-making provide an extremely difficult climate for the participation of private industry. Regulatory and technology policy decisions are slow and susceptible to unanticipated change. In an industry where capital costs are high, construction lead times long, and investment return slow, the delays and instabilities make private investment difficult to justify.

4. Though changes toward more streamlined organization and regulatory procedures are possible, the heterogeneity of views on nuclear fuel cycle issues will remain a key factor in policy formulation. It would be unrealistic to estimate nuclear economics or timetables without full consideration of extensive review procedures and public participation.

5. The rate at which NRC issues regulations and criteria, and at which judicial review is obtained in cases tested in court, is a limiting factor in fuel cycle activities of ERDA and private industry. This may in part be a result of NRC being understaffed or underfunded, but may also be a product of poorly chosen priorities in the past.

6. To achieve non-proliferation goals such as those outlined in the White House statement of October 29, 1976, and in preliminary announcements of current Administration policy, it is important to have a unified national nuclear policy in those areas which have international ramifications. Interagency and congressional coordination and cooperation will be needed to achieve this unified policy.

7. Because of the possible impact of domestic technology decisions on international discussions of plutonium use and on nonproliferation treaty obligations, foreign policy considerations have entered strongly into domestic nuclear technology strategy formation. The existing regulatory framework for domestic facilities does not provide an orderly pathway for these considerations to enter into licensing decisions.

These specific conclusions lead in turn to the following general conclusion:

Coordination of fuel cycle policy and its implementation should be improved. Consideration should be given to the establishment of a coordinating mechanism in the Executive Office of the President. This entity would not have operational responsibilities, but instead have a charter broad enough to look for problems, issues, and opportunities which cut across individual agency responsibility, and authority sufficient to catalyze action among the operational units. Creation of a Department of Energy, in itself, is insufficient for this purpose becaure other key organizations, the NRC, EPA and State Department in particular, play a strong role in energy policy decisions. A White House Energy Council, or the new Office of Science and Technology Policy, might provide a base for such a mechanism. The system of "checks and balances" will be most viable if each of the independent units can depend on some coordinating mechanism to consider optimization of the overall system.

There is a further need for a forum for input and exchange of views among the diverse participants in the nuclear fuel cycle, many of which normally interact primarily in adversary or contractorcontractee relationships. The high-level coordinating office could also provide support and overview for such a forum.

It should be noted that the purpose of this coordinating mechanism would not be to preempt the role of Congress and the public in nuclear policy, nor to subordinate regulation to economic and technical pressures. Its primary purpose would be to avoid dispersion and duplication of effort, arrange priorities, suggest the timing of activities, establish communication among the diverse organizational elements, and otherwise provide for a coordinated program with an identifiable and reliable time schedule. 


\section{B. Historical Evolution of Civilian Nuclear Development and Regulation}

By the mid 1950's a national objective had been defined to develop nuclear power as a major source of electricity. It was decided as a matter of policy that major parts of this industry, reactor manufacture and operation, fuel rod fabrication, and eventually spent fuel reprocessing, would be in the hands of the private sector. The federal government would be responsible for licensing, regulation, and guidance of the overall technology, and for uranium enrichment.

Within this overall framework, the Atomic Energy Act of 1954 (Ref. 1) was an initial attempt to institute clear, centralized, and welldefined allocation of responsibility in civilian nuclear development and regulation. A basic preemption of regulatory authority from state to federal jurisdiction was established. In the years that followed, however, a variety of conditions have led to a series of authoritydiluting measures, with only occasional attempts at reclarification. Many of these measures were based on widely accepted new perceptions of the implications of a large scale nuclear industry, but the contradictory currents they sometimes represented have led to many of the organizational tensions existing today.

The seeds of authority dilution were sown in 1957 amendments to the 1954 Act, allowing formal agreements for limited transfer of the preempted regulatory authority from the Atomic Energy Commission back to individual states. This was permitted in matters concerning " 1) by-product material, 2) source material, and 3) special nuclear materials in quantities not sufficient to form a critical mass." In the 1970 's, however, states have attempted to assert their authority far beyond these narrow areas, of ten seeking to impose general regulations much more stringent than those of the federal government. In a prime example, the state of Minnesota's Pollution Control Agency was able to secure Utility agreement in 1970 to a reactor off-gas storage system in order to reduce plant emissions by $95 \%$ below otherwise allowable limits (Ref. 2), even though the courts later upheld the basic federal authority in this area. This trend has continued in a variety of approaches, the most notable being the several state ballot initiatives of 1976 and the California legislature's requirements that certain aspects of waste disposal technology be demonstrated as a condition for further power plant construction within the State (Ref. 3). Many of the most recent state actions have not yet been fully tested against the 1954 law and its interpretation in the courts, though the traditional state control over land-use is as much a part of the claimed basis for authority as any implications of the Atomic Energy Act. It should be made clear that in a strictly legal sense, the federal preemption of authority for regulation of radioactive emissions and determination of ambient standards should be well established. However, in the more political arena, state authorities can be potent adversaries to federal intentions if conflict develops.

Within the federal establishment, the 1970 Clean Air Amendments (Ref. 4) and the creation of the Environmental Protection Agency (Ref. 5) were a serious challenge to the authority of the AEC over radiation control. The Clean Air legislation gave to EPA authority to regulate "hazardous air pollutants" which are defined as any pollutants which "may cause or contribute to an increase in mortality or an increase in serious...illness". Congressional critics of the AEC immediately interpreted this as giving EPA authority over radioactive emissions (Ref. 6). The EPA-administered solid waste legislations of that same year (Ref. 7), and the Water Pollution amendments of 1972 (Ref. 8), were similarly interpreted. Equally important, the executive reorganization of 1970 (Ref. 5), which created EPA, transferred to it the non-medical ionizing radiation aspects of the Bureau of Radiological Health from the Department of Health, Education, and Welfare, the Division of Radiation Protection Standards from the AEC, and all functions of the independent Federal Radiation Council. The thrust of these transfers was to vest in EPA the authority to determine generally acceptable levels of environmental radioactivity. However, the extent to which EPA could regulate emissions to ensure that these levels were not exceeded was left unresolved.

A conflict developed as EPA prepared in 1973 to set radiation standards for the entire nuclear fuel cycle (Ref. 9). The Executive Office of the President stepped in when the Office of Management and Budget conveyed a Presidential decision that "EPA should discontinue its preparations for issuing, now or in the future, any standards for types of facilities; and that EPA should continue, under its current authority, to have responsibility for setting standards for the total amount of radiation in the general environment...an ambient standard which would have to reflect AEC's findings as to the practicability of emission controls (Ref. 10)." This blunt directive seemed to stem the diffusion of authority, but EPA continued to exert strong influence through its review of environmental impact statements.

The full development, during the 1970's, of the environmental impact statement (EIS) procedures mandated by the National Environmental Policy Act of 1970 (NEPA) (Ref. 11) had, in fact, made centralized policy-making very difficult regardless of executive branch organization. Federal and non-federal agencies, as well as private citizens and groups, had the opportunity for detailed review and public comments concerning policy plans, with the possibility of court challenge to the adequacy of impact assessment. The Council on Environmental Quality, though never staffed to perform a real coordinating function, gained considerable power in its role as advisor to the President concerning adequacy and acceptability of environmental impact statements.

The separation of the AEC into ERDA and the Nuclear Regulatory Commission in 1974 (Ref. 12) once again moved toward a non-centralized nuclear establishment. By vesting nuclear regulatory authority in an independent regulatory agency, an attempt was made to draw a clear distinction between the design, development, and promotion of a nuclear energy industry, and the regulation of its operations. Though a clear definition of responsibility was attempted, ambiguity entered at several points via disagreements between the House and Senate and vague language used to find a compromise (Ref. 13). One key element of ambiguity is the question of the need to license ERDA demonstration facilities, as opposed to purely commercial ventures. This issue remains unresolved, and blurs the role of ERDA as a technology developer and of NRC as a detached licensing authority. The entire notion of separability of development and licensing authority has proven to be difficult in practice, because of the need to consider licensing criteria at a very early stage of technical development.

President Carter's energy reorganization plan of April 1977 proposes to bring an element of centralization into the over-all energy structure by combining the Federal Energy Administration, ERDA, the Federal Power Commission, and a few other elements into a Department of Energy. However, the independence of NRC, ERDA and, naturally, state authority, would be preserved. The basically decentralized organizational structure related to nuclear fuel cycle decisons would thus remain.

Policies of international trade and technology transfer in nuclear matters have been evolving in parallel to the domestic institutional developments. The Atomic Energy Act of 1954 and the broad authority of the Joint Committee on Atomic Energy originally created an unprecedented centralization of authority in the Executive branch and Congress over both the development and international traffic in nuclear technology. In recent years, however, basic foreign policy goals, and weapon proliferation concerns, have begun to bring other voices into U.S. decision making. Executive branch decisions against the sale of reprocessing technology in connection with reactor trade represented a major change in the momentum of developing international trade, and created a major division between the attitudes of the U.S. and other nuclear supplier nations. In 1976, Congress considered, but did not pass, legislation which would have required in one case a new set of criteria for NRC approval of foreign nuclear technology sales, or in another specific findings by the Secretary of State. In both cases, criteria for allowable exports revolved around discouraging reprocessing in countries not currently possessing this capability.

In late October, 1976, the Ford Administration announced a policy (Ref. 14) very close to that which had been discussed in Congress. As a candidate and as President, Mr. Carter has taken a similar position. In each case, nuclear non-proliferation goals have become the major consideration in international nuclear technology transfer decisions. Further, the action of both administrations in linking domestic reprocessing and uranium enrichment decisions to international negotiations concerning proliferation and plutonium use has 
intertwined foreign policy considerations with domestic nuclear energy decisions.

In January and February, 1977, the Congress in effect ratified the decentralization of nuclear planning and operations, adopting the philosophy that nuclear matters were better understood in the broader categories of energy development policy, foreign policy, regulatory policy, and military affairs (Ref. 15). The jurisdiction of the Join Committee on Atomic Energy was drastically reduced, dividing its legislative functions in the House among the separate Science and Technology, Interior, Armed Services, and International Relations Committees. In the Senate, the jurisdiction was similarly divided among the Environment and Public Works, Armed Services, Foreign Relations, and Energy and National Resources Committees. Legislation terminating all statutory authority of the Joint Committee was passed by the Senate in April of 1977.

\section{Current Status of Jurisdiction at Interfaces in Nuclear Development and Regulation}

Historically, ebbs and flows of authority have occurred in the various agencies, but most of the conflicts still exist. Key areas of agreement and of ambiguity are evident in the following interfaces:

1) ERDA-NRC. ERDA is responsible for planning, developing, and demonstrating nuclear technologies. NRC, as an independent regulatory agency, is responsible for licensing nuclear facilities, and arranging for inspection and enforcement of regulations. Decisionmaking in ERDA is of ten colored by its tradition as a technology innovator; whereas, the regulatory responsibility of NRC may lead it in some instances to push in quite opposite directions. Ambiguities occur when licensing and regulatory actions of NRC override other considerations in technical planning; NRC then becomes the key determinant in technical choices and development directions. Plutonium utilization criteria involved in the GESMO decision is one example. Conversely, ERDA development choices may preclude regulatory options which may have been open in principle to NRC. For example, many ERDA military nuclear waste operations are outside NRC jurisdiction by virtue of the same Act even though they have much in common with those for commercial wastes under NRC regulation. We currently have a situation where ERDA is pursuing full-scale development planning for a salt bed repository in Carlsbad, New Mexico for its military wastes. Yet the design and construction of that repository are not integrated into the commercial waste repository development plan.

Though NRC is attempting to frame its regulatory criteria in terms sufficiently broad and performance oriented so as to leave room for a variety of technical approaches, in some cases these criteria may in fact imply facility design specifications beyond the narrowest interpretation of licensing authority. The fact that certain ERDA demonstration facilities (the LMFBR and the high level terminal waste disposal repository) are to be licensed by NRC, as specifically provided in the Energy Reorganization Act (Ref. 12) places NRC in an important role regarding these technologies at a very early stage of development.

2) $E R D A-N R C-E P A$ : EPA's authority is generally considered to be that of stating required conditions outside the boundary of nuclear facililties, with ERDA and NRC providing the technical and regulatory framework needed to maintain those conditions. Analogous to the ERDA-NRC conflicts, however, are cases where a statement of required ambient conditions may very strongly determine either the technical or the regulatory approach. Gaseous fuel cycle effluents could become an example. This situation has tended to bring EPA into decisions about internal details of facility design. The October 1976 statement of nuclear policy by the Ford Administration (Ref. 16) emphasized EPA's role in waste management design criteria, with greater stress on a more narrow enforcement role for NRC. This may tend to reverse, to a considerable extent, the waning of EPA's authority brought about by the OMB memo of 1973 (Ref. 10).

EPA's decision-making processes may also lead, of course, to quite different approaches from that of NRC or the technology innovation thrust of ERDA. NRC, for instance, must deal directly and regularly as an enforcement agency with the nuclear industry. It is not unexpected, then, that notions of practicality and enforceability of specific regulations should be stronger in NRC's considerations than in those of EPA, where the more general concerns of protection of public or ecological health form the dominant focus.

The problems of the giant Seabrook nuclear project in New Hampshire present an excellent example of this different emphasis in decision-making (Ref. 17). EPA, after reviewing its own earlier preliminary decision approving the plant's cooling system, decided that the heated discharge water could cause serious harm to the coastal ecosystem, and refused to grant an exception to the ban on once-through cooling systems which is included in the Federal Water Pollution Control Act. On the other hand, NRC, owing to concern over the aesthetic impact of massive cooling towers as well as their fogging characteristics, had specifically forbidden this alternative. The impasse on this basic design factor was reached long after planning stages were completed, and four months after construction had begun on the basis of the earlier preliminary approval. The utility, NRC, and EPA all considered their actions on the basis of their perceived goals and responsibilities, but with very different underlying assumptions in the decision-making process they reached basically incompatible policy views.

3) States-NRC-EPA: Two conflicting trends seem to be occurring in the roles being played by individual states. Because of costs involved, states are becoming less enthusiastic about their position as routine regulators of small amounts of by-product materials, the authority available to them by the "Agreements" program of the Atomic Energy Act of 1954. Similarly, a greater awareness of the relative hazard of some so-called "low-level wastes" buried in shallow trenches in state-regulated commercial sites has made NRC and EPA less enthusiastic about putting such materials under state control. On the other hand, the political impact of the broader elements of nuclear power policy has made many states acutely interested in regulation of many details of nuclear facility operation. Many claims of authority of this type are legally based on traditional state land-use jurisdictions backed by the political potency of state institutions. The extent of state authority in regulating nuclear facility design, siting, and operation is a major unresolved issue at this time, and will only be decided when some of the existing state regulatory attempts are fully tested in the courts or in political decision making.

State regulatory thinking has been quite appropriately much closer to local health, environment, and economic pressures than to overall national energy strategies. Further increase in State authority will obviously increase the role of these local pressures and dilute the coherence of any national planning.

4) State Department-NRC-ERDA: A new potential for jurisdictional ambiguity has arisen concerning nuclear export markets and nuclear weapons proliferation fears. Though authority for negotiation and implementation of agreements for nuclear exports historically rested with the former AEC alone (Ref. 1), both Congress and the Executive in recent years had begun to view these as parts of overall foreign policy. In one context this policy leans toward limiting weapons spread, but sometimes in contradiction, it seeks also to assure valuable export markets. The independent Arms Control and Disarmament Agency has also begun to voice its concerns over the relation of civilian nuclear equipment design to nuclear weapons proliferation. "Insofar as the means for limiting diversion of civilian nuclear capabilities to weapons applications focuses on technical design features, these foreign policy concerns impact on both NRC and ERDA activities. The resolution of authority in this area rests on decisions as to the priority of nonproliferation over other goals; with these decisions not yet resolved the jurisdictional authority remains murky. The presidential election debate of 1976 appeared to move toward a consensus, however, that nonproliferation constraints would be given higher priority than has been true in the past.

A more fundamental organizational problem is becoming manifest at the interface of foreign and domestic nuclear policy. Even the choices and schedules for domestic commercial nuclear developments are seen as impacting broad foreign policy nonproliferation goals. Conversely, traditional flexibility of criteria for foreign policy decisions may inevitably conflict with the need for a clear and predictable regulatory code in a domestic commercial industry. Even the formal provisions of the non-proliferaiton treaty link domestic 
technology choices to foreign policy goals. An obligation is implied for the U.S. to fully develop and share its civilian nuclear technology, and decisions to cut-off development options on other than technical grounds could be interpreted as abrogations of this obligation. Yet, it is not simple to interject considerations of the delicacy of international negotiations into licensing procedures for domestic commercial facilities, or the preparation of generic or specific environmental impact statements. For the case of the AGNS reprocessing plant, the apparent need for government financing of necessary completion steps gives the President or Congress a practical veto on its operation through their role in the budget process. However, were the facility already completed with private funds, there might be no direct mechanism under existing law to allow foreign policy considerations alone to prevent licensing and operation. The exclusion of international issues from the preparation of the GESMO statement illustrates that this organizational problem goes beyond the specific case of the AGNS plant operation.

5) Industry-NRC-ERDA: The nuclear manufacturing industry and utilities have sought, from ERDA, a clear and reliable indication of technology development directions and investment. Because of the high development costs in nuclear technology, industry is unable to support completely its own development program and to a considerable extent is a captive of ERDA planning. Industry decisions on capital commitments ultimately can be made only in response to ERDA priorities, though ERDA recognizes the need to solicit industry views in setting those priorities. Similarly, industry has pressed strongly for a reliable and stable regulatory climate from NRC. Capital costs and amortization times are so long for nuclear facilities that changes in construction or operating requirements can completely negate conventional economic planning.

In the area of power reactors, regulation and development have proceeded in a relatively orderly interactive manner. Flaws in early reactor designs have been recognized and accounted for in subsequent regulation. Where necessary, they have been corrected, but in all cases, subsequent designs have built on the existing base. Designers, construction firms, and utililties have been able to work within the framework as a tightly regulated but profitable industry. Even here, however, there have been serious problems. The regulatory decisions are slow enough that capital requirements in advance of power returns are beyond the capabilities of many utilities. The Sacramento Municipal Utility District in California is a classic case; Rancho Seco \#2 was cancelled because the utility could not afford the capital for a new plant (Ref. 18). The delayed Seabrook plant already discussed is another; costly equipment stands idle while the regulatory impasse continues (Ref. 17). Virginia Electric and Power has recently canceled a unit in which it had already made considerable investment; once again the demands for capital had outrun the expected timing of returns (Ref. 19). In the latter case especially, uncertainties in future demand coupled with regulatory delays to exacerbate the capital cost problem.

With fuel reprocessing units, the experience has been even more confusing. One of these, the Nuclear Fuel Services, Inc. (NFS) facility West Valley, N.Y. was built and operated in conformance with existing regulations in the 1960 's. Changes in those regulations, enacted after the plant had been licensed and was operating, required extensive and costly changes. NFS recognized the need for some of these, and initiated steps to reduce emissions and establish a cleaner operation. Nevertheless, continuing changes that would have been required were judged by NFS to be sufficiently costly as to be impractical, and the company withdrew its application to modify and reactivate the West Valley Plant.

Similarly, the AGNS plant at Barnwell, S.C. was built as a commercial venture with full expectation of meeting regulatory requirements existing when the investment commitment was made, probably incorporating a modest number of reasonably predictable alterations. Moreover, it was built in response to ERDA planning which projected and promoted a large reprocessing industry entirely in the private commercial sector. Nuclear weapons proliferation and other concerns have subsequently changed technical strategies and greatly broadened regulatory emphasis, however, and the plant has not been licensed to operate even on a provisional basis. The current status of requirements leaves the future of Barnwell undecided. Its management justifiably is reluctant to increase its investment in the face of the existing uncertainities and NRC is not yet able to provide assurances with regard to licensing and future operations.

These two examples illustrate an extremely difficult situation for private industry. The inability of the government agencies to provide relative stabililty in guidelines and regulations is a strong detractor to the private sector making commitments to investment in new nuclear technology on a closed nuclear fuel cycle. Though many other industries operate in a closely regulated climate, the capital intensiveness and long amortization time for nuclear facilities compound the problem of justifying private investment.

An additional problem at the industry-NRC interface is ilfustrated by the NFS plant. The high-level liquid reprocessing wastes were allowed to accumulate there in temporary tank storage. Now that NFS has decided to cease operations, eventual restoration of this site would require these wastes to be solidified and conveyed to terminal storage, as well as decommissioning of the entire plant. The cost for the waste solidification alone is estimated at several hundreds of million dollars, far beyond the assets of the NFS corporation (Ref. 20). It appears thus that these costs will be borne by the public, and the existing 4 million dollar "decomissioning fund" stands only as a monument to our inability to grasp the full ramifications of certain technological activities(Ref. 20). The NFS decomissioning problem has vividly raised the question of whether private fuel cycle participants will be required to post very large bonds to assure financing for plant maintenance or dismantling in the event of insolvency of the owners.

6) Commercial-Military: An important motivation for spent fuel processing is to provide plutonium useful for the start-up of a breeder technology. Plutonium reserves exist within the military establishment; their availability in the long term would facilitate the future option of a breeder economy without using commercial spent fuel recycle and hence reduce the urgency for commercial reprocessing.

Similarly, some of those concerned with the safeguarding of nuclear materials from terrorist attack have suggested a much larger role for the military in the security of nuclear installations and transportation of nuclear materials. The very strong historical separation of the military from direct participation in civilian activities argues against both of these concepts, however. That the military and civilian nuclear waste management programs are kept separated quite ostentatiously within ERDA is just one manifestation of the bias against mixing these sectors. Any degree of mixing military support into civilian programs would probably be seen by many as a basic sign of failure of the fundamental concepts of the civilian nuclear power industry.

\section{7) U.S. Agencies and Industry-International Agencies and} Foreign Institutions

Because of the complex structure of its domestic nuclear establishment, it is of ten difficult for the U.S. to interact on nuclear matters with foreign governments, corporations or international agencies. The independent regulatory checks and reviews on the actions of any single U.S. entity make it difficult to provide definite commitments to more centralized foreign organizations. This problem is shared, of course, by other U.S. industries in world markets, but is probably greater by degree in the nuclear field giving to the broader concerns over nuclear matters. Illustrating this broader concern, the U.S. has at least one entity, the Arms Control and Disarmament Agency (ACDA), which has no parallel in the rest of the world. Thus the agency must use indirect means for playing an international role in nuclear policy.

Considerations concerning U.S. use of the French waste solidification process is just one example of the difficulties inherent at the domestic-international interface. The French were indeed willing to sell this technology, and its adaptation here could have saved both development time and costs. However, because of the public nature of NRC licensing procedures, the U.S. felt it had to require that all details of the process be publicly released and made available from federal files to anyone who would wish to inspect them. From the French point of view, this would have involved giving away a technology developed at great cost and with considerable 
commercial value. As a result, a potentially useful technology transfer did not occur.

On a far larger scale, the importance of non-proliferation concerns in the U.S. clearly limits technology transfer and exchange activities of both ERDA and private industry. The Congress, State Department, ACDA, NRC, and the general public all may have strong voices in international decisions, and make it difficult for any foreign institution to count on any single U.S. agency being an accurate and reliable spokesman for U.S. Policy.

\section{Advantages and Problems of the Current Framework of Dispersed Responsibility}

The above discussion shows that there presently exists a divided and somewhat ambiguously defined authority for development and operation of the civilian nuclear fuel cycle. A number of advantages and problems in technology management stem from this division and ambiguity:

\section{D1. Advantages of the Current Framework}

The primary benefit of the current system is the independent review of nuclear policy and planning that exists in the several and diverse authorities. Public distrust of nuclear policy, which developed during the era when the AEC acted as both regulator and promoter, may be relieved by vigorous open and independent action by NRC, ERDA, and EPA. This factor alone may be worth the inefficiencies and confusion caused by the dispersed jurisdictions. In addition, the independent review may in some cases enhance the chances of avoiding costly harm to health or environment, and may even lead to more imaginative technical developments than might otherwise exist. In any case, the tradition of independent regulatory authorities, with both state and federal roles, is a strong one in our society and probably will not be lightly discarded.

\section{D2. Problems of the Current Framework}

a) Though the division of responsibility provides opportunity for independent review of decisions, it also tends to promote conflicting or contradictory suboptimization of technical systems. Historically, for instance, ERDA has naturally tended toward favorable economics and energy efficiency in reactor design; the Arms Control and Disarmament Agency, in contrast, has viewed such design problems predominantly in terms of minimizing nuclear proliferation risks. In many planning and policy problems, the various participants -industry, EPA, ERDA, NRC, individual states, etc. -- all tend to see the issue largely in terms of their own primary mission and goals. When they act individually on this basis, the sum of these actions may result in a far from optimal system design.

b) The ambiguity of responsibility leads to costly delays and false starts in research, development, and construction programs. ERDA cannot optimize the use of its technical resources without knowing the licensing criteria of NRC -- yet the independent regulatory role of NRC implies that ERDA's and NRC's schedules and planning must evolve independently. Similarly, ERDA and NRC plans can be upset by imposition of individual state, EPA, or State Department authority. Recent General Accounting Office criticisms of the use of the Lossof-Fluid Test Facility epitomize the problem of optimizing technical actions when goals and operation are responsibilities of separate agencies (Ref. 21). Delays in definition of a waste repository demonstration can also be attributed in part to the lack of correlation of ERDA and NRC plans. Plans are further complicated by the need to consider the political climate in individual states as one of the constraints in waste repository siting.

c) The division of responsibility taxes resources of skilled and experienced manpower. In areas where the technical expertise is limited, and multiple agencies, each desiring independent support, compete for this pool, severe shortages can result. As one example, almost all of the nuclear waste disposal experience was concentrated in ERDA after the division of the AEC. Despite the regulatory significance of this area, NRC was left with an extrenely limited internal technical program and had difficulty even in locating potential outside contractors whose personnel were not already heavily committed to work with the ERDA waste program. d) The division of responsibility may result in each unit failing to perceive some problems in its planning, or passing over them on the assumption that these problems will be faced elsewhere in the organizational structure. Mason Willrich's (Ref. 22) report to ERDA has cited this difficulty in the waste management field, where temporary storage, treatment, packaging and transportation responsibility fall upon independent components private industry, and repository handling and long-term responsibilities fall upon the federal government.

e) The division and ambiguity of authority within governmental agencies is making private participation in nuclear fuel cycle development extremely problematic. In the present jurisdictional environment, private investments must not only face all the normal uncertainties of market forces in long lead-time investments, but must also be reconciled with the possibility of sudden and major changes in technical emphasis or regulation. Expectation of private financing of nuclear fuel cycle facilities may prove to be unrealistic as long as the present division of responsibility for fuel cycle development and regulation exists within government.

\section{E. Implications For Fuel Cycle Decisions}

Given the organizational arrangement of dispersed nuclear development and operational responsibility, independent regulatory authority, and constant attention by the public to detailed technical factors, several characteristics of the U.S. nuclear program seem inevitable. On the one hand, it will be slow in development schedules, slow and inertial in its ability to adapt to changing conditions, and high in capital and possibly also operational cost. On the other hand, individual elements themselves will probably be extremely safe, relatively benign in environmental impact, and highly reliable.

An example of the great inertia of our existing system can be taken from current consideration of alternative reactor types to meet newly perceived nuclear nonproliferation or resource efficiency goals. Though the existing Canadian heavy water (CANDU) reactor has been cited as advantageous in some situations, even proponents realize that U.S. licensing requirements would probably require design changes, studies, and procedures which could take more than a decade to implement. Similarly, since the chief private developer of the High Temperature Gas-Cooled Reactor, Gulf General Atomic halted its marketing program, the time required to re-activate that option for practical application has been rapidly growing. In this technically and economically complex field there is no simple way to transfer technology between the private and public sector, or maintain at readiness currently non-marketable options in the anticipation of changing economic, technological or political constraints. The economic structure of the U.S. utility industry emphasizes conservatism even further. The utililties must invest in future growth to achieve the investment tax credits which are a large component of their cash flow income. However, their rate structure is largely such that these investments must be made in extremely reliable and proven technologies. Thus this particular investment potential will certainly be directed toward continuation and expansion of the proven lightwater reactor cycle.

Similarly, developments in reprocessing concepts illustrate the pressure on capital costs as a price for reliability and safety. The AGNS plant, as compared to the NFS facility, is designed for an entirely new level of safety with respect to seismic stability, operational radioactive emissions, and waste handling, but it will probably be an order of magnitude more expensive if completed. Nonetheless, new, even more conservative designs already exist which could again raise that cost threefold or more (Ref. 23). There will always be considerable pressure to invest only in the safest and therefore costliest design in each separately analyzed and administered component of the fuel cycle, without optimizing total investment for maximum system safety. This is only one example of the frequent suboptimization of individual nuclear fuel cycle segments in the decentralized U.S. planning system. The comparison of investment in protection of the biosphere from high level nuclear wastes, with the much more limited investment in protection from the comparable hazard of uranium mill tailings, is another vivid example of suboptimization, which is discussed in Chapter V. 


\section{F. Organization of the Fuel Cycle Management}

Alternative schemes of organization certainly exist. With the problems of decentralized authority quite evident, many ideas have been offered involving re-creation of a more centralized system. The simplest of these ideas would be a reunification of the ERDA nuclear fuel cycle division with NRC. More comprehensive thinking has involved a "Department of Energy" which could provide an umbrella for ERDA, the Federal Energy Administration (FEA), and possibly other regulatory authorities. The legislation proposed by President Carter represents this point of view in combining ERDA, FEA, and the Federal Power Commission. However, while the President's plan would lead to some centralization of policy making, as noted above EPA, NRC, and individual states would retain their current roles, along with that of the courts via NEPA proceedings and other opportunities for litigation.

A possible alternative or additional scheme of policy coordination could involve an entity within the Executive Office of the Presient, an office without administrative or operational responsibility but instead functioning as a coordinating staff for the President himself. Such an office could address itself to identifying gaps between individual agency programs, to identifying or resolving conflicts and contradictions among them, and to setting priorities among diverse efforts. A White House Energy Council, or possibly the new Office of Science and Technology Policy, could fulfill this function. In the absence of a specifically defined unit, in some cases the Office of Management and Budget must inevitably play this role as it did in the case of the 1973 memorandum (Ref. 10) intervening in the EPA-AEC conflict. However, many would argue that in a particularly importan policy area, a more visible and specifically designated entity is needed. This would provide an identifiable focus for policy decision and discussion.

Certainly many of the problems at the go:ernment-industry interface could be resolved by maintaining all of the fuel cycle, save possibly mining, milling and reactor operation, in government hands. In this manner, broad public concerns over fuel cycle policy could enter directly into decision making without involving competitive commercial economics. The problems industry has had with uncertainties in government regulatory and development policy may make this organizational change a necessity, rather than a matter of choice. Because of limited government operational capability in many of these areas, private industry's role would undoubtedly be preserved as contracting builders or operators of facilities. The advantages of such a change would, of course, have to be weighed against the additional capital demands on the federal government, and the loss of the efficiency incentives of competitive market economics.

It should be noted, however, that organizational matters of this kind involve values beyond that of energy investment efficiency, and instead represent basic philosophies of political and economic institutions which are not likely to be lightly set aside by their proponents. For this reason, simple administrative reforms are not likely to change totally the heterogeneity of inputs and diversity of points of view in nuclear fuel cycle choices. Problems in coordinating nuclear policy formulation and implementation among the several agencies are likely to remain an inevitable consequence of that diversity.

\section{References}

1. P.L. 83-703, 68 Stat. 919.

2. $\quad$ "The Debate Goes On: Are Radiation Standards Adequate?" Nuclear News, 13 28-32 (1970).
3. "Nuclear Initiative: Californians Vote 'No' but Legislature Acts", Science 192, 1317-1319.

4. Section 112, P.L. 91-604

5. Reorganization Plan No. 3, 1970.

6. "Science Report/Attack on federal radiation standards threatens nuclear development programs," National Journal, Nov. 14, 1970, 2483-2497.

7. Section 203, P.L. 89-272, 84 Stat. 1676 .

8. P.L. 92-500, 86 Stat. 816

9. "White House cuts down on EPA's nuclear powers," Electrical World, Jan. 15, 1974, 27.

10. Roy Ash, Office of Management and Budget, Memorandum for Administrator Train., Chairman Ray, Dec. 7, 1973.

11. P.L. $91-190$

12. P.L. $93-438$.

13. Section 202, P.L. 93-438, and pp. 33-34, Conference Report on P.L. 93-438.

14. Office of the White House Press Secretary, Fact Sheet, President's Non-proliferation Statement, Oct. 28, 1976

15. H. Res. 4, Jan. 4, 1977, Rules of the House of Representatives, 95th Congress, 1st Session; S. Res. 4, Feb. 4, 1977, Committee Reform Amendments of 1977; Congressional Record, House, Jan. 4, 1977, pp. H5-7; Congressional Quarterly, Feb. 12, 1977, pp. 279-284.

16. Office of the White House Press Secretary, Fact Sheet, President's Nuclear Waste Management Plan, Oct. 27, 1976.

17. "The Seabrook Dilemma: Finding Some Way Out", Nucleur Industry, April 1977, 3-12.

18. Wm. C. Wolbridge, General Manager, Sacramento Municipal Utility District, Report in Future Generation, Jan. 8, 1976.

19. "Pepco Cancels Controversial Nuclear Plant", Washington Star, June 9, 1977, pg. 1.

20. "Issues Related to the Closing of the Nuclear Fuel Services, Inc., Reprocessing Plant at West Valley, N.Y.", U.S. General Accounting Office, Washington, D.C., Report EMD-77-27, March 8, 1977.

21. "This Country's Most Expensive Light Water Reactor Safety Test Facility," Report to the Committee on Government Operations, U.S. Senate, by the Comptroller General of the United States, RFD-76-68, May 26, 1976.

22. Mason Willrich, "Radioactive Waste Management and Regulation," Report to the U.S. ERDA, November, 1976.

23. E. I. DuPont de Nemours and Co., LWR Assistance Task Force Monthly Report, Savannah River Plant, Aiken, S.C.; DPSPLWR-76--13-8, Aug., 1976;-9, Sept., 1976;-10, Oct., 1976;-11, Nov., 1976;-12, Dec., 1976. 
The nuclear fuel industry is made up of a number of steps, each requiring an operational facility which has its own environmental impacts and in which both radioactive and nonradioactive wastes are generated. In this section the model fuel-cycle facilities are reviewed in terms of capacities, waste generation rates, and type of waste produced. To provide the reader with an orientation on waste management activities, numbers are provided from the ERDA fuel cycle model, as referenced in GESMO and the NRC Task Force Report on Waste Management; where necessary, the environmental impacts associated with reprocessing and waste management activities are normalized to a reference reactor year (RRY).

\section{Mining}

Uranium is present to the extent of about $4 \mathrm{ppm}$ of the earth's surface; however, most deposits are of such low grade $(<.001 \%)$ that extraction of the metal is uneconomic. The principal deposits in the United States are located in the Colorado Plateau which includes portions of Arizona, Colorado, New Mexico, and Utah. The majority of the ores mined in this region contain 0.1 to 0.5 w/o uranium. Most of the uranium minerals from this region are of the carnotite $\left(\mathrm{K}_{2} \mathrm{O} \cdot 2 \mathrm{UO}_{3} \cdot \mathrm{U}_{2} \mathrm{O}_{5} \cdot \mathrm{xH}_{2} \mathrm{O}\right)$ and austenite $\left(\mathrm{CaO} \cdot 2 \mathrm{UO}_{3} \cdot \mathrm{PxO}_{5} \cdot \mathrm{H}_{2} \mathrm{O}\right)$ forms. Relatively high grade ores are available from sources outside the country. These ores are approximately 1 to $4 \%$ uranium, primarily in the form of pitchblende or uranite, of variable composition ranging between $\mathrm{UO}_{2}$ and $\mathrm{U}_{3} \mathrm{O}_{8}$.

Uranium ore is obtained from both underground and open-pit mining operations. Wastes from underground mines consist mainly or rock removed in creating shafts and passageways. Wastes from openpit operations consist largely of overburden removed to expose the ore body. A model mine size has been defined in GESMO as having an annual production of 18.1 tons of natural $\mathrm{U}_{3} \mathrm{O}_{8}(14.0 \mathrm{MTU})$ for an underground facility and 181 tons (140 MTU) for an open-pit mine. In either operation, wastes are expected to contain only relatively small amounts of uranium. Mine waste should have essentially the background radioactivity typical of the region.

\section{Milling}

Milling consists of at least four general steps: (1) crushing and/or grinding, (2) chemical dissolution of the ore, (3) physical separation of dissolved ore from the undissolved solid waste, and (4) separation and consolidation of the $\mathrm{U}_{3} \mathrm{O}_{8}$ product, referred to as yellowcake.

Leaching processes at the uranium mill recover more than $90 \%$ of the contained uranium but dissolve only a very small fraction of the finely ground ore. The mills currently discharge these undissolved solids (mill tailings) along with nearly uranium-free process water and its contained chemicals to an impoundment area (tailings pond) near the mill.

Uranium and uranium daughter products are released as airborne particulates and gases from the ore piles, the tailings retention system, and the ore curshing and grinding and ventilation system in the form of dust. Virtually all of the ${ }^{222} \mathrm{Rn}$ daughter product is released as gas. The solid waste tailings contain about $85 \%$ of the radioactive materials originally in the ore. This includes concentrations of ${ }^{226} \mathrm{Ra}$ and ${ }^{230} \mathrm{Th}$. While stored in the tailings retention pond, natural radioactive decay continues resuling in further discharge of radioactive effluents to the atmosphere. Thus such tailings can be an important long-term source of radioactivity, so that some stabilization procedure is necessary for environmental protection. On the basis of assumptions from GESMO and WASH-1248 of $0.1 \% \mathrm{U}_{3} \mathrm{O}_{8}(807 \mathrm{MTU})$, the ratio of 1300 for waste to product masses gives an annual waste stream of $1.37 \times 10^{6}$ tons of $525,000 \mathrm{~m}^{3}$.

\section{Hexafluoride Conversion}

Before sending natural uranium to the enrichment plant it is common practice to convert the $\mathrm{U}_{3} \mathrm{O}_{8}$ yellow cake from the mills to readily volatilized $\mathrm{UF}_{6}$. To produce $\mathrm{UF}_{6}$ production facilities contain large quantities of chemicals but very low levels of radioactivity. The wastes are largely composed of $\mathrm{CaF}_{2}$ ash from dry-process plants, sludge from plants using wet processes, and $\mathrm{CaF}_{2}$ chemical wastes from treating scrub liquors at both types of plants. The $\mathrm{CaF}_{2}$ ash is presently drummed and shipped offsite ot a burial facility. While low-level sludges may be handled similarly, at present they are stored or buried onsite. The model $U_{6}$ plant using either process has an annual capacity of 15,000 MTU (natural). The wet-process model plant produces $3900 \mathrm{~m}^{3}$ of wastes while the dry-process model will produce $2480 \mathrm{~m}^{3}$. In either case, wastes are either disposed of in a licensed burial gorund or are buried onsite.

\section{Enrichment}

The enrichment process raises the isotopic concentration of U-235 in $U_{6}$ feed material from the conversion plants. The annual production rate depends on several factors, including the isotopic concentrations of the feed, tails, and product as well as the plant's efficiency. Assuming nominal U-235 concentration of slightly over $3 \%$ in the product and $0.3 \%$ in the enrichment plant tails, the annual capacity of the model plant is approximately 2,400 MTU.

Enrichment is presently performed in plants using the gaseous diffusion process. Wastes generated result primarily from equipment cleanout and uranium recovery. Liquid wastes are impounded in holding ponds where most of the uranium is collected in sludges of low-level radioactivity that are periodically removed and buried onsite. In addition to wastes generated by the enrichment plants, approximately 35 million $\mathrm{m}^{3}$ of nonradioactive sludges (water and solids) result from operation of the fossil-fueled power plant that supplies power for the enrichment processes.

Future expansions of enrichment capacity are expected to include centrifuge facilities. A gas centrifuge enrichment plant is expected to generate large quantities of nonreusable parts and materials from failed machines. The annual waste from a model centrifuge plant is estimated to be about 56 times greater than from a model diffusion plant of the same capacity. The GESMO model plant has an annual capacity of 8750 MTSWU and generates $100 \mathrm{~m}^{3}$ of waste from a diffusion plant and $5600 \mathrm{~m}^{3}$ from a centrifuge facility. GESMO assumes a ratio of 4 diff usion plants to 2 centrifuge plants to conclude that a waste volume of $1900 \mathrm{~m}^{3}$ is obtained for an average enrichment plant.

\section{Uranium Fuel Fabrication}

A fuel fabrication plant converts enriched $U_{6}$ to $U O_{2}$, into pellets loads the pellets into fuel rods, and assembles the loaded rods into fuel assemblies. The most significant waste generated in these plants is $\mathrm{CaF}_{2}$, which is formed during the conversion operation of the rate of one metric ton for each metric ton of uranium processed. The uranium content of the $\mathrm{CaF}_{2}$ is estimated to be aobut 0.01 $\mu \mathrm{C} / \mathrm{gm}$. The current practice is to package or store the waste in bulk form onsite. As stated in GESMO, a model fabrication plant will process 1500 MTU annually.

\section{Power Reactors}

For the reactor referenced in WASH-1248, wastes generated at the reactor are averaged so as to represent the approximate industry mix of $2 / 3$ PWRs and $1 / 3$ BWRs. The quantity of low-level wastes produced by LWRs is the same for all three fuel-cycle options. Based on a review of reactor licenses' semi-annual oeprating reports through December 1975, a 1000-MWe PWR generates annually approximately $323 \mathrm{~m}^{3}$ of packaged wet wastes (spention-exchange resin, filters, filter sludge, and evaporator bottoms), and $116 \mathrm{~m}^{3}$ of dry and compacted solid waste. A 1000-MWe BWR produces annually about $850 \mathrm{~m}^{3}$ of packaged wet wastes and $133 \mathrm{~m}^{3}$ of dry and compacted solid waste. Using the ratio of $2 / 3$ PWRs and $1 / 3$ BWRs, waste volumes of 500 $\mathrm{m}^{3}$ of resins and $120 \mathrm{~m}^{3}$ of dry compacted solids are obtained for the industry-averaged LWRs. An additional $1 \mathrm{~m}^{3}$ of waste generated in the LWR consists of discarded equipment, in-core instrumentation, and control-rod blades. These wastes contain some 4600 curies of fission products and induced activity. 


\section{Spent Fuel Assemblies as Waste}

In the case of the'no-recycle option, an additional waste stream, the spent fuel assemblies, is also produced. Given that a PWR fuel assembly is nominally 85 inches square and 14 feet long, and assuming that it is overpacked or encapsulated in a 13-inch-diameter casing approximately 16 feet long, each assembly is $15 \mathrm{cu} \mathrm{ft}$ in volume. An annual reload for PWR averages 78 assemblies (@ $450 \mathrm{kgU}$ per assembly) giving approximately $1225 \mathrm{cu} \mathrm{ft}$ or $35 \mathrm{~m}^{3}$. Values of 1090 cu ft or $31.2 \mathrm{~m}^{3}$ are similarly obtained for a BWR, using $200 \mathrm{~kg}$ per assembly and 175 assemblies per year; an assembly is $14 \mathrm{~cm}$ square by $535 \mathrm{~cm}$ long. For a throwaway option, these assemblies would be placed in permanent disposal at a Federal Waste Repository. A stowaway no-recycle option would require indefinitely recoverable disposal.

The temporary storage of spent fuel, whether at a reactor, an independent spent fuel storage facility, a reprocessing plant, or a repository, will generate waste resins from water cleanup systems. These are assumed to be constant for all types of fuel and amount annually to $21 \mathrm{~m}^{3}$ for a $3500-$ MTHM storage pool. Because the spent fuel in the no-recycle option is stored for 10 years by NRC regulations, some $2.1 \mathrm{~m}^{3}$ of such waste are generated per RRY. Less than $1 \mathrm{~m}^{3}$ would be generated for the comparable uranium-only recycle option.

\section{Fuel Reprocessing}

In the uranium and uranium/plutonium recycle options the spent fuel discharged from power reactors is reprocessed for its fissile and fertile values. Opening the fuel rods results in the release of radioactive gases, some of which are released to the atmosphere in normal plant operation. Of particular concern are ${ }^{14} \mathrm{C},{ }^{85} \mathrm{Kr},{ }^{129} \mathrm{I}$, and tritium; these off-gases are the subject of considerble review and discussion by EPA and the NRC and will be considered in Chapter V.

The bulk of the radioactivity from nonvolatile fission products and unrecovered actinides results in the several types of wastes described below. The model fuel reprocessing plant's annual capacity is $2000 \mathrm{MTHM}$. The volume of such wastes is essentially independent of which recycle option is used, but the isotopic composition of the radioactive wastes is altered if plutonium recycle is adopted. Of course in the uranium-only-recycle option, the plutonium becomes a waste to be managed.

\section{High-Level Wastes}

High-level liquid radioactive waste (HLW) is defined in Appendix F, 10 CFR Part 50, as "those aqueous wastes resulting from the operation of the first cycle solvent extraction system, or equivalent, and the concentrated wastes from subsequent extraction cycles, or equivalent, in a facility for reprocessing irradiated reactor fuels". The HLW are solidified, cast, formed, or otherwise contained in a primary container which is provided with an overpack or secondary shell as the outer canister. The reference design of the canister, including overpack, is a right cylinder some 15 inches in diameter and 10 to 12 feet long (15 $\mathrm{ft}^{3}$ or approximatley $\left.0.3 \mathrm{~m}^{3}\right)$. It is assumed to hold about $6.3 \mathrm{ft}^{3}\left(0.18 \mathrm{~m}^{3}\right)$ of solidified waste. Technology for both calcining or vitrifying of the HLW is available for use; a choice has not yet been made. Since it is estimated that each metric ton of fuel reprocessed from either of the recycle options will produce about $2 \mathrm{ft}^{3}$ of calcine or 2.t cu ft of vitrified HLW, each canister will hold the waste from 3.14 or 2.52 metric tons of processed fuel, depending on which waste form is approved by the NRC. Thus 11 to 14 canisters occupying 6 to $8 \mathrm{~m}^{3}$ will be generated annually for each model 1000 MWe power reactor.

For purposes of its assessment, the NRC assumes that following the first cycle of decontamination and the partitioning of the plutonium and uranium streams at the reprocessing plant, the resulting plutonium would be converted to $\mathrm{PuO}_{2}$ of about $95 \%$ purity $(5 \%$ fission products) and encapsulated. For criticality reasons the capsules are then supported by spaces in 30-gal containers, each capable of holding $6 \mathrm{~kg}$ of the materials (approximately 47 canisters per year, or $6 \mathrm{~m}^{3}$ per model reactor). For the uranium-only recycle the GESMO assumes that the plutonium-bearing canisters will be disposed of in federally operated geologic repositories together with the separated and solidified HLW. Alternatively, the solvent extraction process can leave the $\mathrm{Pu}$ with HLW stream, so that the waste $\mathrm{Pu}$ and fission products may be solidified and managed together. With this alternative, one must deal with suitable criticality and accountability controls.

\section{Fuel Element Hulls and Hardware}

In the first step of the reprocessing operation, structural components of fuel assemblies are removed and the fuel rods are chopped into short pieces, so that the $\mathrm{UO}_{2}$ or mixed fuel can be leached from them with nitric acid. This operation produces the feed solution for the processing operation and leaves the cladding undissolved. These cladding pieces, called hulls, after additonal washing with nitric acid solution, are expected to contain less than $0.1 \%$ of the uranium and plutonium present in the fuel, along with small quantities of fission products and products of neutron activation of the metal. Approximately $0.5 \mathrm{~m}^{3}$ of hulls and fuel-assembly hardware result from the processing of each metric ton of heavy metal, regardless of fuel cycle operation, giving approximately $15 \mathrm{~m}^{3}$ of hulls per reactor on an annual basis. As presently designed, reprocessing plants plan for interim storage of these wastes in vaults or concrete containers. Because of the residual plutonium contained in these wastes, it is planned that the hulls will eventually be transferred to a federal geologic repository.

\section{Transuranic (TRU) Wastes}

Reprocessing plants operating under either of the recycle options will generate miscellaneous plutonium-beaing wastes of several types including laboratory wastes (e.g., small tools and gloves), chemical wastes from cleaning the off-gases, failed equipment, filters, and plutonium extraction solvent, wastes from the plutonium nitrateplutonium nitrate-plutonium oxide conversion section of the plant, and certain wastes from the process, for preparing $\mathrm{UF}_{6}$ from recovered ruanium. The volumes of these wastes is estimated to be nearly $30 \mathrm{~m}^{3}$ annually per reactor without compaction or incineration, nearly independent of the choice of recycle option. The wastes will contain about $0.3 \%$ of the plutonium throughput.

The plutonium conversion facilities generate transuranic wastes consisting largely of filters, gloves, discarded process components, and solidified process wastes. It is estimated that these wastes would contain about $0.2 \%$ of the plutonium throughput, or approximately 300 grams per $\mathrm{m}^{3}$. By using the mean of values estimated for an existing reprocessing plant design, the total annual volume of plutonium-bearing waste generated would approach $44 \mathrm{~m}^{3}$ per reactor. The separation facilities would produce minor amounts of solidified aqukeous wastes fom the solvent and off-gas cleaning systems. These wastes would be expected to contain 300 grams of plutonium with the uranium-only-recycle or about 305 grams of plutonium if both plutonium and uranium are recycled. Though some wastes may contain transuranic concentrations sufficiently low to permit their burial in a licensed facility, it is generally assumed that these relatively low-level transuranic wastes will be sent to a federal geologic repository.

\section{Chemical Wastes--UF 6 Conversion}

Each model reactor year the $U_{F}$ facility at a reprocessing plant is expected to produce an estimated 1100 metric tons of chemical wastes (i.e., spent electrolyte, $\mathrm{CaF}_{2}$ from treating fluoride scrub liquors, calcine discharges from the uranyl nitrate-to- $\mathrm{UO}_{2}$ conversion step, and potassium uranate muds) contain about $42 \mathrm{~kg}$ of uranium but negligible radioactivity. These wastes normally would be shipped to a licensed commercial facility for burial, though onsite burial at the reprocessing plant may be possible.

\section{Mixed Oxide Fuel Fabrication}

The MOX fuel plants, which produce fuel rods containing uranium and plutonium are incremental operations resulting from plutonium recycle. The GESMO model plant has an annual capacity of $360 \mathrm{MT}(\mathrm{U}, \mathrm{Pu}) \mathrm{O}_{2}$. The solid wastes for disposal total approximately $10,000 \mathrm{ft}^{3}\left(290 \mathrm{~m}^{3}\right)$ and contain $22 \mathrm{~kg}$ of $\mathrm{PuO}_{2}$, or aobut $0.1 \%$ of $\mathrm{PuO}_{2}$ throughput. This waste volume is equivalent to $8 \mathrm{~m}^{3} / \mathrm{RRY}$ and would be sent to the federal geologic repository followign a suitable procedure for compaction. 


\section{Waste Repositories}

Two categories of waste repositories are assumed. They differ with respect to their locations in the biosphere. One category is surface or near-surface repositories or burial grounds that are used for disposal of low-level wastes of negligible transuranic content. The second category is repositories in continental geological formations which will be used for containing solidified HLW, plus all other wastes having appreciable concentrations of transuranic radionuclides including plutonium. Spent-fuel elements in the no-recycle option would also be sent to such a repository. The discussion of Chapters $V$ and VII treats the issues for both types in detail.

\section{Projected Annual Waste Generation}

Annual waste quantities generated by the ERDA model fuel-cycle facilities operating at full capacity are given in NUREG-0116 for the options of no-recycle and uranium-only-recycle. Their tables also give the average annual capacity requirements, annual waste volumes and annual radioactivity for the individual fuel cycle components to support a single 1000-MWe LWR over its lifetime for the alternative fuel cycles. This data can be combined with LWR model growth estimates to give the average annual waste-generation levels for a model LWR industry. These latter figures are summarized in Table AI-1, to illustrate possible volumes of accumulated wastes from a balanced LWR power system by the year 2000 for the no-recycle and uranium-only-recycle options for the ERDA model assumed in the GESMO. Relative to waste volume typical of industry the volumes shown are quite modest. The discussion Chapter VII will reveal that the nuclear waste volumes themselves are not a major limitation to the practice of the nuclear fuel cycle; the only exception is transuranic low-level waste for which volume reduction is an important option. The most significant issues arise from the viability and long-term predictability of technical alternatives and institutional procedures to cope with these wastes.
TABLE AI-1. Cumulative volumes of waste inventory in the year $2000\left(\mathrm{~m}^{3}\right)$ (NUREG-0116).

\begin{tabular}{lcc}
\hline Type of Waste & \multicolumn{1}{c}{ Fuel Cycle Option } \\
& No Recycle & U Recycle \\
\hline Mil1 Tailings & $7.8 \times 10^{8}$ & $6.9 \times 10^{8}$ \\
Spent Fuel & $55,000^{\mathrm{a}}$ & $6,000^{\mathrm{c}}$ \\
High-Leve1 & $\mathrm{b}$ & $6,500^{\mathrm{d}}$ \\
Transuranic & $\mathrm{b}$ & $76,500^{\mathrm{e}}$ \\
Hulls and Hardware & $\mathrm{b}$ & 52,000 \\
$\quad$ (Transuranic) & & $3.8 \times 10^{6}$ \\
Low-Leve1 Reactor Waste & $3.8 \times 10^{6}$ & \\
$\quad$ (Nontransuranic) & & 300,000 \\
Other Low-Leve1 & 310,000 & 183,000 \\
(Nontransuranic) & & \\
Chemical & 179,000 & \\
\hline
\end{tabular}

a 400,000 spent-fuel assemblies

b Not produced with no-recycle.

c 37,000 spent fuel assemblies in pool storage awaiting processing.

d Volume of HLW in 37,000 canisters.

e Includes plutonium wastes.

The material in this appendix has been drawn from two principal sources:

1. NUREG-0002 Final GESMO: Health, Safety and Environment

2. NUREG-0116 Environmental Survey of the Reprocessing and Waste Management Portions of the LWR Fuel Cycle. 


\section{General Features of HTGR Fuel Reprocessing}

The technology required to reprocess HTGR discharge fuel from high-temperature gas-cooled reactors (HTGR) differs significantly from the Purex technology and from the Thorex technology described in the main text for fuels from light-water reactors. The HTGR fuel actinides are thorium and highly enriched (93\%) uranium, resulting separation in chemistry different from that involved in uraniumplutonium separation. The HTGR separation technology is a combination of the Thorex and Purex processes, but with the following additional features unique to HTGR fuel:

1. The fuel actinides are contained in graphite blocks, which must be crushed and burned or otherwise treated to expose the actinides for dissolution.

2. Considerable quantities of ${ }^{14} \mathrm{C}$ are contained in the graphite blocks, so the total $\mathrm{CO}_{2}$ and $\mathrm{CO}$ evolved in combustion would have to be collected and treated as long-lived wastes.

3. Each fuel block will contain one of three different types of fissile particles, as well as fertile thorium fuel particles. Blocks of each fissile type must be processed separately. The fissile and fertile particles from each graphite block must be elutriated into two different fractions for dissolution and reprocessing. This finally results in four different streams of separated uranium, one of which is to be discarded to control the build-up of ${ }^{236} \mathrm{U}$ and consequent successive neutron absoprtion.

2. Head-End and Separations Technology for HTGR Fuel (Abraham, et al., 1975; Notz, 1976)

The HTGR fuel consists of 200-micron fissile particles of uranium carbide coated with pyrolytic carbon and silicon carbide and 500-micron particles of thorium oxide coated with carbon. The particle coatings reduce fission product release, and the silicon-carbide coating also preserves the integrity of the fissile particles during graphite combustion. These particles are embedded in a graphite matrix contained in prismatic fuel blocks about $79 \mathrm{~cm}$. tall and $40 \mathrm{~cm}$. across the flats of their hexagonal cross section. The fuel blocks are penetrated with holes for helium coolant and for handling, control absorbers, etc. One of the unique problems in HTGR fuel reprocessing is burning the large amount of carbon to remove it from the relatively small amount of fuel material.

The fuel blocks are first crushed for size reduction and then burned with oxygen in a fluidized bed. Relatively large amounts of ${ }^{14} \mathrm{C}$ are present in the graphite, formed by neutron activation of carbon and by $(n, p)$ reactions with residual nitrogen in the graphite. Therefore, the ${ }^{14} \mathrm{C}$ evolved as ${ }^{14} \mathrm{CO}_{2}$ and ${ }^{14} \mathrm{CO}$ during combustion along with the other combustion gases requires that all the $\mathrm{CO}_{2}$ and $\mathrm{CO}$ be recovered and treated as long-lived radioactive waste if ${ }^{1{ }^{4} \mathrm{C}}$ is to be controlled.

The ash from the fluidized combustor consists of the original $\mathrm{UC}_{2}$ particles still coated with silicon carbide and oxide particles of $\mathrm{UO}_{2}$ $\mathrm{ThO}_{2}$ from the incineration of the original $\mathrm{ThO}_{2}$ particles coated with pyrolytic carbon. Although the sizes of these fertile and fissile particles are about the same after graphite combustion, the thoria particles are about three times heavier because of the larger diameter of their actinide kernel and because the $\mathrm{SiC}$ and inner carbon coatings of the fissile particles still remain. The fissile and fertile particles are separated into two fractions by elutriation with carbon dioxide. The thoria particles, now containing fission products and bred uranium, are to be processed by Thorex separation technology, and those fissile uranium particles containing recoverable uranium, fission products, and some neptunium and plutonium are to be processed by Purex separation technology.

The purpose of making the size separation of the fissile and fertile particles from each block is to develop a means of controlling the build-up of neutron-absorbing ${ }^{236} \mathrm{U}$. The fissile particles used to fabricate each graphite fuel block are one of three different types of uranium: (1) highly enriched $(93.5 \%){ }^{235} \mathrm{U}$ make-up uranium, (2) $235,236,238 \mathrm{U}$ remaining after one cycle of irradiation of make-up uranium and recovered for recycle, and (3) ${ }^{233,234,235,236} \mathrm{U}$ recovered from irradiated thorium and recycled. Fuel blocks with different sources of fissile particles msut be processed separately through graphite combustion and particle classification, so that the three different groups of fissile particles can be collected and treated separately. The particles of uranium remaining from the first-cycle irradiation of make-up uranium are to be processed for uranium recycle. The particles of irradiated bred uranium are to be processed and the recovered uranium is to be combined with uranium recovered from thorium and recycled. The uranium particles remaining after the second irradiation cycle of initial make-up uranium contain a relatively high concentration of ${ }^{236} \mathrm{U}$ and are to be discarded to highlevel wastes. The calculated quantities of actinides for each of these streams for a $1000 \mathrm{MW}$ HTGR are given by Pigford, et al. (Pigford and Yang, 1977; Pigford, et al., 1975).

Because elutriation does not produce a quantitative separation, there will be some crossover of fertile and fissile particles, and this crossover will be increased as a result of broken particles. Crossover contaminates the recovered ${ }^{233} \mathrm{U}$ with ${ }^{236} \mathrm{U}$ neutron poison and increases the loss of ${ }^{233} \mathrm{U}$ when fissile particles are retired. It is estimated that about $10 \%$ of the fissile particles may cross over into the fertile stream and as much as $9 \%$ of the fertile particles may cross over into the fissile stream, resulting in a possible penalty to the HTGR fuel cycle (Abraham, et al., 1975).

The thoria particles are dissolved in /"Thorex reagent", consisting of $13-M$ nitric acid, $0.05-M$ hydrofluoric acid, with $0.075-M$ cadmium nitrate for criticality control, held for 24 hours at the leachant boiling temperature. Remaining solids are principally the silicon-carbide coated fissile particles from elutriation crossover and are removed by centrifuging.

The SiC-coated fissile particles are curshed to crack the silicon carbide coating, followed by burning of the inner carbon layers with oxygen in a fluidized bed. The dissolvent is denitrated by boiling and steam stripping to $1-M \mathrm{HNO}_{3}$. Although an acid-deficient feed is optimum for thorium extraction and decontamination, the feed must be kept acidic at greater than $0.5-M \mathrm{HNO}_{3}$ to aovid precipitation of zirconium. Also, acid-deficient conditions favor formation of plutonium polymers. Formic acid is added during denitration to stabilize ruthenium in a reduced valence state and thus minimize the formation of volative $\mathrm{RuO}_{4}$.

Uranium, thorium, and plutonium are coextracted in a solvent extraction column by contacting with concentrated TBP in $n$ dodecane. Because of the relatively weak nitrate-TBP complexing of thorium, a solution of $30 \%$ TBP in $n$-dodecane is used as extractant, and concentrated $(13-M)$ nitric acid solution is added below the feed point of the extraction column to maintain adequate nitrate concentration to promote complexing. The organic extract is scrubbed with $1-M$ nitric acid, introduced at the top of the column to aid in fission-product decontamination.

The high fission-product acitivty in this first extraction column causes substantial radiolytic damage to the organic solvent, producing dibutyl phosphate (DBP) and other undesirable decomposition products which increase the thorium contamination of uranium streams and which increase product losses. Dibutyl phosphate forms a strong thorium complex which precipitates at low acidity and impairs the throughput characteristics of sieve-plate pulse columns. Centrifugal contactors may mitigate this problem by reducing the radiation exposure time of the solvent, but the centrifugal contactor is more sensitive to suspended solids remaining in the dissolvent and to the presence of a second organic phase, rich in thorium and TBP, which forms at high thorium concentrations.

To reduce plutonium contamination of the thorium and uranium 
products, the plutonium is partitioned by scrubbing the organic extract with a dilute nitric acid solution containing ferrous iron to reduce plutonium to inextractable $\mathrm{Pu}^{+3}$ and also containing hydrazine to scavenge nitrate. Concentrated nitric acid added to the aqueous stream near the bottom of the column promotes back-extraction of uranium and thorium into a fresh organic stream entering the bottom of the column.

Although it is possible that satisfactory plutonium partitioning could be obtained in the first extraction column using a reducing acid scrub solution, the degree of decontamination is uncertain because nitrite formed by the more extensive radioloysis in this column results in extractable compounds with trivalent plutonium. It is possible that radiolytic nitrite in this column may be adequately scavenged with hydrazine, thereby making a separate plutonium partitioning column unnecessary.

The organic product containing complexed thorium and uranium is contacted with $0.21-M$ nitric acid to strip the thorium, which forms less stable nitrate-TBP complexes than does uranium. Fluoride a $0.005-M$ is added to overcome the competing effect of dibuty phosphate, which if present from radiolysis and hydrolysis of TBP impairs thorium-uranium separation. Because most of the fissionproduct zirconium follows the thorium, the aqueous thorium product is carried through a second solvent-extraction cycle. Since the plutonium has now been removed, the thorium stream can be denitrated to acid deficiency, which promotes better solvent-extraction separation from zirconium.

The uranium remaining in the organic stream from thoriumuranium partitioning is contacted with dilute $(0.01-M)$ nitric acid to recover the uranium. The aqueous uranium product is then decontaminated with additional solvent extraction separations. If dibutyl phosphate is allowed to build-up in the organic entering the thorium-uranium partitioning column, the thorium carryover in the organic will contaminate the uranium product and may also result in thorium-DBP precipitation in the uranium stripping column. The control of DBP formation in thorium extraction systems requires continued investigation.

When the SiC-coated fission particles are crushed to crack the coating, and the inner carbon layers burned with oxygen in a fluidized bed, the $\mathrm{UO}_{2}$ ash is dissolved in "Purex reagent". This consists of 2.5 $M$ nitric acid with $0.075-M$ cadmium nitrate for criticality control The liquid is then centrifuged to remove remaining $\mathrm{SiC}$ hulls and any undissolved fission products. The dissolvent is adjusted to Purex feed conditions by adding nitric acid to $3.6-M$ and ferrous sulfamate to $0.01-M$, and then contacted with $5 \%$ ТВP in $n$-dodecane. The extract is scrubbed with nitric acid solution containing ferrous sulfamate to maintain plutonium in the trivalent state. The raffinate contains fisison products, neptunium, and plutonium. The uranium is then stripped from the organic with 0.01- $M$ nitric acid. This Purex process for the HTGR fissile particles differs from the Purex technology for low enriched $\mathrm{UO}_{2}$ fuel from light water reactors in that there is no plutonium separation. However, there remains some uncertainty as to the adequacy of plutonium decontamination of the uranium product without a separate plutonium partitioning column.

The irradiation exposure of the HTGR fissile particles is about 95 $\mathrm{Mw}(\mathrm{t})$ day $/ \mathrm{kg}$, as contrasted to about $30 \mathrm{Mw}(\mathrm{t})$ day $/ \mathrm{kg}$ for $\mathrm{LWF}$ fuel This difference in irradiation exposures is a reflection of the greate uranium enrichnment in HTGR fissile particles. The principle consequence of the greater enrichments is the necessarily smaller size of extraction columns and other equipment for criticality control.

\section{Handling of Volatile Radioactive Wastes}

The relative amounts (Pigford and Yang, 1977) of ${ }^{3} \mathrm{H},{ }^{14} \mathrm{C},{ }^{85} \mathrm{Kr}$, and ${ }^{129} \mathrm{I}$ from the HTGR and from a uranium-fueled light water reacto are compared in Table AII-1. The off gas also contains small quantities of ${ }^{220} \mathrm{Rn}$, a decay daughter of the ${ }^{232} \mathrm{U}$ present in uranium recovered from irradiated thorium.

The presently proposed HTGR fuel reprocessing includes processes for recovery of each of these radioactive species, anticipating requirements of fuel reprocessing technology during the late 1980's or early 1990's when HTGR fuel reprocessing might become a commercial reality. The most significant difference between LWR and HTGR reprocessing associated with radioactive gases arises from the large quantities of $\mathrm{CO}_{2}$ released from fuel incineration. The very
TABLE AII-1. Estimated major volatile fission and activation products (180 days after reactor discharge) (Pigford and Yang, 1977).

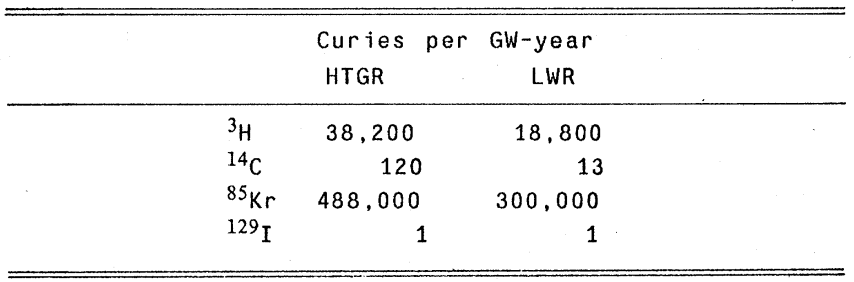

large volume of carbon dioxide, containing only small concentrations of radioactive gases, is a serious complication in fuel reprocessing development. The $\mathrm{CO}_{2}$ also interferes with the processes normally used to concentrate and remove ${ }^{85} \mathrm{Kr}$ from air streams, so a new process is under development for this as part of fuel reprocessing.

Because the incinerator gases contain considerable carbon monoxide, the filtered gas is first passed over a catalyst to oxidize $\mathrm{CO}$ to $\mathrm{CO}_{2}$. At the same time, that portion of the tritium which may be in the form of HT is oxidized to HTO. Elemental radioiodine is removed from the $\mathrm{CO}_{2}$ by adsoprtion on a bed of lead zeolite, followed by a bed of silver zeolite for final elemental iodine cleanup and removal of methyl iodide. Tritiated water is removed on molecular sieves. Because of the low concentration of HTO in the $\mathrm{CO}_{2}$ gas, it may be necessary to inject steam or water vapor upstream of the absorbent bed as a carrier for HTO removal.

The technology for radon removal is under development, but a practical solution may be the use of solid adsorbent to delay the 55sec ${ }^{220} \mathrm{Rn}$ about $10 \mathrm{~min}$, long enough for radioactive decay.

The noble gases are removed by absoprtion in liquid $\mathrm{CO}_{2}$ at high pressure and low temperature. Fractionation of the liquid $\mathrm{CO}_{2}$ adsorbent yields purified krypton gas, which is compressed into a container and stored. About $1 \%$ of the krypton follows the $\mathrm{CO}_{2}$ offgas stream.

The $\mathrm{CO}_{2}$ is finally reacted with a lime solution to precipitate calcium carbonate, which is filtered, dried, and packaged for disposal as a low-level waste. Incineration of HTGR discharge fuel from one gigawatt year of operation results in the production of $410,000 \mathrm{~kg}$. of $\mathrm{CO}_{2}$. This forms $930,000 \mathrm{~kg}$. of $\mathrm{CaCO}_{3}$, which contains ${ }^{14} \mathrm{C}$ at the activity level of 170 nanocuries per gram. The volume of the ${ }^{14} \mathrm{C}$ contaminated $\mathrm{CaCO}_{3}$ per gigawatt year is $550 \mathrm{cu}$. $\mathrm{ft}$.

\section{Summary}

Although the commercialization of Purex technology for $\mathrm{UO}_{2}$ fuel reprocessing will generally benefit all future fuel reprocessing operations, it is clear that considerable further development will be required to commercialize the unique technology contemplated for HTGR fuel reprocessing. The head-end treatment involving the incineration of large quantities of graphite, the several gas-solid and liquid-solid separations, and the transport of finely divided solids are all problems which pose difficulties for reliable maintenance-free remote reprocessing technology for HTGR fuels.

The material in this appendix has been drawn from the following sources:

1. Abraham, L., Carney, H. C., Pence, D., Pierce, V., Strausberg, S., "Flow Sheet Review for Production Reprocessing (120) and Production Refabrication (220) Requirements", General Atomic Report GA-A13751 (Draft) (November 1975).

2. Notz, K. J., "An Overview of HTGR Fuel Recycle", ORNL-TM4747 (January 1976)

3. Pigford, T. H., Cantrell, R. T., Ang, K. P., Mann, B. J., "Fuel Cycles for $1000 \mathrm{Mw}$ High Temperature Gas Cooled Reactor", EPA Contract 68-01-0561, Report EEED-105 (March 1975).

4. Pigford, T. H. and Yang, C. S., "Thorium Fuel Cycles", EPA Contract 68-01-1962, UCB-NE3227 (June 1977) 
The principal actinides involved in using thorium-uranium fuel are shown in the actinide chain of Figure $3 A-11$ of Chapter III. The important reactions are the fission of ${ }^{233} \mathrm{U}$ and ${ }^{235} \mathrm{U}$ and the absorption of neutrons in ${ }^{232} \mathrm{Th}$ to form ${ }^{233} \mathrm{U}$.

The relatively long 27.0 -day half life of ${ }^{233} \mathrm{~Pa}$, the precursor of ${ }^{233} \mathrm{U}$, affects the time that irradiated fuel must be stored prior to reprocessing. If the discharge fuel is stored only for 150 days, as is frequently specified for sufficient decay of ${ }^{131} \mathrm{I}$, some of the ${ }^{233} \mathrm{~Pa}$ will remain during reprocessing. The high radioactivity of protactinium will contribute to the problem of decontatninating the uranium product after it is separated from the fission products and thorium. Also, if protactinium is not recovered the loss of undecayed ${ }^{233} \mathrm{~Pa}$ represents loss in the production of ${ }^{233} \mathrm{U}$ for recycle. For these reasons, present flowsheets for uranium-thorium reprocessing specify a one-year preprocessing holdup of discharge fuel, as compared with 150 days for irradiated $\mathrm{UO}_{2}$ from light-water reactors. This results in greater fuel-cycle inventory of fissile material for the thorium cycle, it delays the time to reach an equilibrium fuel cycle, and it increases the total uranium ore requirements for the thorium cycle.

However, this disadvantage of thorium fueling as compared with uranium fueling will become apparent only when fuel reprocessing and fissile recycle are operative for both concepts. Although commercial technology for $\mathrm{UO}_{2}$ fuel reprocessing is available and could, at the earliest, become operative during 1977, the backlog of discharged $\mathrm{UO}_{2}$ fuel is so great that even with a reasonable expansion of the reprocessing industry a delay of about two years from between $\mathrm{UO}_{2}$ discharge and reprocessing is likely for the duration of this century. Therefore, the relatively long reprocessing storage time required for thorium fuel is a disadvantage only for the long term, and it should not affect the comparative economics of uranium and thorium fueling during this century.

Another problem of the thorium fuel cycle results from the radioactivity of $72-y r 232 \mathrm{U}$, and its daughters. As shown in Section $\mathrm{A} 3 \mathrm{c}$ of Chapter III, ${ }^{232} \mathrm{U}$ is formed by $(\mathrm{n}, 2 \mathrm{n})$ reaction with ${ }^{232} \mathrm{Th}$, and by a $(n, 2 n)$ reaction with ${ }^{233} \mathrm{U}$; it is also formed by the chain initiating with a $(n, \gamma)$ reaction in $235 \mathrm{U}$ or ${ }^{230} \mathrm{Th}$ (a trace element introduced as a by product of uranium mining). Although significant alpha activity results from $232 \mathrm{U}$ in the $233 \mathrm{U}$ to be recovered and recycled, more of a problem results from the ${ }^{232} \mathrm{U}$ daughters. The $232 \mathrm{U}$ decay daughter is $1.91-\mathrm{yr}{ }^{228} \mathrm{Th}$, a radionuclide which is also formed by the radioactive decay of $232 \mathrm{Th}$. As shown in Figure 3A-6 of Chapter III, the decay daughters of $228 \mathrm{Th}$ are all short-lived, so they reach secular equilibrium with ${ }^{228} \mathrm{Th}$ after a delay time of only a few days. The decay of ${ }^{212} \mathrm{Bi}$ and ${ }^{208} \mathrm{Tl}$ are accompanied by very energetic and penetrating gammas, so gamma shielding is required when fabricating fuel from recycled uranium containing ${ }^{232} \mathrm{U}$.

Although chemical reprocessing yields essentially pure uranium, storage after separation and time elapsed in shipping to fabrication allow the build-up of ${ }^{228} \mathrm{Th}$ and its decay daughters. Consequently, the gamma activity in separate uranium containing ${ }^{232} U$ increases continuously. with storage time, until it reaches a maximum at about ten years after separation. The calculated growth in activity and gamma dose rate for uranium metal containing $100 \mathrm{ppm}{ }^{232} \mathrm{U}$ is shown in Figure 11 of Chapter VIII. Once uranium has been separated from thorium by Thorex partitioning, there is considerable incentive to complete the uranium purification and fuel refabrication quickly to avoid the increasing gamma radiation due to the build up of $228 \mathrm{Th}$. Hydrogenous shielding is also necessary because of the high-energy neutrons from alpha decay in recycled uranium. The alphas from the decay of ${ }^{233} \mathrm{U},{ }^{232} \mathrm{U}$, and ${ }^{228} \mathrm{Th}$ interact with light elements such as oxygen and carbon to form neutrons, so the neutron activity also increases with storage time.

The actual ${ }^{232} \mathrm{U}$ content in recycled uranium depends upon the reactor lattice and neutron spectrum, as well as the content of ${ }^{230} \mathrm{Th}$ in the make-up thorium. A typical concentration of $500 \mathrm{ppm}{ }^{232} \mathrm{U}$ in recycled uranium has been predicted for an HTGR fuel cycle and as high as 9,000 ppm for a U-Th LWR fuel cycle (Pigford and Yang, 1977).
The ${ }^{228} \mathrm{Th}$ appearing with the separated thorium results in appreciable radioactivity in the thorium. Consequently, it is not practicable to recycle the recovered thorium until it has been stored for about $10 \mathrm{yr}$.

Uranium-thorium fuel usually contains ${ }^{235} \mathrm{U}$ from fissile make-up and in the recycled uranium. The high burnup and uranium recycle result in considerable production ${ }^{237} \mathrm{~Np}$, according to the reactions shown in Figure $3 \mathrm{~A}-10$ of Chapter III. The ${ }^{237} \mathrm{~Np}$ then forms relatively large activities of $236 \mathrm{Pu}$ and $238 \mathrm{Pu}$. These plutonium activities are important because of the problems of decontaminating uranium from plutonium when reprocessing the uranium. Also, even though fissile plutonium is formed by neutron absorption in the ${ }^{238} \mathrm{U}$ accompanying the highly enriched $235 \mathrm{U}$ make-up, the high activities of $236 \mathrm{Pu}$ and ${ }^{238} \mathrm{Pu}$ will probably discourage any attempt to recover and utilize the fuel value of plutonium in the discharge fuel.

Relatively little ${ }^{239} \mathrm{Pu},{ }^{240} \mathrm{Pu},{ }^{241} \mathrm{Pu}, \mathrm{Am}$, and $\mathrm{Cm}$ are formed in the irradiation of thorium-uranium fuel. Therefore, these radionuclides contribute less to the alpha activity and to the theoretical ingestion "hazard" of high-level and transuranic wastes than in the case of uranium fuels. These are the radionuclides which are the greatest contributors to activity and theoretical toxicity after about 1000 years of waste isolation, when the fission products have decayed.

However, as discussed in Section A3 of Chapter III, after about 100,000 years of isolation the theoretical ingestion toxicity of the wastes is governed by ${ }^{226} \mathrm{Ra}$, formed by

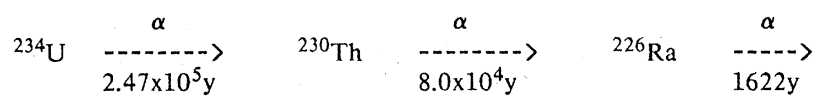

and

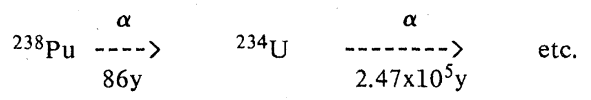

Because ${ }^{234} \mathrm{U}$ is formed in the irradiation of recycled ${ }^{233} \mathrm{U}$, fractional losses of uranium to the radioactive wastes result in considerable long.term production of ${ }^{226} \mathrm{Ra}$. Also, the large ${ }^{238} \mathrm{Pu}$ formation is a further contributor to long-term ${ }^{226} \mathrm{Ra}$. Therefore, these actinide reactions in uranium-thorium fuel result in a relatively large growth in the theoretical hazard of the radioactive wastes after storage periods of a few hundred thousand years.

Following the long-term buildup and decay of ${ }^{226} \mathrm{Ra}$, which peaks at about 200,000 years, the main contributor to the waste ingestion hazard is ${ }^{225} \mathrm{Ra}$, a daughter from the decay of ${ }^{233} \mathrm{U}$ :

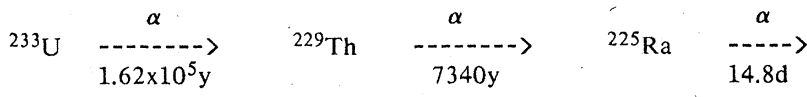

Although much of the ${ }^{225} \mathrm{Ra}$ results from the decay of ${ }^{233} \mathrm{U}$ lost directly to the wastes in reprocessing and fabrication, more results from the formation and decay of ${ }^{233} \mathrm{U}$ formed in the wastes by the decay of $237 \mathrm{~Np}$ :

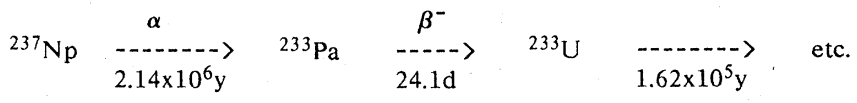

Consequently, the actinide content and theoretical ingestion hazard of the radioactive wastes from uranium-thorium fuel are relatively small for waste disposal times of 1000 to $100,000 \mathrm{yr}$ but are relatively large for waste disposal times of 100,000 to a million years. 
For Pu-Th fueling the irradiation of the make-up plutonium results in considerable production of americium and curium, and those actinides result in much higher theoretical waste ingestion hazard during the period of a few thousand years after fission-product decay. The effect of uranium recycle is to produce similar quantities of ${ }^{233} \mathrm{U}$ ${ }^{234} \mathrm{U}$, and ${ }^{237} \mathrm{~Np}$ in the wastes, as discussed above for $\mathrm{U}-\mathrm{Th}$ fueling.
The material in this appendix has been drawn from the following source:

1. Pigford, T. and Yang, C., Thorium Fuel Cycles, UCB-NE3227, June, 1977. 
We may define a number of integral quantities involving the dose rate $\mathrm{R}(\mathrm{t})$ resulting from a unit release of radioactive isotope.

Let a radionuclide be released at time $t$ and at a rate $I(T) C_{i} y^{-1}$ for a period $d t$. The resultant dose rate at time $t^{\prime}$ is $r\left(t^{\prime}-t\right) I(t) d t$ where $r\left(t^{\prime}-t\right)$ is the dose rate $\left(\right.$ rem $\left.y^{-1}\right)$ at time $t^{\prime}$ due to unit release $(\mathrm{Ci})$ at time $t$. Then if there is continuous release for $0<t<T$, the resulting dose rate at $\mathrm{t}=\mathrm{T}$ is

$$
R(T)=\int_{0}^{T} r\left(t^{\prime}-t\right) I(t) d t
$$

while the total dose over the same period is

$$
D(T)=\int_{0}^{T} \int_{0}^{t^{\prime}} d t^{\prime} r\left(t^{\prime}-t\right) I(t) d t
$$

We consider two special cases:

1. $\mathrm{I}(\mathrm{T})=\mathrm{Q} \delta(\mathrm{t})$, i.e., $\mathrm{Q} \mathrm{Ci}$ released instantaneously at $\mathrm{t}=0$ then

$$
D_{i}(T) \equiv Q \int_{0}^{T} d t^{\prime} r\left(t^{\prime}\right)
$$

$D_{i}(\infty)$ is the quantity conventionally defined as the Dose Commitment in rem resulting from the instantaneous release of $\mathrm{Q} \mathrm{Ci}$ of isotope. $D_{i}(T)$ is the Incomplete or Truncated Dose Commitment for the same conditions.

2. If instead we consider $\mathrm{I}(\mathrm{t})=\mathrm{Q} \mathrm{Ci} \mathrm{y}^{-1}$ for $\mathrm{O}<\mathrm{t}<\mathrm{T}$; then the dose rate at $\mathrm{T}$ is

$$
R_{c}(T)=Q \int_{0}^{T} r\left(t^{\prime}-t\right) d t=Q \int_{0}^{T} r(x) d x=D_{i}(T)
$$

Thus $R_{c}(T)$ (rem $y^{-1 / C i ~} y^{-1}$ ), the dose rate increment due to the continuous release of isotope at a constant rate $Q\left(C i y^{-1}\right)$, is identical to the Truncated Dose Commitment $D_{i}(T)$ (rem/Ci) resulting from the single instantaneous release of $\mathrm{Q} \mathrm{Ci}$. In particular, the equilibrium dose rate increment, $R_{c}(\infty)$ equates the infinite dose commitment, $D_{i}(\infty)$.

For cases of interest to us, isotopes with stable daughters, the Dose Rate Increment is the maximum which will occur. In other cases, the maximim can readily be determined from the explicit decay scheme. For instance, in cases where the isotope released has the longest halflife in the decay chain and long operation is considered, the Dose Rate Increment will closely approximate the maximum.

Figure 5.C-1 illustrates the equivalence. The envelope of all curves is the dose conmitted to time $T$ as the result of release of isotope, while the individual curves correspond to operation for varying periods followed by termination of release.

\section{1. ${ }^{14} \mathrm{C}$}

The nuclide, ${ }^{14} \mathrm{C}$, which decays by beta emission with a half-life of $5,730 \mathrm{y}$ is produced naturally in the upper atmosphere by cosmic ray neutrons in the reaction ${ }^{14} \mathrm{~N}(\mathrm{n}, \mathrm{p}){ }^{14} \mathrm{C}$. It is produced in the $\mathrm{LWR}$ fuel cycle by ${ }^{14} \mathrm{~N}(n, p)$ reaction on nitrogen impurities in the fuel or its cladding; by ${ }^{17} \mathrm{O}(\mathrm{n}, \alpha)$ reactions with oxygen in the fuel or in the coolant, and by ${ }^{13} \mathrm{C}(\mathrm{n}, \gamma)$ in carbon impurities. The latter production would be particularly important for high carbon HTGR fuel. It has been recognized only recently that ${ }^{14} \mathrm{C}$ may be an effluent of concern in the LWR cycle. That produced in the fuel may be released during fuel reprocessing, although its chemical form and movement within the reprocessing plant are not yet well understood. If released as a gas, its form would probably be $\mathrm{CO}$ or $\mathrm{CO}_{2}$. Under such conditions offgas treatment could be included in reprocessing plant design to prevent ${ }^{14} \mathrm{C}$ release. If produced as carbon or carbide it would follow insolubles into $\mathrm{HLW}$. Likewise, the amount of ${ }^{14} \mathrm{C}$ produced outside the fuel and released at operating reactors is not well known. It is conceivable that the amount produced and released at reactors is important in comparison with that potentially released in reprocessing.

Observations of the excess ${ }^{14} \mathrm{C}$ in the troposphere released in the several nuclear explosions of the last few decades show that the excess ${ }^{14} \mathrm{C}$ concentration in the atmosphere decrease relatively rapidly and that the ${ }^{14} \mathrm{C}$ concentration in mammals follows that in the troposphere. We adopt a model in which the rapid decay is provided by an exchange of ${ }^{14} \mathrm{C}$ in a pool comprising the troposphere, land biosphere and humus with $\mathrm{C}$ in a second pool in the deep ocean. The $C$ content of the first pool is taken as $2 \times 10^{18} \mathrm{~g}$, that of the second pool as $44 \times 10^{18} \mathrm{~g}$ and the characteristic half-time for transfer as 30 y.

The nuclide, ${ }^{14} \mathrm{C}$ distributes in the mammal with body carbon and thus delivers a whole body, low-LET radiation dose. The characteristic dose rate is $1.7 \times 10^{8} \mathrm{rem} / \mathrm{yr}$ per $\mathrm{Ci} / \mathrm{g}$ carbon.

1.1 Calculation of Person-rems Associated with Release of ${ }^{14} \mathrm{C}$ to the Atmosphere

Let

$$
\begin{aligned}
& M=\text { mass of carbon in the relevant exchangable pool }(\mathrm{g}) \\
& \mathrm{k}=\text { the dose rate constant in (rem/year) } / \mathrm{pCi} / \mathrm{g}=\left(1.7 \times 10^{-4}\right) \\
& \mathrm{P}=\text { average population size }=\left(4 \times 10^{9}\right. \text { persons) } \\
& \mathrm{R}=\text { dose rate to human tissue (rem/year) } \\
& \mathrm{S}=\text { activity release in Curies } \\
& \mathrm{T}=\text { mean life of } \mathrm{C}-14 \text { in the exchangeable pool (years) } \\
& \mathrm{D}=\text { dose commitment over all time (person-rem) }
\end{aligned}
$$

Then

$$
\begin{aligned}
& R=\frac{10^{12} S k P e^{-t / T}}{M} \\
& D=\int_{0}^{T} R(t) d t=\frac{10^{12} S k P T}{M}
\end{aligned}
$$

if $P$ is assumed constant.

It is assumed that there are two pools, one consisting of the atmosphere, land biosphere, and humus with a carbon content of $2 \times$ $10^{18} \mathrm{~g}$. It is also assumed that the mean residence time of a $\mathrm{C}-14$ atom is 30 years, and that it is removed to the second pool consisting of the oceans with a carbon content of $44 \times 10^{18} \mathrm{~g} \mathrm{C}$, and that there is no physical removal from this pool before decay.

The results of this calculation give population dose commitments from pools 1 and 2 of:

$D_{1}=11$ person-rem $/ \mathrm{Ci}$
$\mathrm{D}_{2}=120$ person-rem/Ci 
or a total of 131 person-rem/Ci, of which about 8 percent is delivered to the extant generation. On a per capita basis the average dose is highest to the first generation and is about 2 nanorad per capita, or the equivalent of roughtly 1 second's worth of average background.

The average global whole body dose rate as a function of time for a continuous release rate of $1 \mathrm{Ci} / \mathrm{y}$ is listed in Table $5 . \mathrm{C}-1$. The gonadal dose rate due to cosmogenically produced ${ }^{14} \mathrm{C}$ is $0.7 \mathrm{mrem} / \mathrm{yr}$. The excess due to nuclear explosions was $0.4 \mathrm{mrem} / \mathrm{yr}$ as of 1968 .

\section{2. ${ }^{85} \mathrm{Kr}$}

The nuclide, ${ }^{85} \mathrm{Kr}$, is a fission product which decays by beta emission with a half-life of 10.6 years. The beta emission is accompanied inf requently ( 0.5 percent) by gamma emission. Since it is a noble gas, it remains uncombined with other materials and resides primarily in voids within the fuel and in the fuel element fission gas plenum. This nuclide is produced in the light fission fragment so that its yield/fission depends strongly on the mass of the fissioning nucleus. Its production by thermal neutron on ${ }^{233} \mathrm{U}:{ }^{235} \mathrm{U}:{ }^{239} \mathrm{Pu}$ is .0058: $.0029: .0014$ per fission.

The release of ${ }^{85} \mathrm{Kr}$ from the fuel cycle can occur primarily during reprocessing. The amount released from operating reactors is negligible in comparison. The $\mathrm{Kr}$ remains in the atmosphere since uptake by the oceans is small with 97 percent in the atmosphere and 3 percent in oceans at equilibrium (NCRP, 1975b).

Upon release from a specific facility, $\mathrm{Kr}$ moves downwind in a plume. The local concentrations of air depend on the current weather conditions and conditions of local terrain. The plume moves generally eastward as a result of prevailing westerly winds and completes its first passage around the earth in about 30 days. Its lateral spread is slow and about two years is required for the north-south mixing to achieve a uniform global atmospheric concentration. The concentration in the first 30 days is quite site-specific, and in the first two years is somewhat latitude-dependent; subsequently the concentration may be considered uniform over the earth's surface. The per capita dose rate at the beginning of year 3 following release is $5 \times 10^{-15} \mathrm{rad}^{-1} \mathrm{Ci}^{-1}$. The whole-body dose is the same magnitude as the dose to most organs except for the skin. Estimates of dose rate per unit concentration have been given as:

\section{$1.5 \times 10^{4}(\mathrm{NCRP}) \mathrm{rem} \mathrm{y}^{1} / \mathrm{Ci} \mathrm{m}^{-3}$ (whole body or gonads). \\ $1.8 \times 10^{6}(\mathrm{NCRP}) \mathrm{rem} \mathrm{y}^{1} / \mathrm{Ci} \mathrm{m}^{-3}$ (skin).}

The whole body dose rate as a function of time for continuous release of ${ }^{85} \mathrm{Kr}$ at a constant rate of $1 \mathrm{Ci}^{-1}$ is given in Table 5.C-1. The gonadal dose rate due to ${ }^{85} \mathrm{Kr}$ produced in earlier nuclear explosions was $2 \times 10^{-4} \mathrm{mrem} / \mathrm{yr}$ as of 1268 .

\section{3. ${ }^{3} H$ (Tritium)}

The nuclide, ${ }^{3} \mathrm{H}$, decays by beta emission with a half-life of 12.3 $\mathrm{y}$. It is produced in nature by spallation of $\mathrm{O}$ and $\mathrm{N}$ by cosmic rays. This nuclide is produced in reactor fuel cycles primarily by ternary fisison. In addition ${ }^{3} \mathrm{H}$ may be produced in the primary coolant at PWR reactors by ${ }^{10} \mathrm{~B}\left(\mathrm{n},{ }^{3} \mathrm{H}\right)$ reactions in boron used as a chemical shim and by ${ }^{2} \mathrm{H}(\mathrm{n}, \alpha)^{3} \mathrm{H}$ in HWR. Tritium is bound in the fuel and released in part during the reprocessing step. The fraction of tritium appearing in the gaseous effluent waste stream depends on plant design and on off-gas treatment.

Tritium released in the atmosphere-soon mixes with atmospheric and surface water. Observations, made on the concentrations of ${ }^{3} \mathrm{H}$ in surface waters following atmospheric release, showed intiial concentrations of approximately $1.6 \times 10^{-21} \mathrm{Ci}{ }^{3} \mathrm{H} / \mathrm{g} \mathrm{H} \mathrm{H}_{2} \mathrm{O}$ per $\mathrm{Ci}{ }^{3} \mathrm{H}$ released. A depletion half-time of about 3 years was observed for the concentration as ${ }^{3} \mathrm{H}$ moved into larger reservoirs. A lower limit of $1 \mathrm{x}$ $10^{-22} \mathrm{Ci}{ }^{3} \mathrm{H} / \mathrm{g} \mathrm{H}_{2} \mathrm{O}$ may be inferred by assuming that the ${ }^{3} \mathrm{H}$ mixes instantaneously and uniformly with the ocean waters in the northern hemisphere. The characteristic dose rate is $1 \times 10^{8} \mathrm{rem} / \mathrm{y}$ per $\mathrm{Ci}^{3} \mathrm{H} / \mathrm{g}$ water.

The dose rates as a function of time for the release of ${ }^{3} \mathrm{H}$ for concentration models based on observation and on hypothetical marine release are given in Table 5.C.1. The gonadal dose rate due to ${ }^{3} \mathrm{H}$ from nuclear explosions was $1 \times 10^{-6} \mathrm{mrem} / \mathrm{yr}$ as of 1968 .

\section{4. ${ }^{129} I$}

The nuclide, ${ }^{129} \mathrm{I}$, is a beta-emitting fission product with a halflife of 16 million years. It is released from the fuel primarily during reprocessing. Again our knowledge of the behavior of atmospherically released ${ }^{129}$ I comes from observations following nuclear explosions.

This nuclide released to the atmosphere moves into two pools. First it will quickly come into equilibrium by mixing with the pool of stable iodine in the circulating waters of the northern hemisphere. This pool contains about $10^{22} \mathrm{~g}$ of water with a stable 1 concentration of $0.064 \mathrm{ppm}$. Subsequently there is further dilution as it mixes with a second larger pool consisting of the deep ocean water with a volume about 60 times that of the circulating water. The mixing half time for this latter process is 20 to 40 years. The initial concentration of ${ }^{129} \mathrm{I}$ in stable I is $1.6 \times 10^{-15} \mathrm{Ci} 129 \mathrm{I} / \mathrm{g} \mathrm{I}$. Within several hundred years the concentration would reach $2.6 \times 10^{-17} \mathrm{Ci}{ }^{129} \mathrm{l} / \mathrm{g} \mathrm{I}$.

These specific activities allow an estimate to be made of the resultant dose. ${ }^{129} \mathrm{I}$ concentrates in the thyroid and delivers dose as a result of beta emission. A specific activity of $1 \mathrm{pCi}{ }^{129} \mathrm{l} / \mathrm{g} \mathrm{I}$ in thyroid tissue delivers a dose rate varying from $1 \times 10^{-7} \mathrm{rad} \mathrm{y}^{-1}$ to an infant to $4.2 \times 10^{-7} \mathrm{rad} y^{-1}$ to an adult. We will use a nominal value of $2 \times 10^{-7} \mathrm{rad}^{-1}$. Thus the release of $1 \mathrm{Ci}$ of ${ }^{129} \mathrm{I}$ results in an initial dose rate of $3 \times 10^{-10} \mathrm{rad}^{-1}$ to the nominal thyroid and decreases to about $5 \times 10^{-12} \mathrm{rad} \mathrm{y}^{-1}$ within 100 to 200 years.

The dose rate to the thyroid as a function of time at a constant rate of $1 \mathrm{Ci}^{-1}$ is given in Table 5.C-1. It is interesting to note that the specific activity of ${ }^{129} \mathrm{I}$ released in fisison is $\sim 10^{-4} \mathrm{Ci} / \mathrm{g}$ I. Thus the maximum thyroid dose is about $30 \mathrm{rem} / \mathrm{y}$ even if no dilution with stable $I$ in the environment were to occur.

\section{5. ${ }^{222} R n$}

\subsection{Physical Properties}

${ }^{222} \mathrm{Rn}$, a noble gas which decays by alpha emission with a half-life of 3.85 days, is a member of the ${ }^{238} \mathrm{U}$ series and is formed directly by the decay of ${ }^{226} \mathrm{Ra}$. Two of its short-lived daughter products are alpha emitters. These daughter products, which are formed in air by radon decay, can be subsequently inhaled.

\subsection{Sources and Environmental Distribution}

All land masses are sources of radon which enters the air by diffusion from the ground. Interestingly, oceanic waters are low in radium concentration and accordingly radon in air over oceans is generally low. Average concentrations of radon in air in the U.S. range between 100 and $800 \mathrm{pCi} / \mathrm{m}^{3}$, whereas radon over oceanic air is generally below $10 \mathrm{pCi} /$ liter (Harley, 1975; Wilkening, 1975). In the U.S., taking the continental area as $7.7 \times 10^{12} \mathrm{~m}^{2}$ and the average radon evolution rate from soil of $4.2 \times 10^{-12} \mathrm{Ci} / \mathrm{m}^{2} / \mathrm{sec}$ gives a continental evolution of $3.2 \mathrm{Ci} / \mathrm{sec}$ or $2.8 \times 10^{5} \mathrm{Ci} /$ day; at equilibrium this is $1.5 \times 10^{6} \mathrm{Ci}$. However, not all the radon evolved will be over the continental U.S. since some will be transported out to sea. Because of atmospheric transport rates and the half-life of ${ }^{222} \mathrm{Rn}$, the major radiological impact of ${ }^{222} \mathrm{Rn}$ will be confined to the continental mass where it was evolved. In short, ${ }^{222} \mathrm{Rn}$ is not a global exposure problem.

It will be useful to compare natural evolution rates with source terms associated with man's disturbance of the natural environment such as storage of uranium mill tailings or ${ }^{222} \mathrm{Rn}$ released in ventilation air from operation of uranium mines (Shearer, 1969).

Although there is not much information about radon indoors relative to outdoors, the little information that exists suggests that indoor radon is generally higher than outdoor radon. In the case where buildings are constructed with materials containing elevated amounts of radium, or for example, overfill material containing radium, such as occurred in Grand Junction, Colorado, indoor radon concentrations may be substantially greater than those outdoors. Concentrations several hundred times background (hundreds of $p$ $\mathrm{Ci} /$ liter) have been observed in a few houses in Grand Junction (Culot, 1973; Spitz, 1974). 
NCRP has speculated that indoor radon concentrations in normal areas should not exceed outdoor concentrations by more than 10 percent if the ventilation rate or turnover rate is four times per hour (NCRP, 1975c; Spitz, 1974). An increase of ten percent corresponds to $18 \mathrm{mrem} /$ year increase in lung dose. However, since increased thermal insulation in housing construction decreases air turnover, it will be interesting to see the degree to which energy conseryation will increase indoor radon daughter exposure. We use the ten percent estimated by NCRP as a measure of the indoor contribution here. Accordingly this translates into an increaase of about $10 \mathrm{mrem} / \mathrm{year}$ over the dose that would be received for residents in a very wellventilated structure relatively free of ${ }^{226} \mathrm{Rn}$ in the materials of construction. We also note that Beninson, et al. (Beninson, 1977a) estimate the indoor contribution to be as high as $400 \mathrm{mrem} /$ year.

\subsection{Dosimetry and Biological Effects}

Most of the radiation dose from the inhalation of ${ }^{222} \mathrm{Rn}$ and its daughters comes from the daughter products. The resulting alpha dose is among the highest of the natural sources of radiation dose to man, estimated to average $100 \mathrm{mrem} / \mathrm{yr}$ to the whole lung. The nuclide, ${ }^{220} \mathrm{Rn}$, is a daughter of ${ }^{232} \mathrm{Th}$, but has a half-life of 55 seconds. The concentration of ${ }^{220} \mathrm{Rn}$ ground level air is generally less than $1 / 10$ that of ${ }^{222} \mathrm{Rn}$, and the lung dose from it is considered small compared to that from ${ }^{222} \mathrm{Rn}$. Estimates of the dose to the bronchial epithelium, which is considered the target cell where lung cancers originate, vary over 2 orders of magnitude ranging between 0.1 and 20 rems per "working level month" (WLM). (BEIR used a value of $0.5 \mathrm{rad} / W L M$ or $5 \mathrm{rem} / \mathrm{WLM}$. Note that "rems" calculated to portions of an organ in this way are not comparable numerically [or even biologically] to rems averaged over a whole organ.) A WLM is defined as the exposure to radon in equilibrium with its daughters at $100 \mathrm{pCi} /$ liter for 170 hours per month. This rather awkward unit results from its use in occupational exposure circumstances in uranium mines (FRC, 1967); and converting a WLM from occupational to environmental exposure requires multiplying by the number of hours in the month divided by 170 , or roughly a factor of 4 .

Estimates of the biological effects of ${ }^{222} \mathrm{Rn}$ daughters are based on epidemiologcal studies of uranium miners who were active underground in the 1940s, 1950s, and early 1960s. They were mostly adult males many of whom were heavy smokers, who were exposed not only to ${ }^{222} \mathrm{Rn}$ and daughters but to mine ore dust, diesel fumes, and a variety of other contaminants found in the air of uranium mines. It is not unreservedly accepted that radon daughters alone are major contributors to the occurrence of lung cancer in these miners. The BEIR report estimates that the absolute risk is approximately $10^{-}$ $6 / \mathrm{yr} / \mathrm{rem}$, or, over a 30 -year period, $3 \times 10^{-5} / \mathrm{rem}$.

In terms of WLM, a linear extrapolation of the uranium miner data gives an estimate of $10^{-4}$ premature deaths from lung cancer per miner per WLM (WASH1224, 1974). It should be noted that most of the evidence in miners comes from exposures exceeding $480 \mathrm{WLM}$. If one assumes that the natural background exposure is 0.0004 working levels, then 70-year exposure in man would give approximately 1.3 WLMs, as a natural background (or $0.02 \mathrm{WLM} / \mathrm{yr}$ ). This figure for average natural background in WLMs is not well established, and it may be in error by a factor of 5 . For instance the miner information, which is based on documented exposures, generally in excess of several hundred WLMs, would require an extrapolation of two to three orders of magnitude in cumulative exposure and a somewhat larger extrapolation in dose rate. In addition, conditions of normal exposure are not equivalent to those in mines and there is neglect of the interaction of other substances in eliciting carcinogenic response, a well-established phenomena and highly suggested in the uranium miner information (Saccomanno, 1969; Nelson, 1969).

There is little doubt that above 400 WLMs an increased incidence of lung cancer in uranium miners has been demonstrated (Lundin $1969,1971)$. In the range between 100 and 400 WLM the data is equivocal. Some authors think a definite response in man has been established and others do not (NIOSH, 1971; Pohl, 1976). The dosimetric information (i.e., evaluation of past exposures) is presently insufficient to decide where the truth lies in this region. It is important to have better estimates of the exposure in this range because this group is the only major human group showing cancer for internal high LET exposure other than the dial painters and patients administered thorium dioxide (Thorotrast) as a radiographic contrast agent. The past dosimetric history of the uranium miners should be established by comprehensively studying the ${ }^{210} \mathrm{~Pb}$ burdens of former miners. $210 \mathrm{~Pb}$ is a long-lived nuclide $\left(\mathrm{T}_{1 / 2}=22\right.$ years $)$ which accumulates in the skeleton and is an index of uranium miners past exposures to radon daughters (Cohen, 1973).

6. ${ }^{226} R a$

\subsection{Physical Properties}

${ }^{226} \mathrm{Ra}$ is an alkaline earth which decays by alpha emission with a half-life of 1600 years. It is a member of the ${ }^{238} \mathrm{U}$ series and is supported immediately by its parent, ${ }^{230} \mathrm{Th}$ with a half-life of 76,000 years and ultimately by the 4.5 billion year half-life ${ }^{238} \mathrm{U}$. It decays into ${ }^{222} \mathrm{Rn}$, a noble gas, whose properties are described in Section 5 of this appendix.

\subsection{Sources of Environmental Distribution}

Radium is found ubiquitously in soils and rocks in concentrations of several $\mathrm{pCi} / \mathrm{gm}$. The average concentration in soil from 200 locations in the U.S. is about $0.6 \mathrm{pCi} / \mathrm{gm}$ and the concentrations in rocks range from slightly below this on the average to several times exceeding this, excluding ores which may have uranium contents up to $80 \%$, generally in equilibrium with the radium.

Radium is found in varying amounts in foods and the daily intake in the U.S. ranges between 1 and $2 \mathrm{pCi} /$ day. It is also found in waters and the concentrations in surface waters range generally from insignificant up to $1 \mathrm{pCi} / \mathrm{l}$. The average concentration in surface water is generally less than $.1 \mathrm{pci} / 1$. Well waters may contain up to tens of $\mathrm{pCi} / 1$ in cerrain areas having highly mineralized formations.

The major route of exposure to man, is from intake in food, and drinking water, and the accumulation in the body is generally taken to be 23 times the daily intake. The major locus of deposition in the body is the skeleton although for chronic exposure half the total body burden may be in the soft tissues. The dose to the skeleton is 16 $\mathrm{mrem} / \mathrm{yr}$ to osteocytes and 7 to surfaces for natural skeletal burdens of about $40 \mathrm{pCi}$. The dosimetry of radium is very well investigated because of the historical experience with occupational exposure to radium by radium dial painters.

\subsection{Biological Effects}

Biological effects of radium are among the hest understood in animals and man of any of the internal emitters. There are a total of 54 osteosarcomas and other tumors originating in bone which have been identified in former radium dial painters and correlated with dose (Evans, 1969). The epidemiological study of former radium dial painters underlies the standards for all bone-seeking radionuclides, including plutonium.

The information on the radium dial painters can be used to establish the limits to risk from radium irradiation in man which along with animal experimentation is used to convert the human epidemiological information with radium to other nuclides which man may be exposed to, particularly artificial emitters which are bone seekers.

If certain assumptions are made about the shape of the dose response the radium dial painter information may be used to make an estimate of the efficiency of radium in inducing osteosarcomas as a function of dose. The dose response appears to be non-linear with a plateau. At the lower doses one may assume that the response is linear and $f$ it the data. This has been done by the BEIR who conclude that for a linear model the response is $5 \times 10^{-6}$ potential excess cancers/rem.

\section{Plutonium}

Plutonium is a man-made element, produced primarily by neutron activation of ${ }^{238} \mathrm{U}$. Plutonium so produced can be used for fuel in the light-water reactor cycle. Accordingly, it is appropriate to review briefly the physical and biological properties of plutonium as well as the present status of the world's contamination with $\mathrm{Pu}$. We describe it in somewhat greater detail than for some other nuclides because of publicly expressed concerns. 


\subsection{Physical Properties}

Most plutonium radionuclides are alpha emitters with the exception of ${ }^{241} \mathrm{Pu}$, which is a beta emitter. The greatest alpha radioactivity for plutonium nuclides present in high burnup LWR fuel is due to ${ }^{238} \mathrm{Pu}$, while most of the mass is due to ${ }^{239} \mathrm{Pu}$ and ${ }^{240} \mathrm{Pu}$. The exact isotopic composition depends primarily on burnup. Increased burnup typically leads to the production of $\mathrm{Pu}$ of higher specific alpha activity $(0.5$ to $1 \mathrm{Ci} / \mathrm{y})$ than that from ${ }^{239} \mathrm{Pu}(0.06 \mathrm{Ci} / \mathrm{y})$ The major internal hazard associated with plutonium is from the alpha emission. Occupational exposure to alphas is controlled by confinement and respiratory protection. Neutron emisssion from $(\alpha, n)$ reactions on light nuclei such as oxygen and fluorine, is the major external hazard associated with occupational exposure.

\subsection{Environmental Distribution and Behavior}

Although plutonium does not occur naturally in any significant amounts, $\mathrm{Pu}$ contamination exists in the environment as a result of nuclear weapons testing (Bennett, 1975). Approximately $440,000 \mathrm{Ci}$ are estimated to have been produced and distributed locally and globally from weapons tests. Space nuclear applications are responsible for $17,000 \mathrm{Ci}$ of ${ }^{238} \mathrm{Pu}$, distributed on a worldwide basis. Table 5.C-2 summarizes the status of the world's contamination. In the U.S. there are about $16,000 \mathrm{Ci}$ present in surface soil and representative concentrations in surface soils on the order of $0.05 \mathrm{p}$ $\mathrm{Ci} / \mathrm{gm}$, which is roughly 1 percent of the natural actinide alpha emitters in soils (see Table 5.C-2).

There have been a large number of review articles regarding plutonium in recent years. WASH-1359 provides a set of references describing plutonium in the environment, its biological behavior and its biological effects (WASH1359, 1974). Other reveiws include Volume 36 of the Handbook of Experimental Pharmacology (Hodge, 1973) and the British Medical Research Council publication on the "Toxicity of Plutonium" (MRC, 1975). The question of nuclear power is not specifically addressed since most plutonium in the environment results from weapons activities. An understanding of the behavior of plutonium, which has been introduced into the environment by weapons testing and space nuclear applications is useful for predicting the behavior of any plutonium that might be released attendant to the nuclear fuel cycle.

In the generic environmental statement for the LMFBR, an analysis was made of the environmental release, transport, return to man, and the biological effects from plutonium in the breeder reactor cycle (WASH1535, 1974). The isotopic composition of breeder reactor plutonium is sufficiently similar so that the conclusions of the study may be valid for LWR type plutonium; namely, the release of plutonium in the breeder cycle at release rates of $1 \mathrm{mCi} / \mathrm{Mwe}-\mathrm{y}$ would not result in significant likelihood of health effects. The report concluded that using a set of assumptions which tended to overpredict the expected accumulation in man and the expected effect, one cancer might be produced through all time $(T=\infty)$ from atmospheric release of $1 \mathrm{Ci}$ of plutonium to the environment. The source of this release was considered to be primarily the fuel reprocessing plant, and a midwest location was assumed for the analysis.

Plutonium is considered one of the most toxic of the radionuclides even though most chemical forms are poorly absorbed from the mammalian GI tract. The general consensus is that, for environmental contamination, inhalation is the major route of important exposure, although, under certain conditions, ingestion may not be negligible as a route to man (Durbin, 1974). It is generally accepted that GI uptake for soluble plutonium is about $3 \times 10^{-5}$ although uptake in excess of this is known to occur for chelated compounds (Bair, 1977).

The biological effects of plutonium have been studied intensively beginning shortly after its discovery. By 1949 the basic metabolic properties of plutonium were well understood (poor absorption, poor excretion, long retention, alpha emitters able to induce osteosarcoma) (Durbin, 1973; ICRP, 1972). Periodically since that time questions of the biological effects of inhaled plutonium in both soluble and insoluble forms in lungs have arisen. In particular there has been debate on the degree to which the likelihood of biological effects depends on the nonuniformity as well as the magnitude of lung dose (Richmond, 1970). It has generally been the conclusion, based on animal experiments, that nonuniform irradiation does not produce a higher frequency of effect than more uniform irradiation (NCRP, 1975d).

Particular mention must be made of the hot particle problem. It is clear historically that the question of hot particles has never been neglected. The Tamplin-Cochran petition (Tamplin, 1974) with arguments based on the Geesaman hypothesis (one cancer per $10^{3}$ particles) seems to be a tenuous argument based on a threshold assumption. Furthermore, the high rate of carcinogenic induction from particulate $\mathrm{Pu}$ in lungs, which would have resulted were this assumption true, has not been observed in experimental animals. For this and other reasons the NCRP (NCRP-46, 1975), the National Academy of Sciences (NAS/NRC, 1974), and the Nuclear Regulatory Commission (NUREG-0002, 1976) have concluded that the petition by the NRDC to reduce inhalation dose limits for plutonium by a factor of 110,000 is without significant merit. We believe that these deliberations have been just. However, continuing review of these standards by NCRP, ICRP, UNSCEAR, and others convened for the purpose may well lead to recommendations for an adjustment of the limit, albeit by a much smaller factor.

It is instructive to review very briefly the plutonium exposure experience. All people on the face of the earth contain some plutonium exposure experience due to plutonium from weapons testing. The per capita body burden in northern latitudes is between 1 and $3 \times 10^{-12} \mathrm{Ci}$ (Campbell, 1973). The population burden for the U.S., $4 \times 10^{-3} \mathrm{Ci}$, exceeds the amount in occupationally exposed persons, estimated at several $100 \mu \mathrm{Ci}$. (Wrenn, 1975). There do not appear to have been any observed effects in man from plutonium inhalation that are unequivocally ascribed to plutonium. Evidence would be most readily obtained in a group of 25 employees from Los Alamos exposed significantly relative to the protection guides (by a factor of several) who have been under medical and health surveillance since 1945. No indication of plutonium-related carcinogenesis has been found in this group (Richmond, 1975; Hemplemann, 1973).

It is interesting to note the degree of reaccumulation of plutonium from nuclear weapons testing from the environment to humans. The per capita reaccumulation is on the order of $10^{-17}, 4$ parts in 100 million of $\mathrm{Pu}$ released has gotten back to man after being in the environment in excess of a decade. Most of this is due to the initial inhalation when plutonium is transported from the stratosphere and troposphere to the surface of the earth. (Bennett, 1975).

The degree to which ingestion is responsible for part of our plutonium burden is small. Bennett has compared measured burden of $\mathrm{Pu}$ in human tissue $(3.2 \mathrm{pCi})$ with that predicted using the ICRP 19 model and the measured concentrations in air $(2.6 \mathrm{pCi})$. Since the maximum plutonium concentration occurred from 1962 to 1965 , this burden requires little or no contribution from ingestion. Limited measurements of plutonium in food support this analysis. The low natural thorium burdens found in man are consistent with the evidence of low accumulation in food. That is, thorium, which is chemically and biologically similar to plutonium and in addition occurs in almost all surface soils at concentrations of $\mathrm{ppm}$, is also not reaccumulated from the environment to a signficiant degree (Wrenn, 1975; Price, 1973).

There is no disagreement that plutonium is a highly toxic element and that per unit mass must be handled with great care. However, hazard is distinct from toxicity, and not only includes toxicity but also the manner in which materials are used, and the likelihood of exposure. Although the toxicity cannot be readily modified, the hazard from plutonium can be limited by engineering design, and administrative and regulatory controls. It seems unlikely that this material will be handled in a way which does not provide extremely effective confinement, and accordingly it does not appear that releases

* There is an abundance of experimental literature in animals that allows one to assess the degree to which plutonium may produce damage in the lung. There is some evidence that the expected effects may have been underestimated in the past, and accordingly it may be that the permissible levels will be reduced by a factor between 2 and 10 in the future (Stannard, 1973). 
to the environment from routine operations poses one of the major important routes of exposure of the general public from the nuclear fuel cycle (LWR). However, the occupational exposures which wlll occur in the process of fabricating mixed oxide fuel with plutonium is an important area deserving of attention. The mixed oxide fabrication plants which have been experimentally operated do not give us cause for great concern. However, we have seen no information or design of plutonium nitrate-to-oxide conversion facilities, and note that careful attention to detail will be required to minimize occupational exposure.

The material in this appendix has been drawn from the following sources:

1. Beninson, 1977a

Beninson, D. J., A. Bouville, B. J. O'Brien, and J. O. Snihs. "Dosimetric Implications of the Natural Sources of Irradiation." In: International Symposium on Areas of High Natural Radioactivity." Brazilian Academy of Sciences, Rio de Janeiro (1977).

2. Bennett, 1975

Bennett, B. G. "Fallout ${ }^{239} \mathrm{Pu}$ Dose to Man." Fallout Program Quarterly Summary Report, HASL-278. 1975.

3. Campbell, 1973

Campbell, E. E., M. G. Milligan, W. D. Moss, H. F. Schulte and J. F. McInray. "Plutonium in Autopsy Tissue." Los Alamos Sceintific Lab. Doc. LA-4875. 1973.

4. Cohen, et al., 1973

Cohen, N., T. Jaakkola, and M. E. Wrenn. Lead-210 Concentration in the Bone, Blood and Excreta of a Former Uranium Miner, Health Physics 24: 601-609 (1973).

5. Culot, 1973

Culot, M.V.J., H. G. Olson, K. J. and Schiager. "Radon Progeny Control in Buildings", Uranium Mill Tailings, Colorado State University, Fort Collins, Colorado (1973).

6. Durbin, 1973

Durbin, P. W. "Metabolism and Biological Effects of the Transplutonium Elements." Uranium, Plutonium, Transplutonic Elements. (H. C. Hodge, J. N. Stannard, and J. B. Hursh, eds.), New York: Springer-Verlag, pp. 739-896. 1973.

7. Durbin, 1974

Durbin, P. W. "Transfer of Plutonium from Soil to Plants: A Review of the Problem." Berkeley, University of California, Document UCID-3689. 1974.

8. Evans, et al., 1969

Evans, R. D., A. T. Keane, R. J. Kolenhow, R. W. Neal and M. M. Shanahan. Radiogenic tumors in the radium and mesothorium case studies at MIT. In: "Delayed Effects of Bone Seeking Radionuclides" (C. W. Mays, W. S. S. Jee, and R. D. Lloyd, eds.), pp. 157-194. Univ. of Utah Press, Salt Lake City. 1969.

9. FRC, 1967

Federal Radiation Council. "Guidance for the Control of Radiation Hazards in Uranium Mining" (Staff Report of FRC). Rep. No. 8, revised. FRC, Washington, D.C. 1967.

\section{Harley, 1975}

Harley, J. H. "Environmental Radon." In: The Noble Gasses, Moghissi, A. A. and R. E. Stanley, Eds. (U.S. Government Printing Office) Washington, D.C. (1975).

11. Hemplemann, 1973

Hemplemann, L. H., W. H. Langham, C. R. Richmond and G. L. Voelz. "A Twenty-seven Year Study of Selected Los Alamos Plutonium Workers." Health Phys. 25:461-479 (1973).
12. Hodge, et al., 1973

Hodge, H. C., J. N. Stannard, and J. B. Hursh. "UraniumPlutonium, Transplutonic Elements," In: "Handbook of Experimental Pharmacology," vol. 36, New York: Springer Verlag (1973).

13. ICRP, 1972

International Commission on Radiation Protection (ICRP). "The Metabolism of Compounds of Plutonium and Other Actinides." ICRP Publication No. 19. Pergamon Press, Oxford. 1972.

14. Lundin, 1969

Lundin, F. E., Jr., J. W. Lloyd, E. M. Smith, V. E. Archer and D. A. Holaday. Mortality of uranium miners in relation to radiation exposure, hard rock mining and cigarette smoking, 1950 through Sept., 1967. Health Phys. 16:571 (1969).

15. Lundin, 1971

Lundin, F. E., Jr., J. K. Wagoner and V. E. Archer. "Radon Daughter Exposure and Respiratory Cancer: Quantitative and Temporal Aspects." NIEHS-NIOSH Joint Monograph No. 1. Public Health Service, U.S. Dept. of Health, Education and Welfare, Washington, D.C. 1971.

16. MRC, 1975

Medical Research Council. "The Toxicity of Plutonium." Medical Research Council Committee on Portection against Ionizing Radiations, Her Majesty's Stationery Office, 1975.

17. NAS/NRC, 1974

NAS/NRC. Health Effects of Alpha Emitting Particles in the Respiratory Tract, Report of the Ad Hoc Committee on Hot Particles of the Advisory Committee on the Biological Effects of Ionizing Radiations, Washington, D. C. (1974).

18. NCRP, $1975 \mathrm{~b}$

National Council on Radiation Protection and Measurement. "Krypton-85 In the Atmosphere - Accumulation, Biological Significance, and Control Technology." NCRP Rep. No. 44, Washington, D.C. 1975

19. NCRP, $1975 \mathrm{c}$

National Council on Radiation Protection and Measurement. "Natural Background Radiation in the United States." NCRP Rep. No. 45, Washington, D.C. 1975.

20. NCRP, 1975d

National Council on Radiation Protection and Measurement. "Alpha Emitting Particles in Lungs." NCRP Rep. No. 46, Washington, D.C. 1975.

21. NIOSH, 1971

National Institute for Occupational Health and Safety (NIOSH). Radon Daughter Exposure and Respiratory Cancer: Quantitative and Temporal Aspects, National Institute of Environmental Health Science, Joint Monograph No. 1 (1971).

22. Nelson, 1969

Nelson, N. Some biological effects of radiation in relation to other environmental agents. AEC Symp. Ser. 16 (1969).

23. NUREG-0002, 1976

U.S. Nuclear Regulatory Commission (NUREG-0002). Final Generic Environmental Statement on the Use of Recycle Plutonium in Mixed Oxide Fuel in Light Water Cooled Reactors; Ch. II. "Background and Experience with Plutonium," Ch. III. "Projected Plutonium Recycle Industry," and Ch. IV. "Environmental Impact Due to the Implementation of Plutonium Recycle." August, 1976 (GESMO).

24. Pohl, 1976

Pohl, R. O. "Health Effects of Radon-222 from Uranium Mining." Search 7:350 (1976). 
25. Price, 1973

Price, K. R. "A Review of Transuranic Elements in Solids, Plants, and Animals." J. Environ. Quality 21:62-66 (1973).

26. Richmond, 1970

Richmond, C. R., J. Langham and R. S. Stone. Biological response to small discrete highly radioactive sources. Health Phys. 18:401 (1970).

27. Saccomonno, 1969

Saccomonno, G. "Uranium Miners Health in Radiation Standards for Uranium Mining," Hearings before the Subcommittee on Research, Development and Radiation of the JCAE Congress at U.S. Part I., pp. 301-315, 1969.

28. Shearer, 1969

Shearer, S. D., Jr., and C. W. Sill. Health Phys. 17:77-88 (1969) and USPHS DER 69-1, "Evaluation of Radon-222 Near Uranium Tailings Piles." (1969).

29. Spitz, 1974

Spitz, H. B. and M. E. Wrenn. "The Diurnal Variation of the Radon-222 Concentration in Residential Structures in Grand Junction, Colorado", In: Health and Safety Laboratory Second Workshop on the Natural Radiation Environment, HASL-287 (1974).

30. Stannard, 1973

Stannard, J. N. Toxicology of Radionuclides. Annu. Rev. Pharmacol. 13:325-357 (1973).
31. Tamplin, 1974

Tamplin, A. P. and T. B. Cochran. "Radiation Standards for Hot Particles." National Resources Defense Council, February 1974.

32. WASH1224, 1974

U.S. Atomic Energy Commission. "Comparative Risks, Costs Benefit Studies of Alternative Sources of Electrical Energy." USAEC Report WASH-1224, Washington, D.C. December 1974.

33. WASH1359, 1974

U.S. Atomic Energy Commission. "Plutonium and Other Transuranium Elements: Sources, Environmental Distribution and Biomedical Effects." USAEC Report WASH-1359, Washington, D.C. December 1974.

34. WASH 1535,1974

U.S. Atomic Energy Commission. "Proposed Final Environmental Statement-Liquid Metal Fast Breeder Reactor Program." USAEC Report WASH-1535, Washington, D.C. December 1974.

35. Wilkening, 1975

Wilkening, M.H., W. E. Clements, and D. Stanley. "Radon-222 Flux Measurements in Widely Separated Regions", In: The Natural Radiation Environment II, (Adams, J.A.S., Lowder, W.M. and Gesell, T., Eds.), USAEC, Oak Ridge, Tenn. (1975).

36. Wrenn and Schulte, 1975

Wrenn, M. E. and H. F. Schulte. "lonizing Radiation", In: Industrial Environmental Health, 2nd Edition, pp. 179-217, Academic Press, Inc., New York (1975). 
Plutonium is the best of all the fissile isotopes in achieving high breeding ratios and low doubling times in fast breeders, and ${ }^{233} \mathrm{U}$ technically, is the next best choice although this isotope is rare in nature. Enriched (20 to $22 \%$ ) ${ }^{235} \mathrm{U}$ from isotope separation theoretically could be used for start-up, but operation must shift soon to a $\mathrm{Pu}$ or a ${ }^{233} \mathrm{U}$ cycle, and the penalties associated with ${ }^{235} \mathrm{U}$ start-up are large. The smaller number of neutrons per fission for ${ }^{235} \mathrm{U}$ result in a larger fissile inventory and a lower breeding ratio than with plutonium fueling. Data calculated for ${ }^{235} \mathrm{U}$ start-up of a $1000 \mathrm{Mwe}$ LMFBR designed for introduction as a first-generation commercial breeder are shown in Table AV-1. The initial core loading must contain about $50 \%$ more fissile material than for plutonium start-up As in the case of plutonium start-up, the schedule for partial core replacement and the external holdup time in post-irradiation cooling, reprocessing, and refabrication require a total of $2-1 / 2$ initial core loadings of externally supplied fissile material before the reactor becomes self sustaining with recycled plutonium and ${ }^{235} \mathrm{U}$ recovered from the discharge fuel. On this basis the total start-up requirement of $11,250 \mathrm{~kg}$ of ${ }^{235} \mathrm{U}$ is calculated (Greebler, 1977).

The above calculation assumes that the residual ${ }^{235} \mathrm{U}$ in the discharge core fuel can be recovered and recycled. However, fastbreeder fuel rods contain a stack of enriched core-pellets near the center of each rod. Below and above the stack of core pellets are stacks of axial-blanket pellets made of natural or depleted uranium oxide. To reprocess LMFBR fuel it is comtemplated that fuel assemblies will be chopped and the oxide pellets dissolved in a manner similar to the head-end reprocessing of water-reactor discharge fuel. When the reactor is initially loaded with enriched uranium, the mixing of core and axial blanket pellets in reprocessing will result in isotopic dilution of the fissile uranium remaining in the discharge core fuel. The uranium enrichment is thereby considerably degraded so additional make-up ${ }^{235} \mathrm{U}$ will be required for the first few recycled core loadings, until the plutonium has grown to near-equilibrium concentration. The total requirement of $235 \mathrm{U}$ for start-up with normal head-end reprocessing, is estimated to be about $18,000 \mathrm{~kg}$, as shown in Table $\mathrm{AV}-1$
This large degradation of uranium enrichment in reprocessing could be avoided if a chopping system could be developed that would segregate core pellets from blanket pellets for separate reprocessing. However, cladding hulls and pellets are so severely damaged in chopping that such separation may be quite difficult. Alternatively, thoria pellets could be used for the axial blankets, so that bred $233 \mathrm{U}$ could be recovered and recycled along with the core uranium. However, introduction of thorium seriously complicates the reprocessing and refabrication technology, and substitution of ${ }^{23,3} \mathrm{U}$ for plutonium further delay's the achievement of the higher breeding ratio and shorter doubling time attainable with plutonium fueling. Therefore, $18,000 \mathrm{~kg}$ may be a more realistic estimate of the ${ }^{2.35} \mathrm{U}$ start-up requirement for first-generation breeders.

Comparison with Table 5 of Chapter VIII shows that the uranium ore required to furnish the ${ }^{235} \mathrm{U}$ for start-up, 3110 to 4980 short tons, is roughly two to three times greater than the ore attributable to plutonium start-up (1530 short tons). A sizeable increase in separative work for start-up also is required. Even with the lower estimate of the ${ }^{235} \mathrm{U}$ start-up (Case $\mathrm{A}$, with ${ }^{235} \mathrm{U}$ recycle), the separative work over the three-year period during which the start-up loadings are being prepared is about seven times greater than the separative work over the same initial period for a light water reactor of the same electrical capacity. Consequently, the rate of ${ }^{235} \mathrm{U}$ startup of first-generation breeders may be limited by commercial-scale enrichment capacity.

${ }^{235} \mathrm{U}$ start-up introduces a period of operation at a low breeding ratio. For the General Electric design of a first-generation commercial fast breeder the breeding ratio is estimated to be only slightly above unity for initial loadings with ${ }^{235} \mathrm{U}$. Over a period of about eight years it rises to the mid-cycle values of 1.3, typical of the equilibrium fuel cycle with recycled plutonium. The loss of excess plutonium production during this period delays the availability of bred plutonium for start-up of subsequent breeders. It is estimated that $235 \mathrm{U}$ start-up results in a deficit in breeding-gain plutonium production of about $1700 \mathrm{~kg}$.

TABLE AV-1. Fissile, ore, and enrichment requirements to start a first-generation fast breeder reactor on enriched uranium ( $1000 \mathrm{Mw}$ electrical power, $80 \%$ capacity factor).

A. Uranium in discharge core fuel is reprocessed separately from uranium in axial and radial blankets

$\begin{array}{ll}\text { Fissile }{ }^{235} \mathrm{U} \text { required for fast breeder start-upa } & 11,250 \mathrm{~kg} \\ \text { Uranium ore required for } 20 \% 235 \mathrm{U}: & \\ \quad \begin{array}{l}0.20 \% \text { depleted uranium } \\ 0.25 \% \text { depleted uranium }\end{array} & 3,800 \text { short tons } \mathrm{U}_{3} \mathrm{O}_{8} \\ & \\ \text { Separative work required for } 20 \%, 235 \mathrm{U}: & 2,560 \mathrm{Mg} \\ \quad \begin{array}{l}0.20 \% \text { depleted uranium } \\ 0.25 \% \text { depleted uranium }\end{array} & 2,330 \mathrm{Mg}\end{array}$

B. Fuel elements containing core and axial blanket are chopped and processed without core-blanket separation, so enriched uranium is not recycled.

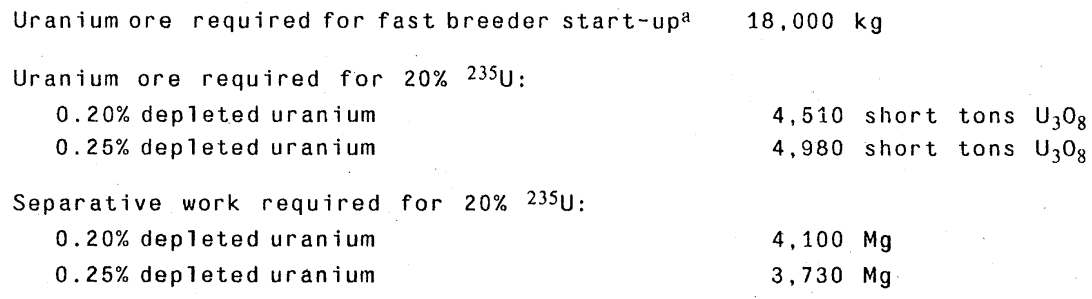

a Based upon $4500 \mathrm{~kg} 235 \mathrm{U}$ for the initial core plus sufficient replacement loadings until reactor is self sustaining on reycle fissile material. Although lower $235 \mathrm{U}$ loadings are possible for a breeder core optimized for $235 \mathrm{U}$ fueling, the purpose here is to start-up a core optimized for steady-state fucling on bred plutonium. (Geebler,
1977 ). 
The costs of plutonium start-up are compared with the costs of ${ }^{235} \mathrm{U}$ start-up in Table AV-2. The ore cost of $\$ 2 \delta / 1 \mathrm{~b} \mathrm{U}_{3} \mathrm{O}_{8}$ and the isotope-separation cost of $\$ 75 / \mathrm{kg}$ of separative work should be applicable as unescalated costs for the 1990's when the first-generation breeders are assumed to be introduced. On this basis, the cost of ${ }^{235} \mathrm{U}$ in uranium of $20 \%$ enrichment is calculated to be $\$ 31 / \mathrm{g}$, almost equally divided between ore costs and isotope separation costs. At the time of breeder introduction the alternative demand for plutonium will be for recycle in the existing base of water reactors, so it is assumed that plutonium is valued as a water-reactor recycle. The LWR material quantities and cost data of Section IV-G lead to a plutonium value (Pigford, 1976) of $\$ 19.90$ per fissile gram, which is $36 \%$ cheaper than the unit cost of $235 \mathrm{U}$. These two fissile costs will be used to calculate the contribution of start-up fissile purchase to the lifetime levelized cost of electrical energy from the breeder.

The superior properties of plutonium for breeder start-up should eventually result in a higher value and higher price of plutonium when breeder start-up places a significant demand upon the plutonium supply. This will eventually result in a larger credit to the fuel cycle costs of light water reactors when plutonium is produced therefrom for breeder start-up. Because electrical energy generation from water reactors will be so credited, the total present value of such credits may be even greater than if the economy of plutonium start-up is credited only to the breeder, as is done in Table AV-2.

As shown in Table AV-2 the total cost of fissile purchase is 2.3 to
3.7 times greater for $235 \mathrm{U}$ start-up than for plutonium start-up. However, a more meaningful comparison is the present-valued contribution to the levelized lifetime fuel cycle cost of breederproduced electrical energy, taking into account the actual times of fissile purchase relative to the time of energy generation and the rapid depreciation of the purchased fissile inventory. The contribution of fissile purchase to the lifetime levelized fuel cycle cost is 2.4 to 3.2 times greater for $235 \mathrm{U}$ start-up than for plutonium start-up. The deficit in breeding-gain plutonium production from the breeder due to $235 \mathrm{U}$ start-up is only 4 to $6 \%$ of the contribution from purchase of starting ${ }^{235} \mathrm{U}$. Thus, the contribution to the cost of electrical energy from $235 \mathrm{U}$ start-up is about 3.4 to $5.1 \mathrm{mills} / \mathrm{kwhr}$ greater than for plutonium start-up. Assuming that the capital costs of firstgeneration breeders will be greater than for water reactors, the net fuel cycle cost of a commercial first-generation breeder must be less than that of the water reactor with uranium-plutonium recycle. The LWR fuel cycle cost was estimated in Section IV-G3b to be about 5 mills/kwhr. A typical estimate (Stauffer, et al., 1975) of the uninflated levelized total fuel cycle cost for a first-generation commercial breeder started up on plutonium, using similar cost parameters, is about 2.0 mills/kwhr. Thus, the additional start-up cost of 3.4 to 5.1 mill kwhr (Table AV-2) if started up with $235 \mathrm{U}$ would constitute a burden to the breeder fuel cycle economy and a large financial burden to the consumers of breeder-produced electrical energy. These increments in start-up costs would increase the levelized total fuel cycle costs by factors of 2.7 and 3.6 , respectively. Of course, all such fuel cycle costs should be seen in perspective with

TABLE AV-2. Economic penalty to start $1000 \mathrm{Mw}$ fast breeder with enriched ${ }^{235} \mathrm{U}$.

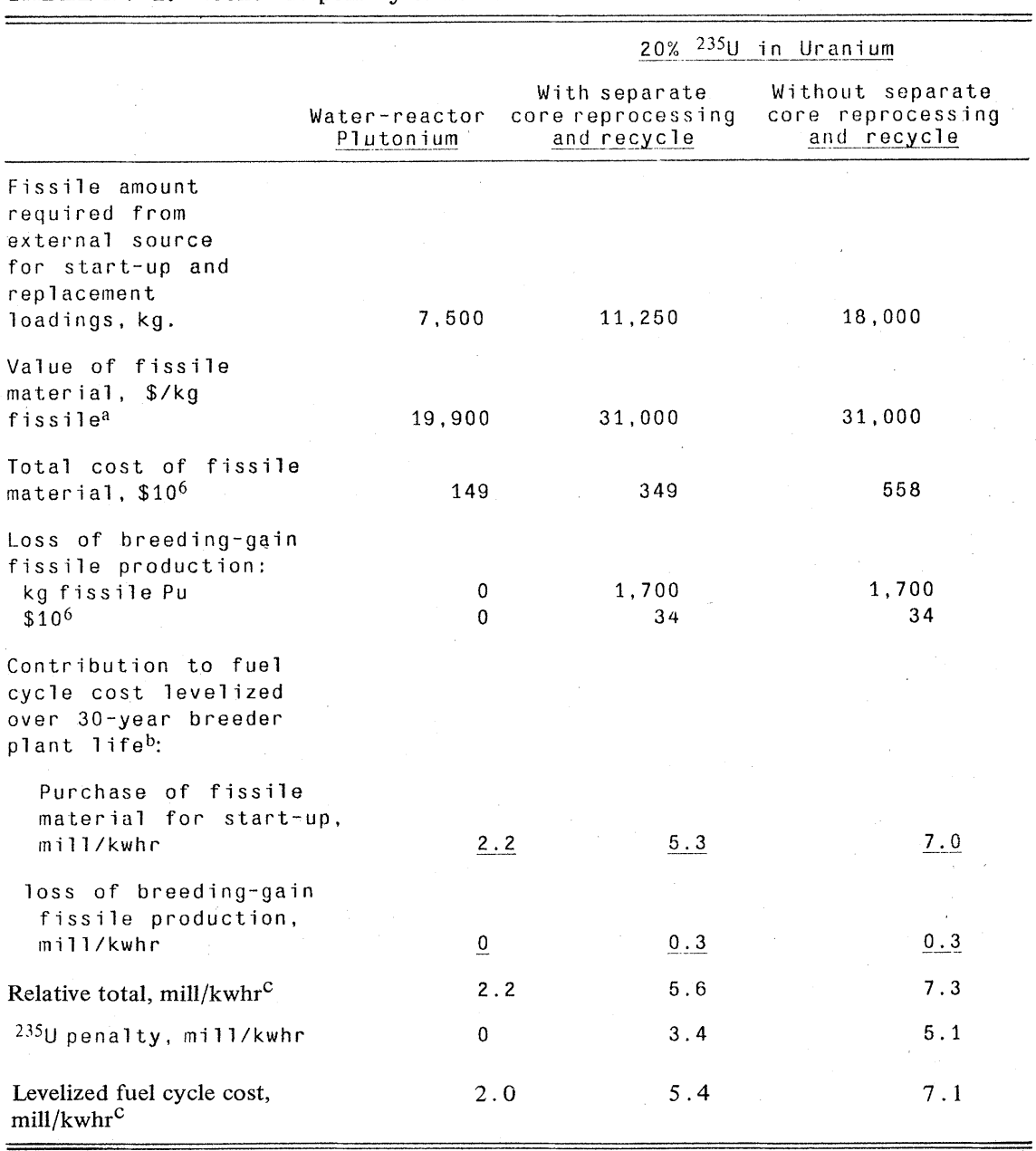

a Plutonium value is calculated for alternative use as a water-reactor fuel.

b Calculated from time schedule of fissile purchases and sale, using utility discount factor of $0.0755 / y$.

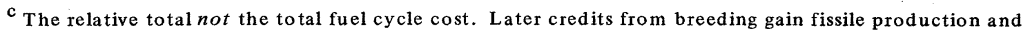
cost of fabrication and reprocessing result in an estimated LMFBR levelized total fuel cycle cost of about $2.0 \mathrm{mill} / \mathrm{kwhr}$ (Stauffer et al, 1975). 
the much larger capital construction costs (see Table 25 of Chapter IV).

In summary the total uranium ore required to fuel water reactors while generating enough plutonium for breeder start-up is about 8490 tons, but only about 1530 tons of this is attributable to breeder startup. If the breeder is started with enriched uranium instead of plutonium, the ore required to produce the start-up uranium is about 3110 tons, if $235 \mathrm{U}$ can be recycled, and 4980 tons if $235 \mathrm{U}$ cannot be recycled. These ore requirements are calculated for $0.25 \%$ tails from isotope separation. They can all be reduced by about $10 \%$ if the tails are of $0.2 \%$ enrichment. The cost of fissile material for breeder startup is estimated to be about 2.3 to 3.7 times greater with enriched uranium than with plutonium fuel. This is due to the greater cost of ${ }^{235} \mathrm{U}$, and the low breeding gain with $235 \mathrm{U}$ start-up.
The material in this appendix has been drawn from the following sources:

1. Greebler, P., (General Electric Co.), Private Communication, March, 1977

2. Pigford, T. H., "The Analysis of the Cost of Electrical Energy from Nuclear Power Plants". UCB-NE 3008 (Rev. 2), Sept. 1976

3. Pigford, T. H., "Start-up of First-Generation Fast Breeders With Plutonium in Enriched Uranium", UCB-NE 3240, April 1977.

4. Stauffer, T. R., Palmer, R. S., Wycoff, H. L., "Breeder Reactor Economics", Breeder Reactor Corporation, July 1975. 


\section{Appendix VI The Possibility of Nuclear Proliferation with ${ }^{231} \mathrm{~Pa}$}

A nation with the capability of producing large quantities of uranium ore can potentially produce $231 \mathrm{~Pa}$ as a by-product of uranium milling. It appears possible that ${ }^{231} \mathrm{~Pa}$ is fissionable with fast neutrons and could be made critical in a non-moderated assembly (Clayton, 1973). ${ }^{231} \mathrm{~Pa}$ occurs naturally as a decay product of ${ }^{235} \mathrm{U}$, according to the reactions:

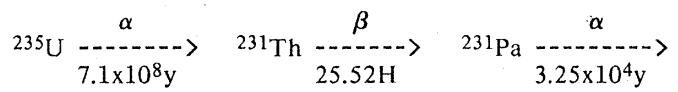

The concentration of ${ }^{231} \mathrm{~Pa}$ in natural uranium is determined by the ratio of half lives and by the $235 \mathrm{U}$ abundance:

$$
\frac{231 \mathrm{~Pa}}{\mathrm{U}}=\frac{3.25 \times 10^{4}}{7.1 \times 10^{8}} \times 0.00715=3.27 \times 10^{-7}
$$

A typical uranium mill producing 1,000 short tons of $\mathrm{U}_{3} \mathrm{O}_{8}$ per year at $90 \%$ uranium recovery could produce as much as $0.252 \mathrm{~kg} / \mathrm{yr}$ of ${ }^{232} \mathrm{~Pa}$ as by product at $100 \%$ recovery. For protactinium recovery factors of $63 \%$ for a recovery process described below, operation of 100 such mills with protactinium recovery for 6.3 years could yield a $100 \mathrm{~kg}$. mass of ${ }^{231} \mathrm{~Pa}$. The protactinium thus produced is slightly less radioactive than ${ }^{239} \mathrm{Pu}$ and much less radioactive than reactor grade plutonium. Spontaneous fission properties are not known.

The protactinium recovery process described by Collins, et al. was actually developed for recovering uranium and protactinium from residual sludges remaining from earlier uranium refining operations at the U.K.A.E.A. Springfield Works. The process steps involved are:
1. Leaching protactinium and uranium from the sludge with cold $4 \mathrm{~N}_{\mathrm{HNO}_{3}}$ in the presence of fluoride

2. Recovering uranium by extraction into 20 percent TBP in kerosene

3. Adding aluminum chloride to the aqueous raffinite and separating a precipitate that carries protactinium

4. Treating the precipitate with cold $\mathrm{NaOH}$ solution and then dissolving it in concentrate hydrochloric acid

5. Recovering protactinium by extraction into di-isobuytl ketone.

In laboratory operations 98 to $99 \%$ of the uranium and 65 to $70 \%$ of the protactinium originally present in the sludge were recovered. The process was successfully applied to the recovery of over $10 \mathrm{~g}$ of protactinium and of nearly 12 tons of uranium with an efficiency of 63 percent for protactinium and $99.4 \%$ for uranium.

The material in this appendix has been drawn from the following sources:

1. Clayton, E. D., "The Nature of Fission and the Criticality Process", BNWL, May 1973.

2. Collins, D. A., Hillary, J. J., Nairn, J. S., and Phillips, G. M., "The Development and Application of a Process for the Recovery of Over $100 \mathrm{~g}$ of Protactinium-231 from a Uranium Refinery Waste Material".

3. Ruby, L., Private Communication, January 1977. 
List of Abbreviations

\begin{tabular}{|c|c|c|}
\hline AEC & $\begin{array}{l}\text { Atomic Energy Commission; a former } \\
\text { federal agency, disbanded by the Energy } \\
\text { Reorganization Act of } 1974\end{array}$ & ICRP \\
\hline APS & American Physical Society & \\
\hline AGNS & Allied-General Nuclear Services & LMFBR \\
\hline BNFP & Barnwell Nuclear Fuel Plant & LWR \\
\hline BNWL & Battelle Northwest Laboratories & MFRP \\
\hline BWR & $\begin{array}{l}\text { Boiling-Water Reactor, a version of an } \\
\text { LWR }\end{array}$ & MOX \\
\hline CANDU & Canadian deuterium-uranium reactor & MT \\
\hline CEQ & Council on Environmental Quality & MTHM \\
\hline Decommissioning & $\begin{array}{l}\text { Taking out of service or ending active } \\
\text { operations }\end{array}$ & $\begin{array}{l}\mathrm{MW}(\mathrm{e}) \\
\mathrm{MW}(\mathrm{t})\end{array}$ \\
\hline EPA & $\begin{array}{l}\text { Environméntal Protection Agency; a } \\
\text { federal agency }\end{array}$ & NAS \\
\hline ERDA & $\begin{array}{l}\text { Energy Research and Development } \\
\text { Administration; a federal agency, successor } \\
\text { to all nonregulatory functions of the } \\
\text { Atomic Energy Commission. }\end{array}$ & NEPA \\
\hline FY & Fiscal Year & NRC \\
\hline GEIS & Generic environmental impact statement. & NWTS \\
\hline GESMO & $\begin{array}{l}\text { Generic environmental impact statement } \\
\text { for mixed oxide fuels (recycle plutonium } \\
\text { in light-water cooled reactors). }\end{array}$ & $\begin{array}{l}\text { OWI } \\
\text { PWR }\end{array}$ \\
\hline HEPA & $\begin{array}{l}\text { High efficiency particulate air filters. } \\
\text { Pleated fiberglass filters with high surface } \\
\text { area and small pore size designed to } \\
\text { remove aerosols with a minimum } \\
\text { efficiency of } 99.97 \% \text { for } 0.3 \text { micrometer } \\
\text { particles. }\end{array}$ & $\begin{array}{l}\text { RCG } \\
\text { RRY }\end{array}$ \\
\hline HLW & High-level wastes & \\
\hline HTGR & High Temperature Gas Reactor & SWU \\
\hline IAEA & International Atomic Energy Agency & TRU \\
\hline
\end{tabular}

International Commission on Radiological Protection

Low-level wastes (containing minimal transuranic elements).

Liquid Metal Fast Breeder Reactor

Light-Water Reactor

Midwest Fuel Recovery Plant.

Mixed oxide reactor fuel (containing both plutonium and uranium)

Metric tons

Metric tons of heavy metal

Megawatts, electrical

Megawatts, thermal

National Academy of Sciences

National Environmental Policy Act of $\overline{1969}$

Nuclear Fuel Services

Nuclear Regulatory Commission; a federal agency, successor to regulatory functions of the Atomic Energy Commission

National Waste Terminal Storage Program

Office of Waste Isolation

Pressurized water reactor, a version of an LWR

Radioactivity Concentration Guide

Reference reactor year. A $1000-\mathrm{MWe}$ reactor, assumed to be operating at $80 \%$ of its maximum capacity for one year. This is equivalent to AFR as used in the original WASH-1248, dated April 1974

Special Nuclear Material

Separative Work Unit

Transuranic 